\title{
Performance evaluations of latex-modified and silica fume modified concrete overlays for bridge decks
}

\author{
Sathish Kumar Raju Konduru \\ West Virginia University
}

Follow this and additional works at: https://researchrepository.wvu.edu/etd

\section{Recommended Citation}

Konduru, Sathish Kumar Raju, "Performance evaluations of latex-modified and silica fume modified concrete overlays for bridge decks" (2009). Graduate Theses, Dissertations, and Problem Reports. 4486. https://researchrepository.wvu.edu/etd/4486

This Thesis is protected by copyright and/or related rights. It has been brought to you by the The Research Repository @ WVU with permission from the rights-holder(s). You are free to use this Thesis in any way that is permitted by the copyright and related rights legislation that applies to your use. For other uses you must obtain permission from the rights-holder(s) directly, unless additional rights are indicated by a Creative Commons license in the record and/ or on the work itself. This Thesis has been accepted for inclusion in WVU Graduate Theses, Dissertations, and Problem Reports collection by an authorized administrator of The Research Repository @ WVU. For more information, please contact researchrepository@mail.wvu.edu. 


\title{
Performance Evaluations of Latex-Modified and Silica Fume Modified Concrete Overlays for Bridge Decks
}

\author{
by \\ Sathish Kumar Raju Konduru \\ Thesis submitted to the \\ College of Engineering and Mineral Resources \\ at \\ West Virginia University \\ in partial fulfillment of the requirements \\ for the degree of \\ Master of Science \\ in \\ Civil and Environmental Engineering \\ Dr. Julio F. Davalos, Ph.D., Chair \\ Dr. Indrajit Ray, Ph.D., Co-Chair \\ Dr. An Chen, Ph.D. \\ Department of Civil and Environmental Engineering
}

Morgantown, West Virginia

2009

Keywords: LMC Overlay, SFMC Overlay, Bonding Slurry, Differential Length Change, Temperature, Pull-Off Bond Strength, ANOVA, Ultrasonic Pulse Velocity, Signal Processing. 


\section{ABSTRACT}

\section{Sathish Kumar Raju Konduru Dr. Julio F. Davalos, Chair and Dr. Indrajit Ray, Co-Chair}

Most of the concrete bridge decks in the cold regions undergo severe reinforcement corrosion due to the transport of chloride ions within the concrete by application of deicing salts on bridge decks in the winter. As a result, protective concrete overlays of about 2-inch thickness are applied on bridge decks. Concrete overlays provide: 1) protection against heavy traffic and the further infiltration of the chloride ions; 2) skid resistance surface; and 3) uniform appearance to extend the service life of bridge decks. Despite these advantages, concrete overlays undergo premature delaminations, edge curling, and corner lifting due to expansion/shrinkage of concrete, temperature changes, and repetitive truck loading.

This research work is a part of Phase-II component of a large-scale project sponsored by WVDOH which is focused on the performance evaluation of Latex Modified Concrete (LMC) and Silica Fume Modified Concrete (SFMC) overlays on Type K substrate concrete deck prototypes. This work evaluated four prototype slabs in two stages with each slab of plan size $1829 \mathrm{~mm}$. (6 ft.) by $2438 \mathrm{~mm}$. (8 ft.). In Stage-I, two bi-layer deck slabs were constructed with the same LMC overlay but with two different bonding conditions. Considering the better bonding condition from Stage-I, Stage-II consisted of two bilayer deck slabs that were constructed with LMC overlay on one slab and SFMC overlay on the other. Pull-Off testing was conducted at different ages for assessing the bond performance of the overlays. The differential length change and differential temperature developed at the interface were continuously monitored using concrete embedment gages and thermocouple loggers, respectively. Debonding due to corner lifting was monitored by installing displacement transducers (LVDT) connected to a data acquisition system. Ultrasonic Pulse Velocity (UPV) testing was conducted to compare the delamination profile at the interface between different types of slabs. Simultaneously with the UPV tester, an oscilloscope was connected to record the time-domain waveform, which was converted into a power spectrum for analysis.

The Stage-I study showed that at 5\% level of significance, both the interface bond strength of the LMC slab with and without bonding slurry were similar. However, the other critical delamination parameters such as differential length changes at the interface and vertical displacement due to corner lifting were much less when bonding slurry was incorporated. Also, the time-domain waveform of LMC with bonding slurry showed less attenuation of wave through the interface compared to no-slurry LMC. Further, the frequency spectrum analysis displayed that LMC with bonding slurry had higher peak magnitude compared to LMC without bonding slurry. Based on the results, the use of bonding slurry was preferred for the Stage-II study. This study showed that in-situ pull-off/bond strength results and nature of failure varied widely due to presence of local voids, compaction, and consolidations.

In Stage-II study where the bonding slurries were used for each case, the results showed that the bond strength values of slab with LMC overlay were higher than those of the slab with SFMC overlay, at $5 \%$ level of significance as analyzed by three-way ANOVA. The vertical displacement due to corner lifting was found to be lower for the slab with LMC overlay. The time-domain signal indicated that the amplitudes of LMC overlay were higher compared to those of SFMC overlay. Further, the power spectrum analysis showed that LMC had higher magnitude of peaks both at center and edge compared to SFMC overlays indicating the strength of the signals were stronger for LMC compared to SFMC.

The overall conclusion of this study is that the bonding slurry has positive effect to reduce delamination and corner lifting; and when slurry is used, the LMC has advantages over SFMC, although both overlay types are viable options. A full-scale study is required in order to conclude the findings and finally develop a performance based specifications for the overlays for the state of West Virginia. 


\section{ACKNOWLEDGEMENTS}

I would like to gratefully acknowledge the enthusiastic supervision of Dr. Julio F. Davalos, a great teacher and advisor who was abundantly helpful and offered invaluable assistance, support and guidance to accomplish this research project. His technical insight was priceless.

My sincere gratitude goes to Dr. Indrajit Ray, my co-advisor, for his enormous support, insightful guidance in the whole research project. His wise advice and suggestion has significantly helped the finishing of my thesis work. Also, I would like to sincerely thank Dr. An Chen for serving as my committee member.

I would like to thank my colleagues Arka Bhattacharya, George Parish, Santiago Velez, Jeremy Meadway, William Sasher, Stephen Wallington, and Ahmad Mohammad for their valuable guidance and support during the entire work. Also, my thanks go to David Turner, Bill Comstock, and Jared Grim for their valuable help during the experimental work. This work would not have been possible without their help and support.

I gratefully acknowledge the financial support provided by West Virginia Department of Transportation-Division of Highways and WVU Research Corp. Incentive Grant Program. I also acknowledge Arrow Concrete Company, Ahern and Associates Inc, and BASF for their generous help and supply of the concrete and materials.

I would like to dedicate this thesis to my family for the love, support and encouragement provided by them. 


\section{TABLE OF CONTENTS}

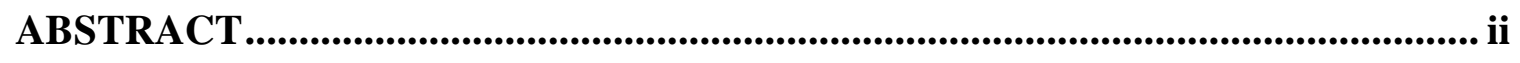

ACKNOWLEDGEMENTS ..............................................................................iii

TABLE OF CONTENTS ......................................................................................... iv

LIST OF TABLES ............................................................................................ viii

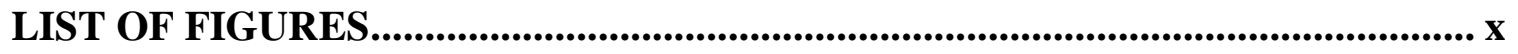

CHAPTER 1： INTRODUCTION ...........................................................................1

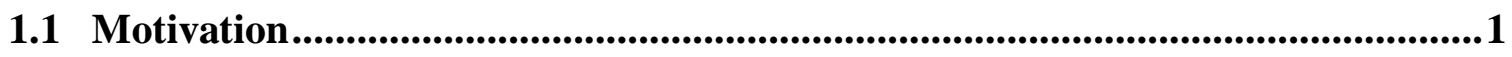

1.2 Objectives......................................................................................................................2

1.3 Scope of the Work.................................................................................................3

1.4 Thesis Organization ............................................................................................4

CHAPTER 2: $\quad$ LITERATURE REVIEW .....................................................5

2.1 Introduction and Background of Concrete Overlays .........................................5

2.2 Types of Concrete Overlays .....................................................................................5

2.3 Performance of Specialized Concrete Overlays.................................................7

2.4 Surface Preparation of Bridge Decks............................................................. 14

2.5 Delamination, Curling, and Cracking of Overlays..........................................17

2.6 Bond Tests and Pull-Off Tests..................................................................... 25

2.7 Ultrasonic Pulse and Signal Processing .......................................................... 34

2.8 Analysis of Variance (ANOVA) .....................................................................44

2.9 Summary of Review ............................................................................................. 52

2.10 Significance of Present Study .............................................................5 52

CHAPTER 3: $\quad$ EXPERIMENTAL PROGRAM ........................................................5 53 


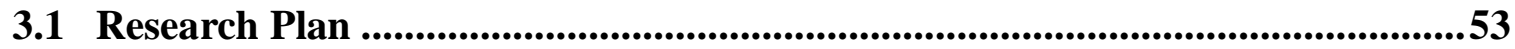

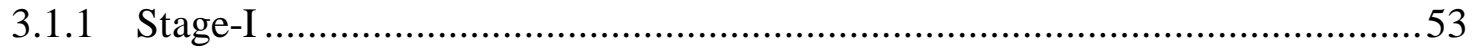

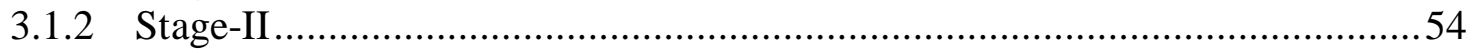

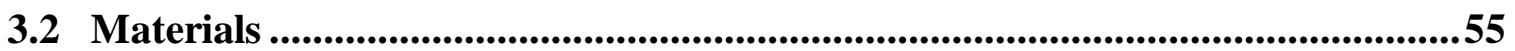

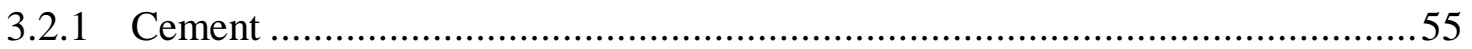

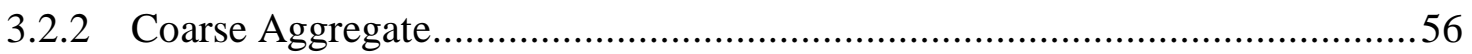

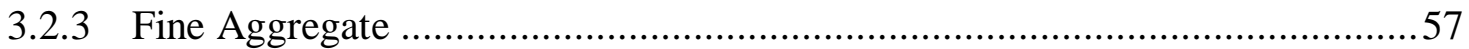

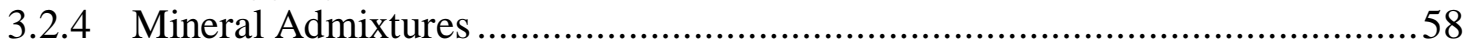

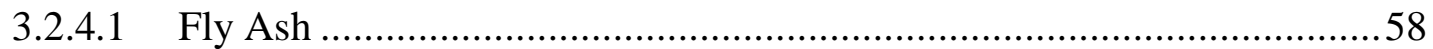

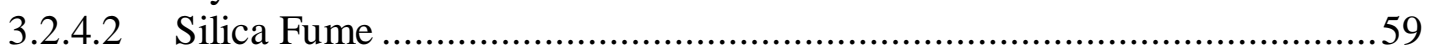

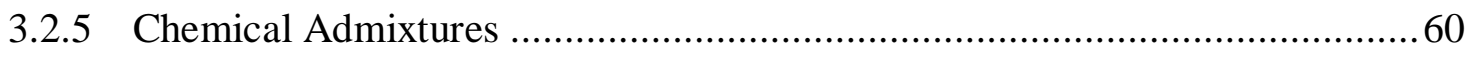

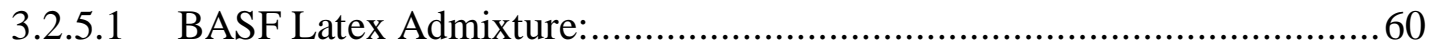

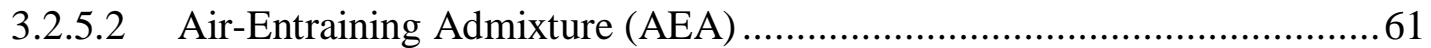

3.2.5.3 High-Range Water Reducing Admixture (HRWRA) .................................61

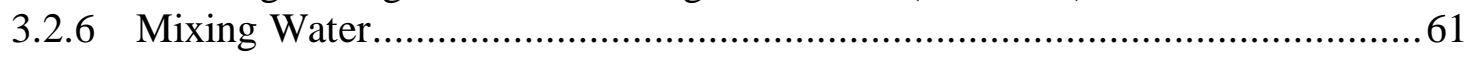

3.3 Mixture Proportions ..................................................................................................61

3.4 Construction Procedure ….....................................................................................66

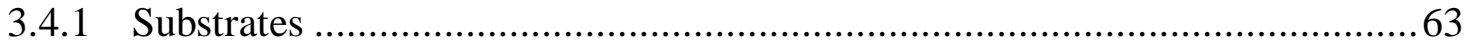

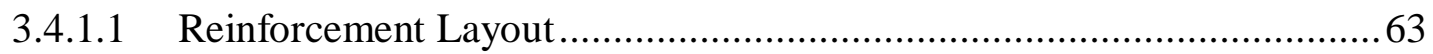

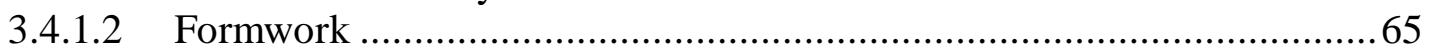

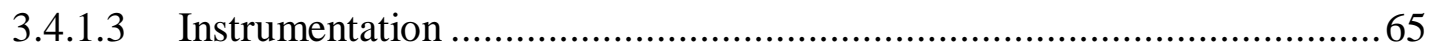

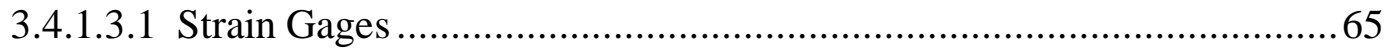

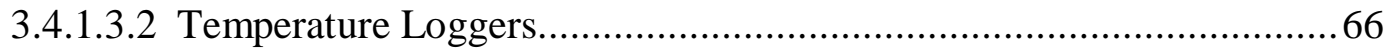

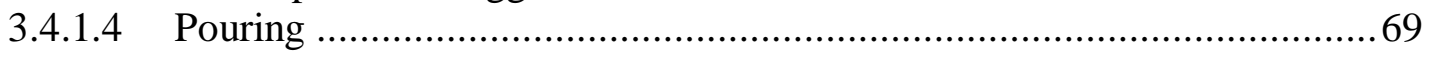

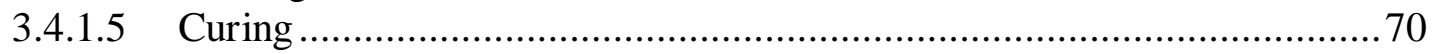

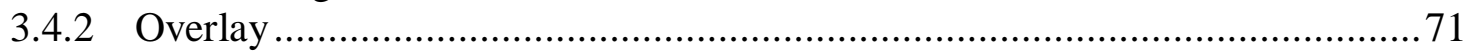

3.4.2.1 Surface Preparation ..........................................................................

3.4.2.2 Formwork and Instrumentation..............................................................

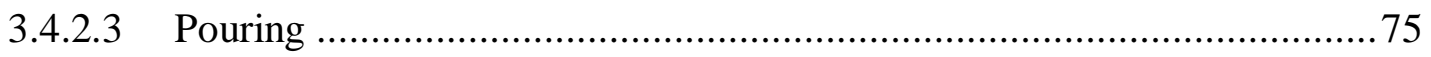

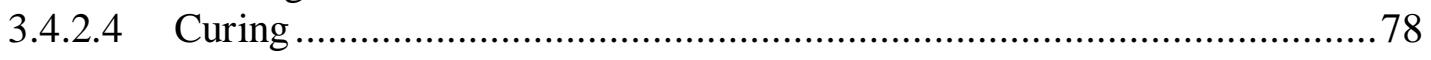

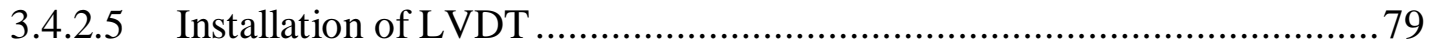

CHAPTER 4: TESTING PROCEDURES ................................................................81

4.1 Compressive Strength...................................................................................................81

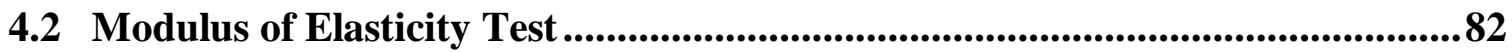

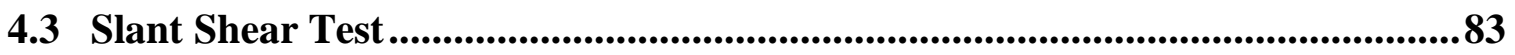

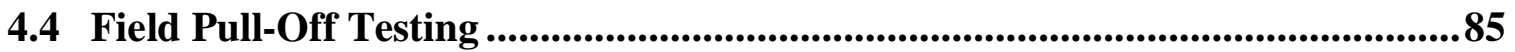

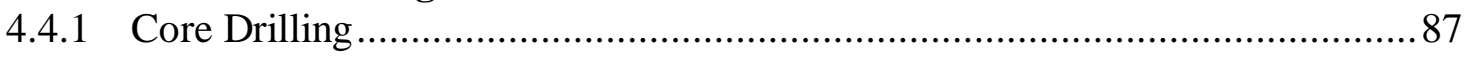

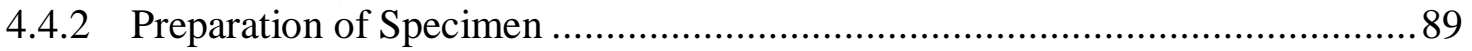

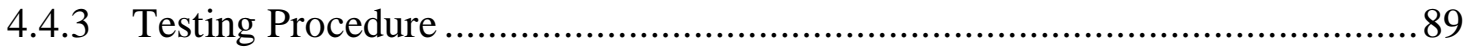




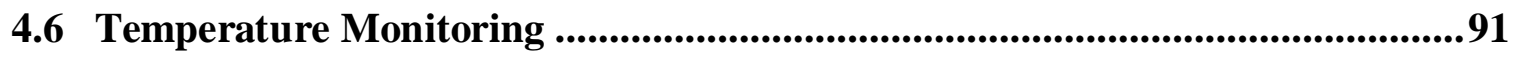

4.7 Ultrasonic Pulse Velocity Testing.................................................................92

4.7.1 Testing Equipment......................................................................... 93

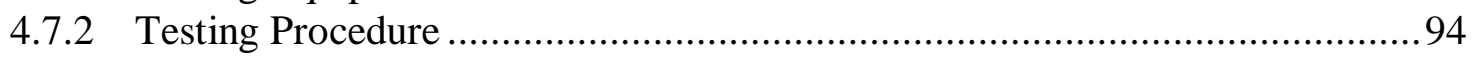

CHAPTER 5: STAGE-I: TEST RESULTS AND DISCUSSIONS .........................98

5.1 Tests Conducted on Specimens ...................................................................98

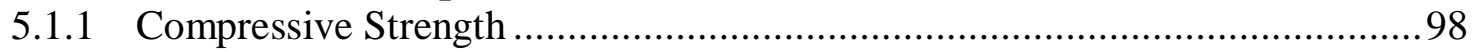

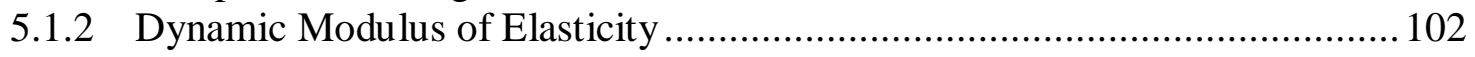

5.2 Tests Conducted on Large-Scale/Prototype Slab ........................................... 103

5.2.1 Pull-Off Testing .................................................................................... 104

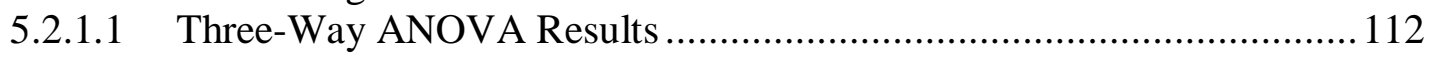

5.2.2 Length Change Monitoring .......................................................... 128

5.2.2.1 Differential Length Change after Overlay Pour.................................... 131

5.2.3 Vertical Displacements Due to Corner Lifting........................................... 137

5.2.4 Indirect Ultrasonic Pulse Velocity Testing ............................................... 138

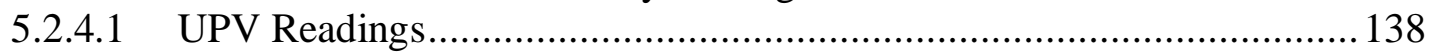

5.2.4.2 Analysis of Signals Collected by Oscilloscope ................................. 142

CHAPTER 6: $\quad$ STAGE-II: TEST RESULTS AND DISCUSSIONS ......................151

6.1 Tests Conducted on Specimens ............................................................. 151

6.1.1 Compressive Strength ........................................................................ 151

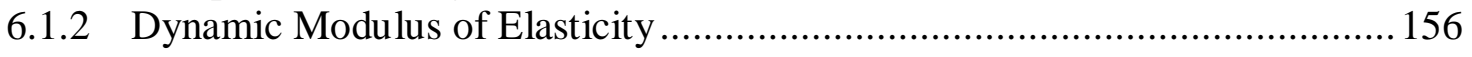

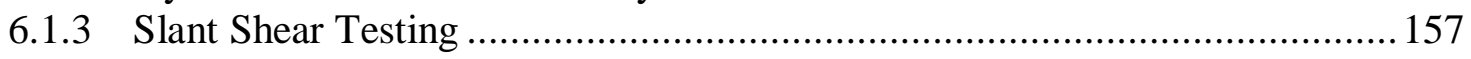

6.2 Tests Conducted on Large-Scale/Prototype Slab ......................................... 158

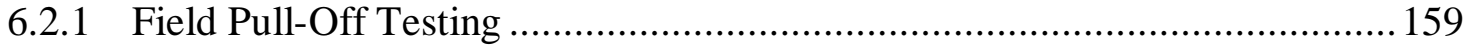

6.2.1.1 Three-way ANOVA Results ......................................................... 168

6.2.2 Length Change Monitoring ................................................................... 184

6.2.2.1 Differential Length Change after Overlay Pour..................................... 185

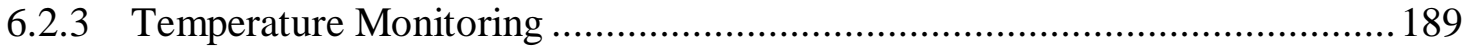

6.2.4 Vertical Displacements Due to Corner Lifting......................................... 190

6.2.5 Direct Ultrasonic Pulse Velocity Testing ............................................ 191

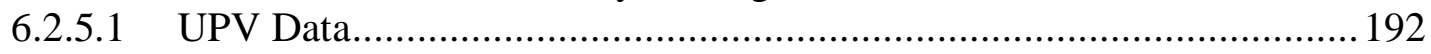

6.2.5.2 Analysis of Signals Collected by Oscilloscope ................................. 198

CHAPTER 7: CONCLUSIONS AND RECOMMENDATIONS .......................... 205

7.1 Conclusions ............................................................................................. 205

7.2 Recommendations.................................................................................. 210 


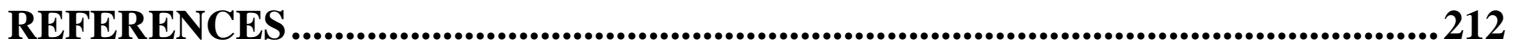

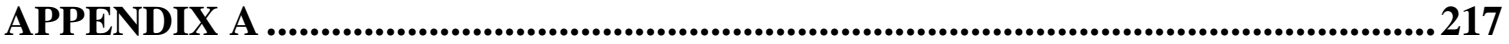

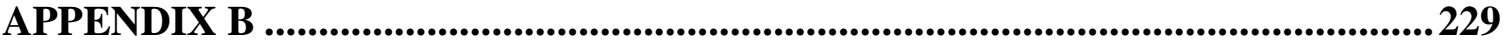




\section{LIST OF TABLES}

Table 2.1: Typical data format for a one way ANOVA (Montgomery (2005)) ...............46

Table 2.2: One-way ANOVA table (Montgomery (2005)) ..............................................4

Table 2.3: Summary of the meaning of three-way ANOVA effects..................................49

Table 2.4: Three-way ANOVA table (Karpinski (2004)) .................................................51

Table 3.1: Physical properties of Type I Portland cement ..............................................56

Table 3.2: Compound compositions of Type I Portland cement .......................................56

Table 3.3: Source and basic properties of coarse aggregates used ....................................57

Table 3.4: Sieve analysis data of coarse aggregates used...............................................57

Table 3.5: Source and basic properties of fine aggregates used ......................................58

Table 3.6: Sieve analysis data of fine aggregates used .....................................................58

Table 3.7: Physical properties of Class F Fly ash ..........................................................59

Table 3.8: Compound composition of Class F Fly ash ....................................................59

Table 3.9: Basic properties of silica fume ................................................................60

Table 3.10: Physical properties of latex admixture used................................................60

Table 3.11: Mixture proportions of substrate and overlay concretes for each cubic meter

of concrete (source: Arrow company and Ahern Associate company).............................62

Table 5.1: Parameters for rectangular hyperbolic fit..................................................... 100

Table 5.2: 35 days pull-off data of LMC and LMC-S slabs in Stage-I........................... 106

Table 5.3: 42 days pull-off data of LMC and LMC-S slabs in Stage-I..........................107

Table 5.4: 49 days pull-off data of LMC and LMC-S slabs in Stage-I..........................108

Table 5.5: 56 days pull-off data of LMC and LMC-S slabs in Stage-I........................... 109

Table 5.6: 90 days pull-off data of LMC and LMC-S slabs in Stage-I..........................110

Table 5.7: Pull off data for LMC bi-layer deck slab in Stage-I .......................................111

Table 5.8: Pull-off data for LMC-S bi-layer deck system in Stage-I .............................. 111

Table 5.9: Class level information for Stage-I pull-off test...........................................115

Table 5.10: Three-way ANOVA table of Stage-I pull-off data .......................................118

Table 6.1: Parameters for rectangular hyperbolic fit................................................... 154

Table 6.2: 7 days pull-off data of LMC and SFMC slabs in Stage-II ............................160

Table 6.3: 14 days pull-off data of LMC and SFMC slabs in Stage-II ...........................161 
Table 6.4: 28 days pull-off data of LMC and SFMC slabs in Stage-II ......................... 162

Table 6.5: 49 days pull-off data of LMC and SFMC slabs in Stage-II ......................... 163

Table 6.6: 56 days pull-off data of LMC and SFMC slabs in Stage-II ......................... 164

Table 6.7: 90 days pull-off data of LMC and SFMC slabs in Stage-II ......................... 165

Table 6.8: Pull off data for LMC bi-layer deck slab in Stage-II..................................166

Table 6.9: Pull off data for SFMC bi-layer deck slab in Stage-II............................... 167

Table 6.10: Class level information for Stage-II pull-off test.................................... 171

Table 6.11: Three-way ANOVA table of Stage-I pull-off data ..................................174

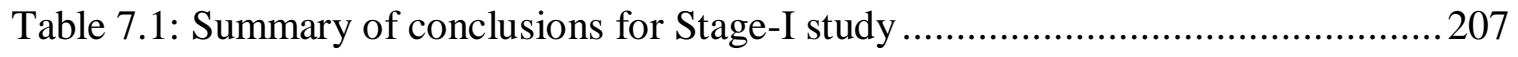

Table 7.2: Summary of conclusions for Stage-II study......................................... 209

Table A.1: 35 days pull-off data of LMC and LMC-S slabs in Stage-I .......................218

Table A.2: 42 days pull-off data of LMC and LMC-S slabs in Stage-I.......................219

Table A.3: 49 days pull-off data of LMC and LMC-S slabs in Stage-I.......................220

Table A.4: 56 days pull-off data of LMC and LMC-S slabs in Stage-I.......................221

Table A.5: 90 days pull-off data of LMC and LMC-S slabs in Stage-I........................222

Table A.6: 7 days pull-off data of LMC and SFMC slabs in Stage-II .........................223

Table A.7: 14 days pull-off data of LMC and SFMC slabs in Stage-II .......................224

Table A.8: 28 days pull-off data of LMC and SFMC slabs in Stage-II ........................225

Table A.9: 49 days pull-off data of LMC and SFMC slabs in Stage-II ........................226

Table A.10: 56 days pull-off data of LMC and SFMC slabs in Stage-II ......................227

Table A.11: 90 days pull-off data of LMC and SFMC slabs in Stage-II ......................228

Table B.1: Indirect UPV table along: G7-M7 for LMC slab and G7-A7 for LMC-S slab

Table B.2: Indirect UPV table along G7-G1 and G7-G13 for both the slabs................231

Table B.3: Indirect UPV table along: G7-M1 and G7-M13 for LMC slab, and G7-A1 and G7-A13 for LMC-S slab. .231

Table B.4: Direct UPV results at 28 days for LMC and SFMC overlays .....................233

Table B.5: Direct UPV results at 56 days for LMC and SFMC overlays .....................234

Table B.6: Direct UPV results at 90 days for LMC and SFMC overlays ....................235 


\section{LIST OF FIGURES}

Figure 2.1 : Concrete surface preparation methods (reproduced from: ICRI guidelines).17 Figure 2.2: a) Shrinkage or temperature induced tensile stresses, and b) Flexural stresses due to moving load (reproduced from: Zhang and $\mathrm{Li}(2002)$ ).........................................20 Figure 2.3: Proposed debonding mechanism (reproduced from: Granju (1996)).............21 Figure 2.4: Curling of the slabs at construction joints (reproduce from: Mailvaganam et al. (2000))

Figure 2.5: Types of bond tests that can be performed in the lab and in-situ (reproduced from: Silfwerbrand (2003)).

Figure 2.6: a) Preparation of specimen for in-place bond test, and b) LOK-test setup (reproduced from: Hindo (1990)).

Figure 2.7: Test method for determining shear bond strength between old mortar (A) and new mortar (B) (reproduced from: Chen et al. (1995) .29

Figure 2.8: Shear bond strength measured by torque test setup (reproduced from: Ali et al. (1998))

Figure 2.9: Types of failure modes (reproduced from: Ali et al. (1998)).

Figure 2.10: Tension test and shear bond test in progress (reproduced from: Delatte et al. (2000))

Figure 2.11: Schematic representation of UPV test method (reproduced from: ASTM C $597(2002 b)$ .36

Figure 2.12: Possible test configurations of the UPV test (reproduced from: Malhotra and Carino (2004))

Figure 2.13: View of the signal in time-domain and frequency-domain (Source: www.ndt-ed.org) .41

Figure 3.1: Summary of the Research Plan ................................................................5

Figure 3.2: Reinforcement layout .............................................................................64

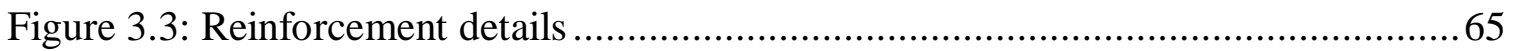

Figure 3.4: Typical embedment gage (Source: www.vishay.com)................................66

Figure 3.5: Typical temperature logger (Source: www.engius.com) ................................67 
Figure 3.6: Plan view of the embedded strain gages and thermocouple loggers in substrate. 68

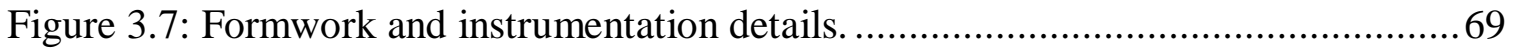

Figure 3.8: Pouring and vibration of substrate concrete....................................... 70

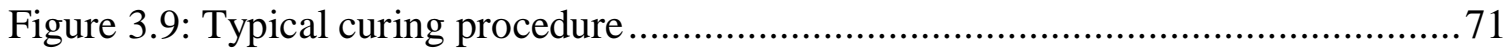

Figure 3.10: BLASTRAC equipment used for shotblasting the surface........................72

Figure 3.11: Typical substrate section after the surface preparation............................72

Figure 3.12: Tying embedment gages to the $25 \mathrm{~mm}$ (1 in) Rebar Chairs. ...................... 73

Figure 3.13: Plan and elevation views of the embedment gages and temperature loggers used in substrate and overlay concretes for typical Stage-I....................................74

Figure 3.14: Overlay concrete supplied in ready mix truck by Ahern Associates Inc.,....76

Figure 3.15: Transferring of overlay concrete from truck using hopper........................76

Figure 3.16: Preparation of bonding slurry...................................................... 77

Figure 3.17: Casting of the overlay concrete................................................... 78

Figure 3.18: Slabs enclosed in a temporarily built tent......................................... 79

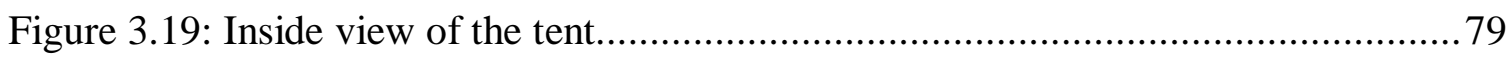

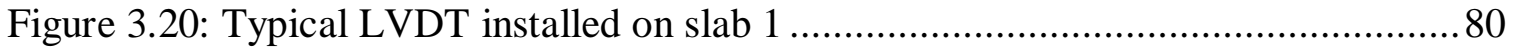

Figure 4.1: Dynamic modulus of elasticity testing in progress. ................................. 83

Figure 4.2: Slant shear test method: (a) half of the cut cylinders ready for pouring substrate concrete; (b)after casting with substrate concrete; (c) other half attached and ready for pouring overlay concrete; (d) slant shear specimen ready for testing...............85

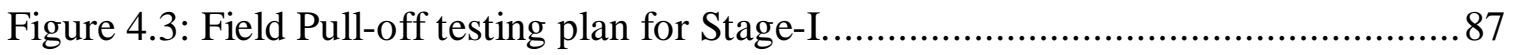

Figure 4.4: Core drilling in progress at a typical location. ....................................... 88

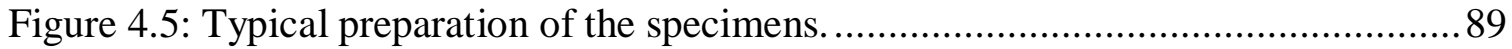

Figure 4.6: Pull-off testing in progress at a typical location....................................90

Figure 4.7: Data acquisition system used for recording strains and vertical corner lifts. .91

Figure 4.8: Monitoring of temperature at the typical location....................................992

Figure 4.9: Gridlines for conducting indirect UPV testing in Stage-I ..........................95

Figure 4.10: Gridlines for conducting direct UPV testing in Stage-II. .........................96

Figure 4.11: Testing equipment used for conducting UPV testing. .............................97

Figure 4.12: UPV testing in progress at typical location in Stage-II. ..........................97 
Figure 5.1: Compressive strength data of Type K substrate and LMC overlay concretes after overlay pour (Stage-I).

Figure 5.2: Rectangular hyperbolic fit plots of: (a) Type K concrete compressive strength; and (b) LMC compressive strength (Stage-I) 101

Figure 5.3: Dynamic modulus of elasticity of Type K substrate and LMC overlay concretes (Stage-I).

Figure 5.4: Typical failure of the samples after pull-off testing ..... 105

Figure 5.5: 35 days pull-off locations on LMC and LMC-S Slabs of Stage-I................106

Figure 5.6: 42 days pull-off locations on LMC and LMC-S slabs of Stage-I ...............107

Figure 5.7: 49 days pull-off locations on LMC and LMC-S slabs for Stage-I.............. 108

Figure 5.8: 56 days pull-off locations on LMC and LMC-S slabs of Stage-I ................109

Figure 5.9: 90 days pull-off locations on LMC and LMC-S slabs of Stage-I ...............110

Figure 5.10: Normal probability plot of residuals for Stage-I pull-off data .................. 117

Figure 5.11: Residual Plot of Stage-I pull-off data ............................................... 117

Figure 5.12: Means for levels of Zone type at each level of Age (Stage-I) ..................121

Figure 5.13: Means for levels of Slab type at each level of Age (Stage-I) ................... 122

Figure 5.14: Means for levels of Zone type at each level of Slab (Stage-I) .................. 123

Figure 5.15: Means for levels of Zone type at each level of Slab type for 35 days age level (Stage-I).

Figure 5.16: Means for levels of Zone type at each level of Slab type for 42 days age level (Stage-I)...... 125

Figure 5.17: Means for levels of Zone type at each level of Slab type for 49 days age level (Stage-I). 126

Figure 5.18: Means for levels of Zone type at each level of Slab type for 56 days age level (Stage-I).

Figure 5.19: Means for levels of Zone type at each level of Slab type for 56 days age level (Stage-I). 128

Figure 5.20: Length change of Type K substrate concrete at typical location 131 Figure 5.21: Locations of embedment strain gages in substrate and overlay concretes (Stage-I); a) Plan view, and b) elevation view. 
Figure 5.22: Comparison of percent length change plots of embedment gages in transverse and longitudinal direction along the edges of LMC and LMC-S slabs (Stage-I)

Figure 5.23: Comparison of percent length change plots of embedment gages in transverse and longitudinal direction along the edges of LMC and LMC-S slabs (Stage-I)

Figure 5.24: Relative displacement of overlay concretes (LMC and LMC-S) recorded by LVDT (Stage-I). 138

Figure 5.25: Locations at which indirect UPV was conducted. 140

Figure 5.26: Indirect UPV plots along: G7-K7 for LMC slab and G7-C7 for LMC-S slab 140

Figure 5.27: Indirect UPV plots along G7-G3 and G7-G11 for both the slabs 141

Figure 5.28: Indirect UPV plots along: G7-K3 and G7-K11 for LMC slab, and G7-C3 and G7-C11 for LMC-S slab.

Figure 5.29: Algorithm for calculating FFT from time-domain signal using MATLAB143

Figure 5.30: Locations at which oscilloscope readings were collected (Stage-I). 145

Figure 5.31: Frequency spectrum plots of LMC and LMC-S bi-layer deck slabs (Stage-I)

Figure 6.1: Compressive strength data (Stage-II) 152

Figure 6.2: Rectangular hyperbolic fit of: (a) Type K concrete compressive strength; (b)

LMC compressive strength; and SFMC compressive strength (Stage-II).......................155

Figure 6.3: Dynamic modulus of elasticity data (Stage-II) .......................................... 157

Figure 6.4: Slant shear strength data (Stage-II). ............................................................158

Figure 6.5: 7 days pull-off locations on LMC and SFMC Slabs of Stage-II...................160

Figure 6.6: 14 days pull-off locations on LMC and SFMC Slabs of Stage-II.................161

Figure 6.7: 28 days pull-off locations on LMC and SFMC Slabs of Stage-II................. 162

Figure 6.8: 49 days pull-off locations on LMC and SFMC Slabs of Stage-II..................163

Figure 6.9: 56 days pull-off locations on LMC and SFMC Slabs of Stage-II................. 164

Figure 6.10: 90 days pull-off locations on LMC and SFMC Slabs of Stage-II............... 165

Figure 6.11: Normal probability plot of residuals for Stage-II pull-off data...................172

Figure 6.12: Residual plot of Stage-II pull-off data .......................................................173 
Figure 6.13: Means for levels of Zone type at each level of Age (Stage-II) ................. 176

Figure 6.14: Means for levels of Slab type at each level of Age (Stage-II) ................... 177

Figure 6.15: Means for levels of Zone type at each level of Slab (Stage-II).................178

Figure 6.16: Means for levels of Zone type at each level of Slab type for 7 days age level (Stage-II)

Figure 6.17: Means for levels of Zone type at each level of Slab type for 14 days age level (Stage-II)

Figure 6.18: Means for levels of Zone type at each level of Slab type for 28 days age level (Stage-II)

Figure 6.19: Means for levels of Zone type at each level of Slab type for 49 days age level (Stage-II)

Figure 6.20: Means for levels of Zone type at each level of Slab type for 56 days age level (Stage-II)

Figure 6.21: Means for levels of Zone type at each level of Slab type for 90 days age level (Stage-II)

Figure 6.22: Locations of embedment strain gages in substrate and overlay concretes (Stage-II); a) Plan view, and b) elevation view. 186

Figure 6.23: Comparison of percent length change plots of embedment gages in transverse and longitudinal direction along the edges of LMC and SFMC slabs

Figure 6.24: Comparison of percent length change plots of embedment gages in transverse and longitudinal direction towards the center of LMC and SFMC slabs 188 Figure 6.25: Average temperature data of substrate concrete, LMC overlay, and SFMC overlay after overlays pour (Stage-II). 190

Figure 6.26: Differential temperature data at typical location of: (a) substrate concrete and LMC overlay, (b) Substrate concrete and SFMC overlay. 190 Figure 6.27: Relative displacement of overlay concretes (LMC and SFMC) recorded by LVDT (Stage-II). 191

Figure 6.28: Locations at which direct UPV testing was conducted (Stage-II) 194

Figure 6.29: Comparision of 28 days UPV plots measured on LMC and SFMC slabs:. 195 Figure 6.30: Comparision of 56 days UPV plots measured on LMC and SFMC slabs.. 196 Figure 6.31: Comparision of 90 days UPV plots measured on LMC and SFMC slabs.. 197 
Figure 6.32: Locations at which oscilloscope readings were recorded (Stage-II) .......... 199

Figure 6.33: Time-domain and power spectrum forms of the virgin signal..................200

Figure 6.34: Comparison of time-domain waveforms for LMC and SFMC bi-layer slabs

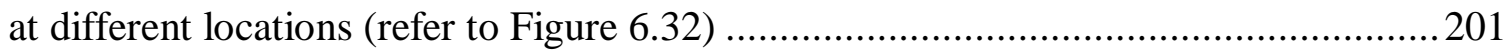

Figure 6.35: Comparison of frequency spectrums for LMC and SFMC bi-layer slabs at different locations (refer to Figure 6.32)

Figure 6.36: Comparison of power spectrums for LMC and SFMC bi-layer slabs at

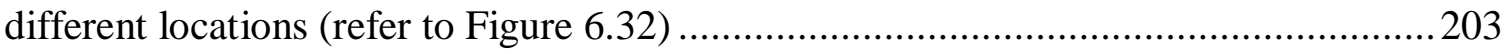

Figure A.1: 35 days pull-off locations on LMC and LMC-Slab of Stage-I ..................218

Figure A.2: 42 days pull-off locations on LMC and LMC-S slabs of Stage-I ..............219

Figure A.3: 49 days pull-off locations on LMC and LMC-S slabs for Stage-I .............220

Figure A.4: 56 days pull-off locations on LMC and LMC-S slabs of Stage-I ..............221

Figure A.5: 90 days pull-off locations on LMC and LMC-S slabs of Stage-I ..............222

Figure A.6: 7 days pull-off locations on LMC and SFMC Slabs of Stage-II................223

Figure A.7: 14 days pull-off locations on LMC and SFMC Slabs of Stage-II ...............224

Figure A.8: 28 days pull-off locations on LMC and SFMC Slabs of Stage-II ..............225

Figure A.9: 49 days pull-off locations on LMC and SFMC Slabs of Stage-II ...............226

Figure A.10: 56 days pull-off locations on LMC and SFMC Slabs of Stage-II .............227

Figure A.11: 90 days pull-off locations on LMC and SFMC Slabs of Stage-II ............228

Figure B.1: Locations at which indirect UPV was conducted ...................................230

Figure B.2: Locations at which direct UPV testing was conducted (Stage-II)...............232 


\section{CHAPTER 1: INTRODUCTION}

\subsection{Motivation}

Most of the concrete bridges in the US are in a need of rehabilitation due to the damage caused by chloride initiated reinforcement corrosion, freeze-thaw cycles, deicing salts, and fatigue due to traffic loads (Babaei and Hawkins (1990); Fitch and Abdulshafi (1998); Paulsson and Silfwerband (1998); Silfwerbrand (2003)). As a result, high performance concrete overlays of thickness usually two-inch are placed on top of the existing structure. This is the most common and effective way of extending the life of concrete bridge decks as shown by recent studies.

Concrete bridge decks can be effectively protected by concrete overlays which: 1) Provide protection against the impact of heavy trucks and further infiltration of salts, acids, gasoline and other contaminants; 2) provide a non skid riding surface; 3) prevent carbonation; 4) create a uniform appearance; and 5) corrects uneven surface created by wear. Despite these advantages, deterioration of concrete overlays occurs mainly due to the temperature changes, freeze and thaw cycles, concrete shrinkage, and repeated loading from the moving traffic. In the bi-layer deck system, the substrate concrete resting freely on its supports undergoes shrinkage whereas the concrete overlay cast on substrate concrete cannot shrink freely due to the restraints from the underlying concrete pavement. Overlay is relatively new compared with substrate and this difference in shrinkage and restraint produces the tensile stresses at the interface. Similarly, the difference in temperature produces the stresses at the interface because of the difference in expansion and contraction of the overlaid concrete compared to the substrate concrete due to the change in temperature. Also, differential shrinkage occurs due to several reasons such as: drying; carbonation of the surface concrete; internal desiccation (autogenous shrinkage); and also due to moisture gradient in the overlay

Concrete overlay systems have been successfully used by the West Virginia Department of Transportation, Division of Highways (WVDOH) for several years to 
protect the bridge decks from environmental exposures and provide a durable riding surface. Details of the overlay systems are defined by the WVDOH Standard Specifications for Roads and Bridges (Section 679, WVDOH, 2000). Overlays can be built on both old and new bridge decks. More cracking and delamination issues are found in the case of overlays on new bridge decks than those on the old decks, because in the case of new decks the surface preparation of substrate concrete is not as aggressive as done for the old decks. In case of new decks typically shot blasting and water jetting is done compared to hydro-demolition for old decks, which is easy to execute. Also, the substrate concrete is not enough matured for old decks.

In order to fulfill the design purpose and achieve optimal performance, a concrete overlay must act compositely with the substrate bridge deck during its service life. Despite the debate regarding the best types of overlays to use there is consensus that a good bond between the concrete overlay and the existing bridge deck is critical to the performance of the bi-layer system. The most important factor affecting the bonding quality is the surface preparation of the bridge deck (Sprinkel 1997). Suprenant (1988) also mentioned that the surface preparation, overlay materials and curing are three major factors, which could affect the concrete-to-concrete bond. This was also observed in the previous research done at West Virginia University (Sun 2004). Other factors, such as the temperature of concrete, curing conditions and the maturity of concrete can also affect the deck characteristics and eventually affect the overall performance of the bi-layer system.

\subsection{Objectives}

The objectives of this research work are as follows:

- To assess the bond performance of concrete overlays by conducting field pull-off testing

- To monitor the differential length change developed at the interface, which is considered as a critical factor for developing shear stresses at the interface between overlay and substrate concrete

- To monitor the vertical displacements due to corner lifting of the overlays 
- To characterize the delaminations developed at the interface by analyzing the waveforms collected by oscilloscope

- To provide guidelines for implementing the concrete overlays in field projects

\subsection{Scope of the Work}

A project on concrete overlays was initiated by WVDOH at WVU in August 2002. This project is divided into three phases. In Phase-I study, current practices of concrete overlays prevalent in West Virginia (WV) and other states was investigated; characterization of various overlay mixtures using different locally available materials and proportions was made; the delamination studies were performed at coupon level with a novel testing methods; preliminary specifications were developed based on the laboratory results; and finally a plan was proposed for field implementation. To predict the delaminations, bond strength testing was not sufficient and hence, Hong (2006) addressed the issues of early age delaminations by measuring the vertical corner lifting, by conducting non destructive testing combined with signal processing techniques, temperature gradients across the depth, and finally he predicted the delamination profiles by mapping the UPV numbers. He conducted experiments on $450 \mathrm{~mm}$ (18 in) long x 200 mm (8 in) wide specimens with $100 \mathrm{~mm}$ (4 in.) thick substrate and $50 \mathrm{~mm}$ (2 in.) thick overlays on top of the substrate. His findings served as an input to start the Phase-II program. In Phase-II program, prototype overlay-substrate slab systems will be constructed, considering the real construction scenario i.e., the substrate and overlay concretes will be constructed following the standards specified by the WVDOH. The width of the slabs

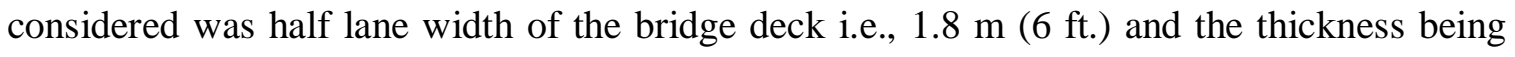
same as that of the WVDOH standard bridge deck. The LMC and SFMC overlays will be evaluated by conducting study on edge curling, bond strength, and delaminations at prototype scale. This research is a Phase-II study funded by WVDOH. Finally, based on the findings of Phase-II study, Phase-III study will be developed to implement the concrete overlays in field and develop a performance based specifications. 


\subsection{Thesis Organization}

This thesis is organized into seven chapters as follows: Chapter 1 describes motivation for the research, objectives of the research, and scope of the work. Chapter 2 provides a detailed literature review on different types and performance of specialized concrete overlays; surface preparation techniques; edge curling, debonding, and delamination of the overlay; bond tests and pull-off testing; ultrasonic pulse velocity and signal processing techniques; and Analysis of Variance (ANOVA). Chapters 3 describes the materials and mixtures proportions used, and construction procedures followed. Chapter 4 discusses about the testing procedures followed. Chapter 5 presents the Stage-I test results and discussions. Chapter 6 presents the Stage-II test results and discussions. Finally, chapter 7 provides the conclusions of the entire study and provides the recommendations for future work. 


\section{CHAPTER 2: LITERATURE REVIEW}

\subsection{Introduction and Background of Concrete Overlays}

Most of the bridge decks, which were originally constructed without consideration of providing sufficient protection practices against reinforcing steel corrosion, are undergoing severe reinforcement corrosion due to the ingress of chloride ions into the concrete during the application of deicing salt on some bridge decks in winter (Babaei and Hawkins (1992)). As a result, bridge decks are usually overlaid with a high strength concrete to provide protection for reinforcement against corrosion, restoring riding quality and hence providing protection for the substrate concrete deck. These special types of high strength concretes are called concrete overlays. By definition concrete overlays are high performance concretes with improved performance, developed by changing properties and composition of normal concrete, placed on the bridge decks to provide protection from electrochemical corrosion of reinforcement due to: deicing salts; abrasive resistance from vehicular load; and increase of the skid resistance.

\subsection{Types of Concrete Overlays}

Most commonly used overlay concretes are: Latex Modified Concrete (LMC), Silica Fume or Microsilica Modified Concrete (SFMC), Low Slump Dense Concrete (LSDC), Fiber Reinforced Concrete (FRC) and Polymer Concrete (PC). The WVDOH uses overlay systems in repairing the old decks and also on the newly constructed bridge decks. The most commonly used overlay systems by WVDOH are LMC and SFMC.

LMC is a high strength concrete produced by adding a white milky-looking liquid, styrene butadiene latex along with antifoam to modify the pore structure of the Portland cement concrete to reduce its permeability and to reduce the excessive air content due to the surfactants in the latex formulation. First LMC was used as a bridge deck overlay material in 1965. 
SFMC overlay is also a high strength concrete produced by adding $5 \%$ to $10 \%$ of silica fume, a byproduct of the ferrosilicon industries, by weight of the Portland cement. Silica fume particles are much finer than the Portland cement; hence fill the microscopic spaces inside the concrete making the concrete a high strength and highly impermeable to chloride ions. Also, the electrical resistivity of SFMC is less, in turn helps to suppress the corrosion reaction in concrete. Commonly, $50 \mathrm{~mm}$ (2 in) thick overlays with 8 inches slump are produced with $12.5 \mathrm{~mm}$ (1/2 in) aggregates. Due to low water to cementitious materials $(\mathrm{w} / \mathrm{cm})$ ratio, 0.35 to 0.4 , wet curing is done immediately to prevent the excessive loss of moisture and in turn reduces the cracking due to plastic and drying shrinkage. SFMC was used in bridge applications since 1970's and in United States; the first SFMC bridge deck overlay was used in 1984.

Low Slump Dense Concrete (LSDC) overlays with zero or very low slump were an early remedy for concrete bridge deck deterioration. With some modifications, similar mixes are still widely used. The mixes typically contain relatively high cement contents and low water-cement ratios. The high cement content and low water content lead to reduced permeability as long as the concrete is well consolidated. This type of concrete bridge deck overlay is commonly called the "Iowa mix" because of extensive use in that state of a low-slump, high-density overlay mix since 1965. Though these mixes use conventional materials and construction equipment, their low slump can make placement and consolidation difficult.

Since the 1960s, fiber-reinforced concrete has been used to increase the durability of transportation structures. The Virginia Department of Transportation (VDOT) used steel fibers in 1974 for a bridge deck overlay and recently used steel and plastic fibers in bridge deck and pavement overlays on an experimental basis. FRC has also been used in overlays in some other states to minimize cracking. Fibers are expected to improve the properties of concrete both in the unhardened and hardened states. In the unhardened state, fibers increase resistance to plastic shrinkage. In the hardened state, fibers improve the strength (impact, tensile, and flexural) and toughness of concrete, depending on fiber type, shape, size, and amount. The most frequently used fibers for bridge deck overlays 
are steel fibers (hooked-end), polypropylene fibers (monofilament and fibrillated), and polyolefin fibers (monofilament).

Polymer concrete (PC) was used as early as 1958 in the United States to produce building cladding. Polymer concrete overlays have been installed on Portland cement concrete bridge decks in many states during the past 25 years. PC consists of aggregate with a polymer binder and contains no Portland cement or water. The most frequently used binders are epoxy, polyester styrene, and methacrylate. The binders are usually twocomponent systems: one component contains the resin and the second contains the curing agent or initiator. The aggregates are usually silica and basalt. Typically, uniformly graded aggregates are used with slurry and premixed overlays, and gap-graded aggregates are used with multiple-layer overlays and are broadcast on the top of slurry and some premixed overlays. Quick curing, high early compressive strength and excellent bond strength are the advantages of PC overlays. Excellent performance can be expected from bridge deck overlays if materials and construction are carefully selected and executed. They can prolong the life of bridge decks for 25 to 35 years.

\subsection{Performance of Specialized Concrete Overlays}

In this section, performance of the different types of specialized concrete overlays on the bridge decks reported by several DOTs and researchers are reviewed.

In the past, concrete decks were protected from electrochemical corrosion of reinforcing steel which occurs mainly due to the heavy use of deicing salts by applying polymer repair surface to the surface of the bridge deck. Polymer concrete by definition is a combination of plastic binder such as epoxy, polyester or methacrylate, and aggregate including silica or basalt (Tarricone (1992)). Though polymer concrete provides increased protection against the corrosion, the main problem due to this application was the increase in dead load and wearing surface thickness. The polymer concrete overlays proved deficient in several performance characteristics such as insufficient skid resistance, freeze-thaw resistance, abrasion resistance. It takes longer curing time, chloride resistance, and moisture insensitivity (Calvo and Meyers (1991)). Due to the 
above mentioned deficiencies several DOTs have been using Latex Modified Concrete (LMC), Silica Fume Modified Concrete (SFMC), Fiber Reinforced Concrete (FRC), and Low Slump Dense Modified Concrete (LSDC) overlays that have a service life of 20 years and more.

Babaei and Hawkins (1990) documented the performance of 12 concrete bridge decks in Washington State that were rehabilitated with LMC and Low Slump Dense Concrete (LSDC) overlays. Recommendations were made by evaluating the factors affecting the serviceability of overlaid bridge decks such as overlay freeze-thaw scaling, surface wear and skid resistance, surface cracking, bond with the substrate deck, and chloride and water intrusion. Based on the evaluations, following conclusions were drawn: 1) the bridge deck concrete overlays need maintenance in the form of resurfacing for every 25 years; 2) the rate of chloride intrusion depends on the extensive application of salts; 3) cracking of the overlays depends on the plastic and drying shrinkage characteristics of the mix used in the overlay, placement, and curing procedures; and finally, 4) LMC and LSDC overlays showed better protection from continued corrosion however the surface cracking can increase the corrosion rate.

Babaei and Hawkins (1992) performed a research on three different types of protective bridge deck systems namely LMC overlay, LSDC overlay and Cathodic Protection (CP) and they selected five LMC, five LSDC and two CP bridge decks on which a detailed field investigations was performed to determine the relative effectiveness of deck systems in preventing reinforcing steel corrosion. Based on the study they concluded that, after an average of seven years of service the average deterioration rate of LMC decks was $0.4 \%$ of the deck area per year and for LSDC decks the average deterioration rate was $0.5 \%$ of the deck area per year. Also, they concluded that the effect of surface cracking on the LMC decks was more severe than that of LSDC decks which is related to the thickness of the overlay. On the basis of their bridge deck life-time costs and unit costs used in their study they finally concluded that a concrete overlay strategy is more cost effective that CP systems. 
Wallace (1987) mentioned that when using Latex Modified Concrete (LMC) for concrete overlays, it is crucial to adhere to the proper placement process. The five critical steps are: 1) prepare; 2) batch; 3) place; 4) finish, and 5) cure. These steps must be followed in sequence and without delay. He explained the importance of the above mentioned steps as follows:

Preparing the deck surface must be done thoroughly. When overlaying old decks, more preparation is required compared to overlaying new decks. To prepare new decks, blast cleaning should remove any hardened curing compound, laitance or other contaminants on the surface of the deck.

Due to the short mixing time of five minutes, LMC must be batched on site. It is recommended that a continuous mobile batcher mixer be used when overlaying bridge and parking decks. This truck is advantageous because it has tanks on board to hold all the raw materials. Due to the required accuracy of the mix proportions, proper monitoring and calibration is recommended.

LMC has a set time of approximately 10 minutes which requires a larger crew working at a faster pace. A typical crew may consist of 17 workers. Before applying the overlay, it is required to prime the deck with latex grout or some of the LMC mix evenly distributing it over the entire deck surface. This should be done simultaneously while placing the LMC so that brushed material does not dry before applying the overlay.

Due to a plastic film that forms on the surface of LMC, pre-saturated burlap must cover the overlay as soon as it has set enough to support the burlap without causing deformations. This will prevent the surface from cracking. It is required to keep the burlap wet for 24 hours and then allowed to air dry for 72 hours before opening to traffic.

Finally, he mentioned that: LMC overlay should not be placed less than a quarter inch thick when rain is expected and LMC overlays should not be placed when the 
temperature is below $40^{\circ} \mathrm{F}$ degrees Fahrenheit or when the surface evaporation rate is more than $7.2 \mathrm{~Pa} / \mathrm{hr}\left(0.15\left(\mathrm{lb} / \mathrm{ft}^{2}\right) / \mathrm{hr}\right)$.

Luther (1988) discussed on the use of silica fume concrete in bridge deck overlays in the United States by several DOTs. The performance of the silica fume concrete as an overlay concrete, reported by several DOTs are reviewed and presented by the author are discussed below:

1. Ohio Department of Transportation (ODOT) placed a first experimental $15.5 \%$ SFMC overlay in 1984 during rehabilitation of Bridge No. ASD-511 along State Route 511 about 2 miles north of Ashland. On this bridge, LMC overlay was applied on one lane and on the other lane SFMC overlay was applied. Inspection of the bridge deck in 1985 by the author showed that no cracks were found and also the inspection in 1987 by the author showed only two cracks in SFMC overlay but there were several cracks found in LMC overlay. A good surface was ensured by performing chain dragging.

2. University of Cincinnati placed two 5\% SFMC overlays on two pedestrian walkways and the placements were successful and hence the use of SFMC was extended to construction of parking garages.

3. Kentucky Department of Transportation (KyDOT) placed a $15.5 \%$ SFMC overlay with minimum thickness of $32 \mathrm{~mm}$ (1.25 in.) thick in 1985. Concrete was placed by using the mortar from the same concrete as a bonding grout. The bridges were inspected regularly, after 4 months no changes were observed, at 9 months few cracks were observed, and more cracks were seen after 2 years. Author mentioned that the SFMC overlay functioned well as a chloride barrier despite of the cracks observed.

4. New York State Department of Transportation (NYSDOT) studied on the air entrained 13.9 percent SFMC in 1984 and concluded that the use of SFMC increased the strength and decreased the permeability of chloride and water. In 1985 two bridges were cast with SFMC, one was a base repair and the other was an overlay. Poor surface closures and plastic shrinkage cracks were observed with both SFMC and LMC when the roller screed was used. An 
inspection in 1987 showed reflection cracks in one lane. Visual inspection indicated better wear resistance and no scaling or pop outs were observed. NYSDOT concluded that the SFMC overlay can be easily handled and finished, and the material can be used as an alternative to LMC and LSDC overlays.

5. Tennessee Department of Transportation (TDOT) used 11.2 percent SFMC in a minimum $100 \mathrm{~mm}$. (4 in.) deep repair of $370 \mathrm{~m}$ (1228 ft.) long span Landon B. Hassler Memorial Bridge. The SFMC used was uniform and exhibited good workability retention; although the concrete was sticky the desired finish was achieved. Again here the SFMC was primarily used to achieve high early strength and hence reducing the time that lanes are closed to traffic.

6. Michigan Department of Transportation (MiDOT) began the laboratory tests in 1985 on 10 percent SFMC. By the late 1986 two four span bridges over I-94 in Detroit received experimental $50 \mathrm{~mm}$ (2 in.) thin-bonded overlays. It was observed that the workability retention was good and surface closure was acceptable. No plastic shrinkage cracking was observed but some tight cracks were observed within 2 weeks except that the hardened concrete showed no spalls or pop outs. Some LSDC placed before showed heavy cracking and LMC generally showed little cracking. It was mentioned that the average shrinkage of SFMC was 0.53 percent whereas for LMC it was 0.41 percent.

7. Maine Department of Transportation (MeDOT) investigated the use of SFMC wearing courses in the place of LMC to achieve good bond and to act as a chloride barrier. For this purpose two bridges were selected for experimental work and 7.2 percent SFMC of $64 \mathrm{~mm}$ (2.5 in.) thick wearing courses was applied on these in later half of 1986. Concrete was placed and wet burlap cured for 7 days and the bridges were opened to traffic in 9 days. They mentioned the concrete was uniform, achieved the desired properties, and was easy to place and finish. They observed no cracks or delaminations. 
8. Virginia Department of Transportation (VDOT) conducted laboratory work on 5 percent SFMC and concluded that 5 percent of SFMC having w/C ratio of 0.4 was suitable to achieve overlays as thin as $38 \mathrm{~mm}$ (1.25 in.) thick. This concrete provided satisfactory strength, low permeability, and satisfactory freezing and thawing durability. After successful laboratory investigation, later in 1987 first SFMC overlay of thickness 38 mm (1.25 in.) was applied by VDOT on a four span bridge near Winchester. VDOT used both 7 percent and 10 percent dose of silica fume in SFMC. Concrete was placed in a standard manner using mortar from the concrete as the bonding grout. They observed only one foot long crack near the location where the overlay thickness changed abruptly and also a zone that was poorly consolidated showed some delamination and was replaced soon. Acceptable frost resistance was observed using this concrete.

Based on the case studies discussed above, he concluded that: SFMC can be successfully used to provide a chloride barrier, to develop high strength, to improve bond strength, and to develop high early strength; SFMC with dosages between 5 and 15.5 percent can be manufactured, placed, finished, and cured using regular equipment and hence the use of SFMC is increasing and is being specified in bridges.

Detwiler et al. (1997) evaluated the performance of bridge deck overlays used on the IL-4 Bridge over I-55 interstate. In 1986, the southbound lane was repaired using a standard Dense Concrete and in 1987, the northbound was repaired by using Silica Fume Concrete, first use of SFMC by the Illinois Department of Transportation (IDOT). Both repairs consisted of partial and full depth repairs followed by $50 \mathrm{~mm}-100 \mathrm{~mm}$ (2 in. - 4 in.) overlay. For SFMC repair bridge deck surface was first prepared by hydrodemolition then the bonding grout containing 1 part of cement plus 1 part of sand plus 15 percent of silica fume by mass was mixed and applied on the surface. The concrete was cured with wet burlap and plastic sheet and continuously kept wet for 7 days. Later, an inspection of SFMC overlay after 3 months showed four short transverse cracks and a few shorter cracks near the drains, authors mentioned these cracks were not significant. Field survey 
was conducted in 1995; observations showed that the performance of SFMC overlay was good, and the surface showed very little abrasion wear. Chain dragging used to detect delaminations, showed overall good bond at the interface with delaminations at 22 locations which were about 2 percent of the overlay. Cores were taken from both the concretes and laboratory testing such as petrographic examination, chloride ion penetration test, and chloride profiles were measured. Based on the test results they obtained, they concluded that, both the dense concrete and SFMC repairs were of high quality. Both performed well under exposure conditions of more than $0.3 \mathrm{MPa}\left(6.3 \mathrm{lb} / \mathrm{ft}^{2}\right)$ of salt applied annually-8 years in case of SFMC and 9 years in case of dense concrete. Chloride ion penetration and chloride profiles showed the chloride penetration of SFMC was lower than dense concrete at a given distance from the surface.

Fitch and Abdulshafi (1998) evaluated the properties of SFMC mixes that have been used by the Ohio Department of Transportation (ODOT) for bridge deck overlays since 1984, which was believed as a first of its kind in United States (Halvorsen (1993)), under field and laboratory conditions. They mentioned that the use of SFMC overlays increased significantly since 1987 at an increasing rate gradually replacing the use of LMC and superplasticized dense concrete. Construction monitoring of 9 overlay projects, visual inspection of 145 decks, and in-depth condition surveys of 288 decks was made to study the properties of silica fume concrete. Conclusions drawn were: the overall performance of 145 bridge decks was good through 1992, with none of these showing any spalling, but the shrinkage cracks were observed; the practice of placing partial or full depth SFMC repairs along with overlay should be avoided because, this practice leads to the development of stress at the interface due to differential volume change; excessive moisture loss should be prevented because, SFMC is more susceptible to drying shrinkage; any liquid material should not be applied to the concrete surface prior to the overlay application, if necessary its application should be controlled, since, this may increase the w/c ratio of the surface paste; both the grouted and ungrouted sets of overlay surface showed optimum bond strength when substrate concrete was dry at the time of overlay; and finally, a thorough investigation should be made to study the bond strength of grouted and ungrouted overlays. 
Issa et al. (2007) evaluated the long term performance of LMC and Micro-Silica Concrete (MSC) overlays with and without fibers under environmental exposure and low-cycle fatigue loading. Based on the experimental observations and analysis of test results they found that the addition of overlay on the full-scale prototype bridge improved the stiffness by forming the full composite action as a result of high bond strength. Bridge deck overlay system maintained integrity without debonding when subjected to 300 low fatigue cycle tests. Finally, they concluded that the successful performance of LMC and SMC overlay construction is ensured by selecting accurate mixture design proportioning and implementation of proper practices such as surface preparation, mixing, placement, finishing, and curing procedures.

\subsection{Surface Preparation of Bridge Decks}

The performance of the bi-layer deck composite system directly depends on the good bond and the full transfer of stresses at the interface of the repair and old concretes. The most important factor for achieving good bond strength is to properly prepare the surface of the bridge deck by attaining well textured surface at the interface line (ICRI, (1996); Sprinkel (1997); Warner et al. (1998)).

Smith (1991) stated that the bond can be weakened if any contaminants or unsound concrete is present at the interface. All the surface contaminants like paints, coatings, tar, and oil should be removed before applying the overlay concrete. Also the surface should be checked for any delaminations or weak areas in the substrate using non destructive testing methods, if any present those areas should be removed and then should be repaired with suitable patching materials. After that, the deck surface should be roughened using the most efficient shotblasting method equipped with vacuum unit since, this method roughens the surface thoroughly without leaving any residue.

Sprinkel (1997) studied on the effect of different types of surface preparation techniques; milling followed by shot blasting technique, Shot blasting technique, and hydro-demolition followed by power-washing, on the bond strength. He recommended 
that for the bridge decks requiring concrete removal depths of less than $6 \mathrm{~mm}(1 / 4 \mathrm{in})$, shotblasting technique can be the effective surface preparation method. Based on the bond strength results on 17 overlays on 26-span bridges, he concluded that the average bond strength of 1.79 MPa (260 psi) was achieved when the surface macrostructure depth was at least $1.5 \mathrm{~mm}, 2.34 \mathrm{MPa}$ (340 psi) was achieved when the macrostructure depths ranged from $1.6 \mathrm{~mm}$ to $2.6 \mathrm{~mm}$, and $0.48 \mathrm{MPa}$ (70 psi) was observed when the macrostructure depth was only $0.48 \mathrm{~mm}$. Finally, excellent bond was achieved by shotblasting technique provided sufficiently roughened and cleaned surface was produced. Also, he mentioned that the milling technique can fracture the base concrete which in turn causes low tensile rupture strengths.

Mailvaganam et al. (1998) recommended that the substrate surface should be saturated before applying the cement based repair materials otherwise there will be a rapid loss of water from the overlay material into substrate concrete which in turn may result in subsequent shrinkage and cracking. Also, they suggested that after the surface preparation is done using impact tools, surface should be further prepared using wet sandblasting or water jetting to remove the damaged layer caused by impact tools otherwise this damaged layer may result in debonding of the repair material from substrate concrete. They recommended that the prepared surface should be inspected to make sure the substrate concrete is sound and structurally durable if not, the surface should be further prepared to remove any unsound concrete. Dust or other deleterious substances not removed after the initial surface preparation must be vacuumed, leaving the surface dust free and clean.

Warner et al. (1998) provided a report about the bond strength performance due to the different surface preparation techniques based on their experience and research. They observed that when the failure occurs in the substrate concrete it occurs near the bond surface usually within 3 to $9 \mathrm{~mm}$ of the interface. They have investigated a large number of overlay failures and observed low tensile rupture strengths from tensile bond tests, which was mainly due to the bad surface preparation. After the tensile bond tests, cores were examined under microscope, they observed severe bruising in the form of microcracks when the pneumatic hammer tools were used while the surface prepared with hydrodemolition showed only one minor crack. They further observed the hydraulic 
cement concrete overlay placed on continuously reinforced concrete pavements in Virginia in 1995 showed lower tensile strengths when the surface was milled and shotblasted compared to the strengths due to only shotblasting technique. Also, the six LMC and SFMC overlays placed on bridges in Virginia in 1994 using pick milling technique exhibited lower tensile bond strengths compared to that of similar overlays placed in 1996 using shotblasting technique only. Based on the conclusions from the case studies, authors mention that the tools that impact directly on the concrete surface will create more damage than the methods that impel a medium like abrasive, small steel shot, or high pressure water since, a single sharp point will affect only small area of the concrete whereas a wide chisel will affect the large area. Finally, authors recommend that any bruising damage observed must be substantially reduced by further preparation with abrasive, shot, or hydroblasting techniques.

International Concrete Repair Institute (ICRI) provided the guidelines for measuring the surface roughness of the prepared surface for overlay pour. The surface roughness is measured by matching the surface with the surface profile chips provided by ICRI. The surface profile is the measure of the average distance from the peaks of the surface to the valleys as observed through a cross sectional view of the surface of the concrete. This dimension is defined pictorially and through physical samples in the ICRI (International Concrete Repair Institute) Technical Guideline No 03732, and is expressed as a Concrete Surface Profile number (CSP 1 through 9). Figure 2.1 shows the corresponding preparation methods. 


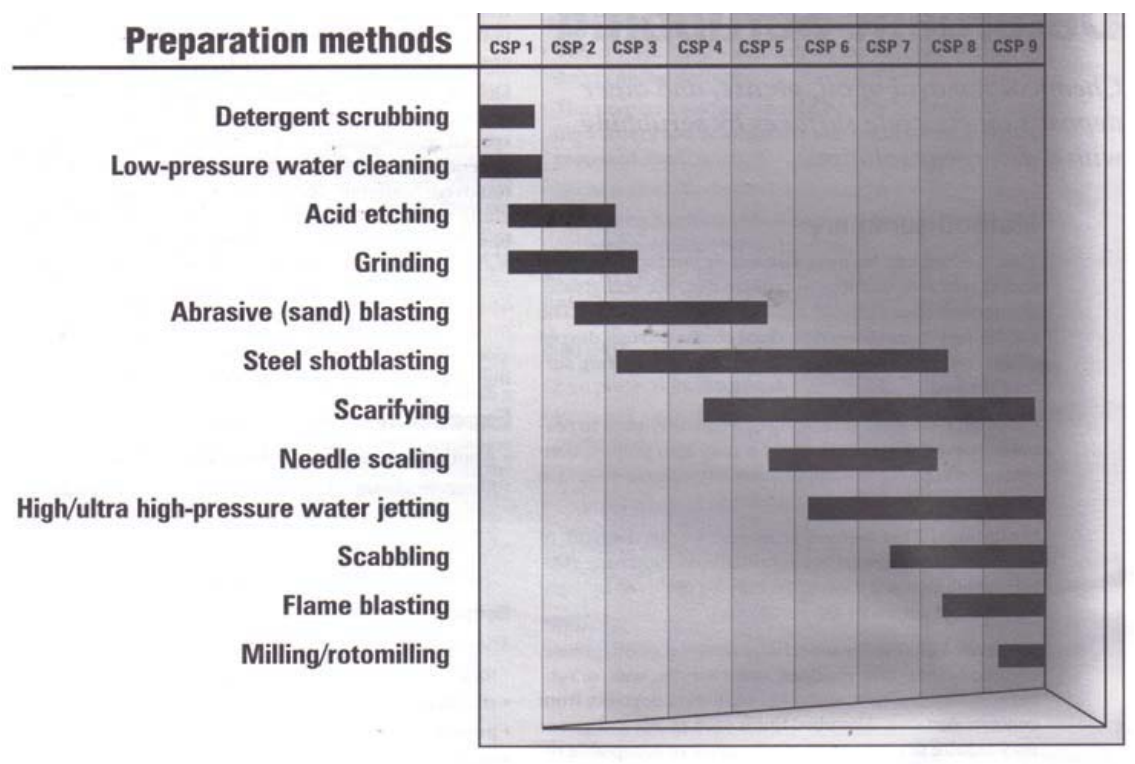

Figure 2.1 : Concrete surface preparation methods (reproduced from: ICRI guidelines)

\subsection{Delamination, Curling, and Cracking of Overlays}

Plastic shrinkage is a potential problem when LMC overlays are placed on a dry, windy day since; LMC has a lower water-cement ratio compared to the conventional concrete, it bleeds. If evaporation rate exceeds the bleeding rate, concrete undergoes plastic shrinkage. LMC's have low bleeding rate and which results in plastic shrinkage. Sprinkel (1988) observed severe plastic shrinkage cracks in the westbound lane of the bridge on Route 5 over the Chickahominy River in Virginia. Thin LMC overlay of thickness $25 \mathrm{~mm}$ ( 1 in) was placed in eight different pours on the bridge deck. He mentioned that when the evaporation rate exceeded $4.8 \mathrm{~Pa} / \mathrm{hr}$ ( $0.1 \mathrm{pound} / \mathrm{square}$ foot/hr), plastic cracks appeared in the LMC overlay studied. He suggested that whenever temperature, humidity, and wind conditions are such that the evaporation rate exceeds 4.8 $\mathrm{Pa} / \mathrm{hr}$ ( 0.1 pound/square foot/hr), proper precautions must be followed such as: building wind breaks, starting early curing, cooling the concrete, and using fog sprays.

Kuhlmann (1991) discussed about the causes of cracking in LMC overlay as follows: Latex Modified Concrete (LMC) exhibit two types of cracks: internally caused and externally caused. 
Internal cracks also known as plastic or shrinkage cracks are due to poor construction practices. LMC is more susceptible to internal cracks because of the lower water content versus conventional concrete. Therefore, LMC must be carefully protected if extreme drying conditions exist. Plastic shrinkage can be reduced in two ways: 1) place concrete when the evaporation rate is low and, 2) place the curing cover close behind the finishing operation. An evaporation rate less than $7.2 \mathrm{~Pa} / \mathrm{hr}\left(0.15\left(\mathrm{lb} / \mathrm{ft}^{2}\right) / \mathrm{hr}\right)$ is desired. Wet burlap and a white polyethylene film is a typical curing cover for a LMC overlay. A white polyethylene film is used to reduce solar heat gain and an undesired increase in temperature of the fresh concrete. LMC is affected by drying shrinkage similar to any other concrete. Latex does not affect drying shrinkage but rather the water-cement interaction. This influence is caused by the amount of water added to the mix and every precaution should be made to avoid adding excess water. Tests have shown that LMC and conventional concrete have the same shrinkage characteristics when using the same water-cement ratio. Trail mixes should be prepared in a laboratory so proper measuring, mixing and testing of the ingredients can be studied.

External cracks are due to structural movements, reflective cracks from the deck, thermal expansion and tearing while finishing. Cracks less than $3.2 \mathrm{~mm}$ (1/8 in.) deep will have little or no effects on the permeability of the LMC overlay. If a crack is deeper than $3.2 \mathrm{~mm}(1 / 8 \mathrm{in}$.), it requires a low viscosity sealer.

Cracks from structural movements occur when excess traffic cause excessive bending in the negative moment region. While LMC's tensile strength is higher than conventional concrete, it is not designed to withstand this excessive loading. These cracks occur in the transverse direction and are spaced approximately $610 \mathrm{~mm}$ (2 ft.) to 1220 $\mathrm{mm}$ (4 ft.) apart.

Reflective cracks result from movement of the deck which reflects through the overlay. Cracks in the deck must be carefully inspected to determine if they are stable before placing the concrete overlay. Proper placement around expansion joints is 
imperative. Placing an overlay over an expansion joint and saw cutting the overlay the following day will result in cracking over the joint and possible debonding next to it. It is required to place an expandable material over the joint and pour the overlay against it. After curing, expandable material is to be removed and the final joint material should be placed.

Thermal cracking occurs when the deck and the overlay expand and contract at different rates. Placing a LMC overlay in the early morning when the deck is the coolest will allow the overlay and the deck to expand together as the ambient air temperature increases throughout the day. Late evening is another popular time for placement due to the same effect.

It is speculated that placing an overlay next to a live lane of traffic has been reason for the appearance of uniform cracks. There is no conclusive field data to confirm this idea but placing the overlay during times of low traffic volume or slow moving traffic should be considered.

The presence of cracks in a LMC overlay does not mean the entire overlay needs to be replaced. The cracks should be studied through measures such as taking cores to determine the effect the crack has on the permeability of overlays. Cracks less than 3.2 mm (1/8 in.) typically do not have a significant effect on the overlay. Anything over 12 $\mathrm{mm}(0.5 \mathrm{in}$.) has a severe effect on the performance of overlays and requires a sealant.

The deterioration of concrete overlays occurs mainly due to the temperature changes, freeze and thaw cycles, concrete shrinkage, and repeated loading from the moving traffic. In the bi-layer deck system, the substrate concrete resting freely on its supports undergoes shrinkage whereas the concrete overlay pavement cast on substrate concrete cannot shrink freely due to the restraints from the underlying concrete pavement. This difference in shrinkage produces the tensile stresses at the interface. Similarly, the difference in temperature produces the stresses at the interface because of the difference in expansion and contraction of the overlaid concrete compared to the 
substrate concrete due to the change in temperature. Further, due to the traffic loading on the pavement induces the bending stresses in the slab and due to the presence of existing cracks in the substrate pavement, maximum bending stresses occur in the overlay pavement near the existing cracks as shown in Figure 2.2. Due to this difference in bending behavior between overlaid pavement and substrate concrete pavement delamination occurs at the interface starting from the existing crack.

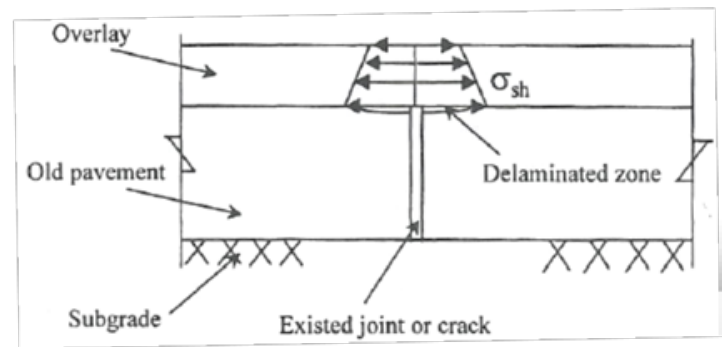

(a)

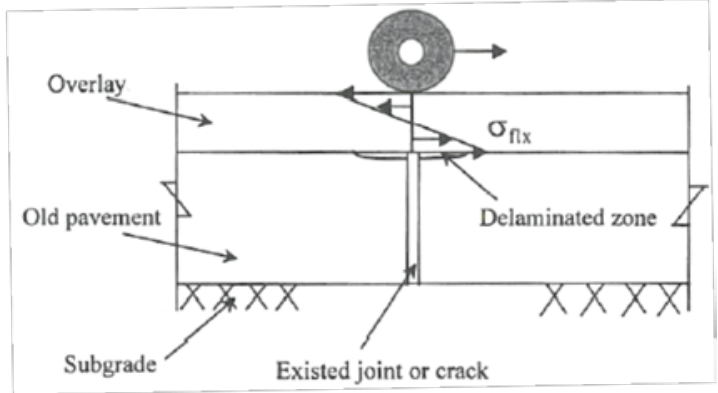

(b)

Figure 2.2: a) Shrinkage or temperature induced tensile stresses, and b) Flexural stresses due to moving load (reproduced from: Zhang and Li (2002))

These cracks developed near the existing crack in the substrate concrete pavement are called reflective cracks in overlaid concrete pavement. Further, these reflective cracks developed in the overlaid concrete pavement undergoes rapid freeze-thaw cycles due to which the water seeped into the cracks freezes and expands which in turn results in the expansion of crack width and depth. After the cracks are developed through the thickness of the overlaid pavement, water moves gradually through the existing cracks into the underlying concrete which is not desirable. The passage of trucks on the overlay cracks results in spalling, or in other words reduces the service life of the bridge.

Zhang and $\mathrm{Li}$ (2002) studied on the reflective cracking by conducting experiments on the application of fiber reinforced engineered cementitious composites (ECC) in the overlaid concrete and Plain Concrete (PC) as the overlay material by investigating the monotonic and fatigue performance under flexural loading. They used two kinds of substrate casting surface, one was a smooth diamond saw cut surface and the other kind 
was a sand-blasted surface. They included a vertical crack in the old concrete and an initial interfacial crack between two concretes was introduced and monitored the deflections due to four-point bending carefully. They concluded that the use of ECC as overlaid material on the substrate concrete significantly increased the load carrying capacity and deformability compared to the PC overlaid beams. They also observed that the certain length of unbonded zone along the interface of ECC overlaid beams above the existing old cracks was required to achieve the deformation level of the layer needed and the debonding at the interface of ECC overlaid beams was enhanced by the smooth cutting surface of the concrete substrate.

Granju (1996) mentioned that the debonding of overlays is mainly caused due to the development of shear stresses at the interface, which are caused as a consequence of the difference in the length change between the overlay concrete and substrate concrete or by external mechanical actions due to traffic loads. He proposed the debonding mechanism as shown in Figure 2.3. He explained that the most critical zone for debonding is not under or near the applied loads but occurs in the sections of maximum negative bending. He mentioned that the joints cutting the overlay act as cracks and in order to prevent these cracks from inducing debonding they should be located above the joints in the base slab.

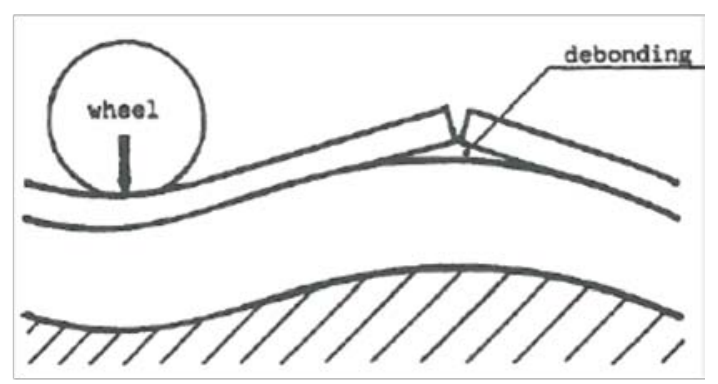

Figure 2.3: Proposed debonding mechanism (reproduced from: Granju (1996))

Curling of the bi-layer deck system is caused mainly due to the differential shrinkage that occurs in an overlay concrete since; the exposed top surface of the overlay concrete shrinks, whereas the underlying substrate concrete does not (Mailvaganam et al. (2000)). This differential shrinkage occurs due to several reasons such as: drying; 
carbonation of the surface concrete; internal desiccation that occurs as the cement paste hydrates (autogenous shrinkage); and also due to moisture gradient in the overlay concrete, since the newly cast concrete dry from top to bottom. If the cement paste and fine aggregates are concentrated at the surface due to bad finishing techniques, it will aggravate the differential shrinkage process. Also, the heat produced due to hardening of fresh concrete will aggravate this process. Curling mainly occurs at the construction joints, cracks, and saw-cut joints as shown in Figure 2.4.

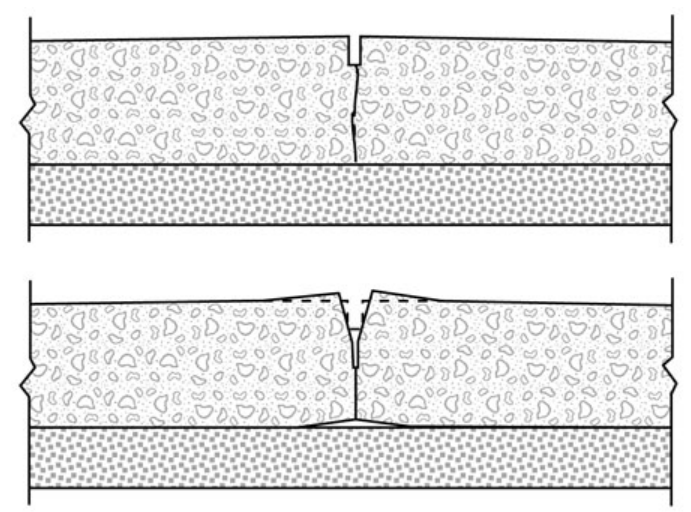

Figure 2.4: Curling of the slabs at construction joints (reproduce from: Mailvaganam et al. (2000))

Lau et al. (1994) studied on the interface shear stresses at the interface between the existing concrete and overlay concrete. The interface shear stresses were calculated by using the finite-element method for different loading conditions. The effects of vertical wheel loads, temperature loading, and wheel loading were included in their study. Also, the computed shear stresses at the interface were compared with the reported values of shear strength of laboratory prepared specimens. It was concluded that the maximum shear stress occurred at the perimeter of the loaded area under the action of normal wheel load and thermal warping whereas, in the case of horizontal braking force, maximum stress occurred at the center of the loaded area. They found that the calculated shear stresses are less than 1/20 of the expected bond strength. It was concluded that not only the wheel loading alone is not so critical in a properly bonded bi-layer system but also the influence of other factors such as environment effects and stress concentrations 
at small defects in the field are significant in determining the performance of interface bonds.

Shin and Lange (2004) studied on the effects of shrinkage, thermal changes and thermal gradients on the early age behavior of bonded concrete overlays by conducting extensive experiments on the high performance concrete (HPC), fiber reinforced concrete (FRC), and Ordinary Portland Concrete (OPC) bonded concrete overlays fabricated in the laboratory. In order to assess the debonding behavior due to volume changes, they developed a finite element modeling. They measured the tensile bond strength, shear bond strength, elastic modulus of elasticity of substrate and overlay concretes, selfweight of overlays and substrates, and a typical value of thermal coefficient and use these parameters as an input for the finite element model. They observed the temperature gradient was relatively small compared to the shrinkage gradient hence they concluded that the temperature gradient was not a major factor for debonding. They observed tensile bond strength of concretes containing high w/c ratio was greater than that of concretes containing lower w/c ratio. They found the debonding length and crack mouth opening displacement of HPC was higher which was due to high shrinkage and low bond strength. Based on the study important recommendation made by them was: appropriate material design (material should be selected so that low shrinkage gradient and high tensile bond strength can be developed at an early age) and construction technique (temperature and humidity conditions should be monitored to prevent the early age failure) should be developed to address the issues like development of tensile bond strength and development of stress gradients in the overlay.

Tang (2000) studied on the cracking and delamination of the $76.2 \mathrm{~mm}$ (3 in.) conventional overlays on the concrete segmental box girder bridge to understand the failure mechanism. He found that the cause of delamination was the combined result of shrinkage of the overlay, nighttime temperature gradient, and inadequate bond strength at the interface. In the early days after placement, temperature induced stress was predominant and then the shrinkage induced stress was predominant. The interface shear stress does not increase with the increase in live load but the penetration of shrinkage 
cracks toward the interface will increase the stress at the interface. The penetration of shrinkage cracks toward the interface can be controlled by providing the appropriate curing procedure.

Hong (2006) studied on the edge curling behavior of the bi-layer system with four different types of overlays: LMC, SFMC, SFMC with Shrinkage Reducing Admixture (SFMC+SRA), and FRC on normal substrate concrete. Pre-notch was induced at one corner to introduce the delamination at the interface. Prismatic rectangular specimen of dimensions $450 \mathrm{~mm}$ (18 in.) by $200 \mathrm{~mm}$ (8 in.) by $100 \mathrm{~mm}$ (4 in.) substrate concrete was cast, and on top of that $50 \mathrm{~mm}$ (2 in.) overlay was cast. The edge curling was monitored by installing LVDT and clip on gages. Based on the results he concluded that the interface crack was initiated at the corner and then propagated through the edges and then into the specimen. He showed that the saturated surface with bonding slurry condition had the best performance followed by the saturated surface. The totally dry surface should be avoided since this showed the largest relative displacement at the interface due to curling effect of overlay. Also, he proved that the specimens at high temperature had the larger relative displacements at the interface.

Yun et al. (2007) analyzed the causes of map and transverse cracking in VeryEarly Strength Latex-Modified Concrete (VES-LMC) and they provided the control methods for minimizing the crack occurrence in the field in Korea. They selected bridge VES-LMC bridge deck overlay for the study and the field instrumentation included the button to monitor the wind speed, relative humidity, concrete temperature, and air temperature. They observed map cracking, occurred mainly due to initial plastic shrinkage and in order to prevent this map cracking they recommended applying the curing compound immediately after placing and before tining. Transverse cracks were observed at very early age which was mainly due to the thermal cracking. To prevent these transverse cracks, they recommended that one should focus on the material properties of VES-LMC material properties such as: low amount of VES cement, possible low early-strength concrete, low hydration temperature, and minimum cement paste volume. 


\subsection{Bond Tests and Pull-Off Tests}

The most common and effective way of extending the life of concrete bridge decks is installing a bonded concrete overlay on the existing structure. The durability of the overlaid concretes depends on the durability of their bond with the substrate concrete i.e., a good bond prevents the water and de-icing salts to penetrate through the interface and hence increases the service life of the bridge deck overlay system and therefore studies should be performed on bond to determine the service life of repaired concrete structures Paulsson and Silfwerband (1998). There are different types of bonding tests developed by researchers to study the bond properties between the overlay and substrate concretes. Silfwerbrand (2003) showed schematically different types of bonding tests that can be performed in the lab and in-situ as shown in the Figure 2.5

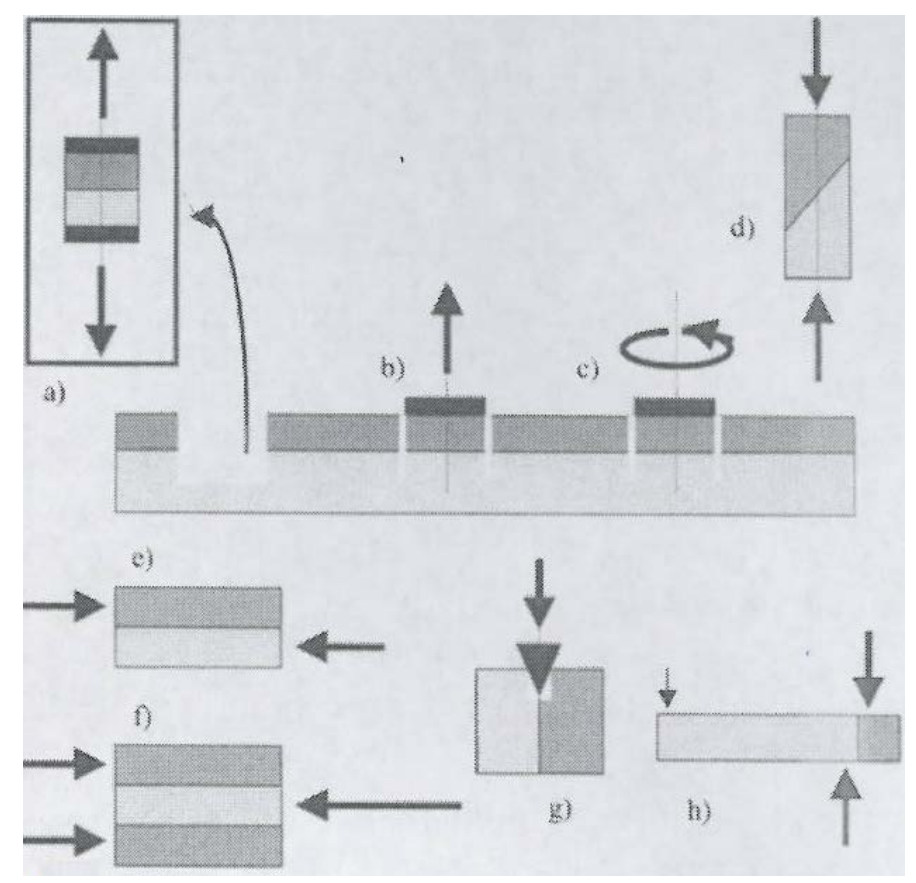

Figure 2.5: Types of bond tests that can be performed in the lab and in-situ (reproduced from: Silfwerbrand (2003))

The tensile bond strength of the interface between overlay concrete and substrate concrete can be measured by applying pure tension loads to the specimens either in the 
laboratory or in-situ. The former one can be performed by cutting the cores in field and carrying them to the laboratory and after further preparation of the specimens direct tensile load is applied as shown in Figure 2.5 (a) and the latter one called pull-off testing can be performed by cutting partial cores into the substrate and applying the tensile load to the prepared specimens directly in the field as shown in Figure 2.5 (b). In both the cases, tensile strength of the overlay concrete, substrate concrete or interface can be measured based on the failure mode of the specimens i.e., the failure at the interface gives the interface tensile bond strength and the failure of overlay concrete or substrate concrete gives the tensile strength of the overlay concrete or the substrate concrete respectively. Figure 2.5(c) shows the shear test method that can be performed in-situ, in this method shear bond strength is calculated by applying a torsional moment to the core. The most common method of determining bond strength in the laboratory is slant shear method as shown in Figure 2.5(d), in this method; bond strength is measured by applying compressive load to the bi-layer specimen repaired in $30^{\circ}$ angle, but the interface is subjected to combined compression and shear. Shear strength can be determined by applying shear forces parallel to the interface as shown in Figure 2.5(e) and Figure 2.5(f). The disadvantage of the methods in Figure 2.5(e) is; this specimen is subjected to bending moment as soon as the load is applied. However, this disadvantage can be rectified by preparing a test specimen consisting of three parts, but this test method has two interfaces and in reality, this kind of repair does not exist. Uni-axial wedge splitting test developed by Tshegg et al. (2000) is shown in Figure 2.5(g), they used this method to measure the fracture properties of the bond. Figure 2.5(h) shows the guillotine test, in this method bond strength is measured by dropping the weight on the protruding interface. Of all the test methods described briefly above, test methods shown in Figure 2.5(b) and Figure 2.5(c) can be used in-situ, whereas the test methods shown in Figure 2.5(a), and Figure 2.5(d) through Figure 2.5(h) can only be performed in laboratory.

The most commonly used bond strength tests include measuring the bond strength through pure tension tests, for instance pull-off test is the most commonly used test insitu and shear bond strength, measured by using the torque tests, can also be performed in-situ but less popular compared to pull-off test method. 
Further, various factors affecting the bond between old and new concrete, different types of bond testing methods performed in field and laboratory, and advantages of using one method over other explained by various researchers are discussed here.

Wall and Shrive (1988) studied on the factors such as; bond materials modulus of elasticity, bond thickness, bond weakness, and casting procedure, which affects the bond between newly placed concrete and old concrete. These factors were studied by conducting numerical finite element analysis and experimental studies on slant shear specimens of dimensions 102 mm (4 in) x102 mm (4 in) x 305 mm (12 in). They found that, the bond material with modulus of elasticity similar to that of the adjacent concrete is desirable, and there was no significant difference in the results due to the slight offset between the new and old concrete occurred during casting procedures. Also, they found that the bond material with more thickness reduced the bond strength; hence the maximum thickness was limited to $3 \mathrm{~mm}$ (1/8 in).

Hindo (1990) devised a new method to determine the in-place direct tensile strength of repair material using the LOK-TEST pullout device. He explained the procedure for performing this test as follows: Initially, a partial core has to be drilled in the test location and the depth of the cut should be extended beyond the interface into substrate concrete to accurately measure the bond strength, followed by attaching a circular steel plate with fast setting epoxy. Once the epoxy is cured, a loading frame is placed on the prepared specimen and loading is applied until the failure and the ultimate load is recorded to calculate the tensile strength. The procedures for core drilling and test set up are as shown in Figure 2.6. 


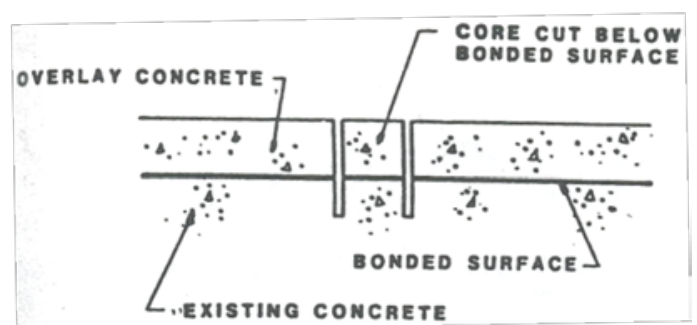

(a)

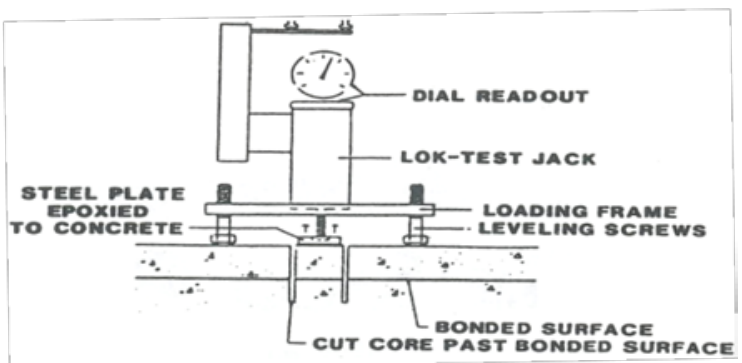

(b)

Figure 2.6: a) Preparation of specimen for in-place bond test, and b) LOK-test setup (reproduced from: Hindo (1990))

He concluded that, this test is a useful tool for performing the bond tests directly in the field and represents the actual field conditions. Hence this method can be used for quality control purpose during construction repairs.

Kuhlmann (1990) studied the bond strength properties on four series of LMC concrete and mortar laboratory specimens by using the pipe-nipple grip test. He claimed that by the visual inspection of the failed specimens we can determine the weakest component of the bi-layer. The pipe-nipple grip test method can be used to measure the bond of LMC at curing times as early as 1 day. The bond of LMC exceeds 0.48 MPa (70 psi) at 1 day, 2.34 MPa (340 psi) at 28 days, and 3.1 MPa (450 psi) at 90 days of curing at room temperature. He recommended studying the effect of overlay thickness on the failure load and mode of failure. He also suggested that the effect of water submersion at various curing times and the surface preparation should be studied.

Chen et al. (1995) measured the shear bond strength between new and old mortar by sandwiching a newly placed mortar between two old mortar pieces cured for 28 days. The sandwich specimens are subjected to shear using the test configuration as shown in the Figure 2.7. 


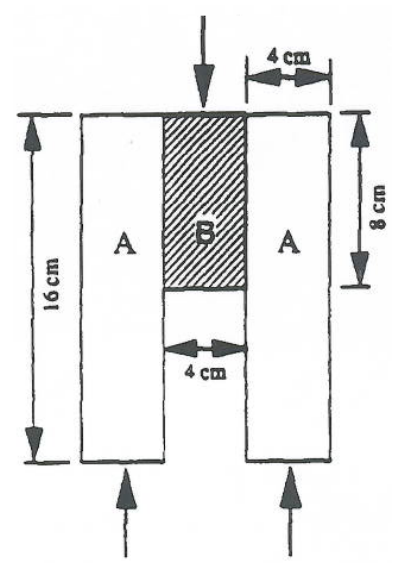

Figure 2.7: Test method for determining shear bond strength between old mortar (A) and new mortar (B) (reproduced from: Chen et al. (1995)

The presence of three parts prevents the development of bending moment, which is not desirable, due to the applied load. However, this test method has some disadvantage since, two interfaces exist and such type of repair hardly exists in reality Silfwerbrand (2003).

Ali et al. (1998) developed a new testing method for measuring the shear bond strength at the interface, where newly cast concrete on old concrete and precast elements bonded to existing concrete structures, in-situ by using torque wrench as shown in the Figure 2.8 below.

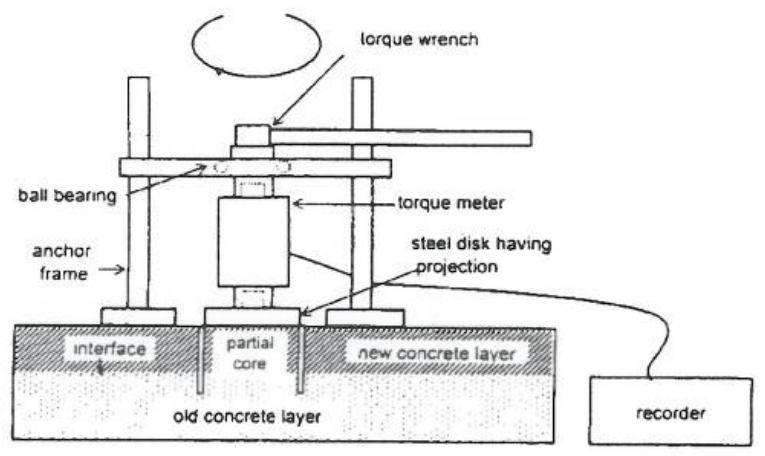

Figure 2.8: Shear bond strength measured by torque test setup (reproduced from: Ali et al. (1998)) 
They performed torque tests and pull-off tests simultaneously on new layer of cement mortar cast on $1000 \mathrm{~mm}$ (3.3 ft.) x $1000 \mathrm{~mm}$ (3.3 ft.) x $100 \mathrm{~mm}$ (0.33ft.) cement mortar slabs, new layer of concrete cast on $1300 \mathrm{~mm}(4.3 \mathrm{ft}$.) x $1100 \mathrm{~mm}(3.67 \mathrm{ft})$ x 100 $\mathrm{mm}(0.33 \mathrm{ft}$.) concrete slabs and precast concrete stiffened by steel pipes as the top concrete layer to investigate the relationship between the shear bond strength and tensile bond strength. They showed the different types of failure modes which are very useful for knowing the conditions of the repair material, old concrete or interface bond as shown in the Figure 2.9 below.
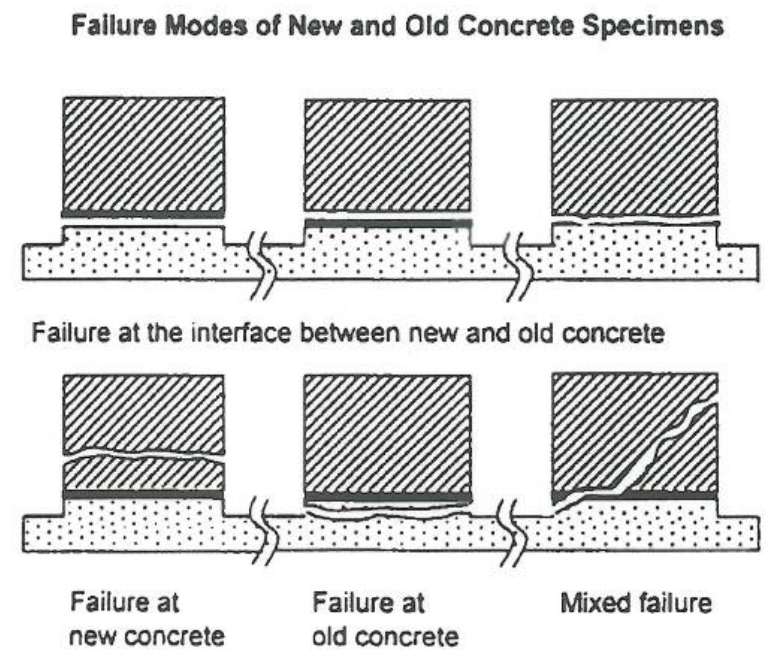

Figure 2.9: Types of failure modes (reproduced from: Ali et al. (1998)).

Based on the results they concluded that without the use of bonding agent, the shear bond strength depended on the degree of surface roughness. The use of bonding agents such as Styrene Butadiene Polymer and epoxy improved the bonding strength at the interface and the failure of specimen never occurred in the interface. Also, when no bonding agent is used or a cement mortar used as a bonding agent developed weak bonding shear strength and the failure occurred at the interface. They observed a linear relationship between shear bond strength and tensile bond strength based on the results.

Delatte et al. (2000) presented the methods for predicting the bond strengths (compressive, splitting, tension and shear bond) development at the early ages based on 
the maturity of the given concrete provided the other variables (curing methods, cement compositions, and specific heat of the aggregates) are controlled. They performed standard compression and splitting tensile tests on $100 \mathrm{~mm}$ (4 in.) x $200 \mathrm{~mm}(8 \mathrm{in}$.) cylinders. The bond tests (tensile and shear bond tests) were carried out on $840 \mathrm{~mm}$ (2.75 ft.) x $530 \mathrm{~mm}$ (1.75 ft.) x $100 \mathrm{~mm}$ (4 in.) bi-layer slabs. Tensile bond tests were carried out by modifying the available pull-off tester by attaching a tester to the bolt cast into the concrete overlay instead of attaching steel disk to the core and they proved this method to be more reliable and produced an interface failure at every instance. The shear bond test was carried out by cutting a full depth $100 \mathrm{~mm}$ (4 in.) core and placing them in a guillotine tester and applying moving load on the overlay half of the cylinder. The tensile and shear bond tests used by them are shown in Figure 2.10 below.
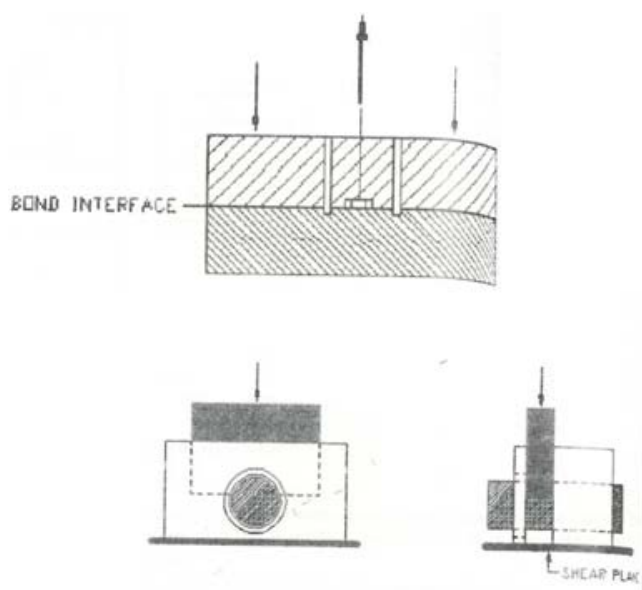

Figure 2.10: Tension test and shear bond test in progress (reproduced from: Delatte et al. (2000))

Simultaneously, they recorded the temperatures by using embedded thermocouples and maturity was calculated by substituting the temperature values in maturity equation. Knowing the maturity, the strength parameter is calculated and the best fits were plotted. Based on their laboratory studies, they concluded that the prediction of the bond strengths can be made by the maturity method and the shear bond strength value is approximately twice of the tensile bond strength. 
Tshegg et al. (2000) studied the fracture properties of the old-new concrete bonds with and without anchor reinforcement by using a uniaxial wedge splitting test developed by them. They concluded that the good bond can be achieved only by the optimized roughening of the old concrete surface and the crack initiation behavior is mainly determined by the type of interface between old and new concrete.

Silfwerbrand (2003) performed pull-off tests and torsion tests on five test slabs in the laboratory and on a bridge between Göteborg and Trollhättan in the west of Sweden. For the laboratory test slabs three different types of removal techniques (water jetting, pneumatic hammers, and sandblasting) were compared and on a bridge, repair was done by water jetting to remove the deteriorated concrete on a bridge. He concluded that the shear bond strength is higher than the tensile bond strength and the ratio between average shear stress and average tensile tests varied between 1.9 and 3.1.

Momayez et al. (2004) developed a new direct shear test to determine bond between existing concrete and repair material. This test method was validated by conducting tests on 192 specimens considering variables like specimen size, maximum aggregate size of repair materials, and type of repair material, interface roughness and age at loadings. They concluded that the average COV of the test was less than $10 \%$ and hence the bi-surface shear is a reliable test to measure the shear stress at the interface. The shear stress is dependent on the size of the specimen, the repair material used, i.e., the larger specimens resulted in smaller shear stresses and the results obtained were higher for stronger materials. Also, they concluded that the increase in surface roughness resulted in increase of shear stresses by 19\% and the use of larger aggregates increased the bond strength slightly. The 14 day strength was $72 \%$ of the 28 day strength and the addition of silica fume increased the bond strength.

The guidelines for measuring the tensile bond strength of the LMC overlays in the field were provided by Yun et al. (2004). They performed FEA by considering various factors: normalized steel disk thickness, normalized overlay thickness, normalized cutting depth, normalized distance from edge of slab, and normalized distance between cores and 
all these factors were considered relative to the core diameter; which effects the bond strength and the results were verified by conducting the experimental work and the experimental results showed good agreement with the FEA results. Based on the results obtained they concluded that, in order to prevent the steel disc interface failure the thickness of the steel disk should be at least 0.3 times the core diameter, also, to attain a pull-off test with the uniform stress distribution at the concrete interface the overlay thickness should be at least 0.4 times the core diameter and they observed, the change in either core distance or edge distance did not affect the stress concentration much at the concrete interface as long as one core distance is maintained. Finally, they recommended the diameter of core should $100 \mathrm{~mm}$ (4 in) and the depth of cut should be between $25 \mathrm{~mm}$ (1 in) and $40 \mathrm{~mm}$ (1.7 in) for optimal performance.

Ray et al. (2005) developed a new direct shear test method at West Virginia University to evaluate the interface properties of four types of high performance concrete overlays on top of normal concrete. Four types of overlays selected were SFMC, LMC, FRC, and Silica Fume Modified Concrete with fly ash (SFMC-FA). Results showed that the successful bond characterization was possible with a new direct shear apparatus. From the consistency of test results and mode of failure observed it was concluded that the proposed test method is effective for the evaluation of bond strength of a commonly used overlay materials.

Ray et al. (2008) studied on the influence of various parameters such as overlay mixture types, surface preparations, application of slurry, age of the substrate, and aggregate type on the shear bond strength. The conclusions made from the study were: Silica Fume Modified Concrete (SFMC) had the strength followed by Latex Modified Concrete (LMC), Fiber Reinforced Concrete (FRC), and Slag Modified Concrete (SLMC); surface preparation CSP \#8 exhibited higher bond strength than CSP \#5; bonding slurry at the interface have little effect on the bond strength; Increase in substrate age increased the bond strength; limestone aggregate improved the bond strength compared to gravel aggregate. 
From the above literature survey, we can conclude that pull-off test is most widely and successfully used test method in field to study the interface bond properties by cutting the partial cores below the substrate concrete. Same test method can be implemented to measure the tensile strength of the overlay material and the substrate concrete depending on the depth of the core cut into the bi-layer system.

\subsection{Ultrasonic Pulse and Signal Processing}

To evaluate the performance of an existing concrete structure, researchers are more and more relying on nondestructive types of testing. Nondestructive testing has been in practice from many decades to detect the defects. ACI 228.2R-98 (1998) defines Nondestructive testing as testing that can be performed on the concrete structures such as in deep foundations, bridges, buildings, pavements, and dams without causing any damage to the structure. Need of conducting non destructive methods on concrete construction are: quality control; trouble shooting of problems; evaluation of condition of older concrete for rehabilitation purpose; and quality assurance of concrete repairs. Several methods of nondestructive testing such as: visual inspection, ultrasonic pulse velocity testing, ultrasonic-echo testing, impact-echo testing, spectral analysis of surface waves, sonic-echo, impulse-response, impedance logging, cross hole sonic logging, and parallel seismic are available. Each test method has their advantages and disadvantages. The selection of the test method depends on the specific purpose.

Of all these test methods Ultrasonic Pulse Velocity (UPV) was selected for this study. UPV test is a nondestructive test which uses mechanical waves resulting in no damage to the concrete structure itself and can be done multiple times in a particular place again and again. This test can be used to detect internal cracking or any other defect inside concrete resulting from aggressive chemical environment and freezing and thawing. Vibrational and stress wave propagation methods were being used by researchers long back. The development of the pulse velocity test method began in Canada and England. Since 1960s, the use of ultrasonic pulse testing increased rapidly and moved to construction sites from the laboratories. 
In UPV test method, an ultrasonic pulse wave is created at a point on the surface of the test subject and the time of travel of the wave from one point to another point is measured. If the distances between the points are known, the pulse velocity can be easily calculated. If the surface of a large solid elastic medium is disturbed by a dynamic load, three types of mechanical waves are created; namely (1) compressional waves, (2) shear waves, and (3) surface waves. Compressional waves are similar to sound waves propagating through air and has the highest velocity between the three mentioned above. For elastic, homogeneous and solid media, the compressional wave velocity is given by:

$$
\begin{gathered}
V=\sqrt{\frac{K E}{\rho}} \\
V=f \lambda
\end{gathered}
$$

Where, $E$ is the dynamic modulus of elasticity, $\rho$ is the density; $K$ is $K=(1-\mu) /(1+\mu)(1-2 \mu)$ and depends on dynamic Poisson's ratio $\mu$. The frequency $f$ and wavelength $\lambda$ of the propagating wave motion are related by Equation 2.2. In a given medium, an increase in wave frequency therefore indicates a decrease in the wavelength and vice versa. As seen from Equation 2.1, the velocity of compressional wave depends on the elastic properties and density of the medium. This is the basic working methodology used in the UPV test. 


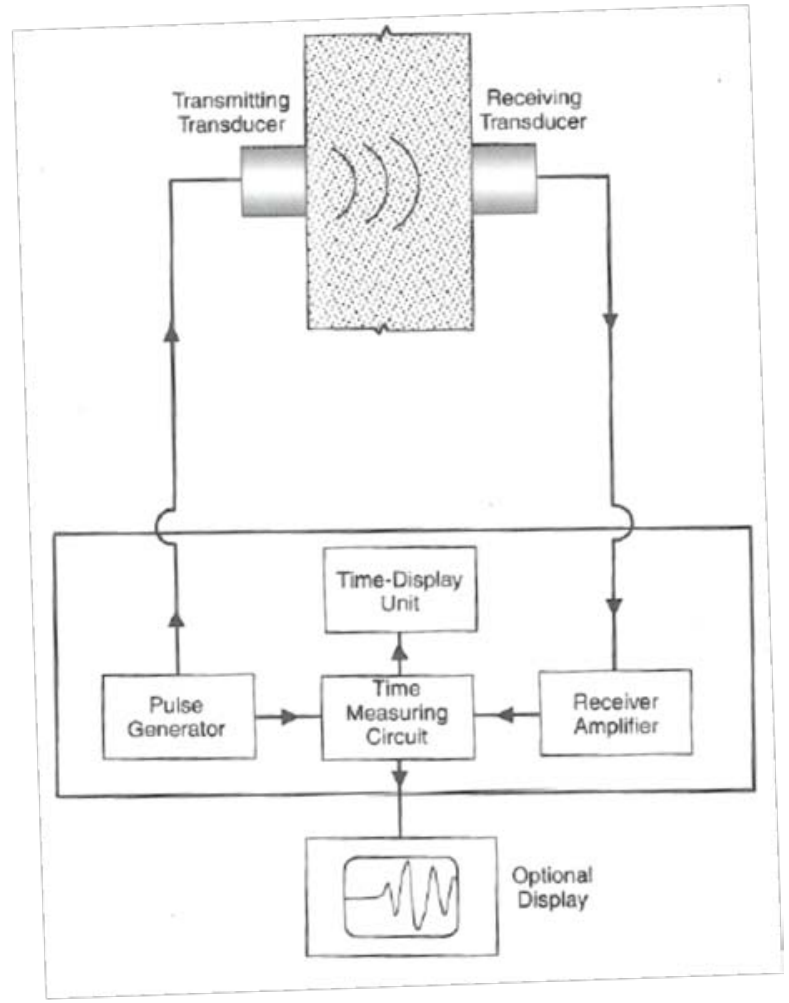

Figure 2.11: Schematic representation of UPV test method (reproduced from: ASTM C 597(2002b)

As seen from Figure 2.11, the transmitting transducer produces and transmits a wave into the concrete and the receiving transducer, at a distance $L$, receives the pulse through the concrete. The UPV test instrument shows the transit time it takes for the wave pulse to travel through the concrete. Hence, the distance $L$ divided by that transit time gives the wave pulse velocity $V$. The pulse through the concrete undergoes scattering at various aggregate-mortar boundaries and is transformed into a complex wave form when it reaches the receiving transducer. To effectively transmit or receive the wave pulse, the transducers must be in full contact with the test medium otherwise air pockets interfere with the propagation and introduces error in measurement. The typical pulse velocity of ordinary concrete varies from 3700 to $4200 \mathrm{~m} / \mathrm{s}$. 


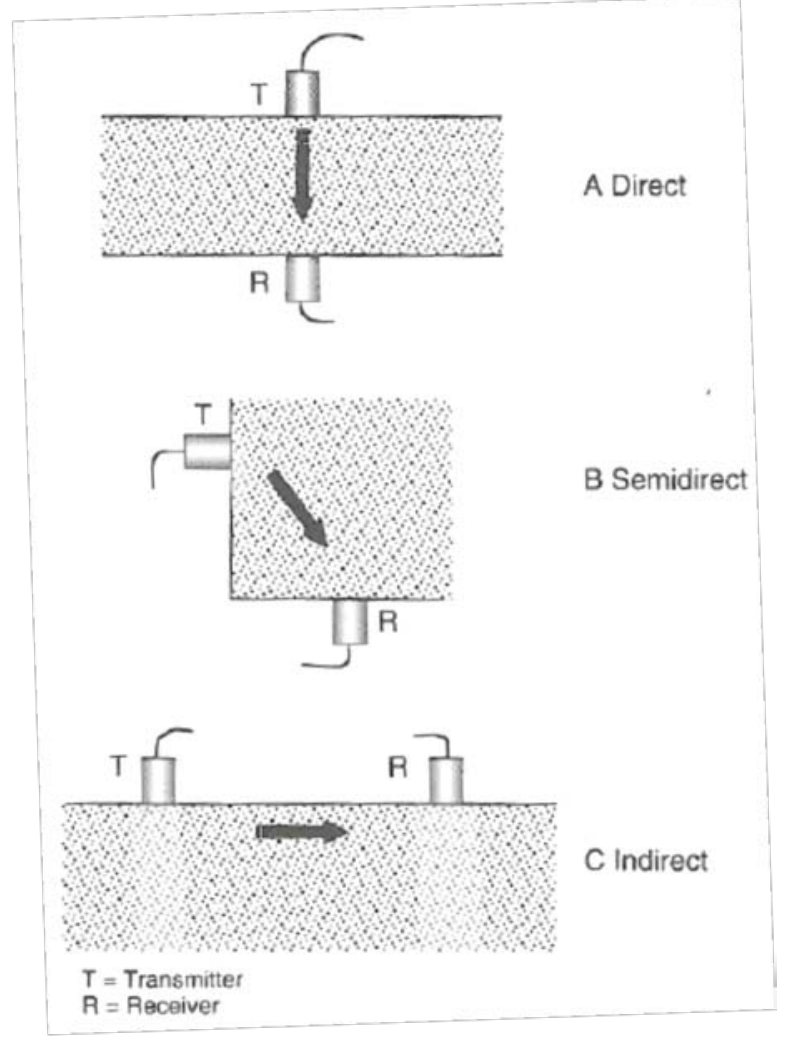

Figure 2.12: Possible test configurations of the UPV test (reproduced from: Malhotra and Carino (2004))

Figure 2.12 represents three possible configurations of the UPV test. These are (1) direct transmission, (2) semi direct transmission, and (3) indirect or surface transmission. Direct transmission is the most satisfactory and desirable process as maximum energy of the pulse is transmitted and received through this process. In the surface transmission process, the pulse propagates in the concrete layer near the surface. Hence, this process can be utilized to detect and estimate the thickness of a surface layer of different quality material.

There are various factors which affect the UPV test; some of them are described in detail here. The pulse velocity is significantly affected by the aggregate size, grading, type and content. Pulse velocity is lowest for concretes with rounded aggregates and highest for crushed granite. Water-cement $(w / c)$ ratio also has some effect on the velocity; as $w / c$ ratio increases, the pulse velocity decreases and vice versa. The effect of 
age of concrete is similar to that of compressive strength development. Admixtures and cement type did not have significant effect on the pulse velocity; although if it affects the rate of hydration, it may indirectly affect the pulse velocity through the concrete. Improper transducer contact is another factor which affects the pulse velocity. If good contact is not ensured, an incorrect pulse velocity reading may result. Pulse velocity of saturated concrete is higher than for air-dry concrete. The influence of moisture on velocity is high in high-strength concrete compared to low-strength concrete because of the difference in pore structure. Although, the path length of the wave should not affect the propagation time as well as the velocity; however in practice, smaller path lengths tend to give more variable and slightly higher velocity because of the inhomogeneous nature of concrete. Pulse velocity, in most of the cases, is not dependent upon the size and shape of a specimen. However, Equation 2.1 is valid for a medium having an infinite extent. That can be satisfied for a finite-dimension test specimen by requiring that the smallest lateral dimension of the specimen must be greater than the wave length of the pulse. One of the most influencing the pulse velocity is the presence of reinforcing bars. The velocity in steel is 1.4 to 1.7 times higher than in concrete, hence, pulse velocity readings tend to be higher in the vicinity of reinforcing bars and produces error. Correction factors should be used on the readings if reinforcements cannot be avoided.

UPV test can be successfully used for quality control as well as for the analysis of deterioration. It may also provide a mean of estimating strength of both in situ and precast concrete. The strength can be roughly estimated using pre-established graphical correlation between pulse velocity and compressive strength. Although it is not unique and affected by many factors, it provides a good non-destructive approach for estimating the strength of an existing structure. This test is very much useful and widely applied for relative assessment of the quality of concrete and its homogeneity. Heterogeneity is caused by deteriorations and imperfectness such as interior cracks, large air voids which causes diffraction of the wave pulse and change time of propagation. It is highly effective in establishing comparative data and qualitative evaluation of concrete. Qualitative comparison include (1) locating cracks, air voids, (2) locating honeycombed concrete, and (3) locating variation of density to evaluate consolidation effectiveness. 
Many researchers have used UPV test as a mean for monitoring the setting and hardening process of cement paste, mortar, and concrete. Evaluation of the rate of setting for different types of cement or admixtures is one of the significant practical uses of this process. Pulse velocity test can be also successfully applied to study the durability of concrete. Deteriorations such as sulfate exposure, alkali aggregate reaction, damages caused by freezing and thawing can be evaluated by this process and can be monitored by conducting repetitive tests on the same concrete element. As the velocity of a wave travelling through an elastic material depends on the elastic constants and density of the material as described by Equation 2.1, it is possible to compute the modulus of elasticity using UPV method if the Poisson's ratio and density is known or assumed properly. Many processes for the estimation of dynamic modulus of elasticity are available in the literature although it is not normally recommended due to error in estimating the Poisson's ratio and inhomogeneous nature of concrete. Another application of this test is to measure the depth of a surface crack. The underlying mechanism for that is the pulse will diffract around a defect whose projected area is perpendicular to the path length is larger than the area of transmitting transducer and the travel time will be higher.

Hence, the UPV test is a useful NDT technique that can be used in laboratories as well as fields with numerous advantageous applications. Although, it has some serious limitations, it adds a new dimension to quality control if concrete is in the field. It is a simple, easy to use, available from wide array of sources, portable and relatively inexpensive. These qualities make it an excellent means for investigating the uniformity of concrete and hence it is widely used as a successful NDT technique around the world.

Jones and Facaoaru (1969) recommended that there should be sufficient acoustical coupling between the concrete and transducers. Typical couplants that can be used for this purpose are solidol, technical Vaseline, liquid soap and kaolin or glycerol paste and when the surface of the concrete is very rough and uneven it should be made smooth before the transducer is fixed. Also, they recommended the minimum path length should be $100 \mathrm{~mm}$ ( 4 in) for concrete with maximum aggregate size less than $30 \mathrm{~mm}$ (1 in.) and $150 \mathrm{~mm}$ (6 in) for concrete with maximum aggregate size less than $45 \mathrm{~mm}(1.75$ 
in.). They provided a guideline for selecting the transducer natural frequency for various path lengths and transversal dimensions. They stated that the use of the ultrasonic pulse technique for locating flaws, voids or other defects in concrete is based on the negligible transmission of ultrasonic energy across a concrete-air interface. If any air filled crack or voids are present in between the transducers they will obstruct the direct ultrasonic beam when its projected area is greater than the area of the transducers and the first pulse arriving at the receiving transducer will be diffracted around the periphery of the defect and the transit time will be longer than in homogeneous concrete.

Yaman et al. (2001) concluded that the indirect UPV testing is statistically similar to the direct UPV testing by conducting experiments on concrete slabs. They mentioned that the above conclusion was made by considering the moisture gradient along the depth and the surface was uniform.

Promboon and Lund (2002) performed Impact Echo and UPV tests on a concrete bridge over Interstate 70 of Denver, Colorado which was repaired with polymer-modified repair mortar for the spalled areas and the epoxy injection was used for cracks to provide the quality assurance of the repairs. They used Impact Echo for locating the preliminary internal cracks at each test point and once the possible crack was found, UPV tests were performed in angular paths to find more information about the location and extent of the unfilled crack. Based on the results obtained they concluded that the use of combination of IE and UPV tests proved to be very effective in locating the unfilled cracks.

Ultrasonic signal-processing technique has been successful in recent years in material testing (Daponte et al. (1995)). Signal processing techniques when applied to the received ultrasonic sound improves our understanding of information contained in the signal. Usually a signal collected from the oscilloscope is viewed in the time-domain signal with ordinate as amplitude or voltage and abscissa as time as shown in Figure 2.13. In most of the cases, this representation is the most logical way to view and analyze them. However, when the frequency content of the signal is of interest time-domain domain signal is converted into frequency-domain and viewed in frequency domain. Ordinate of 
the frequency domain signal is still amplitude but the abscissa is viewed in frequency scale. Frequency domain representation shows how much of the signal's energy is present as a function of frequency. When the signal is a simple sine wave, frequency domain does not provide us much information but, when the signals are more complex, frequency domain provides much information about the signal.

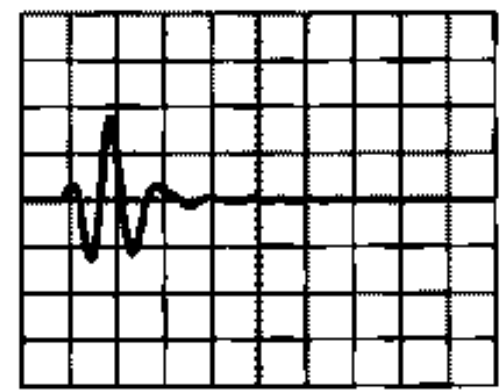

-Time Domain

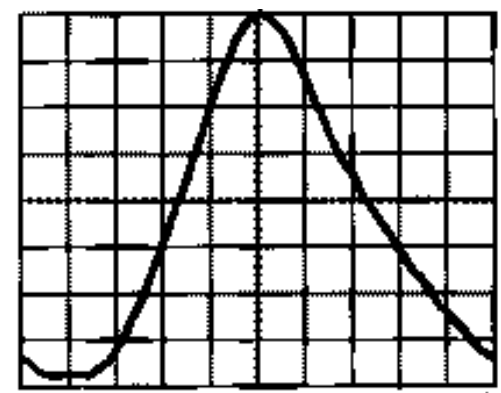

Frequency Domain (Magnitude)

Figure 2.13: View of the signal in time-domain and frequency-domain (Source: www.ndted.org)

The Fourier transform is one of the most power signal analysis and processing tools for transforming the signal defined in time-domain into one defined in the frequency-domain. It was developed by Jean Baptiste Joseph Fourier in 1807 and was named after him. Fourier theory says that any complex waveform can be decomposed into a set of sinusoidal functions with different amplitudes, frequencies, and phases.

According to the Fourier theory, periodic function $F(T)$, with period $T$ can be represented of the form:

$$
F(T)=\frac{a_{o}}{2}+\sum\left(a_{n} \operatorname{Cosn} \varpi_{T} t+b_{n} \operatorname{Sin}_{T} t\right)
$$

Where the coefficients $a_{o}, a_{n}$, and $b_{n}$ are calculated using the formulae:

$$
\begin{aligned}
& a_{o}=\frac{2}{T} \int_{0}^{T} F(T) \cdot d t \\
& a_{n}=\frac{2}{T} \int_{0}^{T} F(T) \cdot \cos n \varpi_{T} t \cdot d t \quad n=1,2, \ldots
\end{aligned}
$$


$b_{n}=\frac{2}{T} \int_{0}^{T} F(T) \cdot \sin n \varpi_{T} t \cdot d t \quad n=1,2, \ldots$

$\varpi_{T}=\frac{2 \pi}{T}$

Equation 2.3 is called the Fourier series and the coefficients are called the Fourier coefficients. Graph of the amplitudes of the Fourier series components can be drawn as a function of frequencies and such a graph is called a spectrum. The component $f_{o}$ defined as $f_{o}=\frac{1}{T}$ is called the fundamental frequency and all the higher components are multiples of $f_{o}$ and are called higher harmonics.

The Fourier Transform $X(f)$ used to transform a continuous time signal into the frequency domain can be expressed as:

$$
X(f)=\int_{-\infty}^{\infty} x(t) e^{-i 2 \pi f t} d t
$$

Where $x(t)$ is a continuous time function.

When both the time and the frequency variables are discrete the Discrete Fourier Transform function is used. This case arises when the digital computers are used to perform the analysis. The Discrete Fourier Transform function can be expressed as:

$$
\begin{aligned}
& X(k)=\sum_{j=1}^{N} x(j) \varpi_{N}^{(j-1)(k-1)} \\
& x(j)=\frac{1}{N} \sum_{k=1}^{N} X(k) \varpi_{N}^{-(j-1)(k-1)}
\end{aligned}
$$

Where, $\varpi_{N}=e^{\frac{(-2 \pi i)}{N}}$ is an Nth root of unity. 
The Fast Fourier Transform (FFT) is a special algorithm which implements the DFT with considerable savings in the computational which means that the computation of the DFT is done with considerable reduction in the number of calculations required. The maximum efficiency of computations is obtained by selecting the number of points to be an integer power of 2 such as 512, 1024, 2048, etc.

Generally, a function $f(t)$ is sampled in many common situations because, when the numerical procedures are to be evaluated on a function using the digital computers they cannot deal with the continuous functions. Let's consider a continuous time function $x(t)$, this continuous time function can be represented as a discrete time signal by using values of $x(t)$ at intervals of $n T_{s}$ to form $x[n]$, here $T_{s}$ is called sampling period. Often a sampling frequency $f_{s}=\frac{1}{T_{s}}$ is specified rather than the sampling period. If the accurate approximation of the signal $x(t)$ has to be made, the sampling period $T_{s}$ has to be small enough if the signal variation is significant because, the significant signal variation implies that higher frequency components are present in the signal. This leads to a phenomenon called aliasing when the sampling frequency is not high enough to sample the signal correctly. This term describes the phenomenon in which the components of the signal at higher frequencies are mistaken for components at lower frequencies and this phenomenon occurs when the continuous time signal has frequencies larger than half of the sampling frequency. In order to avoid this phenomenon, the sampling frequency should be selected greater than or equal to twice the highest frequency present in the signal, this theorem is called Nyquist Sampling Theorem and this sampling frequency is referred to as the Nyquist sampling frequency.

The Power Spectra estimation is the other important signal processing technique that can be used to find the distribution of the power contained in a signal over a finite set of frequency data. This estimation is useful in detecting the signals buried in a wide band noise. The power spectral density (PSD) of a stationary random process $x_{n}$ is mathematically related to the correlation sequence by the discrete-time Fourier transform. In terms of normalized frequency, this is given by: 


$$
P_{x x}(\varpi)=\frac{1}{2 \pi} \sum_{m=-\infty}^{\infty} R_{x x}(m) e^{-j \varpi m}
$$

Where $\varpi=\frac{2 \pi f}{f_{s}}, f_{s}$ is the sampling frequency, $f$ is the physical frequency.

Equation 2.7 can be rewritten as a function of physical frequency as:

$$
P_{x x}(f)=\frac{1}{f_{s}} \sum_{m=-\infty}^{\infty} R_{x x}(m) e^{\frac{-2 \pi j f m}{f_{s}}}
$$

The correlation function $R_{x x}$ can be derived from the PSD by using the inverse discrete Fourier transform and is computed as:

$$
R_{x x}(m)=\int_{-\pi}^{\pi}\left(P_{x x}(\varpi) \cdot e^{j \varpi m}\right) \cdot d \varpi=\int_{-f_{s} / 2}^{f / 2_{s}}\left(P_{x x}(f) \cdot e^{\frac{2 \pi j f m}{f_{s}}}\right) \cdot d f
$$

The average power of the sequence $x_{n}$ over the entire Nyquist interval is represented by:

$$
R_{x x}(0)=\int_{-\pi}^{\pi} P_{x x}(\varpi) \cdot d \varpi=\int_{-f_{s} / 2}^{f / 2_{s}} P_{x x}(f) \cdot d f
$$

The average power of a signal over a particular frequency band $\left[\varpi_{1}, \varpi_{2}\right], 0 \leq \varpi_{1}<\varpi_{1} \leq \pi$, can be found by integrating the PSD over that band:

$$
\bar{P}_{\left[\varpi_{1}, \varpi_{2}\right]}=\int_{\varpi_{1}}^{\varpi_{2}} P_{x x}(\varpi) \cdot d \varpi=\int_{-\varpi_{1}}^{\sigma_{2}} P_{x x}(\varpi) \cdot d \varpi
$$

From Equation 2.11 we can see that $P_{x x}(\varpi)$ represents the power content of a signal over the particular infinitesimal frequency band and hence this is called Power Spectral Density. The units of PSD are power per unit of frequency. In the case of $P_{x x}(\varpi)$ the unit is watts/radian/sample or simply watts/radian whereas the unit of $P_{x x}(f)$, is watts/hertz. Integration of the PSD with respect to frequency yields units of watts as expected for the average power $\bar{P}_{\left[\varpi_{1}, \Phi_{2}\right]}$.

\subsection{Analysis of Variance (ANOVA)}

Always, the experimental data can be presented graphically such as box plots, scatter plots, etc. and the interpretations can be made from the graphs. When we want to compare the difference between the means within the model and between the models, the 
test for equality of means can also be solved by performing t-test. But this method is not the best solution since; doing all pair-wise comparisons is inefficient, it takes a lot of effort to do this test, and also the pair-wise comparison inflates the type-I error. Due to the above mentioned limitations, the best method for testing the equality of several means is the ANOVA. ANOVA has a much wider applications compared to the t-test and hence it is the most useful technique in the field of statistical inference (Montgomery (2005)). ANOVA is used for testing the hypothesis that the means among two or more groups are equal provided the assumptions are satisfied. The two hypotheses that can be inferred from the ANOVA are null hypothesis; if there is no difference between levels means, and alternate hypothesis; if there is a difference between levels means. The assumptions to be tested for performing ANOVA are: 1) the elements of one sample are independent to the elements of those of the other sample, 2) the population variances should be uniform i.e., the equal variance assumption, and 3) the sampled populations should be normally distributed.

Let us consider the one-way ANOVA and formulate the equations for developing the one-way ANOVA table and the same idea can be extended to three-way ANOVA. One way ANOVA has a one factor with $a$ different levels or treatments whereas threeway ANOVA has three different factors $\mathrm{A}, \mathrm{B}$, and $\mathrm{C}$ with each having $a, b$ and $c$ levels or treatments respectively and so on. The observations from each of the treatments are random variables. The typical data for one-way ANOVA looks like in Table 2.1 
Table 2.1: Typical data format for a one way ANOVA (Montgomery (2005))

\begin{tabular}{|c|c|c|c|c|c|c|}
\hline Level & \multicolumn{4}{|c|}{ Observations } & Totals & Averages \\
\hline 1 & $y_{11}$ & $y_{12}$ & .... & $y_{1 n}$ & $y_{1 .}$ & $y_{1 .}$ \\
\hline 2 & $y_{21}$ & $y_{22}$ & $\cdots$ & $y_{2 n}$ & $y_{2 .}$ & $\bar{y}_{2 .}$ \\
\hline 3 & $y_{31}$ & $y_{32}$ & .... & $y_{3 n}$ & $y_{3 .}$ & $\bar{y}_{3 .}$ \\
\hline . & - & - & .... & . & - & - \\
\hline . & • & - & .... & . & - & - \\
\hline . & $\cdot$ & - & .... & - & - & - \\
\hline A & $y_{a 1}$ & $y_{a 2}$ & $\cdots$ & $y_{a n}$ & $y_{a .}$ & $\bar{y}_{a .}$ \\
\hline & & & & & $y_{\text {.. }}$ & $\bar{y}_{\text {.. }}$ \\
\hline
\end{tabular}

From the above table, say in total there are $n$ observations. $y_{i j}$ represents the $j$ th observation taken under level $i, y_{i .}$ represents the sum of all the observations in the $i$ th level, $\bar{y}_{i \text {. }}$ represents the average of the observations in the ith treatment, $y_{\text {.. }}$ represents the grand sum of all the observations, and $\bar{y}_{\text {.. }}$ represents the grand average of all the observations. These can be expressed mathematically as:

$$
y_{i .}=\sum_{j=1}^{n} y_{i j}, \bar{y}_{i .}=\frac{y_{i .}}{n}, y_{. .}=\sum_{i=1}^{a} \sum_{j=1}^{n} y_{i j} \text {, and } \bar{y}_{. .}=\frac{y_{. .}}{N}
$$

Where, $i=1,2, \ldots \mathrm{a}, \mathrm{N}=$ an is the total number of observations.

The one way ANOVA model can be written as:

$$
y_{i j}=\mu_{i}+\varepsilon_{i j}\left\{\begin{array}{l}
i=1,2, \ldots a \\
j=1,2, \ldots n
\end{array}\right.
$$

Where, $y_{i j}$ is the $i j$ th observation,

$\mu_{i}$ is the mean of the $i$ th level, and

$\varepsilon_{i j}$ is the random error arising due to all other sources of variability.

The sum of squares is the very important parameter measured in ANOVA which is used for measuring the overall variability in the data. Total sum of squares can be 
decomposed into sum of squares of treatments and sum of squares of error, derivation for doing this is presented by Montgomery (2005). Total sum of squares can be represented as:

$$
S S_{T}=S S_{\text {Treatments }}+S S_{E}
$$

Where; $S S_{T}=\sum_{i=1}^{a} \sum_{j=1}^{n} y_{i j}{ }^{2}-\frac{y^{2} . .}{N}$,

$$
\begin{aligned}
& S S_{\text {Treatments }}=\frac{1}{n} \sum_{i=1}^{a} y_{i .}{ }^{2}-\frac{y_{. .}{ }^{2}}{N} \text {, and } \\
& S S_{E}=S S_{T}-S S_{\text {Treatments }}
\end{aligned}
$$

After computing sum of squares, mean square between levels and mean squares within levels are computed by dividing the sum of squares with the degrees of freedom. Therefore, Mean square quantities are expressed as:

$$
\begin{gathered}
M S_{\text {Treatments }}=\frac{S S_{\text {Treatments }}}{a-1} \\
M S_{E}=\frac{S S_{E}}{N-a}
\end{gathered}
$$

These mean square values can be compared to test a hypothesis of no difference in means because, $M S_{E}$ estimates $\sigma^{2}$, and if there is no difference in means $M S_{\text {Treatments }}$ also estimates $\sigma^{2}$ but if there is difference in means, the expected value of $M S_{\text {Treatments }}$ is greater than $\sigma^{2}$. The comparison can be made by calculating the $F_{o}$ test statistic value which is distributed as $F$ with a-1 and $\mathrm{N}$-a degrees of freedom. For the null hypothesis, $F$ test statistic is calculated as the ratio of treatments mean square and error mean squares:

$$
F_{o}=\frac{M S_{\text {Treatments }}}{M S_{E}}
$$


From the above equation we can see that if there is no difference in means expected value numerator value is greater than the expected value of denominator and hence we should reject the null hypothesis if the values are too large. For the given level of significance $\alpha$ , between levels degrees of freedom $(a-1)$ and within level degrees of freedom $(N-a)$ the percentage points of the $F$ distribution can be found from the standard $F$ distribution tables. Hence, if $F_{o}>F_{\alpha, a-1, N-a}$, we should reject null hypothesis and accept the alternate hypothesis that there are difference between the levels means.

Based on the above discussions and computations, one-way ANOVA can be summarized into a table as shown in Table 2.2.

Table 2.2: One-way ANOVA table (Montgomery (2005))

\begin{tabular}{lcccc}
\hline Source of variation & $\begin{array}{c}\text { Sum of Squares } \\
\text { (SS) }\end{array}$ & $\begin{array}{c}\text { Degrees of } \\
\text { Freedom }\end{array}$ & $\begin{array}{c}\text { Mean Square } \\
\text { (MS) }\end{array}$ & F $_{\mathbf{0}}$ \\
\hline Between levels & $S_{\text {Treatments }}$ & $a-1$ & $M S_{\text {Treatments }}$ & $F_{o}=\frac{M S_{\text {Treatments }}}{M S_{E}}$ \\
Error (within levels) & $S S_{E}$ & $N-a$ & $M S_{E}$ & \\
Total & $S S_{T}$ & $N-1$ & & \\
\hline
\end{tabular}

The procedures and concepts explained for one-way ANOVA can be extended for three-way ANOVA. Three-way ANOVA has three independent factors A, B, and C with levels $a, b$, and $c$ respectively. Three-way ANOVA interpretation involves the main effects, two way interactions effects, and three way interaction effects.

The summary of the meaning of main effects and interaction effects in 3-way ANOVA are explained clearly by (Karpinski (2004)) and is reproduced here in the Table 2.3. 
Table 2.3: Summary of the meaning of three-way ANOVA effects

Effects Meaning

\title{
Main Effects
}

A

B

C

Two-way

interactions

AB
Comparison of means of factor $\mathbf{A}$ by averaging over levels of $\mathbf{B}$ and $\mathbf{C}$ Comparison of means of factor $\mathbf{B}$ by averaging over levels of $\mathbf{A}$ and $\mathbf{C}$ Comparison of means of factor $\mathbf{C}$ by averaging over levels of $\mathbf{A}$ and $\mathbf{B}$

\begin{abstract}
BC
AC

\section{Three-way}

interaction

ABC

Examines whether the effect $\mathbf{A}$ is the same at every level of $\mathbf{B}$ by averaging over levels of $\mathbf{C}$ and equivalently, examines whether the $\mathbf{B}$ effect is same at every level of $\mathbf{A}$ by averaging over levels of $\mathbf{C}$ Examines whether the effect $\mathbf{B}$ is the same at every level of $\mathbf{C}$ by averaging over levels of $\mathbf{A}$ and equivalently, examines whether the $\mathbf{C}$ effect is same at every level of $\mathbf{B}$ by averaging over levels of $\mathbf{A}$ Examines whether the effect $\mathbf{A}$ is the same at every level of $\mathbf{C}$ by averaging over levels of $\mathbf{B}$ and equivalently, examines whether the $\mathbf{C}$ effect is same at every level of $\mathbf{A}$ by averaging over levels of $\mathbf{B}$

Examines whether two-way $\mathbf{A B}$ interaction is the same at every level of $\mathbf{C}$, equivalently, examines whether two-way $\mathbf{B C}$ interaction is the same at every level of $\mathbf{A}$ and examines whether two-way AC interaction is the same at every level of $\mathbf{B}$
\end{abstract}

Let us assume that the response $Y_{i j k l}$ is influenced by a treatment A with $a$ number of levels, treatment B with $b$ number of levels, and treatment $\mathrm{C}$ with $c$ number of levels and it can be modeled as:

$$
\begin{gathered}
\mathrm{Y}=\text { Model + error } \\
Y_{i j k l}=\mu+\alpha_{j}+\beta_{k}+\gamma_{l}+(\alpha \beta)_{j k}+(\beta \gamma)_{k l}+(\alpha \gamma)_{j l}+(\alpha \beta \gamma)_{j k l}+\varepsilon_{i j k l}
\end{gathered}
$$


Where;

$$
\begin{aligned}
& j=1,2, \ldots, a \text {; } \\
& k=1,2, \ldots, b \text {; } \\
& l=1,2, \ldots, c \text {; } \\
& i=1,2, \ldots, n \text {; } \\
& n \text { = number of observations per level; } \\
& \mu=\text { Overall or grand mean; } \\
& \alpha_{j}=\mu_{. j . .}-\mu_{\ldots .}=\text { the effect of being in level } j \text { of factor A; } \\
& \beta_{k}=\mu_{. . k .}-\mu_{\ldots .}=\text { the effect of being in level } k \text { of factor } \mathrm{B} \text {; } \\
& \gamma_{l}=\mu_{\ldots l}-\mu_{\ldots .}=\text { the effect of being in level } l \text { of factor B; } \\
& (\alpha \beta)_{j k}=\mu_{. j k .}-\left(\mu_{\ldots .}+\alpha_{j}+\beta_{k}\right)=\text { the effect of being in level } j \text { of factor A and level } k \\
& \text { of factor B; } \\
& (\beta \gamma)_{k l}=\mu_{. . k l}-\left(\mu_{\ldots .}+\beta_{k}+\gamma_{l}\right)=\text { the effect of being in level } k \text { of factor B and level } l \text { of } \\
& \text { factor C; } \\
& (\alpha \gamma)_{j l}=\mu_{. j . l}-\left(\mu_{\ldots .}+\alpha_{j}+\gamma_{l}\right)=\text { the effect of being in level } j \text { of factor A and level } l \text { of } \\
& \text { factor C; } \\
& (\alpha \beta \gamma)_{j k l}=\mu_{. j k l}-\left(\mu_{\ldots .}+\alpha_{j}+\beta_{k}+\gamma_{l}+\alpha \beta_{j k}+\beta \gamma_{k l}+\alpha \gamma_{j l}\right)=\text { the effect of being in } \\
& \text { level } j \text { of factor A, level } k \text { of factor B, and level } l \text { of factor } C \text {; } \\
& \varepsilon_{i j k l}=\mu_{i j k l}-\left(\mu+\alpha_{j}+\beta_{k}+\gamma_{l}+\alpha \beta_{j k}+\beta \gamma_{k l}+\alpha \gamma_{j l}+\alpha \beta \gamma_{j k l}\right) \text { is the unexplained part of } \\
& \text { the score }
\end{aligned}
$$

We shall assume errors $\varepsilon_{i j k l}$ are mutually independent and normally distributed random variables with a common variance $\sigma^{2}$ and without the loss of generality we assume that:

$$
\begin{aligned}
& \sum_{j=1}^{a} \alpha_{j}=\sum_{k=1}^{b} \beta_{k}=\sum_{l=1}^{c} \gamma_{l}=\sum_{j=1}^{a}(\alpha \beta)_{j k}=\sum_{k=1}^{b}(\alpha \beta)_{j k}=\sum_{k=1}^{b}(\beta \gamma)_{k l}=\sum_{l=1}^{c}(\beta \gamma)_{k l}=\sum_{i=1}^{a}(\alpha \gamma)_{j k}=\sum_{l=1}^{c}(\alpha \gamma)_{j k} \\
& =\sum_{j=1}^{a}(\alpha \beta \gamma)_{j k l}=\sum_{k=1}^{b}(\alpha \beta \gamma)_{j k l}=\sum_{l=1}^{c}(\alpha \beta \gamma)_{j k l}=0
\end{aligned}
$$


Similar to the one-way ANOVA, sum of squares, mean squares, and $F_{o}$ test statistic values are calculated and tested for the null hypothesis and alternate hypothesis. The sum of squares is partitioned into SS between model, within model, and the SS between models is further partitioned into; SS due to main effects, SS due to 2-way interaction effects, and SS due to 3-way interaction effect. The three-way ANOVA procedure is summarized below in Table 2.4.

Table 2.4: Three-way ANOVA table (Karpinski (2004))

\begin{tabular}{ccccc}
\hline $\begin{array}{c}\text { Source of } \\
\text { variation }\end{array}$ & $\begin{array}{c}\text { Sum of } \\
\text { Squares (SS) }\end{array}$ & $\begin{array}{c}\text { Degree of } \\
\text { freedom (df) }\end{array}$ & $\begin{array}{c}\text { Mean Squares } \\
\text { (MS) }\end{array}$ & $\mathbf{F}_{\mathbf{o}}$ \\
\hline Factor A & $S S_{A}$ & $(a-1)$ & $S S_{A} / d f_{a}$ & $M S_{A} / M S_{W}$ \\
Factor B & $S S_{B}$ & $(b-1)$ & $S S_{B} / d f_{b}$ & $M S_{B} / M S_{W}$ \\
Factor C & $S S_{C}$ & $(c-1)$ & $S S_{C} / d f_{c}$ & $M S_{C} / M S_{W}$ \\
A*B interaction & $S S_{A B}$ & $(a-1)(b-1)$ & $S S_{A B} / d f_{a b}$ & $M S_{A B} / M S_{W}$ \\
B*C interaction & $S S_{B C}$ & $(b-1)(c-1)$ & $S S_{B C} / d f_{b c}$ & $M S_{B C} / M S_{W}$ \\
A*C interaction & $S S_{A C}$ & $(a-1)(c-1)$ & $S S_{A C} / d f_{a c}$ & $M S_{A C} / M S_{W}$ \\
A*B*C interaction & $S S_{A B C}$ & $(a-1)(b-1)(c-1)$ & $S S_{A B C} / d f_{a b c}$ & $M S_{A B C} / M S_{W}$ \\
Between Model & $S S_{B e t}$ & $a b c-1$ & $S S_{B e t} / d f_{B e t}$ & \\
Within Model & $S S_{W}$ & $N-a b c$ & $S S_{W} / d f_{W}$ & \\
Total & $S S_{T}$ & $N-1$ & & \\
\hline
\end{tabular}

The advantages of using three-way ANOVA over one-way ANOVA design (Kennedy and Neville (1986)) are:

1. It is more efficient because, we can test the effects of three factors simultaneously on the measured variable hence, lot of time can be saved and experimental error can be reduced which occurs due to duplicating the same conditions for all the required experiments unlike if it is done by one-way ANOVA design.

2. Interaction effects between three main factors can be determined by using threeway ANOVA.

3. Conclusions can be made on a wide range of situations because; the effect of one factor can be estimated at several levels of the second factor. 


\subsection{Summary of Review}

From the above literature survey we can conclude that:

- LMC and SFMC are the most commonly used overlay types by several Department of Transportation.

- On newly cast bridge decks, shot blasting technique shows better bond strength results.

- Difference in length change between substrate and overlay concretes develops the shear stresses at the interface and results in debonding of overlays

- Of all the bond tests available, field pull-off test proves to be more effective since, this test gives the bond strength values directly in the field and easy to conduct.

- UPV testing combined with the signal processing techniques can be successfully used to characterize the extent of delamination at the interface between overlay and substrate concrete.

\subsection{Significance of Present Study}

Research by Hong (2006), Shin and Lange (2004), and other researchers showed that, the primary reasons for debonding of the overlays is due to the volume changes of the overlay concrete. The debonding profiles were studied at the small scale level. Since the results were limited to the small scale study, this research will be directed towards studying the delamination conditions of the interface using LMC and SFMC overlays. Also, bonding slurry will be applied on one of the slabs to compare the performance of overlay concrete with bonding slurry condition at the interface. 


\section{CHAPTER 3: EXPERIMENTAL PROGRAM}

In this chapter, the research plan, materials and mixture proportions used, the construction procedure followed and the preparation of test specimens are explained in detail.

\subsection{Research Plan}

The research plan is divided into two phases: Stage-I experimental program was conducted to assess the better bonding condition using the most commonly used Latex Modified Concrete (LMC) overlays by WVDOH, whereas Stage-II experimental program was conducted to compare the performance of Latex Modified Concrete (LMC) and Silica Fume Modified Concrete (SFMC) overlays used by the WVDOH based on the better bonding condition selected from the Stage-I experimental program. In both the phases, two substrate slabs of $1829 \mathrm{~mm}$. (6 ft.) wide x $2438 \mathrm{~mm}$. (8 ft.) long x $165 \mathrm{~mm}$ (6.5 in.) thick were cast and after the deck slabs were matured for about 7 weeks, a 50 mm (2 in.) thick overlay was applied over the deck to act as a protective layer to the substrate following the Guidelines of overlay systems defined by the WVDOH standard specifications for roads and bridges (WVDOH 2003, Section 679).

\subsubsection{Stage-I}

In Stage-I experimental program: two prototype overlay-substrate slabs with Latex Modified Concrete overlays (LMC) but with different interface bonding conditions were cast and tested for the best interface bonding condition. Two different interface bonding conditions were considered: 1) Slab-1 without bonding slurry and 2) Slab-2 with bonding slurry. WVDOH standard Type K concrete supplied by Arrow Concrete Co. was used for casting substrate and WVDOH standard Latex Modified Concrete supplied by Ahern \& Associates Inc., was used for casting overlays on two slabs. All the materials and mixture proportions were supplied by the respective companies. 
Before the concrete overlays were cast, the cured substrate concretes were surface finished with the shot-blasting technique followed by the water jetting. After the surface preparation was finished, the surface was saturated by wetting with water. According to the plan, one first slab LMC overlay was directly placed on the prepared surface, whereas on the second slab bonding slurry was prepared first and then the overlay was placed on the prepared bonding slurry. Details about the preparation of bonding slurry are explained in the construction procedures section.

\subsubsection{Stage-II}

In Stage-II experimental program: two prototype overlay-substrate slabs with two different overlays, Latex Modified Concrete Overlay (LMC) and Silica Fume Modified Concrete Overlay (SFMC) with the better interface bonding condition from the Stage-I program was considered and cast. WVDOH standard Type K normal concrete supplied by Arrow Concrete Co. was used for casting substrate and WVDOH standard Latex Modified Concrete and Silica Fume Modified Concretes supplied by Ahern Associates Inc., were used for casting overlays on two slabs. All the materials and mixture proportions were supplied by the respective companies.

Same construction procedure like Stage-I program was followed in Stage-II program, except that the two overlays used on the slabs were made of LMC and SFMC and the same bonding condition was used on both the slabs unlike in Stage-I program. The same bonding condition refers to the better bonding condition concluded from the Stage-I program.

The summary of the research plan is shown in the Figure 3.1: 


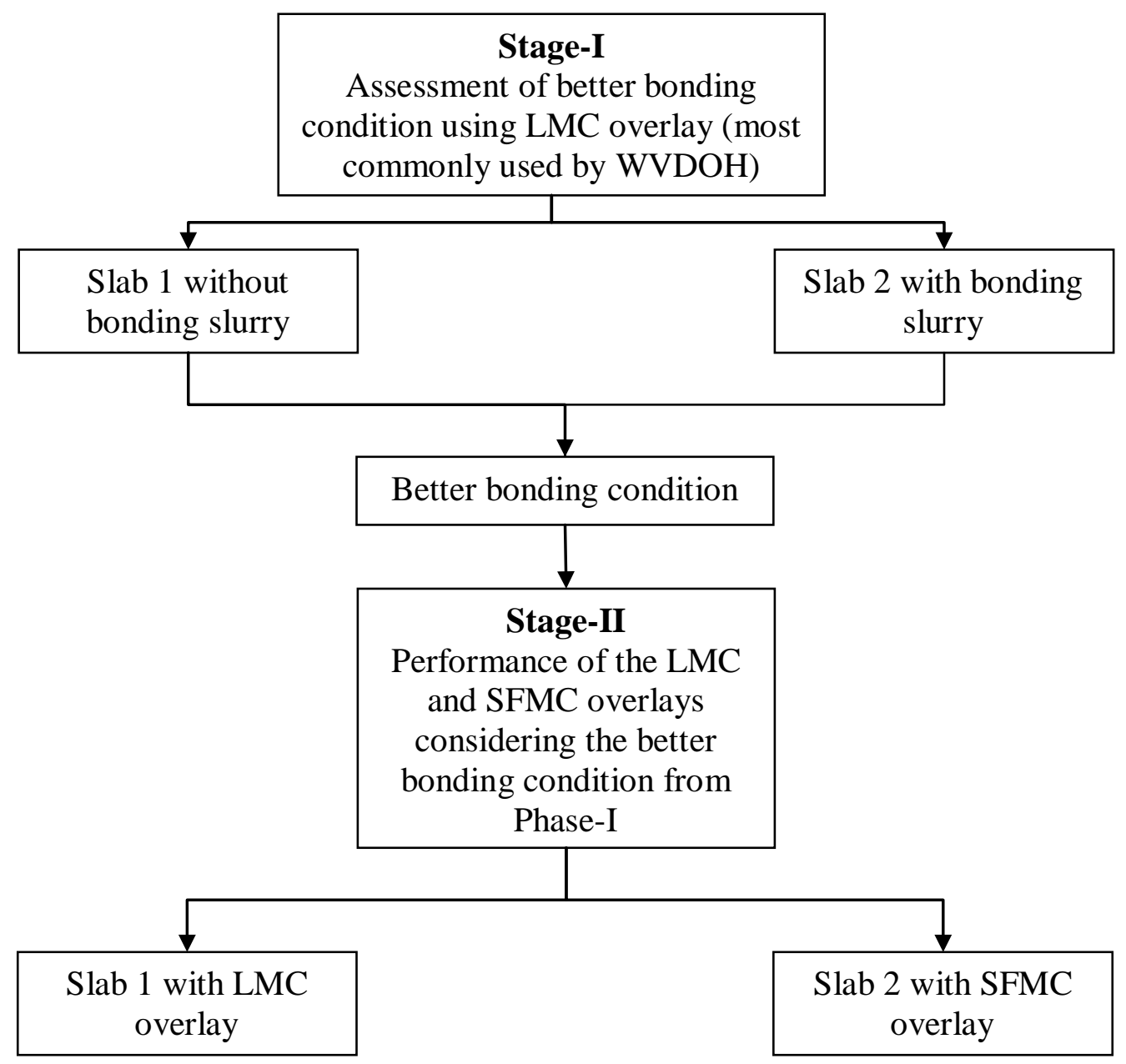

Figure 3.1: Summary of the Research Plan

\subsection{Materials}

All the materials and mix designs required for pouring Type $\mathrm{K}$ substrate concrete, Latex Modified Concrete (LMC) and Silica Fume Modified Concrete (SFMC) Overlays were supplied by Arrow concrete company and Ahern Associates Inc., respectively. Materials and mix designs used by the two companies are explained in this section.

\subsubsection{Cement}

Commercially available Type I Portland cement conforming to ASTM C-150 (Standard Specification for Portland cement) was used for casting substrates, LMC and 
SFMC overlays. The basic physical properties and compound compositions of the Type I Portland cement are shown in Table 3.1 and Table 3.2 respectively.

Table 3.1: Physical properties of Type I Portland cement

\begin{tabular}{|c|c|c|c|}
\hline \multirow{2}{*}{ Specific Gravity } & \multirow{2}{*}{ Fineness } & \multicolumn{2}{|c|}{ Setting time } \\
\cline { 3 - 4 } & & $\begin{array}{c}\text { Initial } \\
\text { (min.) }\end{array}$ & Final (min.) \\
\hline 3.15 & $320 \mathrm{~m}^{2} / \mathrm{kg}$ & 90 & 260 \\
\hline
\end{tabular}

$1 \mathrm{~m}^{2} / \mathrm{kg}=703.07 \mathrm{in}^{2} / \mathrm{lb}$

Table 3.2: Compound compositions of Type I Portland cement

\begin{tabular}{|c|c|}
\hline Compounds & Percentage by mass \\
\hline Tricalcium Silicate $\left(\mathrm{C}_{3} \mathrm{~S}\right)$ & 49.0 \\
\hline Dicalcium Silicate $\left(\mathrm{C}_{2} \mathrm{~S}\right)$ & 25.0 \\
\hline Tricalcium Aluminate $\left(\mathrm{C}_{3} \mathrm{~F}\right)$ & 12.0 \\
\hline Tetracalcium Aluminoferrite $\left(\mathrm{C}_{4} \mathrm{AF}\right)$ & 8.0 \\
\hline Calcium Sulfate $\left(\mathrm{CSH}_{2}\right)$ & 2.2 \\
\hline Calcium Oxide $(\mathrm{CaO})$ & 0.8 \\
\hline Magnesium Oxide $(\mathrm{MgO})$ & 2.0 \\
\hline Others & 1.0 \\
\hline
\end{tabular}

\subsubsection{Coarse Aggregate}

Crushed limestone meeting the quality requirements of ASTM C 33 (Standard Specifications for Concrete Aggregates) was used as coarse aggregate in this study. Some of the physical properties and the sieve analysis results of the coarse aggregates used by the Arrow concrete and Ahern \& Associates Inc., are shown in Table 3.3 and Table 3.4, respectively. 
Table 3.3: Source and basic properties of coarse aggregates used

\begin{tabular}{|c|c|c|}
\hline & $\begin{array}{c}\text { Type K Substrate } \\
\text { Concrete (Arrow } \\
\text { Concrete) }\end{array}$ & $\begin{array}{c}\text { LMC and SFMC } \\
\text { Overlays (Ahern \& } \\
\text { Associates Inc.) }\end{array}$ \\
\hline Source & $\begin{array}{c}\text { Greer Limestone, } \\
\text { Morgantown, WV }\end{array}$ & $\begin{array}{c}\text { Yeager, Wolf Creek, } \\
\text { KY }\end{array}$ \\
\hline Specific Gravity & 2.7 & 2.65 \\
\hline Absorption, \% & 0.7 & 1.37 \\
\hline Unit Weight, $\mathrm{kg} / \mathrm{m}^{3}$ & 1523 & 1596 \\
\hline Material Finer than \#200 Sieve, \% & 1.40 & 1.50 \\
\hline Fineness Modulus, \% & $<1.0$ & - \\
\hline
\end{tabular}

$1 \mathrm{~kg} / \mathrm{m}^{3}=0.062 \mathrm{lb} / \mathrm{ft}^{3}$

Table 3.4: Sieve analysis data of coarse aggregates used

\begin{tabular}{|c|c|c|c|c|}
\hline \multirow{2}{*}{\multicolumn{2}{|c|}{ Sieve Size }} & \multicolumn{2}{|c|}{$\%$ Passing } & \multirow{3}{*}{$\begin{array}{c}\begin{array}{c}\text { ASTM C 33 } \\
\text { Specification } \\
\text { Limit }\end{array} \\
100\end{array}$} \\
\hline & & \multirow{2}{*}{$\begin{array}{l}\text { Greer Limestone, } \\
\text { Morgantown, WV } \\
100\end{array}$} & \multirow{2}{*}{$\begin{array}{c}\text { Yeager, Wolf } \\
\text { Creek, KY }\end{array}$} & \\
\hline $19 \mathrm{~mm}$ & 3/4" & & & \\
\hline $12.5 \mathrm{~mm}$ & $1 / 2 "$ & 99.59 & 100 & 100 \\
\hline $9.5 \mathrm{~mm}$ & 3/8" & 88.14 & 91 & $85-100$ \\
\hline $4.75 \mathrm{~mm}$ & No. 4 & 13.67 & 14 & $10-30$ \\
\hline $2.36 \mathrm{~mm}$ & No. 8 & 1.3 & 4 & $0-10$ \\
\hline $1.18 \mathrm{~mm}$ & No. 16 & 1.02 & 2 & $0-5$ \\
\hline
\end{tabular}

$1 \mathrm{~mm}=0.039$ in

\subsubsection{Fine Aggregate}

Graded river sand of $9.5 \mathrm{~mm}$ (3/8 inch) nominal size conforming to ASTM C 33 (Standard Specification for Concrete Aggregates) was used as fine aggregate in this study. Some of the physical properties and the sieve analysis data of the fine aggregates used by the Arrow Concrete and Ahern Associates Inc., are shown in Table 3.5 and Table 3.6, respectively. 
Table 3.5: Source and basic properties of fine aggregates used

\begin{tabular}{|c|c|c|}
\hline & $\begin{array}{c}\text { Type K Substrate } \\
\text { Concrete (Arrow } \\
\text { Concrete) }\end{array}$ & $\begin{array}{c}\text { Latex Modified } \\
\text { Concrete Overlay } \\
\text { (Ahern \& Associates } \\
\text { Inc.,) }\end{array}$ \\
\hline Source & $\begin{array}{c}\text { Shelly, Newport, } \\
\text { OH }\end{array}$ & $\begin{array}{c}\text { Apple Grove Silica } \\
\text { Sand }\end{array}$ \\
\hline Specific Gravity & 2.60 & 2.64 \\
\hline Absorption, \% & 1.40 & 1.63 \\
\hline Unit Weight, kg/m ${ }^{3}$ & 1684 & 1596 \\
\hline Material Finer than \#200 Sieve, \% & 1.50 & 1.90 \\
\hline Fineness Modulus, \% & 2.84 & 2.70 \\
\hline
\end{tabular}

$1 \mathrm{~kg} / \mathrm{m}^{3}=0.062 \mathrm{lb} / \mathrm{ft}^{3}$

Table 3.6: Sieve analysis data of fine aggregates used

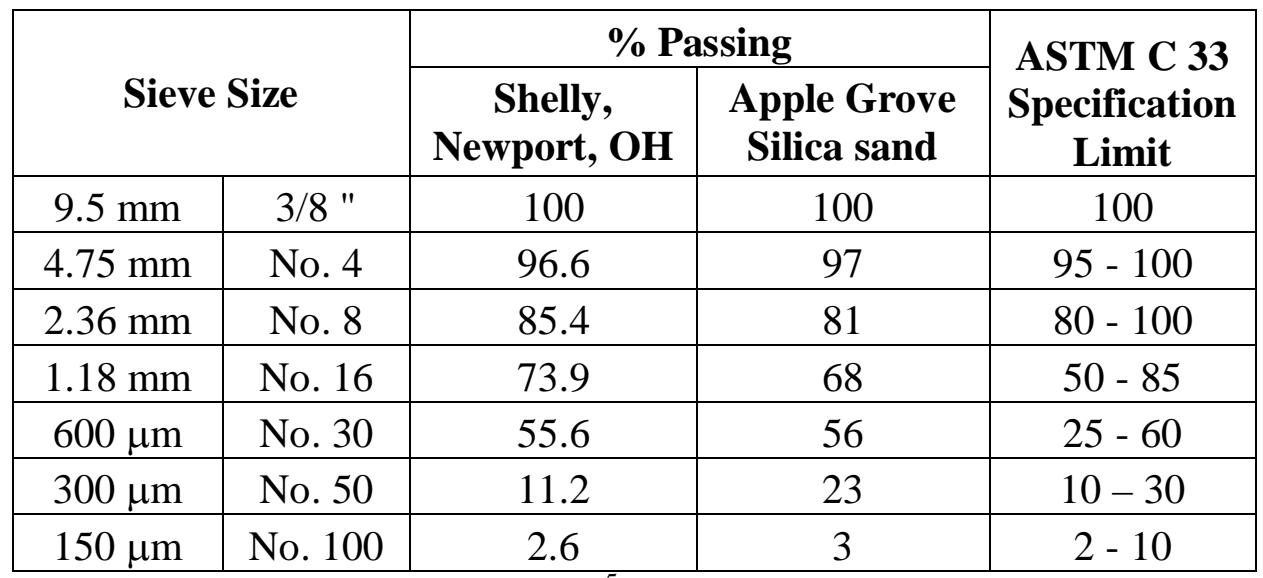

$1 \mathrm{~mm}=0.039$ in, $1 \mu \mathrm{m}=3.937 \times 10^{-5}$ in

\subsubsection{Mineral Admixtures}

\subsubsection{Fly Ash}

Fly ash is the fine inorganic, noncombustible residue obtained after the combustion of ground or powdered coal in power plants. Class F Fly ash conforming to ASTM C 618 (Standard Specification for Standard Specification for Coal Fly Ash and Raw or Calcined Natural Pozzolan for Use in Concrete) was used in this study. Some of the basic physical properties and compound compositions of Fly ash are shown in Table 3.7 and Table 3.8, respectively. 
Table 3.7: Physical properties of Class F Fly ash

\begin{tabular}{|c|c|}
\hline Source & Hatfield power station \\
\hline Specific Gravity & 2.53 \\
\hline Specific Surface, $\mathrm{m}^{2} / \mathrm{kg}$ & 496 \\
\hline Loss of ignition, $\%$ & 3.00 \\
\hline
\end{tabular}

$1 \mathrm{~m}^{2} / \mathrm{kg}=703.07 \mathrm{in}^{2} / \mathrm{lb}$

Table 3.8: Compound composition of Class F Fly ash

\begin{tabular}{|c|c|}
\hline Compounds & Percentage by mass \\
\hline $\mathrm{SiO}_{2}, \%$ & 49.34 \\
\hline $\mathrm{Al}_{2} \mathrm{O}_{3}, \%$ & 22.73 \\
\hline $\mathrm{Fe}_{2} \mathrm{O}_{3}, \%$ & 16.01 \\
\hline $\mathrm{CaO}, \%$ & 3.09 \\
\hline $\mathrm{MgO}, \%$ & 1.06 \\
\hline $\mathrm{Na}_{2} \mathrm{O}, \%$ & 1.15 \\
\hline $\mathrm{K}_{2} \mathrm{O}, \%$ & 1.60 \\
\hline $\mathrm{SO}_{3}, \%$ & 0.97 \\
\hline
\end{tabular}

\subsubsection{Silica Fume}

Silica fume (SF) is a by-product for producing silicon or ferrosilicon alloys. Because of its chemical and physical properties, it is highly reactive Pozzolan of very fine nature, and is able to pack between cement particles. The commercially available silica fume conforming to ASTM C 1240 (Standard Specification for Silica Fume for Use in Hydraulic-Cement Concrete and Mortar) supplied by BASF was used in this study. The specific gravity, specific surface area, and oxide composition are listed in Table 3.9. 
Table 3.9: Basic properties of silica fume

\begin{tabular}{|c|c|}
\hline Specific Gravity & 2.18 \\
\hline $\begin{array}{c}\text { Specific Surface }\left(\mathrm{m}^{2} / \mathrm{kg}\right) \\
\text { (nitrogen absorption method) }\end{array}$ & 21,400 \\
\hline Loss of ignition, \% & 1.64 \\
\hline $\mathrm{SiO}_{2}, \%$ & 99 \\
\hline $\mathrm{Al}_{2} \mathrm{O}_{3}, \%$ & - \\
\hline $\mathrm{CaO}, \%$ & - \\
\hline MgO, \% & - \\
\hline $\mathrm{SO}_{3}, \%$ & 0.156 \\
\hline $\mathrm{Na}_{2} \mathrm{O}, \%$ & 0.57 \\
\hline $\mathrm{K}_{2} \mathrm{O}, \%$ & - \\
\hline $\mathrm{Fe}_{2} \mathrm{O}_{3}, \%$ & - \\
\hline
\end{tabular}

\subsubsection{Chemical Admixtures}

\subsubsection{BASF Latex Admixture:}

Commercially available Styrofan $^{\mathrm{R}} 1186$ latex admixture of type Styrene Butadiene Polymer with Stabilizer and Water manufactured by BASF was used in this study. Physical properties of latex admixture used are shown in Table 3.10.

Table 3.10: Physical properties of latex admixture used

\begin{tabular}{|c|c|}
\hline Source & BASF. \\
\hline Observed Solids, \% by weight & 47.29 \\
\hline Observed Density, $\mathrm{kg} / \mathrm{m}^{3}$ & 1022 \\
\hline Specific gravity & 1.024 \\
\hline
\end{tabular}

$1 \mathrm{~kg} / \mathrm{m}^{3}=0.062 \mathrm{lb} / \mathrm{ft}^{3}$ 


\subsubsection{Air-Entraining Admixture (AEA)}

Commercially available air entraining admixture (AEA) manufactured by BASF was used in this study. AEA was based on neutralized vinsol resin meeting the requirements of ASTM C 260 (Standard Specification for Air-Entraining Admixtures for Concrete).

\subsubsection{High-Range Water Reducing Admixture (HRWRA)}

Commercially available high-range water reducing admixture (HRWRA) of Type F conforming to ASTM C 494 (Standard Specification for Chemical Admixtures for Concrete), manufactured by BASF was used in this study.

\subsubsection{Mixing Water}

Municipal treated water source that comply with the physical and chemical requirements of ASTM C 94 was used in this study.

\subsection{Mixture Proportions}

Three types of concretes were selected for this study: One was Type K Normal Concrete, used for casting substrate and the other two were: Latex Modified Concrete (LMC) and Silica Fume Modified Concretes (SFMC), used for casting overlay on top of the substrates. The West Virginia Department of Highways (WVDOH) approved mixture proportions are summarized in Table 3.11. This is for one cubic meter of concrete based on SSD condition. 
Table 3.11: Mixture proportions of substrate and overlay concretes for each cubic meter of concrete (source: Arrow company and Ahern Associate company)

\begin{tabular}{|c|c|c|c|}
\hline \multirow{2}{*}{ Ingredient } & Substrate & \multicolumn{2}{|c|}{ Overlay } \\
\cline { 2 - 4 } & Type K NC & LMC & SFMC \\
\hline Portland Cement, kg & 335 & 390 & 406 \\
\hline BASF Latex Admixture, kg & - & 125 & - \\
\hline Silica Fume, kg & - & - & 36 \\
\hline Fly Ash, kg & 47 & - & - \\
\hline Coarse Aggregate, kg & 1038 & 798 & 695 \\
\hline Fine Aggregate, kg & 859 & 939 & 933 \\
\hline Water, kg & 158 & 45 & 169 \\
\hline HRWRA, mL & 952 & - & 1769 \\
\hline AEA, mL & - & - & 190 \\
\hline Air content, \% by volume & 5.80 & 5 & 7 \\
\hline Unit Weight, kg/m ${ }^{3}$ & 2437 & 2297 & 2238 \\
\hline w/cm & 0.41 & 0.28 & 0.38 \\
\hline
\end{tabular}

Notes:
- $1 \mathrm{Kg}=2.205 \mathrm{lbs}$
$1 \mathrm{ml}=0.034 \mathrm{fl} . \mathrm{Oz}$
$1 \mathrm{~kg} / \mathrm{m}^{3}=0.0624 \mathrm{lb} / \mathrm{ft}^{3}$

- In LMC overlay, BASF Latex admixture consisted of $47.29 \%$ of solid polymer and rest was water with chemicals.

- In the above table: NC ---Normal Concrete, LMC ---Latex Modified Concrete, SFMC ---Silica Fume Modified Concrete 


\subsection{Construction Procedure}

The procedures followed for constructing the substrates and overlays for both Stage-I and Stage-II experimental program were same. Guidelines of overlay systems defined by the WVDOH standard specifications for roads and bridges (WVDOH 2003, Section 679) were followed to construct the bi-layer deck system. The details of construction procedures are explained in this section.

\subsubsection{Substrates}

In the Stage-I, two substrates were built to study two LMC overlays -one with bonding slurry and the other without bonding slurry, respectively. In the Stage-II, two more substrates were constructed to study the LMC and SFMC overlaid on each substrate, respectively. However, in Stage-II both the overlays were constructed with the better bonding condition concluded form the Stage-I study. All the substrates were cast using Type K normal concrete per WVDOH specifications. The Type K normal concrete was supplied by Arrow Concrete Company through Ready Mix Trucks. Construction procedures of all the substrates are explained in details in the following sections.

\subsubsection{Reinforcement Layout}

In this study, two layers of reinforcement mats were used following the guidelines of WVDOH standard for bridge decks. Bottom reinforcement mat was set to an elevation of $25.4 \mathrm{~mm}$ (1 in.) using $25.4 \mathrm{~mm}$ (1 in.) standard plastic chairs, whereas top reinforcement mat was set to an elevation of $115 \mathrm{~mm}$ (4.5 in.) using $115 \mathrm{~mm}$ (4.5 in.) standard plastic chairs. Rebar elevations are set by plastic chairs conforming to WVDOH standards spaced at $0.9 \mathrm{~m}$ (3 ft.). All rebar's used in this study were of \#4 standard (0.5 in. diameter). As per WVDOH empirical deck design standards, in longitudinal direction 12 bars were evenly placed with $20.3 \mathrm{~cm}$ (8 in.) center to center spacing and in transverse direction 9 bars were evenly spaced with $200 \mathrm{~mm}$ (8 in.) center to center spacing. The details of the reinforcement used in this study are shown in Figure 3.2 and Figure 3.3. 


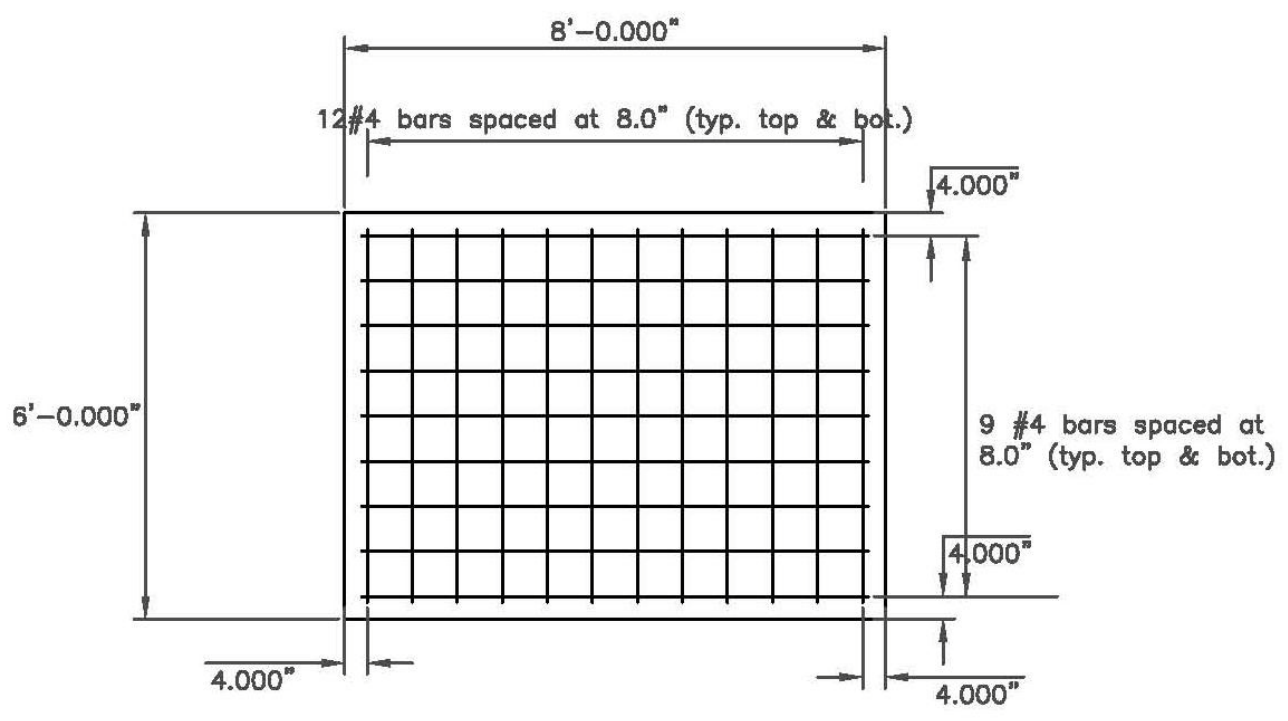

Reinforcement Plan

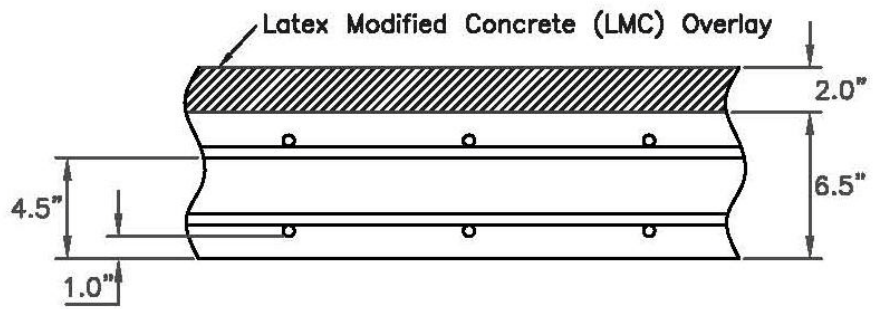

Overlay Deck Elevation

Notes:

All rebar used is $60 \mathrm{ksi}$. \#4 (0.5 in. $\emptyset)$ epoxy coated deformed bar

Top and bottom mats are identical in size of rebar and spacing configuration

Rebar elevations are set by plastic chairs conforming to WVDOH standards spaced at $3^{\prime}-0^{\prime \prime}$

Figure 3.2: Reinforcement layout

Note: $1 \mathrm{in} .=25.4 \mathrm{~mm}, 1 \mathrm{ft}=300 \mathrm{~mm}$, and $1 \mathrm{ksi}=6.9 \mathrm{MPa}$. 


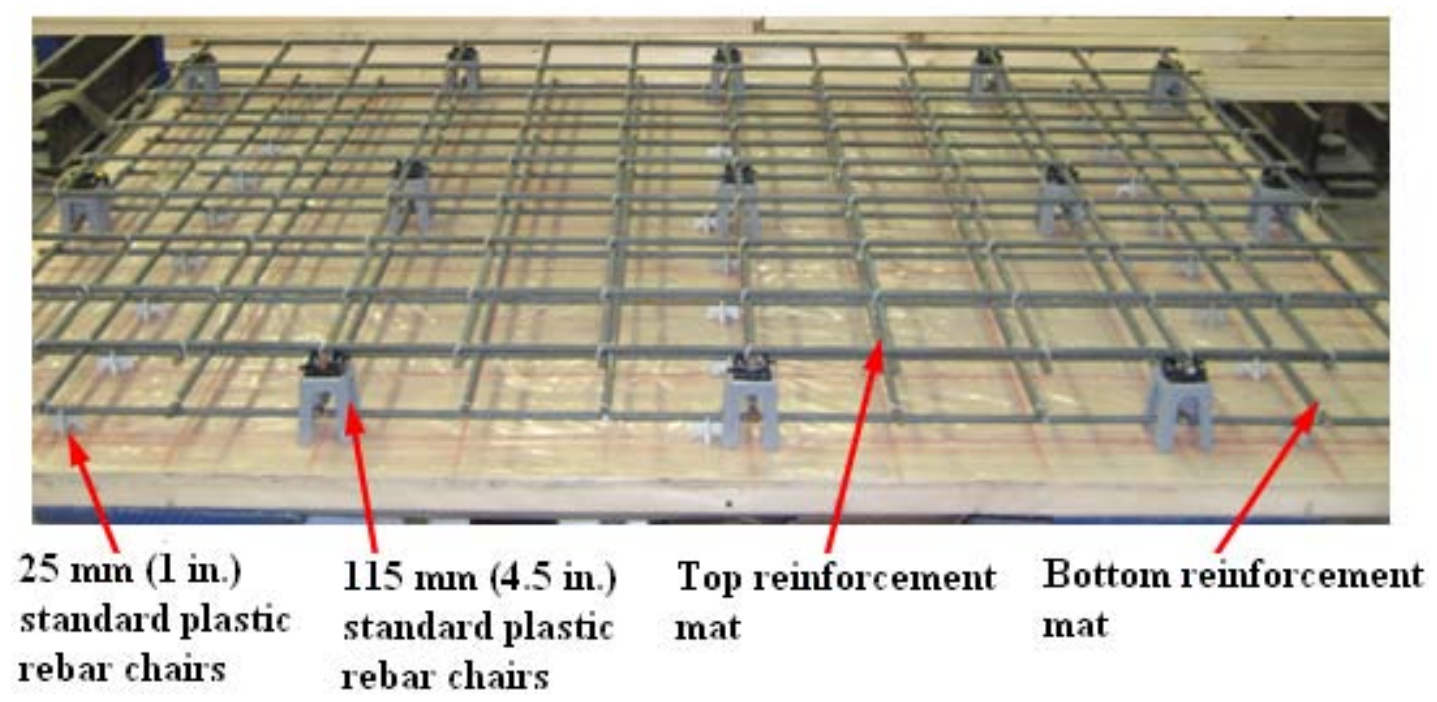

Figure 3.3: Reinforcement details

\subsubsection{Formwork}

After the top and bottom reinforcement mats were installed as discussed in the previous section, wooden formwork of dimensions $2440 \mathrm{~mm}$. (8 ft) long x $1830 \mathrm{~mm}$. (6 $\mathrm{ft}$ ) wide $\mathrm{x} 165 \mathrm{~mm}$ (6.5 in) thick was installed to cast the two substrates.

\subsubsection{Instrumentation}

Special type of strain gages and thermocouple loggers were used in this research program. Specifications and the instrumentation layout are explained below.

\subsection{Strain Gages}

EGP-series embedment strain gages manufactured by Vishay MicroMeasurements Company were used in this study. These embedment strain gages were used for measuring the mechanical strains inside the substrate and overlay concretes. The sensing grid of active gage length $100 \mathrm{~mm}$ (4 in), constructed of a nickel-chromium alloy, was enclosed in a rugged $130 \mathrm{~mm}$ (5 in) outer body, made of proprietary polymer concrete, to resist the mechanical damage during pouring and provides protection from moisture and corrosive attack. Each gage was incorporated with a heavy-duty $3 \mathrm{~m} \mathrm{(10} \mathrm{ft)}$ cable with 22-AWG (0.643-mm dia.) lead wires. EGP-Series Strain Gages were available in both 120-ohm (EGP-5-120) and 350-ohm (EGP-5-350) resistances. 


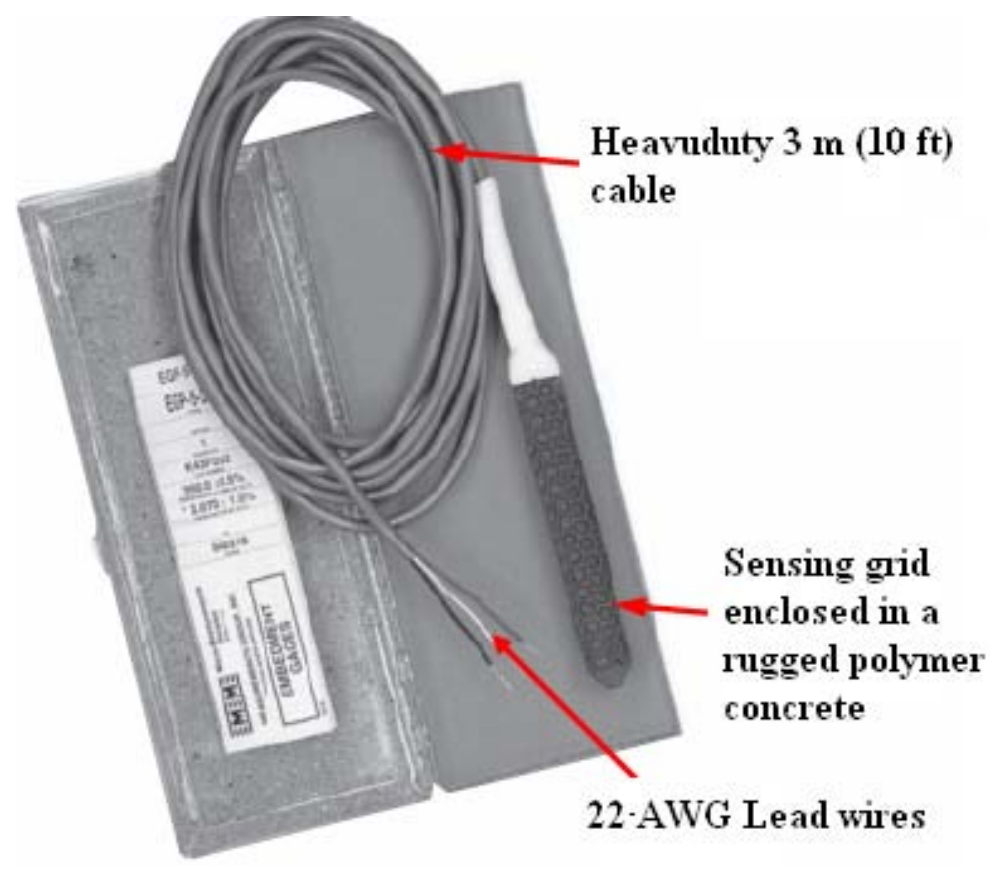

Figure 3.4: Typical embedment gage (Source: www.vishay.com)

As per the plan, the embedment gages were placed in both transverse and longitudinal directions at two different locations and they were tied to the top reinforcement mat. Embedment gages were not tied directly to the reinforcement instead they were tied to the c-shaped rebar's in order to measure the exact strains of the concrete. Embedment gages were installed in both transverse and longitudinal directions to measure the length change of concrete in the respective directions. The depth of the embedment gages was $25 \mathrm{~mm}$. ( $1 \mathrm{in}$.) below the top surface of the substrate, since the clear cover from the top mat of reinforcement was $25 \mathrm{~mm}(1 \mathrm{in}$.). The Plan of the embedment gages used in the substrate concrete is shown in Figure 3.6 below.

\subsection{Temperature Loggers.}

IntelliRock Temperature Loggers manufactured by Engius Company were used in this study. These loggers calculate and store the temperature within the placement where it is embedded and also; they can measure and document the temperature profiles of inplace concrete. The logger contains three components: temperature sensor, 
microprocessor and battery. These components are encased in polyester resin to provide the protection during placing of the concrete. The loggers used in this study are capable of recording temperatures up to 180 days for every one hour. There are also several types of loggers with different capacities manufactured by the supplier. The typical temperature logger used in this study is shown in Figure 3.5 below.

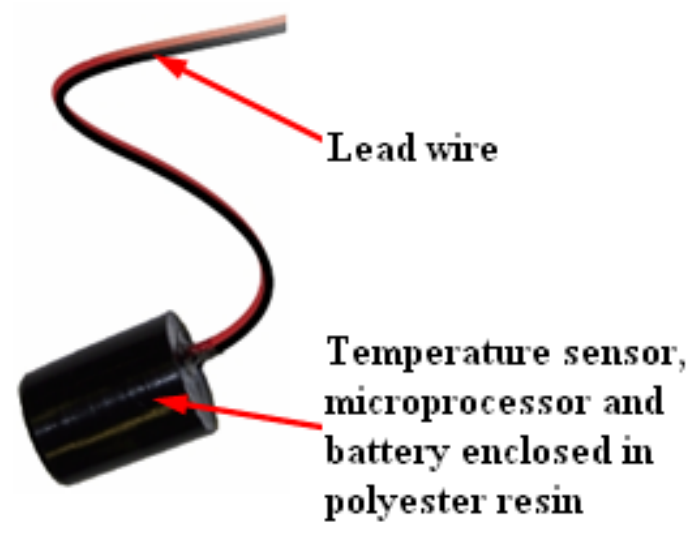

Figure 3.5: Typical temperature logger (Source: www.engius.com)

The temperature loggers were installed at the five different locations: four at the center of four edges and one at the center as per the plan. All the loggers were tied to the top reinforcement and a height of $25 \mathrm{~mm}$ ( 1 in) was maintained at all the locations. Figure 3.6 shows the typical plan of the locations of embedment gages and thermocouple loggers for Stage-I study. Explanation of the terminology used in the figure is explained below the figure. 


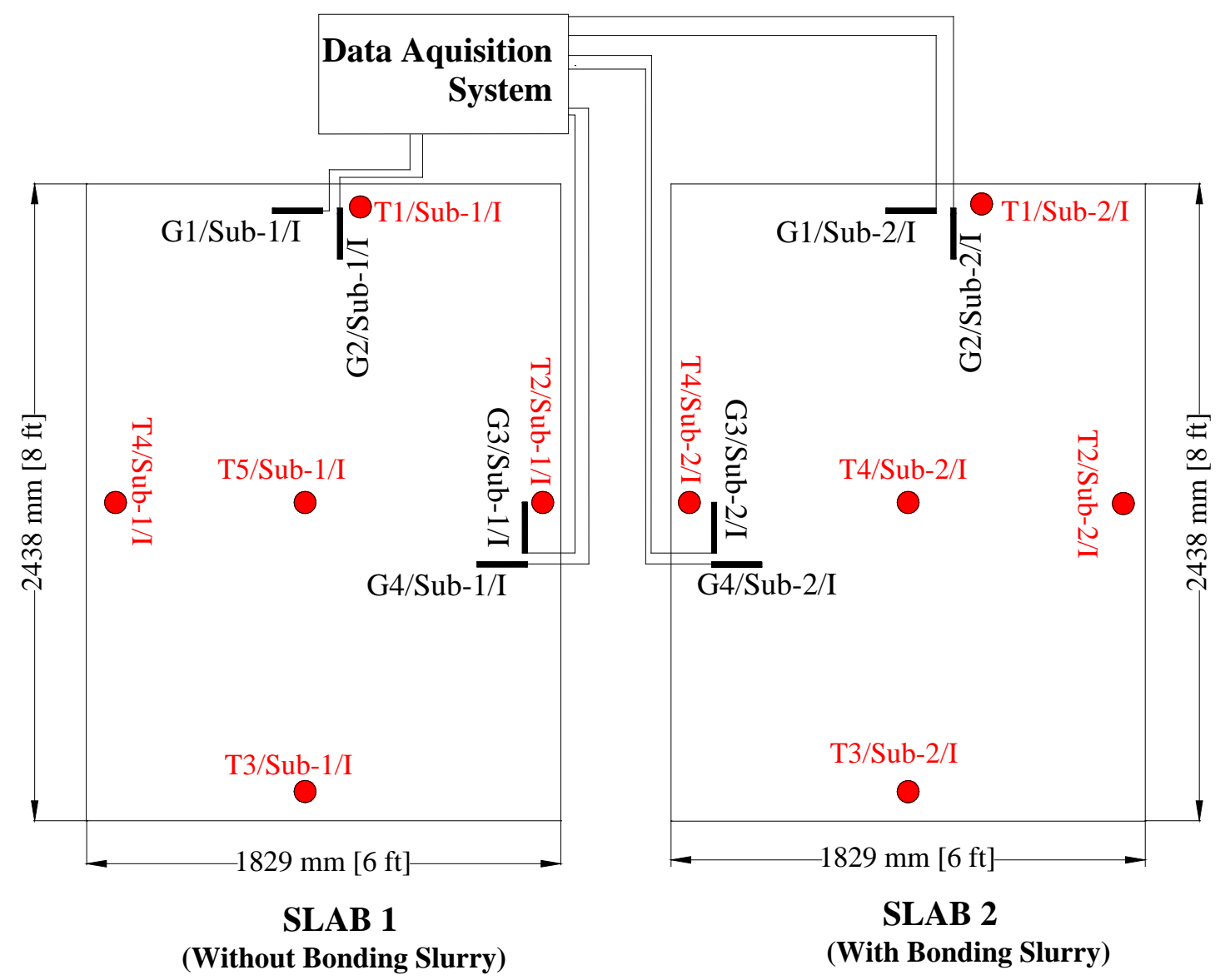

Figure 3.6: Plan view of the embedded strain gages and thermocouple loggers in substrate.

In the above figure, the terminology used was:

- Gx/Substrate type/Sy refers to Substrate Gage $\mathbf{x} /$ Substrate type/ Stage $\mathbf{y}$. Tx/Substrate type/Sy refers to Thermocouple Logger $\mathbf{x} /$ Substrate type/ Stage $\mathbf{y}$. Here $\mathbf{x}$, and $\mathrm{y}$ are variables.

Where, $\mathbf{x}=1$ to 4 --------Embedment Gage Number (refer to above figure for the gage locations)

Substrate type = Sub-1----Type-K Substrate on which LMC overlay was applied without any bonding slurry at the interface. 
Sub-2----Type-K Substrate on which same LMC overlay was applied with bonding slurry at the interface.

$\mathbf{y}=$ I and II ---------Stage Number.

The terminology used here will be reflected in the following chapters.

The details of the formwork and instrumentation are shown in the Figure 3.7 below:

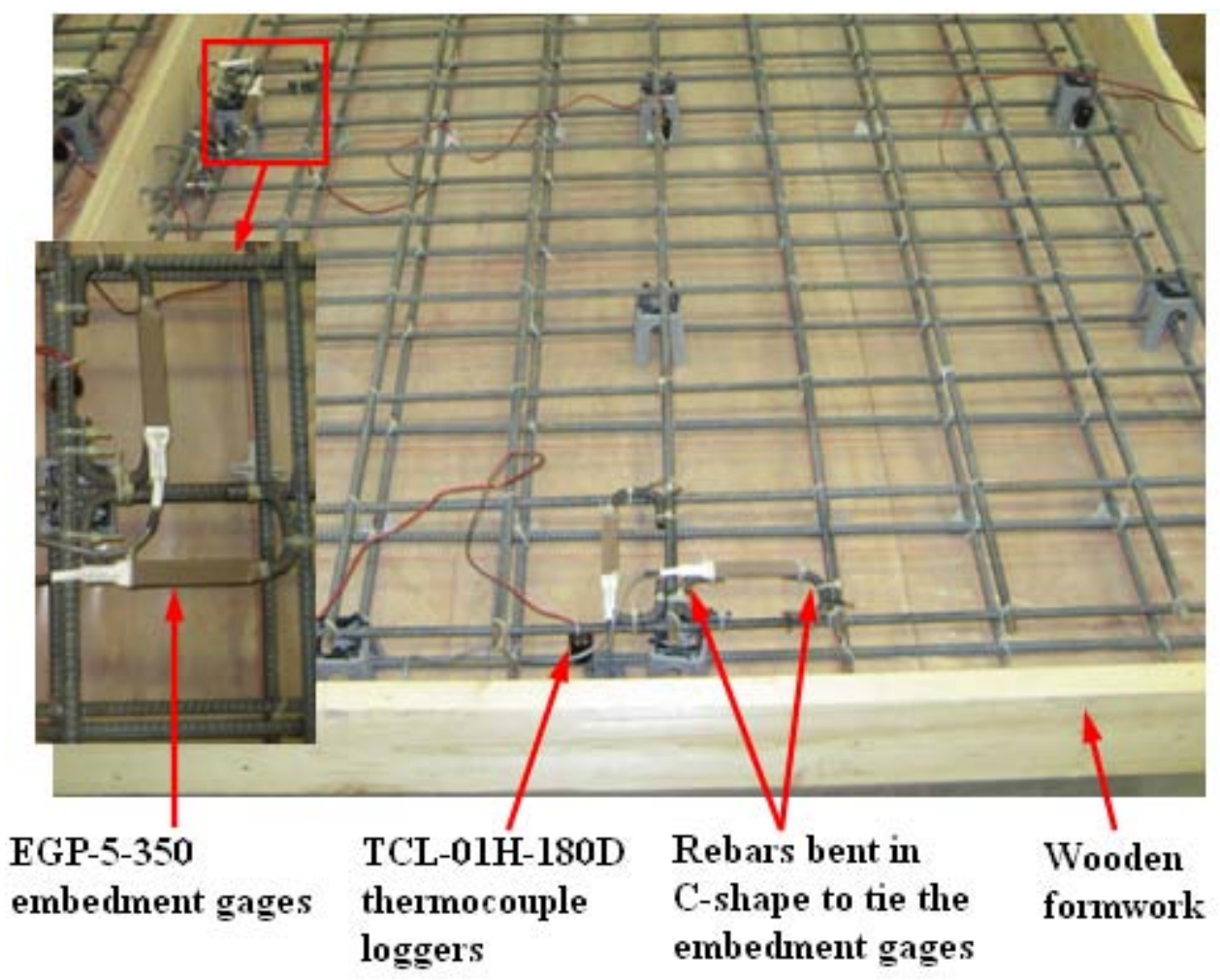

Figure 3.7: Formwork and instrumentation details.

\subsubsection{Pouring}

Before pouring the Type $\mathrm{K}$ normal concrete, supplied by Arrow Concrete Company, the wooden formwork was sprayed with MBT Rheofinish 211 form releasing agent. The concrete was pumped into the prepared substrate formwork as shown in the Figure 3.8 below. Simultaneously, the concrete was vibrated to make sure the concrete is 
evenly distributed around the reinforcement and free of air voids. Near the embedment gages and thermocouples, concrete was carefully placed by placing with the hand to make sure that the embedment gages are completely covered with the concrete and also to prevent the damage caused to the gages and thermocouples during pumping.

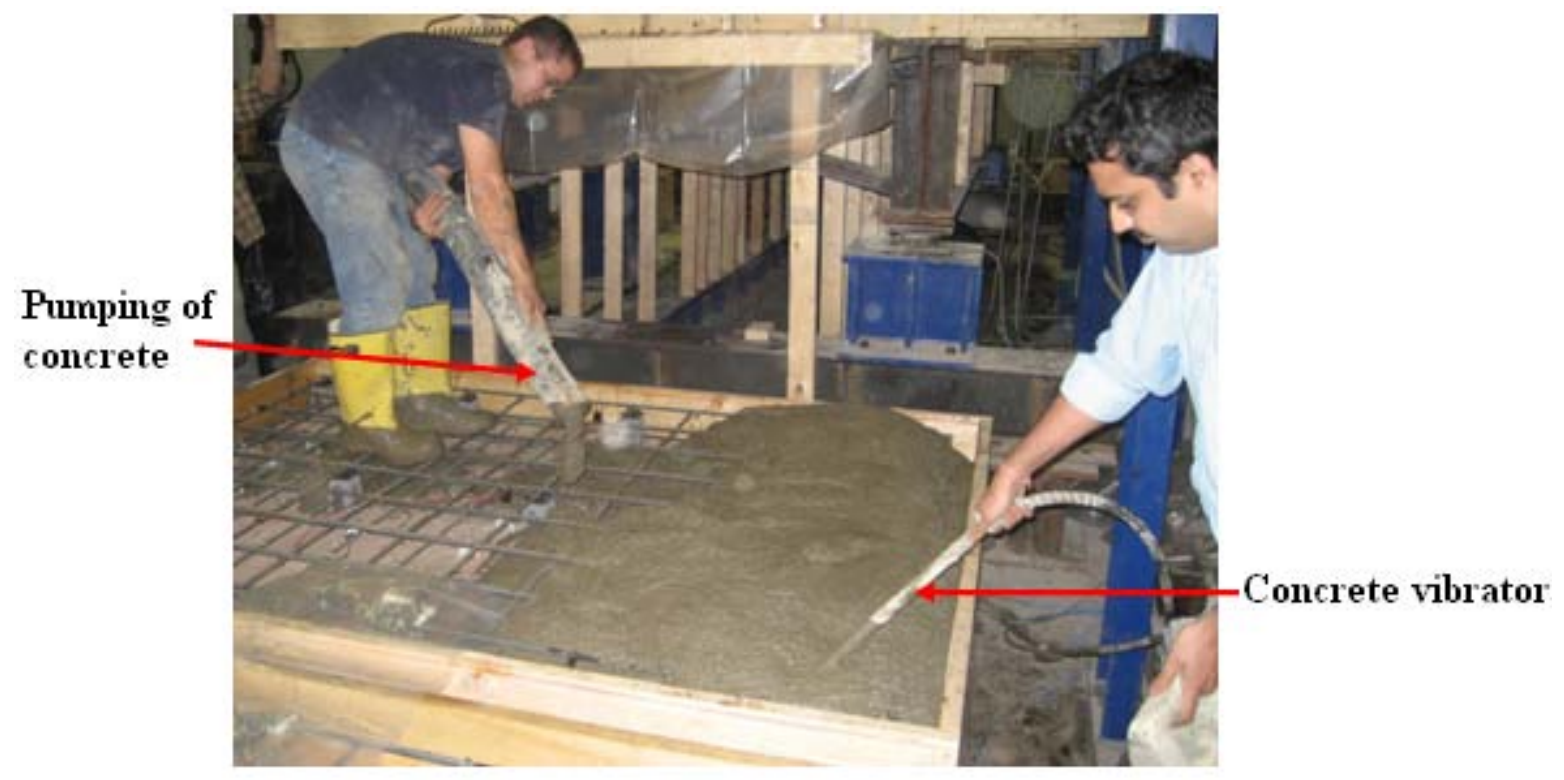

Figure 3.8: Pouring and vibration of substrate concrete

\subsubsection{Curing}

Immediately after pouring the substrate concrete, slabs were covered with clear plastic sheeting in order to retain the heat within the concrete. After the slabs were dry cured for 24 hours, they were covered with wet burlap to prevent the loss of moisture and allowed to cure for 7 days and then the substrate formworks were demolded and air cured for about 7 weeks. The wet burlap curing is shown in Figure 3.9 below. 


\section{Slab covered with wet burlap and plastic sheeting}

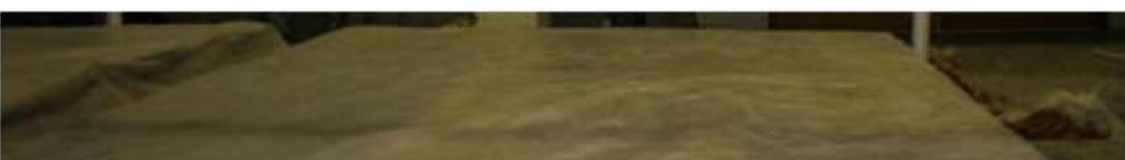

Figure 3.9: Typical curing procedure

\subsubsection{Overlay}

After the surface preparation was finished, $50 \mathrm{~mm}$ (2 in) thick overlays were cast on the top of two substrates. In Stage-I, most commonly used LMC overlays, by WVDOH, were cast with two different bonding conditions: 1) Slab 1 without bonding slurry and 2) Slab 2 with bonding slurry. Whereas in Stage-II program LMC and SFMC overlays were compared with the better bonding condition concluded from Stage-I program. The construction procedures used for both Stage-I and Stage-II programs were similar and were cast following the guidelines of overlay systems defined by the WVDOH standard specifications for roads and bridges (WVDOH 2003, Section 679). The concretes used for casting the overlays were supplied by Ahern Associates Inc., The procedure followed for constructing the overlays is explained in this section.

\subsubsection{Surface Preparation}

The condition of the surface of the substrate concrete is very important for the construction of bonded concrete overlays, Halvorsen (1993). After substrates were matured for about 7 weeks, surface preparation was finished with a shotblasting technique followed by cleaning with magnetic sweeper and stiff bristled brush to remove the steel shot left after shotblasting and all the loose debris, respectively. Finally, the surface was airblast with compressed air to remove all the loose dust particles left on the surface. The shot blaster and magnetic sweeper were manufactured by BLASTRAC Company as shown in Figure 3.10. 


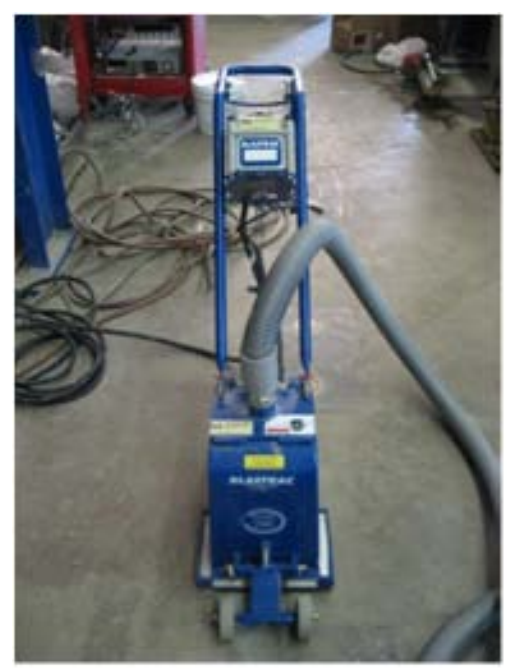

Figure 3.10: BLASTRAC equipment used for shotblasting the surface.

All the required equipments used for this purpose were supplied by MPE Rentals Company in Morgantown. The prepared surface was visually matched with the Concrete Surface Profiles (CSP) referenced plaques provided by International Concrete Repair Institute Technical Guidelines (ICRI 03732: Selecting and Specifying Concrete Surface Preparation for Sealers, coatings and Polymer Overlays). The surface profile between CSP \#4 and CSP \#5 (Figure 3.11) was achieved by using this shotblasting technique.

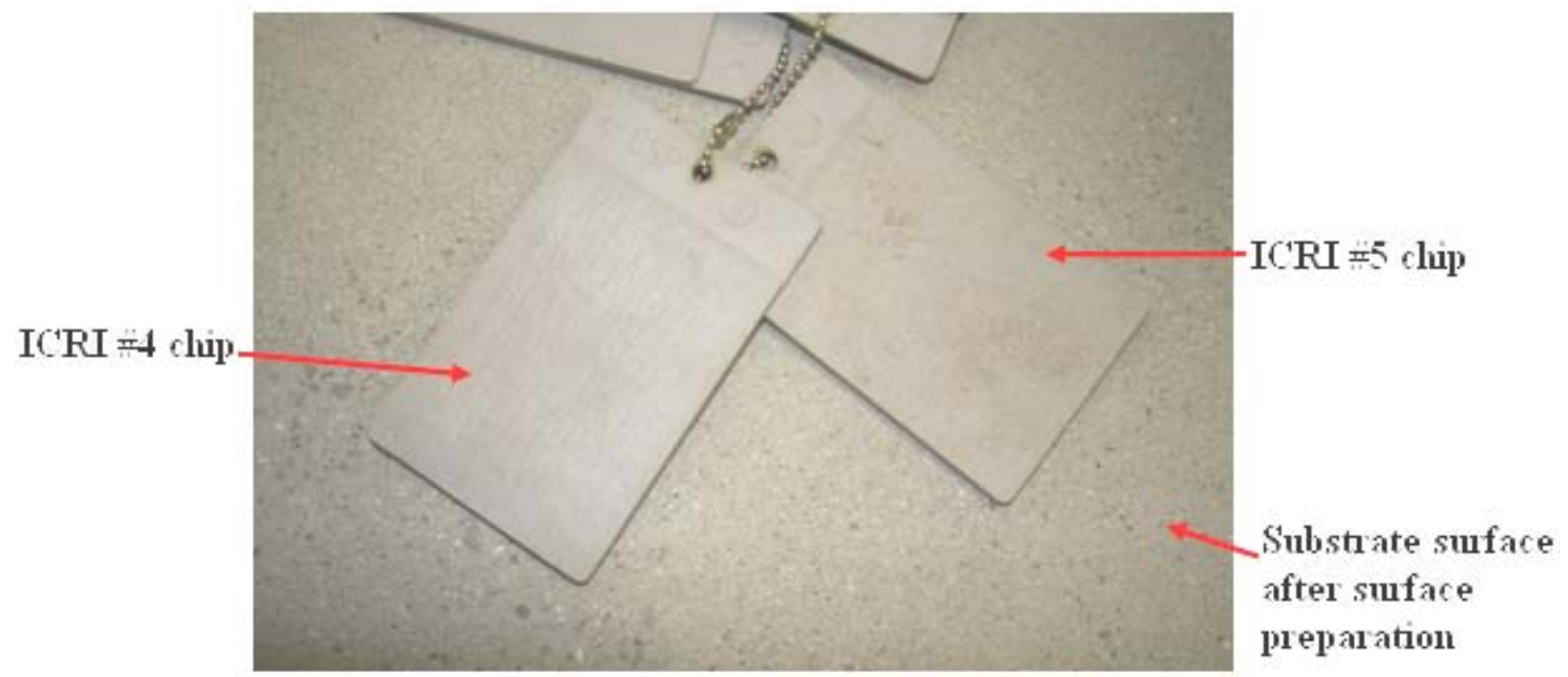

Figure 3.11: Typical substrate section after the surface preparation. 


\subsubsection{Formwork and Instrumentation}

Wooden formwork of dimensions 2439 mm. (8 ft) long x $1829 \mathrm{~mm}$. (6 ft) wide x $50 \mathrm{~mm}$ (2 in.) high was placed on top of the already cast substrate after the surface preparation was finished.

A double layer of plastic sheet was cut in rectangular shape of $300 \mathrm{~mm}(1 \mathrm{ft})$ by $230 \mathrm{~mm}$ (9 in). and were installed at two corners to serve as debonding area for future tests.

For measuring the strains in the overlay concrete and also to monitor the differential length change at the interface, EGP-series embedment gages, similar to the embedment gages used in substrate concrete, were placed exactly above the substrate gages at $25 \mathrm{~mm}$ (1 in.) above the interface using a $25 \mathrm{~mm}$. (1 in.) standard plastic rebar chairs as shown in the below.

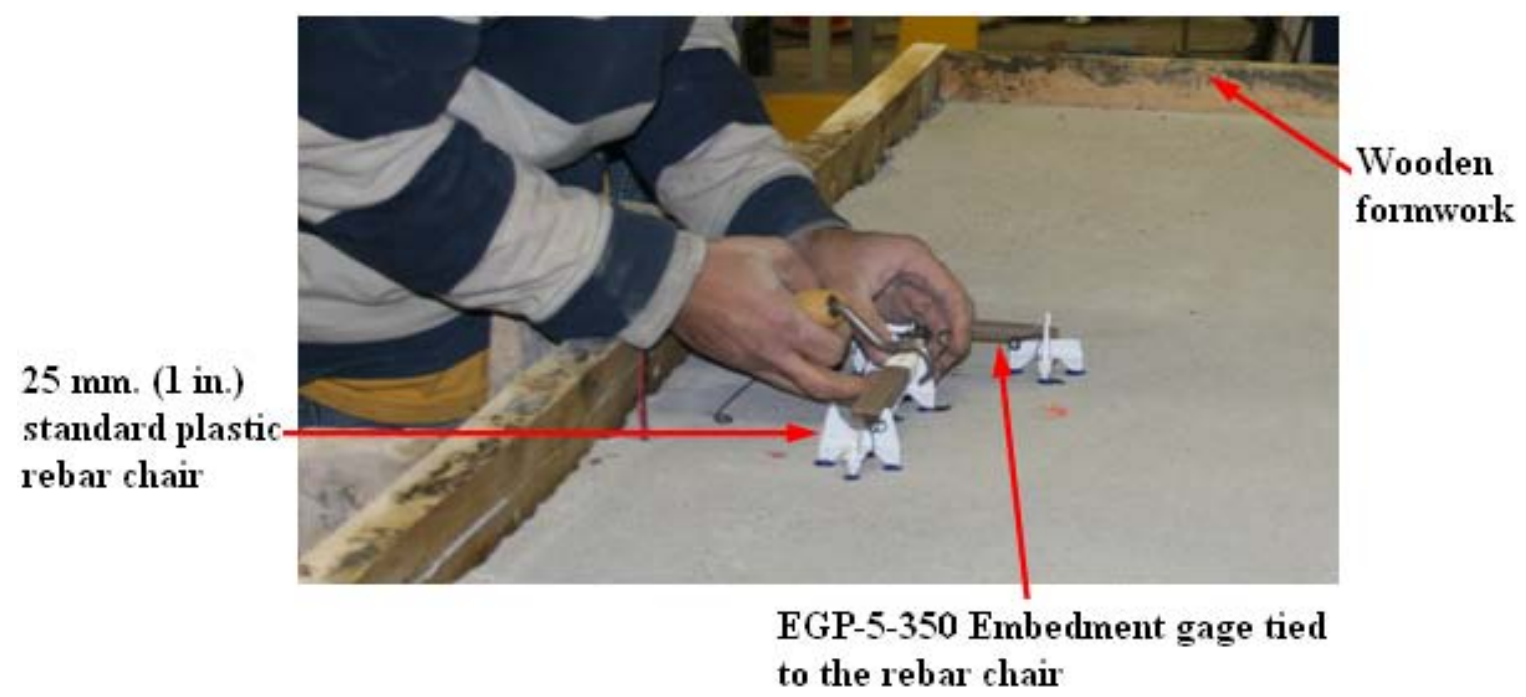

Figure 3.12: Tying embedment gages to the 25 mm (1 in) Rebar Chairs.

The same temperature loggers that were used in substrate concrete were also used in overlay concrete. They were installed at five different locations: four at the center of the edges and one at the center of the slab, and made sure that the thermocouples were placed exactly on top of the thermocouples installed in the substrate concrete. This 
procedure was followed to measure the differential temperatures developed at the interface.

The plan and the elevation views of the strain gages and temperature loggers used is shown in Figure 3.13 below.

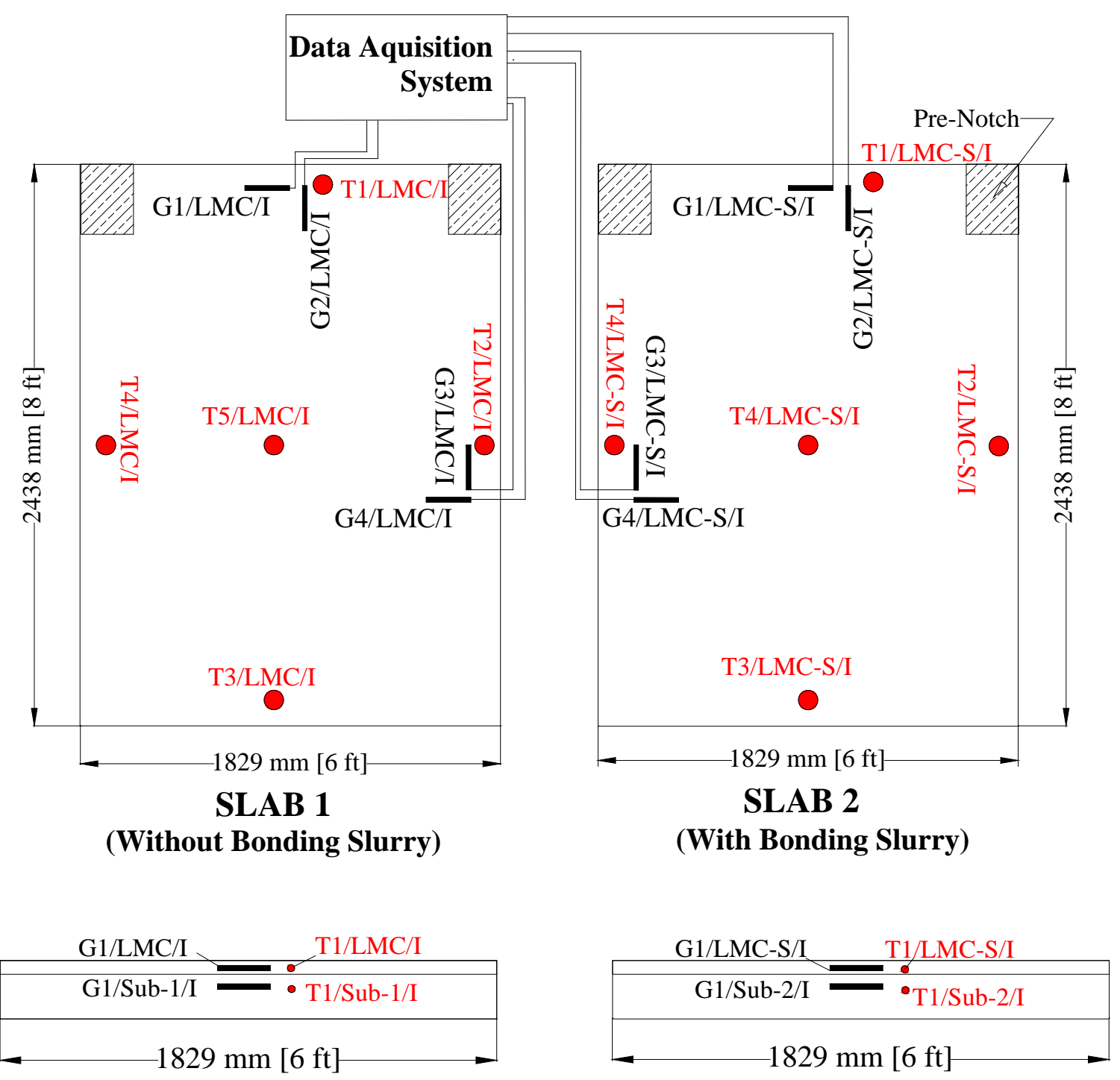

Figure 3.13: Plan and elevation views of the embedment gages and temperature loggers used in substrate and overlay concretes for typical Stage-I. 
In the above figure, the terminology used was:

- $\mathrm{Gx} /$ Overlay type/Sy refers to Overlay Gage $\mathbf{x} /$ Overlay type/ Stage $\mathbf{y}$. Tx/Overlay type/Sy refers to Overlay Temperature Logger $\mathbf{x} /$ Overlay type/ Stage $\mathbf{y}$

Here $\mathrm{x}$ and $\mathrm{y}$ are variables.

Where, $\mathbf{x}=1$ to 4 ---------Embedment Gage Number (refer to the above figure for the location of the gage)

Overlay type = LMC----Slab with LMC overlay without using any bonding slurry at the interface, and LMC-S----Slab with LMC overlay but bonding slurry was used at the interface

$\mathbf{y}=$ I and II ------Stage Number.

\subsubsection{Pouring}

Before placing the overlays, the surfaces of the existing decks were completely saturated with water and the puddle of water was blown out with compressed air. Before pouring the overlay concretes, supplied by Ahern Associates Inc., the prepared surface was well cleaned to make sure that the surface is free of contaminants and the surface was saturated by wetting with water. The overlay concretes were supplied in ready mix truck by Ahern Associates Inc. The overlay truck in which the overlay concretes were supplied is shown in the Figure 3.14 below. 


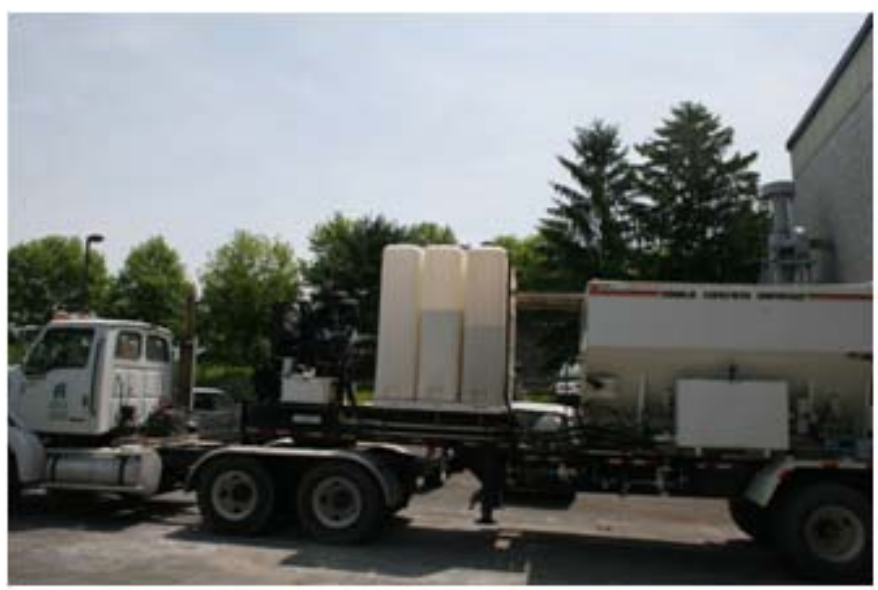

Figure 3.14: Overlay concrete supplied in ready mix truck by Ahern Associates Inc.,

The overlay concretes were transferred from ready mix truck to the slabs using the hopper unlike the substrate concrete as shown in the Figure 3.15 below, since the w/c ratio of High Performance Concrete (HPC) overlays is very low and due to which the workability is very low.

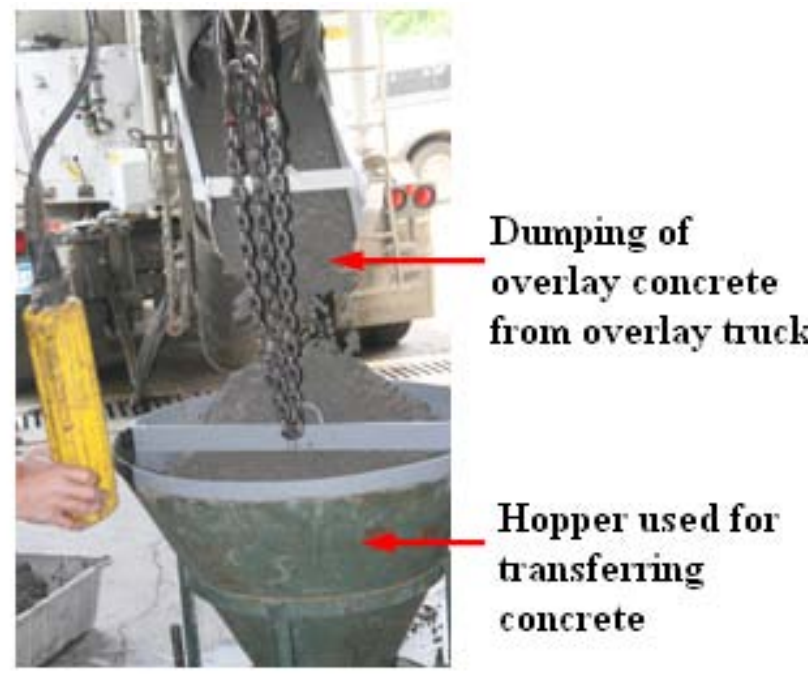

Figure 3.15: Transferring of overlay concrete from truck using hopper.

The procedure followed for preparing the bonding slurry was as follows:

1. First, approximately $6 \mathrm{~mm}(1 / 4 \mathrm{in})$ thick layer of concrete was poured on the saturated surface of the slab. 
2. Then, the concrete was evenly spread on the surface.

3. After that, the concrete was raked with the standard raking instrument to filter out all the coarse aggregates and made sure that the surface is free of the visible coarse aggregate.

4. Even after removal of coarse aggregate, the entire slab surface was covered with slurry - there was no place without it.

5. No extra water was added during the entire process.

6. Special care was taken near the locations of the strain gages to prevent the damage to the strain gages.

The preparation of bonding slurry is shown in Figure 3.16 below.

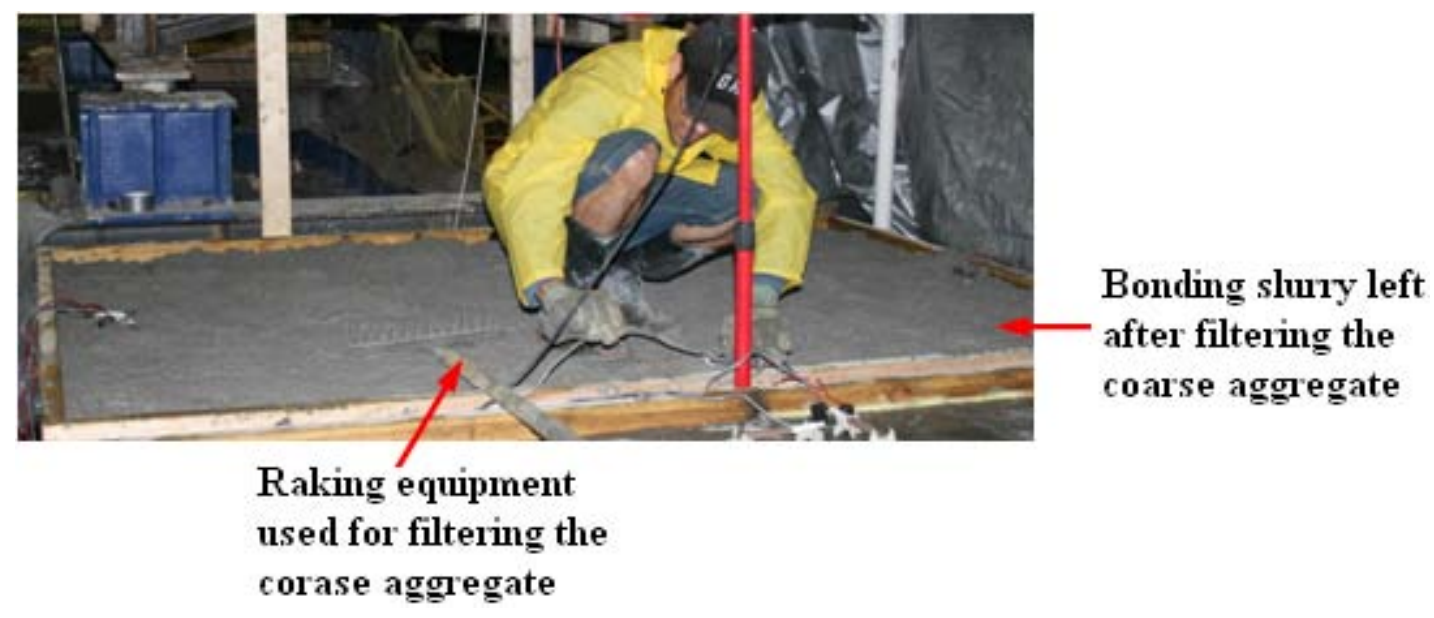

Figure 3.16: Preparation of bonding slurry.

During casting, the overlay concrete was consolidated by a concrete vibrator. Proper care was taken to make sure that the concrete was well placed on the prepared surface. 


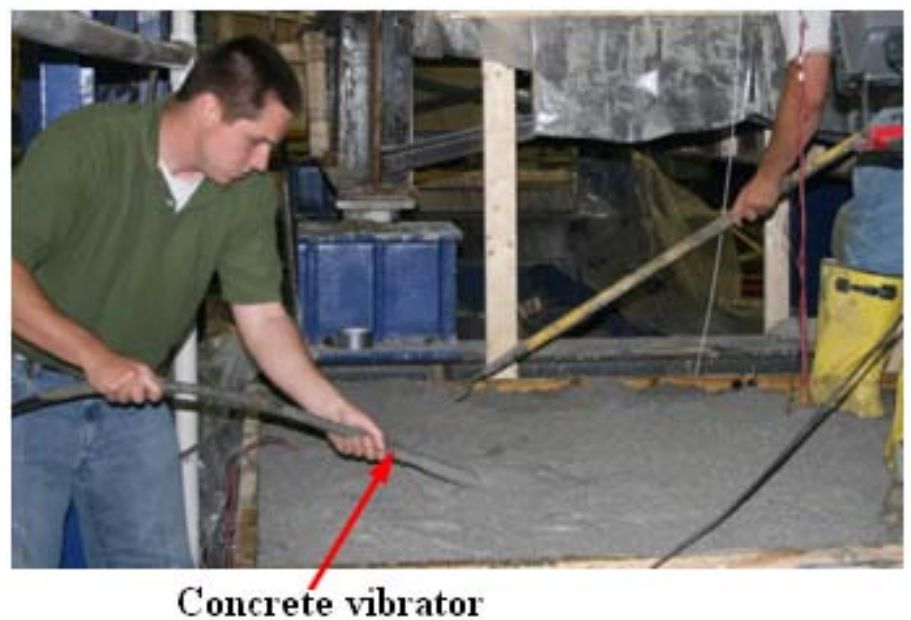

Figure 3.17: Casting of the overlay concrete

\subsubsection{Curing}

Early wet curing of the concrete overlay is very crucial to reduce the evaporation of moisture, Halvorsen (1993). After the overlays were cast, the slabs were cured by covering with wet burlap backed by plastic sheeting for 3 days. After 3 days the side formworks were demolded and the slabs were maintained within large enclosures constructed of PVC pipe skeleton and tarpaulin material, as shown in the Figure 3.18 below. To simulate the harsh field conditions for the bi-layer deck systems, two professional 1200W twin head convertible tripod towers of halogen work lights were installed, to maintain a temperature of $30^{\circ} \mathrm{C}\left(85^{\circ} \mathrm{F}\right)$ to $35^{\circ} \mathrm{C}\left(95^{\circ} \mathrm{F}\right)$, as shown in the Figure 3.19 below. Also, to simulate the air in the field, two regular fans were used to blow the air as shown in the Figure 3.19 below. 


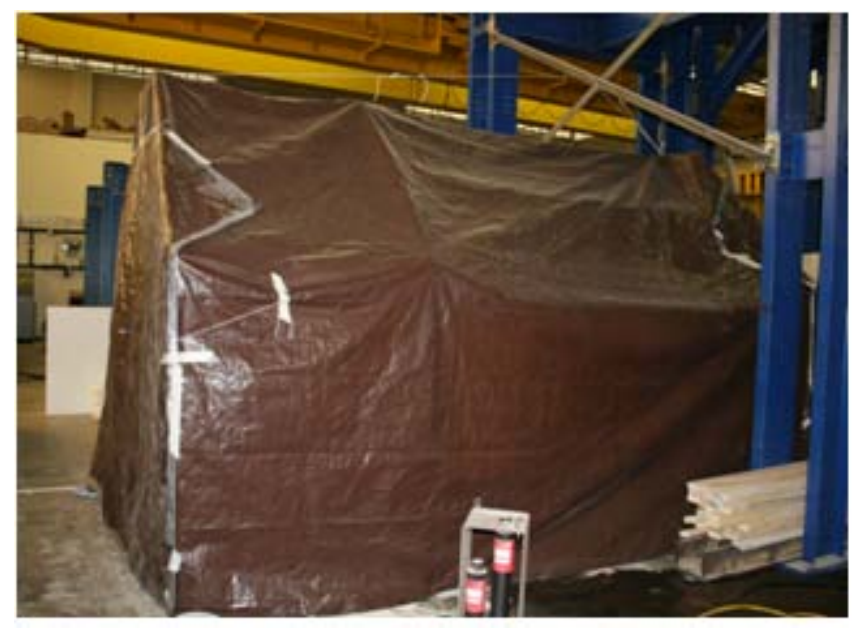

Figure 3.18: Slabs enclosed in a temporarily built tent.

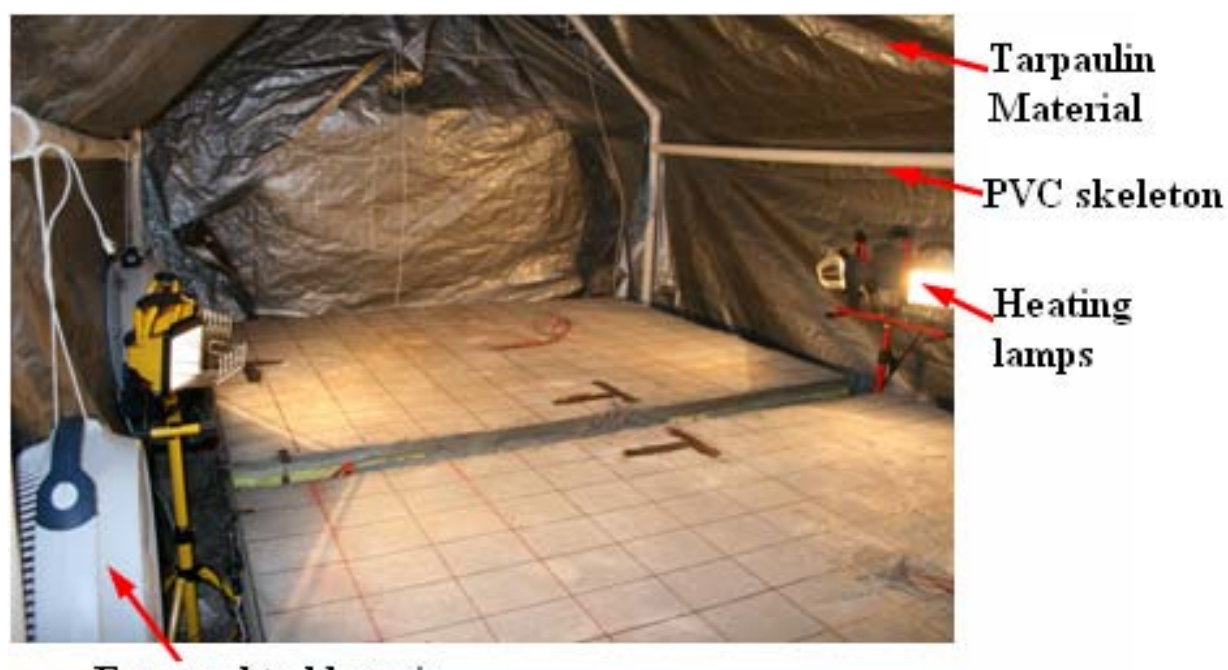

Fan used to blow air

Figure 3.19: Inside view of the tent.

\subsubsection{Installation of LVDT}

After the side formwork was demolded LVDT, manufactured by RDP Company, were installed at the two corners (locations where the plastic sheeting was provided) to monitor the vertical corner lift of the overlays. The LVDT setup at a typical location is shown in the Figure 3.20 below. 
Steel plate attached to the top sufface of overlay

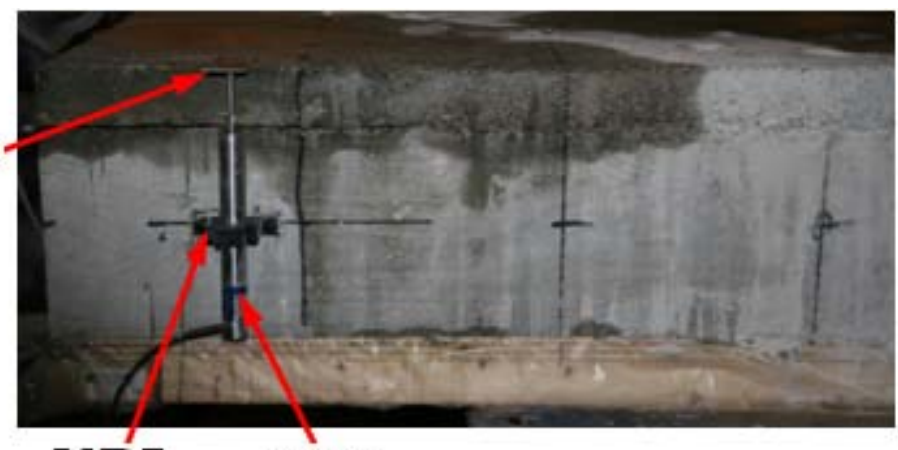

LVDT LVDT

holder

Figure 3.20: Typical LVDT installed on slab 1 


\section{CHAPTER 4: TESTING PROCEDURES}

Different types of tests performed on the small scale specimens and large scale/prototype specimens are explained in details in this section. The testing procedures throughout this study are according to relevant ASTM and AASHTO specifications and standards. All the testing procedures also comply with the requirements of the West Virginia Division of Highways (WVDOH).

\subsection{Compressive Strength}

The compressive strength of the concrete cylindrical specimens (152.4 mm (6 in.) in diameter and $304.8 \mathrm{~mm}$ (12 in.) high), cast along with the substrate and overlay casting, was measured at 7, 14, 28, 56 and 90 days respectively in accordance with ASTM C 39 (2005) (Standard Test Method for Compressive Strength of Cylindrical Concrete Specimens) and AASHTO T 22. This testing was conducted by breaking the cylindrical specimens in 35000 lbs capacity hydraulic type compression machine, manufactured by Gilson Company. The cylindrical specimens were placed on the machine, directly under the loading cell, and they were loaded at a rate of 1.38 MPa (200 Psi). The compressive strength values depends on the size and shape of the specimen, batching, mixing procedures, methods of sampling, molding, age, temperature, and moisture conditions during curing.

Before conducting this test, the top surface of the cylinders was grinded smoothly to make sure the even stress is applied on the cylinder. The failure load was recorded and the compressive strength was calculated by dividing the failure load with the resisting cross-sectional area and reported in MPa (Psi) units.

$$
f_{c}=\frac{P}{A}
$$

Where, $\mathrm{P}$, is the ultimate failure load in $\mathrm{kg}(\mathrm{lb})$

and $\mathrm{A}$, is the resisting cross-sectional area given by $A=\pi r^{2}$ in $\mathrm{mm}^{2}\left(\mathrm{in}^{2}\right)$ 


\subsection{Modulus of Elasticity Test}

The dynamic modulus of elasticity was calculated by measuring the fundamental longitudinal resonant frequencies of concrete cylindrical specimens of dimensions 150 mm (6 in.) in diameter and $300 \mathrm{~mm}$ (12 in.) high using forced resonant method. This test method was conducted following the ASTM C 215 (2002a) (Standard Test Method for Fundamental Transverse, Longitudinal, and Torsional Resonant Frequencies of Concrete Specimens), and was intended primarily for detecting the significant changes in the dynamic modulus of elasticity of laboratory specimens that are undergoing exposure to weathering or other types of potentially deteriorating influences. The testing equipment includes; Magnetic driver, pickup, and sonometer, manufactured by GEOTEST Instrument Corp.

The testing procedure followed for determining the fundamental longitudinal resonant frequency was:

1. At first, the mass of the specimen was measured in $\mathrm{kg}$.

2. Then, the cylindrical specimen was supported at its center on a thick neoprene rubber, which allows the specimen to vibrate freely, cut in desired shape to place the cylinder specimen as shown in Figure 4.1.

3. Then, the magnetic driver was positioned at the center of one end face to make sure the driving force is applied perpendicular to the specimen.

4. After that, the pickup unit was placed on the specimen making sure the direction of the vibration coincides with the pickup sensitivity.

5. Then, using sonometer, the test specimen was forced to vibrate at varying frequencies and simultaneously, the reading given by pickup unit and the oscilloscope pattern are observed for the maximum reading and ellipse shape respectively.

6. The frequency at which the above described conditions are satisfied gives the resonant frequency and this frequency was recorded for further calculations.

7. Finally, the dynamic modulus of elasticity was calculated by using the equation: 


$$
\text { Dynamic } E=D M\left(n^{\prime}\right)^{2}
$$

Where, $\mathrm{D}=5.093\left(\mathrm{~L} / \mathrm{d}^{2}\right), \mathrm{N} . \mathrm{s}^{2} /\left(\mathrm{kg} . \mathrm{m}^{2}\right)$ for a cylinder,

$n^{\prime}=$ fundamental longitudinal frequency, $\mathrm{Hz}$, recorded from step 6 , and

$\mathrm{M}=$ Measured mass of the specimen, $\mathrm{kg}$.

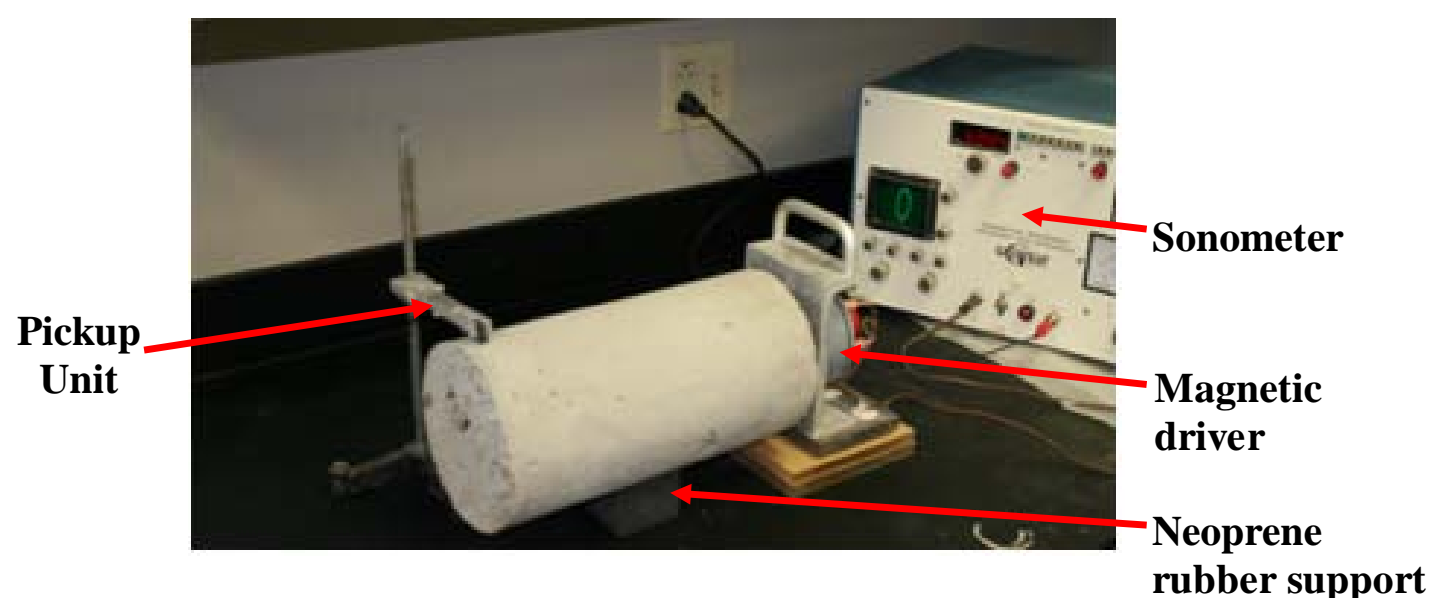

Figure 4.1: Dynamic modulus of elasticity testing in progress.

\subsection{Slant Shear Test}

The most commonly used slant shear strength testing can be used in laboratory to measure the bond strength between repair material and the substrate and was found to be most appropriate to measure the bond properties at the interface (Silfwerbrand (2003; Wall and Shrive (1988). This test method was conducted by following the standard specification ASTM C 1042 (1999) (Standard Test Method for Bond Strength of Latex Systems Used with Concrete by Slant Shear). The standard method is available for 75 mm (3 in.) by $150 \mathrm{~mm}$ (6 in.) cylindrical specimen and the available standard was modified accordingly for $100 \mathrm{~mm}$ (4 in.) by $200 \mathrm{~mm}$ (8 in.) cylindrical specimens.

The procedure followed for testing bond strength using slant shear test method was: 
1. Initially, the $100 \mathrm{~mm}$ (4 in.) by $200 \mathrm{~mm}$ (8 in.) cylindrical specimen molds were machined diagonally at $30^{\circ}$ angle from vertical face and the bottom half of the cut specimen mold was used for pouring substrate concrete.

2. Then, the mold was slightly oiled with water based form releasing agent as shown in Figure 4.2(a).

3. After that, the bottom half specimen molds were placed in a C-channel section to make sure the slant side is positioned up horizontally and the substrate concrete was poured in 3 layers and compacted by following the standard as shown in Figure 4.2(b).

4. Then, the other top half of the specimen mold was attached to the hardened substrate concrete and ready for pouring overlay concrete as shown in Figure 4.2(c).

5. After the specimen mold is ready, overlay concrete was poured in 3 layers and compacted following the standard specifications. For the tests requiring bonding slurry, the thin layer of bonding slurry, prepared from the same overlay concrete, was applied on the prepared surface before pouring the overlay concrete.

6. Then the final slant shear composite sample as shown in Figure 4.2 (d) was tested under compression in universal compression testing machine until the failure of the sample occurs.

7. The failure load and the dimensions of the major and minor axes ( $a$ and $b$ ) of the elliptical bond surface were measured and the shear bond strength was calculated by dividing failure load with the area of the elliptical bonding surface.

$$
\text { Bond strength, } f_{s}=\frac{P}{A}
$$

Where, $\mathrm{P}=$ failure load, and

$$
A=0.7854 a b \text {, Area of the elliptical bond surface, a }
$$
and $\mathrm{b}$ are the lengths of major and minor axes respectively. 


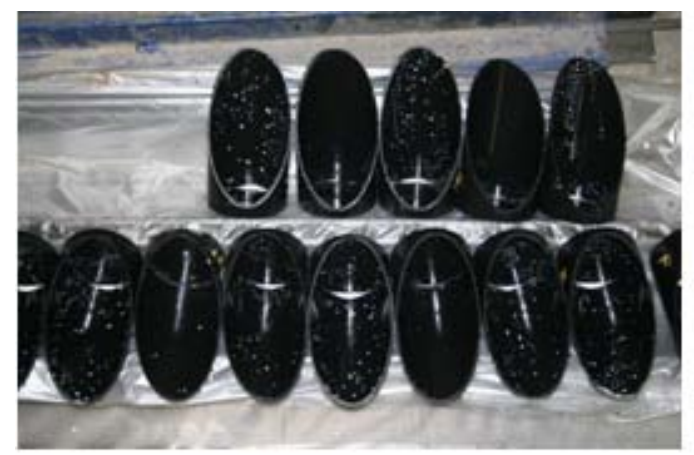

(a)

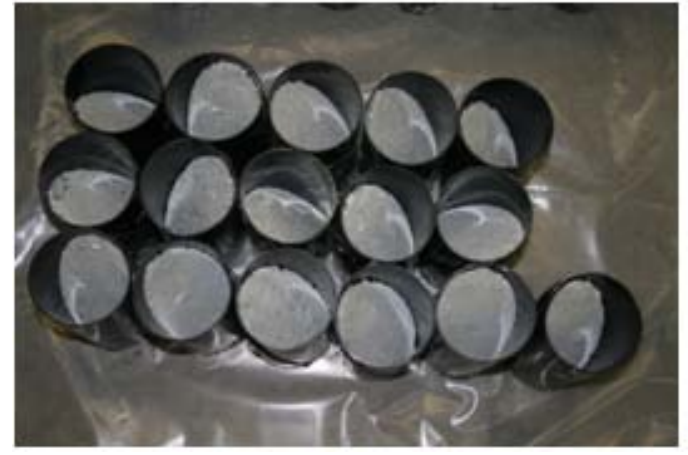

(c)

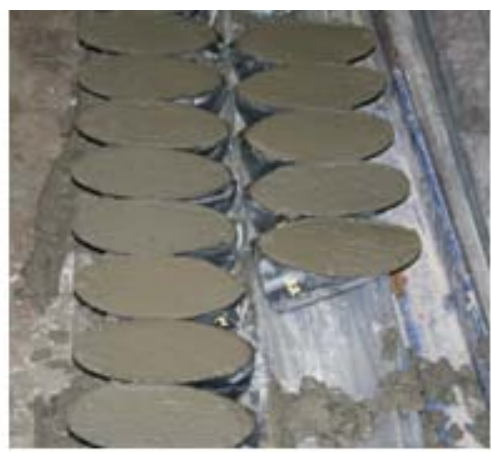

(b)

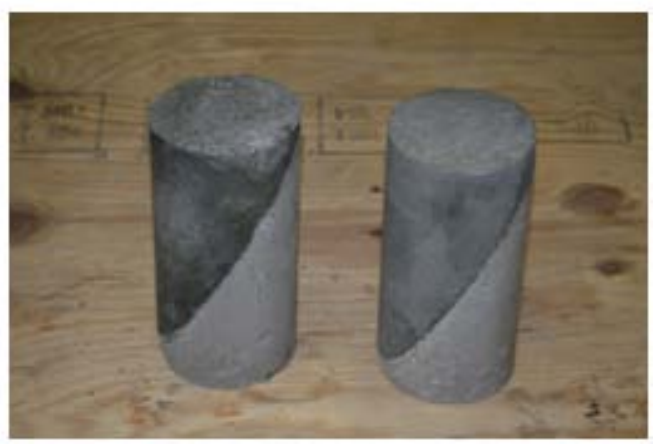

(d)

Figure 4.2: Slant shear test method: (a) half of the cut cylinders ready for pouring substrate concrete; (b)after casting with substrate concrete; (c) other half attached and ready for pouring overlay concrete; (d) slant shear specimen ready for testing.

Note: In all of the above test methods; the curing procedures, surface preparation profiles, and the demolding of formworks are similar to the procedures followed for constructing the large scale slab specimens and done simultaneously along with the same.

\subsection{Field Pull-Off Testing}

The in-situ field pull off testing was performed in accordance with ASTM C 1593 (2004) (Standard Test Method for Tensile Strength of Concrete Surfaces and the Bond Strength or Tensile Strength of Concrete Repair and Overlay Materials by Direct Tension (Pull-off Method)) to measure the tensile strength of the bond at the interface. This testing was carried out by using Dyna Z16 pull-off tester manufactured by Proceq company, which is capable of measuring the tensile force up to $16 \mathrm{KN}$ (3600 lbf). The advantages of this test over the direct tensile testing are Ramakrishnan (2000)): 
- This test will give the immediate tensile strength results, since this was done directly in the field.

- There is no necessity of carrying the specimens to the laboratory.

- For laboratory testing, a weak zone should be developed by cutting notch at the interface, which may result in breakage of the specimen and the specimen may not be valid for bond testing.

According to the testing plan, the slab was divided into two halves: on one half, pull-off testing was performed and on the other half, UPV testing was performed. The region was further divided into two zones namely edge zone (delaminated zone) and center zone (intact zone) to compare the pull-off results between those two regions. The edge zone was assumed to have been delaminated due to the induced pre-notch at the interface and center zone was assumed to be intact zone. The testing plan and the two different zones are clearly shown in the Figure 4.3 below. 


\section{Data Aquisition System}

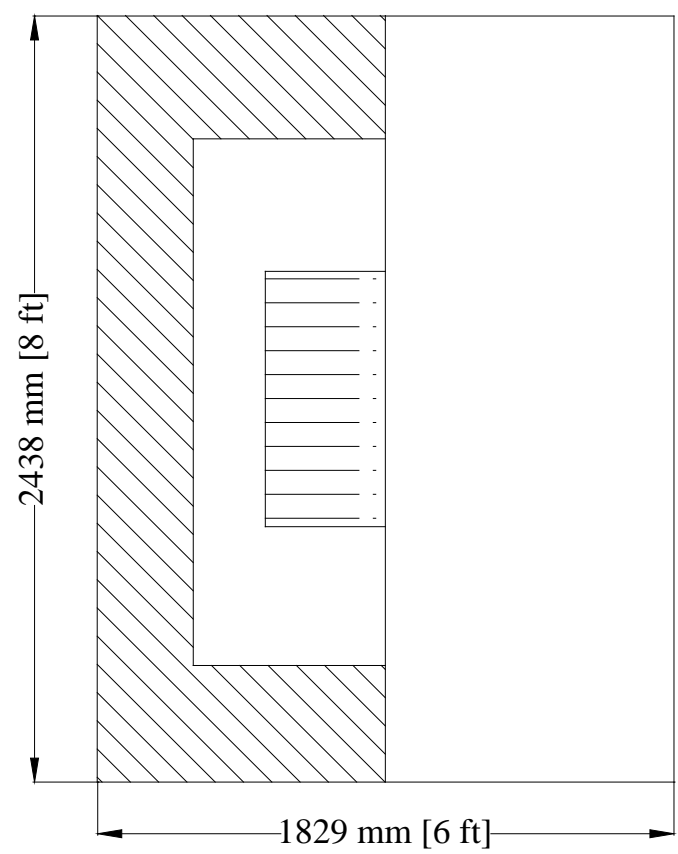

SLAB 1 (Without Bonding Slurry)

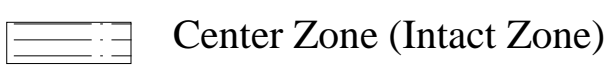

Edge Zone (Delaminated Zone)

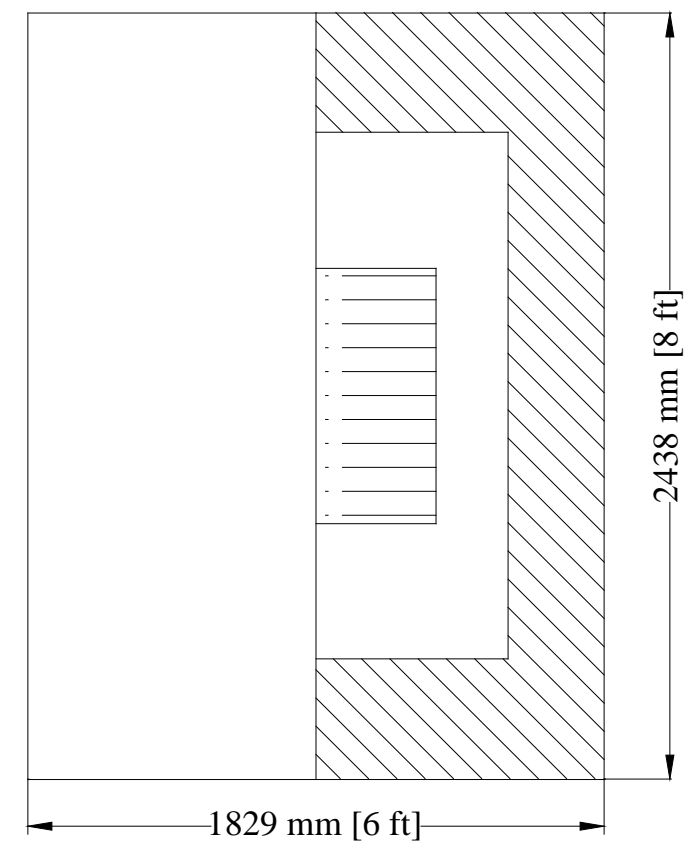

SLAB 2 (With Bonding Slurry)

Figure 4.3: Field Pull-off testing plan for Stage-I.

The Pull-off testing was mainly performed in three steps: 1) cutting the cores 2) preparing the specimens and 3) pulling of the specimens by applying tensile force with Dyna Z16 pull-off tester. All the three steps are explained in details in this section.

\subsubsection{Core Drilling}

In order to conduct the field Pull-off testing, the first step was to cut the cores through overlay concrete into the substrate concrete. Procedure followed for drilling the cores through the overlay concrete to the $12 \mathrm{~mm}$ (1/2 in) deep into the substrate concrete was:

1. Firstly, the diamond drill bit was marked at $65 \mathrm{~mm}$ (2.5 in) and the positions where the cores have to be drilled were located. 
2. Then the area near the located position was made wet with the water to attach the core drilling frame as specified in the user manual provided.

3. After that, Heavy duty core driller equipped with the vacuum pump and 50 mm (2 in.) diamond drill bit was placed on the wet surface and the pump was turned on to create the vacuum with the surface and made sure that the equipment was firmly attached to the surface of the slab.

4. Then, the motor was turned on and the drill bit was slowly driven into the overlay concrete by using the lever arm until the mark on the drill bit matches the top surface of the slab, making sure that the core was cut at least $12 \mathrm{~mm}$ (0.5 in) deep into the substrate.

5. Continuous water supply to the drill bit was maintained using the water tank as long as the machine was operated in order to keep the drill bit cool.

The procedure for core drilling is shown in the Figure 4.4 below.

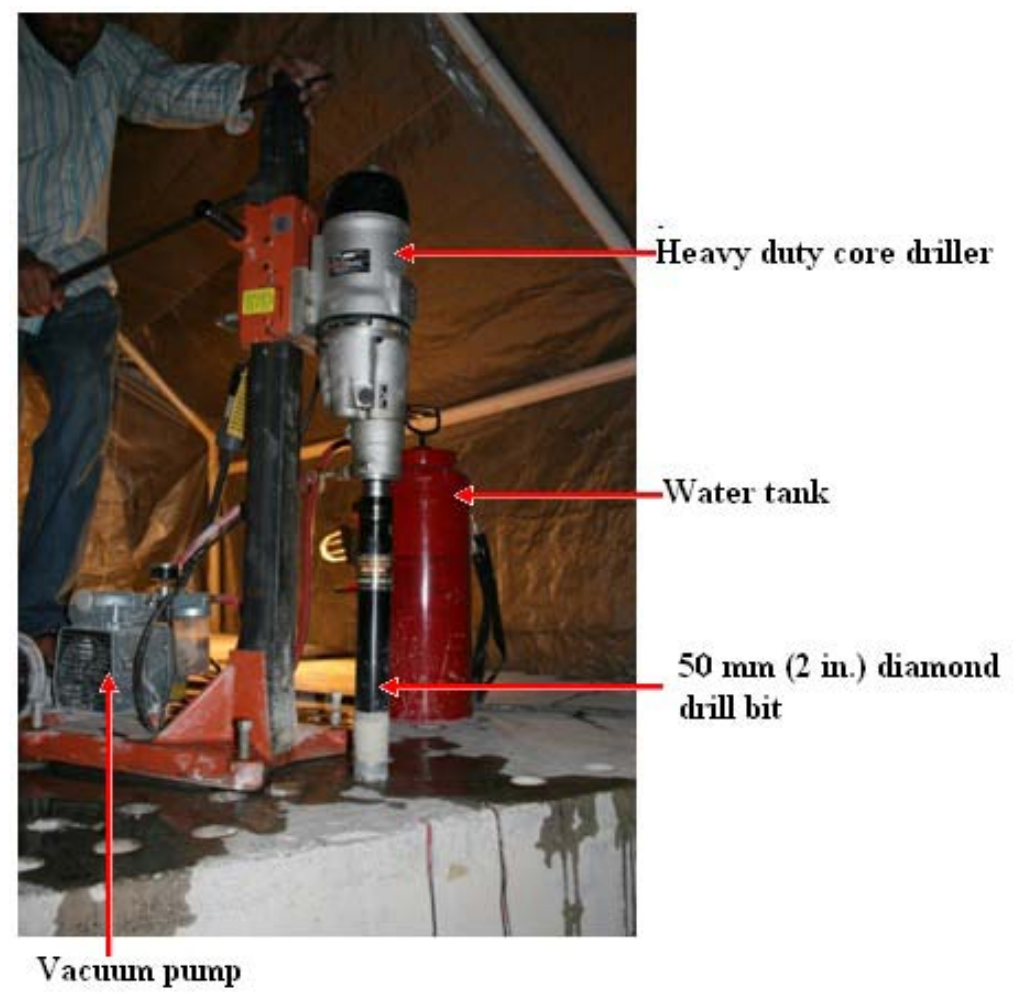

Figure 4.4: Core drilling in progress at a typical location. 


\subsubsection{Preparation of Specimen}

After the cores were drilled at the test locations, the cores were allowed to dry until all the water is evaporated, to attach the $50 \mathrm{~mm}$ ( 2 in.) aluminum test discs on the cut cores. After making sure all the water was dried off, the surface of the test cores was polished with a standard grinder. Then, three parts of epoxy and 1 part of saturant was mixed thoroughly and used as an adhesive to attach the aluminum test discs firmly to the test cores as shown in the Figure 4.5 below. Adequate amount of epoxy was applied to make sure the epoxy does not go through the sides into the hole.

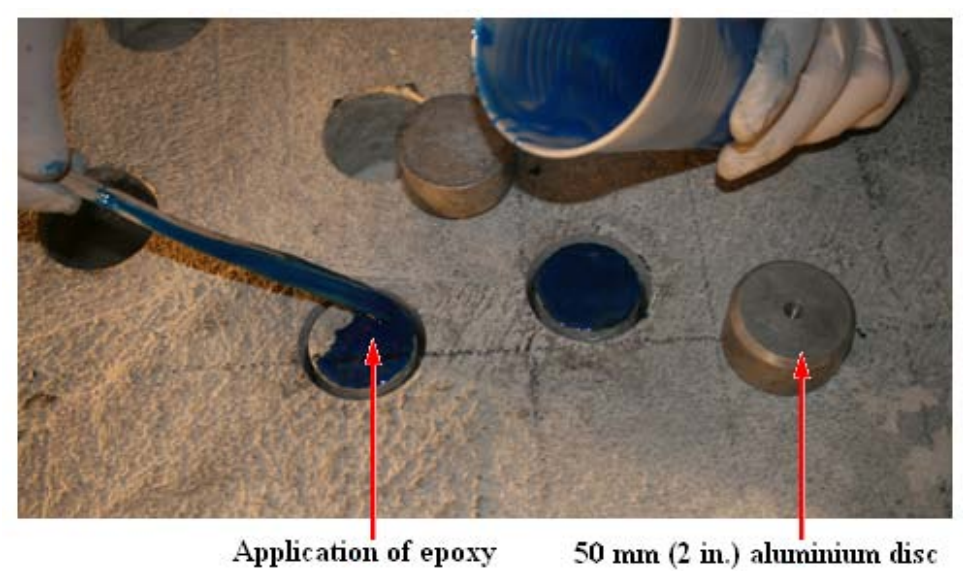

Figure 4.5: Typical preparation of the specimens.

\subsubsection{Testing Procedure}

Procedure followed for conducting the pull-off testing on the prepared specimens was as follows:

1. Aluminum test discs attached to the cut cores were allowed to cure for $24 \mathrm{hrs}$.

2. Then, a small $9 \mathrm{~mm}$ (3/8in) screw with spherical head, provided along with the PROCEQ DYNA Z16 pull-off tester, was screwed into the aluminum test discs.

3. After that, the testing equipment was positioned on top of the test disc and with the help of a notched wheel; the base of the equipment was fixed to the head of the spherical screw.

4. The reading on the manometer was set to zero by pressing both the start button and reset button simultaneously. 
5. Then, the wheel was screwed down until the reading on the manometer changed from zero.

6. Then, the load was applied gradually by turning the crank slowly, making sure the load was applied at a rate of $0.04 \mathrm{MPa} / \mathrm{s}$ (2 psi/s) as per ASTM C1593 standard.

7. The failure load and the failure mode were recorded and after each test, the crank was returned to its original position by turning the crank in opposite direction.

Pull-off testing at typical location is shown in Figure 4.6 below.

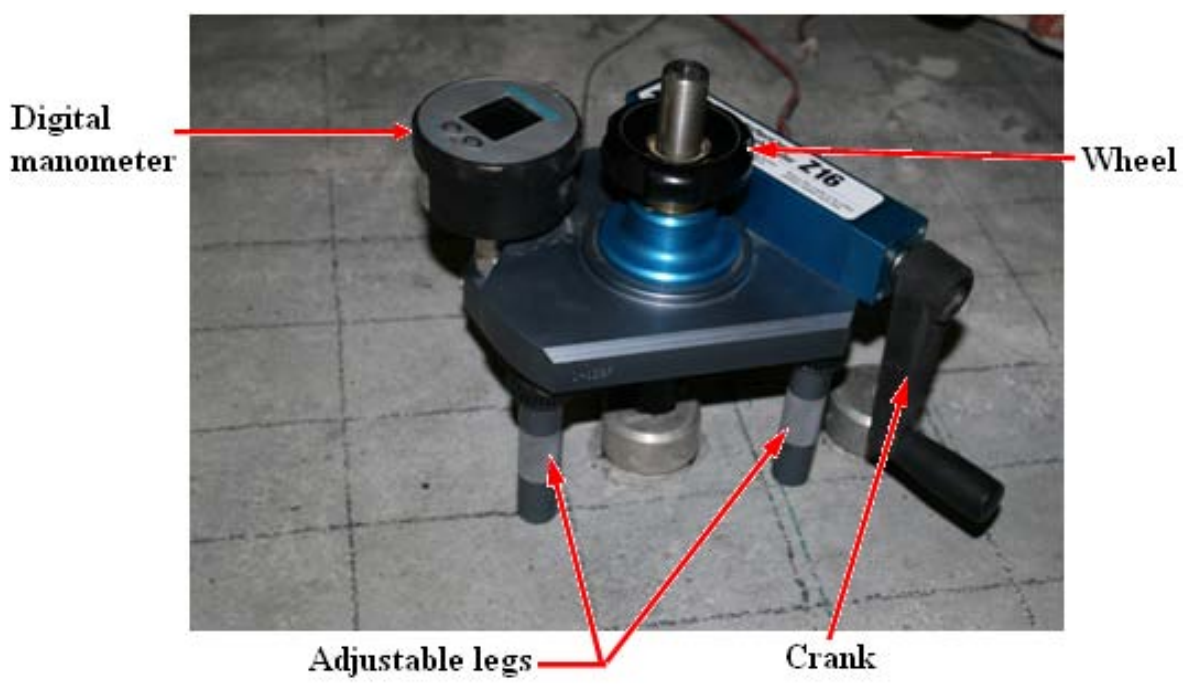

Figure 4.6: Pull-off testing in progress at a typical location.

\subsection{Length Change Monitoring and Vertical Corner Lifts}

All the ends of the embedment gages were soldered to a D-sub connector to develop a quarter bridges following the guidelines for System 5000 Model 5100 scanner provided by Vishay Company. Then, all the embedment strain gages and LVDT were connected to a data acquisition system, System 5000 Model 5100 Scanner (Figure 4.7) from Vishay Intertechnology Company, which was managed by the data management software, StrainSmart installed in a IBM laptop computer. All these equipment were connected to a backup power using UPS (Uninterruptible Power Supply). The data was 
collected for every 15 minutes immediately after the substrate pour and was recorded until the 120 days after the overlay pour.

A total of 8 embedment gages were installed in each bi-layer deck slab: four of them were installed in substrate concrete, $25 \mathrm{~mm}$ (1 in.) below the interface, and other four were installed in overlay concrete, $25 \mathrm{~mm}$ (1 in.) above the interface, to monitor the differential length change developed at the interface (Figure 3.13). 1 LVDT was installed on each slab at the edge where the pre-notch was introduced, to compare the relative vertical displacement of overlay concrete of one slab with the other (Figure 3.20). LVDT was installed across the interface between overlay and substrate concrete so that, the relative vertical displacements of overlay to substrate concrete can be measured.

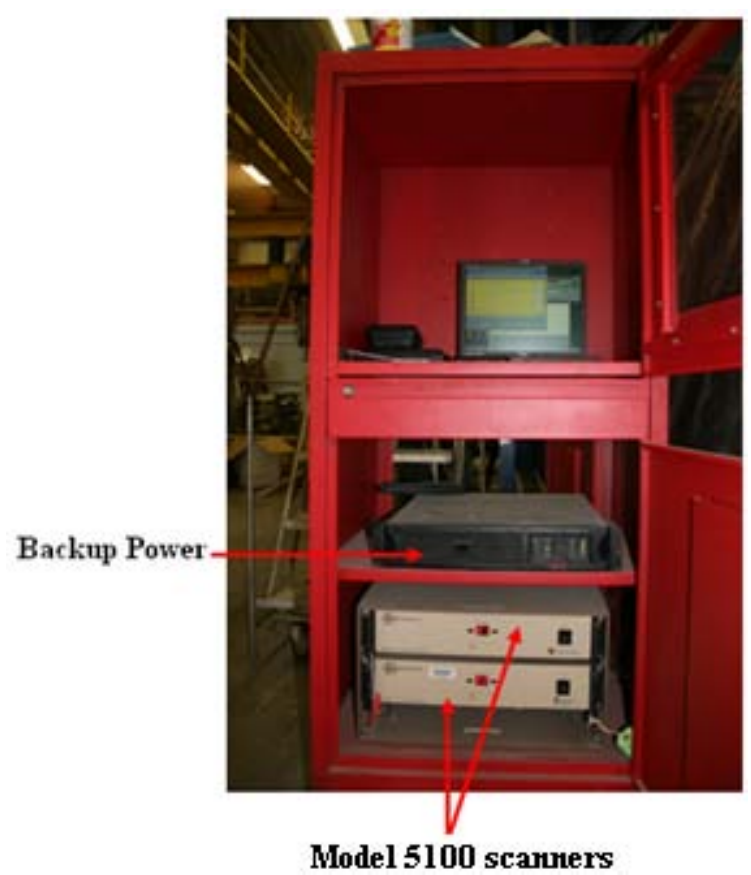

Figure 4.7: Data acquisition system used for recording strains and vertical corner lifts.

\subsection{Temperature Monitoring}

After the substrate concrete was poured, the thermocouple loggers, embedded in the substrate concrete, were started immediately to monitor the heat of hydration and the temperature variation as the slabs mature, using the handheld data logger manufactured by Engius Company as shown in the Figure 4.8 below. The handheld intellirock reader 
was used to communicate with and download the temperature data recorded by the embedded intellirock loggers. Later, the downloaded data from embedded intellirock logger was transferred to the computer by using the data cable and the intellirock software provided by the Engius Company.

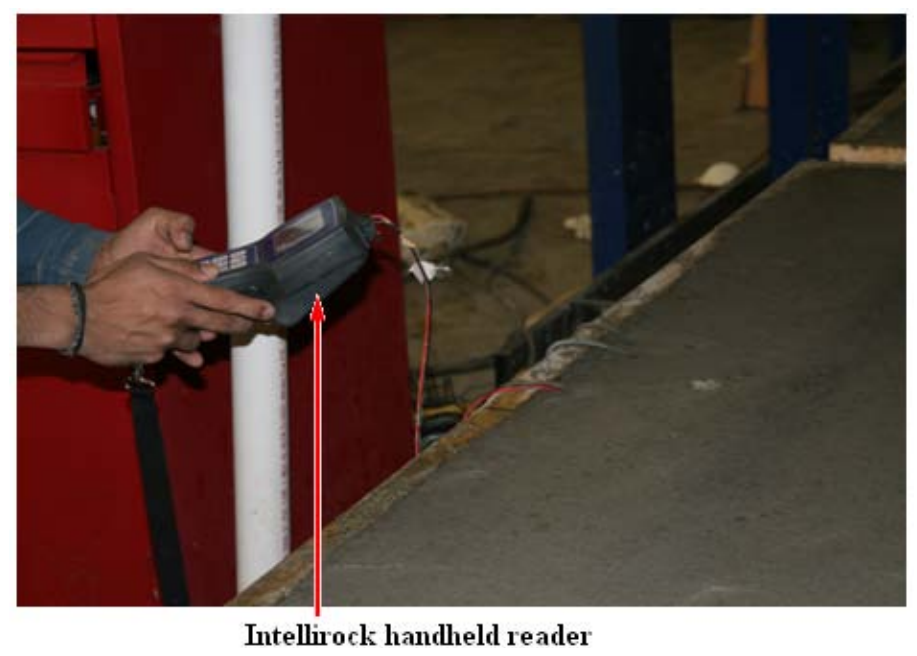

Figure 4.8: Monitoring of temperature at the typical location.

\subsection{Ultrasonic Pulse Velocity (UPV) Testing}

The UPV testing was performed on the other half of the slabs, as mentioned earlier in section 4.4, following the guidelines of (2002a) (Standard Test Method for Pulse Velocity through Concrete) to evaluate the bond condition at the interface. The ultrasonic pulse signal was sent through the bi-layer deck system using transmitter transducer and was received by the receiving transducer; the time of arrival of the first wavefront through the concrete is recorded by the UPV tester to the nearest $0.1 \mu \mathrm{s}$. and by knowing the time of flight and the distance wave travelled, velocity of the wave was calculated. This method was performed based on the concept that higher velocity is obtained when concrete quality is good in terms of homogeneity and density. The oscilloscope was connected to the UPV tester to capture a time-domain signals, which are further analyzed by signal processing techniques. Testing equipment used and the testing procedure followed are explained in this section. 


\subsubsection{Testing Equipment}

The equipments used for conducting the UPV testing and signal processing in this research program were: 1) a pulse velocity tester (V-Meter II), 2) two $82 \mathrm{kHz}$ transducers, and 3) a digital storage oscilloscope.

A pulse velocity tester (V-Meter II), manufactured by James Instruments Company, generates an ultrasonic pulse in the transmitting transducer and measures the transit time taken by the pulse to receive the receiving transducer through the medium. It gives a direct reading of the time of transmission of an ultrasonic pulse passing from a transmitting to a receiving transducer with resolutions of 0.1 micro seconds. It mainly consisted of high voltage pulse generator; which generates a high voltage pulses triggered by charging the capacitance to a high voltage, a built-in microcomputer; central processing unit which controls all the functions and is capable of recording the delay due to the different transducers and corrections are made automatically by subtracting the delay caused, receiving amplifier; which is used for amplifying the received signal, and a master clock; generates the timing pulses for the 0.1 micro sec unit.

The transducers consisted of lead zirconate titanate (PZT4) ceramic piezo electric elements mounted in stainless steel cases. The elements are very tightly held on to the inside face of the case to provide highly efficient acoustic transmission. The shock excitation of the pulse generator causes the transducer to oscillate mechanically at its own natural frequency. Different sizes of piezo electric element and case enable different pulse frequencies to be obtained. The transducers selected for this study were $82 \mathrm{kHz}$ transducers. The adequate amount of coupling agent was applied at each location where the testing was conducted. The transducers were firmly pressed at the test location to make sure the transducers were in complete contact with the test surface so that, all the energy of the pulse is traversed directly through the concrete.

A digital storage oscilloscope, Tektronix TDS1002 60 MHz 2 Channel Digital Real-Time Oscilloscope manufactured by Tektronix Company, was used to record the 
time domain signal of the pulse recorded by the receiving transducer. Also, the displayed time domain signals on the oscilloscope were exported into *.bmp image and *.CSV file formats and these files were saved into a compact flash drive installed in a communication module by pressing the print key on the oscilloscope. Those data was later saved into a computer and further analysis was done by using MATLAB software, powerful analytical and mathematical software.

\subsubsection{Testing Procedure}

According to testing plan, the other half of the slab was used for conducting UPV. In Stage-I, indirect UPV testing was performed to understand the interface bond condition by reading the UPV number and FFT of the signal collected. The gridlines of dimensions $150 \mathrm{~mm}$ (6 in.) in transverse direction by $200 \mathrm{~mm}$ (8 in.) in longitudinal direction, labeled from $G$ to $M$ in transverse direction on slab 1 and from $A$ to $G$ in transverse direction on slab 2 and labeled from 1 to 13 in longitudinal direction on both slabs, were drawn on top surface of the slabs. Transmitting transducer was kept constant at the center of the slab i.e., at G7 and the location of the receiving transducer was varied to measure the UPV number and simultaneously, time-domain signal was captured using oscilloscope. The gridlines drawn for conducting the indirect UPV testing is shown clearly in Figure 4.9 below. 

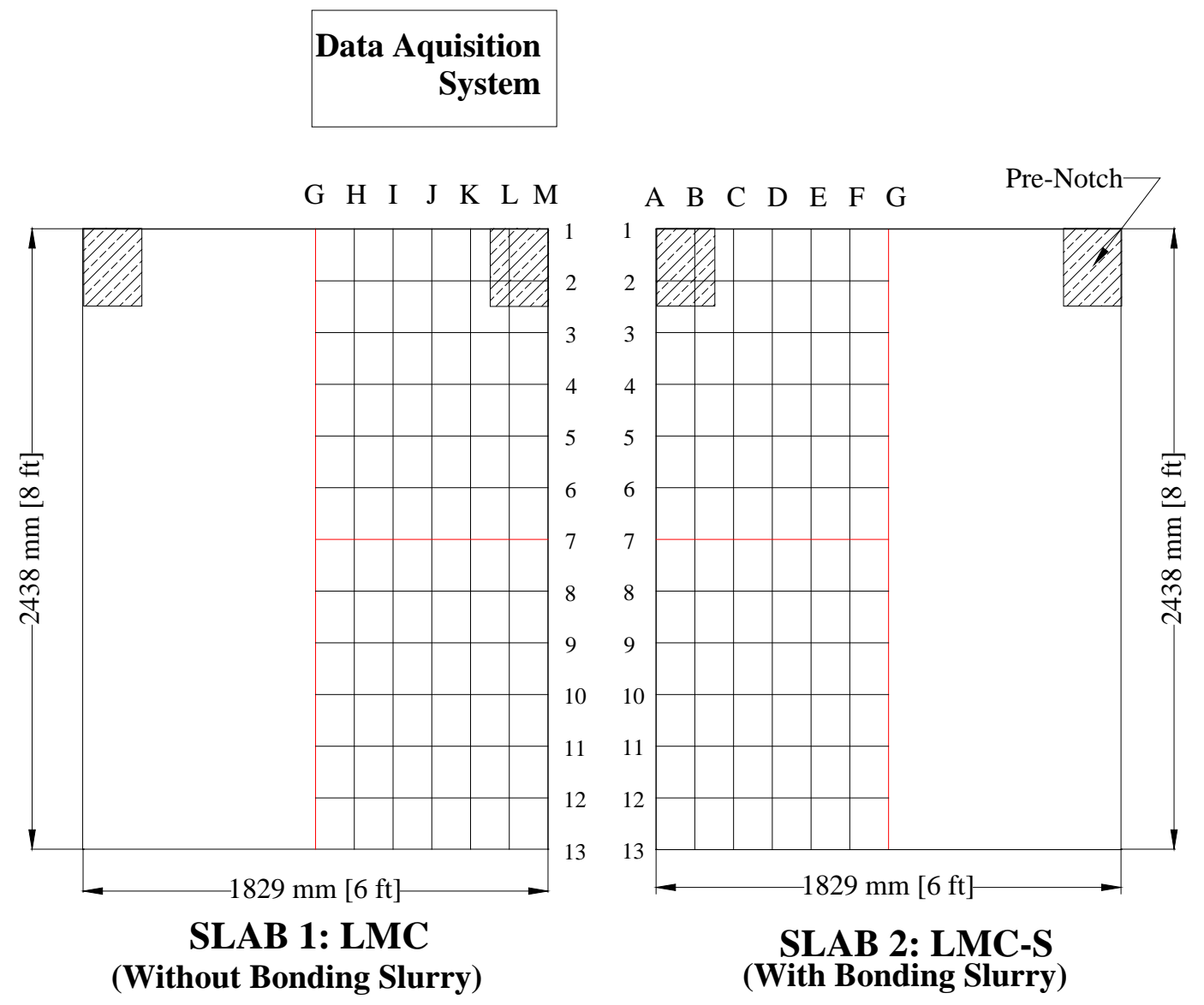

Figure 4.9: Gridlines for conducting indirect UPV testing in Stage-I

In Stage-II, direct UPV testing was performed. The gridlines of dimensions 75 mm (3 in.) in transverse direction by $100 \mathrm{~mm}$ (4 in.) in longitudinal direction, labeled from $\mathrm{M}$ to $\mathrm{Z}$ in transverse direction on slab 1 and from $\mathrm{A}$ to $\mathrm{M}$ in transverse direction on slab 2, and labeled from 1 to 24 in longitudinal direction on both slabs, were drawn on both top and bottom surfaces of the bi-layer slab to exactly maintain the perfect locations of transducers. The transmitting transducer was located at the junction of the grid on top of the slab and the receiving transducer was placed at the matching location at the bottom of the slab, the same procedure was repeated at different locations to record the UPV number and the time-domain signal to compare between LMC and SFMC slabs. The gridlines drawn for conducting the indirect UPV testing is shown in Figure 4.10 below. 


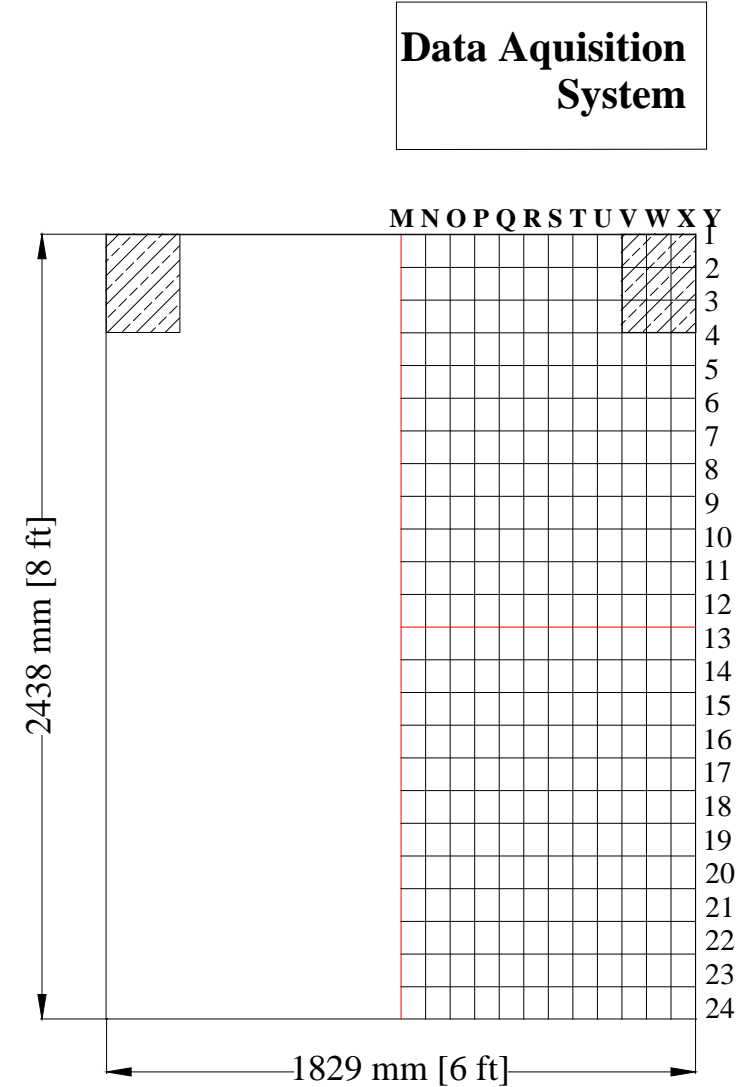

SLAB 1: LMC

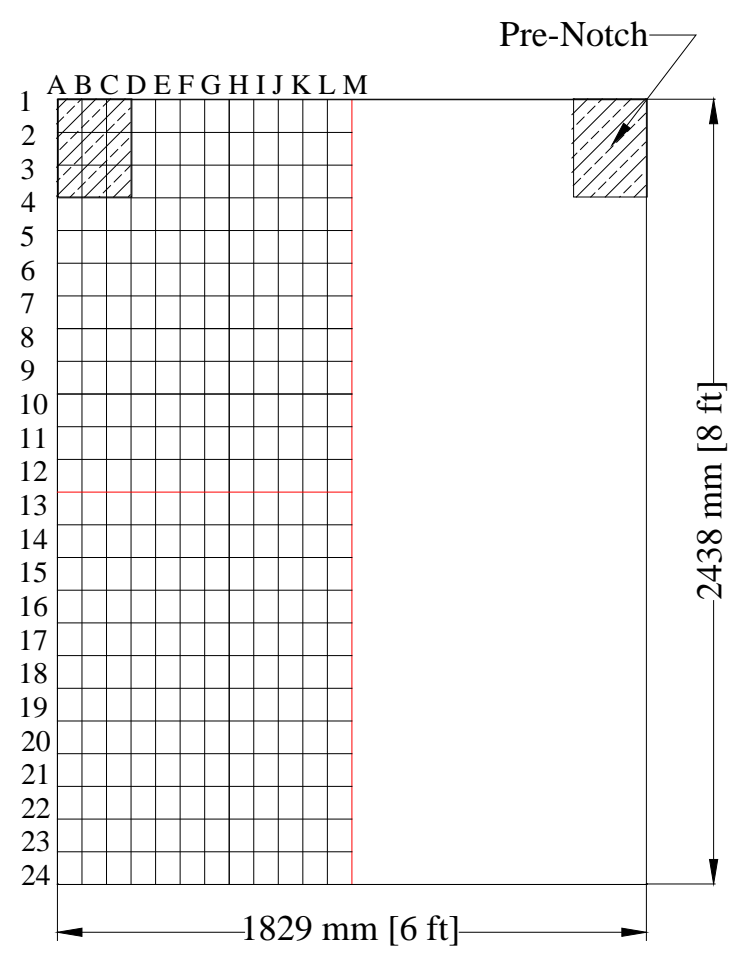

SLAB 2: SFMC

Figure 4.10: Gridlines for conducting direct UPV testing in Stage-II.

Steps followed for conducting the UPV testing through the bi-layer deck system was as follows:

1. After the gridlines were drawn, on the junctions at whom the testing has to be conducted was grinded with regular grinder to smoothen the surface to establish a perfect contact between the transducer and the concrete surface.

2. Then, commercially available petroleum jelly, used as a coupling agent between transducer and concrete surface, was applied at the test locations. 


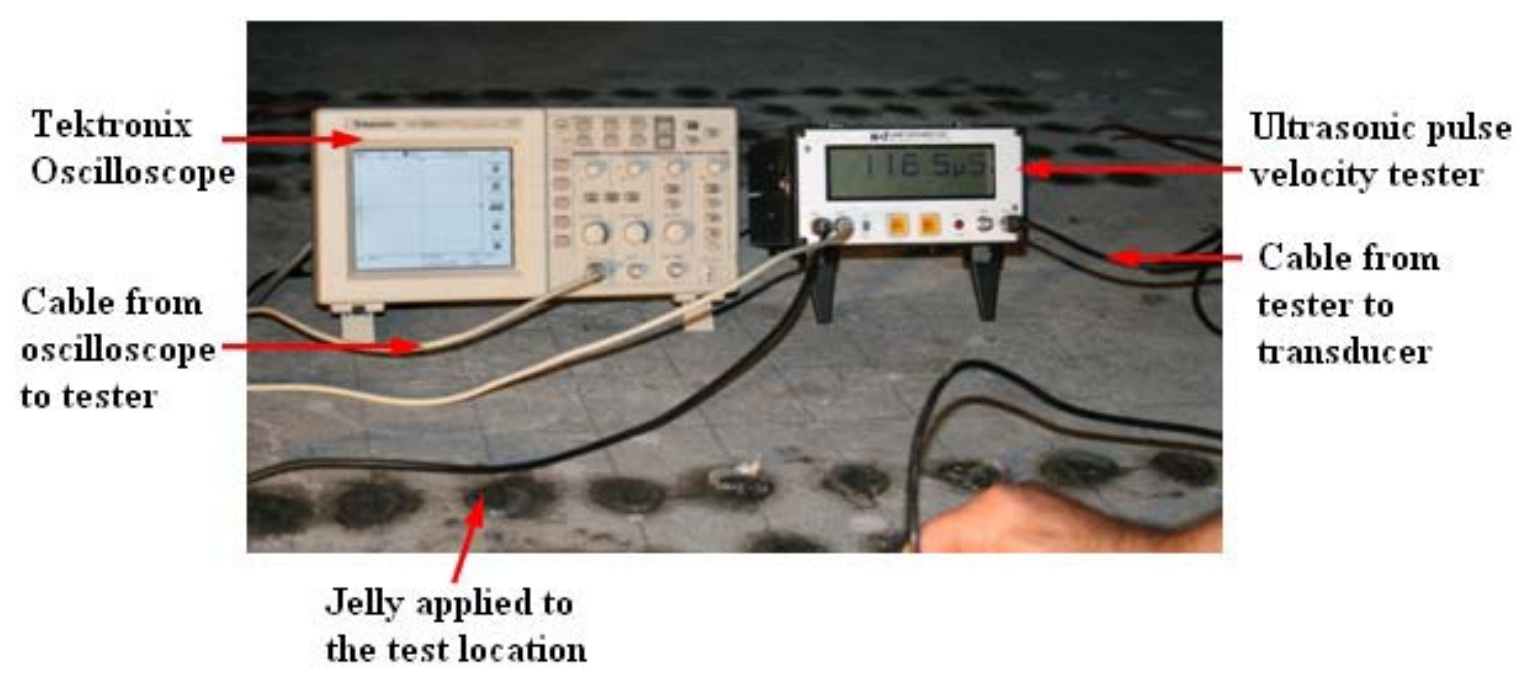

Figure 4.11: Testing equipment used for conducting UPV testing.

3. After that, the transmitting and receiving transducers were pressed firmly to the concrete surface at the test locations.

4. Then, the time of flight of ultrasonic pulse from transmitter to receiving transducer and the pulse waveform in time-domain was recorded using VMeter II and oscilloscope respectively. For each measurement, the time of flight was recorded after the reading on V-Meter II was settled to minimum number and maintained constant to ensure the recorded time of flight is the least transmitting time.

5. Readings along the edges and at the locations where pre-notch was present were discarded.

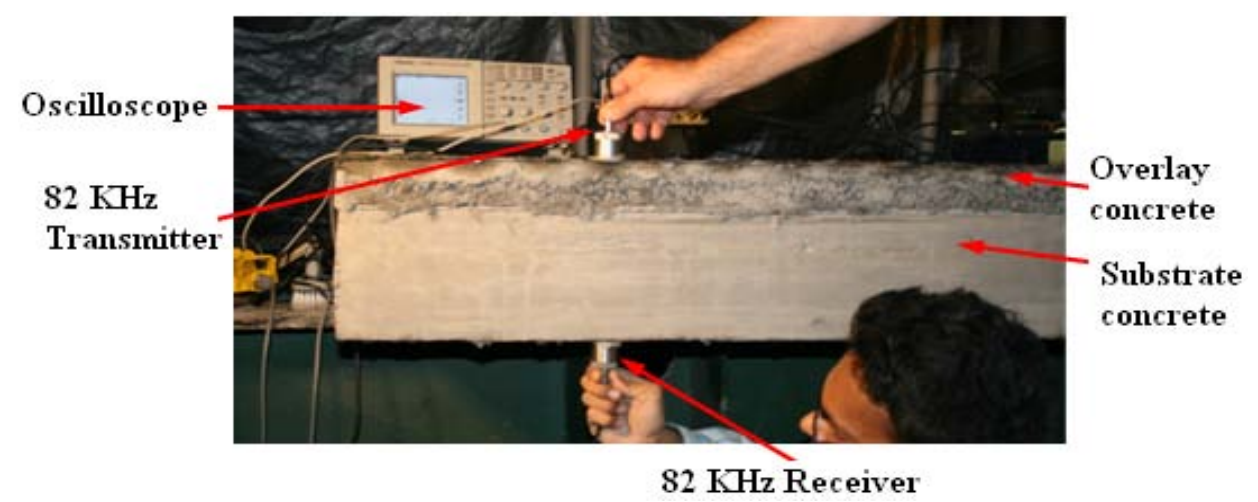

Figure 4.12: UPV testing in progress at typical location in Stage-II. 


\section{CHAPTER 5: STAGE-I: TEST RESULTS AND DISCUSSIONS}

This chapter presents the test results and discussions for Stage-I experiments on small scale and large scale specimens. The experiments on small scale specimens include compressive strength at different ages and dynamic modulus of elasticity at different ages on $150 \mathrm{~mm}$. (6 in.) by $300 \mathrm{~mm}$. (12 in.) cylindrical samples. The experiments on large scale prototype deck slabs include direct tensile bond tests using pull-off tests, shrinkage strain and temperature monitoring of substrate and LMC overlays, vertical corner lift due to curling effect at the pre-notched corners, and indirect UPV testing followed by signal processing to study the delamination at the interface on two $1829 \mathrm{~mm}$. (6 ft.) by 2438 mm. (8 ft.) slabs with two different bonding conditions. The results are presented in tables and figures.

\subsection{Tests Conducted on Specimens}

The compressive strength and dynamic modulus of elasticity tests were carried out on $152 \mathrm{~mm}$. (6 in.) by $305 \mathrm{~mm}$. (12 in.) cylindrical specimens at different ages. Three replicate specimens of each substrate Type K concrete and LMC overlay were cast and tested at each age. The average compressive strength and average dynamic modulus of elasticity was calculated and produced. The compressive strength testing was carried on the same specimens that were used for measuring the dynamic modulus of elasticity.

\subsubsection{Compressive Strength}

The compressive strength of substrate Type K concrete was measured at 28 days after substrate pour and 14, 28, and 56 days after overlay pour. The compressive strength of LMC overlay was measured at 7, 14, 28, and 56 days respectively for quality control purposes and to monitor the strength development with time. The compressive strength was measured by following the standard ASTM C39, (Compressive Strength of Cylindrical Concrete) specifications. 
The average compressive strength values of 3 specimens at each age were calculated and plotted as a bar graph to compare the compressive strengths of Type $\mathrm{K}$ normal concrete and LMC overlay. The compressive strength values for Type K substrate concrete and LMC overlay concrete are shown in Figure 5.1.

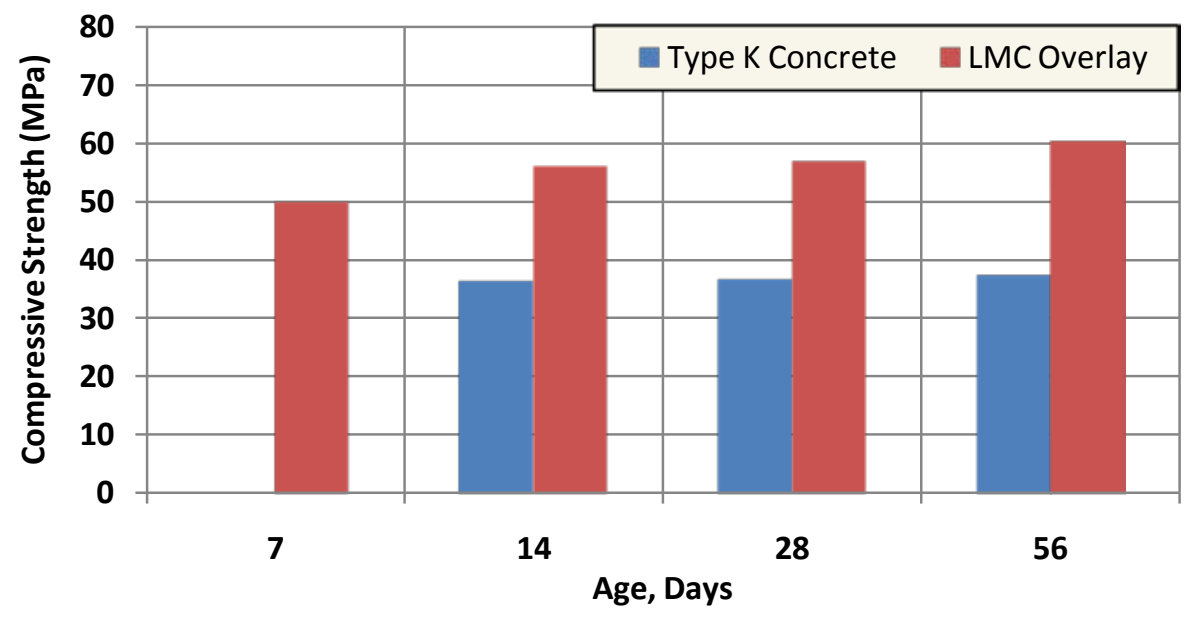

Figure 5.1: Compressive strength data of Type K substrate and LMC overlay concretes after overlay pour (Stage-I).

Figure 5.1 indicates that the compressive strength of both the concretes increased with the increase in age. LMC showed the highest compressive strength compared to that of Type K substrate concrete. Average compressive strength of LMC overlay at 7, 14, 28, and 56 days was $50 \mathrm{MPa}$ (7250 psi), $56 \mathrm{MPa}$ (8120 psi), $57 \mathrm{MPa}$ (8270 psi), and $60 \mathrm{MPa}$ (8700 psi) respectively. LMC overlay mixture satisfied the minimum 28 day compressive strength criteria mentioned by WVDOH, which is $28 \mathrm{MPa}$ (4000 psi). Luo (2002) reported the 28 day compressive strength of LMC as 48 MPa (6950 psi). Sprinkel (2005) reported the 28 day average compressive strength of LMC with Type K cement as 38.7 MPa (5620 psi).

Average compressive strength of Type K normal concrete at 28 days was $36 \mathrm{MPa}$ (5220 psi) and the average compressive strength at 14, 28, and 56 days after overlay pour was $36.3 \mathrm{MPa}$ (5250 psi), 36.6 MPa (5300 psi), and $37 \mathrm{MPa}$ (5350 psi) respectively. 
Rectangular hyperbolic fits of compressive strength was developed, using OriginLab v 7.5, based on the compressive strength results and are shown in Figure 5.2(a) and Figure 5.2(b). The general form of the equation used for generating the rectangular hyperbolic fit is:

$$
Y=\frac{a b x}{1+b x}
$$

Where:

$Y=$ compressive strength at any age, $\mathrm{MPa}$;

$x$ = age in days; and

$a$ and $b$ are constants.

$a$ and $b$ are the constants generated by OrginLab using the best fit equation shown above. Table 5.1 shows the corresponding parameters for Type K substrate concrete and LMC overlay.

Table 5.1: Parameters for rectangular hyperbolic fit

\begin{tabular}{|c|c|c|}
\hline & Type K Normal Concrete & LMC \\
\hline $\mathbf{a}$ & $37.582 \pm 0.257$ & $61.426 \pm 0.954$ \\
\hline $\mathbf{b}$ & $0.589 \pm 0.118$ & $0.629 \pm 0.096$ \\
\hline $\mathbf{R}$ & 0.999 & 0.999 \\
\hline
\end{tabular}




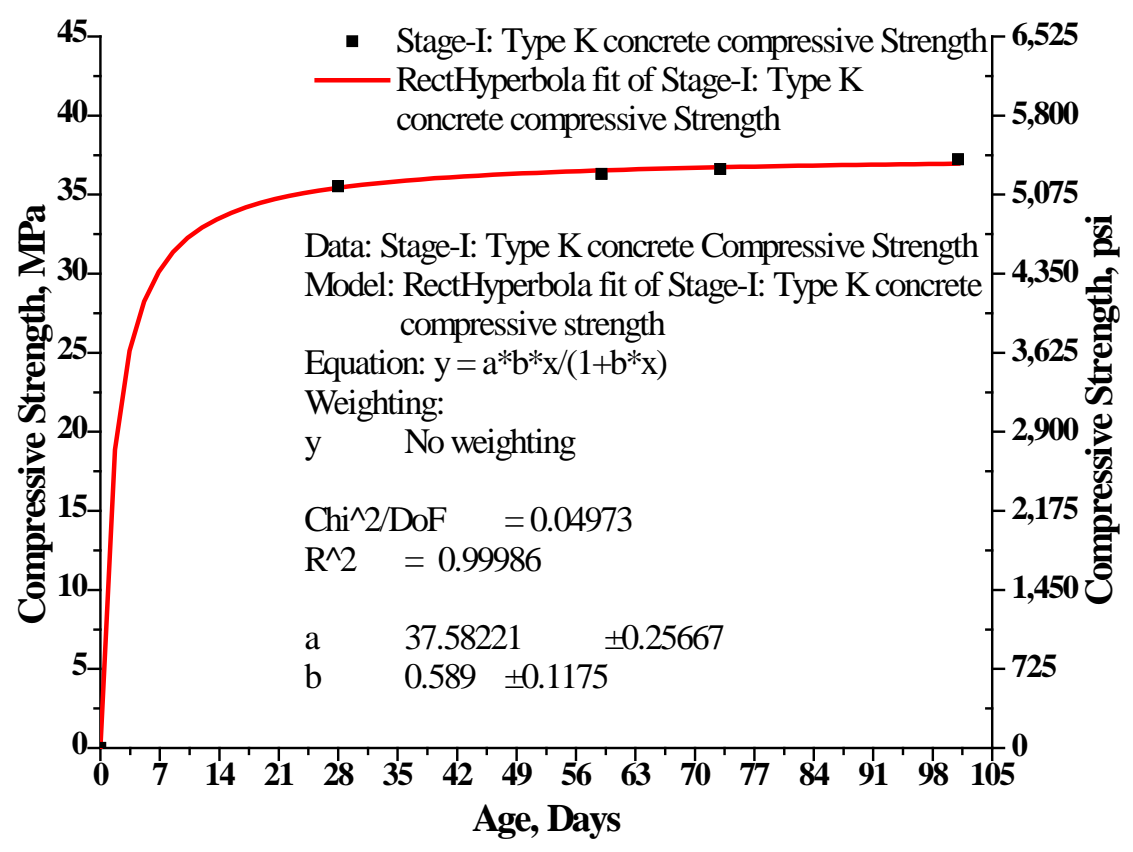

(a)

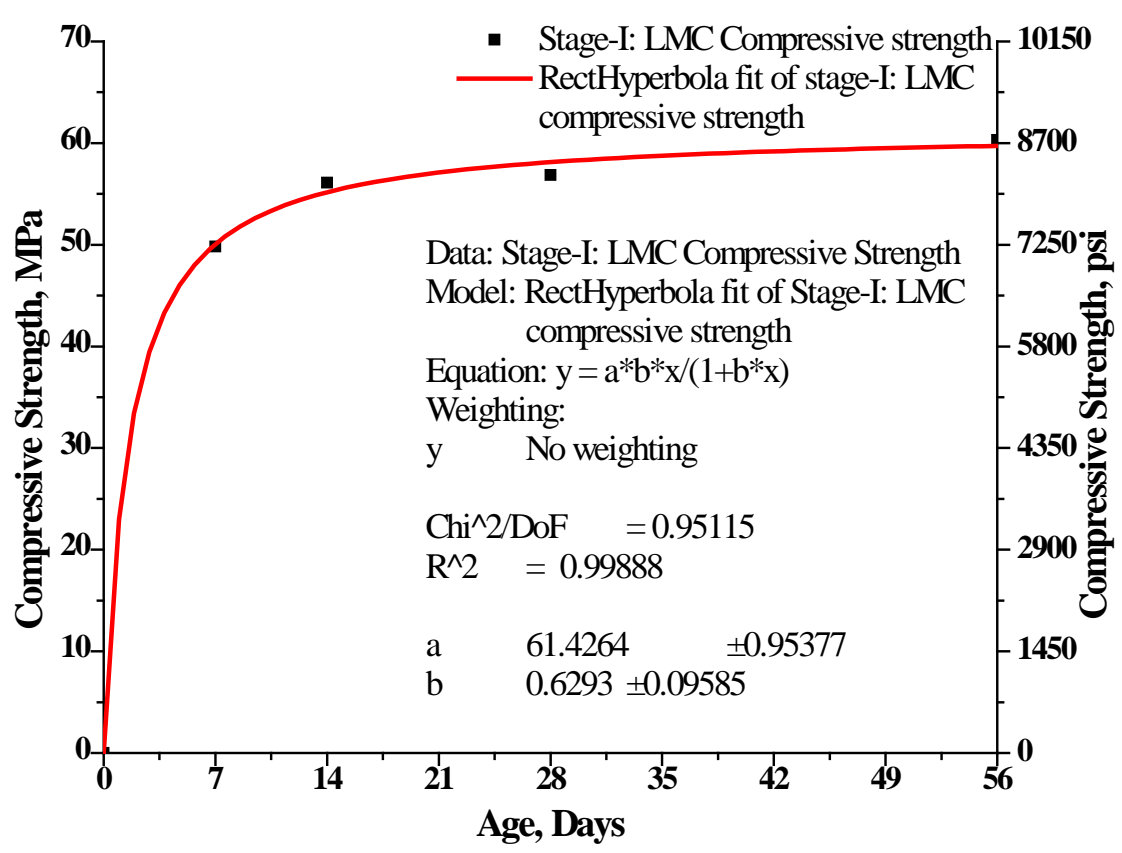

(b)

Figure 5.2: Rectangular hyperbolic fit plots of: (a) Type K concrete compressive strength; and (b) LMC compressive strength (Stage-I) 


\subsubsection{Dynamic Modulus of Elasticity}

The dynamic modulus of elasticity of Type K substrate concrete was measured at 28 days after substrate pour and 14, 28, and 56 days after overlay pour. The dynamic modulus of elasticity of LMC overlay was measured at 7, 14, 28, and 56 days respectively for quality control purposes. Three specimens were tested at each age and the average values are plotted and discussed here. This test was carried out by following the guidelines of ASTM C215 (2002a) (Standard Test Method for Fundamental Transverse, Longitudinal, and Torsional Resonant Frequencies of Concrete Specimens). The test procedure followed is explained in detail in Section 4.2.

The dynamic modulus of elasticity was calculated by using the following equation:

$$
\text { Dynamic } E=D M\left(n^{\prime}\right)^{2}
$$

Where:

$$
\begin{aligned}
& M=\text { Measured mass of the specimen, } \mathrm{kg} \\
& n^{\prime}=\text { fundamental longitudinal frequency, } \mathrm{Hz} \\
& D=5.093\left(L / d^{2}\right), \mathrm{N} \cdot \mathrm{s}^{2} /\left(\mathrm{kg} \cdot \mathrm{m}^{2}\right) \text { for a cylinder specimen } \\
& L=\text { Length of the cylinder, } \mathrm{m} \\
& d=\text { diameter of the cylinder, m }
\end{aligned}
$$




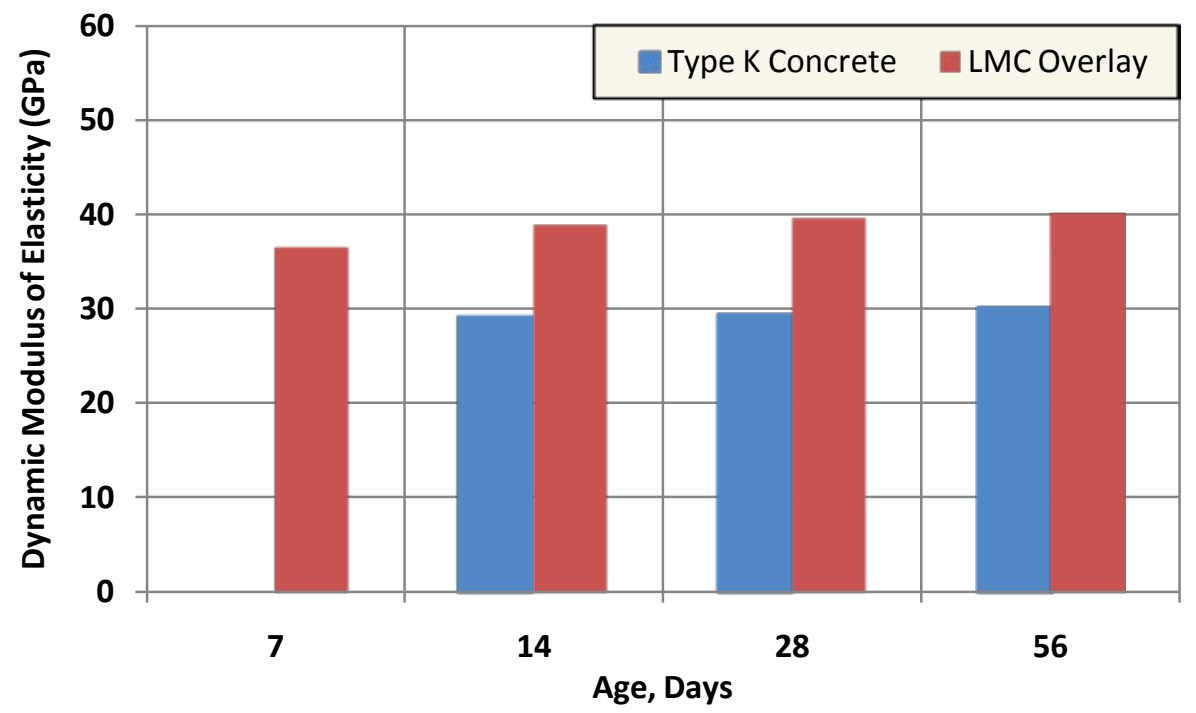

Figure 5.3: Dynamic modulus of elasticity of Type K substrate and LMC overlay concretes (Stage-I)

Figure 5.3 shows the bar diagram of average dynamic modulus of elasticity calculated for Type K substrate concrete and LMC overlay. It is observed that the dynamic modulus of elasticity value increased steadily with time. The value of LMC overlay is always more than the value for Type $\mathrm{K}$ substrate concrete. Dynamic modulus of elasticity value of Type K substrate concrete at 28 days after substrate pour was 28.9 GPa (4120 ksi) and at 14, 28, and 56 days after overlay pour were 29.05 GPa (4215 ksi), 29.32 GPa (4253 ksi), and $30 \mathrm{GPa}$ (4350 ksi) respectively. The dynamic modulus of elasticity value of LMC overlay at 7, 14, 28, and 56 days after overlay pour were 36.42 GPa (5282 ksi), 38.82 GPa (5630 ksi), 39.48 GPa (5725 ksi), and 40.08 GPa (5810 ksi) respectively.

\subsection{Tests Conducted on Large-Scale/Prototype Slab}

In this section, the results of the experiments conducted on large scale prototype deck slabs are discussed. The experiments include direct tensile bond tests using pull-off tests, shrinkage strain and temperature monitoring of substrate and LMC overlays, vertical corner lift due to curling effect at the pre-notched corners, and indirect UPV testing followed by signal processing to study the interface condition on two $6 \mathrm{ft} x 8 \mathrm{ft}$ bilayer deck slabs. LMC overlay was applied on both the slabs but with two different 
bonding conditions: one without bonding slurry and the other with bonding slurry at the interface between substrate deck and overlay concrete.

\subsubsection{Field Pull-Off Testing}

The Pull-Off testing was carried out at 35, 42, 56 and 90 days on $1829 \mathrm{~mm}$. (6 ft.) by $2438 \mathrm{~mm}$. ( $8 \mathrm{ft}$.) slabs to measure the bond strength at the interface. The pull-off testing was carried out by following the ASTM C1593 (2004) (Standard Test Method for Tensile Strength of Concrete Surfaces and the Bond Strength or Tensile Strength of Concrete Repair and Overlay Materials by Direct Tension (Pull-off Method)) standard specifications. Five specimens from the center zone and five specimens from the edge zone were cored and measured for tensile bond strength at each age. The test procedure for conducting field pull-off testing is explained in detail in section 4.4.

The mode of failure at the interface was visually observed and reported for each specimen. Figure 5.4 shows the typical samples after failure under direct tensile test insitu using pull-off testing. The results are presented in tables and figures to compare the performance between two different types of bi-layer deck slabs: one slab with LMC overlay without bonding slurry at the interface and the other slab with the same LMC overlay but bonding slurry was used at the interface. The locations at which the testing was conducted at 35, 42, 49, 56, and 90 days are shown from Figure 5.5 to Figure 5.9,.respectively. Simultaneously, below the figures, bond/tensile strength and the mode of failure are shown in tables for the respective slabs. Values are shown from Table 5.2 through Table 5.6 at 35, 42, 49, 56, and 90 days, respectively for both the slabs. In these tables the interface failure means that more than $70 \%$ of the interface was visible after the failure samples were visually examined. Parallely, substrate failure means that more than $70 \%$ of the substrate concrete attached at the interface after examining the failed samples visually. 


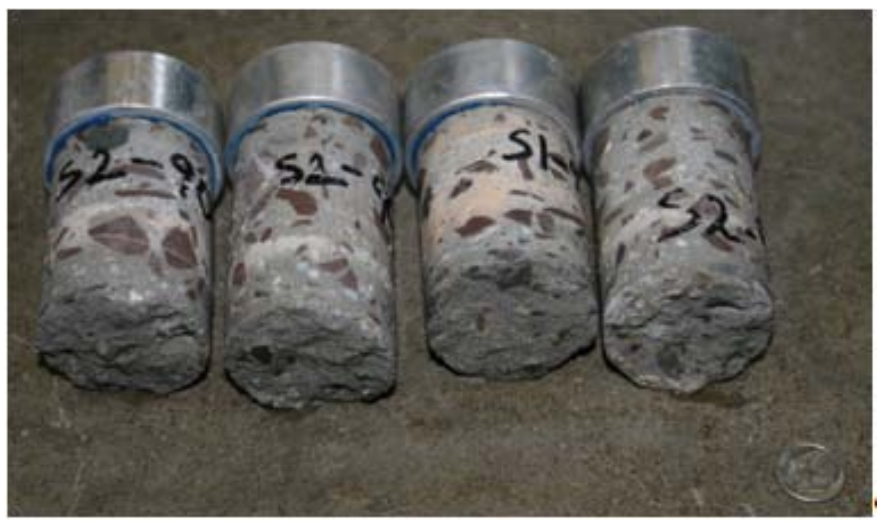

Figure 5.4: Typical failure of the samples after pull-off testing 


\section{Data Aquisition \\ System}

A B C D E F G

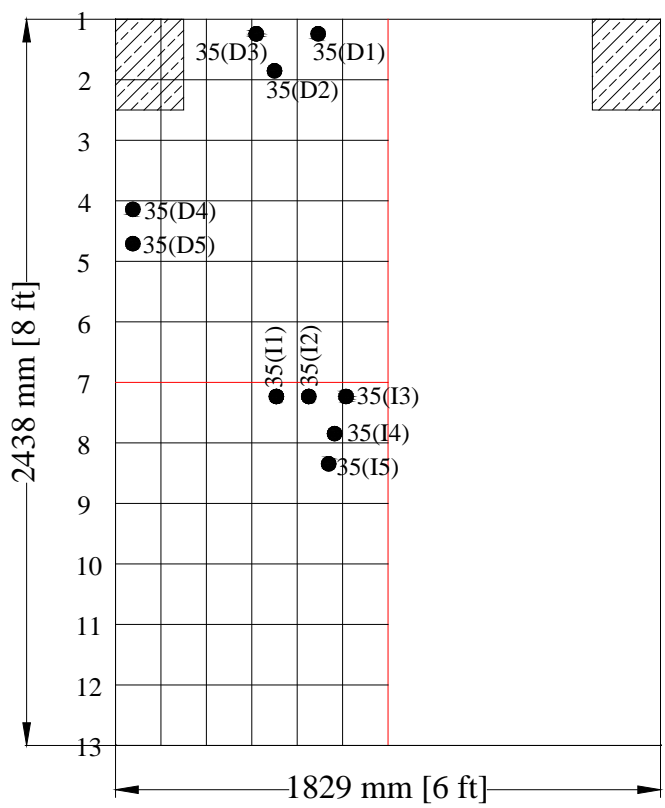

SLAB 1: LMC (Without Bonding Slurry)
Pre-Notch $\longrightarrow$

G H I J K L M

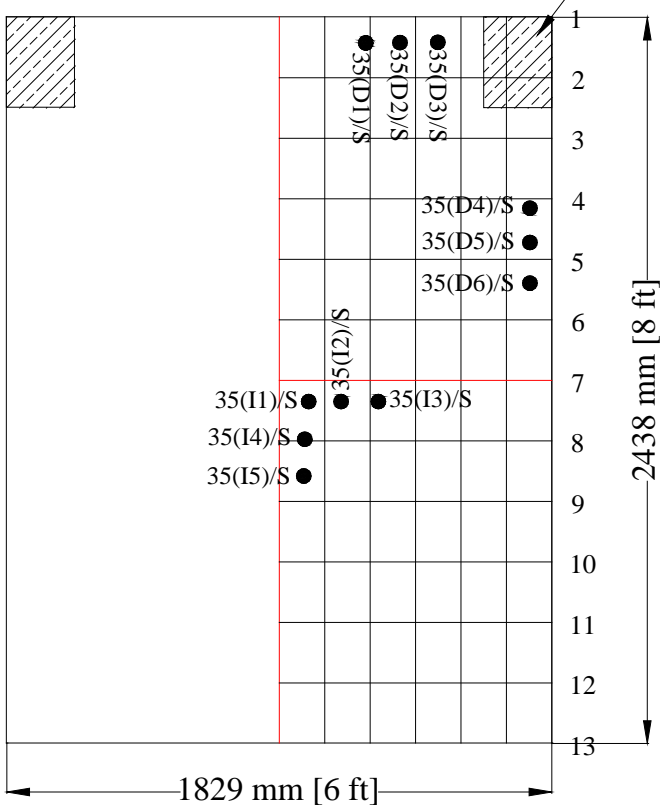

SLAB 2: LMC-S (With Bonding Slurry)

Figure 5.5: 35 days pull-off locations on LMC and LMC-S Slabs of Stage-I

Table 5.2: 35 days pull-off data of LMC and LMC-S slabs in Stage-I

\begin{tabular}{|c|c|c|c|c|c|c|c|}
\hline \multicolumn{4}{|c|}{ Slab 1: LMC (Without Bonding Slurry) } & \multicolumn{4}{|c|}{ Slab 2: LMC-S (With Bonding Slurry) } \\
\hline Zone & $\begin{array}{c}\text { Core } \\
\text { No. }\end{array}$ & $\begin{array}{c}\text { Bond/Tensile } \\
\text { strength } \\
\text { (MPa) }\end{array}$ & Mode of failure & Zone & $\begin{array}{c}\text { Core } \\
\text { No. }\end{array}$ & $\begin{array}{c}\text { Bond/Tensile } \\
\text { strength } \\
\text { (MPa) }\end{array}$ & Mode of failure \\
\hline \multirow{5}{*}{ Edge } & 35(D1) & 1.86 & interface failure & \multirow{5}{*}{ Edge } & 35(D1)/S & - & Failed while drilling \\
\hline & 35(D2) & 1.69 & substrate failure & & 35(D2)/S & 0.69 & substrate failure \\
\hline & 35(D3) & 1.36 & substrate failure & & 35(D3)/S & 1.23 & interface failure \\
\hline & 35(D4) & 2.38 & substrate failure & & 35(D4)/S & 2.11 & interface failure \\
\hline & 35(D5) & 1.05 & interface failure & & 35(D5)/S & 2.51 & overlay failure \\
\hline \multirow{5}{*}{ Center } & 35(I1) & 2.75 & substrate failure & \multirow{5}{*}{ Center } & 35(I1)/S & 0.75 & substrate failure \\
\hline & 35(I2) & 1.99 & interface failure & & 35(I2)/S & 2.78 & interface failure \\
\hline & 35(I3) & 2.46 & interface failure & & 35(I3)/S & 2.44 & interface failure \\
\hline & $35(\mathrm{I} 4)$ & 2.26 & substrate failure & & $35(I 4) / S$ & 2.48 & interface failure \\
\hline & 35(I5) & 2.71 & substrate failure & & 35(I5)/S & 2.58 & interface failure \\
\hline
\end{tabular}

$1 \mathrm{MPa}=145 \mathrm{psi}$ 


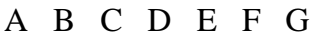

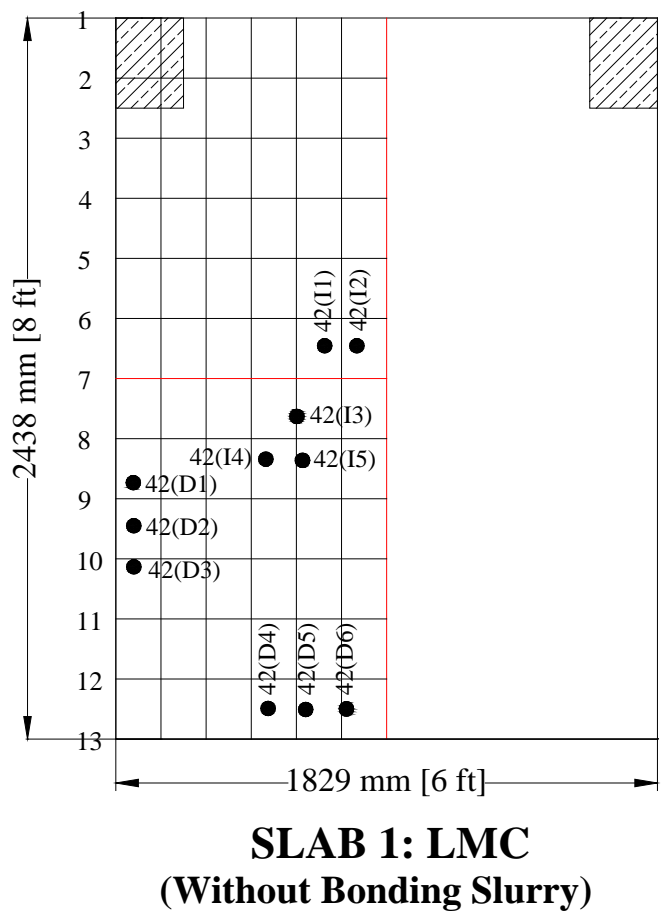

Pre-Notch $\longrightarrow$

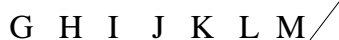

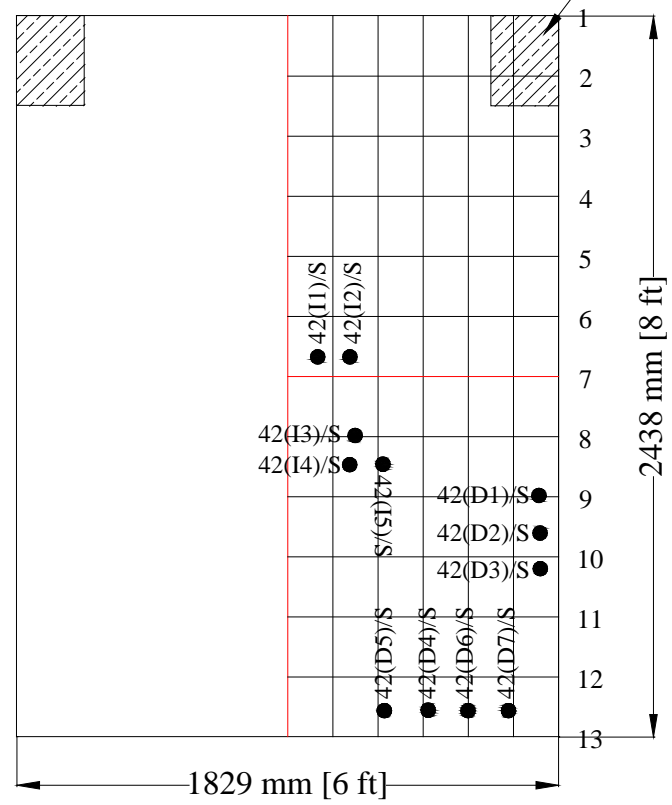

SLAB 2: LMC-S

(With Bonding Slurry)

Figure 5.6: 42 days pull-off locations on LMC and LMC-S slabs of Stage-I

Table 5.3: 42 days pull-off data of LMC and LMC-S slabs in Stage-I

\begin{tabular}{|c|c|c|c|c|c|c|c|}
\hline \multicolumn{4}{|c|}{ Slab 1: LMC (Without Bonding Slurry) } & \multicolumn{4}{|c|}{ Slab 2: LMC-S (With Bonding Slurry) } \\
\hline Zone & $\begin{array}{c}\text { Core } \\
\text { No. }\end{array}$ & $\begin{array}{c}\text { Bond/Tensile } \\
\text { strength } \\
\text { (MPa) }\end{array}$ & Mode of failure & Zone & $\begin{array}{c}\text { Core } \\
\text { No. }\end{array}$ & $\begin{array}{c}\text { Bond/Tensile } \\
\text { strength } \\
\text { (MPa) }\end{array}$ & Mode of failure \\
\hline \multirow{5}{*}{ Edge } & 42(D1) & 1.86 & interface failure & \multirow{7}{*}{ Edge } & 42(D1)/S & 1.12 & interface failure \\
\hline & 42(D2) & 1.99 & interface failure & & 42(D2)/S & 0.67 & substrate failure \\
\hline & 42(D3) & 2.29 & substrate failure & & 42(D3)/S & - & Failed while drilling \\
\hline & 42(D5) & 1.76 & interface failure & & $42(\mathrm{D} 4) / \mathrm{S}$ & - & Failed while drilling \\
\hline & 42(D6) & 2.58 & overlay failure & & 42(D5)/S & - & Failed while drilling \\
\hline \multirow{5}{*}{ Center } & 42(I1) & 2.28 & substrate failure & & 42(D6)/S & 1.19 & interface failure \\
\hline & 42(I2) & 2.13 & interface failure & & $42(\mathrm{D} 7) / \mathrm{S}$ & 2.36 & interface failure \\
\hline & 42(I3) & 2.53 & substrate failure & \multirow{5}{*}{ Center } & $42(\mathrm{I} 1) / \mathrm{S}$ & 2.43 & interface failure \\
\hline & 42(I4) & 1.34 & failure at epoxy bond & & 42(I2)/S & 1.49 & overlay failure \\
\hline & 42(I5) & 2.08 & failure at epoxy bond & & 42(I3)/S & 2.41 & interface failure \\
\hline & & & & & $42(\mathrm{I} 4) / \mathrm{S}$ & 2.02 & substrate failure \\
\hline & & & & & $42(I 5) / S$ & 1.16 & overlay failure \\
\hline
\end{tabular}




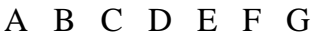

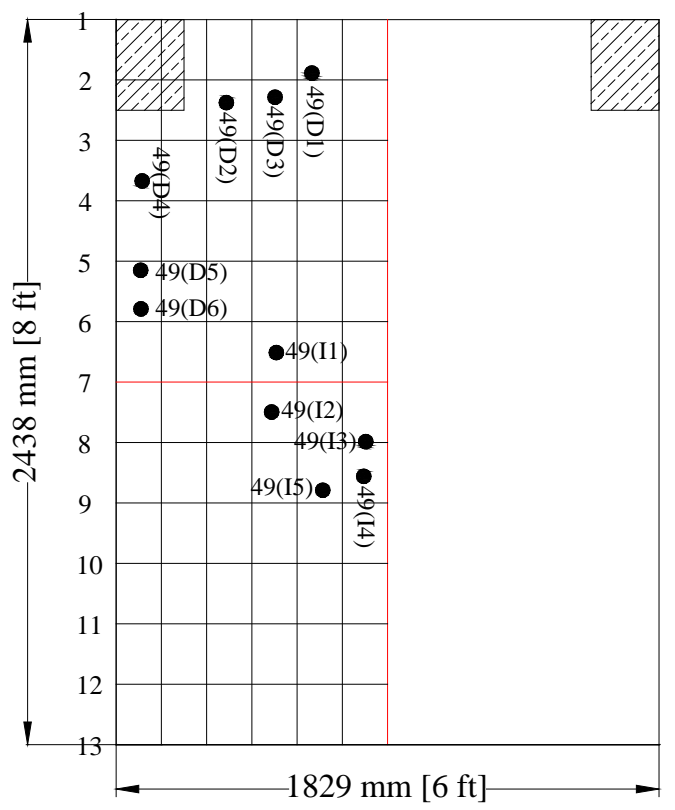

SLAB 1: LMC

(Without Bonding Slurry)
Pre-Notch $\longrightarrow$

G H I J K L M

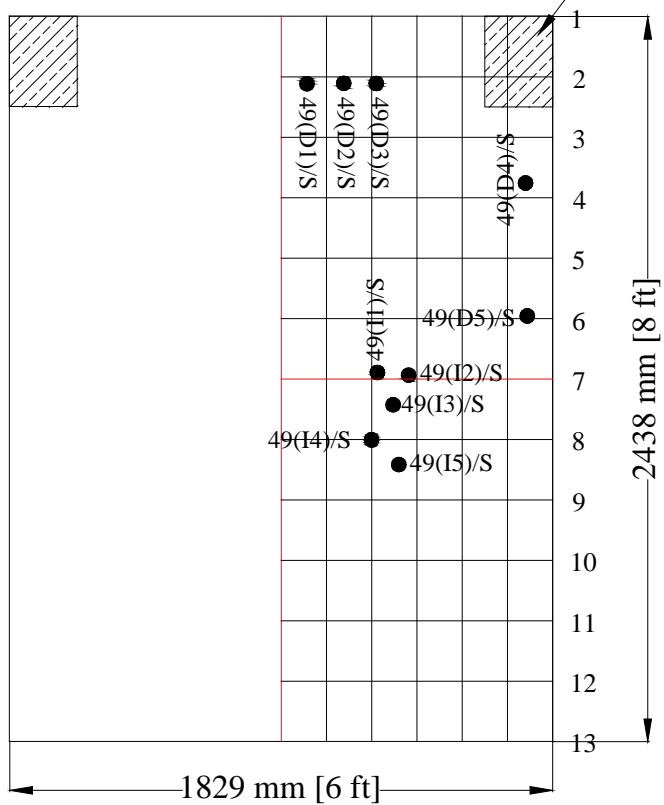

SLAB 2: LMC-S

(With Bonding Slurry)

Figure 5.7: 49 days pull-off locations on LMC and LMC-S slabs for Stage-I

Table 5.4: 49 days pull-off data of LMC and LMC-S slabs in Stage-I

\begin{tabular}{|c|c|c|c|c|c|c|c|}
\hline \multicolumn{4}{|c|}{ Slab 1: LMC (Without Bonding Slurry) } & \multicolumn{4}{|c|}{ Slab 2: LMC-S (With Bonding Slurry) } \\
\hline Zone & $\begin{array}{c}\text { Core } \\
\text { No. }\end{array}$ & $\begin{array}{c}\text { Bond/Tensile } \\
\text { strength } \\
\text { (MPa) }\end{array}$ & Mode of failure & Zone & $\begin{array}{c}\text { Core } \\
\text { No. }\end{array}$ & $\begin{array}{c}\text { Bond/Tensile } \\
\text { strength } \\
\text { (MPa) }\end{array}$ & Mode of failure \\
\hline \multirow{6}{*}{ Edge } & 49(D1) & 2.26 & interface failure & \multirow{5}{*}{ Edge } & $49(\mathrm{D} 1) / \mathrm{S}$ & 1.44 & substrate failure \\
\hline & 49(D2) & 1.86 & interface failure & & 49(D2)/S & 1.57 & interface failure \\
\hline & 49(D3) & 3.15 & overlay failure & & 49(D3)/S & 2.21 & interface failure \\
\hline & 49(D4) & - & Failed while drilling & & $49(\mathrm{D} 4) / \mathrm{S}$ & 1.50 & substrate failure \\
\hline & 49(D5) & 2.29 & substrate failure & & $49(\mathrm{D} 5) / \mathrm{S}$ & 1.47 & substrate failure \\
\hline & 49(D6) & 2.81 & substrate failure & \multirow{5}{*}{ Center } & 49(I1)/S & 1.74 & interface failure \\
\hline \multirow{5}{*}{ Center } & 49(I1) & 2.41 & interface failure & & $49(\mathrm{I} 2) / \mathrm{S}$ & 1.79 & substrate failure \\
\hline & 49(I2) & 2.66 & substrate failure & & 49(I3)/S & 1.09 & overlay failure \\
\hline & 49(I3) & 2.41 & substrate failure & & $49(\mathrm{I} 4) / \mathrm{S}$ & 0.77 & substrate failure \\
\hline & 49(I4) & 1.36 & interface failure & & 49(I5)/S & 1.67 & substrate failure \\
\hline & 49(I5) & 1.39 & substrate failure & & & & \\
\hline
\end{tabular}

$1 \mathrm{MPa}=145 \mathrm{psi}$ 
A B C D E F

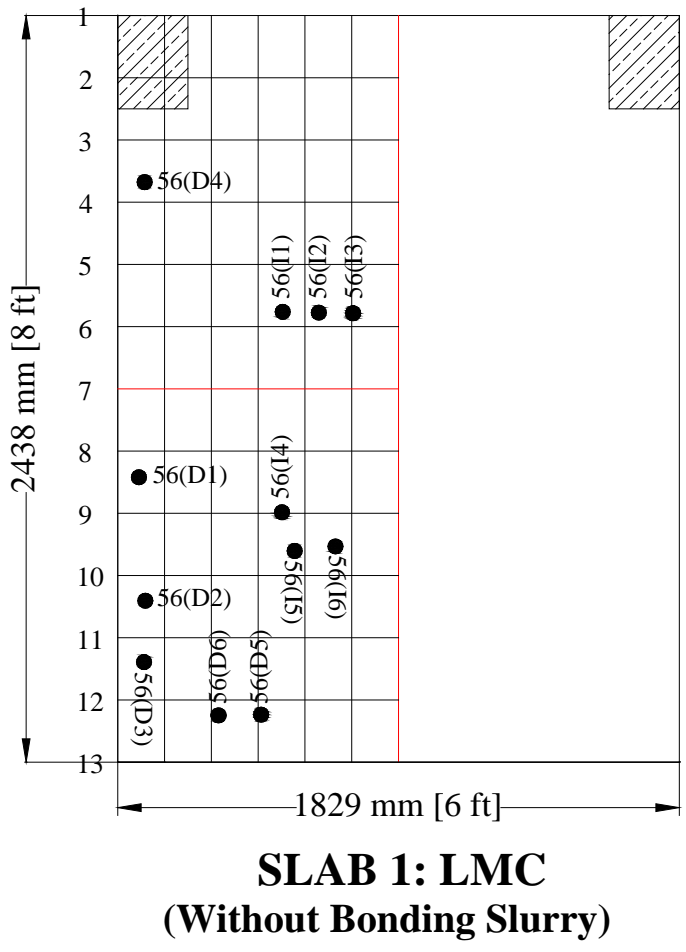

Pre-Notch $\longrightarrow$

G $\quad H \quad I \quad J \quad K \quad L r$

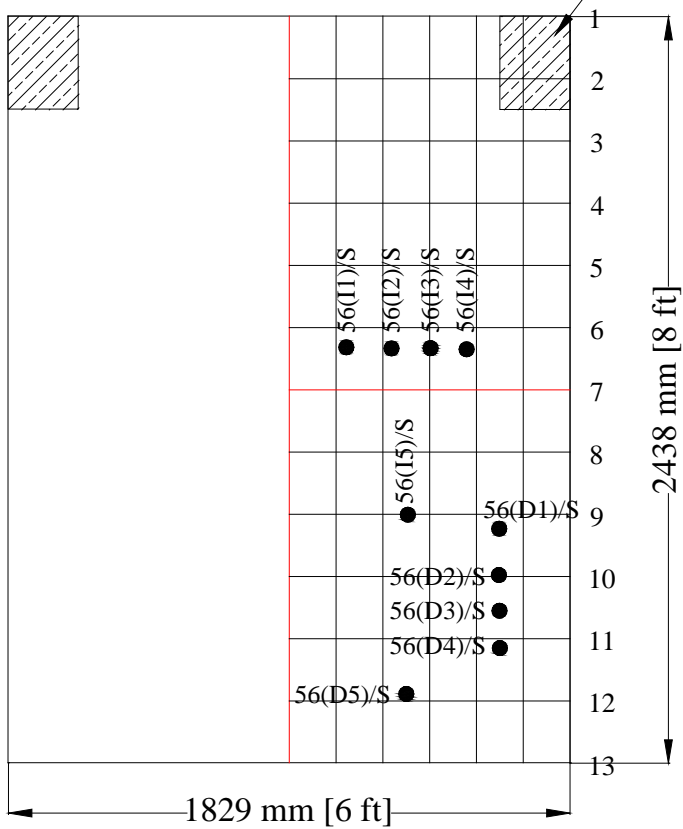

SLAB 2: LMC-S (With Bonding Slurry)

Figure 5.8: 56 days pull-off locations on LMC and LMC-S slabs of Stage-I

Table 5.5: 56 days pull-off data of LMC and LMC-S slabs in Stage-I

\begin{tabular}{|c|c|c|c|c|c|c|c|}
\hline \multicolumn{4}{|c|}{ Slab 1: LMC (Without Bonding Slurry) } & \multicolumn{4}{|c|}{ Slab 2: LMC-S (With Bonding Slurry) } \\
\hline Zone & $\begin{array}{c}\text { Core } \\
\text { No. }\end{array}$ & $\begin{array}{c}\text { Bond/Tensile } \\
\text { strength } \\
\text { (MPa) }\end{array}$ & Mode of failure & Zone & $\begin{array}{c}\text { Core } \\
\text { No. }\end{array}$ & $\begin{array}{c}\text { Bond/Tensile } \\
\text { strength } \\
\text { (MPa) }\end{array}$ & Mode of failure \\
\hline \multirow{5}{*}{ Edge } & 56(D1) & 2.39 & substrate failure & \multirow{5}{*}{ Edge } & $56(\mathrm{D} 1) / \mathrm{S}$ & 1.76 & interface failure \\
\hline & 56(D2) & 2.21 & interface failure & & $56(\mathrm{D} 2) / \mathrm{S}$ & 1.39 & interface failure \\
\hline & 56(D3) & 1.81 & interface failure & & 56(D3)/S & 2.73 & substrate failure \\
\hline & 56(D4) & 1.61 & interface failure & & $56(\mathrm{D} 4) / \mathrm{S}$ & 2.51 & interface failure \\
\hline & 56(D5) & 2.19 & substrate failure & & $56(\mathrm{D} 5) / \mathrm{S}$ & 0.74 & substrate failure \\
\hline \multirow{6}{*}{ Center } & 56(I1) & 2.11 & interface failure & \multirow{5}{*}{ Center } & $56(\mathrm{I1}) / \mathrm{S}$ & 0.57 & substrate failure \\
\hline & 56(I2) & 1.61 & substrate failure & & $56(\mathrm{I} 2) / \mathrm{S}$ & 1.05 & interface failure \\
\hline & 56(I3) & 1.28 & substrate failure & & $56(\mathrm{I} 3) / \mathrm{S}$ & 1.32 & interface failure \\
\hline & $56(\mathrm{I} 4)$ & 2.86 & overlay failure & & $56(\mathrm{I} 4) / \mathrm{S}$ & 1.37 & interface failure \\
\hline & 56(I5) & 2.49 & substrate failure & & 56(I5)/S & 1.22 & interface failure \\
\hline & $56(\mathrm{I} 6)$ & 1.69 & substrate failure & & & & \\
\hline
\end{tabular}

$1 \mathrm{MPa}=145$ psi 


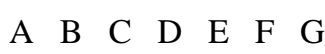

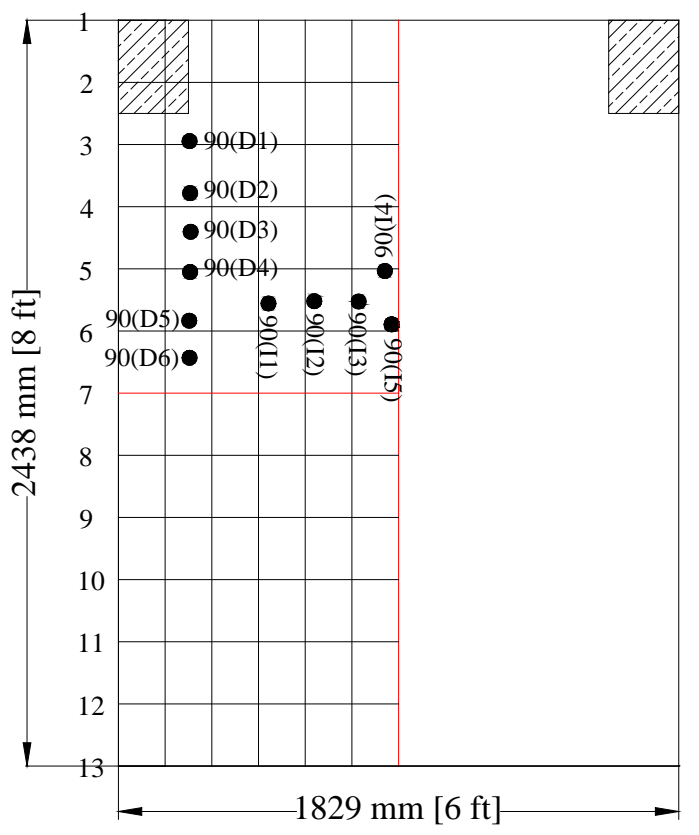

SLAB 1: LMC (Without Bonding Slurry)
Pre-Notch

G H I J K L M

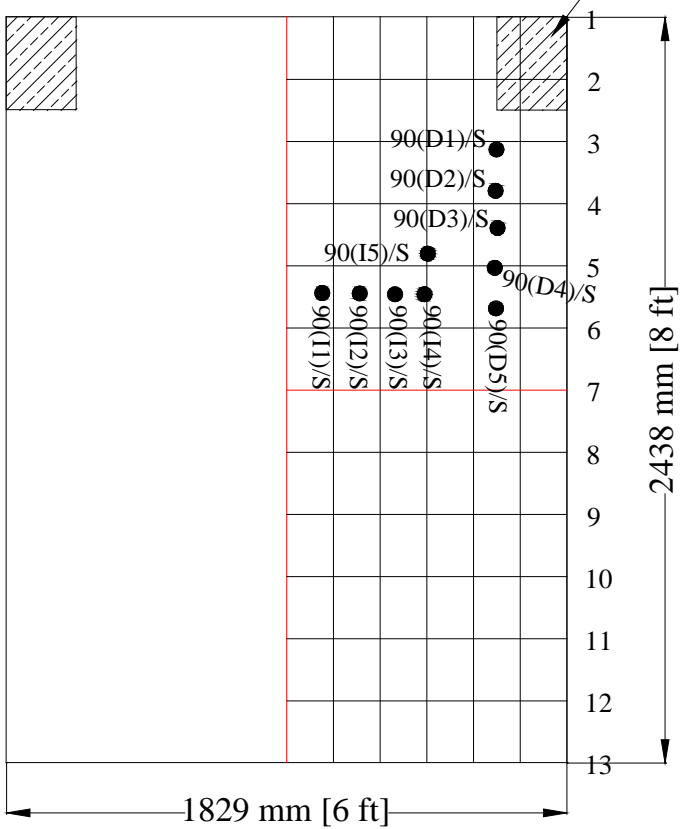

SLAB 2: LMC-S (With Bonding Slurry)

Figure 5.9: 90 days pull-off locations on LMC and LMC-S slabs of Stage-I

Table 5.6: 90 days pull-off data of LMC and LMC-S slabs in Stage-I

\begin{tabular}{|c|c|c|c|c|c|c|c|}
\hline \multicolumn{4}{|c|}{ Slab 1: LMC (Without Bonding Slurry) } & \multicolumn{4}{|c|}{ Slab 2: LMC-S (With Bonding Slurry) } \\
\hline Zone & $\begin{array}{c}\text { Core } \\
\text { No. }\end{array}$ & $\begin{array}{c}\text { Bond/Tensile } \\
\text { strength } \\
\text { (MPa) }\end{array}$ & Mode of failure & Zone & $\begin{array}{c}\text { Core } \\
\text { No. }\end{array}$ & $\begin{array}{c}\text { Bond/Tensile } \\
\text { strength } \\
\text { (MPa) }\end{array}$ & Mode of failure \\
\hline \multirow{6}{*}{ Edge } & 90(D1) & 2.09 & interface failure & \multirow{5}{*}{ Edge } & 90(D1) & 2.23 & interface failure \\
\hline & 90(D2) & 2.06 & interface failure & & 90(D2) & 1.98 & interface failure \\
\hline & 90(D3) & 2.73 & substrate failure & & 90(D3) & 1.47 & substrate failure \\
\hline & 90(D4) & 2.85 & substrate failure & & 90(D4) & 1.89 & substrate failure \\
\hline & 90(D5) & 2.54 & substrate failure & & 90(D5) & 2.48 & interface failure \\
\hline & 90(D6) & 2.63 & substrate failure & \multirow{5}{*}{ Center } & 90(I1) & 1.96 & interface failure \\
\hline \multirow{5}{*}{ Center } & 90(I1) & 2.54 & substrate failure & & 90(I2) & 1.67 & substrate failure \\
\hline & 90(I2) & 2.46 & interface failure & & 90(I3) & 2.69 & interface failure \\
\hline & 90(I3) & 2.59 & substrate failure & & 90(I4) & 3.33 & overlay failure \\
\hline & 90(I4) & 2.19 & interface failure & & 90(I5) & 1.64 & interface failure \\
\hline & 90(I5) & 2.06 & interface failure & & & & \\
\hline
\end{tabular}

$1 \mathrm{MPa}=145 \mathrm{psi}$ 
Table 5.7 and Table 5.8 summarizes the number of cores tested, number of cores failed at the interface, average failure stress at the interface, number of substrate failures, and the average failure stress of the substrate are presented for center zone and edge zone of slab with LMC overlay and slab with LMC-S overlay. The failure stress due to coring, epoxy failure, and overlay failure which are not desirable are not considered and hence are not included in these tables. The failure of specimen in substrate was considered here since, Warner et al. (1998) suggested that failure through the substrate concrete is always desirable, because it is an indicator that the overlay material is stronger than the substrate.

Table 5.7: Pull off data for LMC bi-layer deck slab in Stage-I

\begin{tabular}{|c|c|c|c|c|c|c|c|c|c|c|c|}
\hline & & \multicolumn{10}{|c|}{ Slab 1: LMC (Without Bonding Slurry) } \\
\hline & & \multicolumn{2}{|c|}{35 days } & \multicolumn{2}{|c|}{42 days } & \multicolumn{2}{|c|}{49 days } & \multicolumn{2}{|c|}{56 days } & \multicolumn{2}{|c|}{90 days } \\
\hline & & $\begin{array}{l}\text { Center } \\
\text { Zone }\end{array}$ & $\begin{array}{l}\text { Edge } \\
\text { Zone }\end{array}$ & $\begin{array}{l}\text { Center } \\
\text { Zone }\end{array}$ & $\begin{array}{l}\text { Edge } \\
\text { Zone }\end{array}$ & $\begin{array}{l}\text { Center } \\
\text { Zone }\end{array}$ & $\begin{array}{l}\text { Edge } \\
\text { Zone }\end{array}$ & $\begin{array}{l}\text { Center } \\
\text { Zone }\end{array}$ & $\begin{array}{l}\text { Edge } \\
\text { Zone }\end{array}$ & $\begin{array}{l}\text { Center } \\
\text { Zone }\end{array}$ & $\begin{array}{l}\text { Edge } \\
\text { Zone }\end{array}$ \\
\hline \multicolumn{2}{|c|}{ Number of Cores } & 5 & 5 & 3 & 5 & 5 & 5 & 6 & 5 & 5 & 6 \\
\hline \multicolumn{2}{|c|}{ Average Failure Stress (Mpa) } & 2.43 & 1.67 & 2.31 & 2.10 & 2.05 & 2.47 & 2.01 & 2.04 & 2.37 & 2.48 \\
\hline \multirow{2}{*}{$\begin{array}{l}\text { Interface } \\
\text { failure }\end{array}$} & $\begin{array}{l}\text { Number of } \\
\text { Interface Failures }\end{array}$ & 2 & 2 & 1 & 3 & 2 & 2 & 1 & 3 & 3 & 1 \\
\hline & $\begin{array}{l}\text { Average Failure } \\
\text { Stress (MPa) }\end{array}$ & 2.23 & 1.46 & 2.13 & 1.87 & 1.88 & 2.06 & 2.11 & 1.88 & 2.24 & 2.08 \\
\hline \multirow{2}{*}{$\begin{array}{l}\text { Substrate } \\
\text { failure }\end{array}$} & $\begin{array}{l}\text { Number of } \\
\text { SubstrateFailures }\end{array}$ & 3 & 3 & 2 & 1 & 3 & 2 & 4 & 2 & 2 & 6 \\
\hline & $\begin{array}{l}\text { Average Failure } \\
\text { Stress (MPa) }\end{array}$ & 2.57 & 1.81 & 2.40 & 2.29 & 2.15 & 2.55 & 1.77 & 2.29 & 2.57 & 2.48 \\
\hline
\end{tabular}

$1 \mathrm{MPa}=145 \mathrm{psi}$

Table 5.8: Pull-off data for LMC-S bi-layer deck system in Stage-I

\begin{tabular}{|c|c|c|c|c|c|c|c|c|c|c|c|}
\hline & & \multicolumn{10}{|c|}{ Slab 2: LMC-S (With Bonding Slurry) } \\
\hline & & \multicolumn{2}{|c|}{35 days } & \multicolumn{2}{|c|}{42 days } & \multicolumn{2}{|c|}{49 days } & \multicolumn{2}{|c|}{56 days } & \multicolumn{2}{|c|}{90 days } \\
\hline & & $\begin{array}{l}\text { Center } \\
\text { Zone }\end{array}$ & $\begin{array}{l}\text { Edge } \\
\text { Zone }\end{array}$ & $\begin{array}{l}\text { Center } \\
\text { Zone }\end{array}$ & $\begin{array}{l}\text { Edge } \\
\text { Zone }\end{array}$ & $\begin{array}{l}\text { Center } \\
\text { Zone }\end{array}$ & $\begin{array}{l}\text { Edge } \\
\text { Zone }\end{array}$ & $\begin{array}{l}\text { Center } \\
\text { Zone }\end{array}$ & $\begin{array}{l}\text { Edge } \\
\text { Zone }\end{array}$ & $\begin{array}{l}\text { Center } \\
\text { Zone }\end{array}$ & $\begin{array}{l}\text { Edge } \\
\text { Zone }\end{array}$ \\
\hline \multicolumn{2}{|c|}{ Number of Cores } & 5 & 5 & 5 & 7 & 4 & 5 & 5 & 5 & 5 & 5 \\
\hline \multicolumn{2}{|c|}{ Average Failure Stress (Mpa) } & 2.57 & 1.63 & 1.90 & 1.34 & 1.49 & 1.64 & 1.11 & 1.83 & 2.26 & 2.01 \\
\hline \multirow{2}{*}{$\begin{array}{l}\text { Interface } \\
\text { failure }\end{array}$} & $\begin{array}{l}\text { Number of } \\
\text { Interface Failures }\end{array}$ & 4 & 2 & 2 & 3 & 1 & 2 & 4 & 3 & 2 & 3 \\
\hline & $\begin{array}{l}\text { Average Failure } \\
\text { Stress (MPa) }\end{array}$ & 2.57 & 1.67 & 2.42 & 1.56 & 1.74 & 1.89 & 1.24 & 1.89 & 2.10 & 2.23 \\
\hline \multirow{2}{*}{$\begin{array}{l}\text { Substrate } \\
\text { failure }\end{array}$} & $\begin{array}{l}\text { Number of } \\
\text { SubstrateFailures }\end{array}$ & - & 1 & 1 & - & 3 & 3 & - & 1 & 1 & 2 \\
\hline & $\begin{array}{l}\text { Average Failure } \\
\text { Stress (MPa) }\end{array}$ & - & 0.69 & 2.02 & - & 1.41 & 1.47 & - & 2.73 & 1.67 & 1.68 \\
\hline
\end{tabular}


In Table 5.7 and Table 5.8 the number of cores tested, number of cores failed at the interface, average failure stress at the interface, number of substrate failures, and the average failure stress of the substrate were presented for center zone and edge zone of slab with LMC overlay and slab with LMC overlay with bonding slurry at the interface. Sprinkel and Ozyildirim (2000) qualified the bond strength test results as:

If bond strength values are $>=2.1 \mathrm{MPa}$ (300 psi), excellent

1.7 MPa to 2.1 MPa (250 to $299 \mathrm{psi}$ ), very good

1.4 MPa to 1.7 MPa (200 to $249 \mathrm{psi}$ ), good

$0.7 \mathrm{MPa}$ to $1.4 \mathrm{MPa}$ (100 to $199 \mathrm{psi}$ ), fair and

$0 \mathrm{MPa}$ to $0.7 \mathrm{MPa}$ (0 to $99 \mathrm{psi}$ ), poor

Also, Sprinkel (2005) and Sprinkel (2004) mentioned the lower quality limit for bond strength should be $1.03 \mathrm{MPa}$ (150 psi) at 28 days. As we can see form our results all the interfacial bond strength values are greater than the lower quality limit of $1.03 \mathrm{MPa}$ (150 psi) and all the values are close to or greater than 1.7 MPa (250 psi). Hence the condition of the interface can be considered as good or very good.

Hence, from the above tables we can just qualitatively describe the bond condition. But general conclusions cannot be made to compare the bond strength values between the two slabs. Because, high variance in the values is observed between the individual results and simple mean cannot be calculated. Hence, three-way ANOVA was considered to conduct the in depth study and the conclusions were drawn based on threeway ANOVA. The results and discussions following the three-way ANOVA are discussed in the following section. Only the interface failure values were considered to perform three-way ANOVA.

\subsubsection{Three-Way ANOVA Results}

The three-way ANOVA procedure explained in Section 2.8 was selected in this study for understanding the effect of three factors (Age, Zone Type, and Slab Type) on the Pull-Off bond strength. Since, the development of three-way ANOVA table is a 
tedious process and involves lot of math; the calculations were performed on computer, using a powerful statistical software package called Statistical Analysis System (SAS) version 9.0.

Using the SAS help documentation, the SAS code was developed to perform the three way ANOVA table. The steps involved in developing the SAS code were:

1. The factors and different levels or treatments in the respective factors were defined by coding the levels within the factors using the Proc format function;

2. Defining data table in a systematic format using the Data function;

3. Then, the normal probability and residual plots were developed and analyzed to see if the data was following the assumptions for performing three-way ANOVA;

4. After checking for the assumptions three-way ANOVA table was developed using Proc GLM function. Here Proc GLM was used instead of Proc ANOVA because, the number of observations per level or treatment for different factors are not same;

5. Proc Summary function was used to print the summary of the class level information from Step 1 and the ANOVA table developed in Step 4;

6. After developing the three-way ANOVA table, the influence of various factors on the pull-off strength was studied by plotting the two-way and threeway interaction plots; and

7. The final conclusions were made by studying the graphs developed in Step 6 .

In the following discussion, SAS code is presented simultaneously with the output and interpretation of the results for better understanding of the effects of selected factors on the pull-off strength.

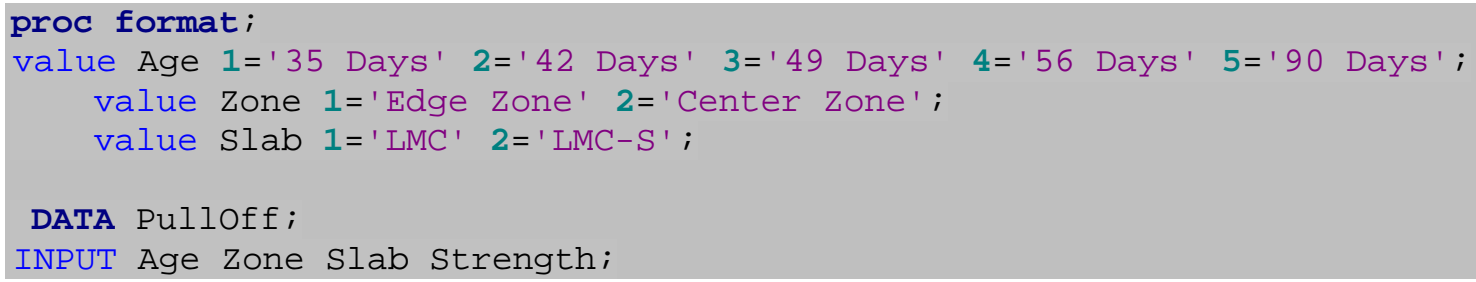




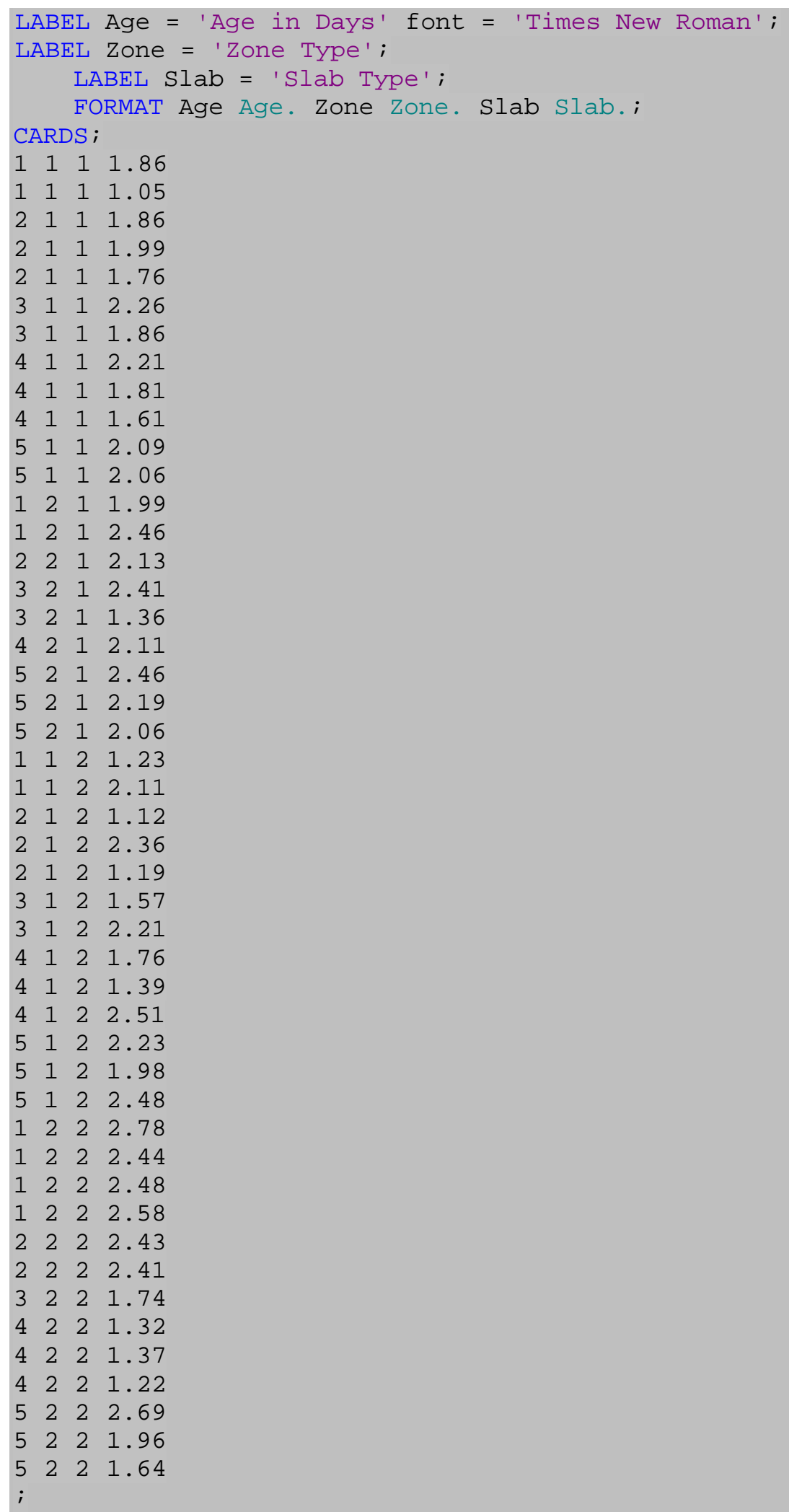

1111.86

11111.05

$\begin{array}{lllll}2 & 1 & 1 & 1.86\end{array}$

$\begin{array}{llll}2 & 1 & 1 & 1.99\end{array}$

$\begin{array}{llllll}2 & 1 & 1 & 1.76\end{array}$

$\begin{array}{llll}3 & 1 & 1 & 2.26\end{array}$

30111.86

$\begin{array}{llll}4 & 1 & 1 & 2.21\end{array}$

$\begin{array}{lllll}4 & 1 & 1 & 1.81\end{array}$

$\begin{array}{lllll}4 & 1 & 1 & 1.61\end{array}$

$\begin{array}{llll}5 & 1 & 1 & 2.09\end{array}$

$\begin{array}{lllll}5 & 1 & 1 & 2.06\end{array}$

$\begin{array}{llll}1 & 2 & 1 & 1.99\end{array}$

$\begin{array}{llll}1 & 2 & 1 & 2.46\end{array}$

221213

$\begin{array}{lllll}3 & 2 & 1 & 2.41\end{array}$

$\begin{array}{llll}3 & 2 & 1 & 1.36\end{array}$

$\begin{array}{lllll}4 & 2 & 1 & 2.11\end{array}$

$\begin{array}{llll}5 & 2 & 1 & 2.46\end{array}$

$\begin{array}{llll}5 & 2 & 1 & 2.19\end{array}$

$\begin{array}{llll}5 & 2 & 1 & 2.06\end{array}$

1121.23

1122.11

$\begin{array}{llll}2 & 1 & 2 & 1.12\end{array}$

$\begin{array}{llll}2 & 1 & 2 & 2.36\end{array}$

$\begin{array}{llll}2 & 1 & 2 & 1.19\end{array}$

$\begin{array}{llll}3 & 1 & 2 & 1.57\end{array}$

$\begin{array}{llll}3 & 1 & 2 & 2.21\end{array}$

4121.76

$\begin{array}{llll}4 & 1 & 2 & 1.39\end{array}$

$\begin{array}{llll}4 & 1 & 2 & 2.51\end{array}$

$\begin{array}{lllll}5 & 1 & 2 & 2.23\end{array}$

$\begin{array}{llll}5 & 1 & 2 & 1.98\end{array}$

$\begin{array}{llll}5 & 1 & 2 & 2.48\end{array}$

1222.78

$\begin{array}{llll}1 & 2 & 2 & 2.44\end{array}$

$\begin{array}{llll}12 & 2 & 2.48\end{array}$

$\begin{array}{llll}1 & 2 & 2 & 2.58\end{array}$

$\begin{array}{llll}2 & 2 & 2 & 2.43\end{array}$

$\begin{array}{llll}2 & 2 & 2 & 2.41\end{array}$

$\begin{array}{llll}3 & 2 & 2 & 1.74\end{array}$

$\begin{array}{llll}4 & 2 & 2 & 1.32\end{array}$

$\begin{array}{llll}4 & 2 & 2 & 1.37\end{array}$

$\begin{array}{llll}4 & 2 & 2 & 1.22\end{array}$

$\begin{array}{llll}5 & 2 & 2 & 2.69\end{array}$

$\begin{array}{llll}5 & 2 & 2 & 1.96\end{array}$

$\begin{array}{llll}5 & 2 & 2 & 1.64\end{array}$

;

Note: In the data table above the outliers of the data were not included. 
Above written code defines the factors, levels or treatments within factors, and data table. The data table is defined in such a way that column 1 coding represents the level or treatments of Age factor, column 2 coding represents the level or treatment of Zone factor, column 3 coding represents the level or treatment of Slab factor, and column 4 represents value of the pull-off strength observation for the particular case. Each row in the input data table represents the individual observation for the respective case.

The three factors selected were 1) Age, 2) Zone Type and 3) Slab Type. Age factor has 5 levels or treatments namely; 35 days, 42 days, 49 days, 56 days, and 90 days. Zone Type factor has two levels or treatments namely; Center Zone and Edge Zone. Slab factor has two levels or treatments namely; LMC (Slab with LMC overlay without using bonding slurry at the interface) and LMC-S (slab with LMC overlay with bonding slurry condition at the interface). The independent variables were the age, zone type, and slab type factors and the dependent variable was Pull-Off strength. The summary of the class level information is shown in Table 5.9.

Table 5.9: Class level information for Stage-I pull-off test

\begin{tabular}{|c|c|c|}
\hline Class/Factor & Levels/Treatments & Values \\
\hline Age & 5 & 35 Days 42 Days 49 Days 56 Days 90 \\
Days
\end{tabular}

Code written here is used for developing the normal probability plot, residual plot, and ANOVA table. Normal Probability and residual plots are plotted for checking the model adequacy and after checking the model adequacy, three-way ANOVA was developed.

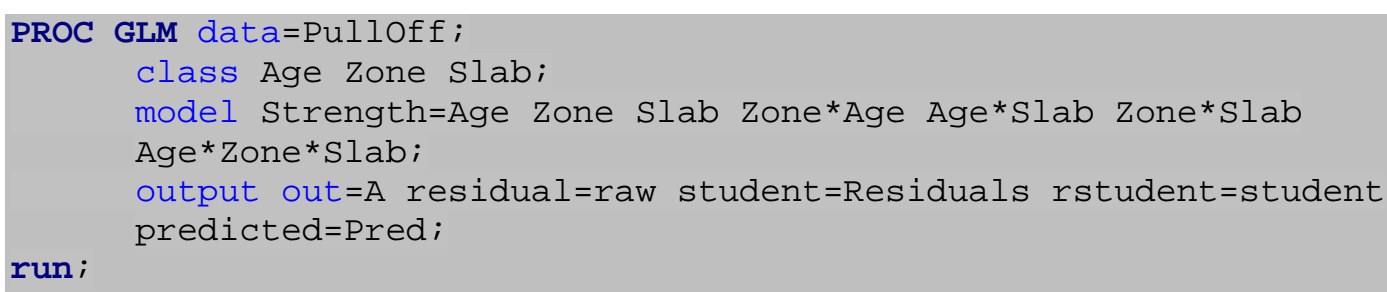




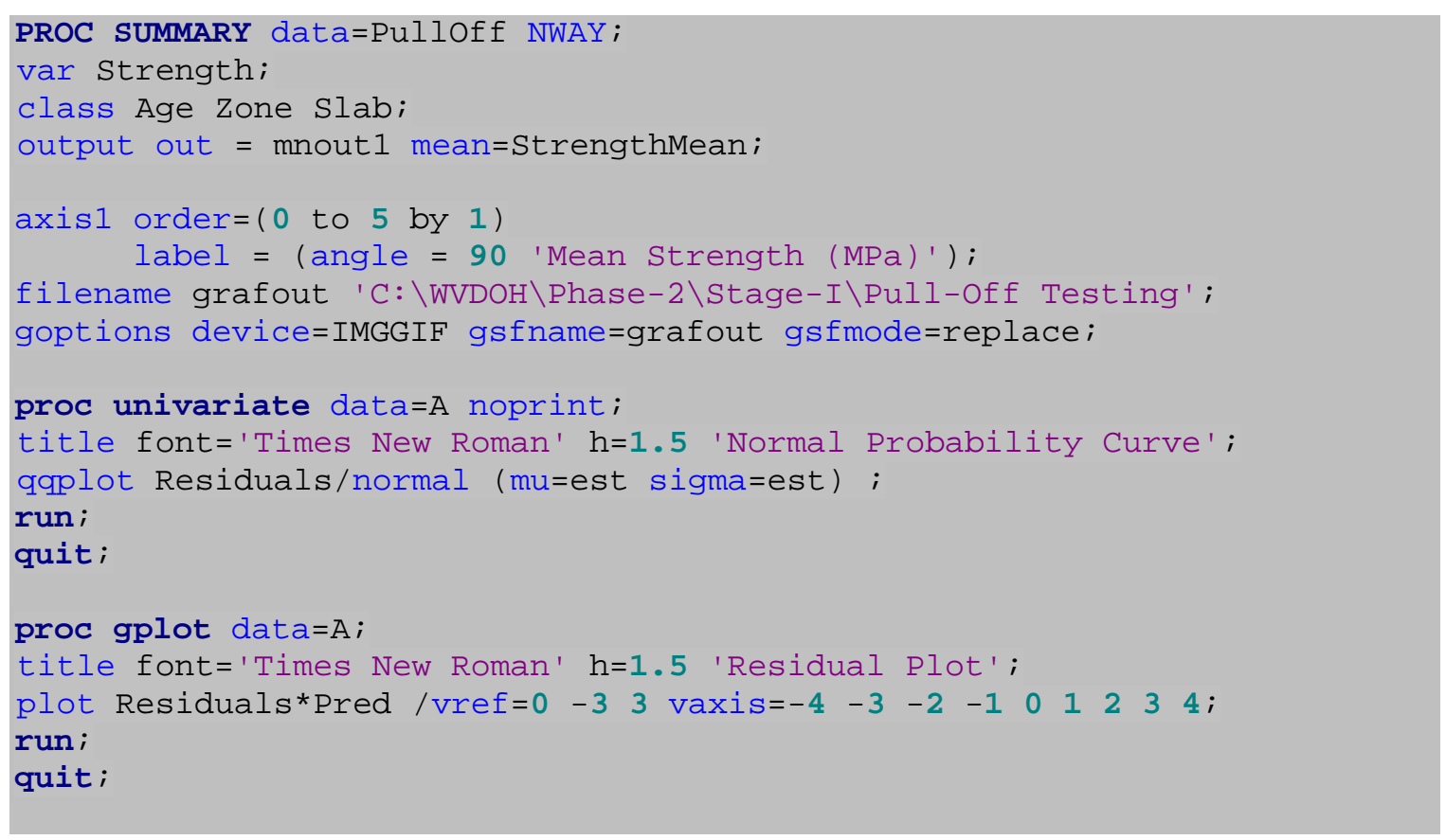

Here, normal probability plot of the residuals is constructed to validate the assumption of normality. This plot means that the underlying error distribution is normal and this plot will resemble a straight line. More emphasis should be placed on the central values of the plot than on the extremes. From the Figure 5.10, we can see that there is nothing unusual in the normal probability plot i.e., by examining this plot we can see that all the data is concentrated at the center and hence underlying error distribution is normal. Therefore, the assumption of normal distribution is valid. 
Normal Probability Curve

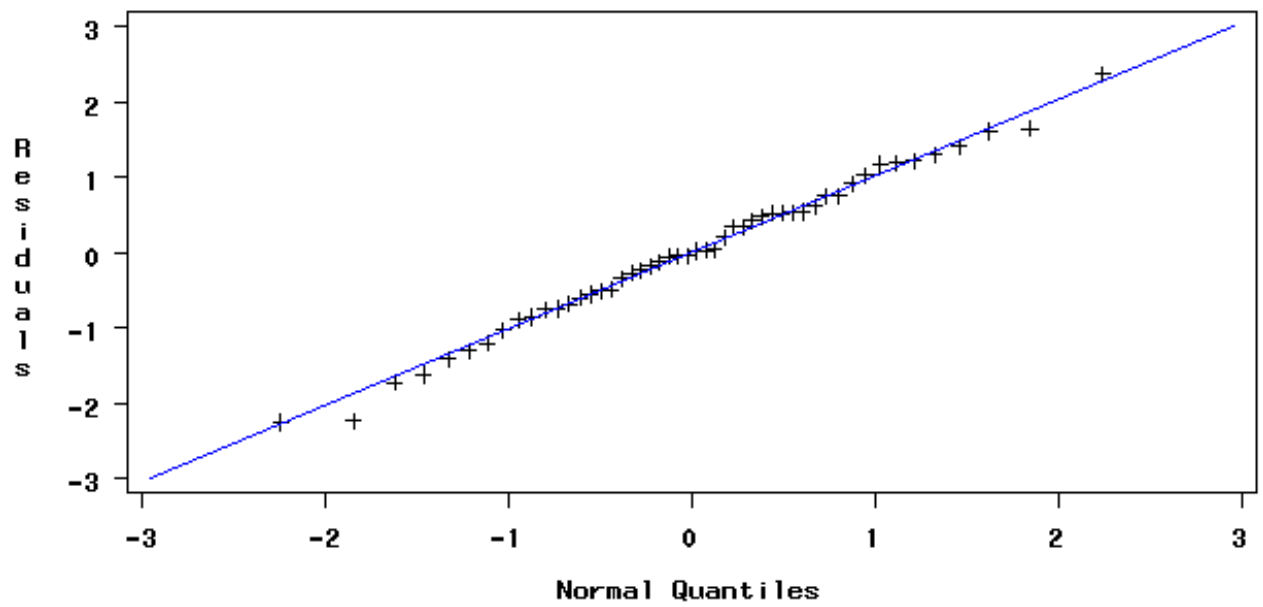

Figure 5.10: Normal probability plot of residuals for Stage-I pull-off data

Also, the assumption of uniform variance is checked by plotting the residual plot of the experimental data. The residual plot is developed by plotting the residuals versus predicted or fitted values. This plot should not show any obvious pattern i.e., the residuals should be structureless which means that the residuals should be unrelated to any other variable including the predicted value. From the Figure 5.11 we can see that the residual plot is structureless and hence the assumption, homogeneity of variances is validated.

Residual Plot

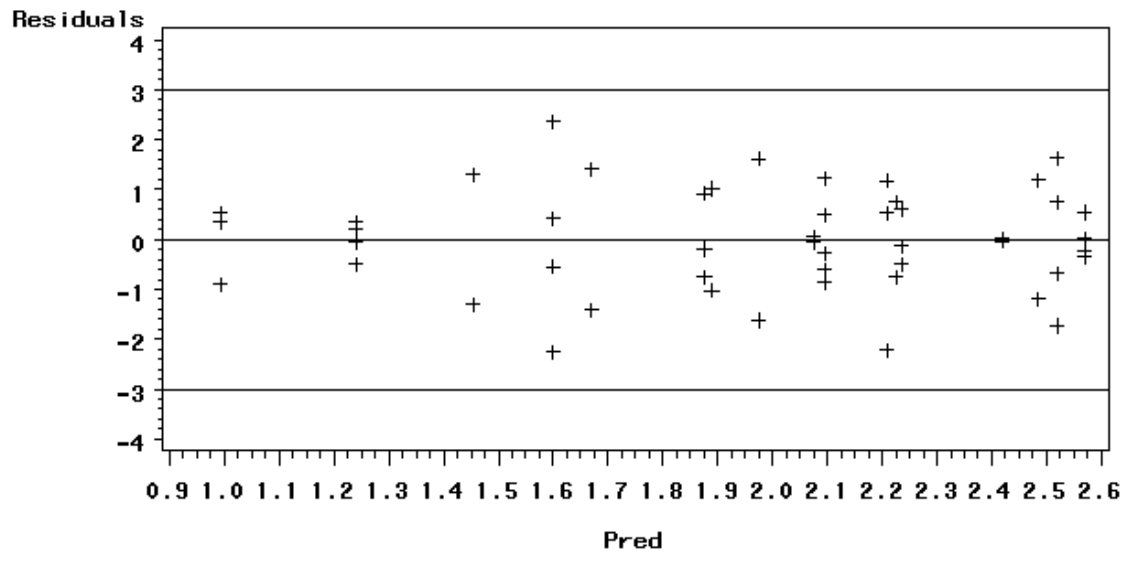

Figure 5.11: Residual Plot of Stage-I pull-off data 
After checking for the assumptions of normal distribution and the uniform variance, ANOVA table is computed to interpret the effects of Age, Zone type, and slab type on the pull-off bond strength. The treatments sum of squares are partitioned into 7 components namely,

1. Sum of squares of A, the Age effect

2. Sum of squares of $B$, the zone effect

3. Sum of squares of $\mathrm{C}$, the slab effect

4. Interaction between Age effect and Zone effect, A x B interaction

5. Interaction between Age effect and Slab effect, $\mathrm{A} \times \mathrm{C}$ interaction

6. Interaction between Zone effect and Slab effect, B x C interaction

7. Interaction between Age, Zone, and Slab effects, A x B x C interaction

Table 5.10: Three-way ANOVA table of Stage-I pull-off data

\begin{tabular}{|c|c|c|c|c|c|}
\hline Source & DF & $\begin{array}{c}\text { Sum of } \\
\text { Squares }\end{array}$ & Mean Square & $F_{o}$ Value & Pr>F \\
\hline Age & 4 & 0.669 & 0.167 & 1.03 & 0.407 \\
\hline Zone & 1 & 0.465 & 0.465 & 2.87 & 0.1015 \\
\hline Slab & 1 & 0.0316 & 0.0316 & 0.20 & 0.6618 \\
\hline Age*Zone & 4 & 1.738 & 0.435 & 2.69 & 0.0525 \\
\hline Age*Slab & 4 & 0.52 & 0.130 & 0.80 & 0.533 \\
\hline Zone*Slab & 1 & 0.0126 & 0.0126 & 0.08 & 0.782 \\
\hline Age*Zone*Slab & 4 & 0.547 & 0.137 & 0.85 & 0.509 \\
\hline $\begin{array}{c}\text { Model (Between } \\
\text { Treatments) }\end{array}$ & 19 & 5.24 & 0.276 & 1.70 & 0.10 \\
\hline $\begin{array}{c}\text { Error (Within Treatments) } \\
\text { Agrected Total }\end{array}$ & 27 & 4.366 & 0.161 & & \\
\hline Correct & 9.60 & & & \\
\hline
\end{tabular}

In this study, $5 \%$ level of significance $(\alpha=0.05)$ was considered and the conclusions were made based on this. The discussions and conclusions that can be made based on the Table 5.10 are as follows: 
1. Clearly, the Probability value of the model test statistic $F_{o}$ is 0.10 , which is greater than 0.05 , hence we accept null hypothesis and reject alternate hypothesis i.e., the treatment means does not differ significantly.

2. Since, the p-value of test statistic $F_{o}$ for Age effect is 0.407 which is greater than 0.05, we can conclude that the five corresponding population means does not differ significantly. The effect of age on the pull-off strength is not significant i.e., the bond strength at the interface did not change considerably with the increase in age of the slabs within the range of experiment.

3. For the Zone effect, the ANOVA showed the p-value of test statistic $F_{o}$ is 0.1015 which is greater than 0.05 , we conclude that the effect of zone on the bond strength is not significant. There is a no significant change in the bond strength value when the two zones were compared i.e., Zone type did not significantly affect the bond strength at the interface.

4. Similarly, for the slab effect p-value of test statistic $F_{o}$ is less than 0.6618 which is greater than 0.05 implies that the two population means did not differ significantly. The effect of slab on the bond strength was not significant, which implies that the bond strength for the two different types of slabs is same.

Further in depth interpretations can be made by considering the two-way and three-way interactions.

5. Age by Zone two-way interaction being significant (p-value greater than 0.05 ) implies that the difference between population means of bond strength due to five different age levels for the Center Zone is not significantly different from the difference between population means of bond strength due to five different age levels for the Edge Zone.

6. Age by Slab two-way interaction being non significant (p-value greater than 0.05 ) implies that the difference between population means of bond strength due to five different age levels for the slab with LMC overlay is not significantly different from the difference between population means of bond strength due to five different age levels for the Slab with LMC-S (LMC overlay with bonding slurry condition at the interface) overlay. 
7. Similarly, Zone by Slab two-way interaction being non significant (p-value greater than 0.05) implies that the difference between population means of bond strength due to Center Zone and Edge Zone effects for the slab with LMC overlay is not significantly different from the difference between population means of bond strength due to Center Zone and Edge Zone effects for the Slab with LMC-S (LMC overlay with bonding slurry condition at the interface) overlay.

8. The three-way interaction Age by Zone type by slab type being non significant (pvalue greater than 0.05 ) implies that the age by zone interactions is the same for two types of slabs, Age by Slab interaction is the same for two different zones, and zone by slab interaction is the same for five different levels of age.

Two-way and three-way interaction effects can be better understood by plotting and examining the interactions. Code written here is used for developing the two-way interaction plots.

PROC SUMMARY data=Pulloff NWAY;

var Strength;

class Age Zone;

output out = mnout2 mean=StrengthMean;

proc gplot data=mnout2;

title1 height=2 font='Times New Roman' '2-Way Interaction';

title2 height=1.5 font='Times New Roman' 'Zone By Age';

plot StrengthMean *Age=Zone/vaxis=axis1;

symbol1 $\mathrm{v}=$ diamond $\mathrm{h}=2 \mathrm{i}=$ join $\mathrm{c}=\mathrm{red}$;

symbol2 $\mathrm{v}=$ star $i=j$ join $h=2 \mathrm{c}=$ black; 
2-Way Interaction

Zone By Age

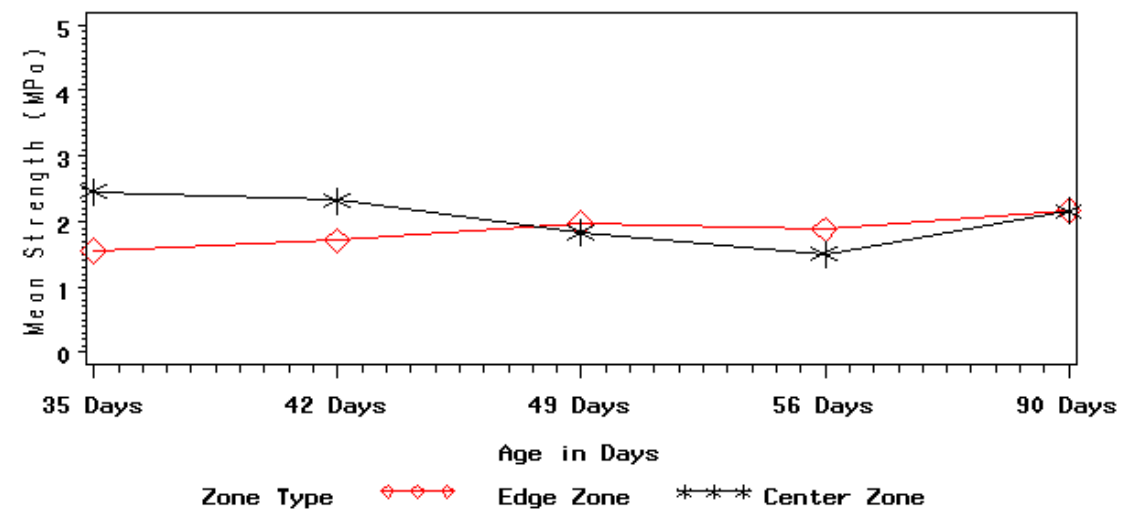

Figure 5.12: Means for levels of Zone type at each level of Age (Stage-I)

Figure 5.12 is plotted by taking age factor in the $\mathrm{x}$-axis and plotting the means of bond strength for each level of zone type, i.e., corresponding to center zone and edge zone. We can conclude that the overall performance of center zone was better than edge zone for different levels of age factor. Center zone was performing better than edge zone for 35 days and 42 days but, equal performance is observed for both the center zone and edge zone for 49, 56 and 90 days respectively.

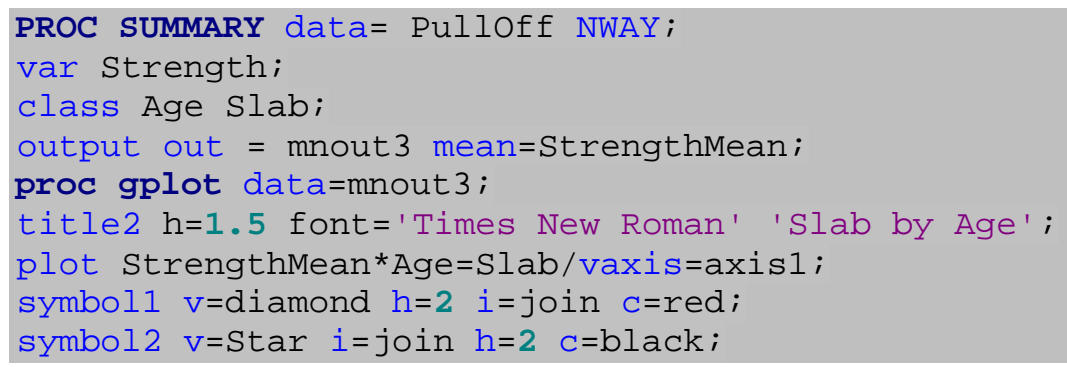


2-Way Interaction

Slab by Age

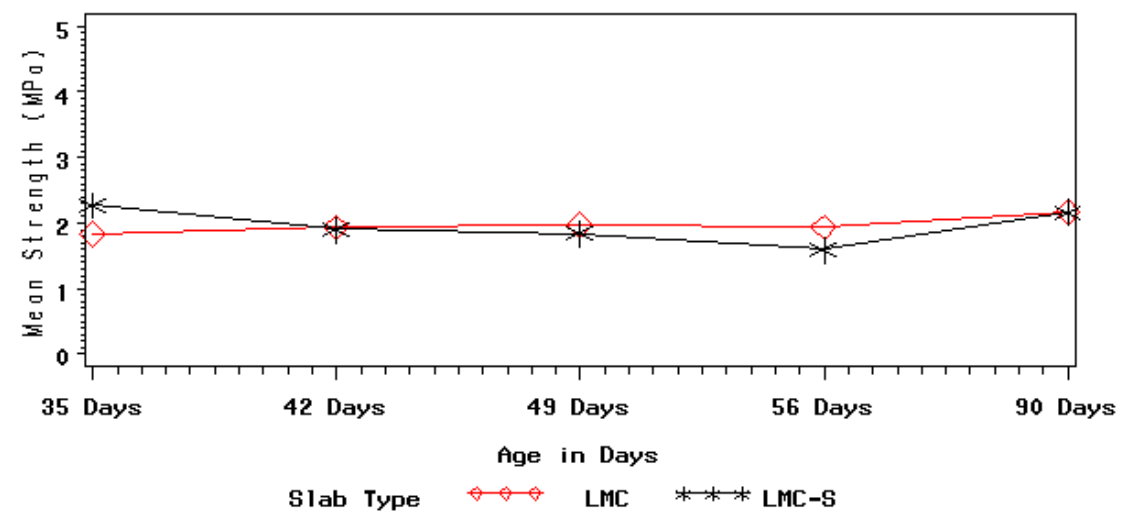

Figure 5.13: Means for levels of Slab type at each level of Age (Stage-I)

Figure 5.13 is plotted by taking age factor in the $\mathrm{x}$-axis and plotting the means of bond strength for each level of slab type, i.e., corresponding to slabs with LMC and LMC-S overlays. By looking at the plot we can further conclude that the bond strength of slab with LMC overlay was similar to that of slab with LMC-S overlay for different levels of Age.

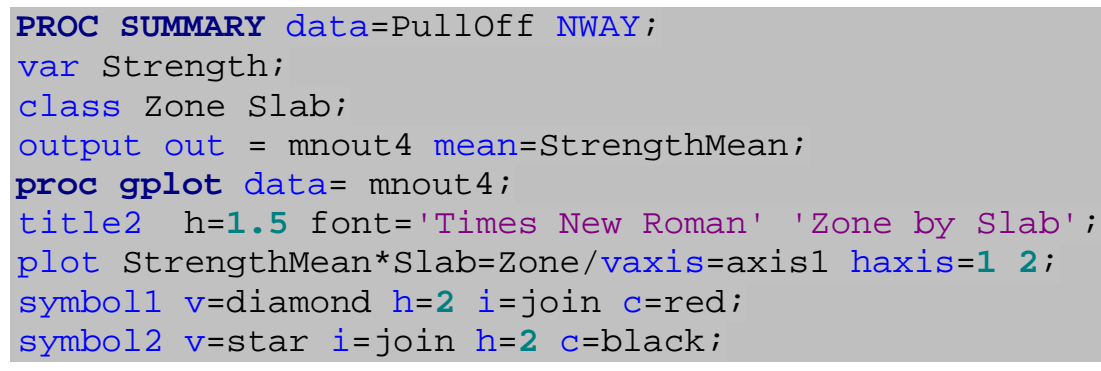


2-Way Interaction

Zone by Slab

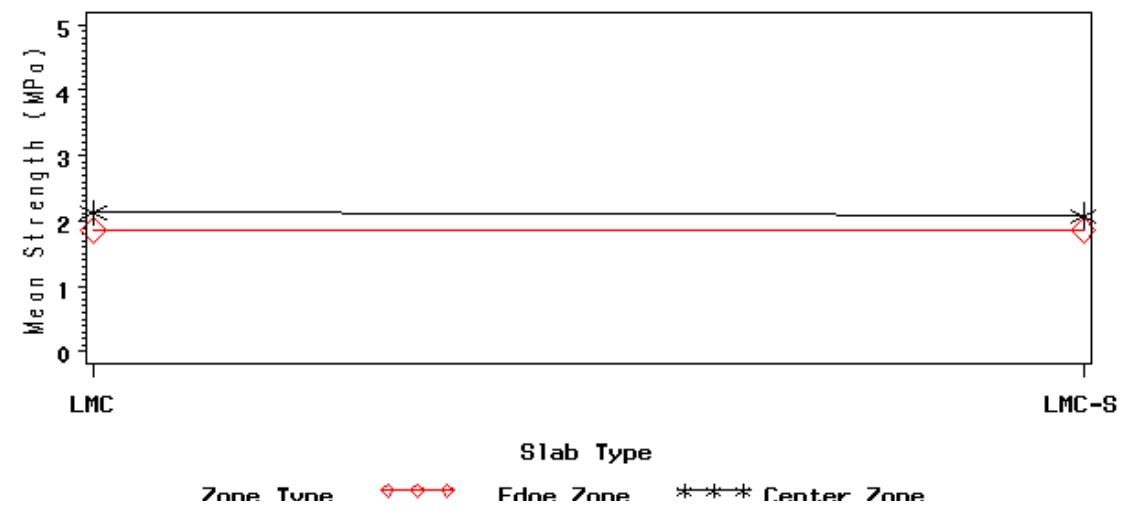

Figure 5.14: Means for levels of Zone type at each level of Slab (Stage-I)

Figure 5.14 is plotted by taking slab factor in the $\mathrm{x}$-axis and plotting the means of bond strength for each level of zone type. By looking at the plot we can further conclude that the bond strength of center zone was better than that of edge zone for two different types of slabs.

Code written here is for developing the three-way interaction plots. For Age by Zone by Slab three-way interaction, we consider the Zone by Slab interaction for each level of age and interpretation of the results was made based on the graphs developed.

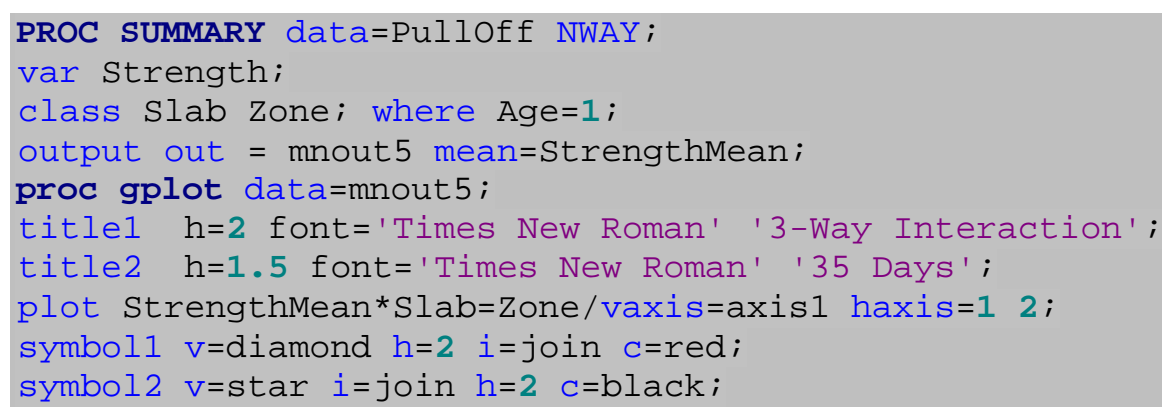


3-Way Interaction

35 Days

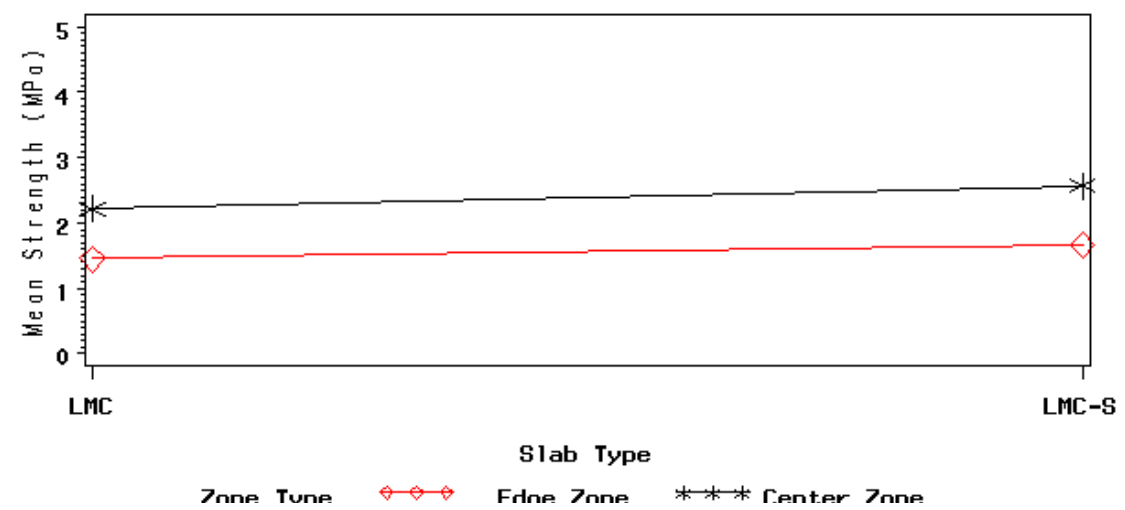

Figure 5.15: Means for levels of Zone type at each level of Slab type for 35 days age level (Stage-I)

Figure 5.15 is plotted by taking Slab factor in the x-axis and plotting the means of bond strength at each level of zone type for 35 days of age level. By looking at the plot we can further conclude that the effect of center zone was better than the effect of edge zone for 35 days of age. The effect of center zone and edge zone of both the slabs on the performance of bond strength was almost same for 35 days of age.

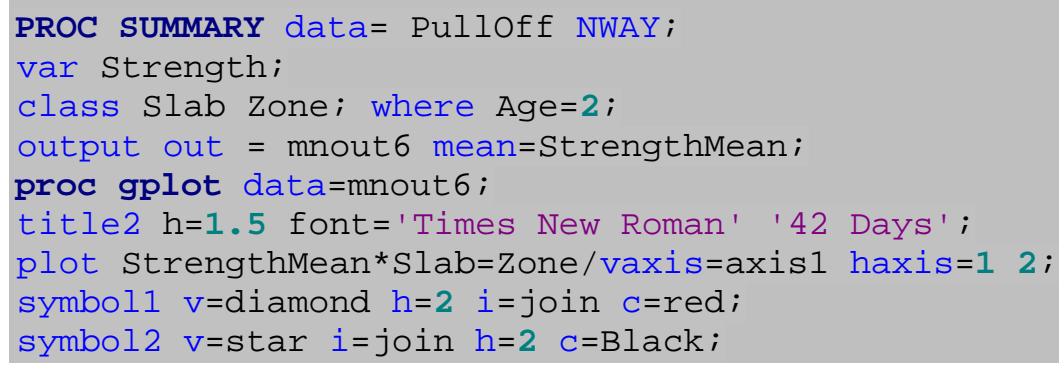


3-Way Interaction

42 Days

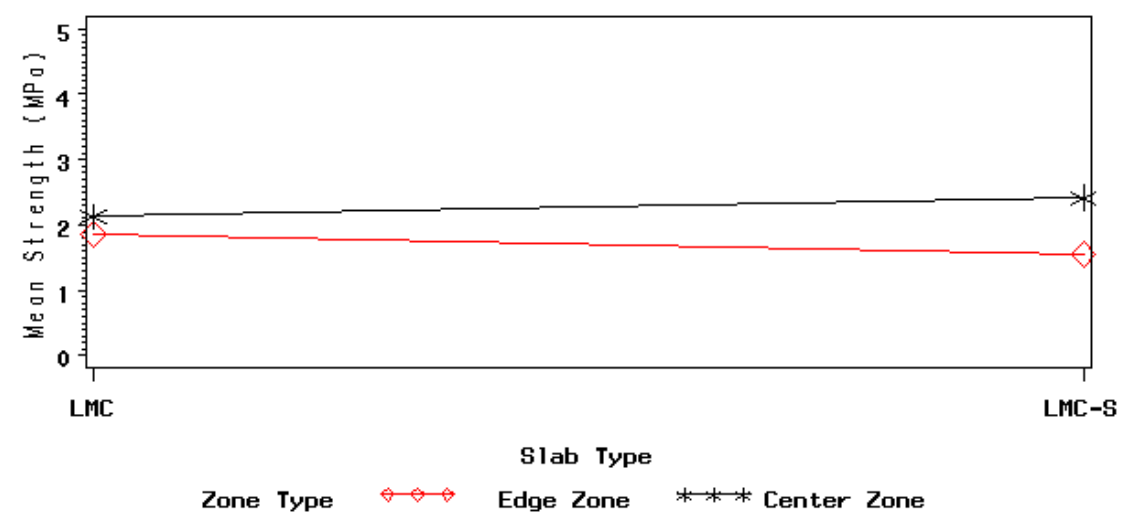

Figure 5.16: Means for levels of Zone type at each level of Slab type for 42 days age level (Stage-I)

Figure 5.16 is plotted by taking Slab factor in the x-axis and plotting the means of bond strength at each level of zone type for 42 days of age level. By looking at the plot we can further conclude that the effect of center zone was almost same for both the slabs but the effect of edge zone on LMC slab is more than that of LMC-S slab. The difference in strength between center and zone for LMC slab is almost negligible but the difference of strength between center and zone for LMC-S slab is more than LMC slab.

PROC SUMMARY data=Pulloff NWAY;

var Strength;

class Slab Zone; where Age $=3$;

output out = mnout 7 mean=StrengthMean;

proc gplot data=mnout7;

title2 h=1.5 font='Times New Roman' ' 49 Days';

plot StrengthMean*Slab=Zone/vaxis=axis1 haxis=1 2;

symbol1 $\mathrm{v}=$ diamond $\mathrm{h}=2 \mathrm{i}=$ join $\mathrm{c}=\mathrm{red}$;

symbol2 $v=$ star $i=j o i n ~ h=2 ~ c=b l a c k$; 
3-Way Interaction

49 Days

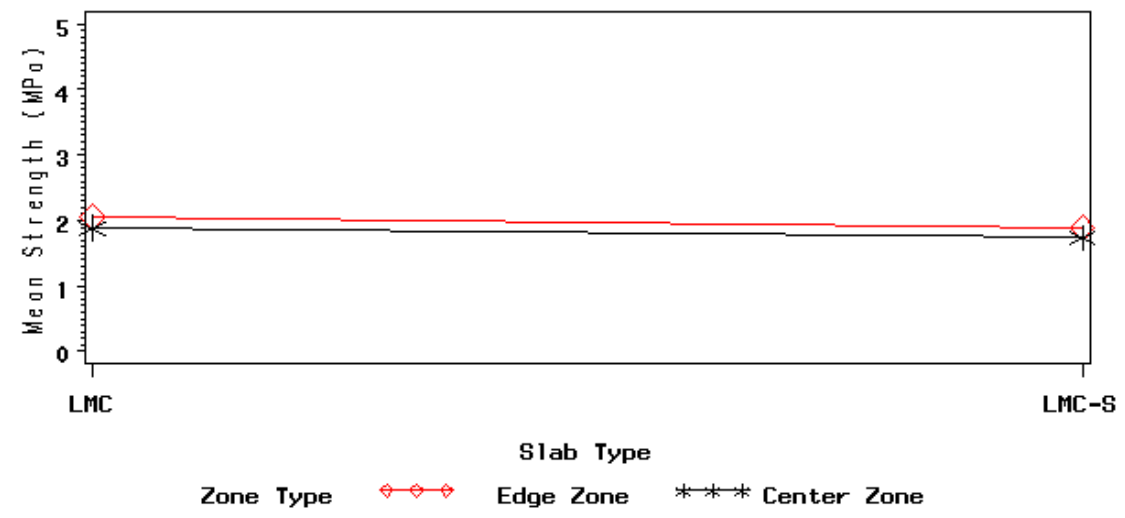

Figure 5.17: Means for levels of Zone type at each level of Slab type for 49 days age level (Stage-I)

Figure 5.17 is plotted by taking Slab factor in the x-axis and plotting the means of bond strength at each level of zone type for 49 days of age level. By looking at the plot we can conclude that the effect of both the center zone and edge zone was almost same for both the slabs at 49 days of age level.

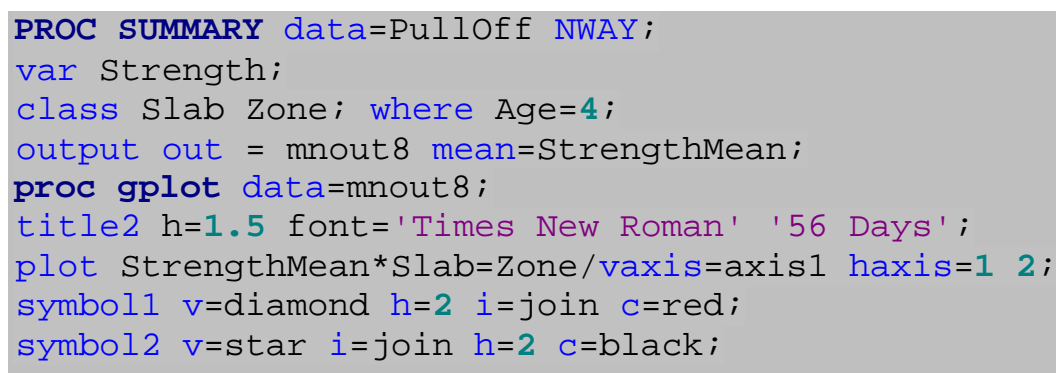


3-Way Interaction

56 Days

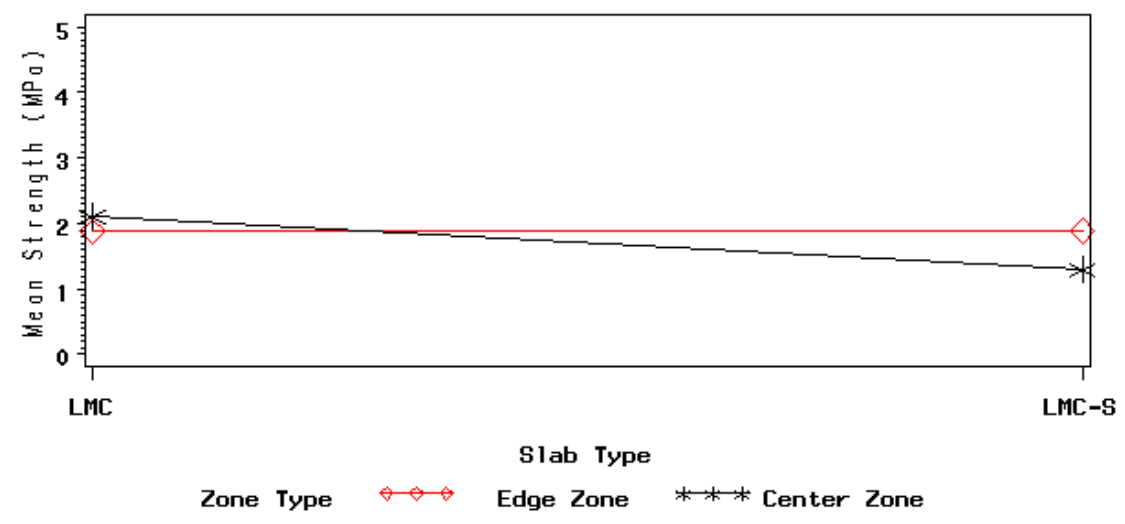

Figure 5.18: Means for levels of Zone type at each level of Slab type for 56 days age level (Stage-I)

Figure 5.18 is plotted by taking Slab factor in the x-axis and plotting the means of bond strength at each level of zone type for 56 days of age level. By looking at the plot we can conclude that the effect of the edge zone was almost same for both the slabs, but the strength at the center zone of LMC slab was more than that of LMC-S slab. Further, we can see that the strength at center and edge zones of LMC-S overlay was almost same at 56 days of age level.

PROC SUMMARY data=Pulloff NWAY;

var Strength;

class Slab Zone; where Age $=5$;

output out = mnout9 mean=StrengthMean;

proc gplot data=mnout9;

title2 h=1.5 font='Times New Roman' '90 Days';

plot StrengthMean*Slab=Zone/vaxis=axis1 haxis=1 2;

symbol1 $\mathrm{v}=$ diamond $\mathrm{h}=2 \mathrm{i}=\mathrm{join} \mathrm{c}=\mathrm{red}$;

symbol2 $\mathrm{v}=$ star $i=j o i n ~ h=2$ c=black;

run;

quit; 


\section{3-Way Interaction}

90 Days

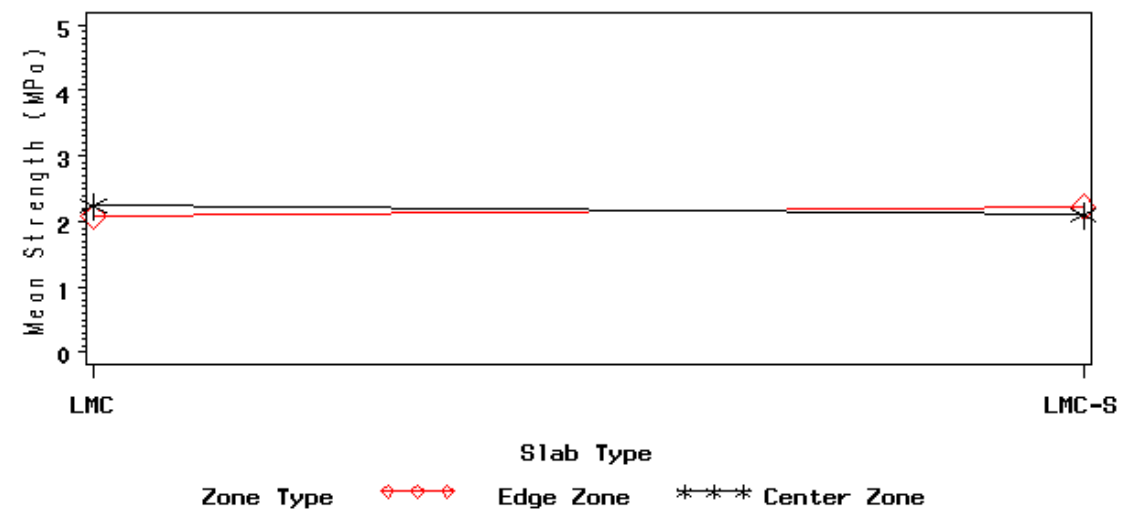

Figure 5.19: Means for levels of Zone type at each level of Slab type for 56 days age level (Stage-I)

Figure 5.19 is plotted by taking Slab factor in the x-axis and plotting the means of bond strength at each level of zone type for 90 days of age level. By looking at the plot we can conclude that the effect of both the center zone and edge zone was almost same for both the slabs at 90 days of age level.

In summary, the overall performance of slab with LMC overlay was similar to that of the slab with LMC-S overlay. Hence we can conclude that the use of bonding slurry at the interface will not affect the bond strength.

\subsubsection{Length Change Monitoring}

Concrete undergoes volume changes due to several reasons such as shrinkage, drying, etc and hence in this study, length change of both the substrate and overlay concretes was monitored by embedding the special type of concrete embedment gages in the concretes as discussed in section 3.5. In order to measure the differential length change developed at the interface (main cause for curling of the overlay concrete Mailvaganam et al. (2000)); strain gages were installed $25 \mathrm{~mm}$ (1 in.) below the interface in substrate concrete and $25 \mathrm{~mm}$ ( $1 \mathrm{in}$.) above the interface. Details of the type of 
embedment gages used in this study are explained in detail in section 3.5.1.3 and the testing procedure is explained in detail in section 4.5.

Figure 5.20 shows the length change profile of a substrate Type $\mathrm{K}$ concrete at typical location recorded by embedment gage. In this graph, the length change of the substrate Type K concrete is shown for the entire age of the substrate i.e., length change up to the overlay pour and after the overlay pour.

This graph can be easily used to distinguish the effects of construction, heat of hydration, thermal expansion, shrinkage and curing process of the Type $\mathrm{K}$ substrate concrete. This graph shows the percentage length change plot of the entire period of the Stage-I study i.e., before the overlay pour and after overlay pour. The conclusions that can be drawn from the Figure 5.20 are as follows:

1. After the substrate concrete was poured the percentage change in length suddenly increased from 0 to $0.014 \%$, which is mainly due to the heat of hydration process that occurred within 24 hrs of casting;

2. After that the percentage change in length was almost maintained constant for 7 days, because the substrate concretes were cured under wet-burlap. This constant reading can only be observed up to 7 days after substrate pour because the substrate slabs were wet-cured for 7 days as per WVDOH standard specifications. Due to this wet curing the loss of moisture from the slab is minimized since the heat of hydration was retained within the slab which is helpful for curing;

3. Then, after 7 days we can see the gradual drop in percent length change value, which accounts for stopping of the wet-curing process and the removal of formwork. Since, the slabs were opened to air for dry curing, the loss of moisture is allowed from all the sides except from the bottom. Due to this, the concrete shrinks or contract and hence the drop in percent length change can be observed clearly from the graph;

4. Later, after 6 weeks all the embedment gages were stopped to prepare the surface preparation for overlay construction. Hence, no reading is observed 
between $6^{\text {th }}$ and $7^{\text {th }}$ week after substrate pour. This is considered as a buffer period for construction and is shown in figure;

5. Again, after the overlay construction in $7^{\text {th }}$ week, the substrate strain gages were started again to monitor the percent length change of concrete due to the overlay application;

6. As we can see from the figure, the expansion of the substrate concrete is again observed after overlay construction. This phenomenon is observed because, due to the application of the overlay concrete, the rate of evaporation from the top surface area is restricted and the evaporation is only possible from the sides of the slabs. Due to this additional moisture diffused into the substrate concrete, it expands again and once the rate of evaporation is stabilized, the expansion of the slab is maintained constant. But, after 28 days of overlay, expansion of the slab is again observed, the reason for this behavior is: when coring procedure is done for conducting pull-off testing, water is added continuously to the slab, which results in the increase of moisture and hence the concrete expands again;

7. Also, the other reason that accounts for the expansion of the slabs after overlay construction is due to the application of high heat to the bi-layer system which leads to the thermal expansion. 


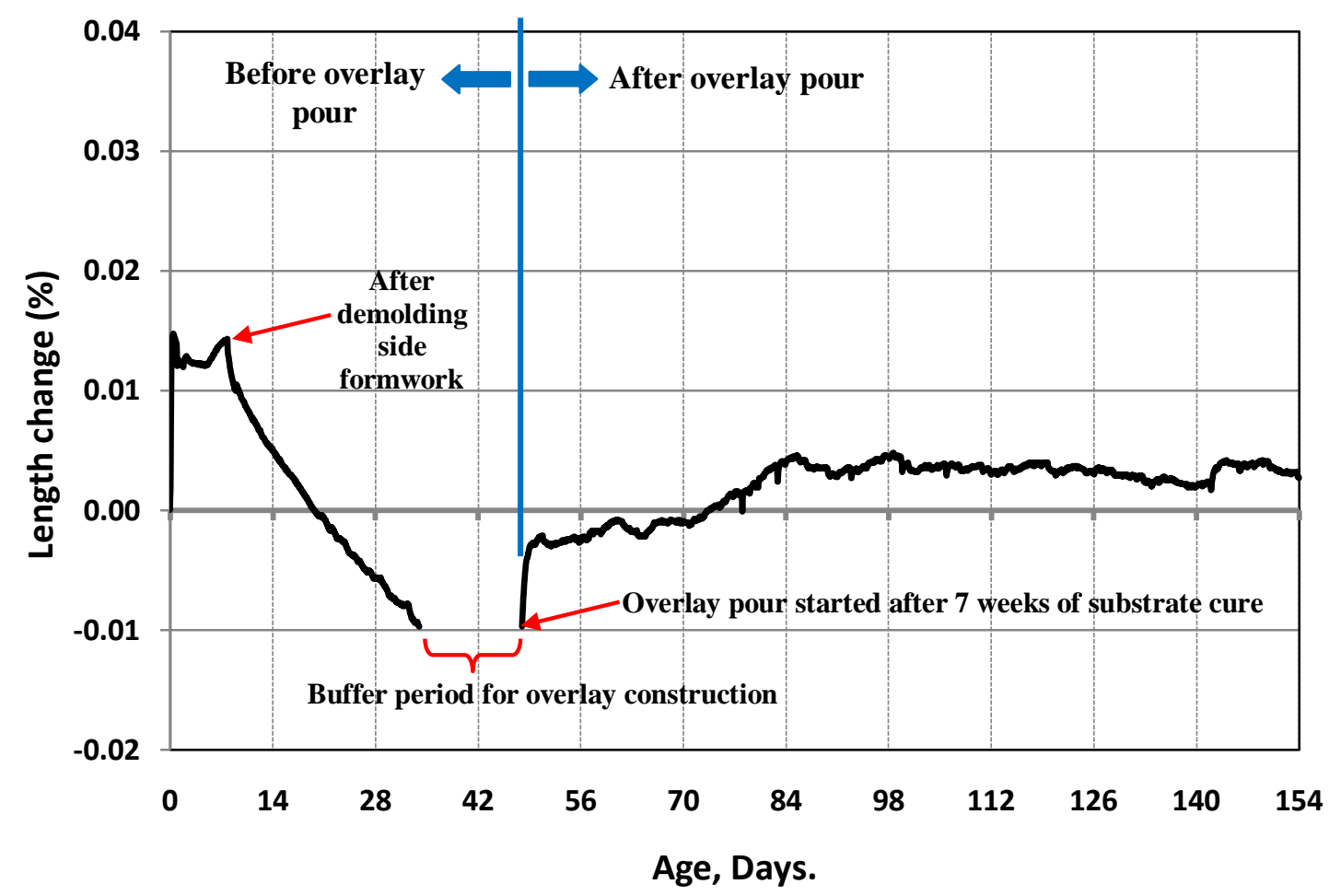

Figure 5.20: Length change of Type K substrate concrete at typical location

Similar pattern of length change behavior of Type $\mathrm{K}$ substrate concrete was observed at all the locations except, the values were different at different locations. This depends on the intensity of rate of evaporation, material properties of the overlay concrete, thermal expansion, bonding condition and hence the delamination. All the plots of substrate and overlay concrete at different locations are shown from Figure 5.22(a) to Figure 5.22(d) for slab with LMC overlay and from Figure 5.23(a) to Figure 5.23(d) for slab with LMC-S overlay.

\subsubsection{Differential Length Change after Overlay Pour}

Similar to the substrate concrete, embedment gages were installed in overlay concrete, 25mm (1 in.) above the interface, to monitor the percent length change of the overlay concrete. This way the differential length change was monitored between the overlay and substrate concretes at the interface. Monitoring of differential length change is important, because this change leads to the development of shear stresses at the 
interface which were considered as the main cause for delamination (Kim et al. (2003); Mailvaganam et al. (2000); and Zhang and Li (2002)).

The plan and elevation views of the location of strain gages in substrate and overlay concretes are shown in Figure 5.21(a) and Figure 5.21(b). The plots of the percent length change of the different strain gages with age are shown below the Figure 5.21. All the figures in the left side corresponds to the percent length change plots of the embedment strain gages in the slab with LMC overlay whereas, the right side figures represents the percent length change plots of the embedment strain gages in slab with LMC overlay with bonding slurry at the interface. That is, Figure 5.22(a), Figure 5.22(c), Figure 5.23(a), and Figure 5.23(c) shows the percent length change plots of substrate and LMC overlays of slab without bonding slurry (LMC). Whereas Figure 5.22(b), Figure 5.22(d), Figure 5.23(b), and Figure 5.23(d) shows the percent length change plots of substrate and overlay concrete of slab with bonding slurry at the interface (LMC-S). In all these plots, red line refers to the percent length change plot of overlay concrete and the black line refers to the percent length change plot of substrate concrete. Reference to the Figure 5.21 is always necessary when reading the Figure 5.22 and Figure 5.23. Description of the terminology used in Figure 5.21 is explained clearly in section 3.5.1.3 and section 3.5.2.2. 


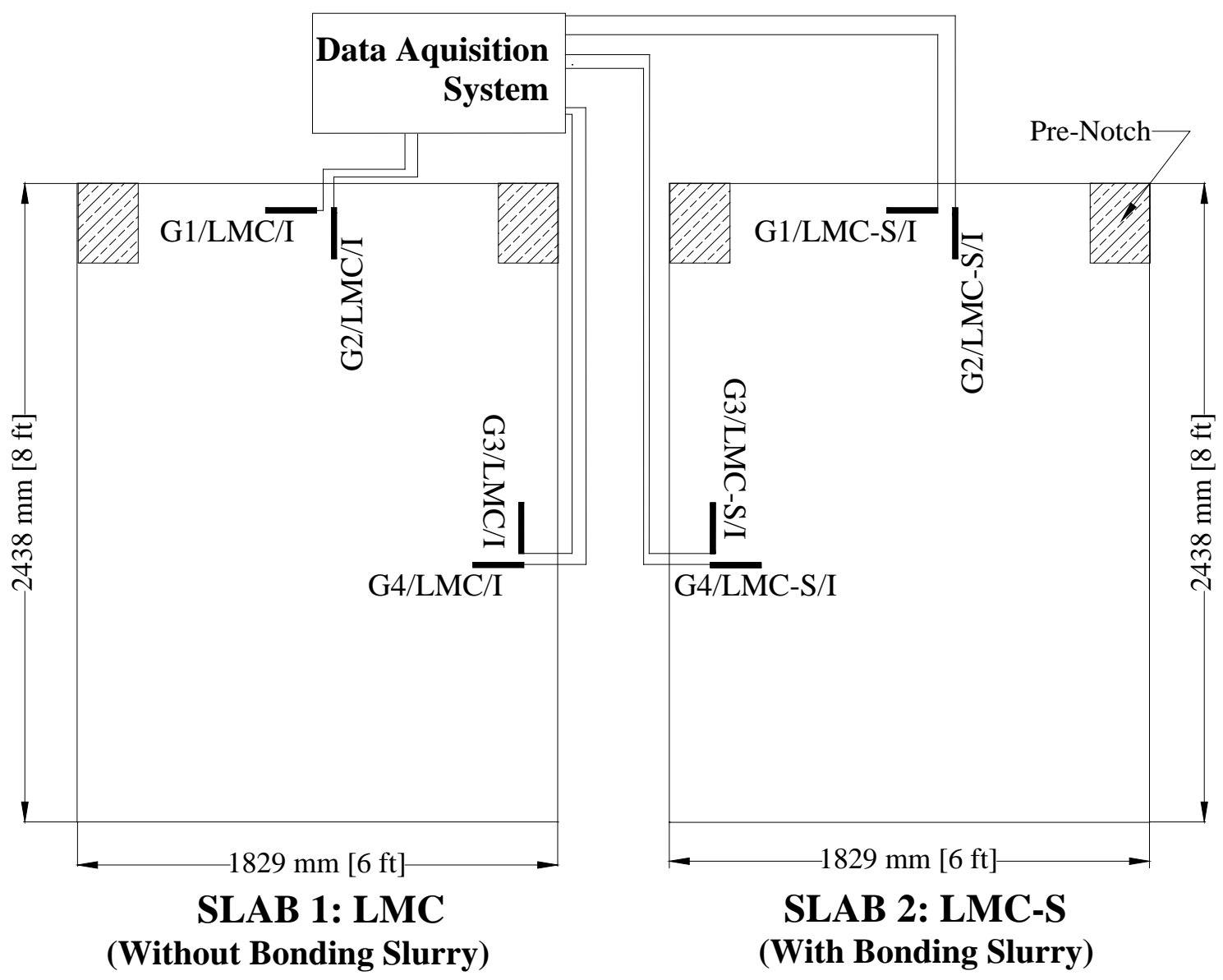

(a)
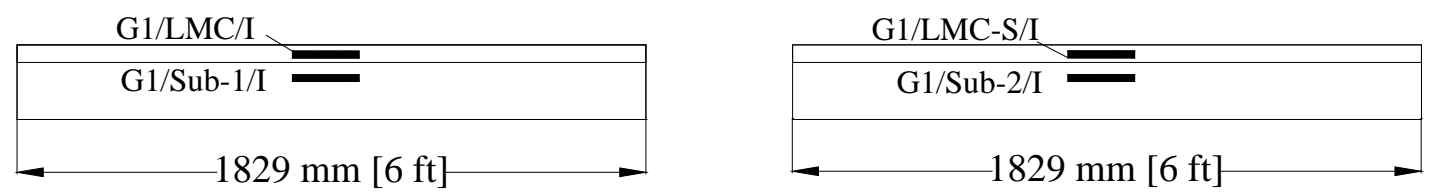

(b)

Figure 5.21: Locations of embedment strain gages in substrate and overlay concretes (Stage-I); a) Plan view, and b) elevation view

As per the plan, embedment gages were installed in both substrate and overlay concretes in transverse and longitudinal directions facing towards the pre-notch and center, to study the differential length change, as shown in Figure 5.21. For example, G1/Sub-1/I and G1/LMC/I strain gages were installed 25 mm (1 in.) below and 25 mm (1 in.) above the interface respectively in the transverse direction along the edge on slab 
with LMC overlay without bonding slurry at the interface as shown in the Figure 5.21. Similarly, G1/Sub-2/I and G1/LMC-S/I strain gages were installed $25 \mathrm{~mm}$ (1 in.) below and $25 \mathrm{~mm}$ (1 in.) above the interface respectively in the transverse direction along the edge on slab with LMC overlay with bonding slurry at the interface as shown in the Figure 5.21. This installation was selected to study the effect of bonding slurry on the differential length change developed at the interface. Similar to the configuration explained, strain gages were also installed in longitudinal direction to study the effect of using bonding slurry at the interface on shear stresses.

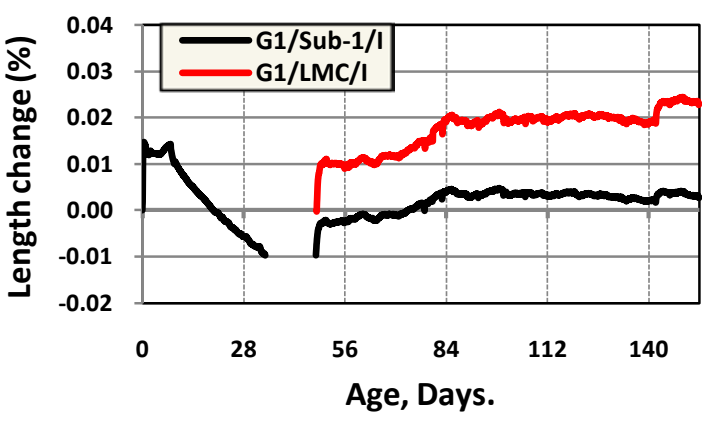

(a)

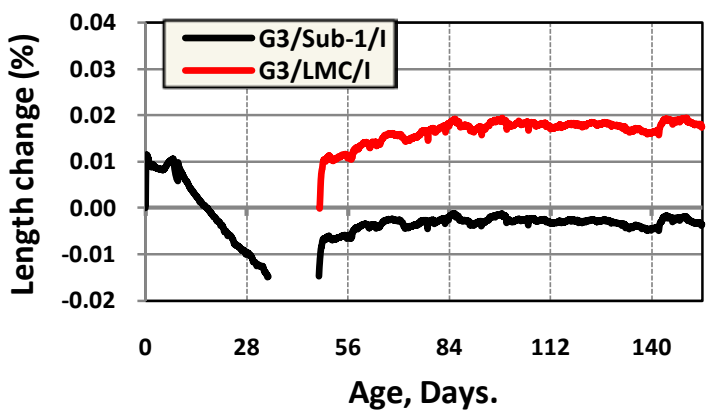

(c)

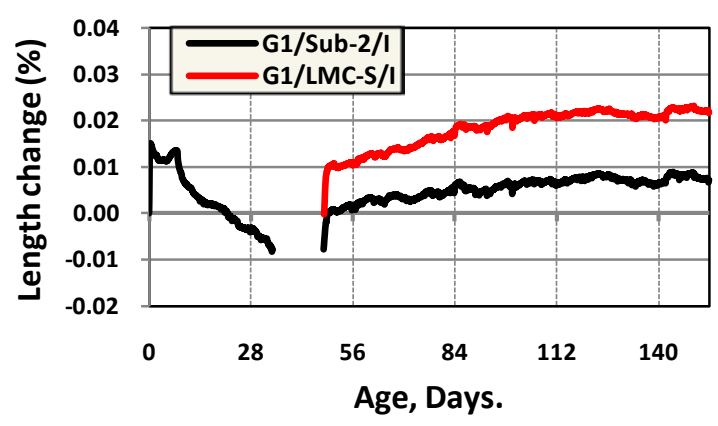

(b)

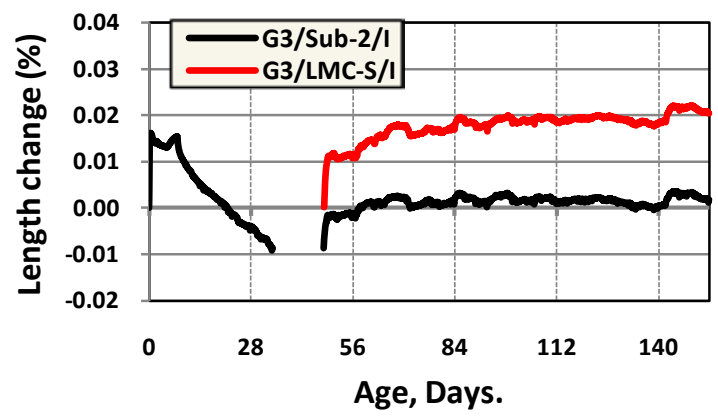

(d)

Figure 5.22: Comparison of percent length change plots of embedment gages in transverse and longitudinal direction along the edges of LMC and LMC-S slabs (Stage-I) 


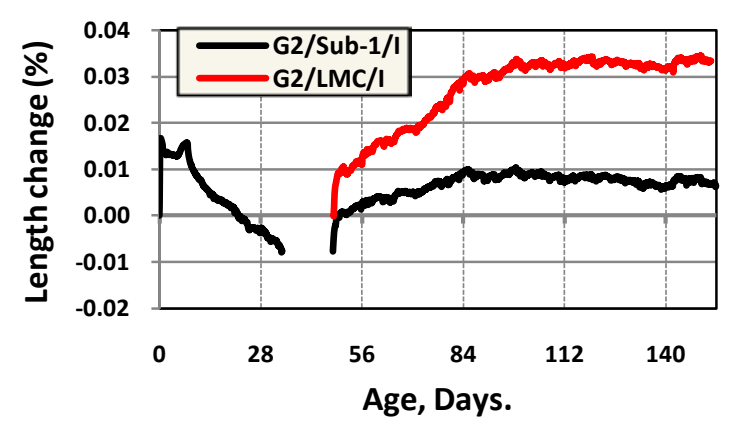

(a)

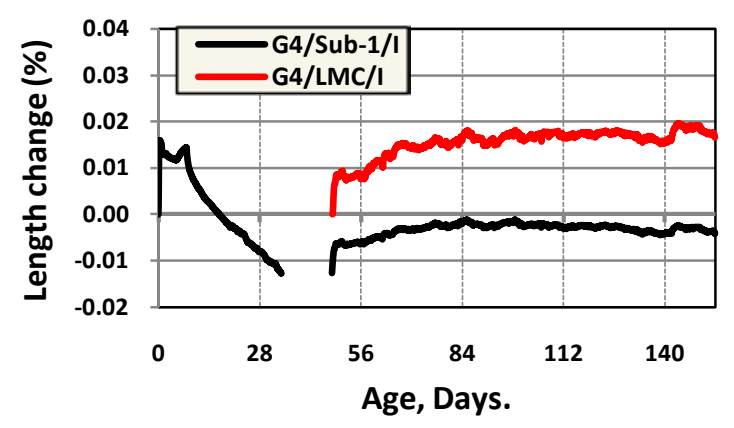

(c)

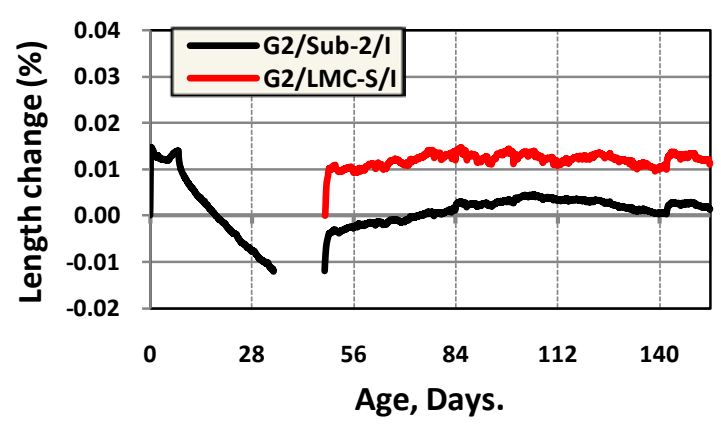

(b)

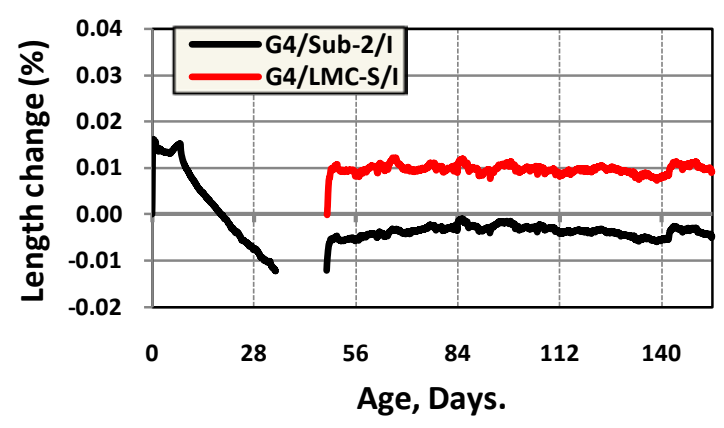

(d)

Figure 5.23: Comparison of percent length change plots of embedment gages in transverse and longitudinal direction along the edges of LMC and LMC-S slabs (Stage-I)

Based on the results as shown in Figure 5.22 and Figure 5.23, following conclusions were drawn:

1. By comparing, differential length change of LMC overlay without bonding slurry in transverse direction along the edge (Figure 5.22(a)) and differential length change of LMC overlay with bonding slurry in transverse direction along the edge (Figure 5.22(b)), we can see that the differential length change developed at the interface for LMC overlay with bonding slurry was less. Hence, we can conclude that the differential stress developed at the interface was less when bonding slurry was applied at the interface.

2. Also by comparing, differential length change of LMC overlay without bonding slurry in transverse direction facing towards the center (Figure 5.22(c)) and differential length change of LMC overlay with bonding slurry in transverse direction facing towards the center (Figure 5.22(d)), we can see that the differential length change developed at the interface for LMC overlay with 
bonding slurry was less. Hence, we can conclude that the differential stress developed at the interface was less when bonding slurry was applied at the interface.

3. By comparing, differential length change of LMC overlay without bonding slurry in longitudinal direction along the edge (Figure 5.23(a)) and differential length change of LMC overlay with bonding slurry in longitudinal direction along the edge (Figure 5.23(b)), we can see that the differential length change developed at the interface for LMC overlay with bonding slurry was less. Hence, we can conclude that the differential stress developed at the interface was less when bonding slurry was applied at the interface.

4. Also, by comparing differential length change of LMC overlay without bonding slurry in transverse direction facing towards the center (Figure 5.23(c)) and differential length change of LMC overlay with bonding slurry in transverse direction facing towards the center (Figure 5.23(d)), we can see that the differential length change developed at the interface for LMC overlay with bonding slurry was less. Hence, we can conclude that the differential stress developed at the interface was less when bonding slurry was applied at the interface.

In all the cases, high differential length change was observed in both the transverse and longitudinal direction along the edges of LMC and LMC-S slabs compared to that of the transverse and longitudinal directions towards the center. Hence we can conclude that the intensity of delaminations is more at the edges.

In summary, we can conclude that when bonding slurry was applied at the interface, the differential length change observed at the interface at all the locations discussed above was less when compared to the one without bonding slurry. Also, we can say that the composite action of the bi-layer deck slab was more when bonding slurry was applied. 


\subsubsection{Vertical Displacements Due to Corner Lifting}

Vertical displacements occurred due to corner lifting of the overlay concrete was measured by installing LVDT at the corners of two slabs. This was done to compare the effect of bonding slurry on the vertical corner lifting of the overlays. The LVDT were started only after all the formwork was demolded and all the instrumentation was installed i.e., after 3 days of overlay curing. In vertical corner lift versus time graph, the time zero indicates the onset of the measurements. No data could be recorded immediately after 3 days of overlay pour.

Two types of surface bonding conditions were selected in this Stage-I study. One slab was constructed with saturated surface condition but without bonding slurry at the interface and the other one was constructed with saturated surface and the bonding slurry applied at the interface. In this stage-I, on both the slabs LMC overlay was applied to study the effect of bonding slurry condition at the interface. The average temperature maintained in the enclosed room was around $35^{\circ} \mathrm{C}\left(95^{\circ} \mathrm{F}\right)$. Figure 5.24 shows the vertical corner lift versus the age for LMC without bonding slurry and with bonding slurry at the interface. 


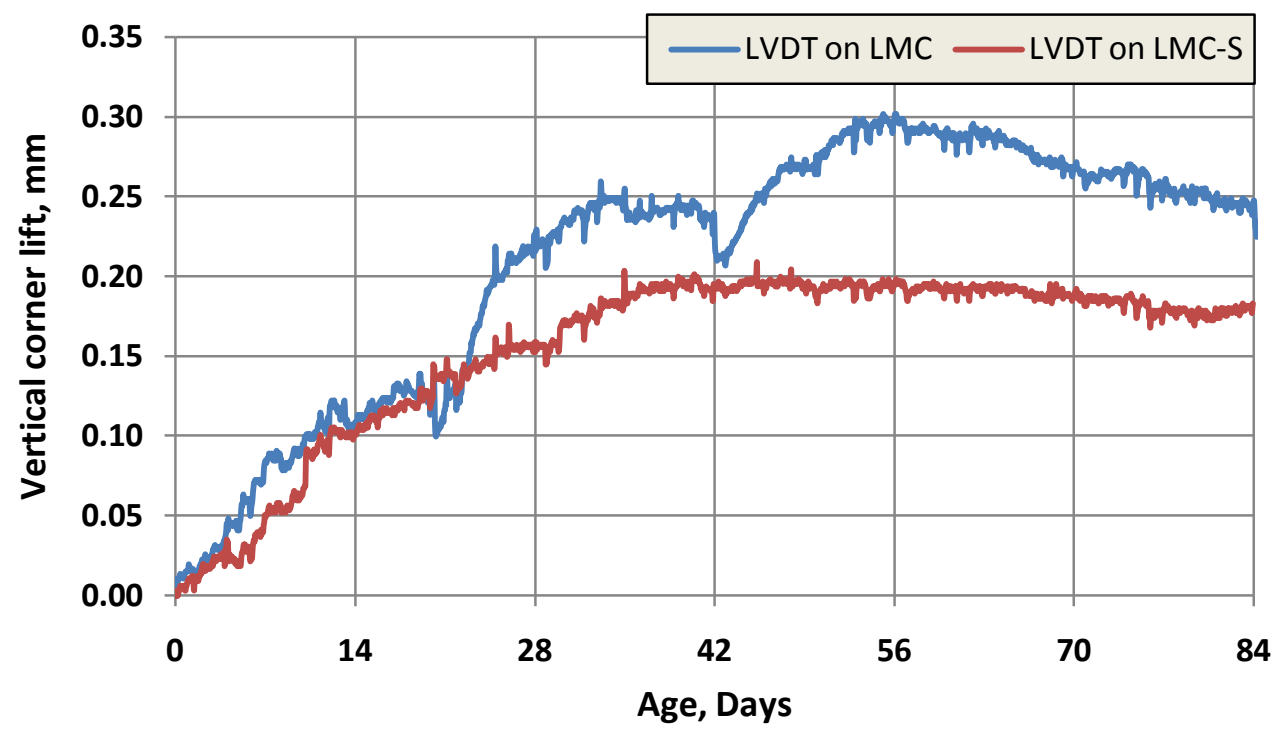

Figure 5.24: Relative displacement of overlay concretes (LMC and LMC-S) recorded by LVDT (Stage-I)

From the Figure 5.24, it is observed that the saturated surface with bonding slurry at the interface had the lowest crack opening at the corner when compared to the one without bonding slurry. Hong (2006) observed that SFMC overlay with dry substrate surface had the largest relative displacements at the interface; followed by saturated substrate surface; and saturated substrate surface with slurry being the least by conducted the experiments on small scale samples. Hence, we can conclude that the use of bonding slurry with saturated surface condition at the interface would potentially decrease the probability of cracking due to vertical corner lift of the overlays.

\subsubsection{Indirect Ultrasonic Pulse Velocity Testing}

Indirect UPV testing was carried out at different locations to predict the delamination profile at the interface. The testing procedure for conducting this testing is explained in detail in section 4.7.

\subsubsection{UPV Readings}

The gridlines were drawn on the half of the slab on which UPV testing was carried out. In longitudinal direction the gridlines were spaced $200 \mathrm{~mm}$ (8 in.) whereas in 
transverse direction gridlines were spaced at $150 \mathrm{~mm}$ (6 in.). Gridlines in longitudinal direction were named from 1 to 13 whereas in transverse direction they were named from $\mathrm{G}$ to $\mathrm{M}$. Indirect testing was carried out by fixing the transmitting transducer location at the center of the slab (G7) and varying the location of receiving transducer at various locations as shown in Figure 5.25. In this figure, red circle shows the location of transmitting transducer whereas, the black circles shows the locations of receiving transducer.

Several cases were selected and the UPV reading was measured for each case and compared between the two slabs. In first case, the transmitting transducer was fixed at location G7 and the location of receiving transducer was varied from; H7 to K7 on LMC bi-layer deck slab and from F7 to C7 on LMC-S bi-layer deck slab in transverse direction. Then, the transmitting transducer was fixed at location G7 and the receiving transducer was varied from; G6 to G3 and G8 to G11 on LMC bi-layer deck slab, and G6 to G3 and G8 to G11 on LMC-S bi-layer deck slab in longitudinal direction. Finally, the transmitting transducer was fixed at location G7 and the receiving transducer was varied from; H6 to K3 and H8 to K11 on LMC bi-layer deck slab, and F6 to C3 and F8 to C11 on LMC-S bi-layer deck slab in diagonal direction towards the corner. For each location, the time required for ultrasonic signal to travel from transmitting to receiving transducer was captured and UPV was computed. In all the three cases mentioned above, the time Vs distance plots were generated and compared between the two slabs and the conclusions were drawn based on these graphs. Time recorded at each location is shown in Appendix B. When reading Figure 5.26 through Figure 5.28 always reference to Figure 5.25 should be made to clearly understand the location numbers. 


\section{Data Aquisition \\ System}

$\begin{array}{llllllll}G & H & \text { I } & \text { J } & \text { K } & \text { L } & \text { M }\end{array}$
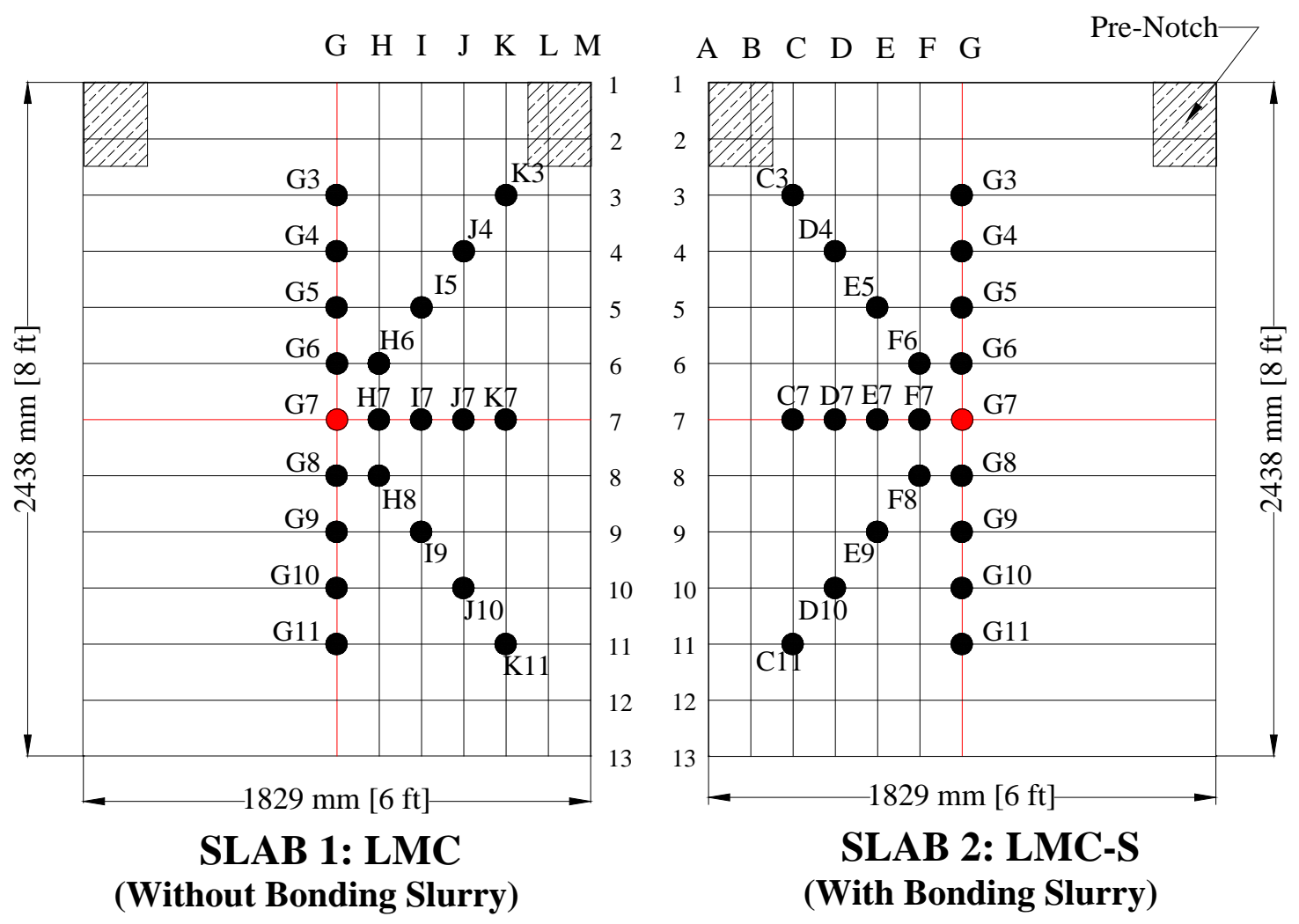

Figure 5.25: Locations at which indirect UPV was conducted

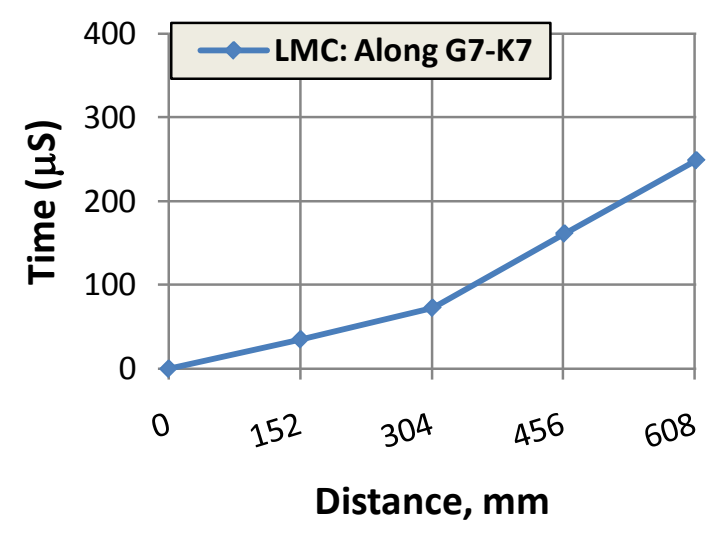

(a)

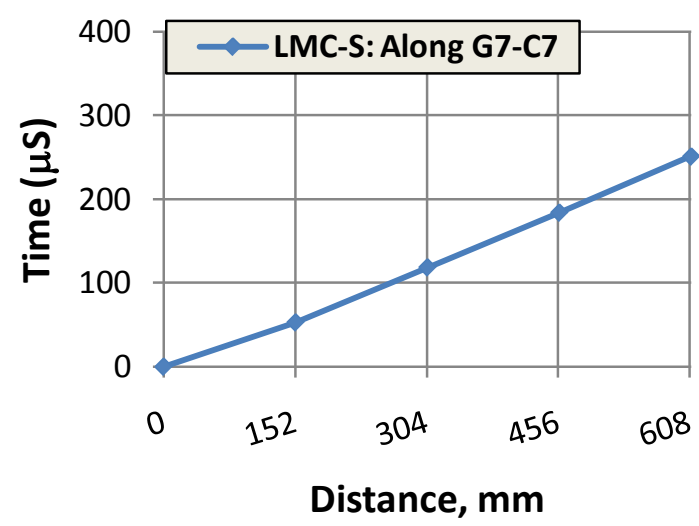

(b)

Figure 5.26: Indirect UPV plots along: G7-K7 for LMC slab and G7-C7 for LMC-S slab 
From Figure 5.26(a) and Figure 5.26(b), constant slope is observed in the case of slab with LMC-S overlay when compared to that of slab with LMC overlay when measured at the same locations. That is, the homogeneity of the concrete is better in the case of slab with LMC-S overlay than that of the slab with LMC overlay.

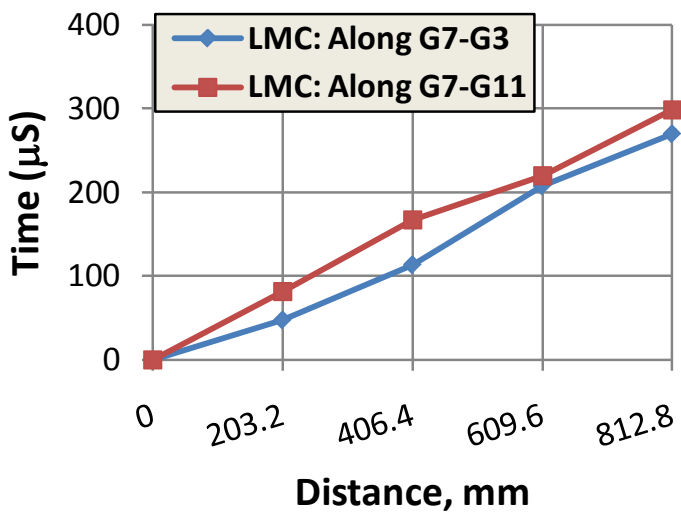

(a)

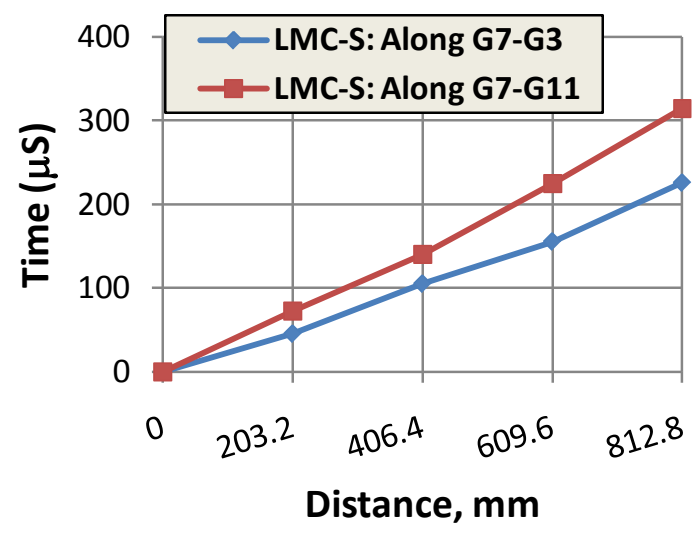

(b)

Figure 5.27: Indirect UPV plots along G7-G3 and G7-G11 for both the slabs

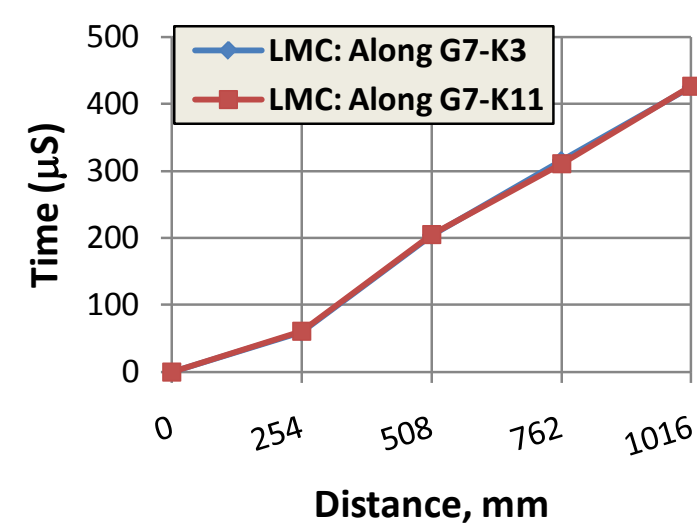

(a)

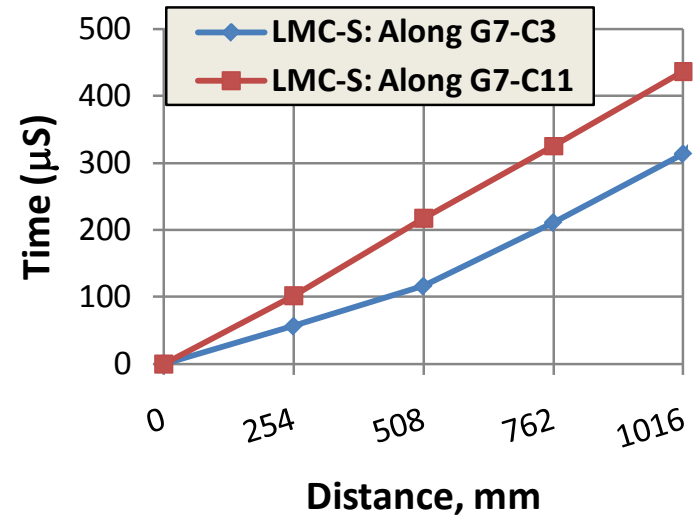

(b)

Figure 5.28: Indirect UPV plots along: G7-K3 and G7-K11 for LMC slab, and G7-C3 and G7-C11 for LMC-S slab

Also, from Figure 5.27 and Figure 5.28, constant slope is observed in the case of slab with LMC-S overlay when compared to that of slab with LMC overlay. The time required for the signal to travel from transmitting to receiving transducer in the case of slab with LMC-S overlay is always less than that of the slab with LMC overlay. Hence 
from the above figures we can conclude that in all the cases less delamination is observed when bonding slurry was applied at the interface.

\subsubsection{Analysis of Signals Collected by Oscilloscope}

Oscilloscope was connected to the UPV tester to record the time domain signal simultaneously with the time. Later this time domain signal was saved into compact flash in EXCEL format and was transferred into computer using this compact flash drive in order to convert the waveform into Fast Fourier Transform (FFT) using MATLAB, powerful computing and analytical software. Signals are converted to FFT form since, when the signals are more complex; frequency domain provides much information about the signal.

Step by step procedure for converting the time domain signal into FFT using MATLAB is as follows:

1. Initially, from the sampling interval selected for time domain signal, sampling frequency is calculated by taking the inverse of the sample interval of time domain signal;

2. Then, the amplitude data recorded by oscilloscope in EXCEL format is imported into MATLAB using simple array read format;

3. After that, length of the time domain signal is calculated by using the length function in order to calculate the Nyquist interval;

4. After calculating the length of the signal, Nyquist interval is calculated by calculating the next power of 2 from the length of the time-domain signal;

5. Then, the FFT of the time domain amplitude is calculated by using in-built fft function in MATLAB;

6. After that, the frequency range of interest is defined in this step, it is calculated by using the length of the time-domain signal;

7. Then, only one-sided FFT amplitude is computed based on the Nyquist theorem, since this theorem states that the values at higher frequencies are the reflections of the values at the lower frequencies and these values should not be mistaken; 
8. After computing the one-sided FFT amplitude and frequency range, both arrays are transformed into same dimensions using transpose functions;

9. Then, both the arrays of same dimension are concatenated into one single array with first column as frequency and the second column as computed onesided amplitude value;

10. Finally, the concatenated array is exported into excel by using xlswrite function and saved this file into a folder of interest.

Above described steps are shown in algorithm format in Figure 5.29.

- Sampling frequency is calculated from the time-domain signal

- Amplitude data was imported from time-domain signal

2

3

- Length of the amplitude data in step 2 is calculated using length function

- Nyquist interval is calculated by calculating the next power of 2 from the length of time-domian signal

- FFT of the time-domain signal amplitude is calculated by using the standard FFT equation

- Frequency Range is defined

- One-sided absolute value of FFT amplitude calculated in Step 5 is computed based on Nyquist theorem

- Frequency range from step 6 and one-sided FFT amplitude from step 7 are concatenated into a single array by using horzcat function

- FFT data from Step 8 is then exported into Microsoft EXCEL by using 9 xlswrite function

Figure 5.29: Algorithm for calculating FFT from time-domain signal using MATLAB 
Code used for converting the time-domain signal into FFT is as follows:

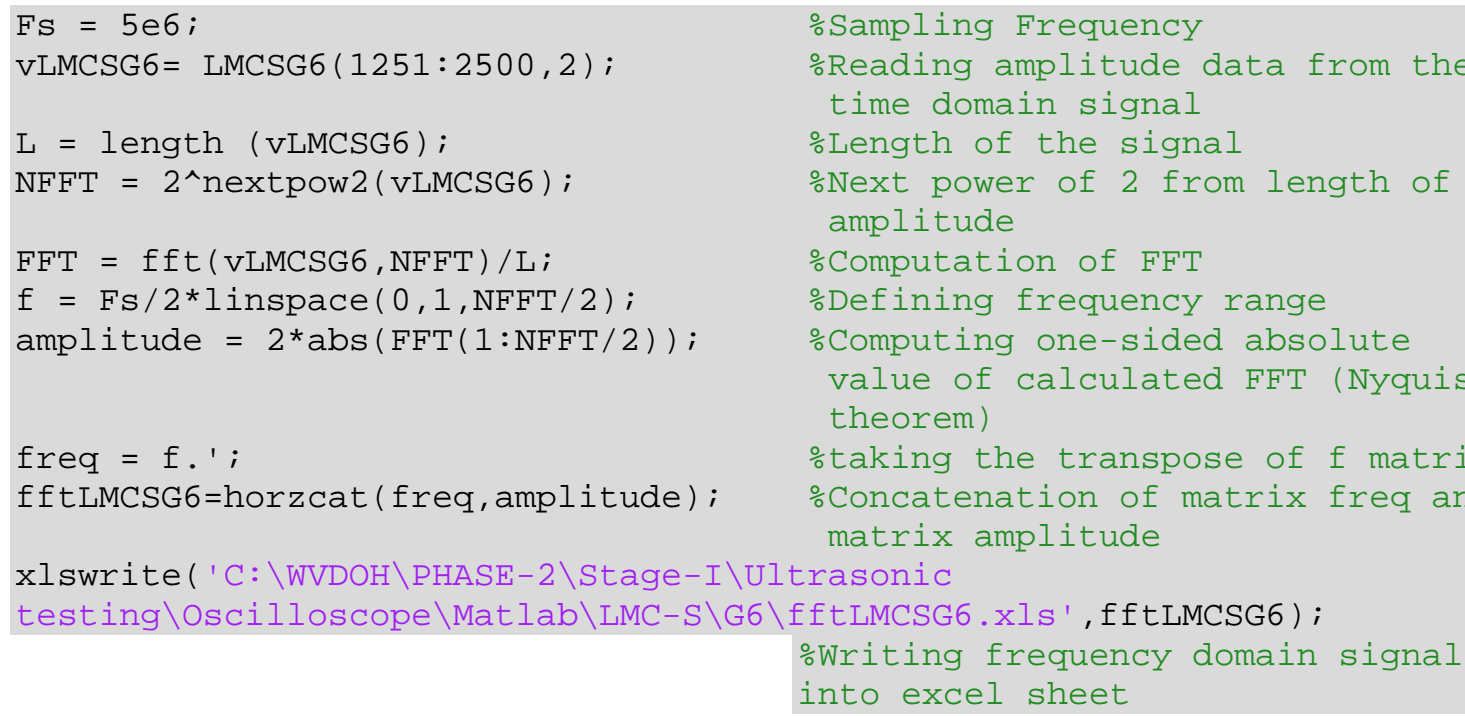

Figure 5.30 shows the locations at which time-domain waveforms were collected simultaneously along with pulse time using oscilloscope. Time-domain waveform was not recorded at the edges and the corners as shown in the above figure, i.e., signals were not recorded at locations G2, G1, L2, M1, L7, M7, G12, G13, L12, and M13 on slab with LMC overlay and G2, G1, B2, A1, B7, A7, G12, G13, B12, and A13 on slab with LMC$\mathrm{S}$ overlay since, the power of the signal was negligible when recorded at the above mentioned locations. 


\section{Data Aquisition}

System

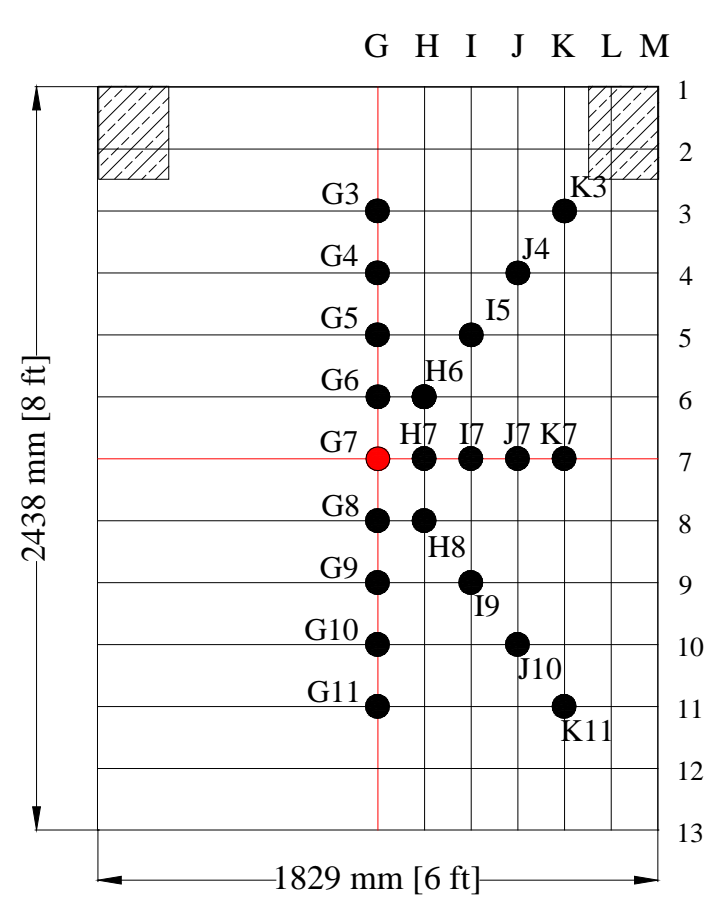

SLAB 1: LMC (Without Bonding Slurry)

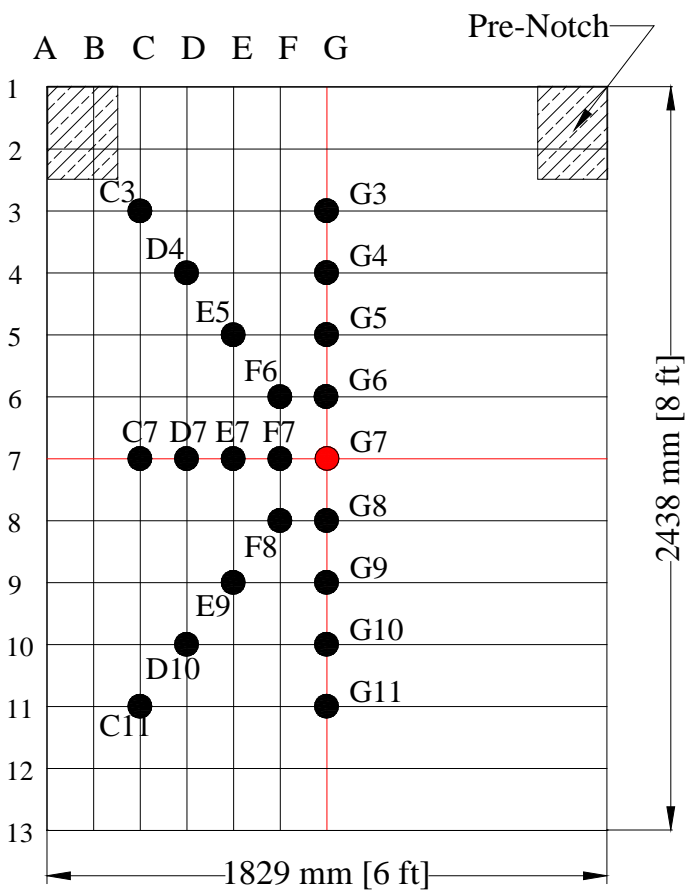

SLAB 2: LMC-S (With Bonding Slurry)

Figure 5.30: Locations at which oscilloscope readings were collected (Stage-I)

In all the figures plotted below, we can see that when the distance between the transmitting transducer and the receiving transducer increases, the magnitude of the peak of the signal reduces and almost becomes negligible. Maximum peak is observed when the transducers are closely spaced and vice versa. At all the locations, maximum peak is observed around the $82 \mathrm{kHz}$ if any since, $82 \mathrm{kHz}$ transducer was used in this study. Comparisons between two slabs are made based on the peaks recorded at different locations. When reading the Figure 5.31, always reference has to be made to Figure 5.30 for better understanding of the terminology.

After converting and exporting the time-domain waveform into FFT as explained above. The exported FFT in excel format is further used to plot the FFT's as shown from Figure 5.31(a) through Figure 5.31(j). All the figures in the left hand side are plotted for 
the slab with LMC overlay whereas the figures in the right hand side are plotted for the slab with LMC-S overlay. That is, Figure 5.31(a), Figure 5.31(c), Figure 5.31(e), Figure 5.31(g), and Figure 5.31(i) shows the FFT plots for slab with LMC overlay whereas Figure 5.31(b), Figure 5.31(d), Figure 5.31(f), Figure 5.31(h), and Figure 5.31(j) shows the FFT plots for slab with LMC-S overlay.

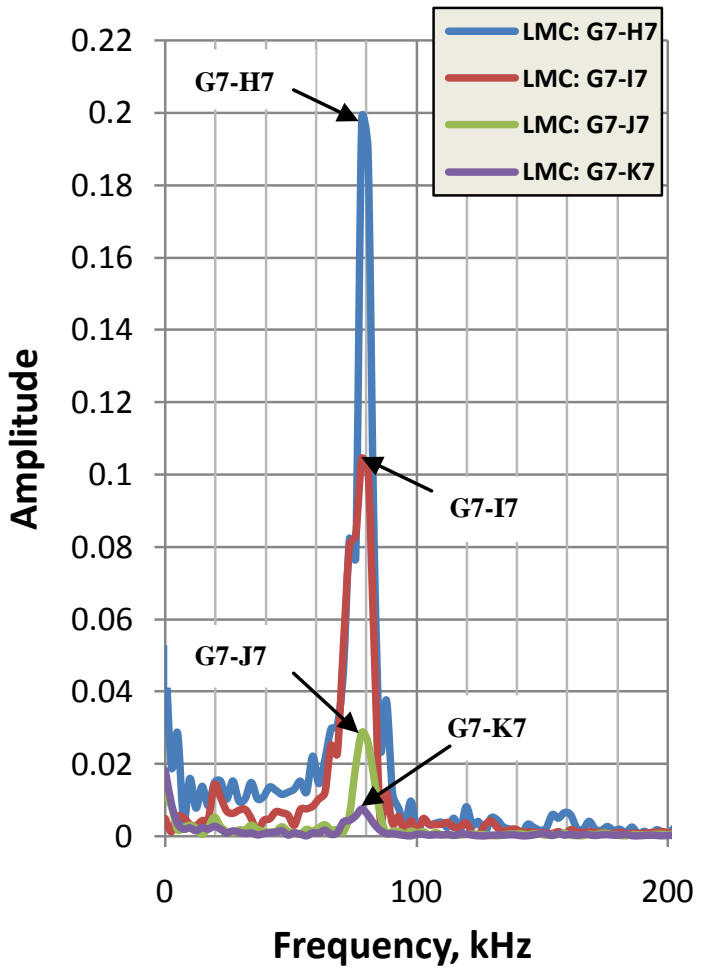

(a)

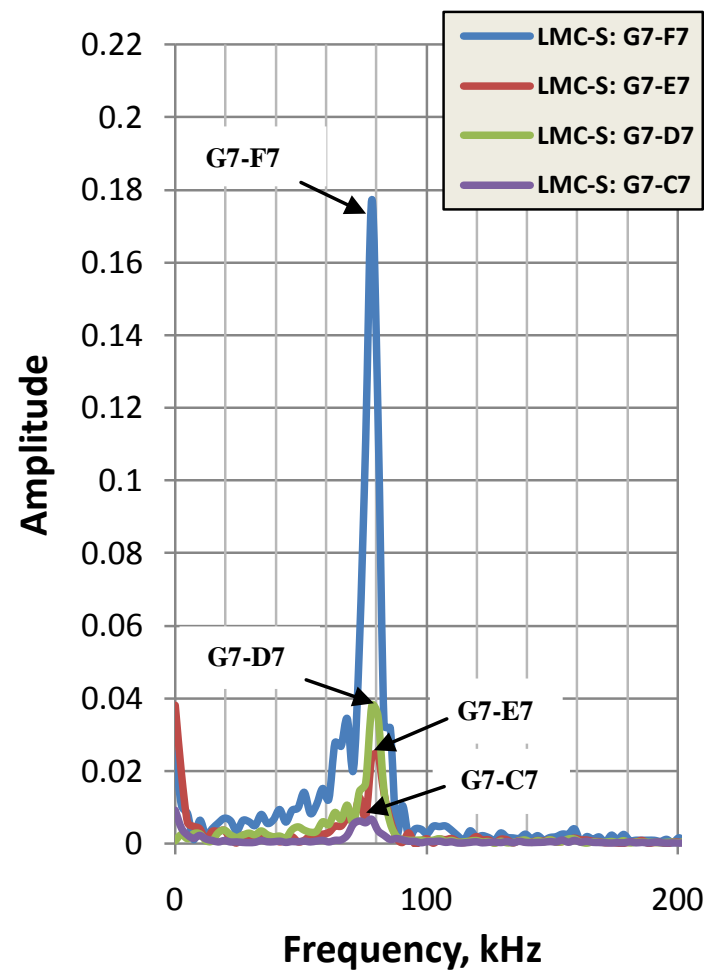

(b) 


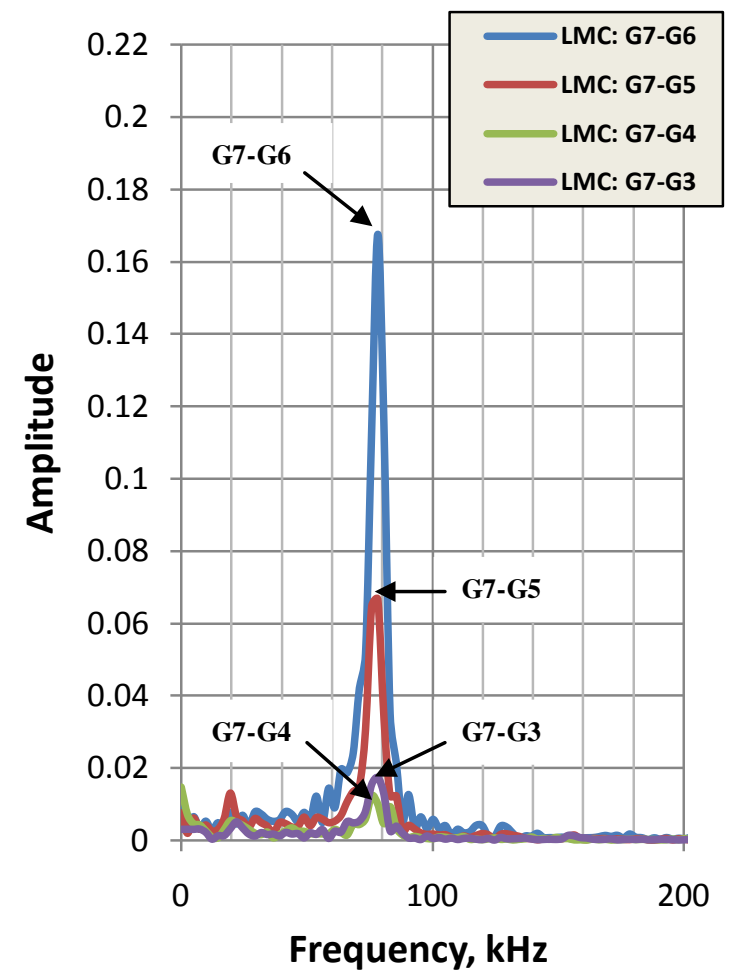

(c)

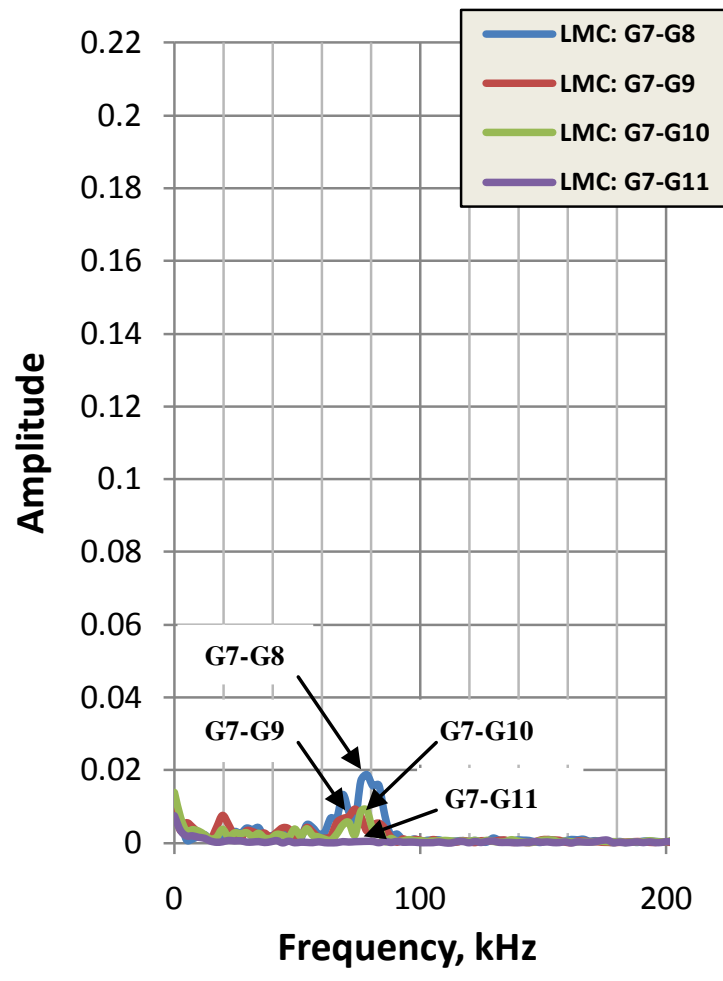

(e)

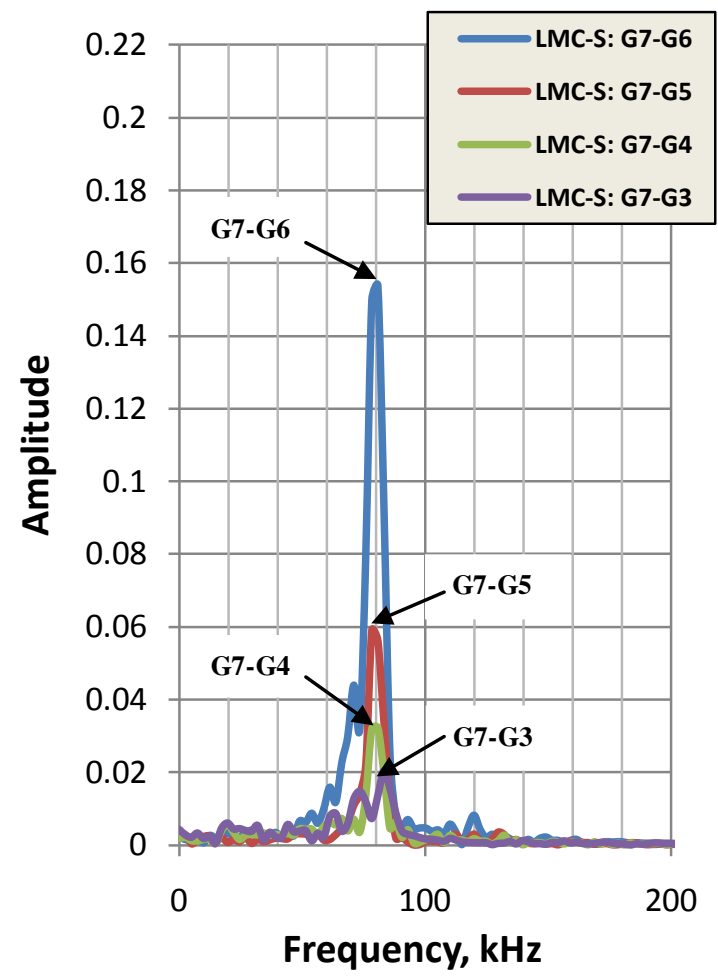

(d)

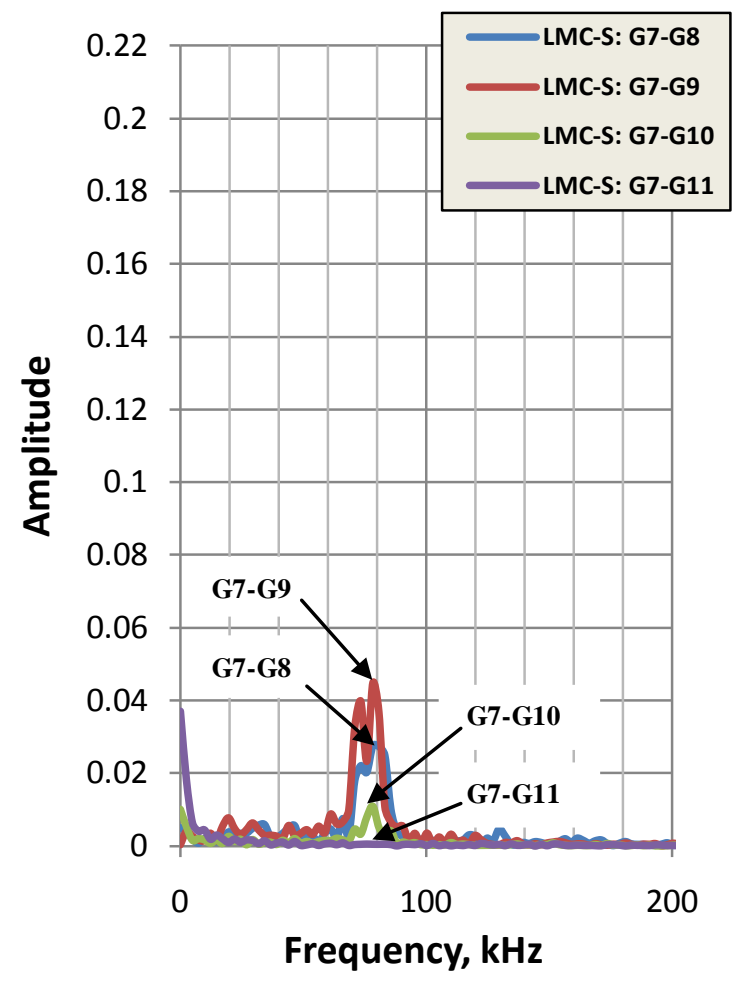

(f) 


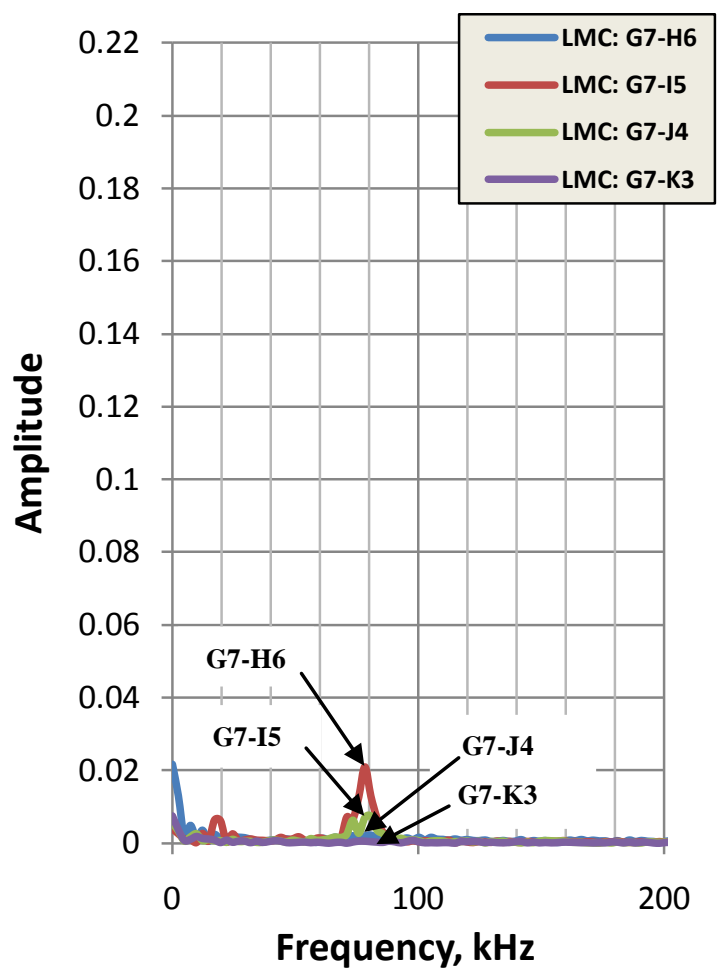

(g)

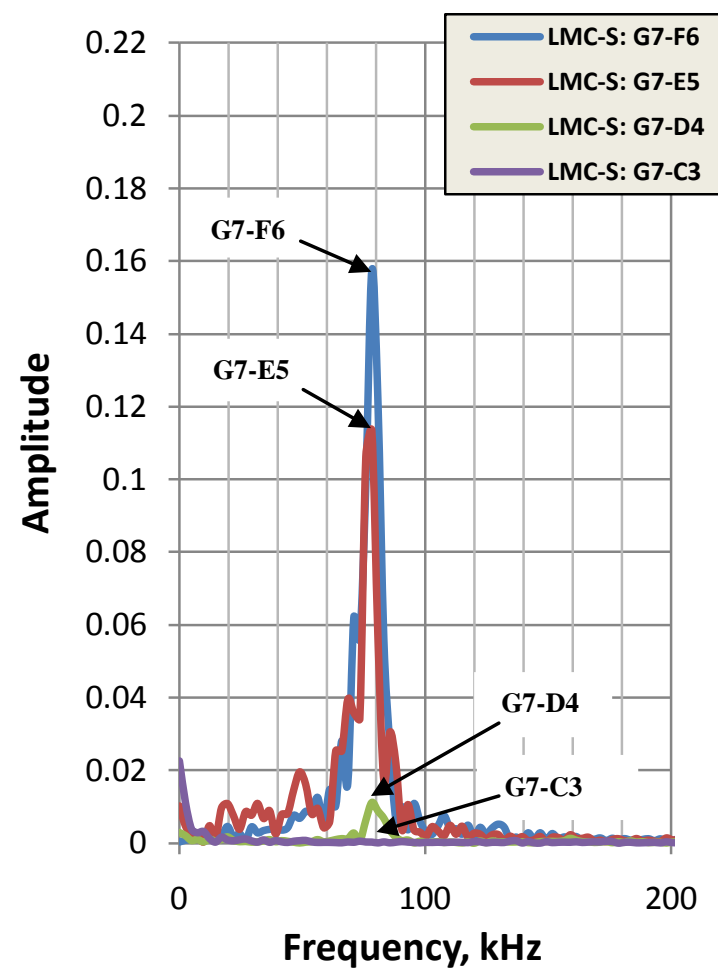

(h) 


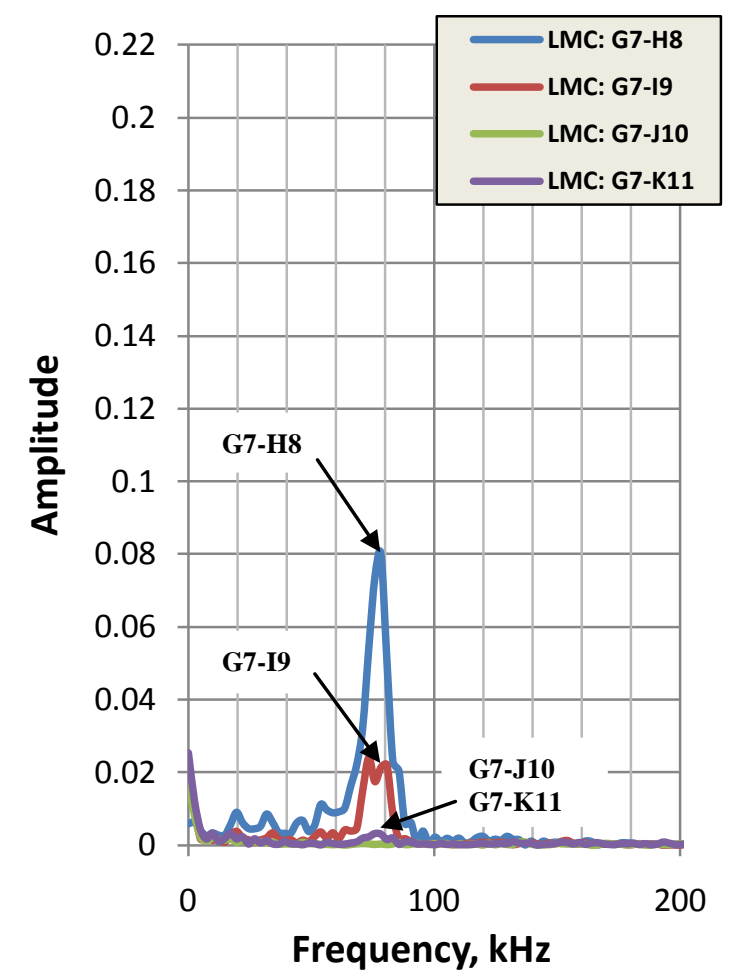

(i)

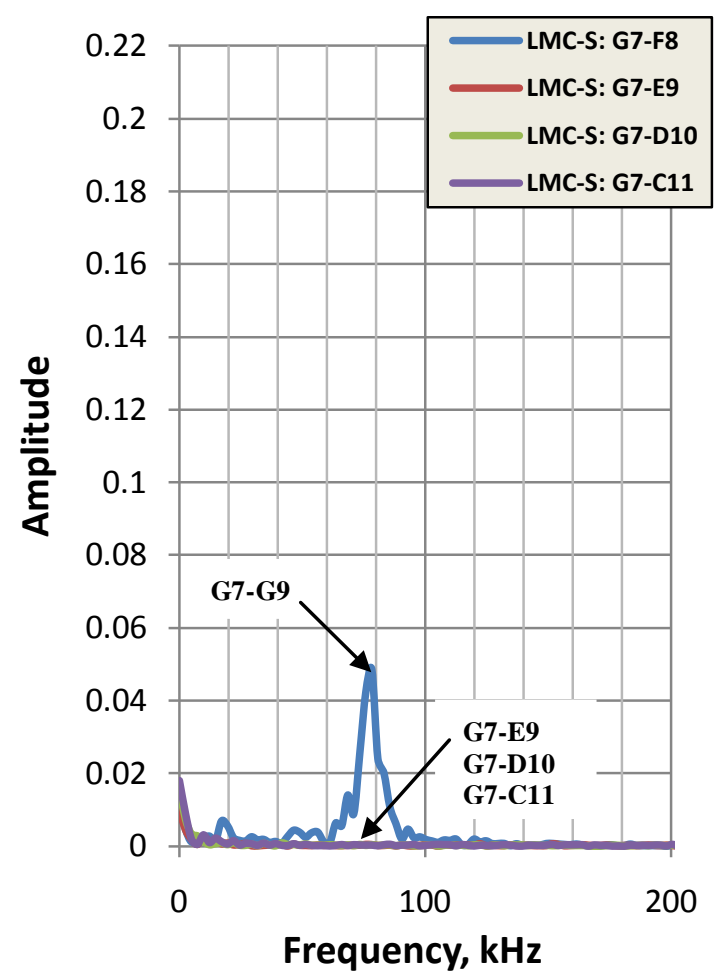

(j)

Figure 5.31: Frequency spectrum plots of LMC and LMC-S bi-layer deck slabs (Stage-I)

Figure 5.31(a) and Figure 5.31(b) shows the FFT plots of the time-domain waveform when the location of the receiving transducer is changed from $\mathrm{H} 7$ to $\mathrm{K} 7$ on slab with LMC overlay and from F7 to C7 on slab with LMC-S overlay. On comparing these figures, we can see that the peak values of the signals at the locations H7, I7, J7, and K7 on slab with LMC overlay were $0.2,0.1,0.03$, and 0.01 respectively whereas the peak values at the locations F7, E7, D7, and C7 on the slab with LMC-S overlay were $0.18,0.025,0.04,0.01$ respectively. In the case of slab with LMC-S overlay the peak value at the third location (0.04) was greater than that at second location $(0.025)$ on the same slab and also greater than that at third location (0.03) of the slab with LMC overlay, which means that less delamination is observed in case of slab with LMC-S overlay.

On comparing Figure 5.31(c), Figure 5.31(d), Figure 5.31(e), and Figure 5.31(f), we can observe that the peak values at all the locations were more for slab with LMC-S 
overlay when compared to that of slab with LMC overlay. Also, we can see that the high peak values were observed when the receiving transducer is moved towards the side with pre-notch unlike that observed when the receiving transducer is moved towards the side without pre-notch. This unexpected phenomenon is observed because the embedded thermocouple was on the path of the signal. Similar results can be observed when comparing the Figure 5.31(g), Figure 5.31(h), Figure 5.31(i), and Figure 5.31(j). Higher peak value was also observed in the case of slab with LMC-S overlay when the receiving transducer was moved in diagonal direction towards the pre-notch as seen from Figure 5.31(g) and Figure 5.31(h).

From the above discussion, we can conclude that the use of bonding slurry at the interface reduced the delamination and increased the homogeneity of the bi-layer deck slab, which is also observed in the case from UPV plots. 


\section{CHAPTER 6: STAGE-II: TEST RESULTS AND DISCUSSIONS}

This chapter presents the test results and discussions for Stage-II experiments on small scale and large scale specimens. The experiments on small scale specimens include compressive strength at different ages, dynamic modulus of elasticity at different ages, and slant shear testing at different ages on $100 \mathrm{~mm}$ (4 in.) by $200 \mathrm{~mm}$ (8 in.) cylindrical samples. The experiments on large scale prototype deck slabs include direct tensile bond tests using pull-off tests, differential length change monitoring and temperature monitoring of substrate and LMC, SFMC overlays, vertical corner lift due to curling effect at the pre-notched corners, and indirect UPV testing followed by signal processing to study the delamination at the interface on two $1829 \mathrm{~mm}$ (6 ft.) by $2438 \mathrm{~mm}(8 \mathrm{ft}$.) slabs. On both the slabs respective bonding slurry was applied at the interface because the Stage-I results showed that the performance was better when the bonding slurry was applied at the interface. The results are presented in tables and figures.

\subsection{Tests Conducted on Specimens}

The compressive strength and dynamic modulus of elasticity tests were carried out on $100 \mathrm{~mm}$ (4 in.) by $200 \mathrm{~mm}$ (8 in.) cylindrical specimens at different ages. Three replicate specimens of each substrate Type K concrete, LMC overlay, and SFMC overlays were cast and tested at each age. The average of compressive strength and average dynamic modulus of elasticity was calculated and produced. The compressive strength testing was carried on the same specimens that were used for measuring the dynamic modulus of elasticity.

\subsubsection{Compressive Strength}

The compressive strength of substrate Type K concrete was measured at 3, 7, 14, and 28 days after substrate pour and 7, 14, 28, 56 and 90 days after overlay pour whereas, the compressive strength of LMC and SFMC overlays was measured at 7, 14, 28, 56 and 90 days respectively for quality control purposes and to monitor the strength development 
with time. The compressive strength was measured by following the standard ASTM C39, (Compressive Strength of Cylindrical Concrete) specifications.

The average compressive strength values of 3 specimens at each age were calculated and plotted as a bar graph to compare the compressive strengths of Type $\mathrm{K}$ normal concrete, LMC overlay, and SFMC overlay. The compressive strength values for Type K substrate, LMC overlay, and SFMC overlay concretes are shown in Figure 6.1.

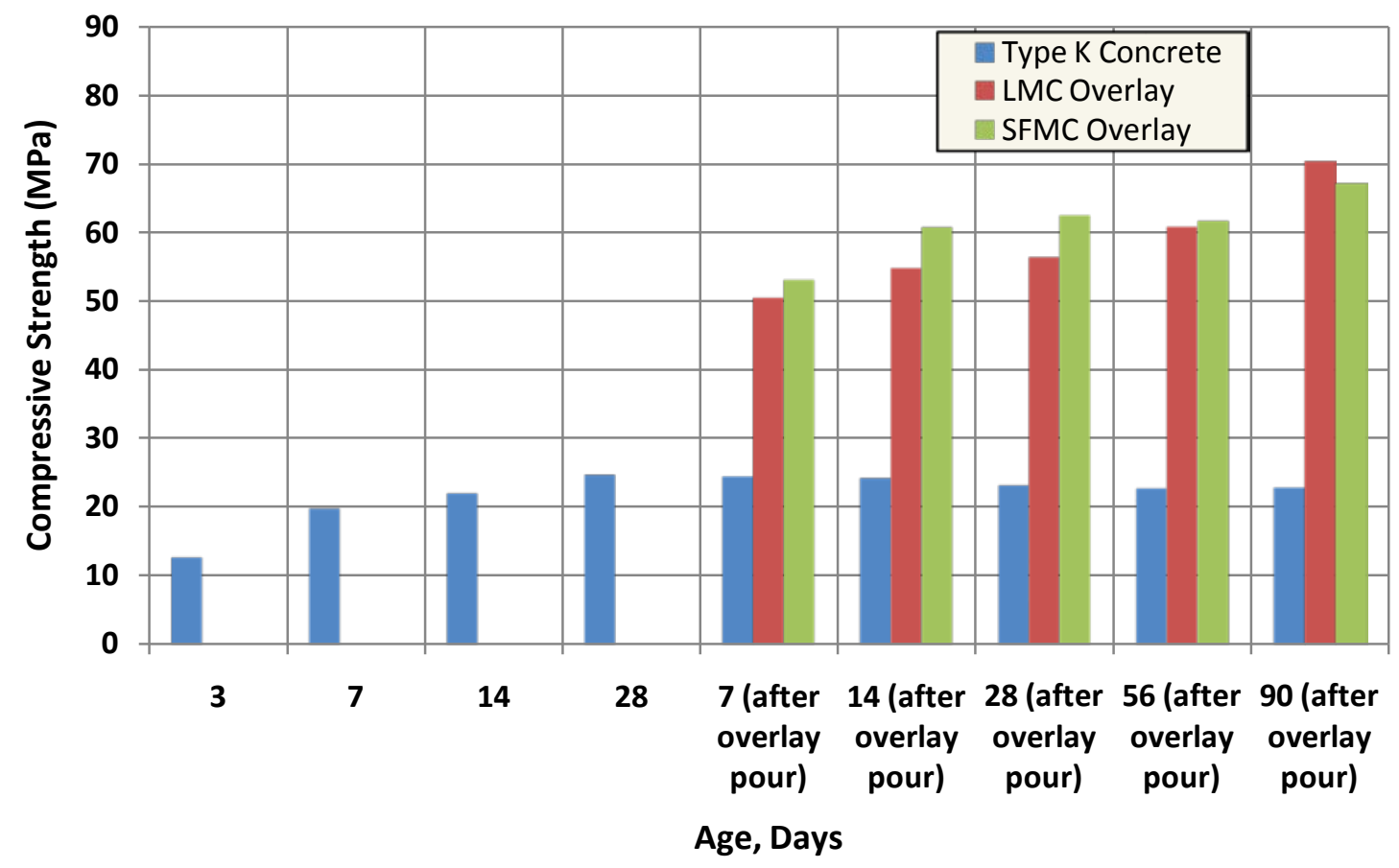

Figure 6.1: Compressive strength data (Stage-II)

Figure 6.1 indicates that the compressive strength of both the LMC and SFMC overlays was greater than that of substrate type K concrete. The compressive strength of all the concretes increased with age. The compressive strength of SFMC overlay was always greater than that of LMC overlay and Type K substrate concretes. LMC showed the highest compressive strength compared to that of Type K substrate concrete.

The compressive strength of SFMC overlay was 52.9 MPa (7673 psi), $61 \mathrm{MPa}$ (8845 psi), $62 \mathrm{MPa}$ (8992 psi), $62 \mathrm{MPa}$ (8992 psi), and $67 \mathrm{MPa}$ (9718 psi) at 7, 14, 28, 56 , and 90 days respectively. SFMC overlay mixture satisfied the minimum 28 day 
compressive strength criteria mentioned by WVDOH, which is $28 \mathrm{MPa}$ (4000 psi). Fitch and Abdulshafi (1998) reported the 28 day compressive strength of SFMC range from 52.57 MPa (8200 psi) to $78.41 \mathrm{MPa}$ (11380 psi). These values indicate the high compressive strengths of SFMC overlay compared to LMC overlay and Substrate Type K concrete.

Average compressive strength of LMC overlay at 7, 14, 28, 56, and 90 days was 50.6 MPa (7330 psi), 54.7 MPa (7930 psi), 56.3 MPa (8168 psi), 60.8 MPa (8800 psi), and 70.3 MPa (10000 psi) respectively. LMC overlay mixture satisfied the minimum 28 day compressive strength criteria mentioned by WVDOH, which is $28 \mathrm{MPa}$ (4000 psi). Luo (2002) reported the 28 day compressive strength of LMC as $48 \mathrm{MPa}$ (6950 psi). Sprinkel (2005) reported the 28 day average compressive strength of LMC with Type K cement as 38.7 MPa (5620 psi).

Rectangular hyperbolic fits of compressive strength was developed, using OriginLab v 7.5, based on the compressive strength results and are shown in Figure 6.2(a), Figure 6.2(b), and Figure 6.2(c). The general form of the equation used for generating the rectangular hyperbolic fit is:

$$
Y=\frac{a b x}{1+b x}
$$

Where:

$Y=$ compressive strength at any age, $\mathrm{MPa}$;

$x=$ age in days; and

$a$ and $b$ are constants.

$a$ and $b$ are the constants generated by OriginLab using the best fit equation shown above. Table 6.1 shows the corresponding parameters for Type $K$ substrate concrete, LMC overlay, and SFMC overlay. 
Table 6.1: Parameters for rectangular hyperbolic fit

\begin{tabular}{|c|c|c|c|}
\hline & $\begin{array}{c}\text { Type K Normal } \\
\text { Concrete }\end{array}$ & LMC & SFMC \\
\hline $\mathbf{a}$ & $24.408 \pm 0.589$ & $66.262 \pm 3.256$ & $66.286 \pm 1.412$ \\
\hline $\mathbf{b}$ & $0.455 \pm 0.0878$ & $0.385 \pm 0.147$ & $0.600 \pm 0.137$ \\
\hline $\mathbf{R}$ & 0.988 & 0.989 & 0.998 \\
\hline
\end{tabular}

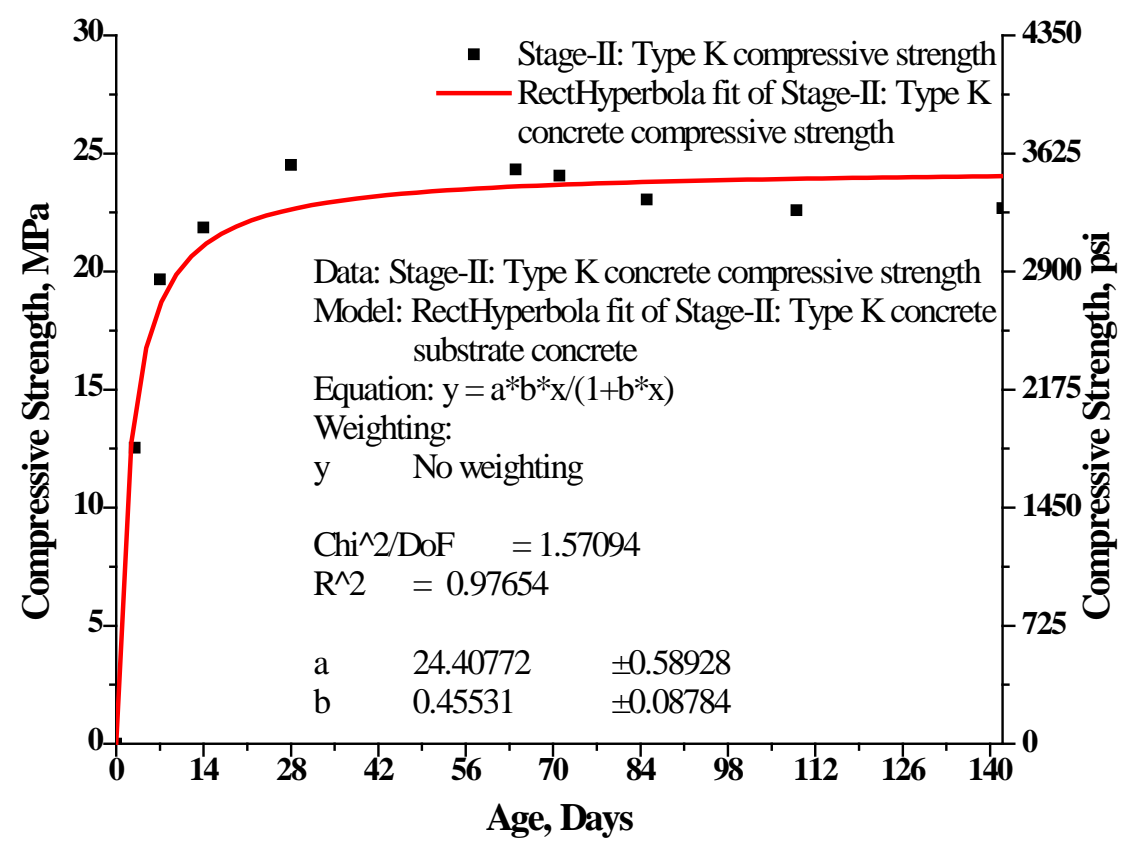

(a) 


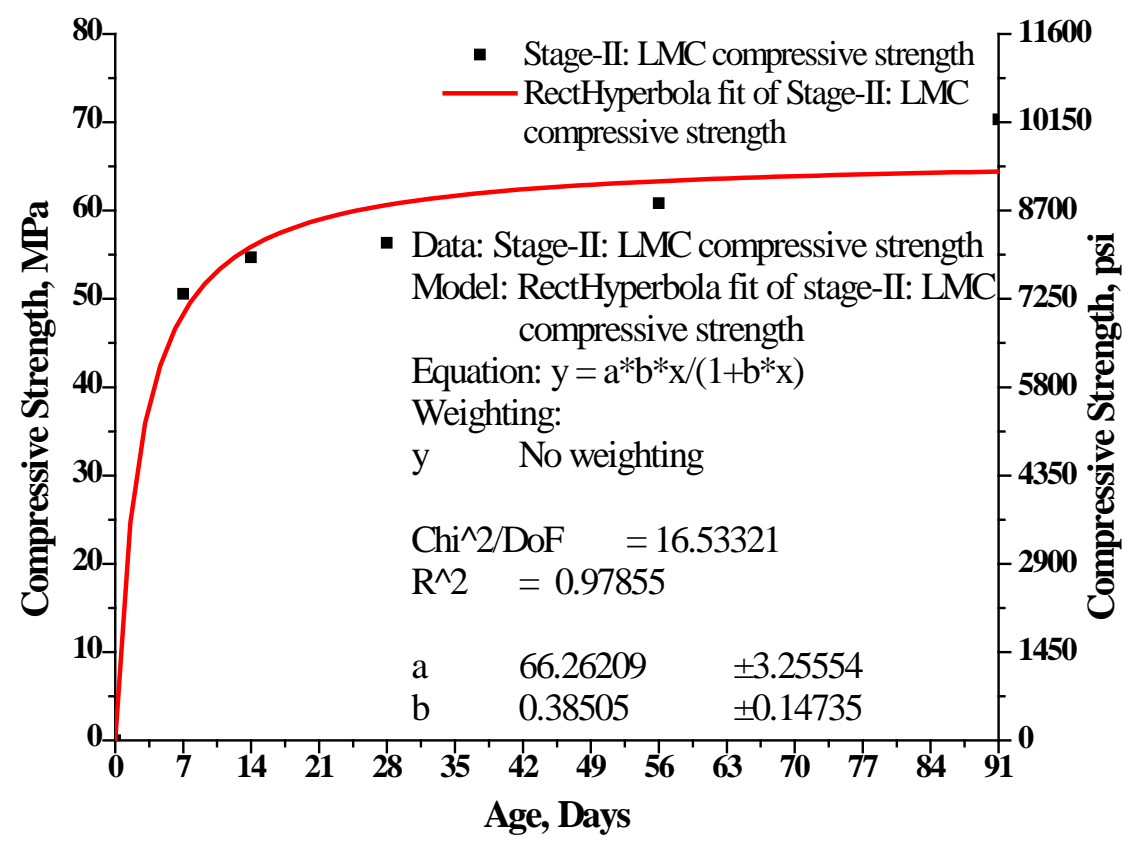

(b)

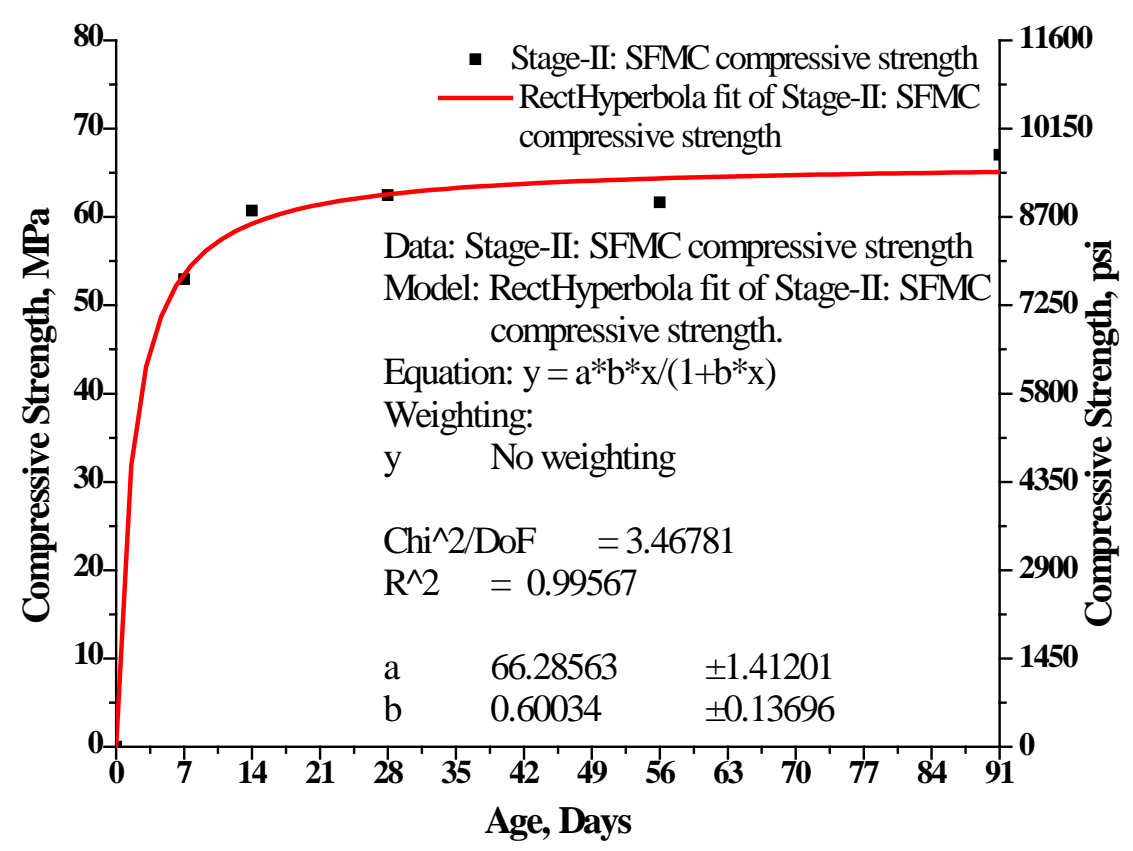

(c)

Figure 6.2: Rectangular hyperbolic fit of: (a) Type K concrete compressive strength; (b) LMC compressive strength; and SFMC compressive strength (Stage-II) 


\subsubsection{Dynamic Modulus of Elasticity}

The dynamic modulus of elasticity of Type K substrate concrete was measured at 14 and 28 days after substrate pour and 7, 14, 28, 56, and 90 days respectively after overlay pour whereas, the dynamic modulus of elasticity of LMC and SFMC overlay was measured at 7, 14, 28, 56, and 90 days respectively after overlay pour, following the guidelines of ASTM C215 (2002a) (Standard Test Method for Fundamental Transverse, Longitudinal, and Torsional Resonant Frequencies of Concrete Specimens). The test procedure followed is explained in detail in Section 4.2.

The dynamic modulus of elasticity was calculated by using the following equation:

$$
\text { Dynamic } E=D M\left(n^{\prime}\right)^{2}
$$

Where:

$$
\begin{aligned}
& \mathrm{M}=\text { Measured mass of the specimen, } \mathrm{kg} \\
& n^{\prime}=\text { fundamental longitudinal frequency, } \mathrm{Hz} \\
& \mathrm{D}=5.093\left(\mathrm{~L} / \mathrm{d}^{2}\right), \mathrm{N} \cdot \mathrm{s}^{2} /\left(\mathrm{kg} \cdot \mathrm{m}^{2}\right) \text { for a cylinder specimen, } \\
& \mathrm{L}=\text { Length of the cylinder, } \mathrm{m} \\
& \mathrm{d}=\text { diameter of the cylinder, } \mathrm{m}
\end{aligned}
$$




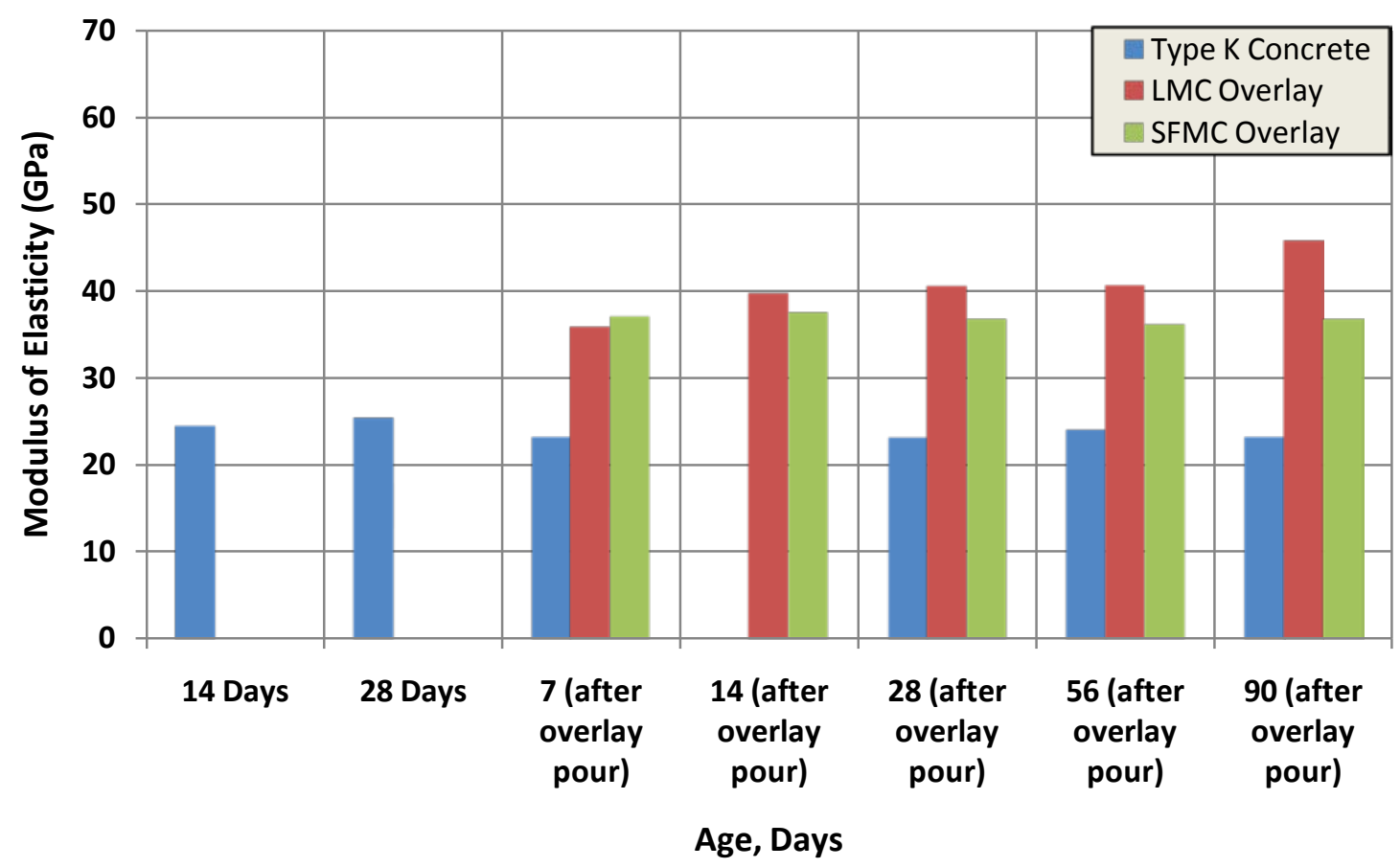

Figure 6.3: Dynamic modulus of elasticity data (Stage-II).

Figure 6.3 shows the bar diagram of average dynamic modulus of elasticity calculated for Type K substrate concrete, LMC overlay, and SFMC overlay. It is observed that the dynamic modulus of elasticity value increased steadily with time. The value for LMC overlay and SFMC overlay is always more than the value for Type $\mathrm{K}$ substrate concrete.

\subsubsection{Slant Shear Testing}

The bond strength at the interface was measured in the laboratory scale by fabricating the bi-layer cylindrical specimens with interface at the $30^{\circ}$ angle. After the molds were fabricated the compressive load was applied in the standard compression machine until the failure of the specimens. This test method was conducted by following the standard specification ASTM C 1042 (1999) (Standard Test Method for Bond Strength of Latex Systems Used with Concrete by Slant Shear). The construction procedure and testing procedures followed for conducting this testing are explained in detail in section 4.3. 


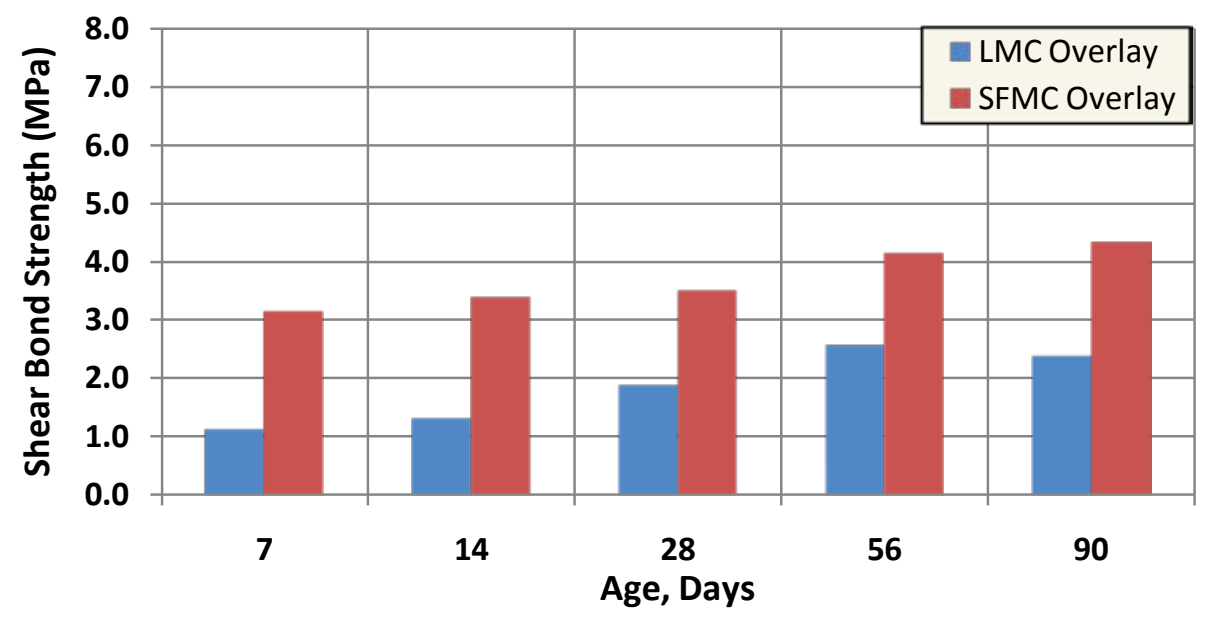

Figure 6.4: Slant shear strength data (Stage-II).

Figure 6.4 shows the bar diagram plot of the bond strength values measured by slant shear test method. We can see that the SFMC overlays showed the higher bond strength values compared to that of LMC overlay but, the bond strength value of SFMC overlay does not purely indicate the interface bond strength because in the case of SFMC overlay most of the specimens failed in mixed mode that is the top half of the specimens was initially failed in compression and then the failure plane was propagated through the interface. Modifications should be made to this test method in order to evaluate the slant shear strength more accurately. This test may be used as quality control test for field match cured specimens.

\subsection{Tests Conducted on Large-Scale/Prototype Slab}

In this section, the results of the experiments conducted on large scale prototype deck slabs of Stage-II are discussed. The experiments include direct tensile bond tests using pull-off tests, differential length change and temperature monitoring of substrate concrete, LMC overlay, and SFMC overlay, vertical corner lift due to curling effect at the pre-notched corners, and indirect UPV testing followed by signal processing to study the interface condition on two 1829 mm (6 ft.) by 2438 mm (8 ft.) bi-layer deck slabs. LMC overlay was used on slab 1 and the SFMC overlay was applied on slab 2. Bonding slurry was used at the interface for both the slabs. LMC bonding slurry was used at the interface 
on slab with LMC overlay and SFMC slurry was used at the interface on slab with SFMC overlay.

\subsubsection{Field Pull-Off Testing}

The Pull-Off testing was carried out at 7, 14, 28, 49, 56, and 90 days on $1829 \mathrm{~mm}$ (6 ft.) by $2438 \mathrm{~mm}$ (8 ft.) slabs to measure the bond strength at the interface. The pull-off testing was carried out by following the ASTM C1593 (2004) (Standard Test Method for Tensile Strength of Concrete Surfaces and the Bond Strength or Tensile Strength of Concrete Repair and Overlay Materials by Direct Tension (Pull-off Method)) standard specifications. The slabs were divide into two zones namely center zone (intact zone) and edge zone (delaminated zone) and three specimens from the center zone and seven specimens from the edge zone were cored and measured for tensile bond strength at each age. The mode of failure at the interface was visually observed and reported for each specimen. The results are presented in tables and figures to compare the performance between two different types of bi-layer deck slabs: one slab with LMC overlay with LMC bonding slurry at the interface whereas the other slab with SFMC overlay with SFMC bonding slurry at the interface.

Similar to the Stage-I study; the mode of failure at the interface was visually observed and reported for each specimen. The results are presented in tables and figures to compare the performance between two different types of bi-layer deck slabs: one slab with LMC overlay with LMC bonding slurry at the interface and the other slab with the SFMC overlay with SFMC bonding slurry at the interface. The locations at which the testing was conducted at 7, 14, 28, 49, 56, and 90 days shown from Figure 6.5 to Figure 6.10.respectively. Simultaneously, below the figures, bond/tensile strength and the mode of failure are shown in tables for the respective slabs. Values are presented in tables from Table 6.2 through Table 6.7 for 7, 14, 28, 49, 56, and 90 days respectively for both the slabs. In these tables the interface failure means that more than $70 \%$ of the interface was visible after the failure samples were visually examined. 


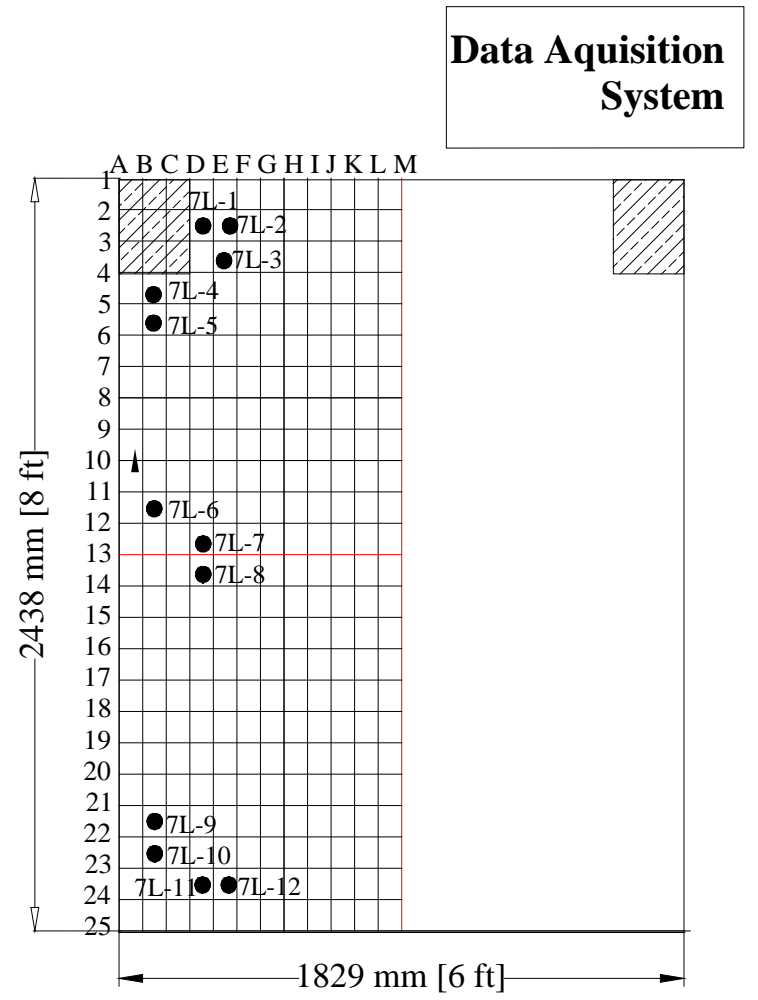

SLAB 1: LMC

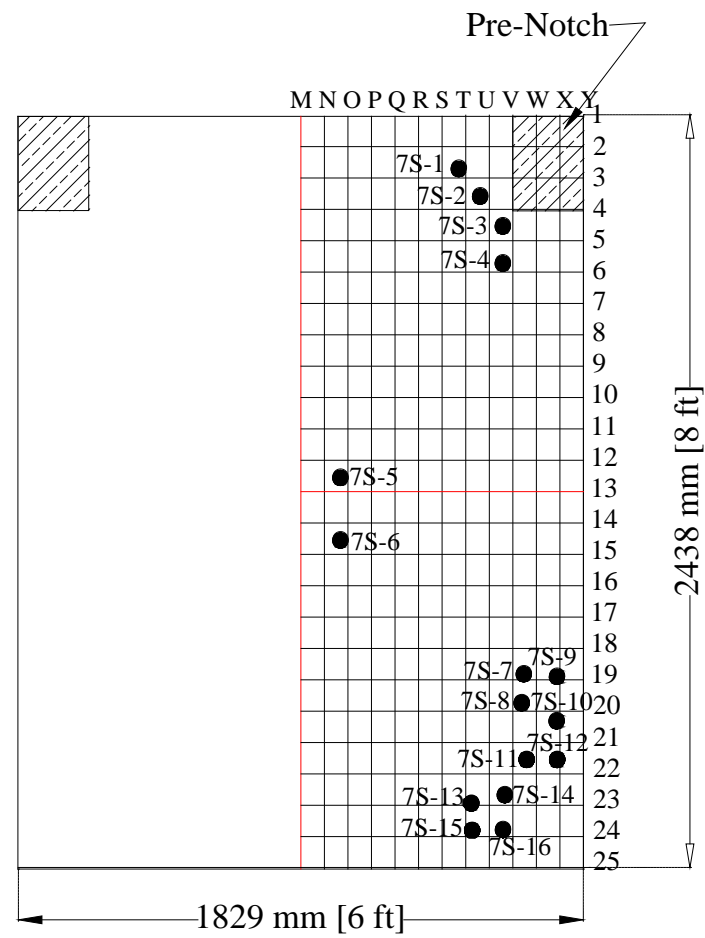

SLAB 2: SFMC

Figure 6.5: 7 days pull-off locations on LMC and SFMC Slabs of Stage-II I

Table 6.2: 7 days pull-off data of LMC and SFMC slabs in Stage-II

\begin{tabular}{|c|c|c|c|c|c|c|c|}
\hline \multicolumn{4}{|c|}{ Slab 1-LMC } & \multicolumn{4}{|c|}{ Slab 2-SFMC } \\
\hline Zone & $\begin{array}{c}\text { Core } \\
\text { No. }\end{array}$ & $\begin{array}{c}\text { Bond/Tensile } \\
\text { strength } \\
\text { (MPa) }\end{array}$ & Mode of failure & Zone & $\begin{array}{c}\text { Core } \\
\text { No. }\end{array}$ & $\begin{array}{c}\text { Bond/Tensile } \\
\text { strength } \\
\text { (MPa) }\end{array}$ & Mode of failure \\
\hline \multirow{9}{*}{ Edge } & 7L-1 & 0.00 & failed while coring & \multirow{8}{*}{ Edge } & 7S-1 & 0.80 & interface failure \\
\hline & 7L-2 & 0.92 & substrate failure & & $7 \mathrm{~S}-2$ & 0.50 & interface failure \\
\hline & 7L-3 & 1.17 & substrate failure & & $7 \mathrm{~S}-3$ & 0.94 & interface failure \\
\hline & 7L-4 & 0.79 & interface failure & & $7 \mathrm{~S}-4$ & 1.36 & interface failure \\
\hline & 7L-5 & 1.67 & epoxy bond failure & & $7 \mathrm{~S}-7$ & 0.92 & interface failure \\
\hline & 7L-9 & 1.67 & substrate failure & & $7 \mathrm{~S}-8$ & 1.27 & interface failure \\
\hline & 7L-10 & 1.34 & interface failure & & $7 S-9$ & 0.00 & failed while coring \\
\hline & 7L-11 & 0.97 & epoxy bond failure & & $7 \mathrm{~S}-10$ & 0.00 & failed while coring \\
\hline & 7L-12 & 1.39 & interface failure & \multirow{2}{*}{ Center } & 7S-5 & 1.02 & epoxy bond failure \\
\hline \multirow{3}{*}{ Center } & 7L-6 & 2.01 & substrate failure & & 7S-6 & 1.46 & interface failure \\
\hline & 7L-7 & 1.64 & substrate failure & & & & \\
\hline & 7L-8 & 1.24 & substrate failure & & & & \\
\hline
\end{tabular}




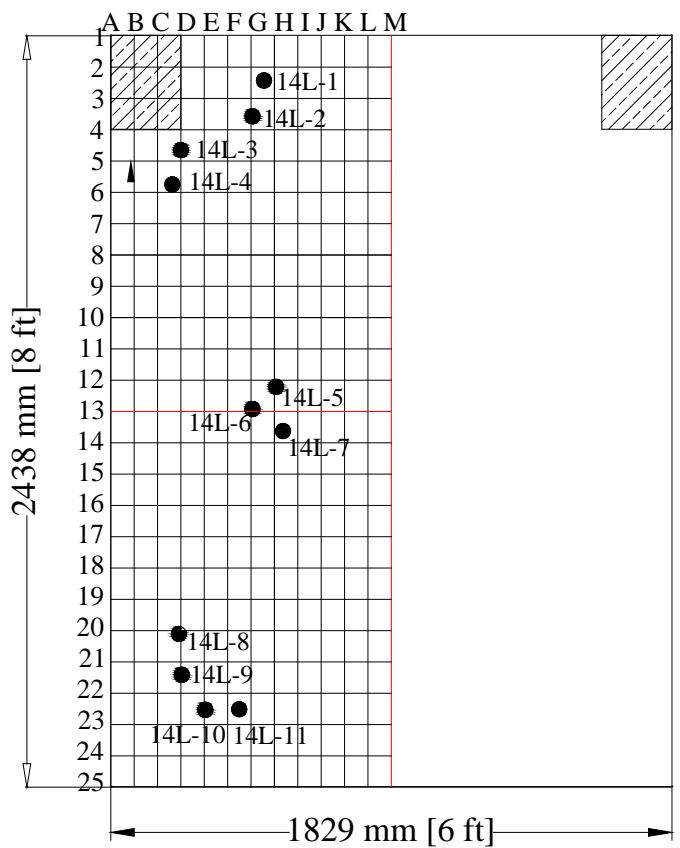

1 SLAB 1: LMC

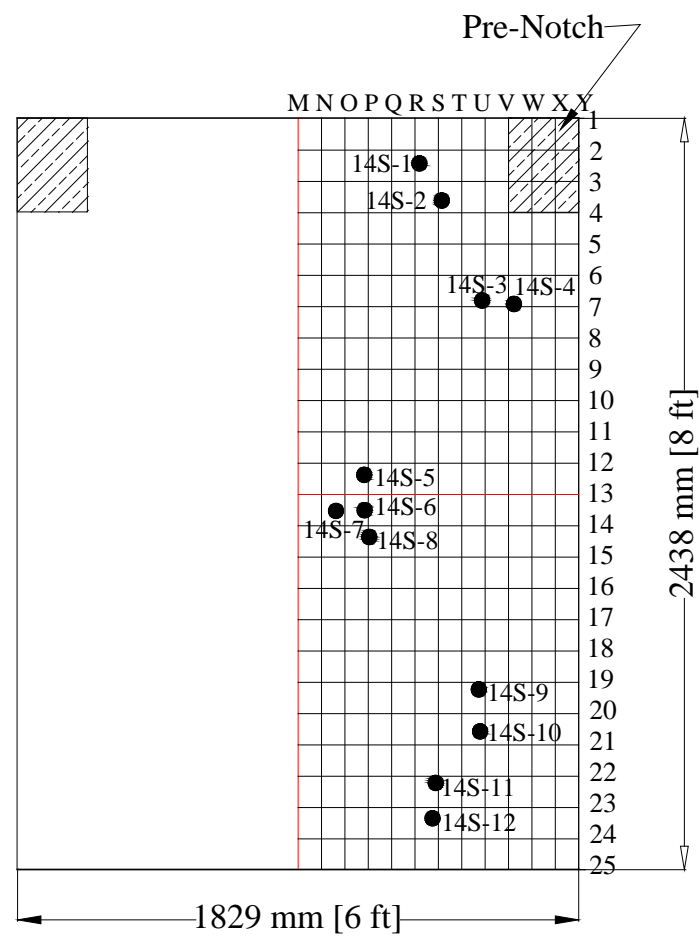

SLAB 2: SFMC

1

Figure 6.6: 14 days pull-off locations on LMC and SFMC Slabs of Stage-II

Table 6.3: 14 days pull-off data of LMC and SFMC slabs in Stage-II

\begin{tabular}{|c|c|c|c|c|c|c|c|}
\hline \multicolumn{4}{|c|}{ Slab 1-LMC } & \multicolumn{4}{|c|}{ Slab 2-SFMC } \\
\hline Zone & $\begin{array}{l}\text { Core } \\
\text { No. }\end{array}$ & $\begin{array}{c}\text { Bond/Tensile } \\
\text { strength } \\
\text { (MPa) }\end{array}$ & Mode of failure & Zone & $\begin{array}{c}\text { Core } \\
\text { No. }\end{array}$ & $\begin{array}{c}\text { Bond/Tensile } \\
\text { strength } \\
\text { (MPa) }\end{array}$ & Mode of failure \\
\hline \multirow{8}{*}{ Edge } & 14L-1 & 1.30 & substrate failure & \multirow{8}{*}{ Edge } & 14S-1 & 0.00 & interface failure \\
\hline & 14L-2 & 1.17 & interface failure & & $14 S-2$ & 0.14 & interface failure \\
\hline & $14 \mathrm{~L}-3$ & 1.17 & interface failure & & $14 S-3$ & 0.64 & interface failure \\
\hline & 14L-4 & 0.38 & interface failure & & $14 S-4$ & 0.28 & interface failure \\
\hline & 14L-8 & 1.26 & interface failure & & $14 S-9$ & 0.67 & interface failure \\
\hline & 14L-9 & 1.76 & interface failure & & $14 \mathrm{~S}-10$ & 0.70 & interface failure \\
\hline & 14L-10 & 1.20 & interface failure & & $14 \mathrm{~S}-11$ & 0.69 & interface failure \\
\hline & 14L-11 & 1.27 & interface failure & & $14 \mathrm{~S}-12$ & 0.47 & interface failure \\
\hline \multirow{3}{*}{ Center } & 14L-5 & 1.29 & interface failure & \multirow{4}{*}{ Center } & $14 S-5$ & 2.41 & overlay failure \\
\hline & 14L-6 & 1.19 & interface failure & & 14S-6 & 2.74 & overlay failure \\
\hline & 14L-7 & 1.20 & interface failure & & 14S-7 & 2.51 & overlay failure \\
\hline & & & & & 14S-8 & 3.82 & overlay failure \\
\hline
\end{tabular}



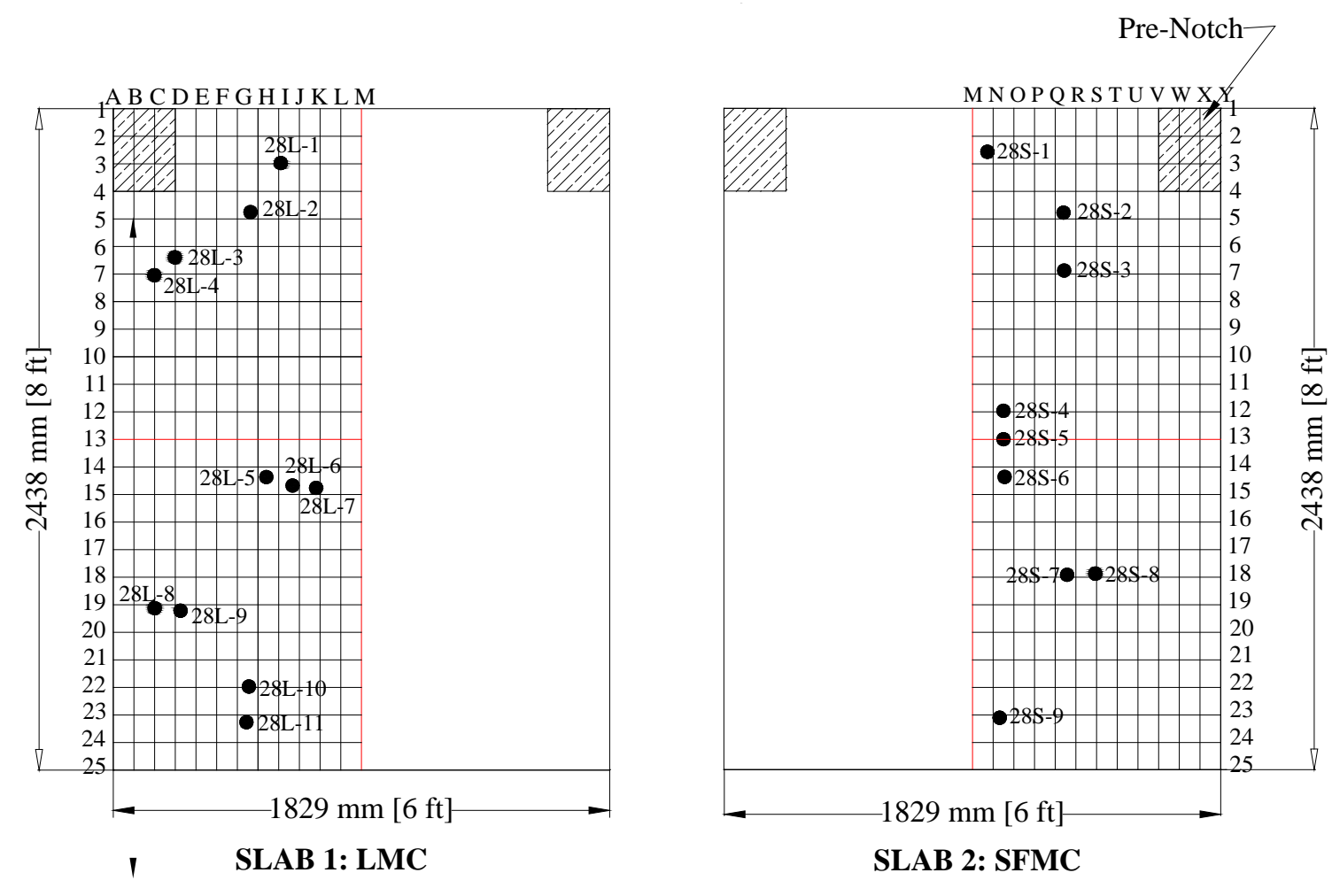

1

Figure 6.7: 28 days pull-off locations on LMC and SFMC Slabs of Stage-II

Table 6.4: 28 days pull-off data of LMC and SFMC slabs in Stage-II

\begin{tabular}{|c|c|c|c|c|c|c|c|}
\hline \multicolumn{4}{|c|}{ Slab 1-LMC } & \multicolumn{4}{|c|}{ Slab 2-SFMC } \\
\hline Zone & $\begin{array}{c}\text { Core } \\
\text { No. }\end{array}$ & $\begin{array}{c}\text { Bond/Tensile } \\
\text { strength } \\
\text { (MPa) }\end{array}$ & Mode of failure & Zone & $\begin{array}{c}\text { Core } \\
\text { No. }\end{array}$ & $\begin{array}{c}\text { Bond/Tensile } \\
\text { strength } \\
\text { (MPa) }\end{array}$ & Mode of failure \\
\hline \multirow{8}{*}{ Edge } & $28 \mathrm{~L}-1$ & 1.09 & interface failure & \multirow{6}{*}{ Edge } & $28 \mathrm{~S}-1$ & 1.07 & substrate failure \\
\hline & $28 \mathrm{~L}-2$ & 1.64 & substrate failure & & $28 S-2$ & 1.59 & interface failure \\
\hline & $28 \mathrm{~L}-3$ & 2.24 & interface failure & & $28 S-3$ & 1.39 & substrate failure \\
\hline & $28 \mathrm{~L}-4$ & 1.92 & interface failure & & $28 \mathrm{~S}-7$ & 0.15 & interface failure \\
\hline & $28 \mathrm{~L}-8$ & 1.67 & substrate failure & & $28 S-8$ & 0.72 & substrate failure \\
\hline & $28 \mathrm{~L}-9$ & 0.99 & interface failure & & $28 \mathrm{~S}-9$ & 0.79 & interface failure \\
\hline & 28L-10 & 1.12 & interface failure & \multirow{3}{*}{ Center } & $28 S-4$ & 1.39 & interface failure \\
\hline & 28L-11 & 1.46 & substrate failure & & $28 S-5$ & 1.86 & substrate failure \\
\hline \multirow{3}{*}{ Center } & 28L-5 & 1.00 & interface failure & & $28 S-6$ & 1.57 & substrate failure \\
\hline & 28L-6 & 0.87 & interface failure & & & & \\
\hline & 28L-7 & 1.27 & interface failure & & & & \\
\hline
\end{tabular}



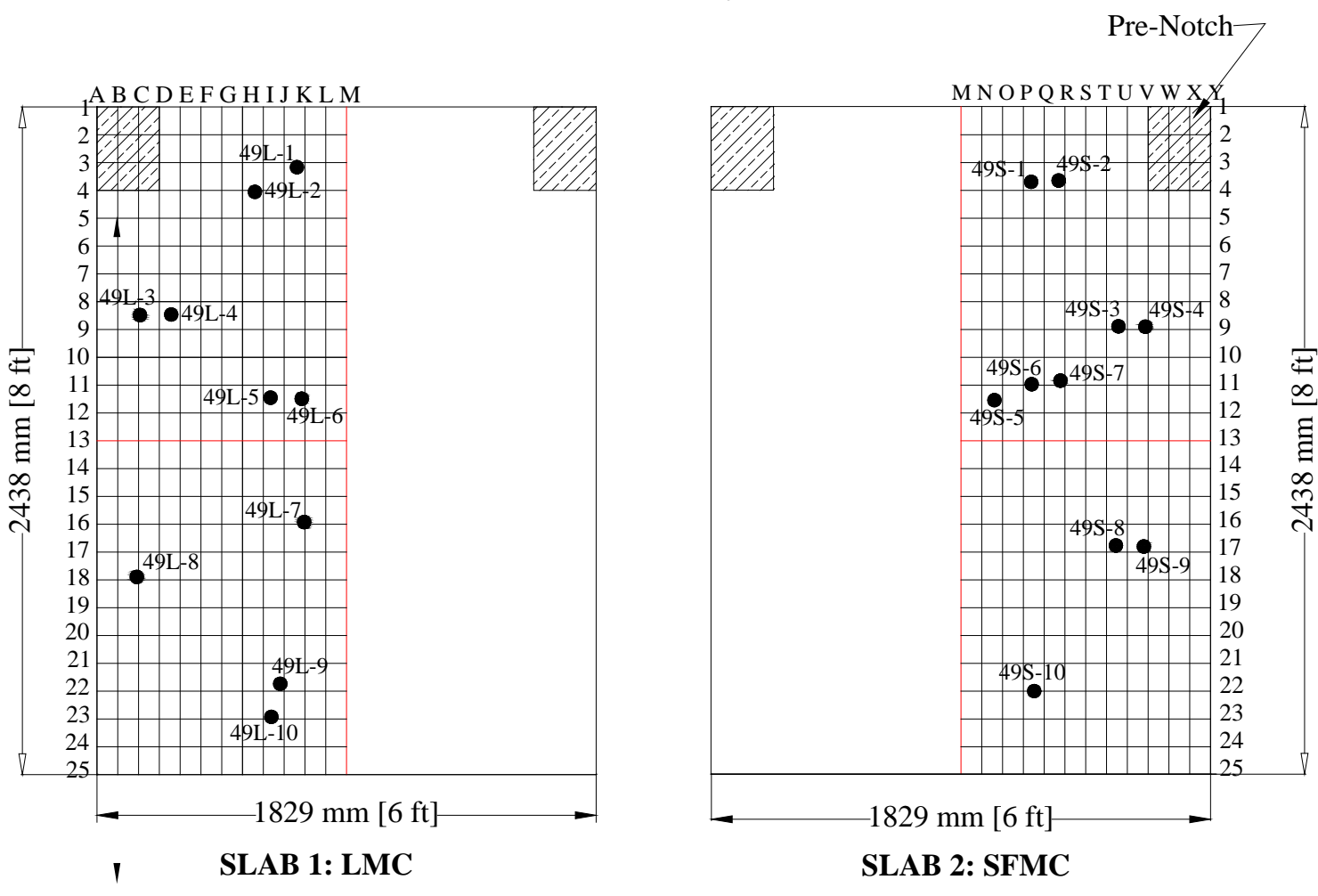

SLAB 2: SFMC

Figure 6.8: 49 days pull-off locations on LMC and SFMC Slabs of Stage-II

Table 6.5: 49 days pull-off data of LMC and SFMC slabs in Stage-II

\begin{tabular}{|c|c|c|c|c|c|c|c|}
\hline \multicolumn{4}{|c|}{ Slab 1-LMC } & \multicolumn{4}{|c|}{ Slab 2-SFMC } \\
\hline Zone & $\begin{array}{c}\text { Core } \\
\text { No. }\end{array}$ & $\begin{array}{c}\text { Bond/Tensile } \\
\text { strength } \\
\text { (MPa) }\end{array}$ & Mode of failure & Zone & $\begin{array}{c}\text { Core } \\
\text { No. }\end{array}$ & $\begin{array}{c}\text { Bond/Tensile } \\
\text { strength } \\
\text { (MPa) }\end{array}$ & Mode of failure \\
\hline \multirow{7}{*}{ Edge } & 49L-1 & 0.22 & interface failure & \multirow{7}{*}{ Edge } & 49S-1 & 1.14 & interface failure \\
\hline & 49L-2 & 2.11 & substrate failure & & 49S-2 & 0.57 & interface failure \\
\hline & $49 \mathrm{~L}-3$ & 1.32 & substrate failure & & $49 S-3$ & 2.01 & interface failure \\
\hline & 49L-4 & 1.00 & interface failure & & $49 S-4$ & 0.13 & interface failure \\
\hline & $49 \mathrm{~L}-8$ & 1.56 & substrate failure & & 49S-8 & 0.64 & interface failure \\
\hline & 49L-9 & 1.79 & interface failure & & $49 S-9$ & 0.20 & interface failure \\
\hline & 49L-10 & 1.89 & interface failure & & 49S-10 & 0.47 & interface failure \\
\hline \multirow{3}{*}{ Center } & 49L-5 & 1.81 & interface failure & \multirow{3}{*}{ Center } & 49S-5 & 1.52 & interface failure \\
\hline & 49L-6 & 1.33 & interface failure & & 49S-6 & 1.24 & interface failure \\
\hline & 49L-7 & 1.05 & interface failure & & 49S-7 & 0.35 & interface failure \\
\hline
\end{tabular}



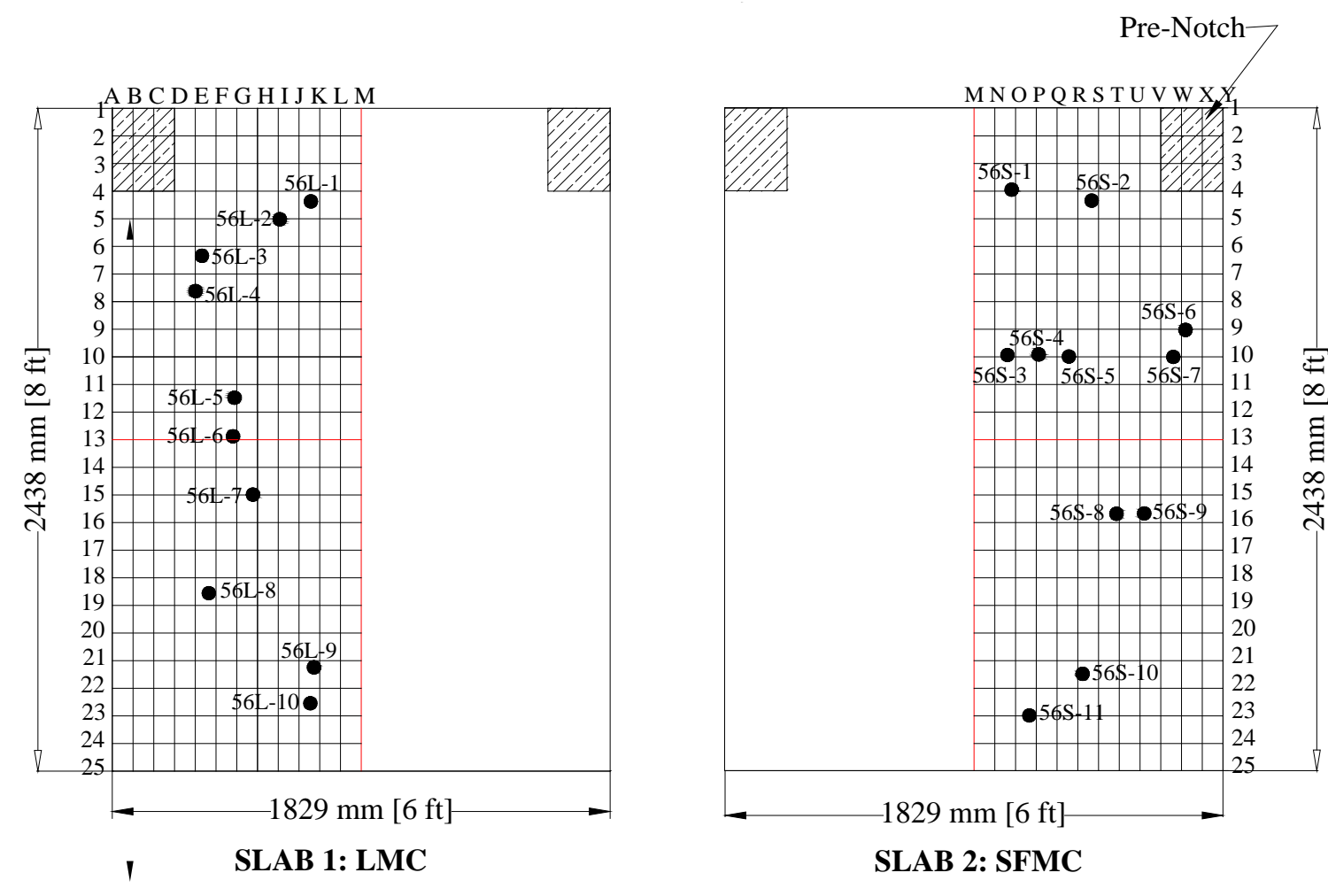

SLAB 2: SFMC

1

Figure 6.9: 56 days pull-off locations on LMC and SFMC Slabs of Stage-II

Table 6.6: 56 days pull-off data of LMC and SFMC slabs in Stage-II

\begin{tabular}{|c|c|c|c|c|c|c|c|}
\hline \multicolumn{4}{|c|}{ Slab 1-LMC } & \multicolumn{4}{|c|}{ Slab 2-SFMC } \\
\hline Zone & $\begin{array}{l}\text { Core } \\
\text { No. }\end{array}$ & $\begin{array}{l}\text { Bond/Tensile } \\
\text { strength } \\
\text { (MPa) }\end{array}$ & Mode of failure & Zone & $\begin{array}{l}\text { Core } \\
\text { No. }\end{array}$ & $\begin{array}{l}\text { Bond/Tensile } \\
\text { strength } \\
\text { (MPa) }\end{array}$ & Mode of failure \\
\hline \multirow{7}{*}{ Edge } & $56 \mathrm{~L}-1$ & 1.26 & substrate failure & \multirow{8}{*}{ Edge } & 56S-1 & 1.29 & substrate failure \\
\hline & $56 \mathrm{~L}-2$ & 1.04 & epoxy bond failure & & $56 \mathrm{~S}-2$ & 0.38 & interface failure \\
\hline & $56 \mathrm{~L}-3$ & 1.56 & interface failure & & $56 \mathrm{~S}-6$ & 0.30 & interface failure \\
\hline & $56 \mathrm{~L}-4$ & 0.64 & interface failure & & $56 \mathrm{~S}-7$ & 0.17 & interface failure \\
\hline & $56 \mathrm{~L}-8$ & 0.65 & interface failure & & $56 \mathrm{~S}-8$ & 0.24 & interface failure \\
\hline & 56L-9 & 0.82 & interface failure & & $56 \mathrm{~S}-9$ & 0.17 & interface failure \\
\hline & 56L-10 & 1.00 & interface failure & & $56 \mathrm{~S}-10$ & 1.11 & interface failure \\
\hline \multirow{3}{*}{ Center } & 56L-5 & 0.84 & interface failure & & $56 \mathrm{~S}-11$ & 1.61 & substrate failure \\
\hline & 56L-6 & 0.77 & interface failure & \multirow{3}{*}{ Center } & $56 S-3$ & 1.02 & interface failure \\
\hline & $56 \mathrm{~L}-7$ & 1.19 & interface failure & & $56 S-4$ & 0.87 & substrate failure \\
\hline & & & & & 56S-5 & 1.24 & substrate failure \\
\hline
\end{tabular}



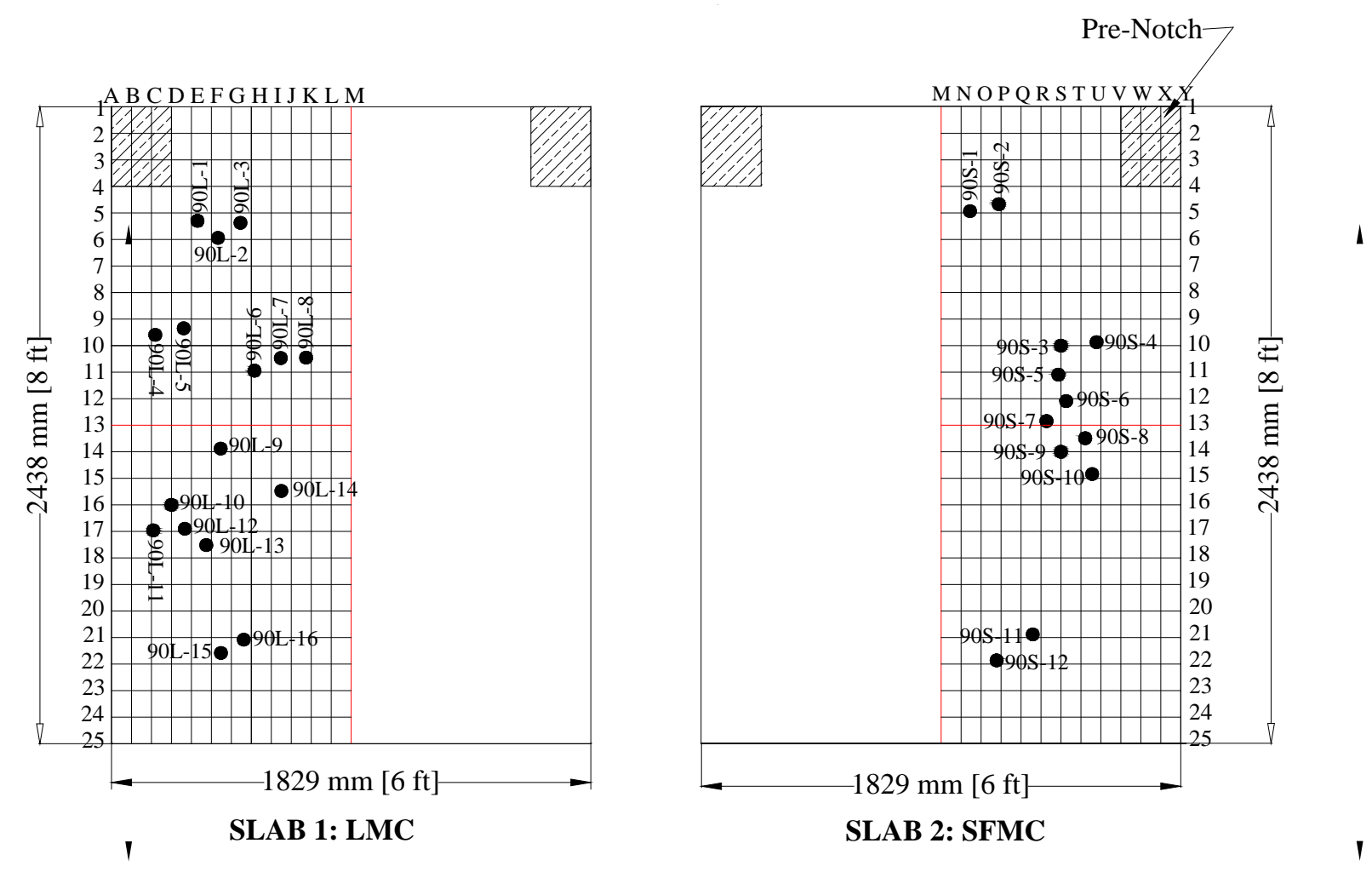

Figure 6.10: 90 days pull-off locations on LMC and SFMC Slabs of Stage-II

Table 6.7: 90 days pull-off data of LMC and SFMC slabs in Stage-II

\begin{tabular}{|c|c|c|c|c|c|c|c|}
\hline \multicolumn{4}{|c|}{ Slab 1-LMC } & \multicolumn{4}{|c|}{ Slab 2-SFMC } \\
\hline Zone & $\begin{array}{l}\text { Core } \\
\text { No. }\end{array}$ & $\begin{array}{c}\text { Bond/Tensile } \\
\text { strength } \\
\text { (MPa) }\end{array}$ & Mode of failure & Zone & $\begin{array}{l}\text { Core } \\
\text { No. }\end{array}$ & $\begin{array}{c}\text { Bond/Tensile } \\
\text { strength } \\
\text { (MPa) }\end{array}$ & Mode of failure \\
\hline \multirow{12}{*}{ Edge } & 90L-1 & 1.84 & substrate failure & \multirow{9}{*}{ Edge } & 90S-1 & 0.97 & interface failure \\
\hline & $90 \mathrm{~L}-2$ & 1.44 & substrate failure & & $90 \mathrm{~S}-2$ & 1.67 & substrate failure \\
\hline & 90L-3 & 1.26 & interface failure & & $90 \mathrm{~S}-3$ & 0.08 & interface failure \\
\hline & 90L-4 & 2.36 & substrate failure & & 90S-4 & 0.00 & failed while coring \\
\hline & 90L-5 & 2.31 & substrate failure & & 90S-5 & 0.00 & failed while coring \\
\hline & $90 \mathrm{~L}-10$ & 0.00 & failed while coring & & 90S-9 & 0.38 & interface failure \\
\hline & 90L-11 & 1.86 & interface failure & & $90 \mathrm{~S}-10$ & 0.55 & interface failure \\
\hline & 90L-12 & 0.00 & failed while coring & & 90S-11 & 0.69 & interface failure \\
\hline & 90L-13 & 0.65 & interface failure & & $90 \mathrm{~S}-12$ & 0.30 & interface failure \\
\hline & 90L-14 & 1.26 & interface failure & \multirow{3}{*}{ Center } & 90S-6 & 0.84 & interface failure \\
\hline & 90L-15 & 1.09 & interface failure & & 90S-7 & 1.17 & interface failure \\
\hline & 90L-16 & 0.77 & interface failure & & 90S-8 & 0.57 & interface failure \\
\hline \multirow{4}{*}{ Center } & 90L-6 & 1.67 & epoxy bond failure & & & & \\
\hline & 90L-7 & 1.40 & substrate failure & & & & \\
\hline & 90L-8 & 0.80 & interface failure & & & & \\
\hline & 90L-9 & 1.14 & interface failure & & & & \\
\hline
\end{tabular}


Table 6.8and Table 6.9 summarizes the number of cores tested, number of cores failed at the interface, average failure stress at the interface, number of substrate failures, and the average failure stress of the substrate are presented for center zone and edge zone of slab with LMC overlay and slab with LMC-S overlay. The failure stress due to coring, epoxy failure, and overlay failure which are not desirable are not considered and hence are not included in these tables. The failure of specimen in substrate was considered here since, Warner et al. (1998) suggested that failure through the substrate concrete is always desirable, because it is an indicator that the overlay material is stronger than the substrate.

Table 6.8: Pull off data for LMC bi-layer deck slab in Stage-II

\begin{tabular}{|c|c|c|c|c|c|c|c|c|c|c|c|c|c|}
\hline & & \multicolumn{12}{|c|}{ Stage-II: Pull-off tests results } \\
\hline & & \multicolumn{12}{|c|}{ Slab 1: LMC } \\
\hline & & \multicolumn{2}{|c|}{7 days } & \multicolumn{2}{|c|}{14 days } & \multicolumn{2}{|c|}{28 days } & \multicolumn{2}{|c|}{49 days } & \multicolumn{2}{|c|}{56 days } & \multicolumn{2}{|c|}{90 days } \\
\hline & & $\begin{array}{l}\text { Center } \\
\text { Zone }\end{array}$ & $\begin{array}{l}\text { Edge } \\
\text { Zone }\end{array}$ & $\begin{array}{l}\text { Center } \\
\text { Zone }\end{array}$ & $\begin{array}{l}\text { Edge } \\
\text { Zone }\end{array}$ & $\begin{array}{l}\text { Center } \\
\text { Zone }\end{array}$ & $\begin{array}{l}\text { Edge } \\
\text { Zone }\end{array}$ & $\begin{array}{l}\text { Center } \\
\text { Zone }\end{array}$ & $\begin{array}{l}\text { Edge } \\
\text { Zone }\end{array}$ & $\begin{array}{l}\text { Center } \\
\text { Zone }\end{array}$ & $\begin{array}{l}\text { Edge } \\
\text { Zone }\end{array}$ & $\begin{array}{l}\text { Center } \\
\text { Zone }\end{array}$ & $\begin{array}{l}\text { Edge } \\
\text { Zone }\end{array}$ \\
\hline \multicolumn{2}{|c|}{ Number of Cores } & 3 & 6 & 3 & 7 & 3 & 8 & 3 & 6 & 3 & 6 & 3 & 10 \\
\hline \multicolumn{2}{|c|}{ Average Failure Stress (Mpa) } & 1.63 & 1.21 & 1.23 & 1.31 & 1.05 & 1.52 & 1.40 & 1.61 & 0.93 & 0.99 & 1.12 & 1.48 \\
\hline \multirow{2}{*}{$\begin{array}{l}\text { Interface } \\
\text { failure }\end{array}$} & $\begin{array}{l}\text { Number of } \\
\text { Interface Failures }\end{array}$ & - & 3 & 3 & 6 & 3 & 5 & 3 & 3 & 3 & 5 & 2 & 6 \\
\hline & $\begin{array}{l}\text { Average Failure } \\
\text { Stress (MPa) }\end{array}$ & - & 1.17 & 1.23 & 1.31 & 1.05 & 1.47 & 1.40 & 1.56 & 0.93 & 0.93 & 0.97 & 1.15 \\
\hline \multirow{2}{*}{$\begin{array}{l}\text { Substrate } \\
\text { failure }\end{array}$} & $\begin{array}{l}\text { Number of } \\
\text { Substrate Failures }\end{array}$ & 3 & 3 & - & 1 & - & 3 & - & 3 & - & 1 & 1 & 4 \\
\hline & $\begin{array}{l}\text { Average Failure } \\
\text { Stress (MPa) }\end{array}$ & 1.63 & 1.26 & - & 1.30 & - & 1.59 & - & 1.66 & - & 1.26 & 1.40 & 1.99 \\
\hline
\end{tabular}

$1 \mathrm{MPa}=145 \mathrm{psi}$ 
Table 6.9: Pull off data for SFMC bi-layer deck slab in Stage-II

\begin{tabular}{|c|c|c|c|c|c|c|c|c|c|c|c|c|c|}
\hline & & \multicolumn{12}{|c|}{ Stage-II: Pull-off tests results } \\
\hline & & \multicolumn{12}{|c|}{ Slab 2: SFMC } \\
\hline & & \multicolumn{2}{|c|}{7 days } & \multicolumn{2}{|c|}{14 days } & \multicolumn{2}{|c|}{28 days } & \multicolumn{2}{|c|}{49 days } & \multicolumn{2}{|c|}{56 days } & \multicolumn{2}{|c|}{90 days } \\
\hline & & $\begin{array}{l}\text { Center } \\
\text { Zone }\end{array}$ & $\begin{array}{l}\text { Edge } \\
\text { Zone }\end{array}$ & $\begin{array}{l}\text { Center } \\
\text { Zone }\end{array}$ & $\begin{array}{l}\text { Edge } \\
\text { Zone }\end{array}$ & $\begin{array}{l}\text { Center } \\
\text { Zone }\end{array}$ & $\begin{array}{l}\text { Edge } \\
\text { Zone }\end{array}$ & $\begin{array}{l}\text { Center } \\
\text { Zone }\end{array}$ & $\begin{array}{l}\text { Edge } \\
\text { Zone }\end{array}$ & $\begin{array}{l}\text { Center } \\
\text { Zone }\end{array}$ & $\begin{array}{l}\text { Edge } \\
\text { Zone }\end{array}$ & $\begin{array}{l}\text { Center } \\
\text { Zone }\end{array}$ & $\begin{array}{l}\text { Edge } \\
\text { Zone }\end{array}$ \\
\hline \multicolumn{2}{|c|}{ Number of Cores } & 1 & 6 & 4 & 4 & 3 & 5 & 2 & 4 & 3 & 3 & 3 & 4 \\
\hline \multicolumn{2}{|c|}{ Average Failure Stress (Mpa) } & 1.46 & 0.97 & $2.87^{(1)}$ & 0.67 & 1.61 & 1.11 & 1.38 & 1.09 & 1.04 & 1.33 & 0.86 & 0.97 \\
\hline \multirow{2}{*}{$\begin{array}{l}\text { Interface } \\
\text { failure }\end{array}$} & $\begin{array}{l}\text { Number of } \\
\text { Interface Failures }\end{array}$ & 1 & 6 & - & 4 & 1 & 2 & 2 & 4 & 1 & 1 & 3 & 3 \\
\hline & $\begin{array}{l}\text { Average Failure } \\
\text { Stress (MPa) }\end{array}$ & 1.46 & 0.97 & - & 0.67 & 1.39 & 1.19 & 1.38 & 1.09 & 1.02 & 1.11 & 0.86 & 0.74 \\
\hline \multirow{2}{*}{$\begin{array}{l}\text { Substrate } \\
\text { failure }\end{array}$} & $\begin{array}{l}\text { Number of } \\
\text { Substrate Failures }\end{array}$ & - & - & - & - & 2 & 3 & - & - & 2 & 2 & - & 1 \\
\hline & $\begin{array}{l}\text { Average Failure } \\
\text { Stress (MPa) }\end{array}$ & - & - & - & - & 1.72 & 1.06 & - & - & 1.05 & 1.45 & - & 1.67 \\
\hline
\end{tabular}

Note: 1) Core depth was not deep enough to initiate the failure at the interface hence, all the cores failed in overlay concrete.

In Table 6.8 and Table 6.9 the number of cores tested, number of cores failed at the interface, average failure stress at the interface, number of substrate failures, and the average failure stress of the substrate were presented for center zone and edge zone of slab with LMC overlay and slab with LMC overlay with bonding slurry at the interface. Sprinkel and Ozyildirim (2000) qualified the bond strength test results as:

If bond strength values are $>=2.1 \mathrm{MPa}$ (300 psi), excellent

1.7 MPa to 2.1 MPa (250 to $299 \mathrm{psi}$ ), very good

1.4 MPa to $1.7 \mathrm{MPa}$ (200 to $249 \mathrm{psi}$ ), good

0.7 MPa to 1.4 MPa (100 to $199 \mathrm{psi}$ ), fair and

$0 \mathrm{MPa}$ to $0.7 \mathrm{MPa}$ (0 to $99 \mathrm{psi})$, poor

Also, Sprinkel (2005) and Sprinkel (2004) mentioned the lower quality limit for bond strength as $1.03 \mathrm{MPa}(150 \mathrm{psi})$ at 28 days. As we can see form our results all the interfacial bond strength values are greater than the lower quality limit of 1.03 MPa (150 psi) and all the values are close to or greater than $1.03 \mathrm{MPa}(150 \mathrm{psi})$. Hence the condition of the interface can be considered as either fair or good. 
Hence, from the above tables we can just qualitatively describe the bond condition. But general conclusions cannot be made to compare the bond strength values between the two slabs. Because, high variance in the values is observed between the individual results and simple mean cannot be calculated. Hence, three-way ANOVA was considered to conduct the in depth study and the conclusions were drawn based on threeway ANOVA. The results and discussions following the three-way ANOVA are discussed in the following section. Only the interface failure values were considered to perform three-way ANOVA.

\subsubsection{Three-way ANOVA Results}

Also, for interpreting the stage-II pull-off results, three-way ANOVA similar to the one performed for Stage-I study was selected here. The procedure for developing the three-way ANOVA table is explained in section 2.8. The effect of three factors (Age, Zone Type, and Slab Type) on the Pull-Off bond strength. SAS 9.0 was selected here to develop the three-way ANOVA. The steps involved in developing three-way ANOVA table are explained clearly in section 5.2.1.1. Procedure of developing three-way ANOVA code in SAS 9.0 and the information of factors (although same as in Stage-I) is again explained here for the clarity of the information.

In the following discussion, SAS code is presented simultaneously with the output and interpretation of the results for better understanding of the effects of selected factors on the pull-off strength.

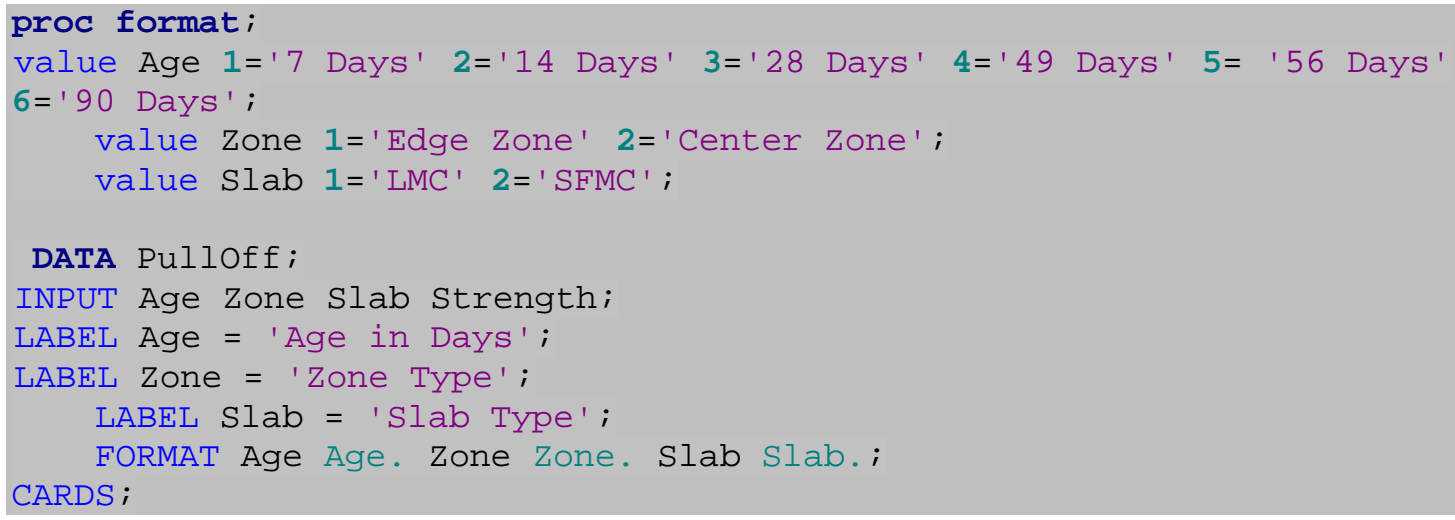




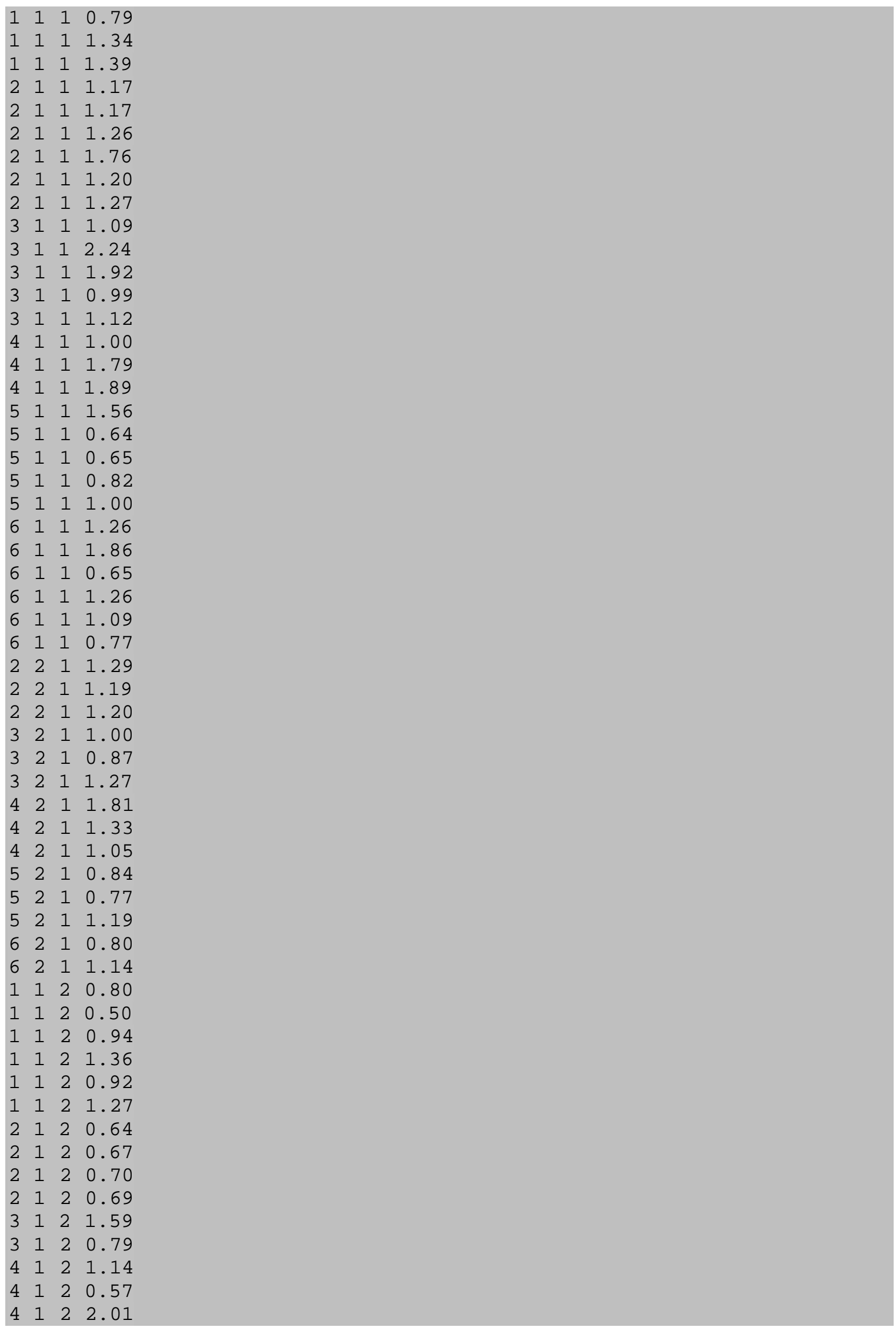


Above written code defines the factors, levels or treatments within factors, and data table. The data table is defined in such a way that column 1 coding represents the level or treatments of Age factor, column 2 coding represents the level or treatment of Zone factor, column 3 coding represents the level or treatment of Slab factor, and column 4 represents value of the pull-off strength observation for the particular case. Each row in the input data table represents the individual observation for the respective case.

The three factors selected were 1) Age, 2) Zone Type and 3) Slab Type. Age factor has 6 levels or treatments namely; 7 days, 14 days, 28 days, 49 days, 56 days and 90 days. Zone Type factor has two levels or treatments namely; Center Zone and Edge Zone. Slab factor has two levels or treatments namely; LMC (Slab with LMC overlay with bonding slurry condition at the interface) and SFMC (slab with SFMC overlay with bonding slurry condition at the interface). The independent variables were the age, zone type, and slab type factors and the dependent variable was Pull-Off strength. The summary of the class level information is shown in Table 6.10. 
Table 6.10: Class level information for Stage-II pull-off test

\begin{tabular}{|c|c|c|}
\hline Class & Levels & Values \\
\hline Age & 6 & 7 Days 14 Days 28 Days 49 Days 56 Days \\
90 Days \\
\hline Zone & 2 & Center Zone Edge Zone \\
\hline Slab & 2 & LMC SFMC \\
\hline
\end{tabular}

Code written here is used for developing the normal probability plot, residual plot, and ANOVA table. Normal Probability and residual plots are plotted for checking the model adequacy and after checking the model adequacy, three-way ANOVA was developed.

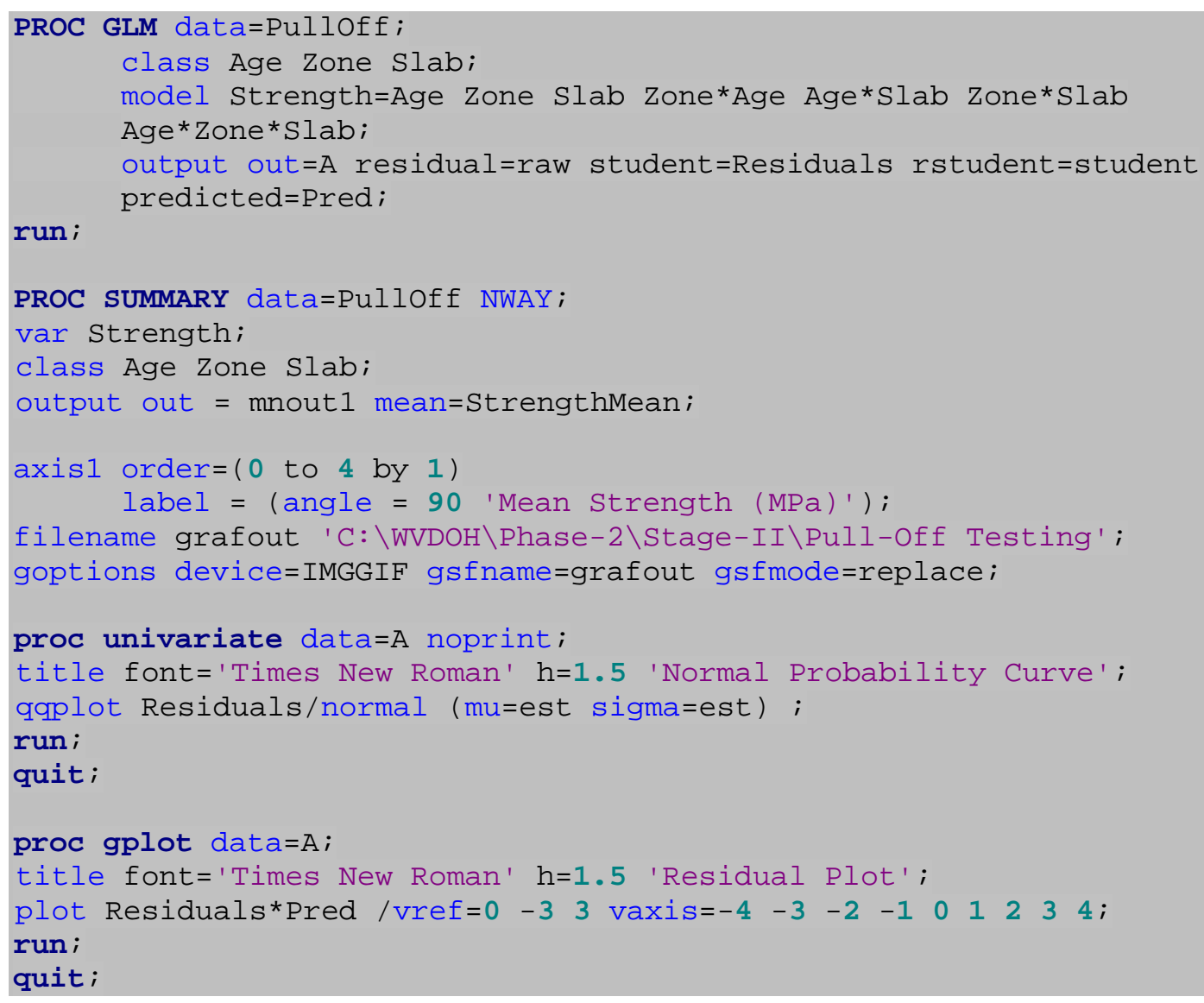

Here, normal probability plot of the residuals is constructed to validate the assumption of normality. This plot means that the underlying error distribution is normal and this plot will resemble a straight line. More emphasis should be placed on the central 
values of the plot than on the extremes. From the Figure 6.11, we can see that there is nothing unusual in the normal probability plot i.e., by examining this plot we can see that all the data is concentrated at the center and hence underlying error distribution is normal. Therefore, the assumption of normal distribution is valid.

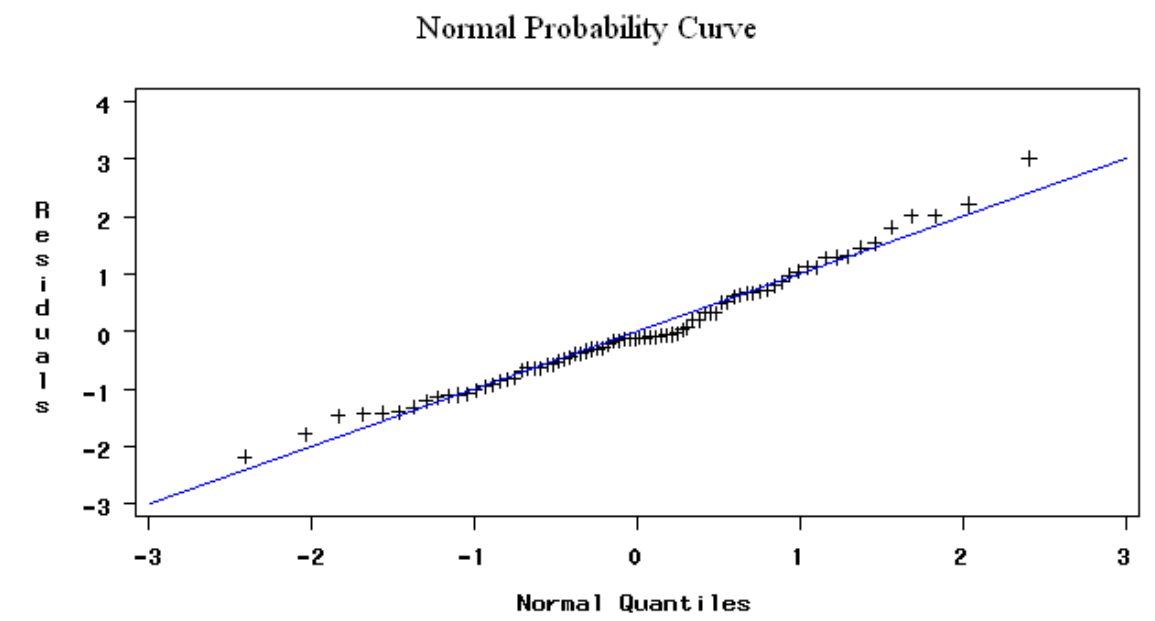

Figure 6.11: Normal probability plot of residuals for Stage-II pull-off data

Also, the assumption of uniform variance is checked by plotting the residual plot of the experimental data. The residual plot is developed by plotting the residuals versus predicted or fitted values. This plot should not show any obvious pattern i.e., the residuals should be structureless which means that the residuals should be unrelated to any other variable including the predicted value. From the Figure 6.12, we can see that the residual plot is structureless and hence the assumption, homogeneity of variances is validated. 
Residual Plot

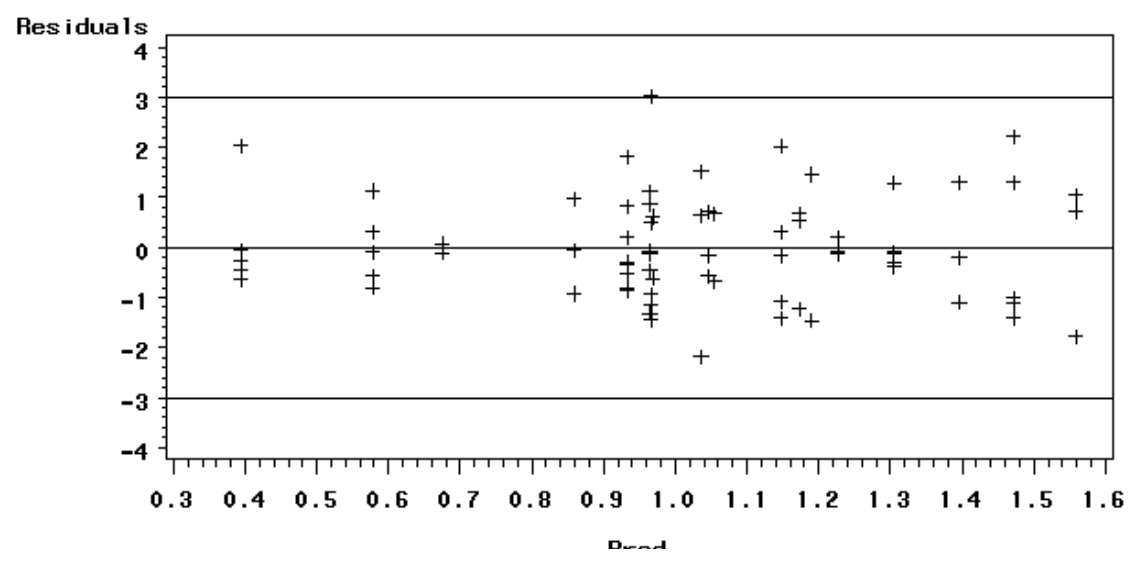

Figure 6.12: Residual plot of Stage-II pull-off data

After checking for the assumptions of normal distribution and the uniform variance, ANOVA table is developed to interpret the effects of Age, Zone type, and slab type on the pull-off bond strength. The treatments sum of squares are partitioned into 7 components namely,

1. Sum of squares of $A$, the Age effect

2. Sum of squares of B, the zone effect

3. Sum of squares of $\mathrm{C}$, the slab effect

4. Interaction between Age effect and Zone effect, A x B interaction

5. Interaction between Age effect and Slab effect, $\mathrm{A} \times \mathrm{C}$ interaction

6. Interaction between Zone effect and Slab effect, B x C interaction

7. Interaction between Age, Zone, and Slab effects, $\mathrm{A} \times \mathrm{B} \times \mathrm{C}$ interaction 
Table 6.11: Three-way ANOVA table of Stage-I pull-off data

\begin{tabular}{|c|c|c|c|c|c|}
\hline Source & DF & $\begin{array}{c}\text { Sum of } \\
\text { Squares }\end{array}$ & $\begin{array}{c}\text { Mean } \\
\text { Square }\end{array}$ & $\begin{array}{c}\mathbf{F} \\
\text { Value }\end{array}$ & Pr>F \\
\hline Age & 5 & 2.847 & 0.569 & 3.81 & 0.0047 \\
\hline Zone & 1 & 0.130 & 0.130 & 0.87 & 0.354 \\
\hline Slab & 1 & 2.529 & 2.529 & 16.93 & 0.0001 \\
\hline Age*Zone & 5 & 0.699 & 0.14 & 0.94 & 0.464 \\
\hline Age*Slab & 5 & 0.481 & 0.096 & 0.64 & 0.668 \\
\hline Zone*Slab & 1 & 0.69 & 0.69 & 4.62 & 0.036 \\
\hline Age*Zone*Slab & 3 & 0.088 & 0.0294 & 0.20 & 0.898 \\
\hline $\begin{array}{c}\text { Model (Between } \\
\text { Treatments) }\end{array}$ & 21 & 7.465 & 0.355 & 2.02 & 0.013 \\
\hline $\begin{array}{c}\text { Error (Within } \\
\text { Treatments) }\end{array}$ & 58 & 8.665 & 0.149 & & \\
\hline Corrected Total & 79 & 16.130 & & & \\
\hline
\end{tabular}

In this study, $5 \%$ level of significance $(\alpha=0.05)$ was considered and the conclusions were made based on this. The discussions and conclusions that can be made based on the Table 6.11 are as follows:

1. Clearly, the Probability value of the model test statistic $F_{o}$ is 0.013 which is less than 0.05 , hence we reject null hypothesis and accept alternate hypothesis i.e., the treatment means differ very significantly.

2. Since, the p-value of test statistic $F_{o}$ for Age effect is 0.0047 which is less than 0.05 , hence we can conclude that the six corresponding population means differ significantly. The effect of age on the pull-off strength is very significant i.e., the bond strength at the interface changed considerably with the increase in age of the slabs within the range of experiment.

3. For the Zone effect, the ANOVA showed the p-value of test statistic $F_{o}$ is 0.354 which is less than 0.05 , we conclude that the effect of zone on the bond strength is not significant. There is a no significant change in the bond strength value when the two zones were compared i.e., Zone type does not significantly affect the bond strength at the interface. 
4. Similarly, for the slab effect p-value of test statistic $F_{o}$ is 0.0001 which is less than 0.05 implies that the two population means differ significantly. The effect of slab on the bond strength was very significant, which implies that the bond strength for the two different types of slabs is significantly different.

Further in depth interpretations can be made by considering the two-way and three-way interactions.

5. Age by Zone two-way interaction being non significant ( $\mathrm{p}$-value greater than 0.05) implies that the difference between population means of bond strength due to six different age levels for the Center Zone is not significantly different from the difference between population means of bond strength due to six different age levels for the Edge Zone.

6. Age by Slab two-way interaction being non significant (p-value greater than 0.05) implies that the difference between population means of bond strength due to six different age levels for the slab with LMC overlay is not significantly different from the difference between population means of bond strength due to six different age levels for the Slab with SFMC overlay.

7. Similarly, Zone by Slab two-way interaction being significant (p-value less than 0.05) implies that the difference between population means of bond strength due to Center Zone and Edge Zone effects for the slab with LMC overlay is significantly different from the difference between population means of bond strength due to Center Zone and Edge Zone effects for the Slab with SFMC overlay.

8. The three-way interaction Age by Zone type by slab type being non significant (pvalue greater than 0.05 ) implies that the age by zone interactions is the same for two types of slabs, Age by Slab interaction is the same for two different zones, and zone by slab interaction is the same for five different levels of age.

Two-way and three-way interaction effects can be better understood by plotting and examining the interactions. Code written here is used for developing the two-way interaction plots. 


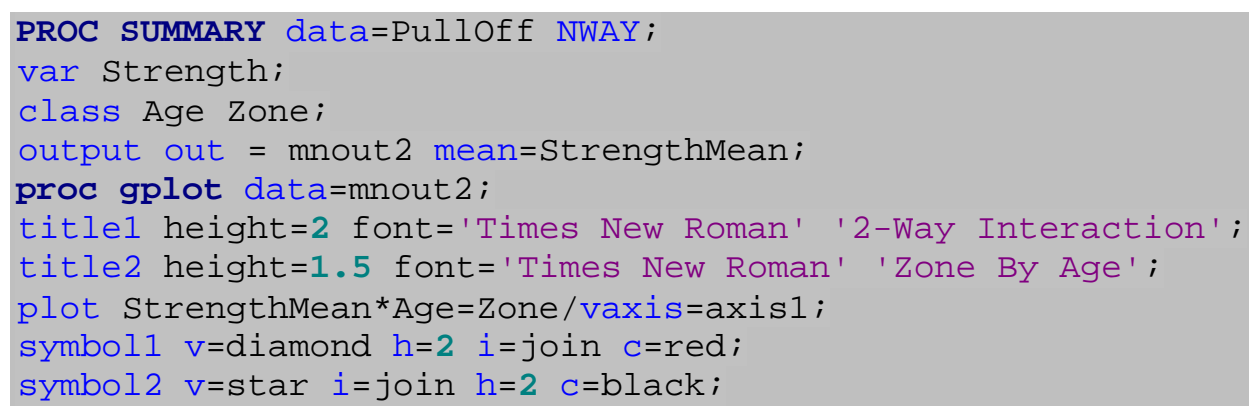

2-Way Interaction

Zone By Age

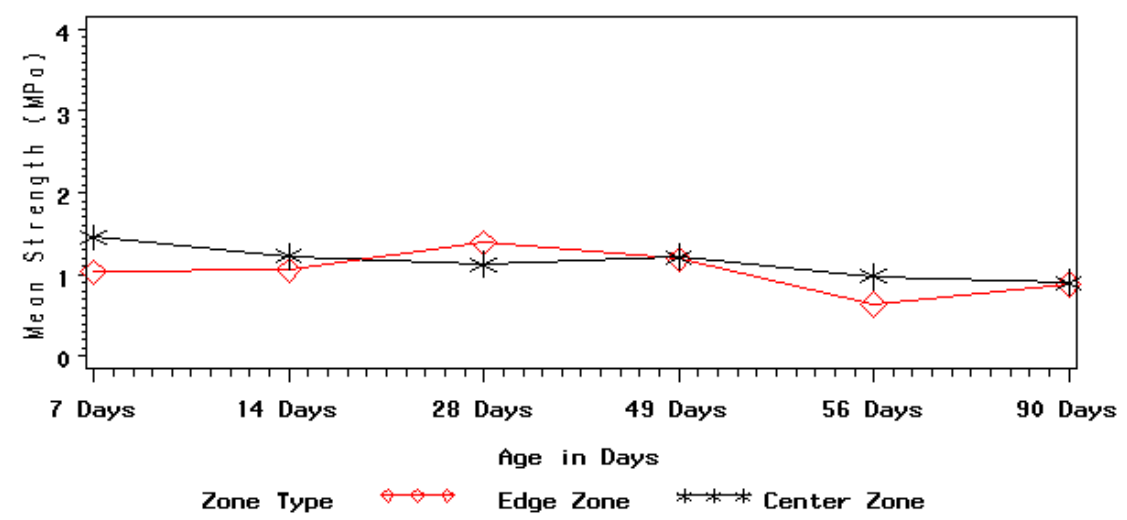

Figure 6.13: Means for levels of Zone type at each level of Age (Stage-II)

Figure 6.13 is plotted by taking age factor in the x-axis and plotting the means of bond strength for each level of zone type, i.e., corresponding to center zone and edge zone. We can conclude that the overall performance of center zone was better than edge zone for different levels of age factor. Center zone was performing better than edge zone for 7 days, 14 days but, equal performance is observed for both the center zone and edge zone for 28, 49 and 90 days i.e., as the slab is maturing with age the performance of edge zone is also increasing.

PROC SUMMARY data $=$ Pulloff NWAY;

var Strength;

class Age Slab;

output out = mnout3 mean=StrengthMean;

proc gplot data=mnout3;

title2 $\mathrm{h}=1.5$ font='Times New Roman' 'Slab by Age';

plot StrengthMean *Age=Slab/vaxis=axis1;

symbol1 $\mathrm{v}=$ diamond $\mathrm{h}=2$ i=join $\mathrm{c}=\mathrm{red}$; 
2-Way Interaction

Slab by Age

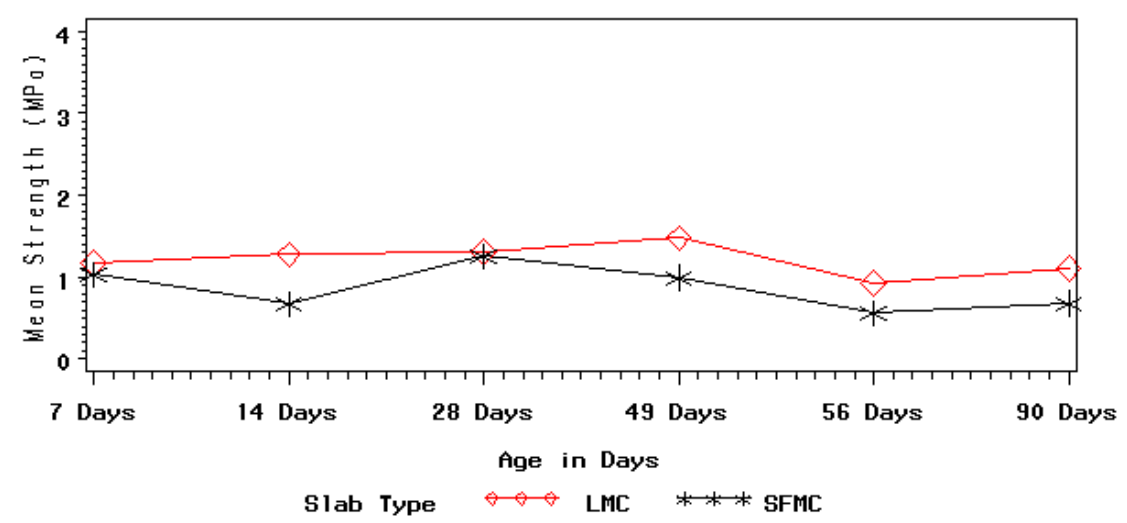

Figure 6.14: Means for levels of Slab type at each level of Age (Stage-II)

Figure 6.14 is plotted by taking age factor in the x-axis and plotting the means of bond strength for each level of slab type, i.e., corresponding to slabs with LMC and SFMC overlays. By looking at the plot we can conclude that the bond strength of LMC slab was greater than that of SFMC slab for different levels of Age. But, nothing can be predicted about the increase or decrease in bond strength due to the maturity of the slabs.

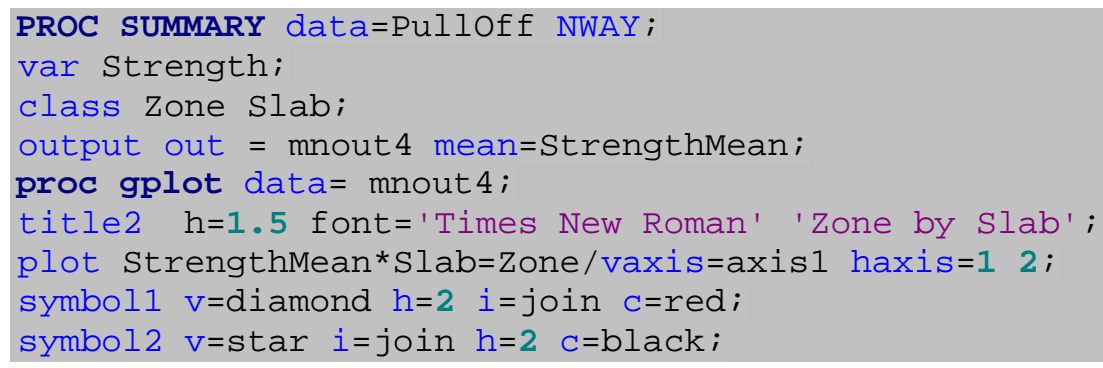


Zone by Slab

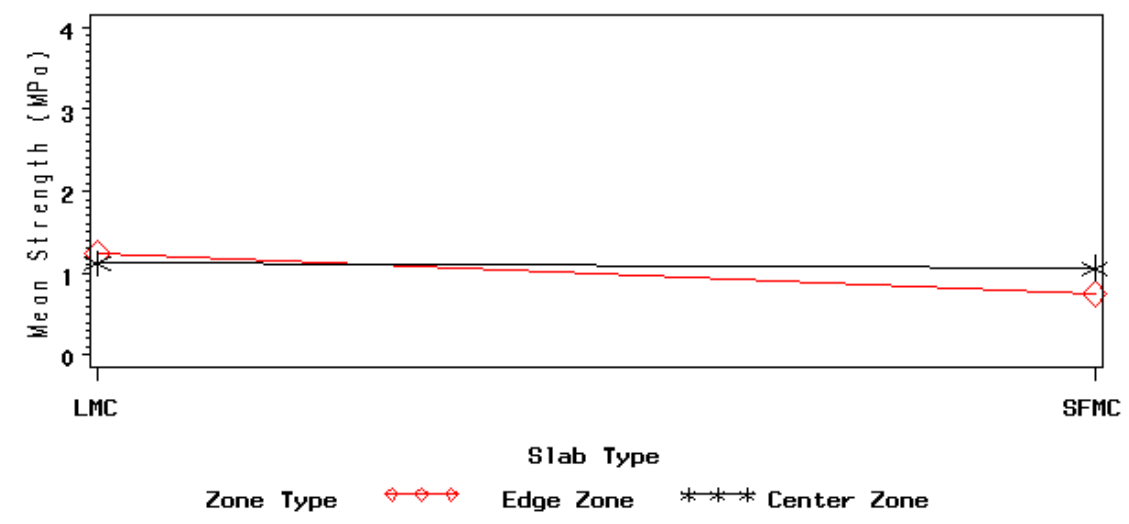

Figure 6.15: Means for levels of Zone type at each level of Slab (Stage-II)

Figure 6.15 is plotted by taking slab factor in the $\mathrm{x}$-axis and plotting the means of bond strength for each level of zone type. By looking at the plot we can further that the bond strength of center zone was better than that of edge zone for SFMC overlay but, the edge zone bond strength was better than center zone but not very significant for LMC overlay. Also, we can see that the overall strength of slab with LMC overlay was more than that of slab with SFMC overlay.

Code written here is for developing the three-way interaction plots. For Age by Zone by Slab three-way interaction, we consider the Zone by Slab interaction for each level of age and interpretation of the results was made based on the graphs developed.

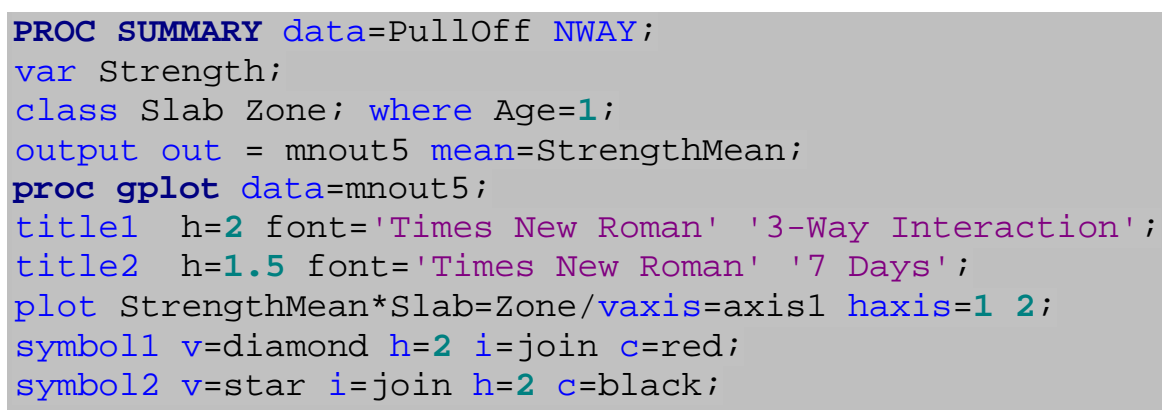


3-Way Interaction

7 Days

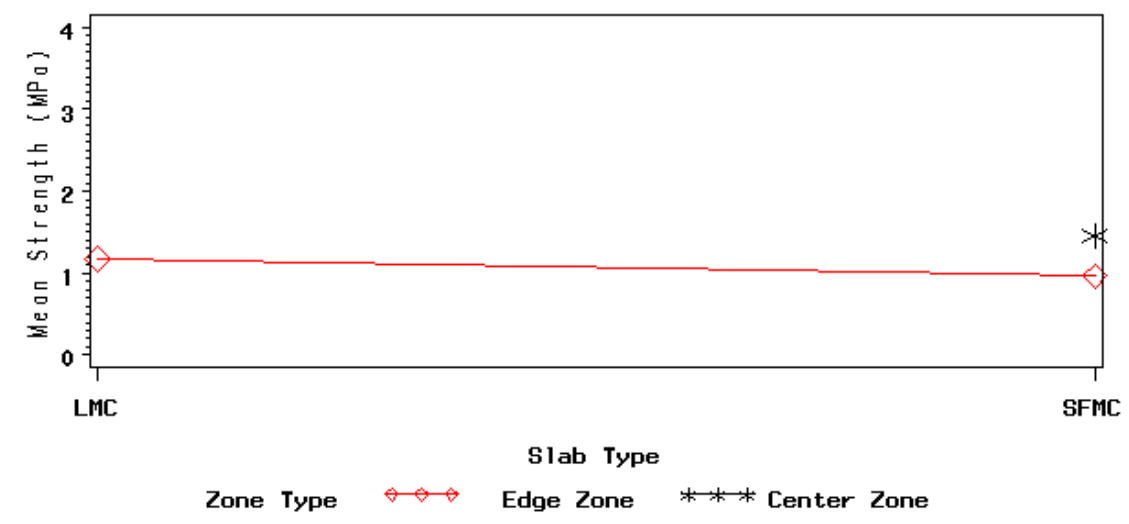

Figure 6.16: Means for levels of Zone type at each level of Slab type for 7 days age level (Stage-II)

Figure 6.16 is plotted by taking Slab factor in the x-axis and plotting the means of bond strength at each level of zone type for 7 days of age level. By looking at the plot we cannot compare the center zone performance for two slabs but the only conclusion that can be made is the edge zone performance of LMC slab is better than SFMC slab. Since, no interface failure was observed when pull-off testing was conducted.

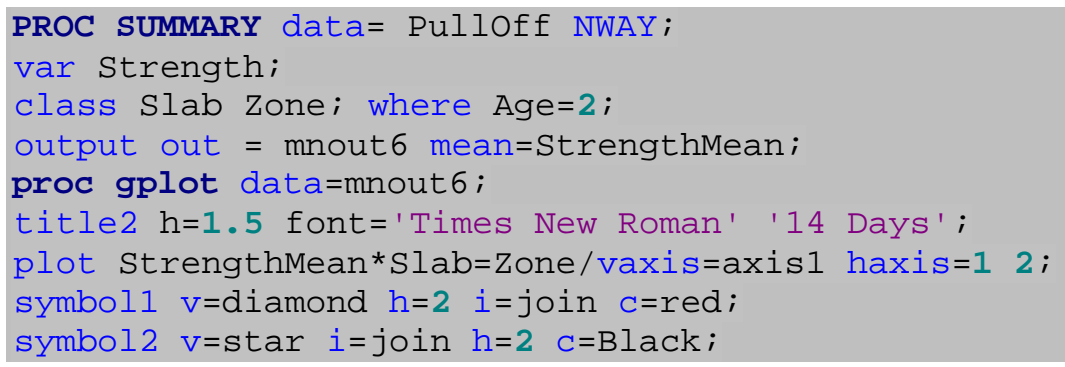


3-Way Interaction

14 Days

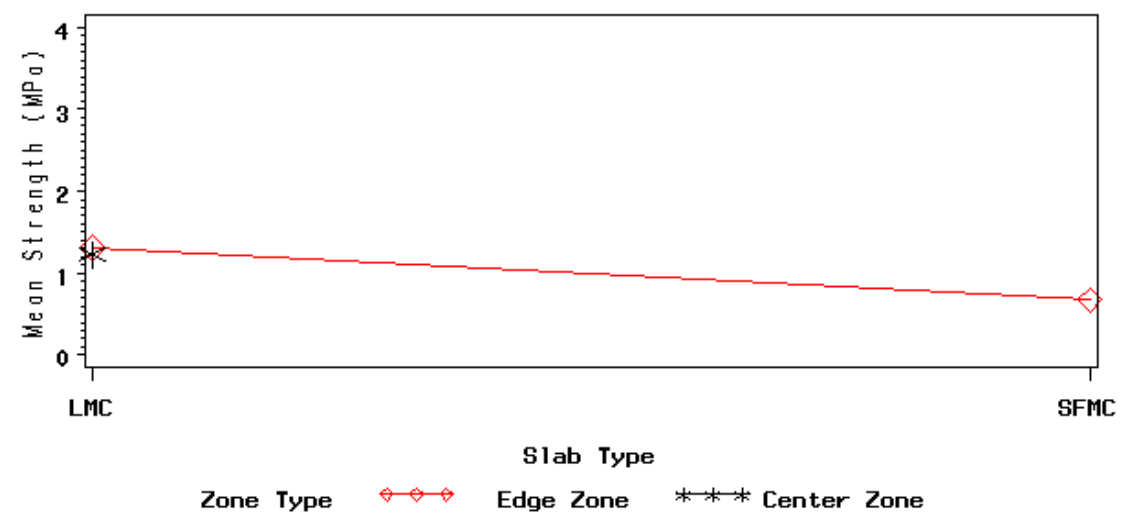

Figure 6.17: Means for levels of Zone type at each level of Slab type for 14 days age level (Stage-II)

Figure 6.17 is plotted by taking Slab factor in the x-axis and plotting the means of bond strength at each level of zone type for 14 days of age level. Nothing can be concluded out of this figure since the depth of the coring was not into the substrate concrete in the center zone on the slab with SFMC overlay and hence all the failures occurred in overlay concrete which was not desirable for this study. The only conclusion that can be made out of this figure is that the performance of edge zone on the slab with LMC overlay was better than that of the slab with SFMC overlay.

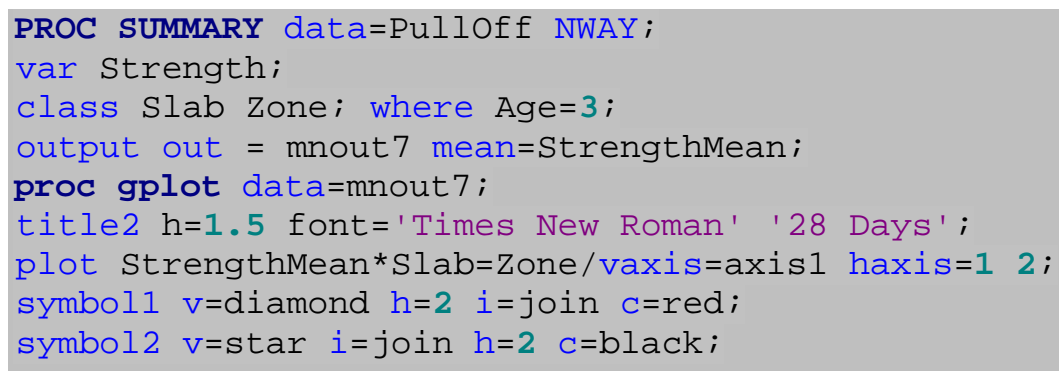


3-Way Interaction

28 Days

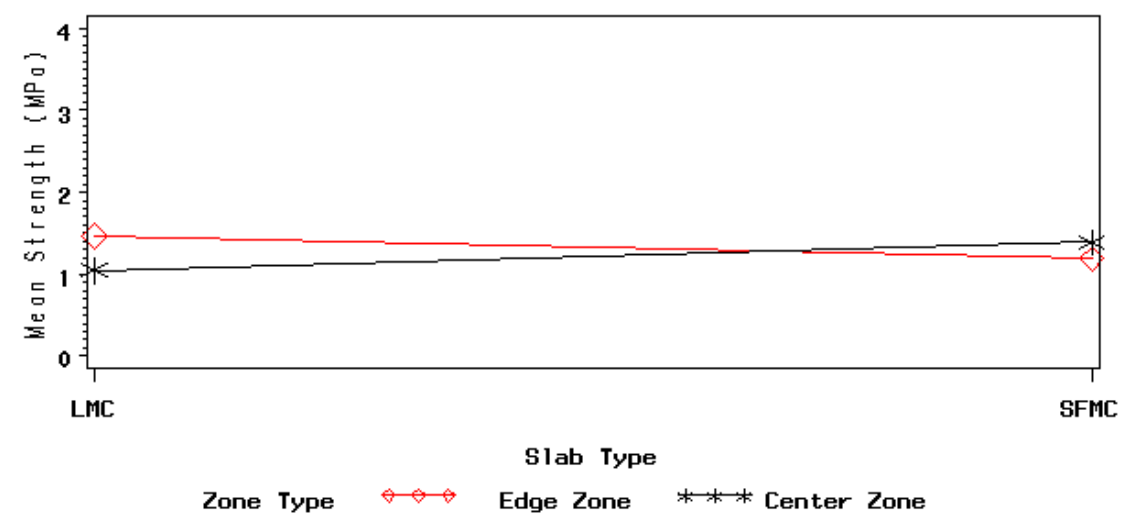

Figure 6.18: Means for levels of Zone type at each level of Slab type for 28 days age level (Stage-II)

Figure 6.18 is plotted by taking Slab factor in the x-axis and plotting the means of bond strength at each level of zone type for 28 days of age level. By looking at the plot we can conclude that the performance of edge zone on slab with LMC overlay was better than that of slab with SFMC overlay. Also, we can observe that the performance of center zone on slab with SFMC overlay was better than that of LMC overlay. But, the difference in strength values at center and edge zones for both the slabs is not very significant.

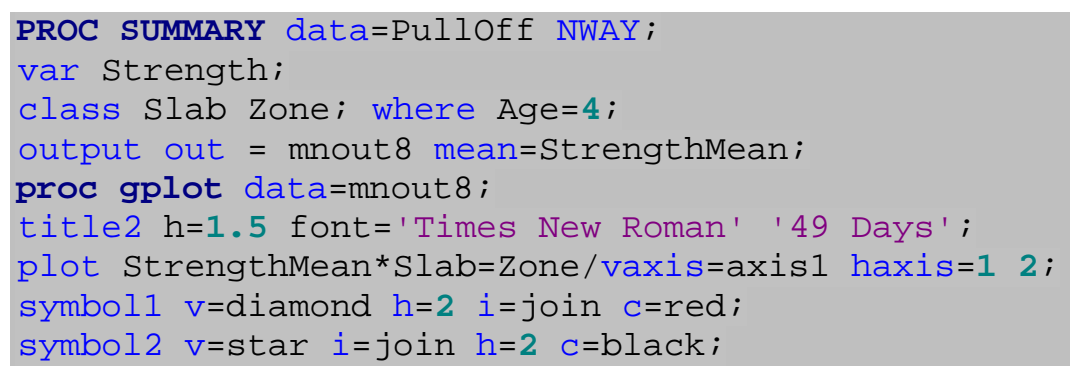


3-Way Interaction

49 Days

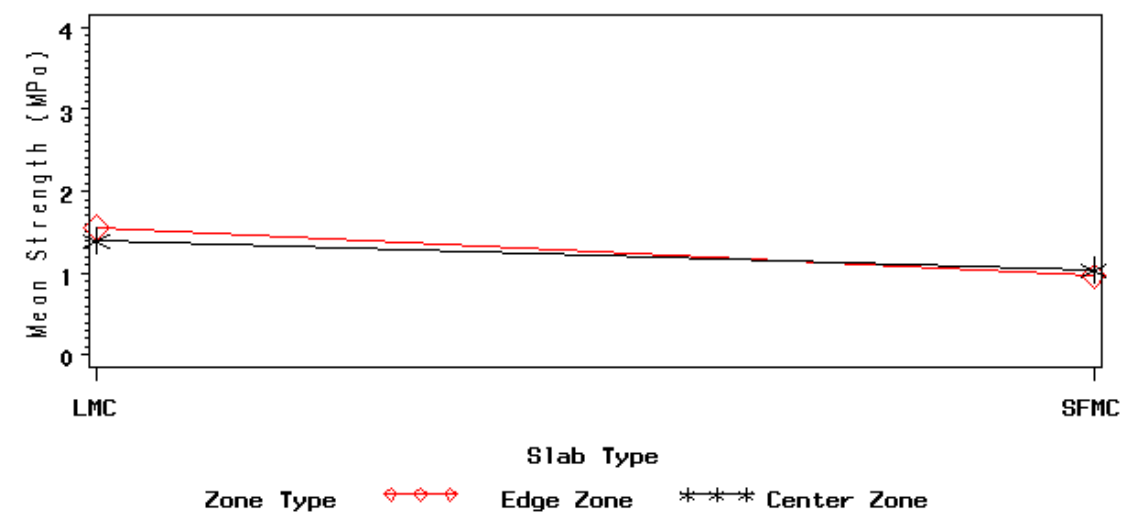

Figure 6.19: Means for levels of Zone type at each level of Slab type for 49 days age level (Stage-II)

Figure 6.19 is plotted by taking Slab factor in the x-axis and plotting the means of bond strength at each level of zone type for 49 days of age level. By looking at the plot we can conclude that the performance of both the center and edge zones on slab with LMC overlay was better than that of slab with SFMC overlay. Performance of center and edge zones is almost same when the individual slabs are considered.

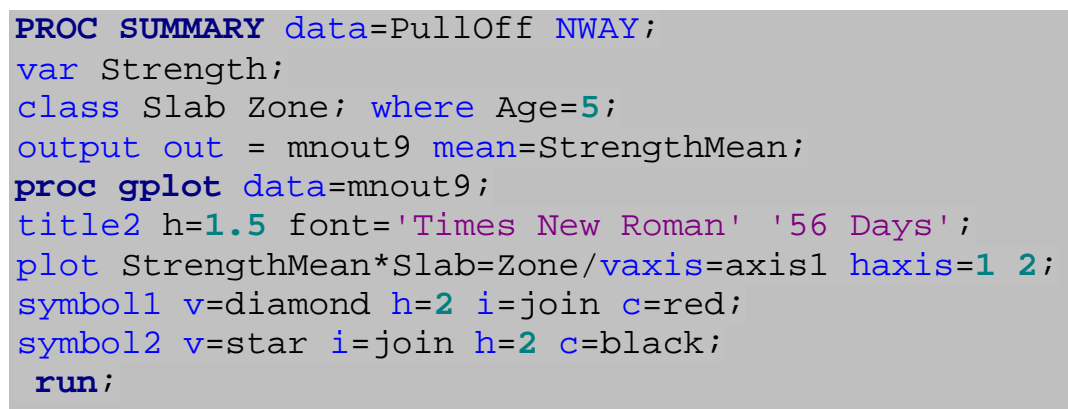


3-Way Interaction

56 Days

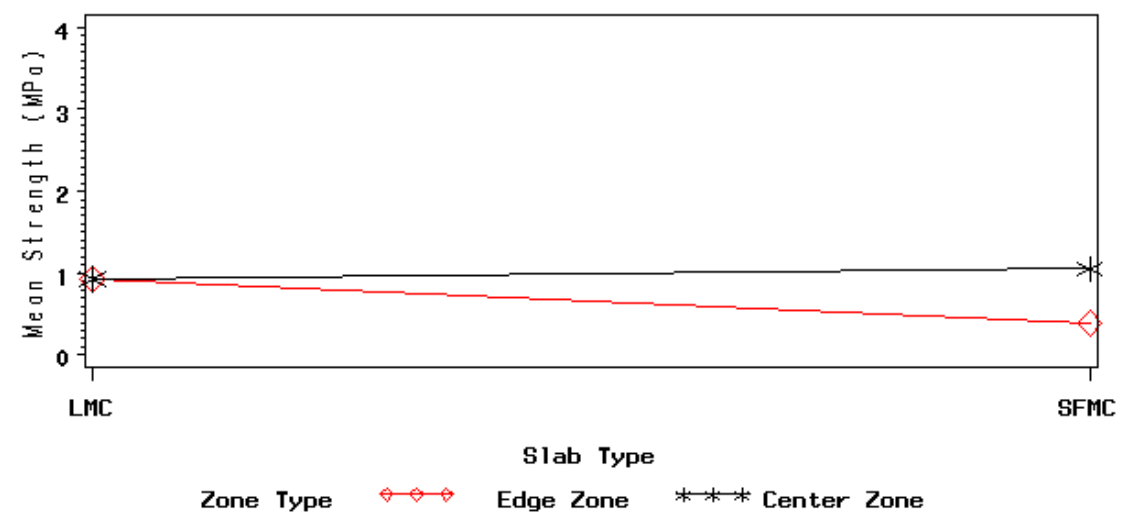

Figure 6.20: Means for levels of Zone type at each level of Slab type for 56 days age level (Stage-II)

Figure 6.20 is plotted by taking Slab factor in the x-axis and plotting the means of bond strength at each level of zone type for 56 days of age level. By looking at the plot we can conclude that the effect of both the center zone and edge zone was almost same for both the slabs at 56 days of age level. The overall performance of two types of slabs was almost same but, the edge zone of slab with SFMC overlay was slightly greater than that of slab with LMC overlay.

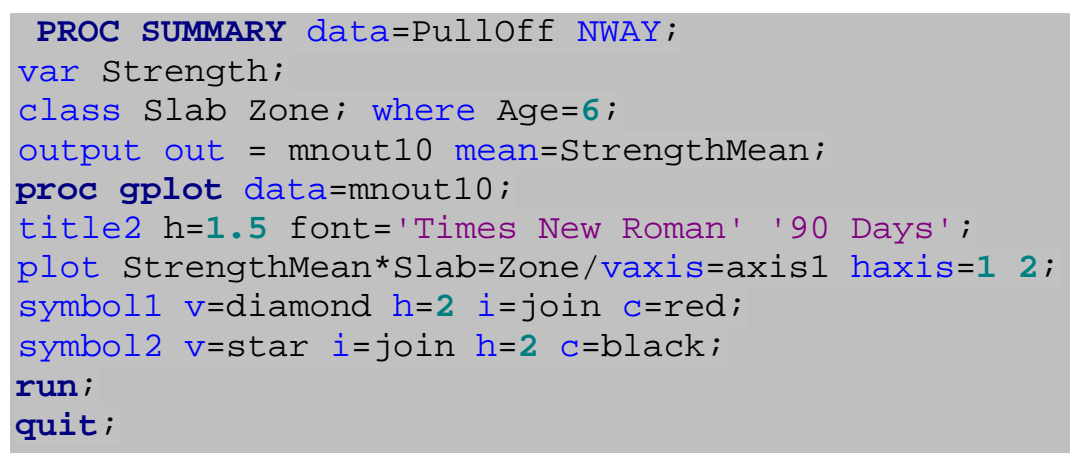




\section{3-Way Interaction}

90 Days

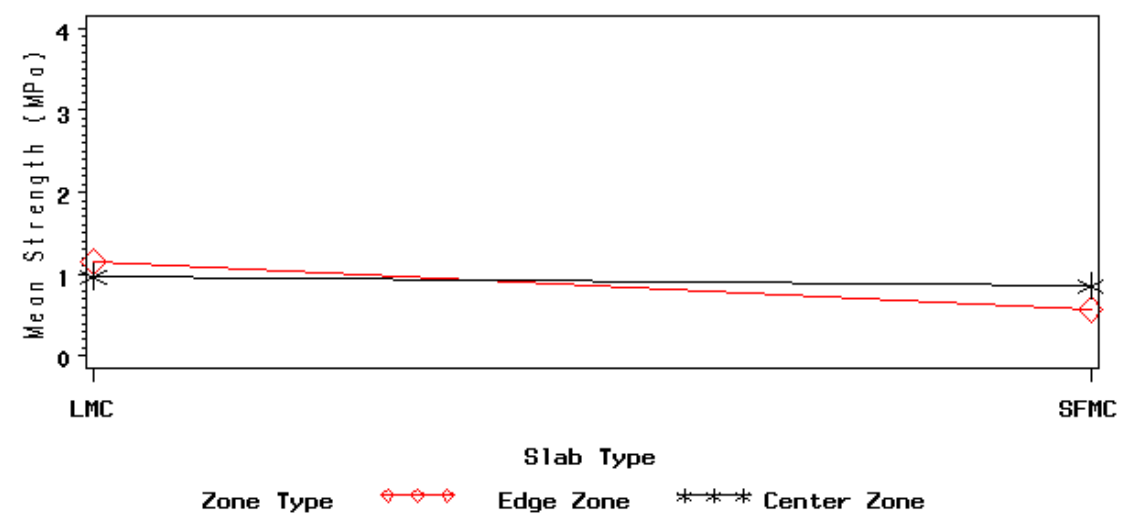

Figure 6.21: Means for levels of Zone type at each level of Slab type for 90 days age level (Stage-II)

Figure 6.21 is plotted by taking Slab factor in the x-axis and plotting the means of bond strength at each level of zone type for 90 days of age level. By looking at the plot we can conclude that the effect of both the center zone and edge zones of slab with LMC overlay is more than that of the slab with SFMC overlay. Both the zones performed equally well when the individual slab is considered at 90 days of age.

From the Figure 6.16 through Figure 6.21 we can see that the overall performance of slab with LMC overlay was better than the slab with SFMC overlay and as the slabs are maturing with age the performance of edge zone is also increasing compared to that of center zone.

\subsubsection{Length Change Monitoring}

Similar to the Stage-I study, length change of the substrate concrete, LMC overlay and SFMC overlays was monitored by embedding the special type of concrete embedment gages in the respective concretes in Stage-II. Details of the type of embedment gages used in this study are explained in detail in section 3.5.1.3 and the testing procedure is explained in detail in section 4.5. 


\subsubsection{Differential Length Change after Overlay Pour}

Differential length change monitoring is very important, because this change leads to the development of shear stresses at the interface which were considered as the main cause for delamination (Kim et al. (2003); Mailvaganam et al. (2000); and Zhang and Li (2002)).The differential length change developed at the interface was monitored by installing embedment strain gages $25 \mathrm{~mm}$ (1 in.) below the interface in substrate concrete and $25 \mathrm{~mm}$ (1 in.) above the interface in overlay concretes.

The plan and elevation views of the location of strain gages in substrate and overlay concretes are shown in Figure 6.22(a) and Figure 6.22(b). The plots of the percent length change of the strain gages at different locations with age are shown below the Figure 6.22. All the figures in the left side corresponds to the percent length change plots of the embedment strain gages in the slab with LMC overlay with LMC bonding slurry at the interface whereas, the right side figures represents the percent length change plots of the embedment strain gages in slab with SFMC overlay with SFMC bonding slurry at the interface. In all these plots, red line refers to the percent length change plot of overlay concrete and the black line refers to the percent length change plot of substrate concrete. Reference to the Figure 6.22 is always necessary when reading the Figure 6.23 and Figure 6.24. 

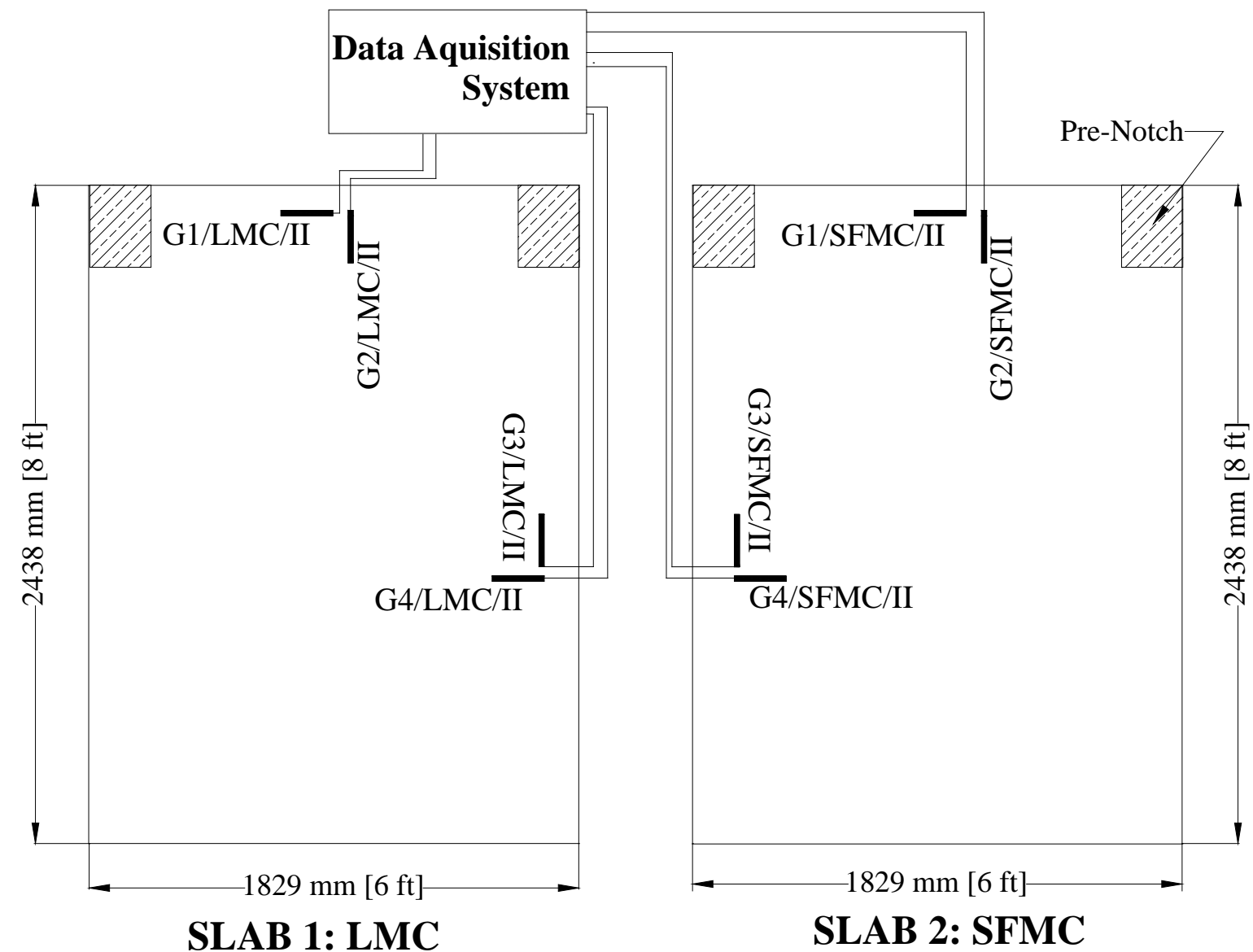

SLAB 2: SFMC

(a)
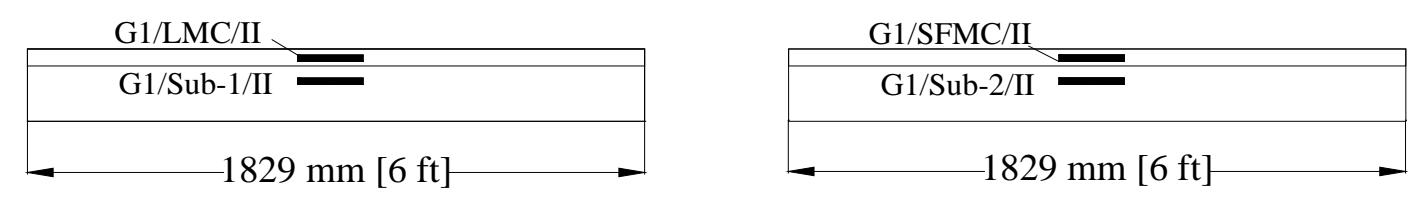

(b)

Figure 6.22: Locations of embedment strain gages in substrate and overlay concretes (Stage-II); a) Plan view, and b) elevation view.

Similar to the Stage-I study, embedment gages were installed in both substrate and overlay concretes in transverse and longitudinal directions facing towards the prenotch and center, to study the differential length change, as shown in Figure 6.22. For example, G1/Sub-1/II and G1/LMC/II strain gages were installed 25 mm (1 in.) below and $25 \mathrm{~mm}$ (1 in.) above the interface respectively in the transverse direction along the 
edge on slab with LMC overlay and LMC bonding slurry at the interface as shown in the Figure 6.22. Similarly, G1/Sub-2/II and G1/SFMC/II strain gages were installed 25 mm (1 in.) below and $25 \mathrm{~mm}$ ( 1 in.) above the interface respectively in the transverse direction along the edge on slab with SFMC overlay with SFMC bonding slurry at the interface as shown in the Figure 6.22. The differential length change developed at the interface are plotted and compared in Figure 6.23 and Figure 6.24.

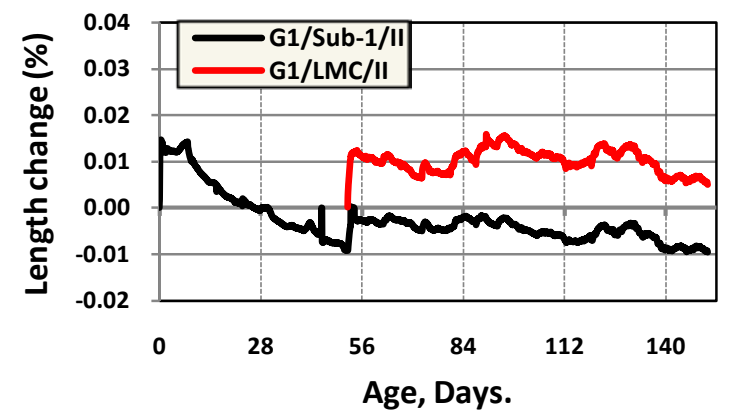

(a)

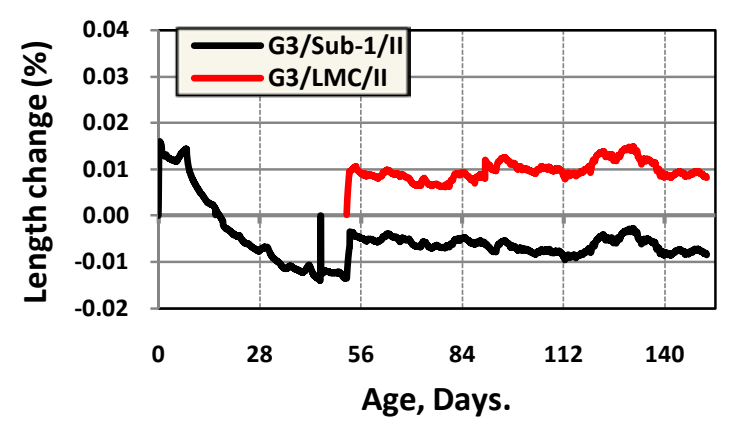

(c)

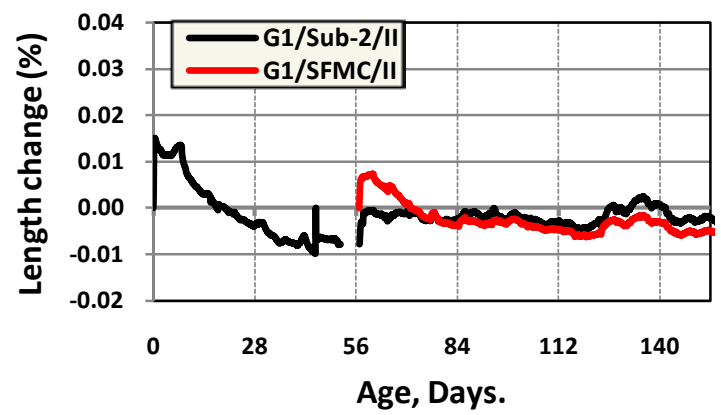

(b)

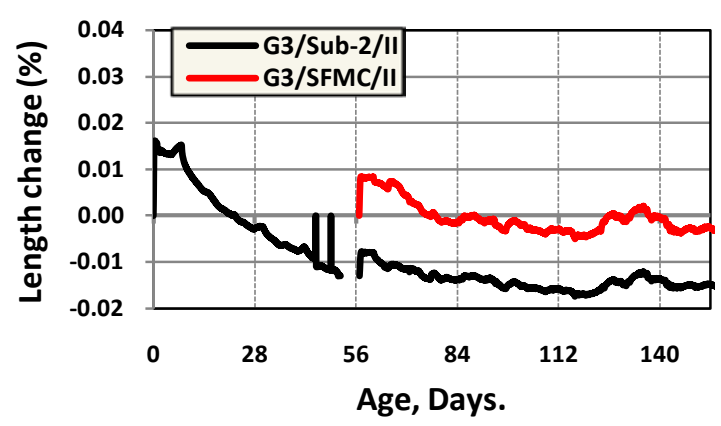

(d)

Figure 6.23: Comparison of percent length change plots of embedment gages in transverse and longitudinal direction along the edges of LMC and SFMC slabs 


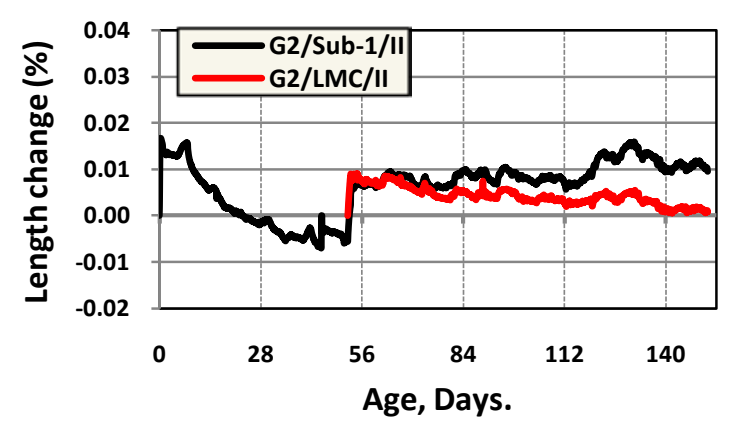

(a)

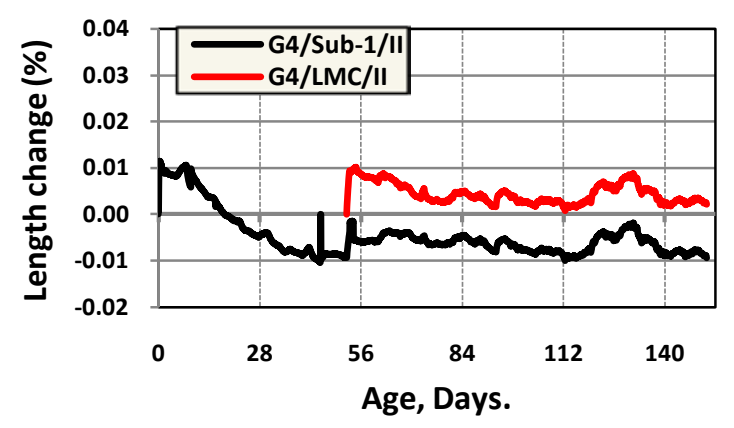

(c)

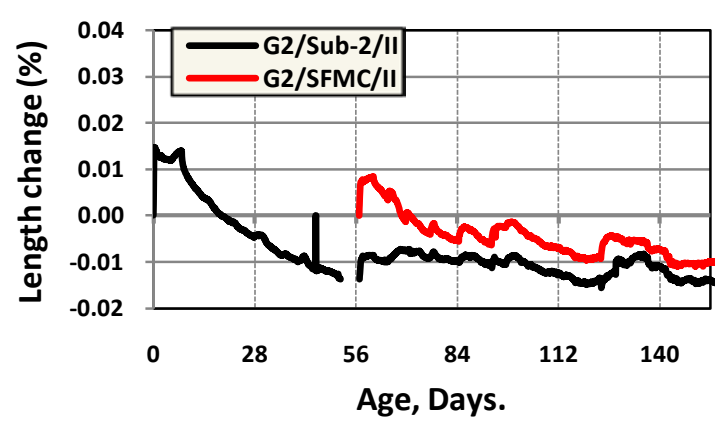

(b)

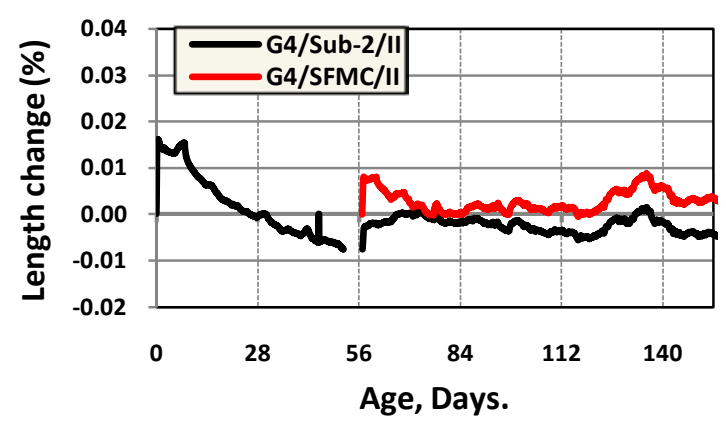

(d)

Figure 6.24: Comparison of percent length change plots of embedment gages in transverse and longitudinal direction towards the center of LMC and SFMC slabs

From the Figure 6.23 and Figure 6.24 we can conclude that the differential length change developed at the interface in transverse and longitudinal directions along the edge is more when compared to that facing towards the center. This can be due to the edge effects and the inserted pre-notch effect.

Here, No general comparison can be made between LMC and SFMC overlays because the shrinkage phenomenon of LMC is different from SFMC. This can be explained as LMC holds water molecules thus in turn retains moisture and does not allow shrinkage. Whereas, SFMC drying is more and volume change is more. LMC is more flexible and hence is less susceptible to volume changes. 


\subsubsection{Temperature Monitoring}

Special type of embedment thermocouple loggers manufactured by Engius company were installed at different locations to monitor the temperature of the Type $\mathrm{K}$ substrate concrete, LMC overlay, and SFMC overlays. Thermocouple loggers were installed at five different locations in both substrate and overlay concretes to monitor the differential temperature developed at the interface. To do so, thermocouple logger in substrate concrete was installed $25 \mathrm{~mm}$ (1 in.) below the interface and thermocouple logger in overlay concrete was installed $25 \mathrm{~mm}$ (1 in.) above the interface. For the terminology used in this section refer to the section 3.4.1.3.2.

Figure 6.25 shows the average temperature plots of substrate concrete, LMC overlay and SFMC overlays. It is observed that the temperature in the SFMC overlay was greater than that of the LMC overlay and substrate concrete. Also, from this we can notice that initially after overlay pour temperature was increased for initial 24 hrs and then the temperature decreased due to the wet curing and then the temperature increase again because of the external heat applied to the slabs.

Figure 6.26 shows the differential temperature changes developed at the interface due to the difference in the temperatures between substrate and overlay concretes at typical location. It is observed that the differential temperature developed at the interface of the SFMC overlay and substrate concrete was more when compared with the slab with LMC overlay. This leads to the development of temperature load at the interface and hence the shear stresses are developed at the interface. 


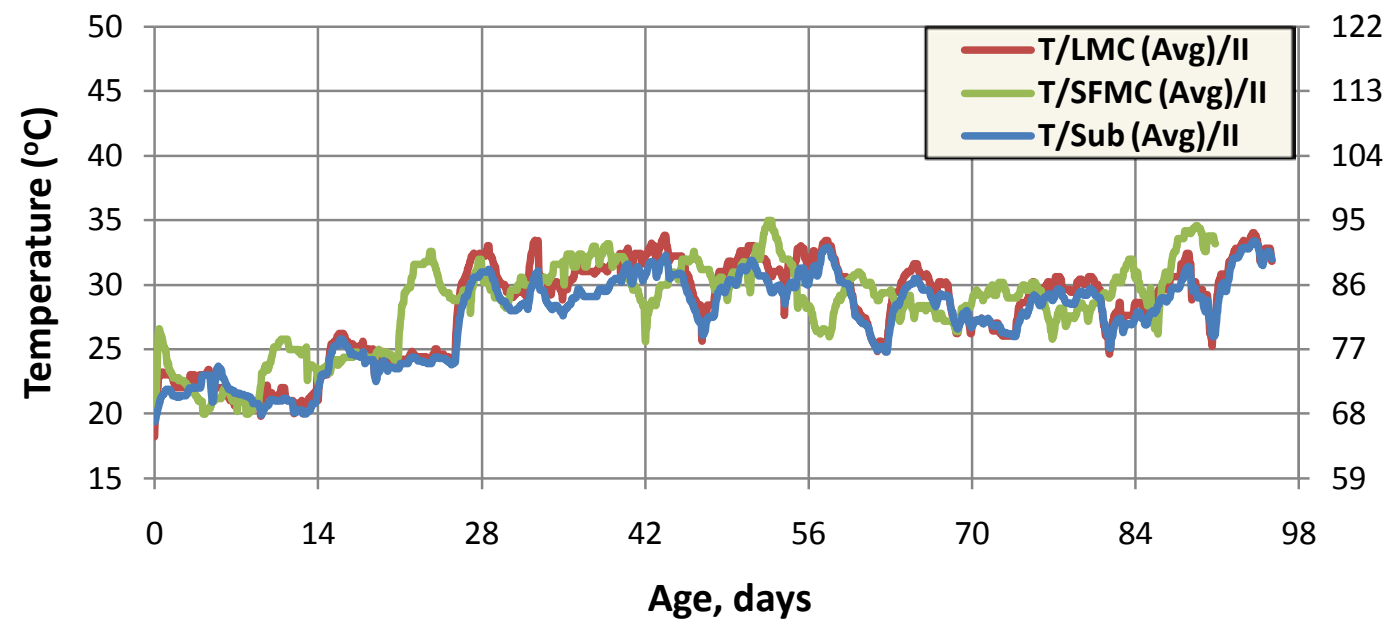

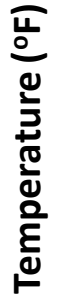

Figure 6.25: Average temperature data of substrate concrete, LMC overlay, and SFMC overlay after overlays pour (Stage-II).

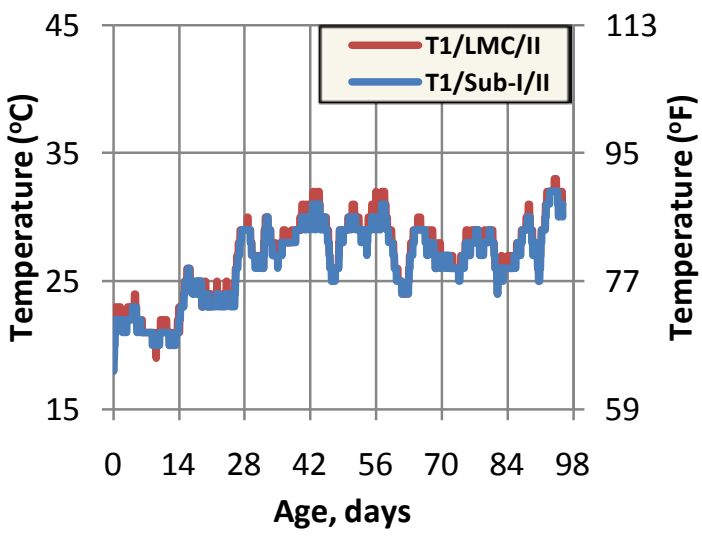

(a)

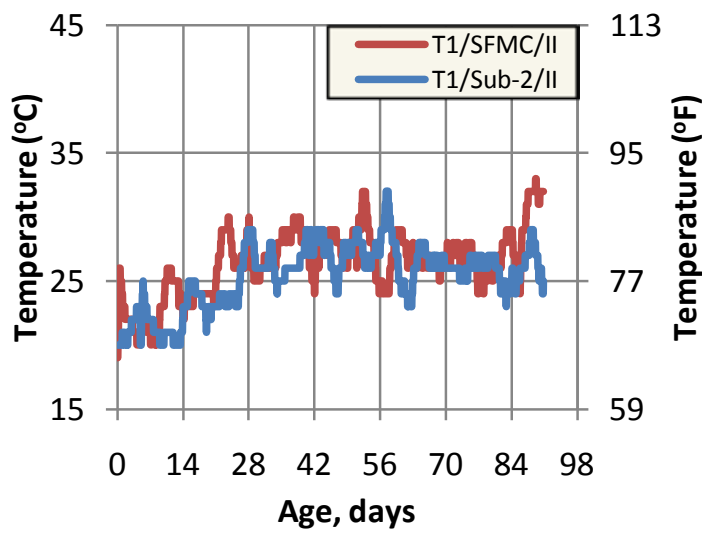

(b)

Figure 6.26: Differential temperature data at typical location of: (a) substrate concrete and LMC overlay, (b) Substrate concrete and SFMC overlay.

\subsubsection{Vertical Displacements Due to Corner Lifting}

Similar to the Stage-I study, vertical displacements occurred due to corner lifting of the overlay concrete was measured by installing LVDT at the corners of two slabs. This was done to compare the vertical corner lifting of LMC overlay with SFMC overlay. The LVDT were started only after all the formwork was demolded and all the instrumentation was installed i.e., after 3 days of LMC overlay curing and after 7 days of 
SFMC overlay curing. In vertical corner lift versus time graph, the time zero indicates the onset of the measurements.

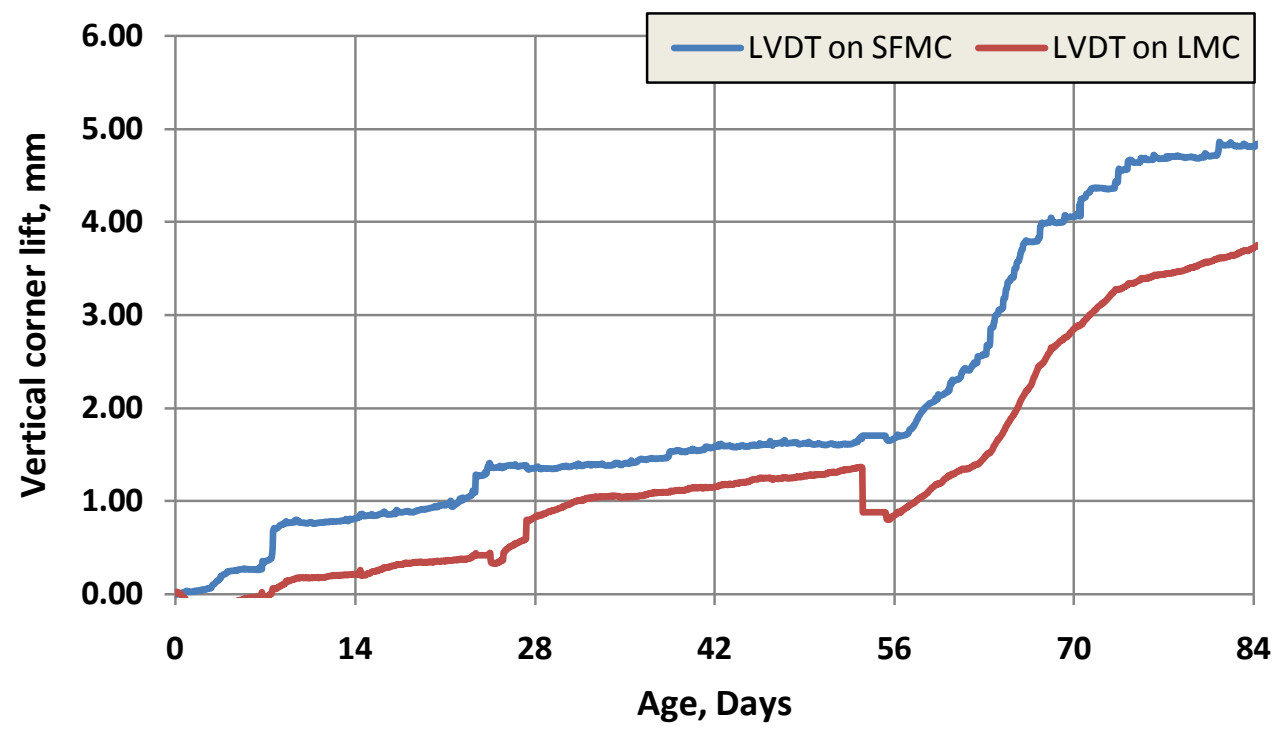

Figure 6.27: Relative displacement of overlay concretes (LMC and SFMC) recorded by LVDT (Stage-II)

From the Figure 6.27, it is observed that the slab with LMC overlay had the lowest crack opening at the corner when compared to the slab with SFMC overlay. Hence, we can conclude that the debonding due to vertical lifting at the corner is less when LMC overlay was used. Therefore, the performance of slab with LMC overlay was superior to that of the slab with SFMC overlay. Also Hong(2006) observed higher vertical corner lift in the SFMC with surface saturated condition at $35^{\circ} \mathrm{C}$ compared to LMC overlay with the similar conditions. This is in agreement with the differential temperature change observed at the interface.

\subsubsection{Direct Ultrasonic Pulse Velocity Testing}

Direct UPV testing was carried out at different locations in this Stage-II study instead of indirect UPV testing to study the delamination profile at the interface. Since direct UPV testing is more reliable to access the delamination at the interface because the signal transmits directly through the interface and therefore gives the exact profile of the 
interface cracking. Slabs were constructed on the elevation in this Stage-II study so that the bottom face was easily accessible and hence direct UPV testing was carried out in this Stage-II study. The testing procedure for conducting this testing is explained in detail in section 4.7.

\subsubsection{UPV Data}

The gridlines were drawn on the half of the slab on which UPV testing was carried out. In longitudinal direction the gridlines were spaced $100 \mathrm{~mm}$ (4 in.) whereas in transverse direction gridlines were spaced at $75 \mathrm{~mm}$ (3 in.). Gridlines in longitudinal direction were named from 1 to 25 whereas in transverse direction: they were named from $M$ to $Y$ for slab with LMC Overlay and from A to M on slab with SFMC overlay. Direct testing was carried out at different locations as shown in Figure 6.28. In this figure, red circle shows the test locations and this zone is assumed as the delaminated zone with pre-notch, blue circles shows the test locations and this zone is assumed as the center zone without any delaminations, and green circles shows the test locations and this zone is assumed as the delaminated zone without any pre-notch.

Time required for the signal to transit directly from transmitting transducer to receiving transducer is shown by UPV tester and recorded to calculate the velocity of the signal. The UPV numbers were calculated and plotted at different locations. The colors used for classifying different zones are used for plotting the UPV numbers for clarity of the plots. The plots are shown in colored boxes with the respective colors for the particular zones. The plots are generated along the lines in respective zones. Two lines (2-2 and 3-3) are selected in the delaminated zone with pre-notch, five lines (11-11, 12$12,13-13,14-14$, and 15-15) are selected in the center zone, and two lines (23-23 and 2424) are selected in the zone without pre-notch. The plots are developed side by side for two different slabs to compare the performance of two different types of overlays used.

All the plots in left hand side shows the direct UPV plots for slab with LMC overlay and the right hand side plots are for slab with SFMC overlay. From Figure 6.29 through Figure 6.31, figures (a),(c), and (e) presents UPV plots on slab with LMC 
overlay in the delaminated zone with pre-notch, center zone, and delaminated zone without pre-notch respectively in each case whereas, figures (b),(d), and (f) presents UPV plots on slab with SFMC overlay in the delaminated zone with pre-notch, center zone, and delaminated zone without pre-notch respectively in each case.

Figure 6.10 shows the UPV plots for slab with LMC overlay and slab with SFMC overlay at 28 days. Observations that can be made from the Figure 6.29 are as follows:

1. Velocities of around $3 \mathrm{Km} / \mathrm{s}$ was observed in the delaminated zone with pre-notch (Figure 6.29 (a) and Figure 6.29 (b)). Within this zone as we move towards the edge from the center (i.e., from $\mathrm{M}$ to $\mathrm{Y}$ on slab with LMC overlay and from $\mathrm{M}$ to A on slab with SFMC overlay) very less velocities were observed at the ends. When the velocities at the ends of slab with LMC overlay are compared with that of slab with SFMC overlay less velocities were observed;

2. Velocities greater than $3.5 \mathrm{Km} / \mathrm{s}$ was observed when the center zone (Figure 6.29 (c) and Figure 6.29 (d)) was considered. Also, in this zone as we move towards the edge less velocities were observed. When two slabs are compared, the performance of slab with LMC overlay was better than that of slab with SFMC overlay;

3. Velocities closer to $3.5 \mathrm{Km} / \mathrm{s}$ is observed when the delaminated zone without prenotch (Figure 6.29 (e) and Figure 6.29 (f)) was considered. When two slabs are compared, the velocities observed at the edges on slab with SFMC overlay was very less than that of slab with LMC overlay;

Similar observations can be seen from Figure 6.30 and Figure 6.31 at 56 and 90 days respectively. Hence, we can conclude that the performance of slab with LMC overlay was always better than that of the slab with SFMC overlay at 28, 56, and 90 days. Direct UPV testing by Hong (2006) on small scale samples showed higher crack front profiles when SFMC saturated with slurry at $35^{\circ} \mathrm{C}$ was used in comparison with the LMC overlay. In all the cases the performance of center zone was always better followed by delaminated zone without pre-notch and then by delaminated zone with pre-notch. 


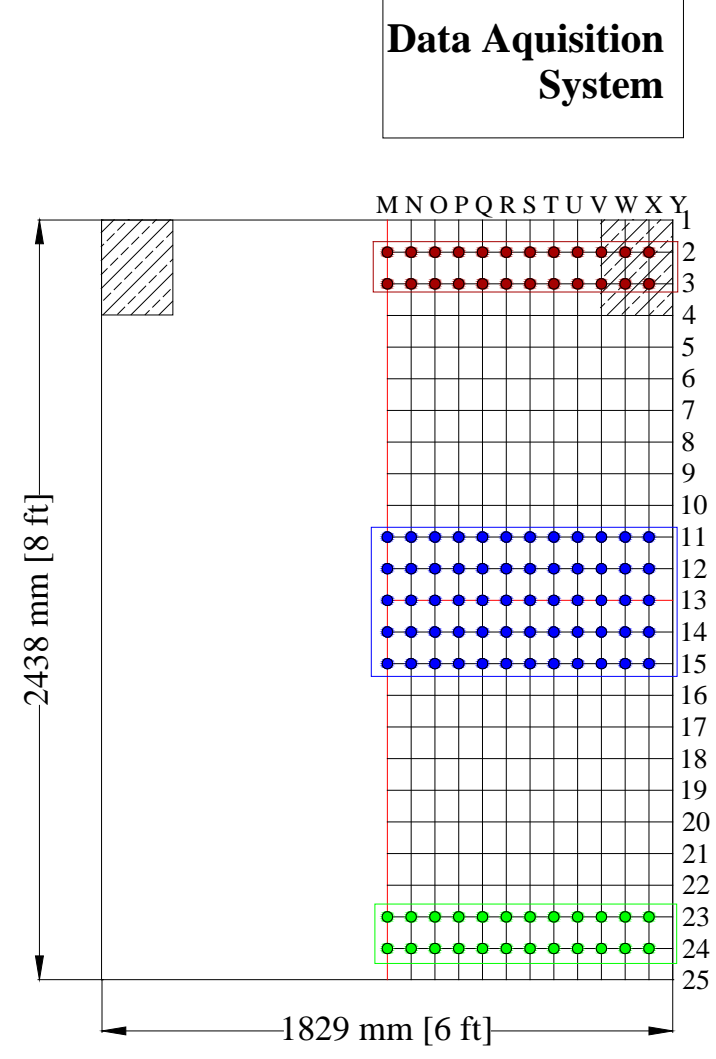

SLAB 1: LMC

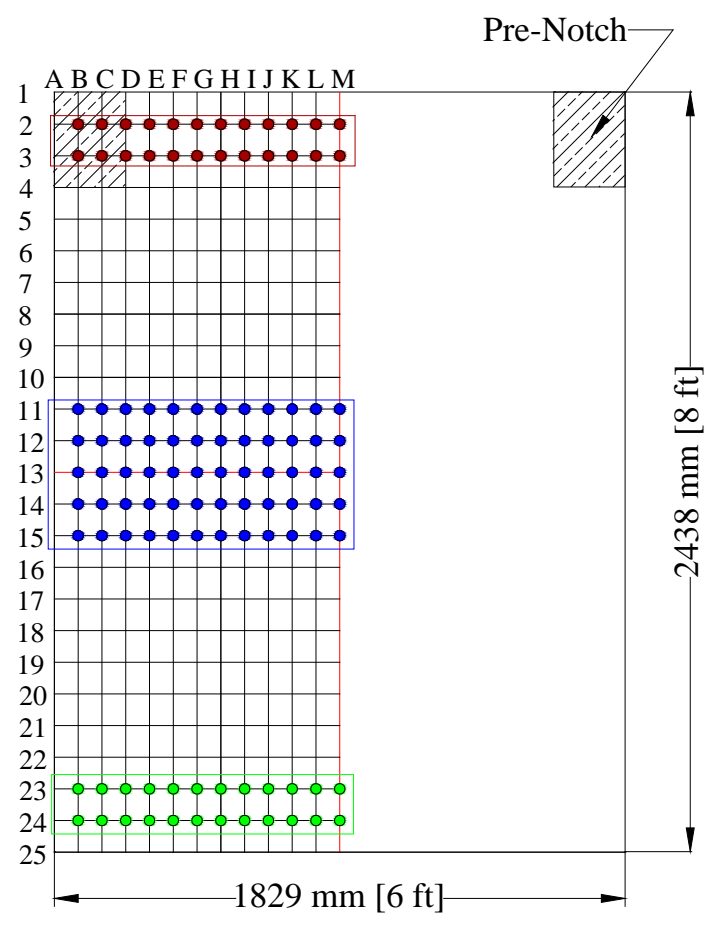

SLAB 2: SFMC

Figure 6.28: Locations at which direct UPV testing was conducted (Stage-II) 
Delaminated Zone with Pre-notch

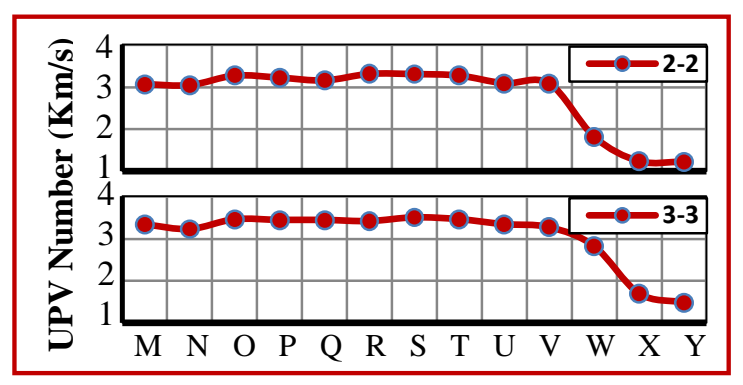

(a)

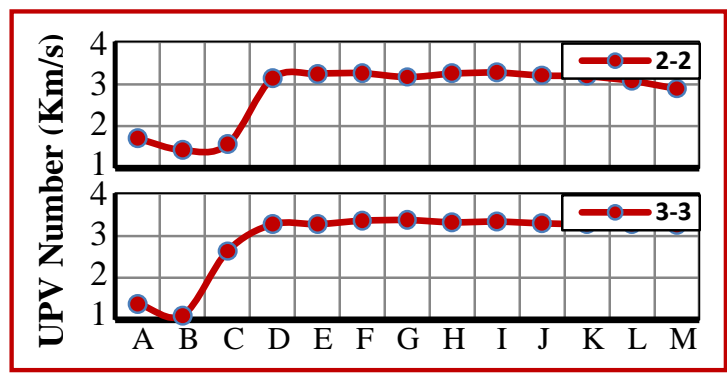

(b)

Center Zone

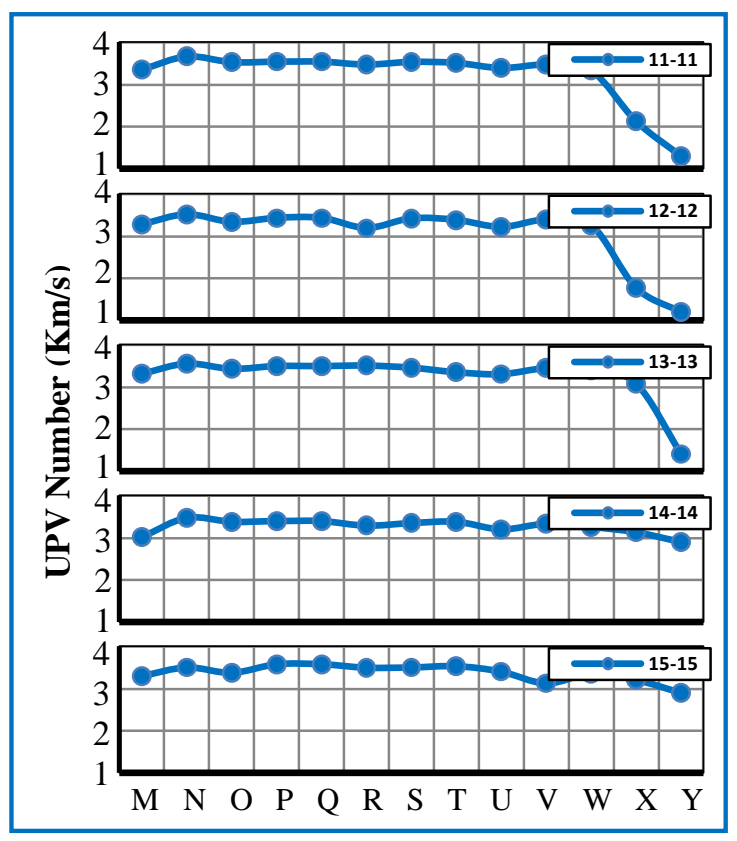

(c)

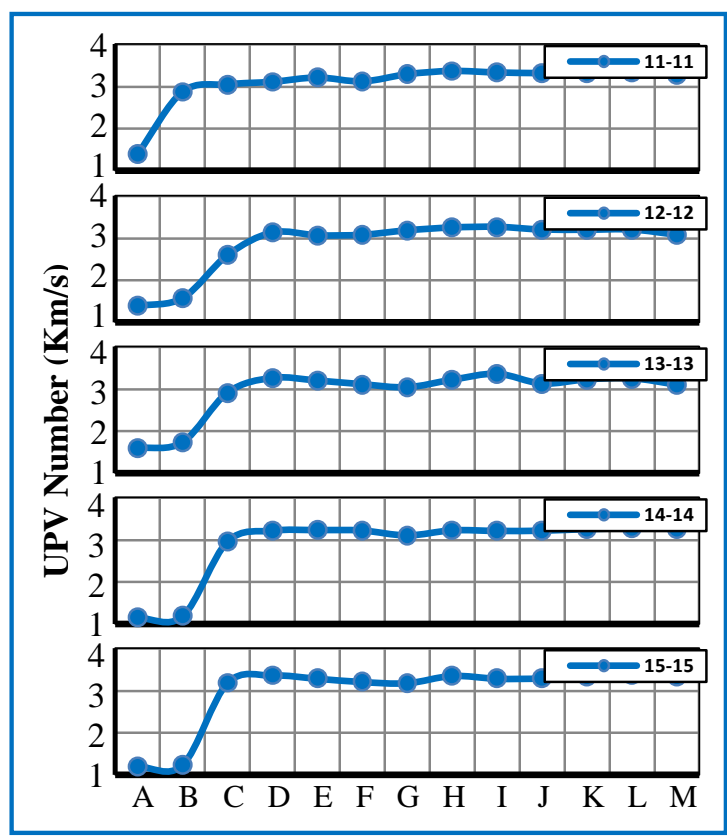

(d)

Delaminated Zone without Pre-notch

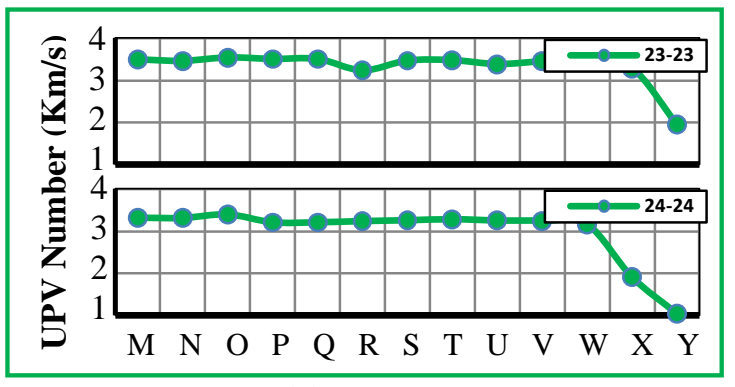

(e)

LMC

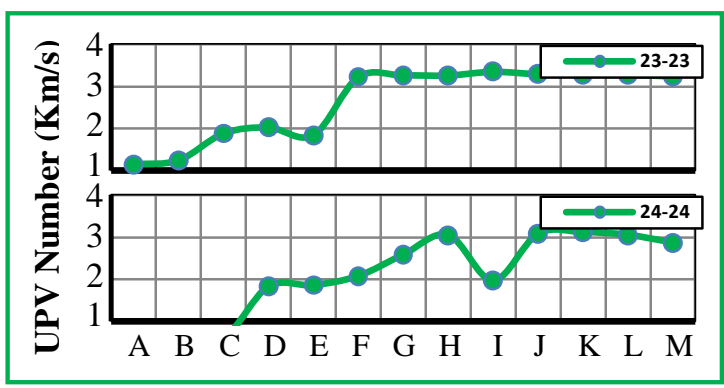

(f)

SFMC

Figure 6.29: Comparision of 28 days UPV plots measured on LMC and SFMC slabs: 
Delaminated Zone with Pre-notch

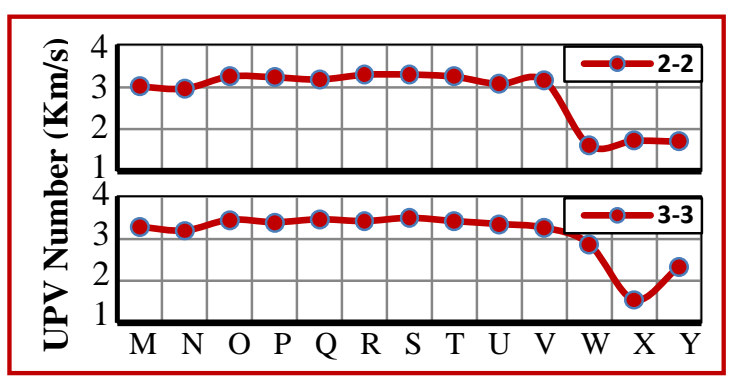

(a)

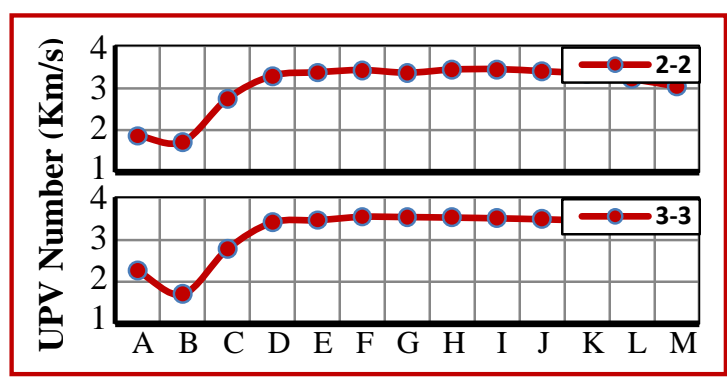

(b)

Center Zone

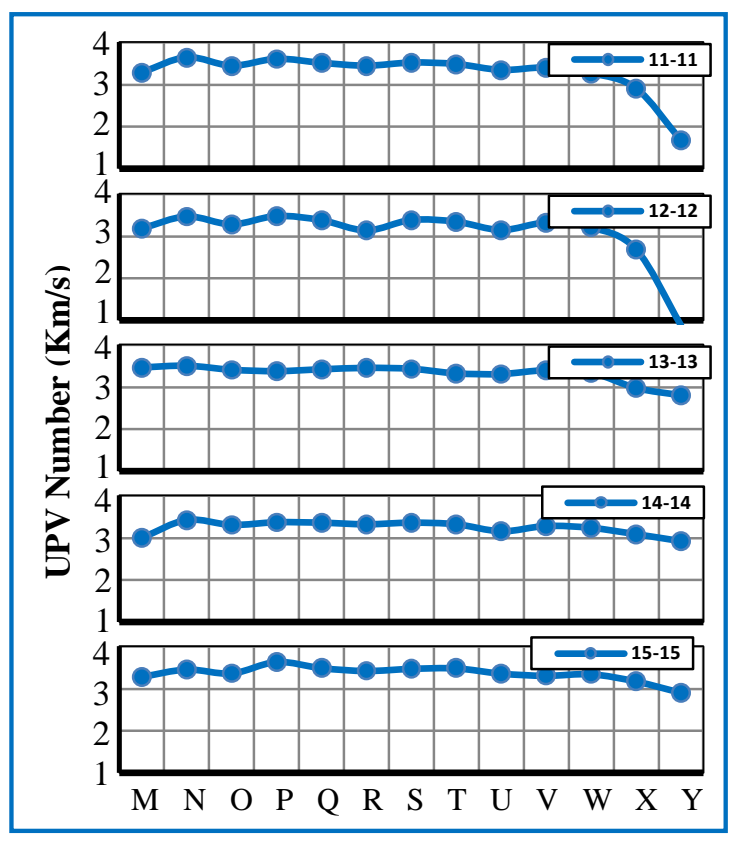

(c)

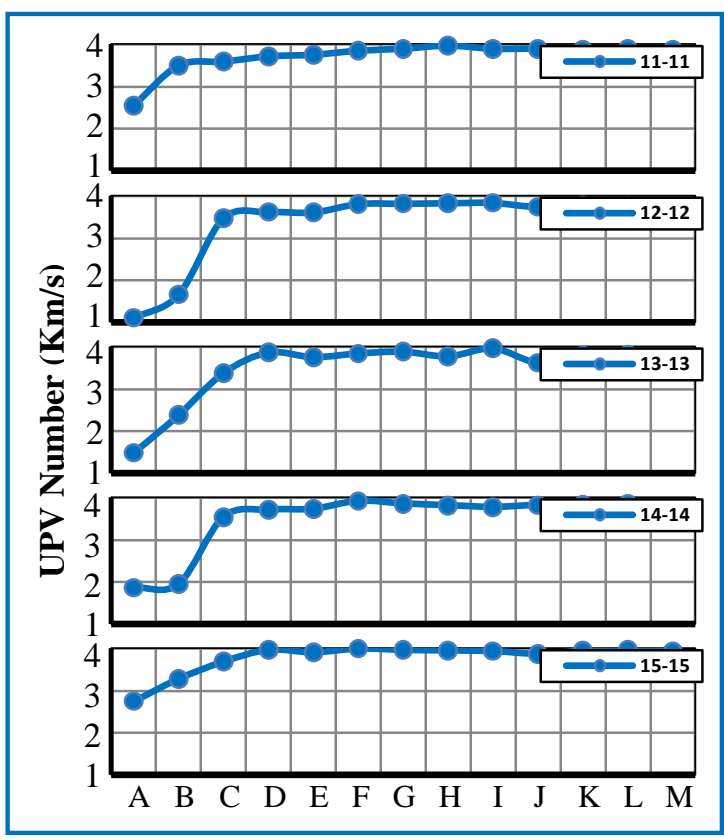

(d)

Delaminated Zone without Pre-notch

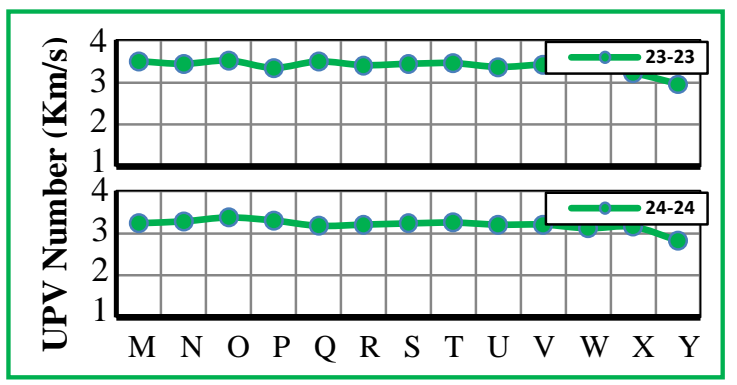

(e)

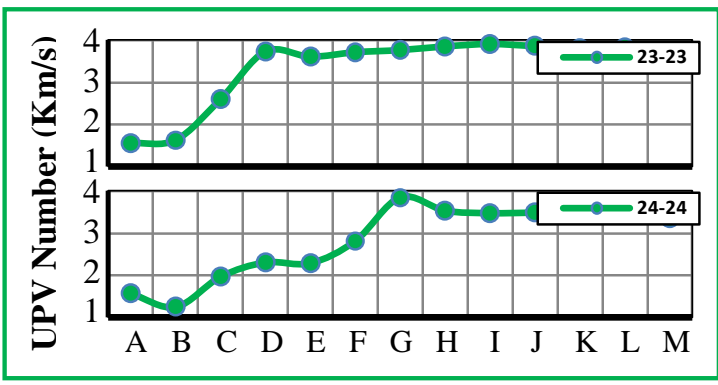

(f)

LMC

SFMC

Figure 6.30: Comparision of 56 days UPV plots measured on LMC and SFMC slabs 
Delaminated Zone with Pre-notch

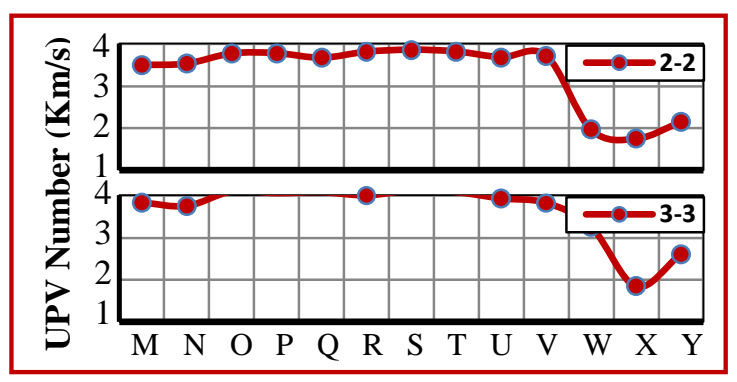

(a)

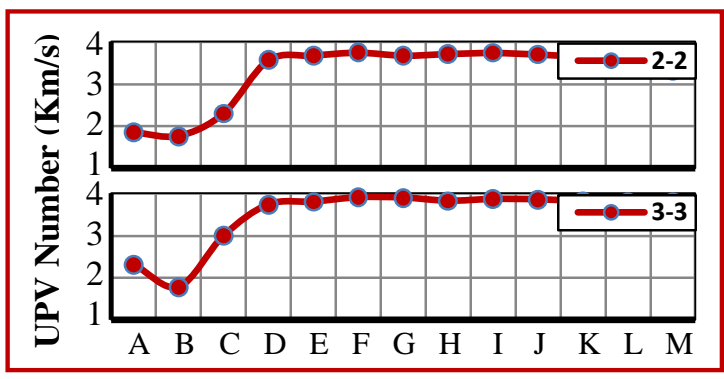

(b)

Center Zone

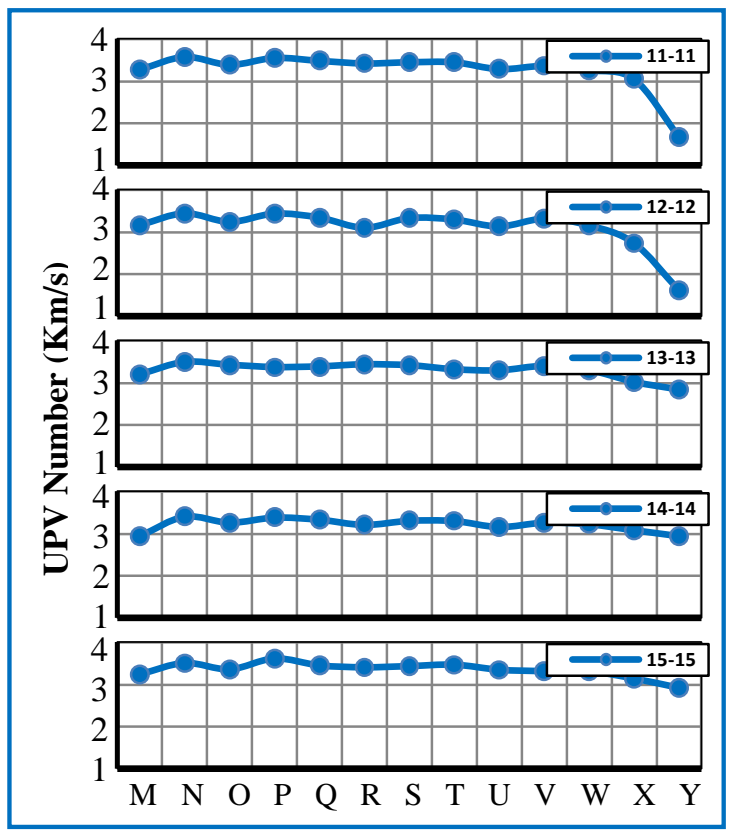

(c)

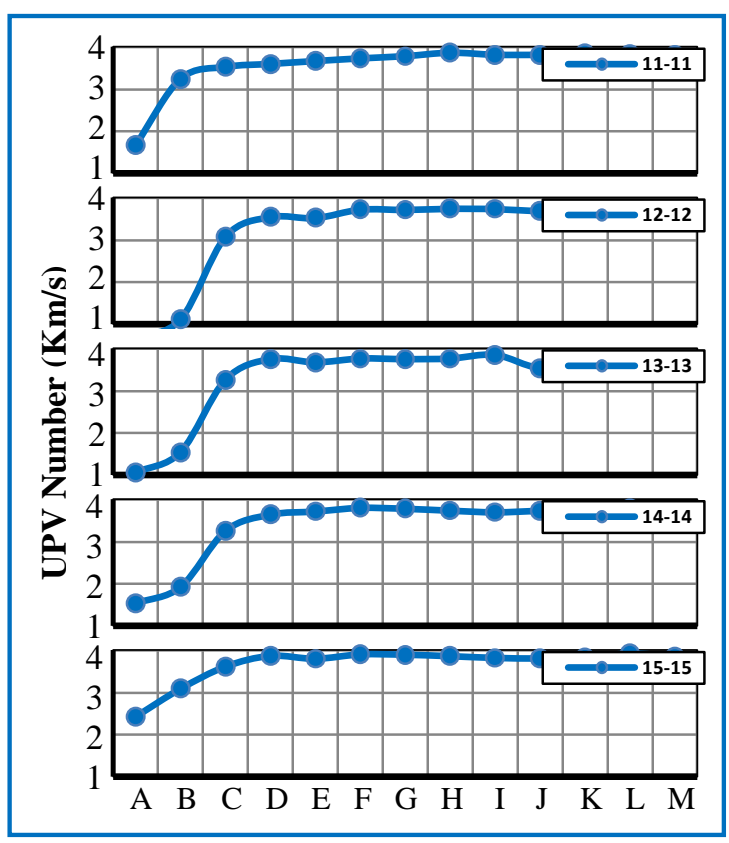

(d)

Delaminated Zone without Pre-notch

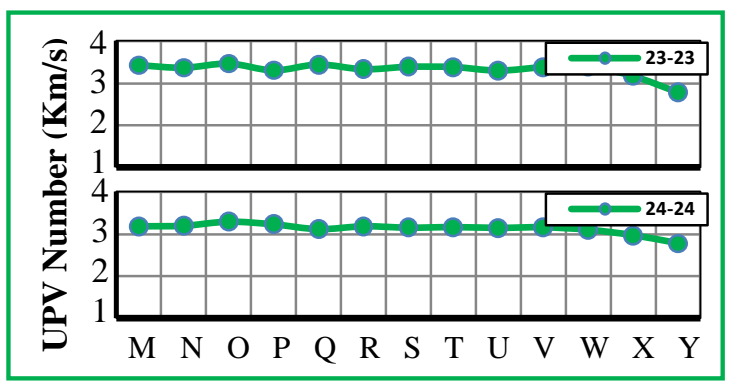

(e)

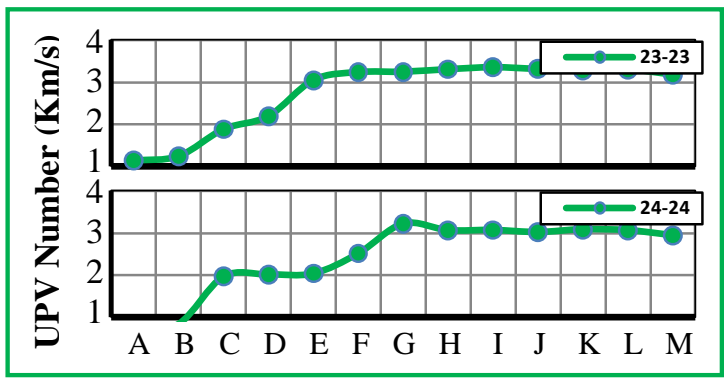

(f)

LMC

SFMC

Figure 6.31: Comparision of 90 days UPV plots measured on LMC and SFMC slabs 


\subsubsection{Analysis of Signals Collected by Oscilloscope}

Similar to the Stage-I study; oscilloscope was connected to the UPV tester to record the time domain signal simultaneously with the time. Later this time domain signal was saved into compact flash in EXCEL format and was transferred into computer using this compact flash drive in order to convert the waveform into Fast Fourier Transform (FFT) and then into power spectrum using MATLAB, powerful computing and analytical software. Signals are converted to FFT form since, when the signals are more complex; frequency domain provides much information about the signal.

The step by step procedure followed for converting the time-domain waveform into FFT is explained in section 5.2.4.2 and the similar steps were followed in this StageII study for converting into FFT. Additionally in this study, time-domain waveform was also converted into power spectrum using FFT function. The additional step added here is calculating the power of the FFT amplitude by multiplying the amplitude with its conjugate value. The advantage of converting into waveform is unwanted frequencies can be neglected and the peak magnitude can be amplified. The code used for converting time-domain waveform into FFT and then into power spectrum is shown below in highlighted text.

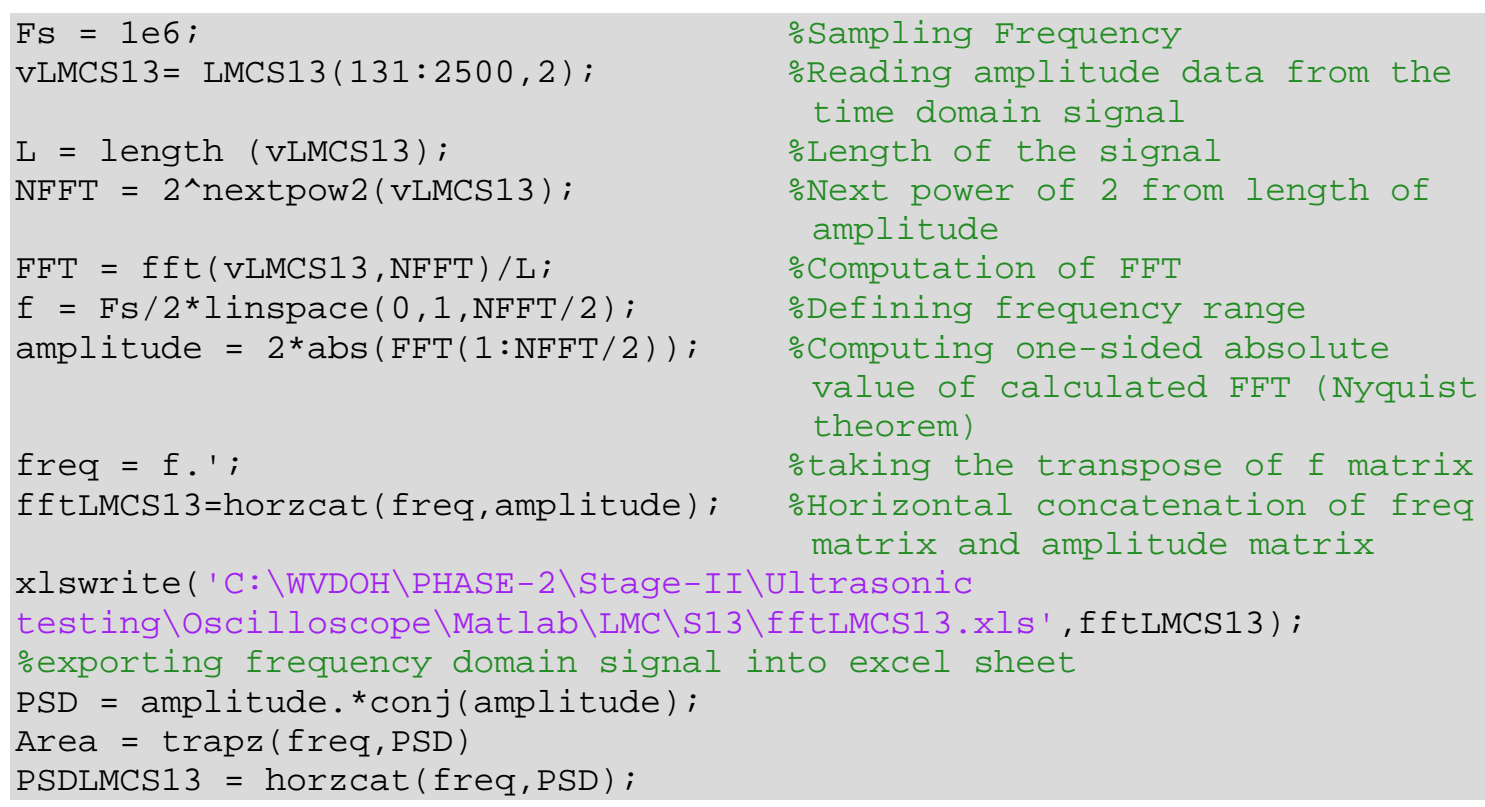


Figure 6.32 shows the locations at which time-domain waveforms were recorded simultaneously along with pulse time using oscilloscope. Signals were recorded at S13, N13, X13, S3, and S24 on the slab with LMC overlay and at locations G13, L13, B13, G3, and G24 on the slab with SFMC overlay. Comparisons are made between slabs and within the slabs.

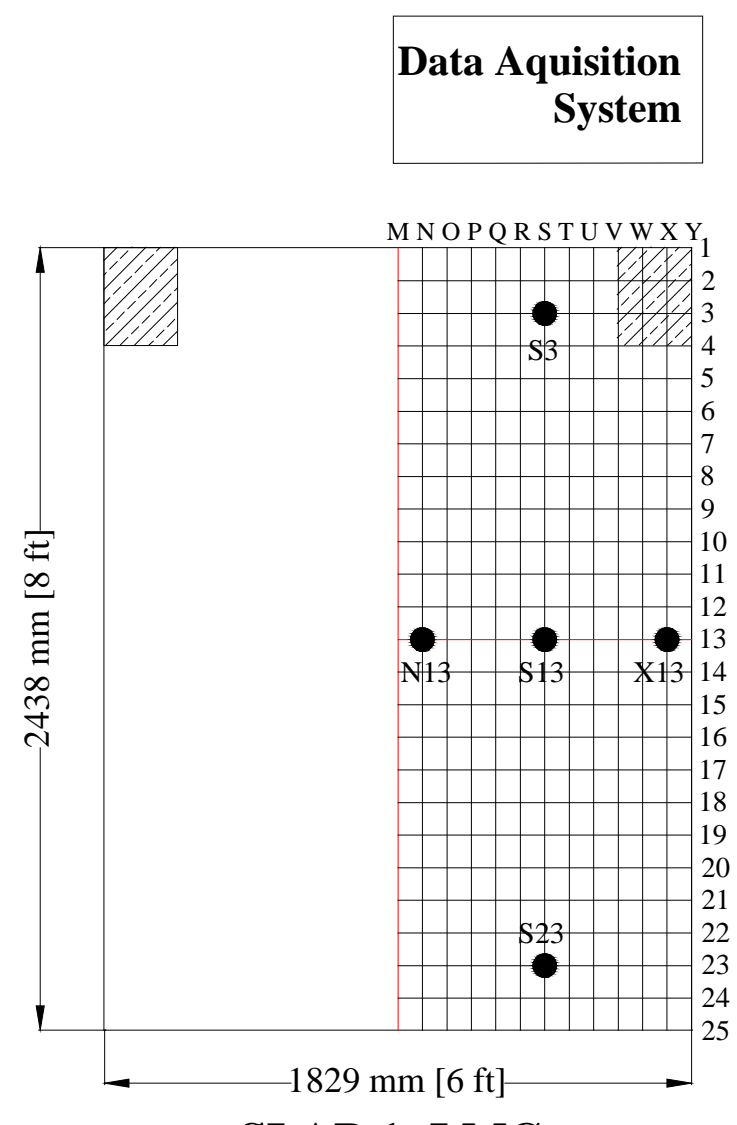

SLAB 1: LMC

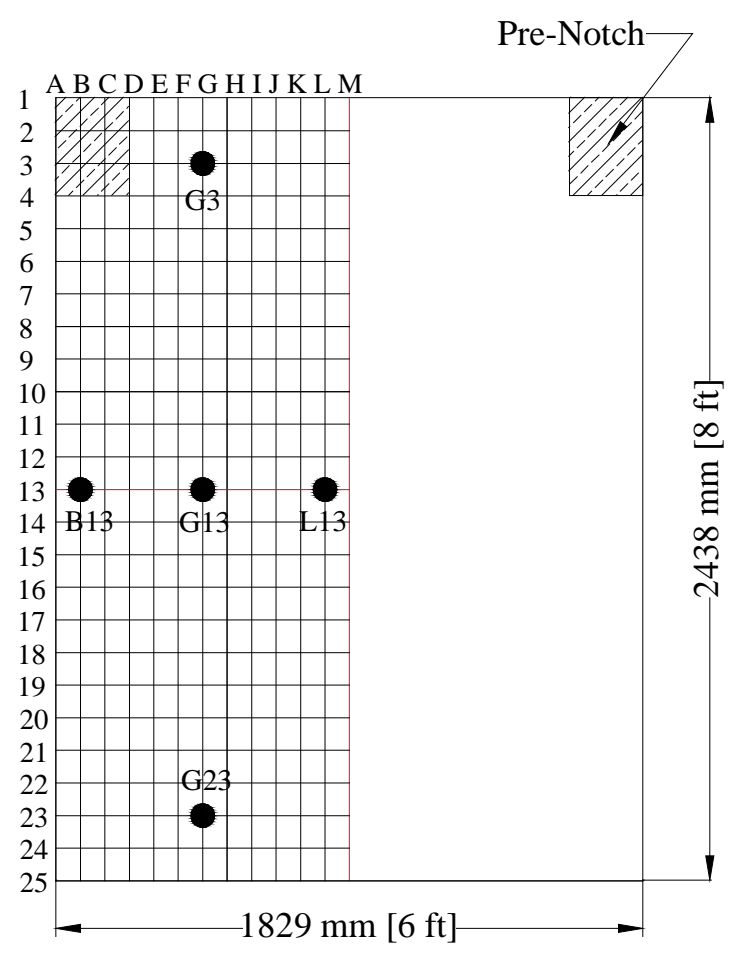

SLAB 2: SFMC

Figure 6.32: Locations at which oscilloscope readings were recorded (Stage-II)

Virgin signal was collected by transmitting the signal directly from transmitting transducer to receiving transducer with only a layer of gel as a medium in between. Time domain signal was collected and transformed into power spectrum using FFT as shown in 
Figure 6.33. Figure 6.33(a) shows the time-domain waveform of virgin signal and Figure 6.33(b) shows the power spectrum of the virgin signal. As expected all the power of the signal is concentrated around the $82 \mathrm{kHz}$ frequency since, $82 \mathrm{kHz}$ transducers were used in this study.

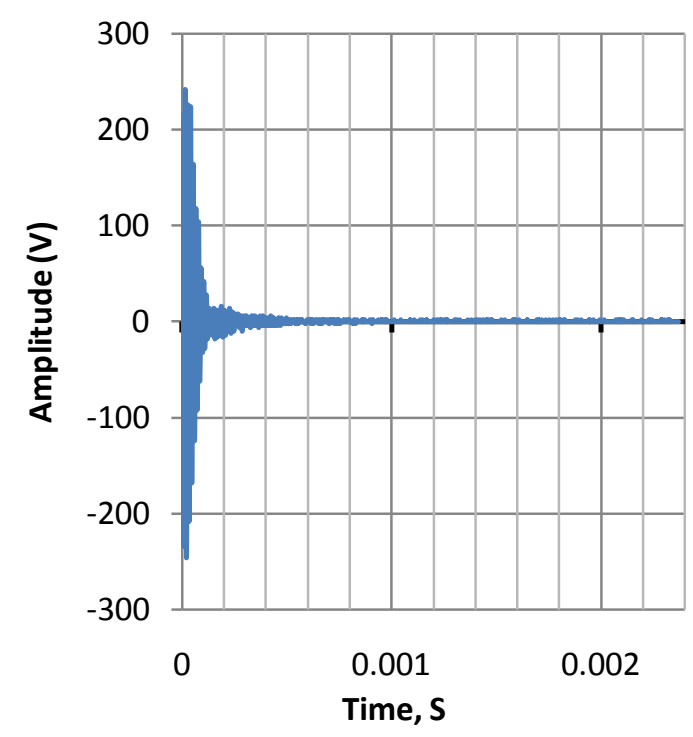

(a)

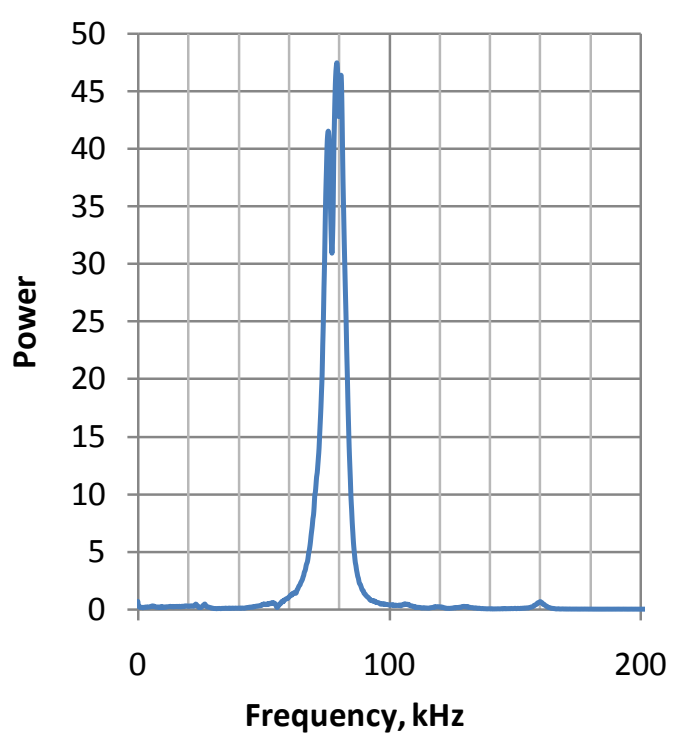

(b)

Figure 6.33: Time-domain and power spectrum forms of the virgin signal

Figure 6.34 shows the time-domain waveforms of the signal collected at the selected locations, Figure 6.35 shows the frequency spectrum of the converted timedomain waveform, and Figure 6.36 shows the power spectrum of the converted timedomain waveform. By comparing Figure 6.33 with Figure 6.34, Figure 6.35, and Figure 6.36, it is observed that the signal strength has decreased significantly from around $250 \mathrm{~V}$ to around $6 \mathrm{~V}$. Also, when we look at the Figure 6.35 it is evident that the transmission through slab induced more frequencies components within $82 \mathrm{kHz}$. This is due to the heterogeneity of the concrete sample. 


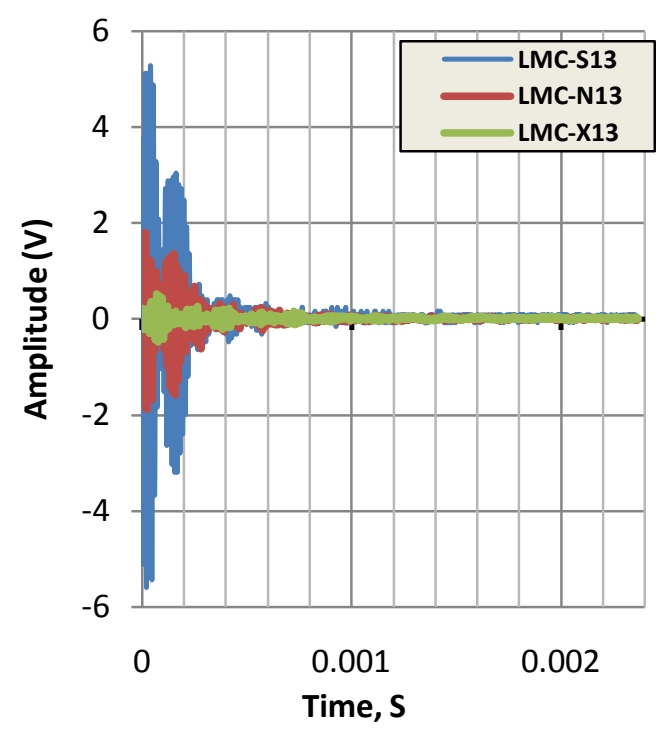

(a)

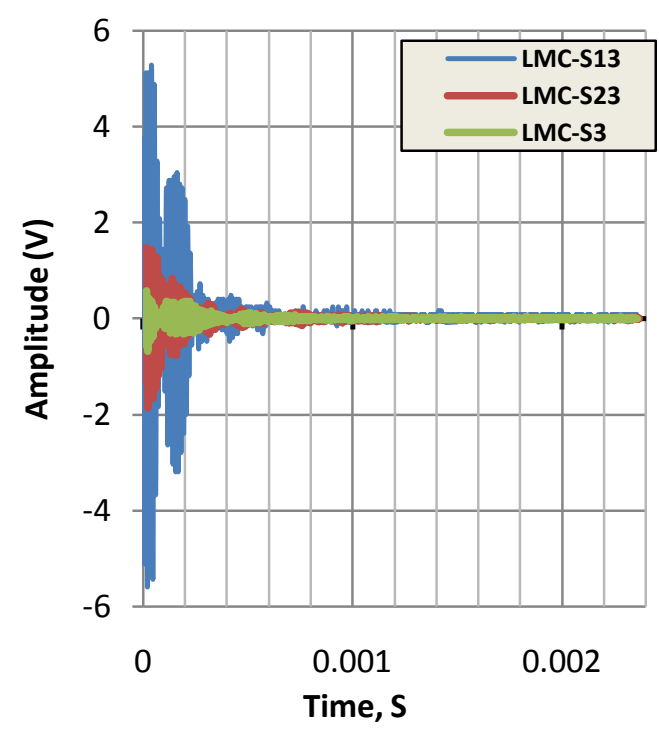

(c)

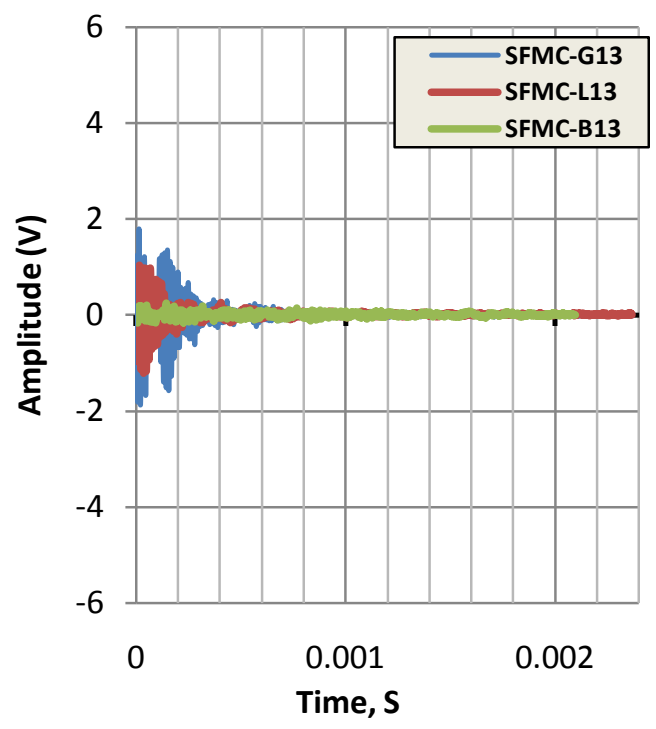

(b)

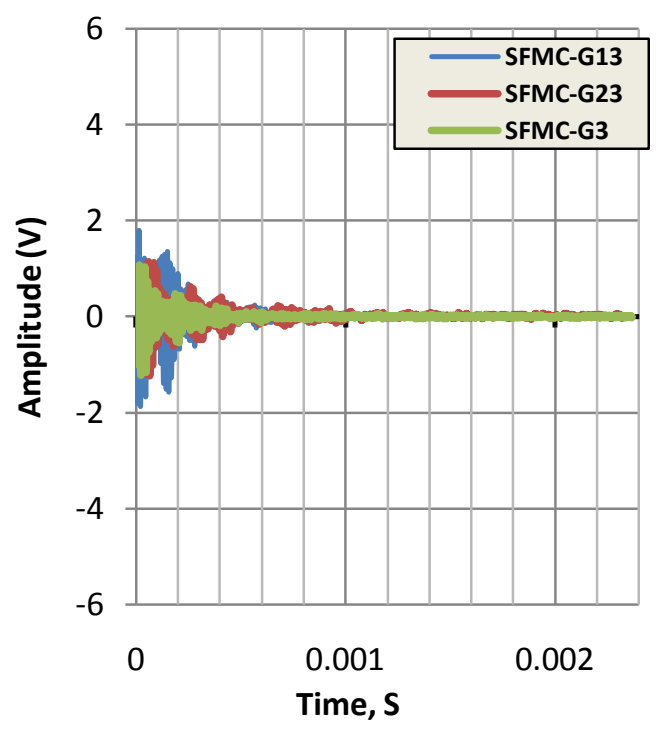

(d)

Figure 6.34: Comparison of time-domain waveforms for LMC and SFMC bi-layer slabs at different locations (refer to Figure 6.32) 


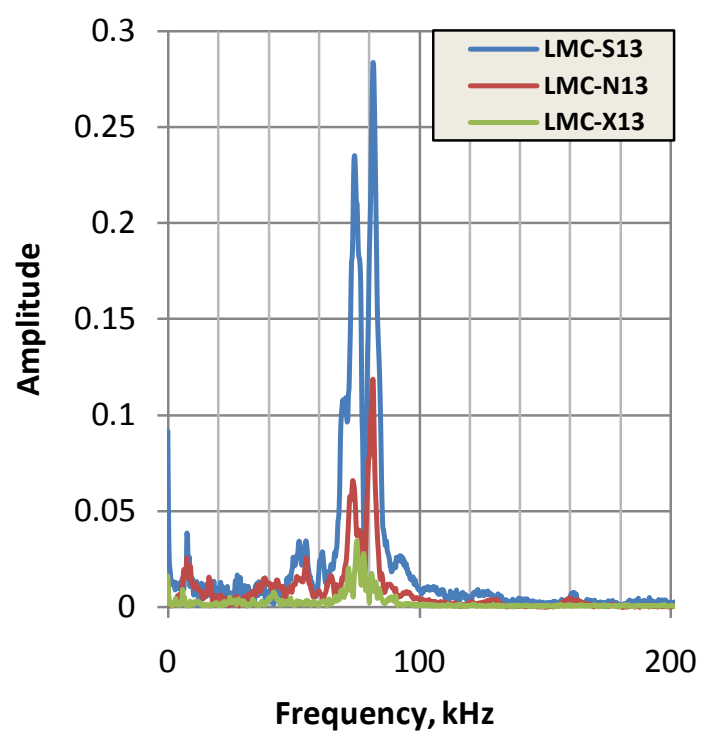

(a)

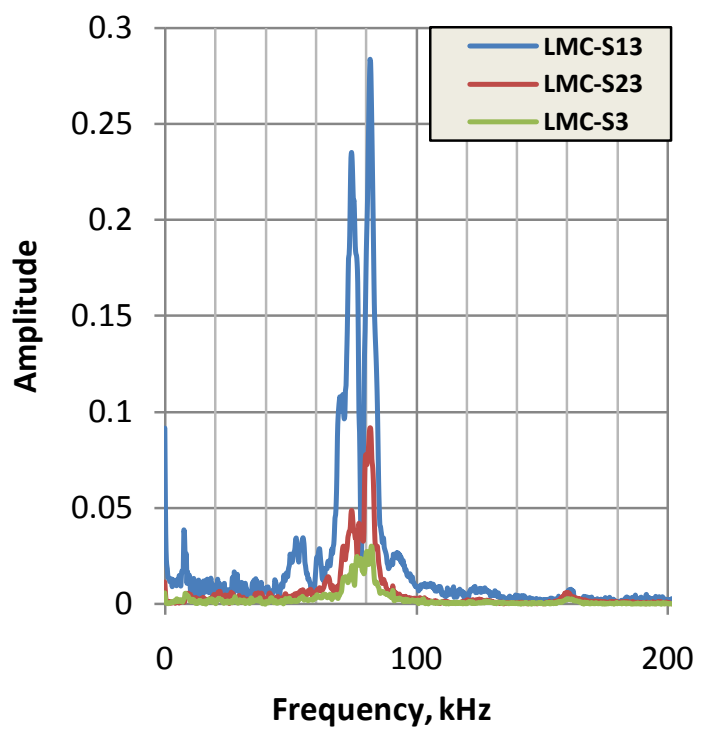

(c)

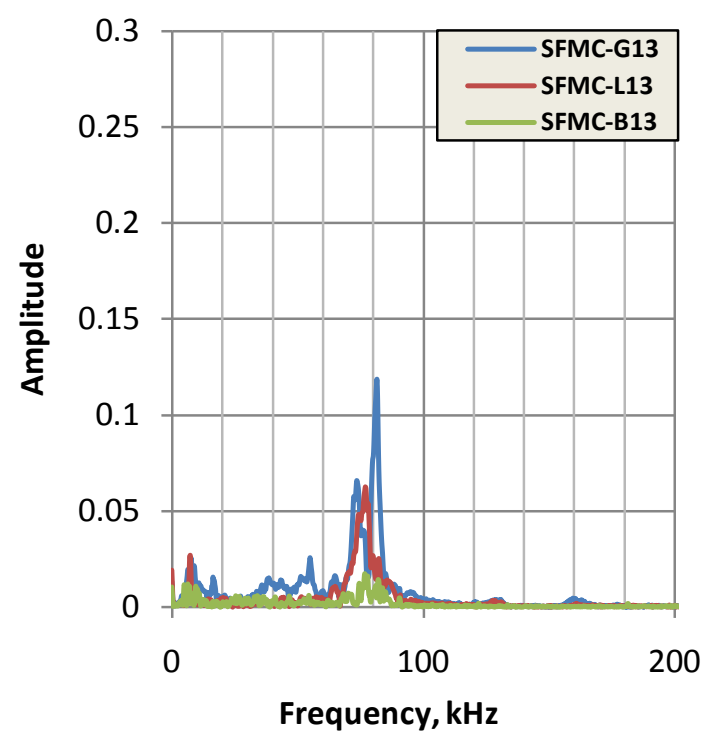

(b)

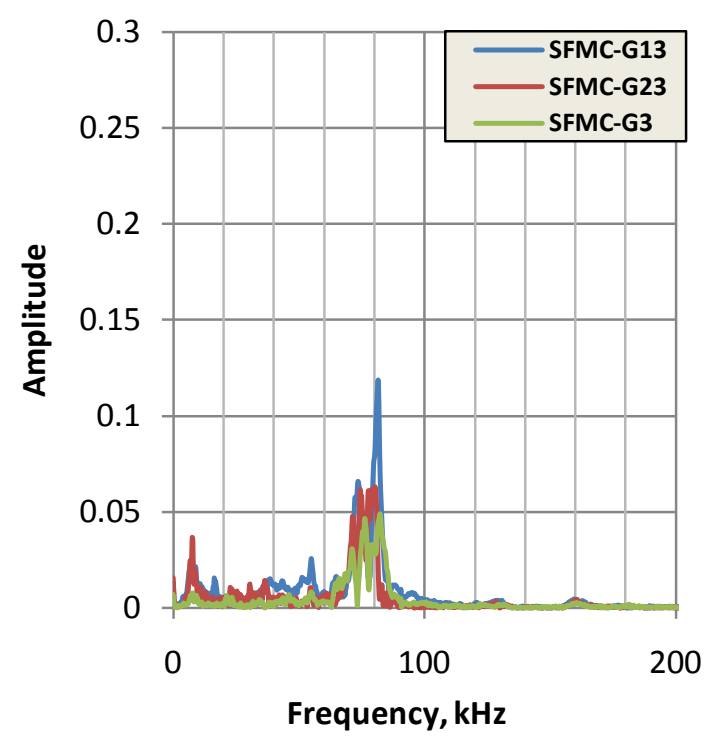

(d)

Figure 6.35: Comparison of frequency spectrums for LMC and SFMC bi-layer slabs at different locations (refer to Figure 6.32) 


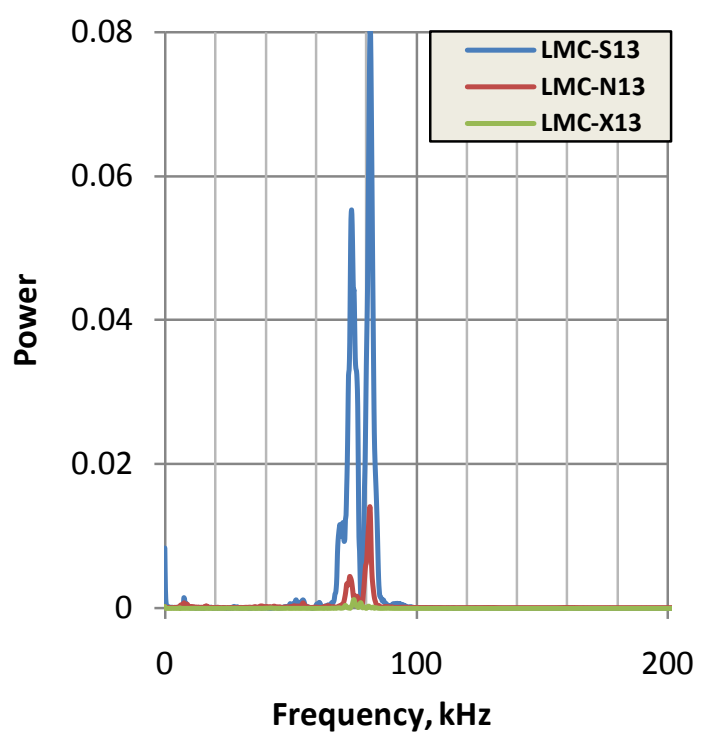

(a)

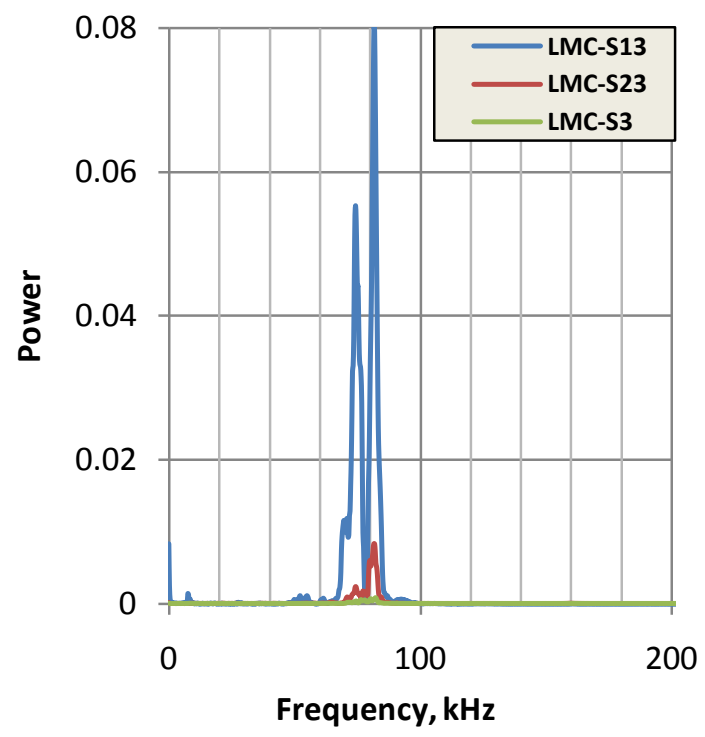

(c)

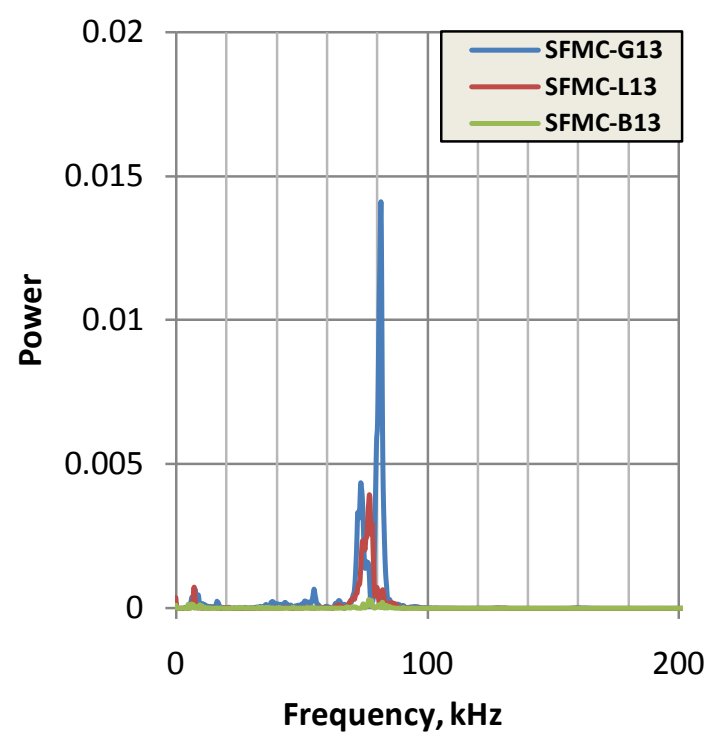

(b)

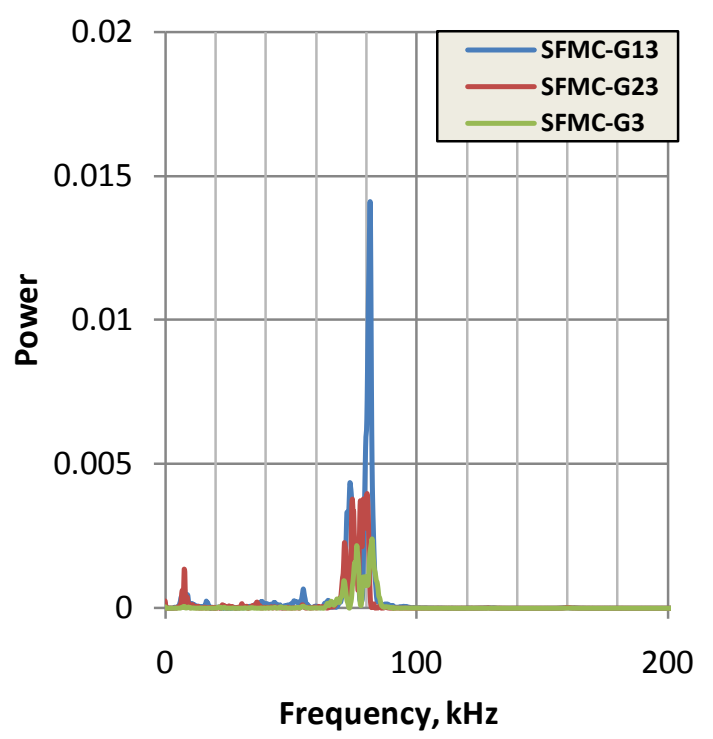

(d)

Figure 6.36: Comparison of power spectrums for LMC and SFMC bi-layer slabs at different locations (refer to Figure 6.32)

Figure 6.34 can be used for comparison of the performance of the two different types of overlay systems by looking at the amplitude of the waveform. Hong (2006) mentioned that the decrease in waveform is attributed to the deflection and diffraction of the signal at the delaminated surface. When comparing the different locations on the slab 
with LMC overlay (Figure 6.34(a) and Figure 6.34(c)) it is observed that the amplitude at location X13 (0.05 V) was least and then followed by location S3 (0.075 V), S23 (1.75 V), N13 (2 V), and S13 (5 V). Hence we can conclude that within the slab with LMC overlay location X13 was more delaminated compared to the other locations.

Similarly, When comparing the different locations on the slab with SFMC overlay (Figure 6.34(b) and Figure 6.34(d)) it is observed that the amplitude at location C13 $(0.025 \mathrm{~V})$ was least when compared to the other locations. Hence we can conclude that within the slab with SFMC overlay location C13 was more delaminated compared to the other locations.

When the amplitudes of the waveform at locations on slab with LMC overlay are compared with the amplitudes at same locations on the slab with SFMC overlay it is observed that the slab with SFMC overlay is more delaminated than slab with LMC overlay. Hence from the time-domain waveforms we can conclude that the performance of slab with LMC overlay was better than the slab with SFMC overlay. Similar conclusion was made from the UPV reading and vertical corner lifting. Higher peaks were observed by Hong (2006) when LMC overlay with saturated surface condition at the interface was used.

The above discussion can be validated by comparing the peak values of FFT and power spectrum at various locations as seen in Figure 6.35 and Figure 6.36. That is, lower the peak value (strength of the signal) indicates that the signal is attenuated and the strength of the signal is lost by the time it reaches receiving transducer. From these power spectrum graphs we can conclude that the less delamination was observed in slab with LMC overlay when compared with slab with SFMC overlay. This conclusion is in agreement with the conclusion drawn from time-domain waveform. 


\section{CHAPTER 7: CONCLUSIONS AND RECOMMENDATIONS}

\subsection{Conclusions}

Following conclusions can be drawn from the Stage-I study:

1. Compressive strength of LMC overlay concrete was always greater than that of Type K substrate concrete;

2. Dynamic modulus of elasticity was always greater than that of Type K substrate concrete;

3. Three-way ANOVA was performed on the pull-off bond strength and the results shows that, at $5 \%$ level of significance the difference in the bond strength values is not very significant;

4. Differential length change developed at the interface due to temperature changes was monitored at the interface by installing the embedment gages in substrate and overlay concretes and the results shows that lesser differential length change was observed when the bonding slurry was used at the interface. Hence we can conclude that when the bonding slurry is applied at the interface, the shear stresses developed at the interface due to volume changes can be reduced and hence delaminations can be reduced;

5. Vertical corner lifting of both slabs was monitored by installing LVDT at the corners and the results shows that the vertical displacements due to vertical corner lifting of the slab with LMC overlay was higher than that of slab with LMC-S overlay. Therefore, we can conclude that the use of bonding slurry has potentially reduced the debonding due to vertical corner lifting;

6. Indirect UPV testing was conducted to monitor the delamination profile at the interface and the results showed the constant slope of the time Vs distance plots in the case of slab with LMC-S overlay. Hence we can conclude that the use of bonding slurry at the interface has showed less delaminations when compared to the slab without bonding slurry;

7. Finally, time domain signals were converted into FFT and from the results obtained, higher peaks were observed in the case of slab with LMC-S overlay 
when compared with that of the slab with LMC overlay. Higher peaks signify the soundness of the interface. Hence we can conclude that the use of bonding slurry at the interface reduced the delamination and increased the soundness of the interface significantly.

8. Table 7.1 in the next page summarizes the conclusions of the Stage-I study. 
Table 7.1: Summary of conclusions for Stage-I study

\begin{tabular}{|c|c|c|c|c|c|c|c|c|}
\hline & \multirow{3}{*}{$\begin{array}{l}\text { Bond/tensile } \\
\text { strength }\end{array}$} & \multicolumn{4}{|c|}{ Differential length change } & \multirow{3}{*}{ Corner lifting } & \multicolumn{2}{|c|}{ UPV } \\
\hline & & \multicolumn{2}{|c|}{ Transverse } & \multicolumn{2}{|c|}{ Longitudinal } & & \multirow{2}{*}{ Indirect UPV plots } & \multirow{2}{*}{ Frequency spectrum } \\
\hline & & edge & center & edge & center & & & \\
\hline $\begin{array}{c}\text { LMC overlay } \\
\text { without } \\
\text { bonding slurry }\end{array}$ & Similar & Higher & Higher & Higher & Higher & Higher & Variable slope & Lower Peak magnitudes \\
\hline $\begin{array}{c}\text { LMC overlay } \\
\text { with bonding } \\
\text { slurry }\end{array}$ & Similar & Lower & Lower & Lower & Lower & Lower & Constant slope & Higher peak magnitudes \\
\hline Explanation & $\begin{array}{c}\text { Difference of } \\
\text { Interface bond } \\
\text { strength values } \\
\text { between LMC } \\
\text { and LMC with } \\
\text { slurry was not } \\
\text { significant at } \\
5 \% \text { level }\end{array}$ & $\begin{array}{r}\text { Always, tl } \\
\text { interfac } \\
\text { which }\end{array}$ & $\begin{array}{l}\text { differentia } \\
\text { vas less fol } \\
\text { ade the us }\end{array}$ & $\begin{array}{l}\text { gth chang } \\
\text { C-S com! } \\
\text { slurry mo }\end{array}$ & $\begin{array}{l}\text { erved at the } \\
\text { to LMC, } \\
\text { ective.. }\end{array}$ & $\begin{array}{c}\text { At the corners, vertical } \\
\text { displacement of the overlay } \\
\text { in comparison with } \\
\text { substrate concrete was } \\
\text { lower for LMC-S slab. } \\
\text { Hence, the corner lifting } \\
\text { was limited by applying } \\
\text { bonding slurry at the } \\
\text { interface. }\end{array}$ & $\begin{array}{l}\text { Constant slopes mean better } \\
\text { interface condition and vice- } \\
\text { versa. Hence, better interface } \\
\text { condition was observed when } \\
\text { bonding slurry was applied at } \\
\text { the interface. }\end{array}$ & $\begin{array}{l}\text { Higher peak magnitude implies } \\
\text { less delaminations are present. } \\
\text { Hence, when bonding slurry } \\
\text { condition was used less } \\
\text { delaminations were observed, } \\
\text { when receiver was moved } \\
\text { diagonally from center towards } \\
\text { pre-notch. }\end{array}$ \\
\hline
\end{tabular}


Following conclusions can be drawn from the Stage-II study:

1. Compressive strength of SFMC overlay concrete was always greater than that of LMC overlay and Type K substrate concrete;

2. Dynamic modulus of elasticity of LMC overlay was greater than that of SFMC overlay and Type K substrate concrete;

3. Three-way ANOVA was performed on the pull-off bond strength and the results shows that, at 5\% level of significance, the slab with LMC overlay showed higher bond strength values compared to the slab with SFMC overlay;

4. Differential length change developed at the interface due to temperature changes was monitored at the interface by installing the embedment gages in substrate and overlay concretes and the results shows that lesser differential length change was observed when the bonding slurry was used at the interface. Hence we can conclude that when the bonding slurry is applied at the interface, the shear stresses developed at the interface due to volume changes can be reduced and hence delaminations can be reduced;

5. Vertical corner lifting of both slabs was monitored by installing LVDT at the corners and the results shows that the vertical displacements due to vertical corner lifting of the slab with LMC overlay was lower than the slab with SFMC overlay;

6. Direct UPV testing was conducted at 28, 56, and 90 days to compare the delamination profile at the interface between two slabs. Results showed higher velocities on the slab with LMC overlay when compared to the slab with SFMC overlay at all the ages. Hence we can conclude that the slab with LMC overlay showed less delaminations at the interface compared to the slab with SFMC overlay;

7. Finally, time domain waveform, frequency spectrum, and power spectrum at the selected locations were compared between the two slabs. Results showed higher amplitude and higher peaks for the slab with LMC overlay when compared to the slab with SFMC overlay. Higher peaks signify the soundness of the interface. Hence we can conclude that the slab with LMC overlay showed less delamination when compared to the slab with SFMC overlay at the same locations.

8. Table 7.2 in the next summarizes the conclusions of the Stage-I study 
Table 7.2: Summary of conclusions for Stage-II study

\begin{tabular}{|c|c|c|c|c|}
\hline & \multirow{2}{*}{$\begin{array}{l}\text { Bond/tensile } \\
\text { strength }\end{array}$} & \multirow[b]{2}{*}{ Corner lifting } & \multicolumn{2}{|r|}{ UPV } \\
\hline & & & Direct UPV plots & Power spectrum \\
\hline $\begin{array}{c}\text { LMC } \\
\text { overlay }\end{array}$ & Higher & Lower & Higher velocities & Higher Peak magnitude \\
\hline $\begin{array}{l}\text { SFMC } \\
\text { overlay }\end{array}$ & Lower & Higher & Lower velocities & Lower peak magnitude \\
\hline Explanation & $\begin{array}{l}\text { Interface bond } \\
\text { strength values } \\
\text { was higher in } \\
\text { case of LMC at } \\
5 \% . \text { significance } \\
\text { level }\end{array}$ & $\begin{array}{l}\text { At the corners, vertical } \\
\text { displacement of the overlay } \\
\text { in comparison with substrate } \\
\text { concrete was lower for LMC } \\
\text { slab }\end{array}$ & $\begin{array}{l}\text { UPV quantifies the } \\
\text { soundness of the } \\
\text { interface. That is higher } \\
\text { the velocity implies the } \\
\text { sound interface with } \\
\text { less delamination and } \\
\text { vice versa. }\end{array}$ & $\begin{array}{l}\text { Higher peak magnitude implies } \\
\text { less delaminations are present. }\end{array}$ \\
\hline
\end{tabular}




\subsection{Recommendations}

Based on the present study following recommendations are made:

1) The new decks need to be matured for atleast about 6 to 7 weeks before surface preparation is started and overlays are poured.

2) The ICRI surface profile of at least number 4 or 5 is recommended for surface preparation of the new decks.

3) The surface should be saturated with water and excess water has to be removed, before pouring the overlay concrete, followed by the application of bonding slurry or agents.

4) Application of bonding slurry at the interface is highly recommended, since the bonding agents provide additional chemical bonding on the top of mechanical bonding by acting as coupling agents. This may not be required for old deck because higher mechanical bonding is ensured by hydro-demolition techniques.

5) The bonding slurry to be used at the interface should be made with same concrete with coarse aggregate removed by raking without exceeding the water/cementitious material ratios. No extra water should be added during this operation.

6) The detailed information of type, step-by-step application, and construction methodology of bonding slurry or agents will be provided after the large-scale study in proposed Phase III program.

7) Slant shear test method can be successfully used as quality control test for field match cured specimens provided the modifications are made to the test method.

8) Special care should be taken at corners and edges as those locations are found to be the most sensitive areas where failure mostly initiates.

9) Present study shows that both LMC and SFMC are viable options for overlays. Also, SFMC + shrinkage reducing admixtures overlays should be verified in the proposed Phase III.

10) Even though chain drag method is commonly used to evaluate the overlay performance after construction, it is recommended to monitor the overlaysubstrate debonding using advanced NDT techniques such as Infrared Thermography (IRT) for overlay-substrate debonding. 
11) The pull-off test is a good measure of bond strength but the variations are too high due to several reasons. The locations of the test sites should not be too closely spaced and it is recommended that this test method should be conducted at structurally less important locations since it is semi-destructive test. Soon after conducting the pull off tests, the cores should be filled up with similar concrete.

12) Finally, based on the Phase-II study, Phase-III study should be conducted in which full scale demonstration bridge needs to be constructed at different locations and evaluated for field performance. On the basis of Phase-III study, performance based specifications needs to be developed mainly emphasizing on the use of local materials and construction practices in lieu of currently used prescription based specifications. 


\section{REFERENCES}

[1] International Concrete Repair Institute, 1996, "Guide for Selecting and Specifying Concrete Surface Preparation for Sealers, Coatings, and Membranes," Guideline No. 03732, Sterling, VA, pp. 34.

[2] ACI Committee 228, 1998, "Nondestructive Test Methods for Evaluation of Concrete in Structures (ACI 228.2R-98) " American Concrete Institute, Farmington Mills, MI, pp. 62.

[3] ASTM C 1042, 1999, "Standard Test Method for Bond Strength of Latex Systems used with Concrete by Slant Shear," Annual Book of ASTM Standards, V. 04.02, West Conshohocken, PA, pp. 556-563.

[4] ASTM C 215, 2002a, "Standard Test Method for Fundamental Transverse, Longitudinal, and Torsional Resonant Frequencies of Concrete Specimens," Annual Book of ASTM Standards, V. 04.02, West Conshohocken, PA, pp. 143-149.

[5] ASTM C 597, 2002b, "Standard Test Method for Pulse Velocity Through Concrete," Annual Book of ASTM Standards, V. 04.02, West Conshohocken, PA, pp. 320-323.

[6] ASTM C 1583, 2004, "Standard Test Method for Tensile Strength of Concrete Surfaces and the Bond Strength or Tensile Strength of Concrete Repair and Overlay Materials by Direct Tension (Pull-off Method)," Annual Book of ASTM Standards, V. 04.02, West Conshohocken, PA, pp. 807-811.

[7] ASTM C 39/C 39M, 2005, "Standard Test Method for Compressive Strength of Cylindrical Concrete Specimens," Annual Book of ASTM Standards, V. 04.02, West Conshohocken, PA, pp. 22-28.

[8] Ali, M.; Kurhara, S.; and Matsui, S., 1998, "Bonding Shear Strength at the Interface between Old and New Concrete," Technology Reports of the Osaka University, 2327, april 15.

[9] Babaei, K., and Hawkins, N. M., 1990, "Performance of Bridge Deck Concrete Overlays," Extending the Life of Bridges, ASTM STP 1100, Maupin, G. W., Brown, B. C. and Lichtenstein, A. G., American Society for Testing and Materials, Philadelphia.

[10] Babaei, K., and Hawkins, N. M., 1992, "Performance of Rehabilitated/Protected Concrete Bridge Decks," Corrosion Forms and Control for Infrastructure, ASTM STP 1137, Chaker, V., American Society for Testing and Materials, Philadelphia.

[11] Calvo, L., and Meyers, M., 1991, "Overlay Materials for Bridge Decks," Concrete International Magazine, July, pp.46-47. 
[12] Chen, P.-W.; Fu, X.; and Chung, D. D. L., 1995, "Improving Bond Strength between Old and New Concrete by Adding Carbon Fibers to the New Concrete," Cement and Concrete Research, V. 25, No. 3, pp. 491-496.

[13] Daponte, P.; Maceri, F.; and Olivito, R. S., 1995, "Ultrasonic Signal-Processing Techniques for the Measurement of Damage Growth in Structural Materials," IEEE Transactions on Instrumentation and Measurement, V. 44, No. 6, December, pp. 10031008.

[14] Delatte, N. J.; Williamson, M. S.; and Fowler, D. W., 2000, "Bond Strength Development with Maturity of High-Early-Strength Bonded Concrete Overlays," ACI Materials Journal, V. 97, No. 2, March-April, pp. 201-207.

[15] Detwiler, R. J.; Kojundic, T.; and Fidjestol, P., 1997, "Evaluation of Bridge Deck Overlays," American Concrete Institute, V. 19, No. 8, August, pp. 43-45.

[16] Fitch, M. G., and Abdulshafi, O. A., 1998, "Field and Laboratory Evaluation of Silica Fume Modified Concrete Bridge Deck Overlays in Ohio," Transportation Research Record, No. 1610, pp. 20-27,

[17] Granju, J. L., 1996, "Thin Bonded Overlays," Advanced Cement Based Materials, V. 4, No., pp. 21-47.

[18] Halvorsen, G. T., 1993, "Bridge Deck Overlays," Concrete Construction Magazine, June, pp.415-419.

[19] Hindo, K. R., 1990, "In-Place Bond Testing and Surface Preparation of Concrete," Concrete International, April, pp.46-48.

[20] Hong, T., 2006, "Edge Curling Effect on Interface Delamination of Concrete Overlays for Bridge Decks," Master of Science, Civil and Environmental Engineering, West Virginia University, Morgantown, WV.

[21] Issa, M. A.; Alhassan, M. A.; and Shabila, H. I., 2007, "Low-Cycle Fatigue Testing of High-Performance Concrete Bonded Overlay-Bridge Deck Slab Systems," Journal of Bridge Engineering, V. 12, No. 4, July/August, pp. 419-428.

[22] Jones, R., and Facaoaru, I., 1969, "Recommendations for Testing Concrete by the Ultrasonnic Pulse Method," Materiaux et Constructions, V. 2, No. 10, pp. 275-284.

[23] Karpinski (2004), A., Factorial ANOVA: Higher Order ANOVAs, Ed,

[24] Kennedy, J. B., and Neville, A. M., 1986, Basic Statistical Methods for Engineers and Scientists, Third Ed, Harper \& Row, Publishers, New York. 
[25] Kim, S.-M.; Nelson, P. K.; Ruiz, M.; Rasmussen, R. O.; and Turner, D., 2003, "Early-Age Behavior of Concrete Overlays on Continuously Reinforced Concrete Pavements," Transportation Research Record, No. 1823, pp. 80-92,

[26] Kuhlmann, L., 1991, "Cracks in Latex-Modified Concrete Overlays - How They Get There, How Serious They Are, and What To Do About Them," Midland, Michigan, 48640.

[27] Kuhlmann, L. A., 1990, "Test Method for Measuring the bond Strength of LatexModified Concrete and Mortar," ACI Materials Journal, V. 87, No. 4, July - August, pp. 387-394.

[28] Lau, C. M.; Fwa, T. F.; and Paramasivam, P., 1994, "Interface Shear Stress in Overlaid Concrete Pavements," Journal of Transportation Engineering, V. 120, No. 2, March-April, pp. 163-177.

[29] Luo, S., 2002, "Evaluations of Concrete Overlays for Bridge Deck Applications," Master of science, Department of Civil and Environmental Engineering, West Virginia University, Morgantown, WV.

[30] Luther, M. D., 1988, "Silica-Fume (Microsilica) Concrete in Bridges in the United States," Transportation Research Record, No. 1204, pp. 11-20,

[31] Mailvaganam, N.; Springfield, J.; Repette, W.; and Taylor, D., 2000, "Curling of Concrete Slabs on Grade," Construction Technology Update, No., pp. http://irc.nrccnrc.gc.ca/pubs/ctus/44_e.html\#ref2.

[32] Mailvaganam, N. P.; Pye, G. B.; and Arnott, M. R., 1998, "Surface Preparation of the Concrete Substrate," Construction Technology, No. 24, pp. http://irc.nrccnrc.gc.ca/pubs/ctus/24_e.html.

[33] Malhotra, V. M., and Carino, N. J., 2004, Handbook on Nondestructive Testing of Concrete, Second Ed.

[34] Momayez, A.; Ramezanianpour, A. A.; Rajaie, H.; and Ehsani, M. R., 2004, "BiSurface Shear Test for Evaluating Bond between Existing and New Concrete," ACI Materials Journal, V. 101, No. 2, March-April, pp. 99-106.

[35] Montgomery, D. C., 2005, Design and Analysis of Experiments, 6 Ed, John Wiley \& Sons, New York.

[36] Paulsson, J., and Silfwerband, J., 1998, "Durability of Repaired Bridge Deck Overlays," Concrete International, February, pp.76-82.

[37] Promboon, Y., and Lund, J., "Nondestructive Evaluation (NDE) Methods for Quality Assurance of Epoxy Injection Crack Repairs." 
[38] Ramakrishnan, V., 2000, "Tensile Bond Strength of a High Performance Concrete Bridge Deck Overlay," Sturgis, South Dakota, Project \# 9904, February.

[39] Ray, I.; Davalos, J. F.; and Luo, S., 2005, "Interface Evaluations of OverlayConcrete Bi-Layer Composites by a Direct Shear Test Method," Cement and Concrete Composites, V. 27, No., pp. 339-347.

[40] Ray, I.; Davalos, J. F.; and Sun, Z., 2008, "Design of Experiment and Statistical Analysis of Bond Strength Test of Concrete," International Journal of Materials and Product Technology, in press.

[41] Shin, H.-C., and Lange, D. A., 2004, "Effects of Shrinkage and Temperature in Bonded Concrete Overlays," ACI Materials Journal, V. 101, No. 5, September-October, pp. 358-364.

[42] Silfwerbrand, J., 2003, "Shear Bond Strength in Repaired Concrete Structures," Materials and Structures, V. 36, No., July, pp. 419-424.

[43] Smith, A., 1991, "Overlaying Concrete Bridge Decks with Polymer Concrete," Concrete Construction Magazine, April, pp.325-332.

[44] Sprinkel, M. M., 1988, "Predicting Plastic Shrinkage Cracking in LMC Overlays," Concrete Construction Magazine, July, pp.672-674.

[45] Sprinkel, M. M., 1997, "Preparing Bridge Decks for Overlays," Concrete Digest, V. 8, No. 5, pp. 242-247.

[46] Sprinkel, M. M., 2004, "Performance Specification for High Strength Concrete Overlays on Bridges," Virginia Transportation Research Council, Charlottesville, VA, VTRC 05-R2, August.

[47] Sprinkel, M. M., 2005, "Latex-Modified Concrete Overlay Containing Type K Cement," Virginia Transportation Research Council, Charlottesville, VA 22903, FHWA/VTRC 05-R26, April.

[48] Sprinkel, M. M., and Ozyildirim, C., 2000, "Evaluation of Hydraulic Cement Overlays Placed on Three Pavements in Virginia," Virginia Transportation Research Council, Charlottesville, VA, VTRC 01-R2, August.

[49] Tang, F.-F., 2000, "Overlay for Concrete Segmental Box-Girder Bridges," Journal of Bridge Engineering, V. 5, No. 4, November, pp. 311-321.

[50] Tarricone, P., 1992, "Overlays on Deck," Civil Engineering Magazine, September, pp.42-45. 
[51] Tshegg, E. K.; Ingruber, M.; Surberg, C. H.; and Munger, F., 2000, "Factors Influencing Fracture Behavior of Old-New Concrete Bonds," ACI Materials Journal, V. 97, No. 4, July-August, pp. 447-453.

[52] Wall, J. S., and Shrive, N. G., 1988, "Factors Affecting Bond between New and Old Concrete," ACI Materials Journal, V. 85, No., March-April, pp. 117-125.

[53] Wallace, M., 1987, "Overlaying Decks with LMC," Concrete Construction Magazine, December, pp.1027-1033.

[54] Warner, J.; Bhuyan, S.; Smoak, W. G.; Hindo, K. R.; and Sprinkel, M. M., 1998, "Surface Preparation for Overlays," Concrete International, V. 20, No. 5, May, pp. 4346.

[55] Yaman, I. O.; Inci, G.; Yesiller, N.; and Aktan, H. M., 2001, "Ultrasonic Pulse Velocity in Concrete using Direct and Indirect Transmission," ACI Materials Journal, V. 98, No. 6, November-December, pp. 450-457.

[56] Yun, K.-K.; Kim, K.-H.; Jeong, W.-K.; and Kim, S.-K., 2007, "Causes and Prevention of Bridge Deck Cracking with Very-Early Strength Latex-Modified Concrete," Transportation Research Annual Meeting, No. 07-0318, pp. 22,

[57] Yun, K.-K.; Kim, S.-H.; Jeong, W.-K.; and Kim, K. W., 2004, "In Situ Criteria of Pull-Off Test for Measuring Bond Strength of Latex-Modified Concrete Overlay," Journal of the Transportation Research Record, No. 1893, National Research Council, Washington D.C., pp. 37-45,

[58] Zhang, J., and Li, V. C., 2002, "Monotonic and Fatigue Performance in Bending of Fiber-Reinforced Engineered Cementetious Composites in Overlay System," Cement and Concrete Research, V. 32, No., pp. 415-423. 
APPENDIX A 


\section{Pull-Off Test Results}

\section{$\underline{\text { Stage-I }}$}

\section{Data Aquisition \\ System}

A B C D E F G

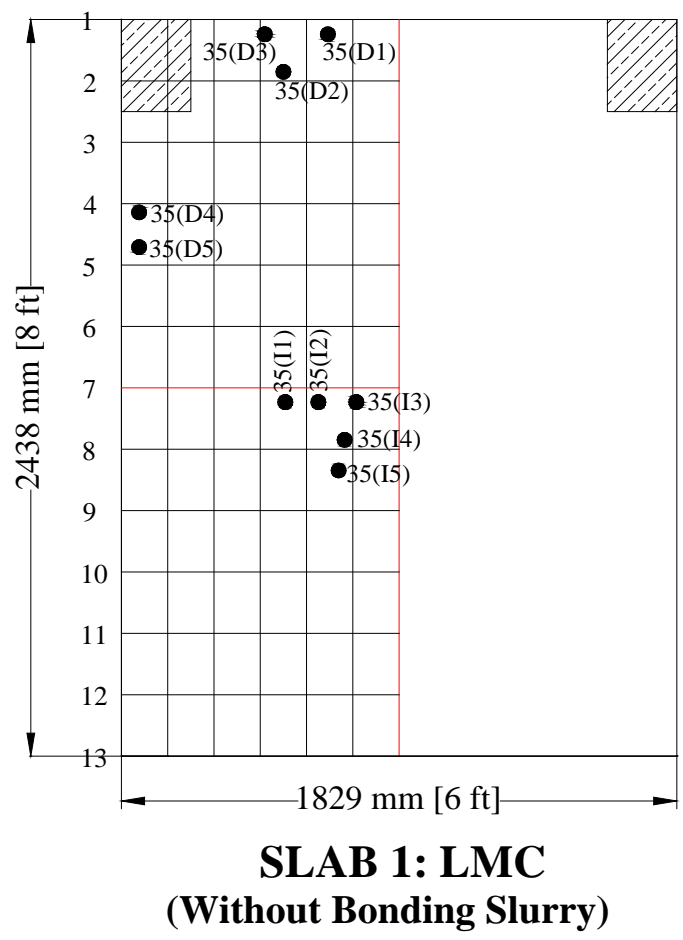

Pre-Notch $\longrightarrow$ G H I J K L M

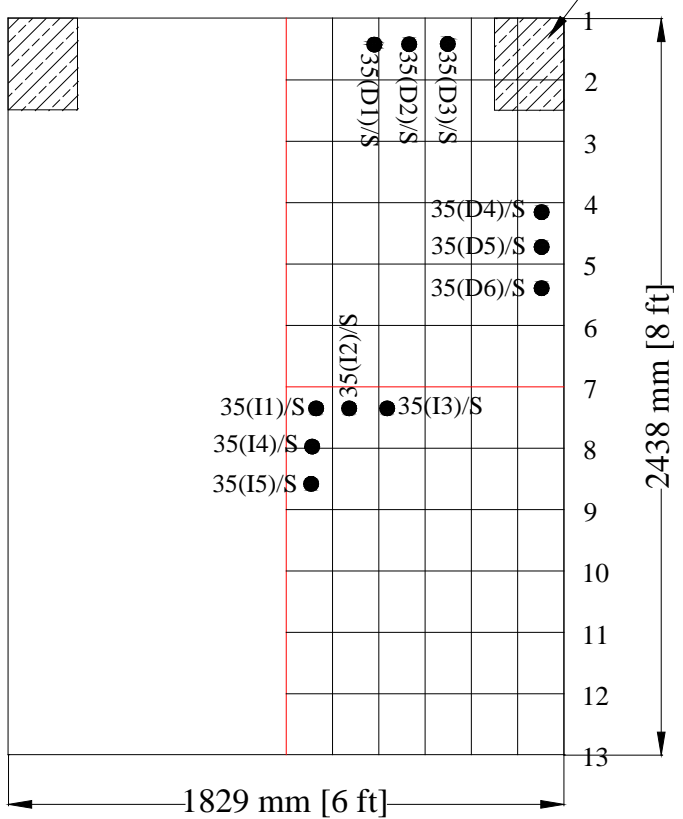

SLAB 2: LMC-S (With Bonding Slurry)

Figure A.1: 35 days pull-off locations on LMC and LMC-Slab of Stage-I

Table A.1: 35 days pull-off data of LMC and LMC-S slabs in Stage-I

\begin{tabular}{|c|c|c|c|c|}
\hline \multicolumn{5}{|c|}{ Slab 1: LMC (Without Bonding Slurry) } \\
\hline $\begin{array}{c}\text { Core } \\
\text { No. }\end{array}$ & $\begin{array}{l}\text { Failure } \\
\text { load (N). }\end{array}$ & $\begin{array}{c}\text { Area } \\
\left(\mathrm{mm}^{2}\right)\end{array}$ & $\begin{array}{l}\text { Tensile } \\
\text { stress } \\
\text { (MPa) }\end{array}$ & $\begin{array}{c}\text { Mode } \\
\text { of } \\
\text { failure }\end{array}$ \\
\hline 35(D1) & 2882 & 1551.8 & 1.86 & $\mathrm{i}$ \\
\hline 35(D2) & 2629 & 1551.8 & 1.69 & $\mathrm{~s}$ \\
\hline 35(D3) & 2104 & 1551.8 & 1.36 & $\mathrm{~S}$ \\
\hline 35(D4) & 3688 & 1551.8 & 2.38 & $\mathrm{~s}$ \\
\hline 35(D5) & 1637 & 1551.8 & 1.05 & $\mathrm{i}$ \\
\hline 35(I1) & 4261 & 1551.8 & 2.75 & 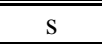 \\
\hline $35(\mathrm{I} 2)$ & 3092 & 1551.8 & 1.99 & $\mathrm{i}$ \\
\hline 35(I3) & 3817 & 1551.8 & 2.46 & $\mathrm{i}$ \\
\hline $35(\mathrm{I} 4)$ & 3505 & 1551.8 & 2.26 & $\mathrm{~s}$ \\
\hline 35(I5) & 4208 & 1551.8 & 2.71 & $\mathrm{~s}$ \\
\hline
\end{tabular}

\begin{tabular}{|c|c|c|c|c|}
\hline \multicolumn{5}{|c|}{ Slab 2: LMC-S (With Bonding Slurry) } \\
\hline $\begin{array}{l}\text { Core } \\
\text { No. }\end{array}$ & $\begin{array}{c}\text { Failure } \\
\text { load } \\
\text { (N). }\end{array}$ & $\begin{array}{c}\text { Area } \\
\left(\mathbf{m m}^{2}\right)\end{array}$ & $\begin{array}{c}\text { Tensile } \\
\text { stress } \\
\text { (MPa) }\end{array}$ & $\begin{array}{l}\text { Mode of } \\
\text { failure }\end{array}$ \\
\hline $35(\mathrm{D} 1) / \mathrm{S}$ & - & - & - & C \\
\hline $35(\mathrm{D} 2) / \mathrm{S}$ & 1063 & 1551.8 & 0.69 & $\mathrm{~S}$ \\
\hline 35(D3)/S & 1913 & 1551.8 & 1.23 & $\mathrm{i}$ \\
\hline $35(\mathrm{D} 4) / \mathrm{S}$ & 3274 & 1551.8 & 2.11 & $\mathrm{i}$ \\
\hline 35(D5)/S & 3897 & 1551.8 & 2.51 & 0 \\
\hline 35(I1)/S & 11170 & "1551.8 & 0.75 & $\bar{S}$ \\
\hline $35(\mathrm{I} 2) / \mathrm{S}$ & 4310 & 1551.8 & 2.78 & $\mathrm{i}$ \\
\hline $35(\mathrm{I} 3) / \mathrm{S}$ & 3790 & 1551.8 & 2.44 & $\mathrm{i}$ \\
\hline $35(\mathrm{I} 4) / \mathrm{S}$ & 3843 & 1551.8 & 2.48 & $\mathrm{i}$ \\
\hline $35(\mathrm{I} 5) / \mathrm{S}$ & 3999 & 1551.8 & 2.58 & $\mathrm{i}$ \\
\hline
\end{tabular}


A $B$ C $D$ D E F

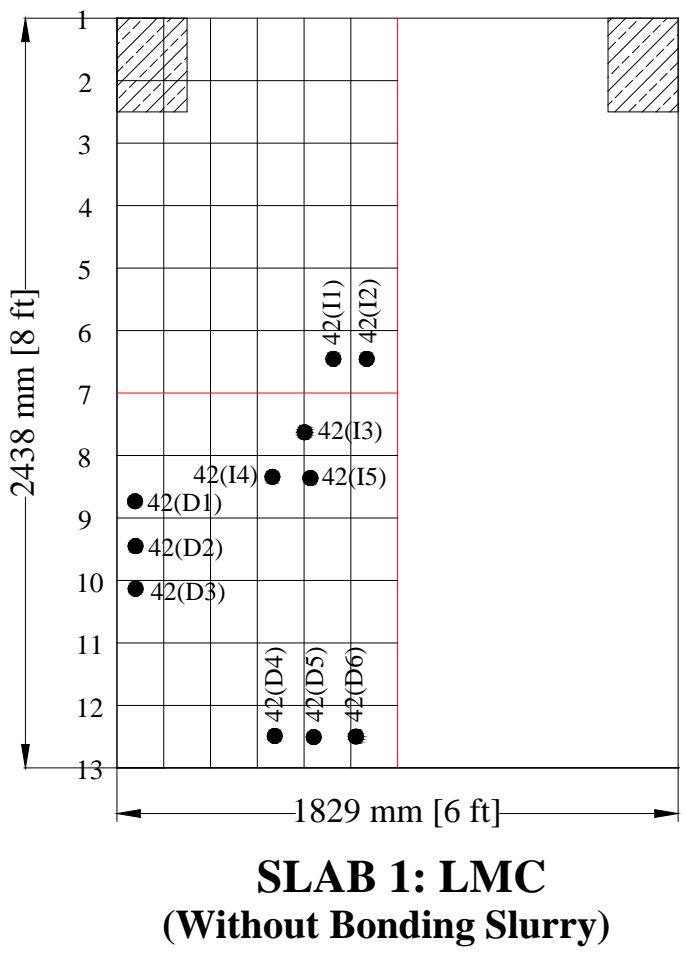

Pre-Notch $\longrightarrow$

G H I J K L M

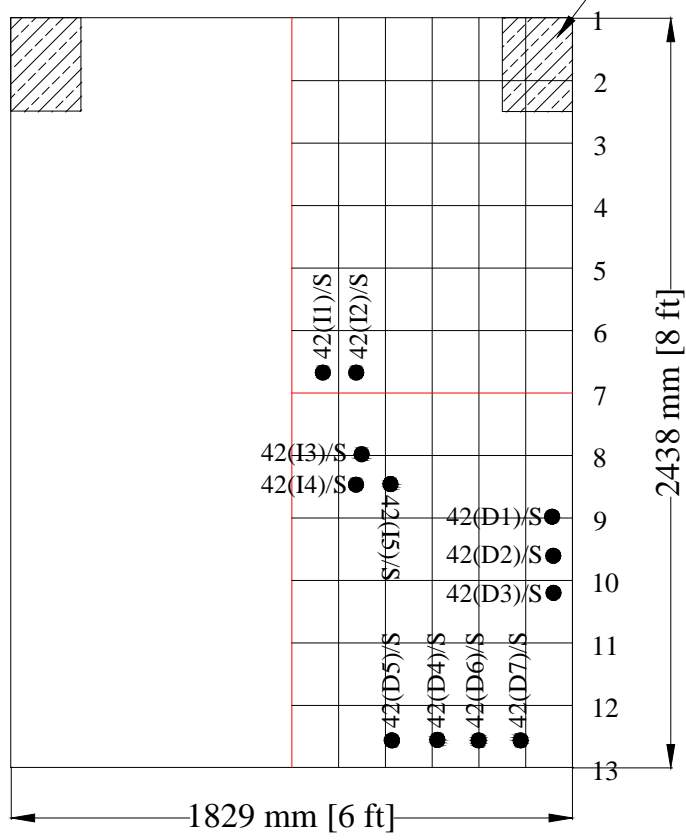

SLAB 2: LMC-S (With Bonding Slurry)

Figure A.2: 42 days pull-off locations on LMC and LMC-S slabs of Stage-I

Table A.2: 42 days pull-off data of LMC and LMC-S slabs in Stage-I

\begin{tabular}{|c|c|c|c|c|}
\hline \multicolumn{4}{|c|}{ Slab 1: LMC (Without Bonding Slurry) } \\
\hline $\begin{array}{c}\text { Core } \\
\text { No. }\end{array}$ & $\begin{array}{c}\text { Failure } \\
\text { load } \\
\text { (N). }\end{array}$ & $\begin{array}{c}\text { Area } \\
\left.\text { (mm }^{2}\right)\end{array}$ & $\begin{array}{c}\text { Tensile } \\
\text { stress } \\
\text { (MPa) }\end{array}$ & $\begin{array}{c}\text { Mode } \\
\text { of } \\
\text { failure }\end{array}$ \\
\hline 42(D1) & 2882 & 1551.8 & 1.86 & i \\
\hline 42(D2) & 3092 & 1551.8 & 1.99 & $\mathrm{i}$ \\
\hline 42(D3) & 3559 & 1551.8 & 2.29 & $\mathrm{~s}$ \\
\hline 42(D5) & 2727 & 1551.8 & 1.76 & $\mathrm{i}$ \\
\hline 42(D6) & 3999 & 1551.8 & 2.58 & $\mathrm{o}$ \\
\hline \hline 42(I1) & 3532 & 1551.8 & 2.28 & $\mathrm{~s}$ \\
\hline 42(I2) & 3301 & 1551.8 & 2.13 & $\mathrm{i}$ \\
\hline 42(I3) & 3923 & 1551.8 & 2.53 & $\mathrm{~s}$ \\
\hline 42(I4) & 2077 & 1551.8 & 1.34 & $\mathrm{e}$ \\
\hline 42(I5) & 3221 & 1551.8 & 2.08 & $\mathrm{e}$ \\
\hline
\end{tabular}

\begin{tabular}{|c|c|c|c|c|}
\hline \multicolumn{5}{|c|}{ Slab 2: LMC-S (With Bonding Slurry) } \\
\hline Core No. & $\begin{array}{c}\text { Failure } \\
\text { load } \\
\text { (N). }\end{array}$ & $\begin{array}{c}\text { Area } \\
\left(\mathbf{m m}^{2}\right)\end{array}$ & $\begin{array}{c}\text { Tensile } \\
\text { stress } \\
\text { (MPa) }\end{array}$ & $\begin{array}{c}\text { Mode } \\
\text { of } \\
\text { failure }\end{array}$ \\
\hline $42(\mathrm{D} 1) / \mathrm{S}$ & 1739 & 1551.8 & 1.12 & $\mathrm{i}$ \\
\hline $42(\mathrm{D} 2) / \mathrm{S}$ & 1041 & 1551.8 & 0.67 & $\mathrm{~s}$ \\
\hline 42(D3)/S & - & - & - & C \\
\hline $42(\mathrm{D} 4) / \mathrm{S}$ & - & - & - & $-\mathrm{C}$ \\
\hline 42(D5)/S & - & - & - & $-\mathrm{C}$ \\
\hline 42(D6)/S & 1846 & 1551.8 & 1.19 & $\mathrm{i}$ \\
\hline 42(D7)/S & 3661 & 1551.8 & 2.36 & $\mathrm{i}$ \\
\hline 42(I1)/S & 3768 & 1551.8 & 2.43 & 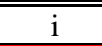 \\
\hline $42(\mathrm{I} 2) / \mathrm{S}$ & 2313 & 1551.8 & 1.49 & 0 \\
\hline $42(\mathrm{I} 3) / \mathrm{S}$ & 3741 & 1551.8 & 2.41 & $\mathrm{i}$ \\
\hline $42(\mathrm{I} 4) / \mathrm{S}$ & 3140 & 1551.8 & 2.02 & $\mathrm{~S}$ \\
\hline $42(\mathrm{I} 5) / \mathrm{S}$ & 1793 & 1551.8 & 1.16 & 0 \\
\hline
\end{tabular}




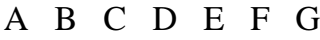

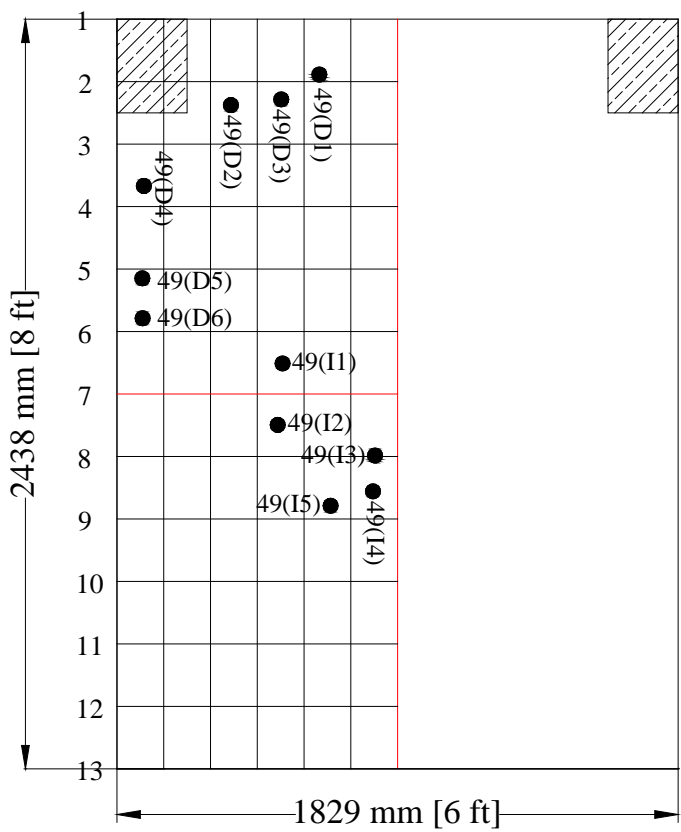

SLAB 1: LMC (Without Bonding Slurry)
G H I J K L M

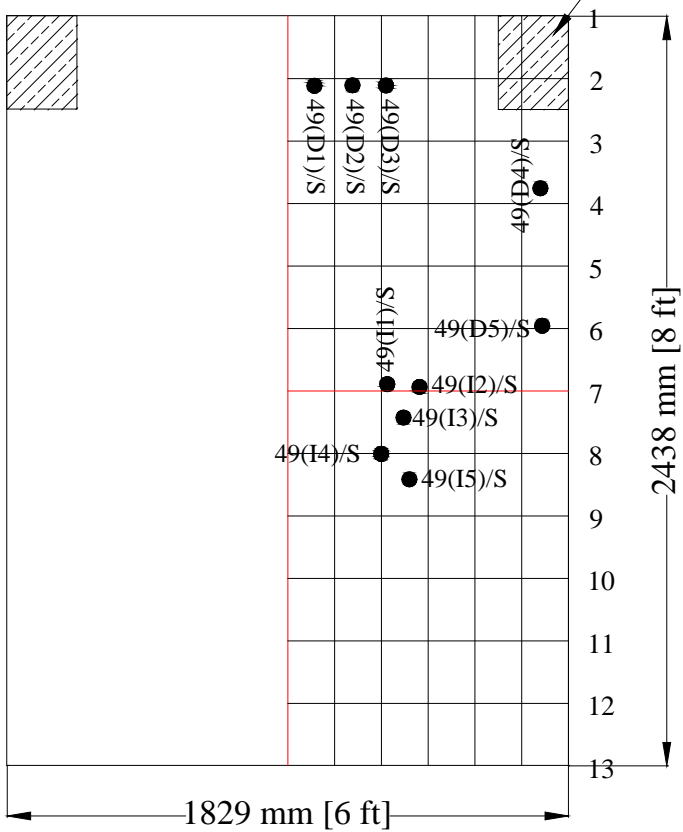

SLAB 2: LMC-S (With Bonding Slurry)

Figure A.3: 49 days pull-off locations on LMC and LMC-S slabs for Stage-I

Table A.3: 49 days pull-off data of LMC and LMC-S slabs in Stage-I

\begin{tabular}{|c|c|c|c|c|}
\hline \multicolumn{5}{|c|}{ Slab 1: LMC (Without Bonding Slurry) } \\
\hline $\begin{array}{c}\text { Core } \\
\text { No. }\end{array}$ & $\begin{array}{c}\text { Failure } \\
\text { load } \\
\text { (N). }\end{array}$ & $\begin{array}{c}\text { Area } \\
\left.\text { (mm }^{\mathbf{2}}\right)\end{array}$ & $\begin{array}{c}\text { Tensile } \\
\text { stress } \\
\text { (MPa) }\end{array}$ & $\begin{array}{c}\text { Mode } \\
\text { of } \\
\text { failure }\end{array}$ \\
\hline 49(D1) & 3505 & 1551.8 & 2.26 & $\mathrm{i}$ \\
\hline 49(D2) & 2882 & 1551.8 & 1.86 & $\mathrm{i}$ \\
\hline 49(D3) & 4884 & 1551.8 & 3.15 & $\mathrm{o}$ \\
\hline 49(D4) & - & - & - & $\mathrm{C}$ \\
\hline 49(D5) & 3559 & 1551.8 & 2.29 & $\mathrm{~s}$ \\
\hline 49(D6) & 4364 & 1551.8 & 2.81 & $\mathrm{~s}$ \\
\hline \hline 49(I1) & 3741 & 1551.8 & 2.41 & $\mathrm{i}$ \\
\hline 49(I2) & 4128 & 1551.8 & 2.66 & $\mathrm{~s}$ \\
\hline 49(I3) & 3741 & 1551.8 & 2.41 & $\mathrm{~s}$ \\
\hline 49(I4) & 2104 & 1551.8 & 1.36 & $\mathrm{i}$ \\
\hline 49(I5) & 2157 & 1551.8 & 1.39 & $\mathrm{~s}$ \\
\hline
\end{tabular}

\begin{tabular}{|c|c|c|c|c|}
\hline \multicolumn{5}{|c|}{ Slab 2: LMC-S (With Bonding Slurry) } \\
\hline Core No. & $\begin{array}{c}\text { Failure } \\
\text { load } \\
\text { (N). }\end{array}$ & $\begin{array}{c}\text { Area } \\
\text { (mm }^{2} \text { ) }\end{array}$ & $\begin{array}{c}\text { Tensile } \\
\text { stress } \\
\text { (MPa) }\end{array}$ & $\begin{array}{c}\text { Mode } \\
\text { of } \\
\text { failure }\end{array}$ \\
\hline 49(D1)/S & 2233 & 1551.8 & 1.44 & $\mathrm{~S}$ \\
\hline 49(D2)/S & 2442 & 1551.8 & 1.57 & $\mathrm{i}$ \\
\hline 49(D3)/S & 3430 & 1551.8 & 2.21 & $\mathrm{i}$ \\
\hline 49(D4)/S & 2335 & 1551.8 & 1.50 & $\mathrm{~S}$ \\
\hline 49(D5)/S & 2286 & 1551.8 & 1.47 & $\mathrm{~S}$ \\
\hline \hline 49(I1)/S & 2700 & 1551.8 & 1.74 & $\mathrm{i}$ \\
\hline 49(I2)/S & 2780 & 1551.8 & 1.79 & $\mathrm{~s}$ \\
\hline $49(\mathrm{I} 3) / \mathrm{S}$ & 1690 & 1551.8 & 1.09 & $\mathrm{0}$ \\
\hline $49(\mathrm{I} 4) / \mathrm{S}$ & 1197 & 1551.8 & 0.77 & $\mathrm{~S}$ \\
\hline $49(\mathrm{I} 5) / \mathrm{S}$ & 2598 & 1551.8 & 1.67 & $\mathrm{~S}$ \\
\hline
\end{tabular}




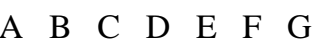

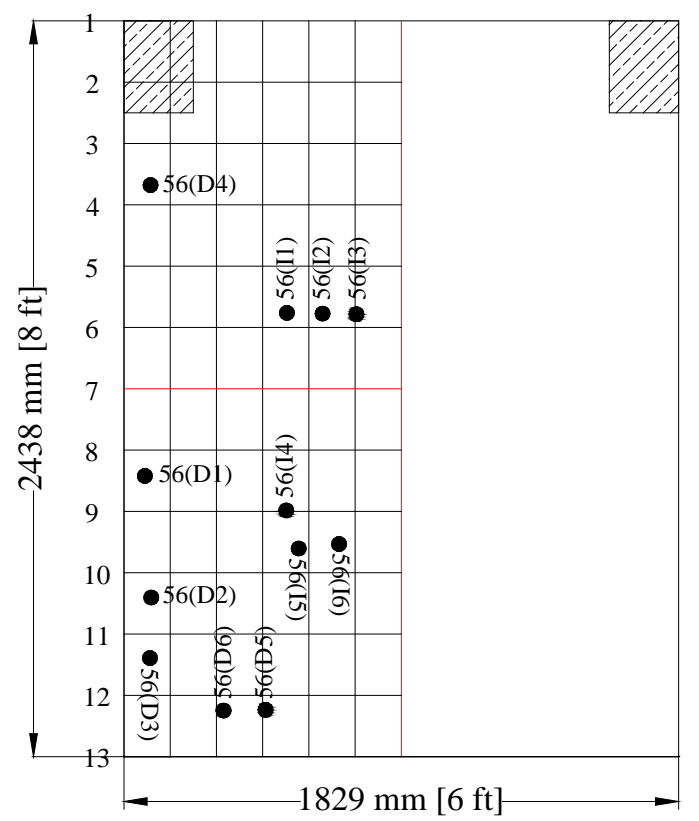

SLAB 1: LMC (Without Bonding Slurry)
Pre-Notch -

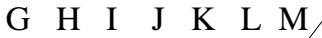

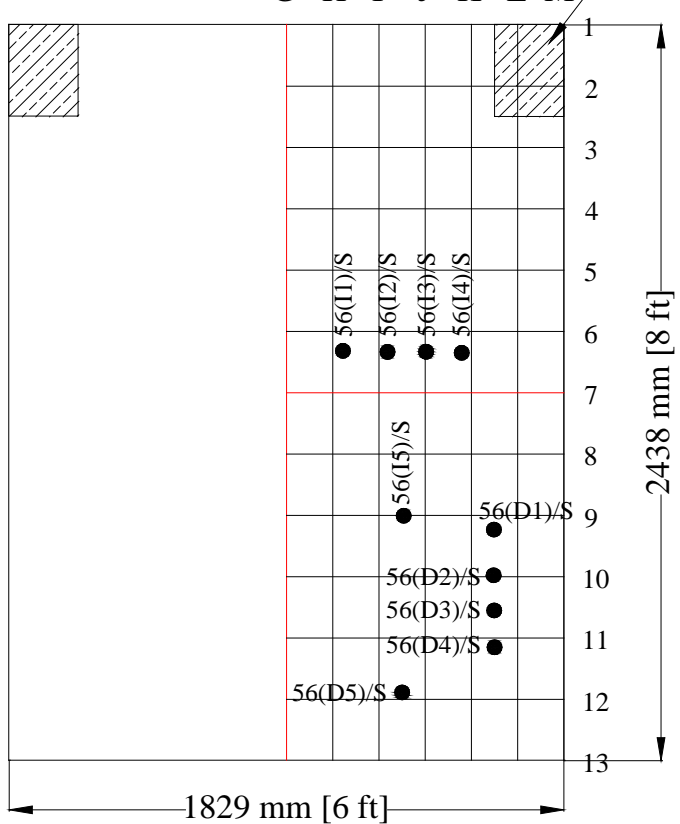

SLAB 2: LMC-S

(With Bonding Slurry)

Figure A.4: 56 days pull-off locations on LMC and LMC-S slabs of Stage-I

Table A.4: 56 days pull-off data of LMC and LMC-S slabs in Stage-I

\begin{tabular}{|c|c|c|c|c|}
\hline \multicolumn{5}{|c|}{ Slab 1: LMC (Without Bonding Slurry) } \\
\hline $\begin{array}{c}\text { Core } \\
\text { No. }\end{array}$ & $\begin{array}{c}\text { Failure } \\
\text { load } \\
\text { (N). }\end{array}$ & $\begin{array}{c}\text { Area } \\
\text { (mm2) }\end{array}$ & $\begin{array}{c}\text { Tensile } \\
\text { stress } \\
\text { (MPa) }\end{array}$ & $\begin{array}{c}\text { Mode } \\
\text { of } \\
\text { failure }\end{array}$ \\
\hline 56(D1) & 3714 & 1551.8 & 2.39 & s \\
\hline 56(D2) & 3430 & 1551.8 & 2.21 & i \\
\hline 56(D3) & 2807 & 1551.8 & 1.81 & i \\
\hline 56(D4) & 2495 & 1551.8 & 1.61 & i \\
\hline 56(D5) & 3403 & 1551.8 & 2.19 & s \\
\hline \hline 56(I1) & 3274 & 1551.8 & 2.11 & i \\
\hline 56(I2) & 2495 & 1551.8 & 1.61 & s \\
\hline $56(I 3)$ & 1984 & 1551.8 & 1.28 & s \\
\hline $56(I 4)$ & 4439 & 1551.8 & 2.86 & o \\
\hline 56(I5) & 3870 & 1551.8 & 2.49 & s \\
\hline 56(I6) & 2624 & 1551.8 & 1.69 & s \\
\hline
\end{tabular}

\begin{tabular}{|c|c|c|c|c|}
\hline \multicolumn{5}{|c|}{ Slab 2: LMC-S (With Bonding Slurry) } \\
\hline Core No. & $\begin{array}{c}\text { Failure } \\
\text { load } \\
\text { (N). }\end{array}$ & $\begin{array}{c}\text { Area } \\
\text { (mm2) }\end{array}$ & $\begin{array}{c}\text { Tensile } \\
\text { stress } \\
\text { (MPa) }\end{array}$ & $\begin{array}{c}\text { Mode } \\
\text { of } \\
\text { failure }\end{array}$ \\
\hline $56(\mathrm{D} 1) / \mathrm{S}$ & 2736 & 1551.8 & 1.76 & $\mathrm{i}$ \\
\hline $56(\mathrm{D} 2) / \mathrm{S}$ & 2157 & 1551.8 & 1.39 & $\mathrm{i}$ \\
\hline $56(\mathrm{D} 3) / \mathrm{S}$ & 4235 & 1551.8 & 2.73 & $\mathrm{~S}$ \\
\hline $56(\mathrm{D} 4) / \mathrm{S}$ & 3897 & 1551.8 & 2.51 & $\mathrm{i}$ \\
\hline $56(\mathrm{D} 5) / \mathrm{S}$ & 1143 & 1551.8 & 0.74 & $\mathrm{~S}$ \\
\hline \hline $56(\mathrm{I} 1) / \mathrm{S}$ & 885 & 1551.8 & 0.57 & $\mathrm{~S}$ \\
\hline $56(\mathrm{I} 2) / \mathrm{S}$ & 1637 & 1551.8 & 1.05 & $\mathrm{i}$ \\
\hline $56(\mathrm{I} 3) / \mathrm{S}$ & 2051 & 1551.8 & 1.32 & $\mathrm{i}$ \\
\hline $56(\mathrm{I} 4) / \mathrm{S}$ & 2131 & 1551.8 & 1.37 & $\mathrm{i}$ \\
\hline $56(\mathrm{I} 5) / \mathrm{S}$ & 1895 & 1551.8 & 1.22 & $\mathrm{i}$ \\
\hline
\end{tabular}




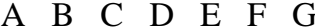

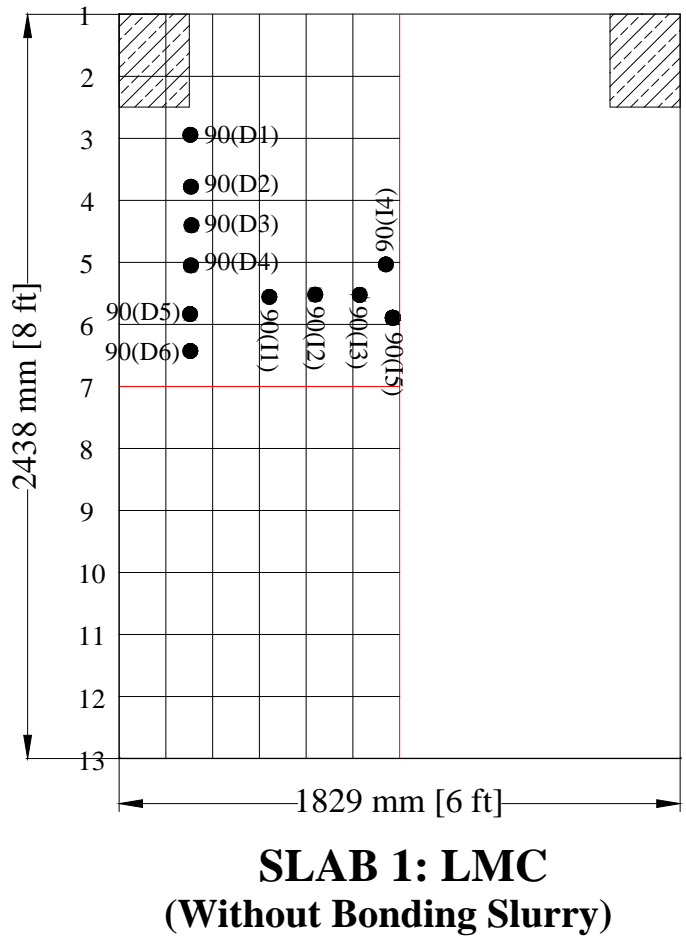

Pre-Notch $\longrightarrow$

$\begin{array}{llllllll}G & H & I & J & K & L & M\end{array}$

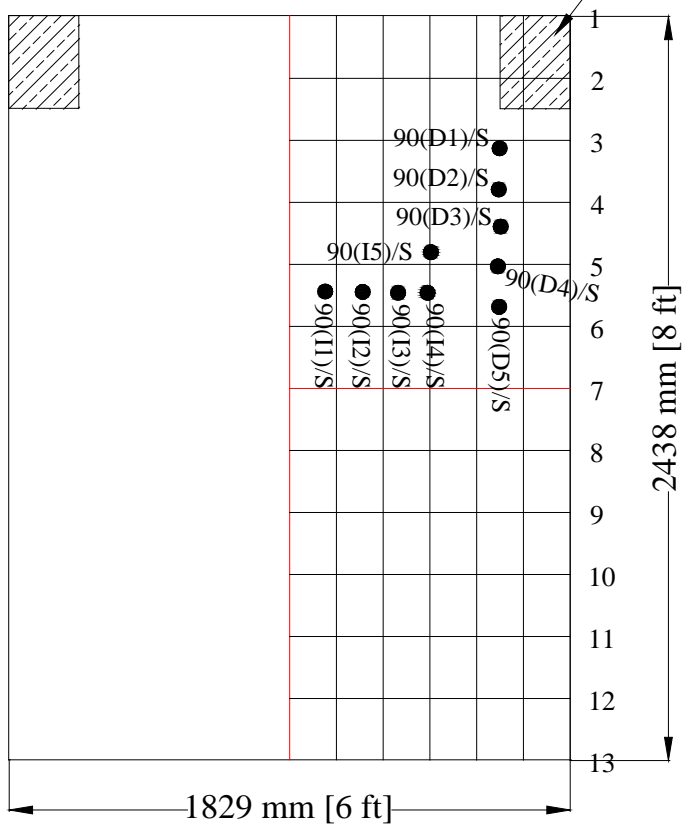

SLAB 2: LMC-S

(With Bonding Slurry)

Figure A.5: 90 days pull-off locations on LMC and LMC-S slabs of Stage-I

Table A.5: 90 days pull-off data of LMC and LMC-S slabs in Stage-I

\begin{tabular}{|c|c|c|c|c|}
\hline \multicolumn{5}{|c|}{ Slab 1: LMC (Without Bonding Slurry) } \\
\hline $\begin{array}{c}\text { Core } \\
\text { No. }\end{array}$ & $\begin{array}{c}\text { Failure } \\
\text { load } \\
\text { (N). }\end{array}$ & $\begin{array}{c}\text { Area } \\
\text { (mm2) }\end{array}$ & $\begin{array}{c}\text { Tensile } \\
\text { stress } \\
\text { (MPa) }\end{array}$ & $\begin{array}{c}\text { Mode } \\
\text { of } \\
\text { failure }\end{array}$ \\
\hline 90(D1) & 3247 & 1551.8 & 2.09 & $\mathrm{i}$ \\
\hline 90(D2) & 3194 & 1551.8 & 2.06 & $\mathrm{i}$ \\
\hline 90(D3) & 4235 & 1551.8 & 2.73 & $\mathrm{~s}$ \\
\hline 90(D4) & 4417 & 1551.8 & 2.85 & $\mathrm{~s}$ \\
\hline 90(D5) & 3946 & 1551.8 & 2.54 & $\mathrm{~s}$ \\
\hline 90(D6) & 4079 & 1551.8 & 2.63 & $\mathrm{~s}$ \\
\hline \hline 90(I1) & 3946 & 1551.8 & 2.54 & $\mathrm{~s}$ \\
\hline $90(\mathrm{I} 2)$ & 3817 & 1551.8 & 2.46 & $\mathrm{i}$ \\
\hline $90(\mathrm{I})$ & 4026 & 1551.8 & 2.59 & $\mathrm{~s}$ \\
\hline $90(\mathrm{I})$ & 3403 & 1551.8 & 2.19 & $\mathrm{i}$ \\
\hline $90(\mathrm{I})$ & 3194 & 1551.8 & 2.06 & $\mathrm{i}$ \\
\hline
\end{tabular}

\begin{tabular}{|c|c|c|c|c|}
\hline \multicolumn{5}{|c|}{ Slab 2: LMC-S (With Bonding Slurry) } \\
\hline Core No. & $\begin{array}{c}\text { Failure } \\
\text { load } \\
\text { (N). }\end{array}$ & $\begin{array}{c}\text { Area } \\
\text { (mm2) }\end{array}$ & $\begin{array}{c}\text { Tensile } \\
\text { stress } \\
\text { (MPa) }\end{array}$ & $\begin{array}{c}\text { Mode } \\
\text { of } \\
\text { failure }\end{array}$ \\
\hline $90(\mathrm{D} 1) / \mathrm{S}$ & 3456 & 1551.8 & 2.23 & $\mathrm{i}$ \\
\hline $90(\mathrm{D} 2) / \mathrm{S}$ & 3065 & 1551.8 & 1.98 & $\mathrm{i}$ \\
\hline $90(\mathrm{D} 3) / \mathrm{S}$ & 2286 & 1551.8 & 1.47 & $\mathrm{~s}$ \\
\hline $90(\mathrm{D} 4) / \mathrm{S}$ & 2936 & 1551.8 & 1.89 & $\mathrm{~S}$ \\
\hline $90(\mathrm{D} 5) / \mathrm{S}$ & 3843 & 1551.8 & 2.48 & $\mathrm{i}$ \\
\hline \hline $90(\mathrm{I} 1) / \mathrm{S}$ & 3038 & 1551.8 & 1.96 & $\mathrm{i}$ \\
\hline $90(\mathrm{I} 2) / \mathrm{S}$ & 2598 & 1551.8 & 1.67 & $\mathrm{~S}$ \\
\hline $90(\mathrm{I} 3) / \mathrm{S}$ & 4181 & 1551.8 & 2.69 & $\mathrm{i}$ \\
\hline $90(\mathrm{I} 4) / \mathrm{S}$ & 5169 & 1551.8 & 3.33 & 0 \\
\hline $90(\mathrm{I} 5) / \mathrm{S}$ & 2544 & 1551.8 & 1.64 & $\mathrm{i}$ \\
\hline
\end{tabular}




\section{$\underline{\text { Stage-II }}$}

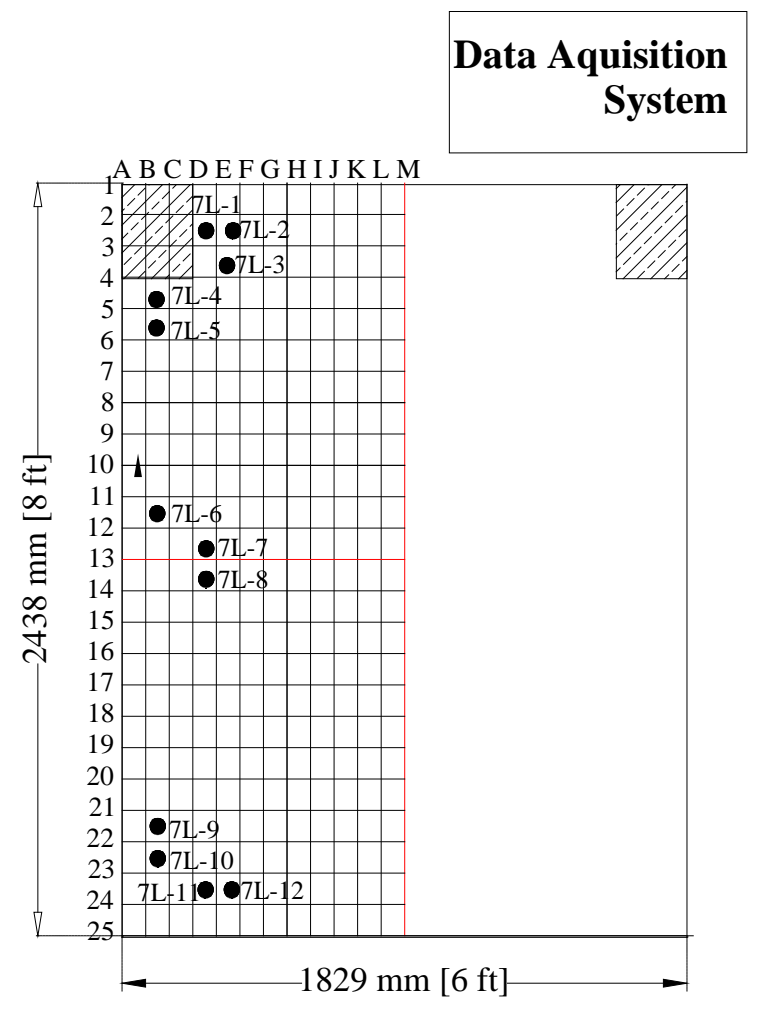

SLAB 1: LMC

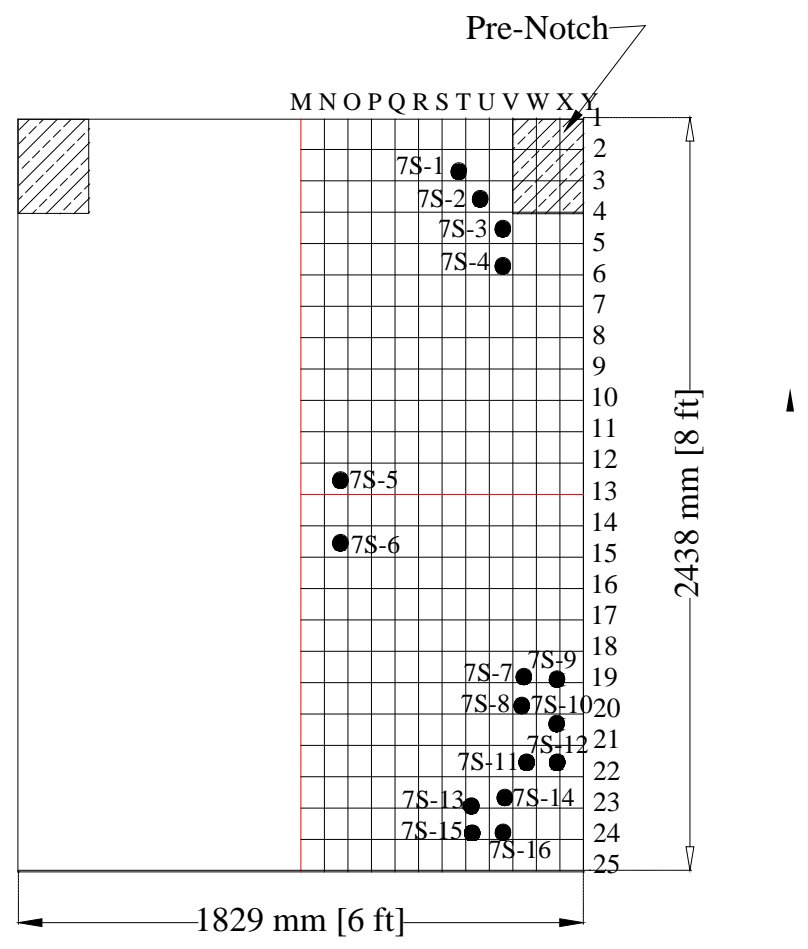

SLAB 2: SFMC

Figure A.6: 7 days pull-off locations on LMC and SFMC Slabs of Stage-II

Table A.6: 7 days pull-off data of LMC and SFMC slabs in Stage-II

\begin{tabular}{|c|c|c|c|c|}
\hline \multicolumn{5}{|c|}{ Slab 1-LMC } \\
\hline $\begin{array}{c}\text { Core } \\
\text { No. }\end{array}$ & $\begin{array}{c}\text { Failure } \\
\text { load } \\
\text { (N). }\end{array}$ & $\begin{array}{c}\text { Area } \\
\mathbf{( m m}^{\mathbf{2}} \mathbf{)}\end{array}$ & $\begin{array}{c}\text { Tensile } \\
\text { stress } \\
\mathbf{( M P a})\end{array}$ & $\begin{array}{c}\text { Mode } \\
\text { of } \\
\text { failure }\end{array}$ \\
\hline 7L-1 & - & 1551.8 & 0.00 & $\mathrm{C}$ \\
\hline 7L-2 & 1428 & 1551.8 & 0.92 & $\mathrm{~s}$ \\
\hline 7L-3 & 1819 & 1551.8 & 1.17 & $\mathrm{~s}$ \\
\hline 7L-4 & 1219 & 1551.8 & 0.79 & $\mathrm{i}$ \\
\hline 7L-5 & 2598 & 1551.8 & 1.67 & $\mathrm{e}$ \\
\hline 7L-6 & 3118 & 1551.8 & 2.01 & $\mathrm{~s}$ \\
\hline 7L-7 & 2544 & 1551.8 & 1.64 & $\mathrm{~s}$ \\
\hline 7L-8 & 1922 & 1551.8 & 1.24 & $\mathrm{~s}$ \\
\hline 7L-9 & 2598 & 1551.8 & 1.67 & $\mathrm{~s}$ \\
\hline 7L-10 & 2077 & 1551.8 & 1.34 & $\mathrm{i}$ \\
\hline 7L-11 & 1508 & 1551.8 & 0.97 & $\mathrm{e}$ \\
\hline 7L-12 & 2157 & 1551.8 & 1.39 & $\mathrm{i}$ \\
\hline
\end{tabular}

\begin{tabular}{|c|c|c|c|c|}
\hline \multicolumn{5}{|c|}{ Slab 2-SFMC } \\
\hline $\begin{array}{c}\text { Core } \\
\text { No. }\end{array}$ & $\begin{array}{c}\text { Failure } \\
\text { load } \\
\text { (N). }\end{array}$ & $\begin{array}{c}\text { Area } \\
\left(\mathbf{m m}^{\mathbf{2}} \mathbf{)}\right.\end{array}$ & $\begin{array}{c}\text { Tensile } \\
\text { stress } \\
\mathbf{( M P a})\end{array}$ & $\begin{array}{c}\text { Mode } \\
\text { of } \\
\text { failure }\end{array}$ \\
\hline $7 \mathrm{~S}-1$ & 1246 & 1551.8 & 0.80 & $\mathrm{i}$ \\
\hline $7 \mathrm{~S}-2$ & 778 & 1551.8 & 0.50 & $\mathrm{i}$ \\
\hline $7 \mathrm{~S}-3$ & 1455 & 1551.8 & 0.94 & $\mathrm{i}$ \\
\hline $7 \mathrm{~S}-4$ & 2104 & 1551.8 & 1.36 & $\mathrm{i}$ \\
\hline $7 \mathrm{~S}-5$ & 1584 & 1551.8 & 1.02 & $\mathrm{e}$ \\
\hline $7 \mathrm{~S}-6$ & 2260 & 1551.8 & 1.46 & $\mathrm{i}$ \\
\hline $7 \mathrm{~S}-7$ & 1428 & 1551.8 & 0.92 & $\mathrm{i}$ \\
\hline $7 \mathrm{~S}-8$ & 1975 & 1551.8 & 1.27 & $\mathrm{i}$ \\
\hline $7 \mathrm{~S}-9$ & - & 1551.8 & 0.00 & $\mathrm{c}$ \\
\hline $7 \mathrm{~S}-10$ & - & 1551.8 & 0.00 & $\mathrm{C}$ \\
\hline
\end{tabular}




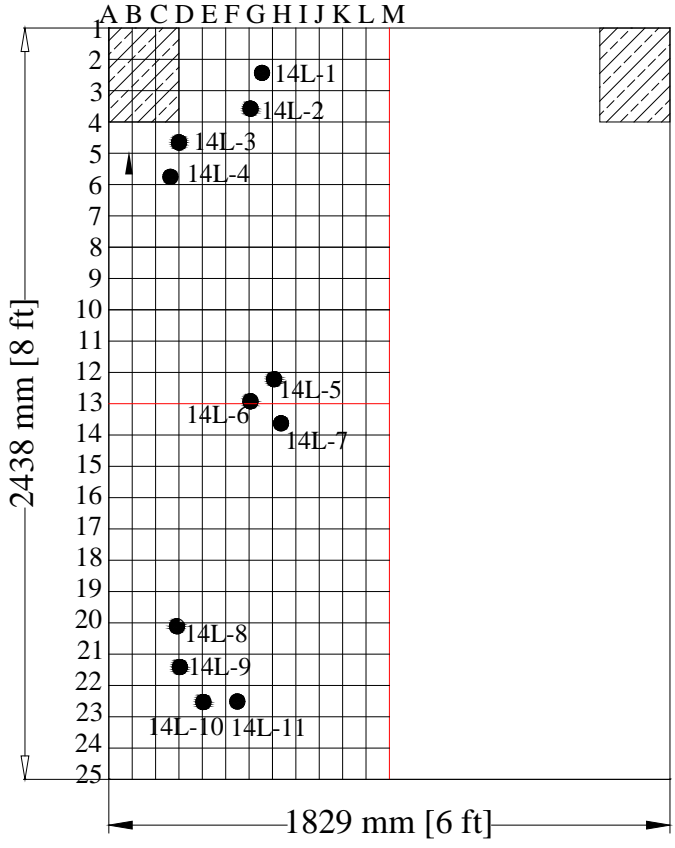

SLAB 1: LMC
Pre-Notch-7

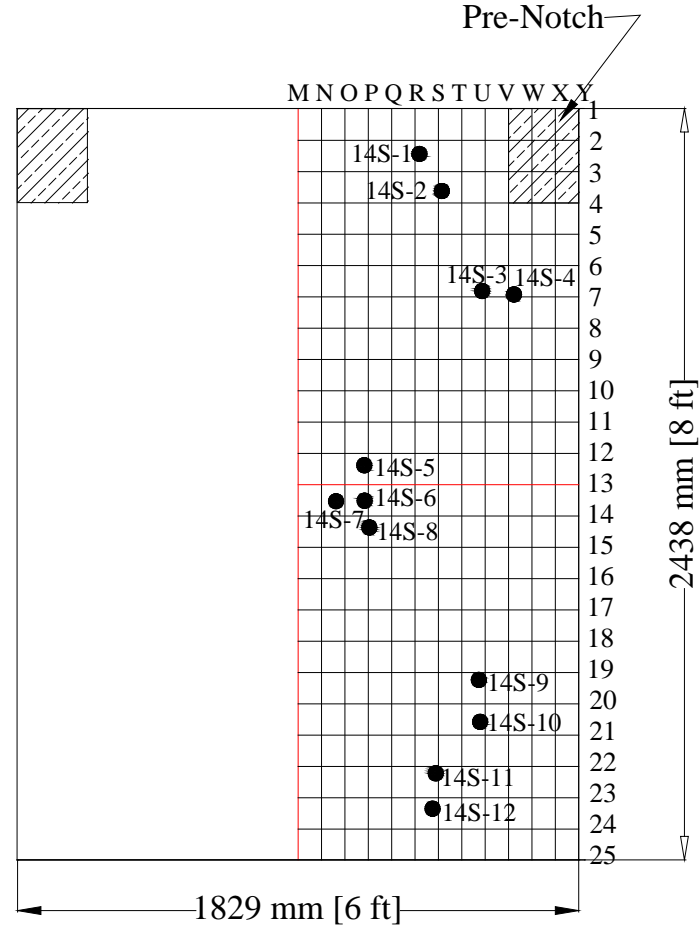

SLAB 2: SFMC

Figure A.7: 14 days pull-off locations on LMC and SFMC Slabs of Stage-II

Table A.7: 14 days pull-off data of LMC and SFMC slabs in Stage-II

\begin{tabular}{|c|c|c|c|c|}
\hline \multicolumn{5}{|c|}{ Slab 1-LMC } \\
\hline $\begin{array}{c}\text { Core } \\
\text { No. }\end{array}$ & $\begin{array}{c}\text { Failure } \\
\text { load } \\
\text { (N). }\end{array}$ & $\begin{array}{c}\text { Area } \\
\left(\mathbf{m m}^{2}\right)\end{array}$ & $\begin{array}{c}\text { Tensile } \\
\text { stress } \\
\text { (MPa) }\end{array}$ & $\begin{array}{c}\text { Mode } \\
\text { of } \\
\text { failure }\end{array}$ \\
\hline 14L-1 & 2024 & 1551.8 & 1.30 & s \\
\hline 14L-2 & 1819 & 1551.8 & 1.17 & $\mathrm{i}$ \\
\hline 14L-3 & 1819 & 1551.8 & 1.17 & $\mathrm{i}$ \\
\hline 14L-4 & 596 & 1551.8 & 0.38 & $\mathrm{i}$ \\
\hline 14L-5 & 2002 & 1551.8 & 1.29 & $\mathrm{i}$ \\
\hline 14L-6 & 1846 & 1551.8 & 1.19 & $\mathrm{i}$ \\
\hline 14L-7 & 1868 & 1551.8 & 1.20 & $\mathrm{i}$ \\
\hline 14L-8 & 1948 & 1551.8 & 1.26 & $\mathrm{i}$ \\
\hline 14L-9 & 2727 & 1551.8 & 1.76 & $\mathrm{i}$ \\
\hline 14L-10 & 1868 & 1551.8 & 1.20 & $\mathrm{i}$ \\
\hline 14L-11 & 1975 & 1551.8 & 1.27 & $\mathrm{i}$ \\
\hline
\end{tabular}

\begin{tabular}{|c|c|c|c|c|}
\hline \multicolumn{5}{|c|}{ Slab 2-SFMC } \\
\hline $\begin{array}{c}\text { Core } \\
\text { No. }\end{array}$ & $\begin{array}{c}\text { Failure } \\
\text { load } \\
\mathbf{( N )}\end{array}$ & $\begin{array}{c}\text { Area } \\
\left(\mathbf{m m}^{2}\right)\end{array}$ & $\begin{array}{c}\text { Tensile } \\
\text { stress } \\
\mathbf{( M P a})\end{array}$ & $\begin{array}{c}\text { Mode } \\
\text { of } \\
\text { failure }\end{array}$ \\
\hline $14 \mathrm{~S}-1$ & 0 & 1551.8 & 0.00 & $\mathrm{i}$ \\
\hline $14 \mathrm{~S}-2$ & 222 & 1551.8 & 0.14 & $\mathrm{i}$ \\
\hline $14 \mathrm{~S}-3$ & 988 & 1551.8 & 0.64 & $\mathrm{i}$ \\
\hline $14 \mathrm{~S}-4$ & 440 & 1551.8 & 0.28 & $\mathrm{i}$ \\
\hline $14 \mathrm{~S}-5$ & 3741 & 1551.8 & 2.41 & o \\
\hline $14 \mathrm{~S}-6$ & 4252 & 1551.8 & 2.74 & o \\
\hline $14 \mathrm{~S}-7$ & 3897 & 1551.8 & 2.51 & o \\
\hline $14 \mathrm{~S}-8$ & 5921 & 1551.8 & 3.82 & o \\
\hline $14 \mathrm{~S}-9$ & 1041 & 1551.8 & 0.67 & $\mathrm{i}$ \\
\hline $14 \mathrm{~S}-10$ & 1090 & 1551.8 & 0.70 & $\mathrm{i}$ \\
\hline $14 \mathrm{~S}-11$ & 1063 & 1551.8 & 0.69 & $\mathrm{i}$ \\
\hline $14 \mathrm{~S}-12$ & 725 & 1551.8 & 0.47 & $\mathrm{i}$ \\
\hline
\end{tabular}



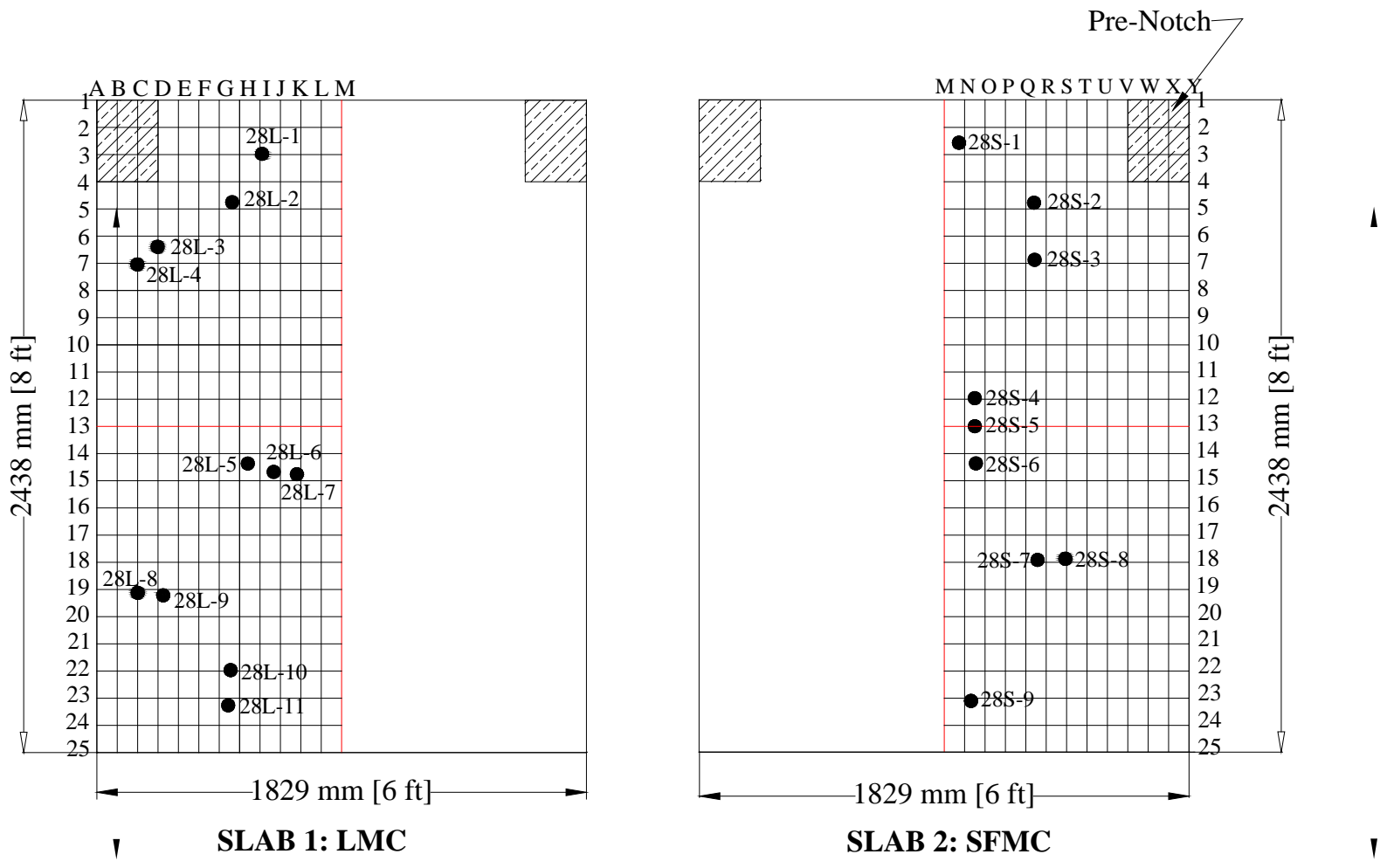

Figure A.8: 28 days pull-off locations on LMC and SFMC Slabs of Stage-II

Table A.8: 28 days pull-off data of LMC and SFMC slabs in Stage-II

\begin{tabular}{|c|c|c|c|c|}
\hline \multicolumn{5}{|c|}{ Slab 1-LMC } \\
\hline $\begin{array}{c}\text { Core } \\
\text { No. }\end{array}$ & $\begin{array}{c}\text { Failure } \\
\text { load } \\
\text { (N). }\end{array}$ & $\begin{array}{c}\text { Area } \\
\mathbf{( m m}^{2} \text { ) }\end{array}$ & $\begin{array}{c}\text { Tensile } \\
\text { stress } \\
\text { (MPa) }\end{array}$ & $\begin{array}{c}\text { Mode } \\
\text { of } \\
\text { failure }\end{array}$ \\
\hline 28L-1 & 1690 & 1551.8 & 1.09 & $\mathrm{i}$ \\
\hline 28L-2 & 2544 & 1551.8 & 1.64 & $\mathrm{~S}$ \\
\hline 28L-3 & 3479 & 1551.8 & 2.24 & $\mathrm{i}$ \\
\hline 28L-4 & 2985 & 1551.8 & 1.92 & $\mathrm{i}$ \\
\hline 28L-5 & 1557 & 1551.8 & 1.00 & $\mathrm{i}$ \\
\hline 28L-6 & 1352 & 1551.8 & 0.87 & $\mathrm{i}$ \\
\hline 28L-7 & 1975 & 1551.8 & 1.27 & $\mathrm{i}$ \\
\hline 28L-8 & 2598 & 1551.8 & 1.67 & $\mathrm{~S}$ \\
\hline 28L-9 & 1530 & 1551.8 & 0.99 & $\mathrm{i}$ \\
\hline 28L-10 & 1739 & 1551.8 & 1.12 & $\mathrm{i}$ \\
\hline 28L-11 & 2260 & 1551.8 & 1.46 & $\mathrm{~s}$ \\
\hline
\end{tabular}

\begin{tabular}{|c|c|c|c|c|}
\hline \multicolumn{5}{|c|}{ Slab 2-SFMC } \\
\hline $\begin{array}{c}\text { Core } \\
\text { No. }\end{array}$ & $\begin{array}{c}\text { Failure } \\
\text { load } \\
\text { (N). }\end{array}$ & $\begin{array}{c}\text { Area } \\
\left(\mathrm{mm}^{2}\right)\end{array}$ & $\begin{array}{c}\text { Tensile } \\
\text { stress } \\
\text { (MPa) }\end{array}$ & $\begin{array}{c}\text { Mode } \\
\text { of } \\
\text { failure }\end{array}$ \\
\hline 28S-1 & 1664 & 1551.8 & 1.07 & $\mathrm{~s}$ \\
\hline $28 \mathrm{~S}-2$ & 2469 & 1551.8 & 1.59 & $\mathrm{i}$ \\
\hline $28 \mathrm{~S}-3$ & 2157 & 1551.8 & 1.39 & $\mathrm{~s}$ \\
\hline 28S-4 & 2157 & 1551.8 & 1.39 & $\mathrm{i}$ \\
\hline $28 S-5$ & 2882 & 1551.8 & 1.86 & $\mathrm{~s}$ \\
\hline 28S-6 & 2442 & 1551.8 & 1.57 & $\mathrm{~s}$ \\
\hline $28 S-7$ & 236 & 1551.8 & 0.15 & $\mathrm{i}$ \\
\hline 28S-8 & 1117 & 1551.8 & 0.72 & $\mathrm{~s}$ \\
\hline $28 S-9$ & 1219 & 1551.8 & 0.79 & $\mathrm{i}$ \\
\hline
\end{tabular}



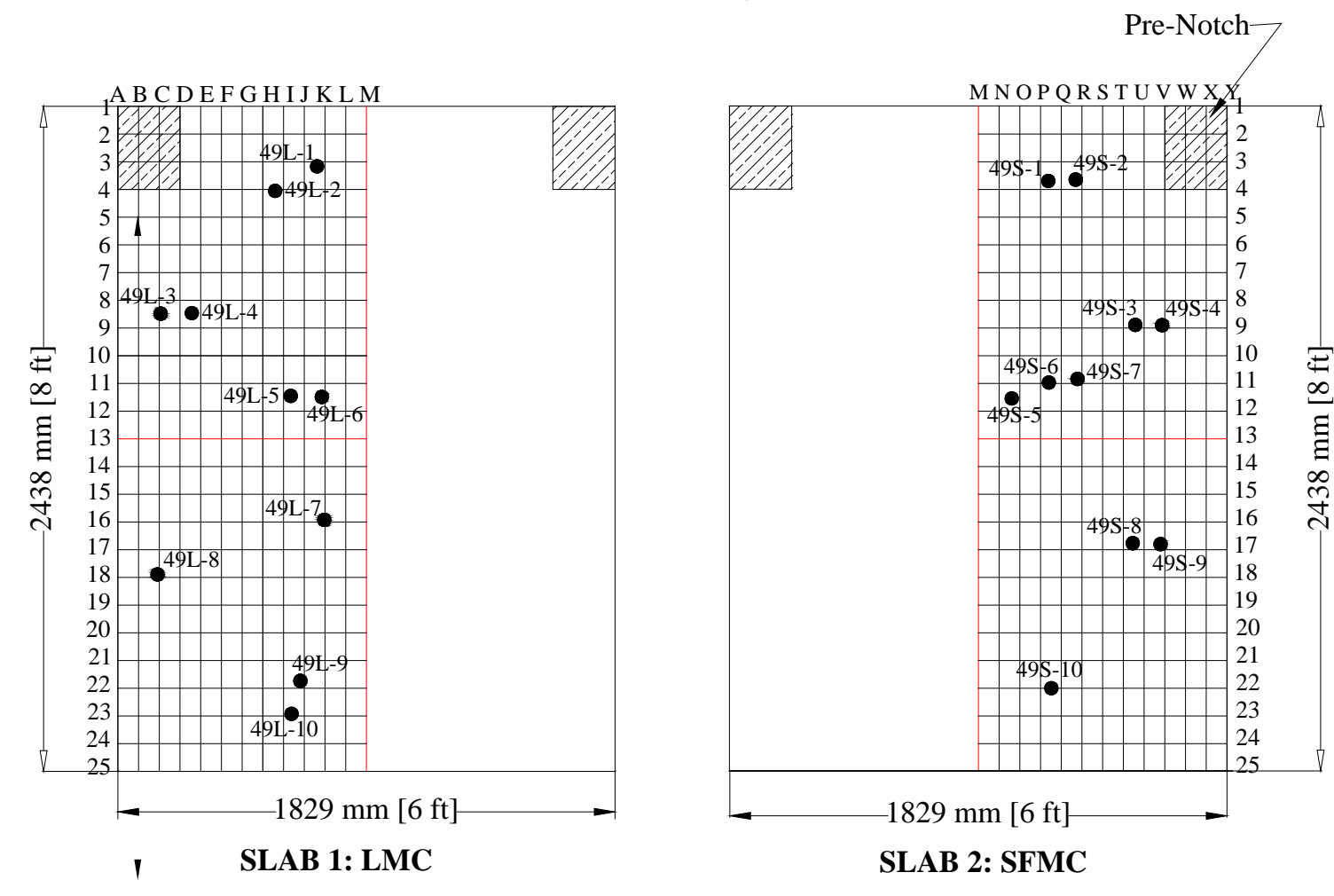

SLAB 2: SFMC

Figure A.9: 49 days pull-off locations on LMC and SFMC Slabs of Stage-II

Table A.9: 49 days pull-off data of LMC and SFMC slabs in Stage-II

\begin{tabular}{|c|c|c|c|c|}
\hline \multicolumn{5}{|c|}{ Slab 1-LMC } \\
No. & $\begin{array}{c}\text { Failure } \\
\text { load } \\
\text { (N). }\end{array}$ & $\begin{array}{c}\text { Area } \\
\left(\mathbf{m m}^{2}\right)\end{array}$ & $\begin{array}{c}\text { Tensile } \\
\text { stress } \\
\text { (MPa) }\end{array}$ & $\begin{array}{c}\text { Mode } \\
\text { of } \\
\text { failure }\end{array}$ \\
\hline 49L-1 & 338 & 1551.8 & 0.22 & $\mathrm{i}$ \\
\hline 49L-2 & 3274 & 1551.8 & 2.11 & $\mathrm{~s}$ \\
\hline 49L-3 & 2051 & 1551.8 & 1.32 & $\mathrm{~S}$ \\
\hline 49L-4 & 1557 & 1551.8 & 1.00 & $\mathrm{i}$ \\
\hline 49L-5 & 2807 & 1551.8 & 1.81 & $\mathrm{i}$ \\
\hline 49L-6 & 2064 & 1551.8 & 1.33 & $\mathrm{i}$ \\
\hline 49L-7 & 1637 & 1551.8 & 1.05 & $\mathrm{i}$ \\
\hline 49L-8 & 2415 & 1551.8 & 1.56 & $\mathrm{~s}$ \\
\hline 49L-9 & 2780 & 1551.8 & 1.79 & $\mathrm{i}$ \\
\hline 49L-10 & 2936 & 1551.8 & 1.89 & $\mathrm{i}$ \\
\hline
\end{tabular}

\begin{tabular}{|c|c|c|c|c|}
\hline \multicolumn{5}{|c|}{ Slab 2-SFMC } \\
\hline No. & $\begin{array}{c}\text { Failure } \\
\text { load } \\
\text { (N). }\end{array}$ & $\begin{array}{c}\text { Area } \\
\left(\mathbf{m m}^{2}\right)\end{array}$ & $\begin{array}{c}\text { Tensile } \\
\text { stress } \\
\text { (MPa) }\end{array}$ & $\begin{array}{c}\text { Mode } \\
\text { of } \\
\text { failure }\end{array}$ \\
\hline 49S-1 & 1766 & 1551.8 & 1.14 & $\mathrm{i}$ \\
\hline 49S-2 & 885 & 1551.8 & 0.57 & $\mathrm{i}$ \\
\hline 49S-3 & 3118 & 1551.8 & 2.01 & $\mathrm{i}$ \\
\hline 49S-4 & 209 & 1551.8 & 0.13 & $\mathrm{i}$ \\
\hline 49S-5 & 2362 & 1551.8 & 1.52 & $\mathrm{i}$ \\
\hline 49S-6 & 1922 & 1551.8 & 1.24 & $\mathrm{i}$ \\
\hline 49S-7 & 547 & 1551.8 & 0.35 & $\mathrm{i}$ \\
\hline 49S-8 & 988 & 1551.8 & 0.64 & $\mathrm{i}$ \\
\hline 49S-9 & 311 & 1551.8 & 0.20 & $\mathrm{i}$ \\
\hline 49S-10 & 1819 & 3832.0 & 0.47 & $\mathrm{i}$ \\
\hline
\end{tabular}



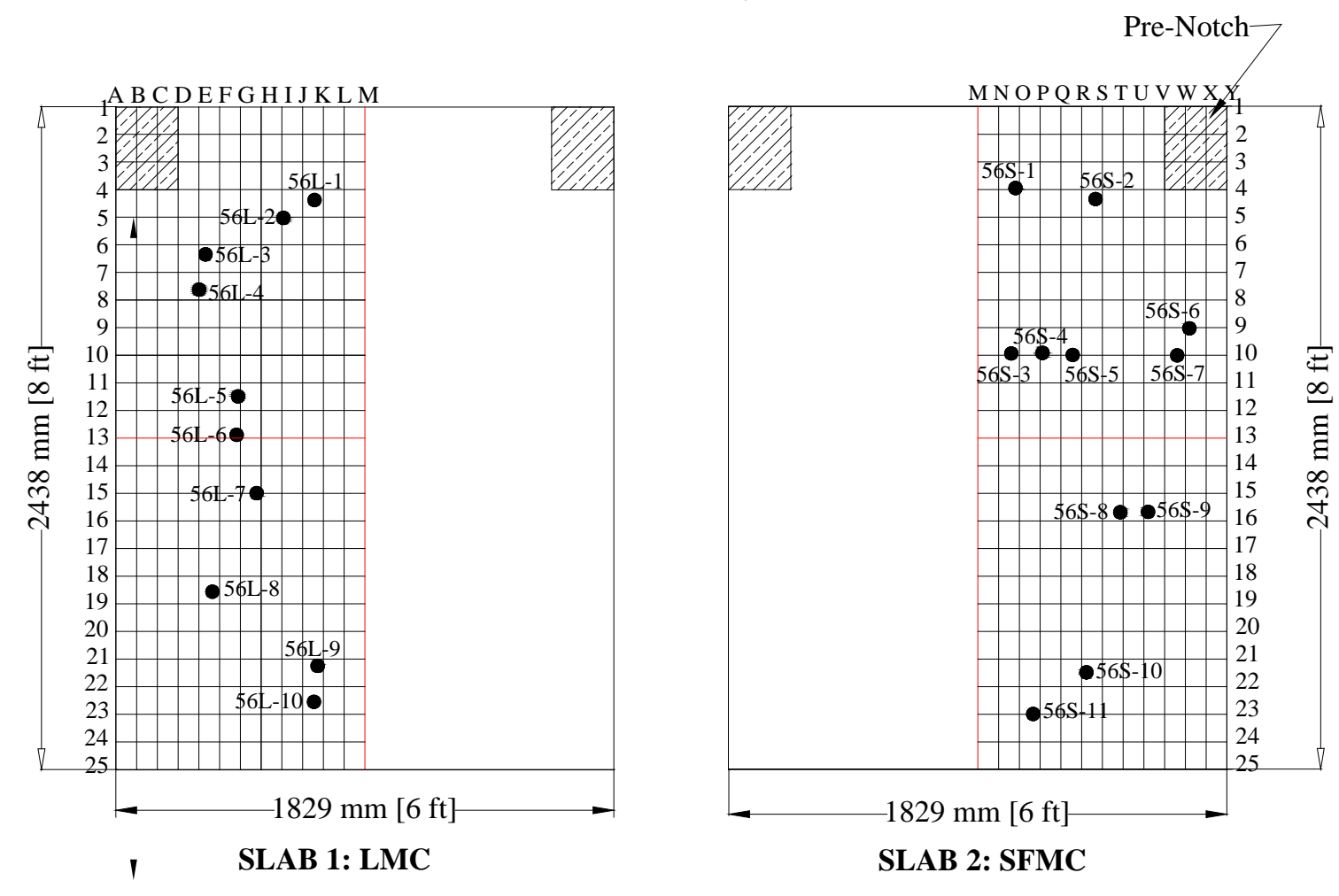

Figure A.10: 56 days pull-off locations on LMC and SFMC Slabs of Stage-II

Table A.10: 56 days pull-off data of LMC and SFMC slabs in Stage-II

\begin{tabular}{|c|c|c|c|c|}
\hline \multicolumn{5}{|c|}{ Slab 1-LMC } \\
\hline No. & $\begin{array}{c}\text { Failure } \\
\text { load } \\
\text { (N). }\end{array}$ & $\begin{array}{c}\text { Area } \\
\left(\mathbf{m m}^{2}\right)\end{array}$ & $\begin{array}{c}\text { Tensile } \\
\text { stress } \\
\text { (MPa) }\end{array}$ & $\begin{array}{c}\text { Mode } \\
\text { of } \\
\text { failure }\end{array}$ \\
\hline 56L-1 & 1948 & 1551.8 & 1.26 & $\mathrm{~s}$ \\
\hline 56L-2 & 1610 & 1551.8 & 1.04 & $\mathrm{e}$ \\
\hline 56L-3 & 2415 & 1551.8 & 1.56 & $\mathrm{i}$ \\
\hline 56L-4 & 988 & 1551.8 & 0.64 & $\mathrm{i}$ \\
\hline 56L-5 & 1299 & 1551.8 & 0.84 & $\mathrm{i}$ \\
\hline 56L-6 & 1197 & 1551.8 & 0.77 & $\mathrm{i}$ \\
\hline 56L-7 & 1846 & 1551.8 & 1.19 & $\mathrm{i}$ \\
\hline 56L-8 & 1014 & 1551.8 & 0.65 & $\mathrm{i}$ \\
\hline 56L-9 & 1272 & 1551.8 & 0.82 & $\mathrm{i}$ \\
\hline 56L-10 & 1557 & 1551.8 & 1.00 & $\mathrm{i}$ \\
\hline
\end{tabular}

\begin{tabular}{|c|c|c|c|c|}
\hline \multicolumn{5}{|c|}{ Slab 2-SFMC } \\
\hline $\begin{array}{c}\text { Core } \\
\text { No. }\end{array}$ & $\begin{array}{c}\text { Failure } \\
\text { load } \\
\text { (N). }\end{array}$ & $\begin{array}{c}\text { Area } \\
\mathbf{( m m}^{2} \text { ) }\end{array}$ & $\begin{array}{c}\text { Tensile } \\
\text { stress } \\
\text { (MPa) }\end{array}$ & $\begin{array}{c}\text { Mode } \\
\text { of } \\
\text { failure }\end{array}$ \\
\hline $56 S-1$ & 2002 & 1551.8 & 1.29 & s \\
\hline $56 S-2$ & 596 & 1551.8 & 0.38 & i \\
\hline $56 S-3$ & 1584 & 1551.8 & 1.02 & i \\
\hline $56 S-4$ & 1352 & 1551.8 & 0.87 & s \\
\hline $56 S-5$ & 1922 & 1551.8 & 1.24 & S \\
\hline $56 S-6$ & 467 & 1551.8 & 0.30 & i \\
\hline $56 S-7$ & 258 & 1551.8 & 0.17 & i \\
\hline $56 S-8$ & 365 & 1551.8 & 0.24 & i \\
\hline $56 S-9$ & 258 & 1551.8 & 0.17 & i \\
\hline $56 S-10$ & 1717 & 1551.8 & 1.11 & i \\
\hline $56 S-11$ & 2495 & 1551.8 & 1.61 & s \\
\hline
\end{tabular}



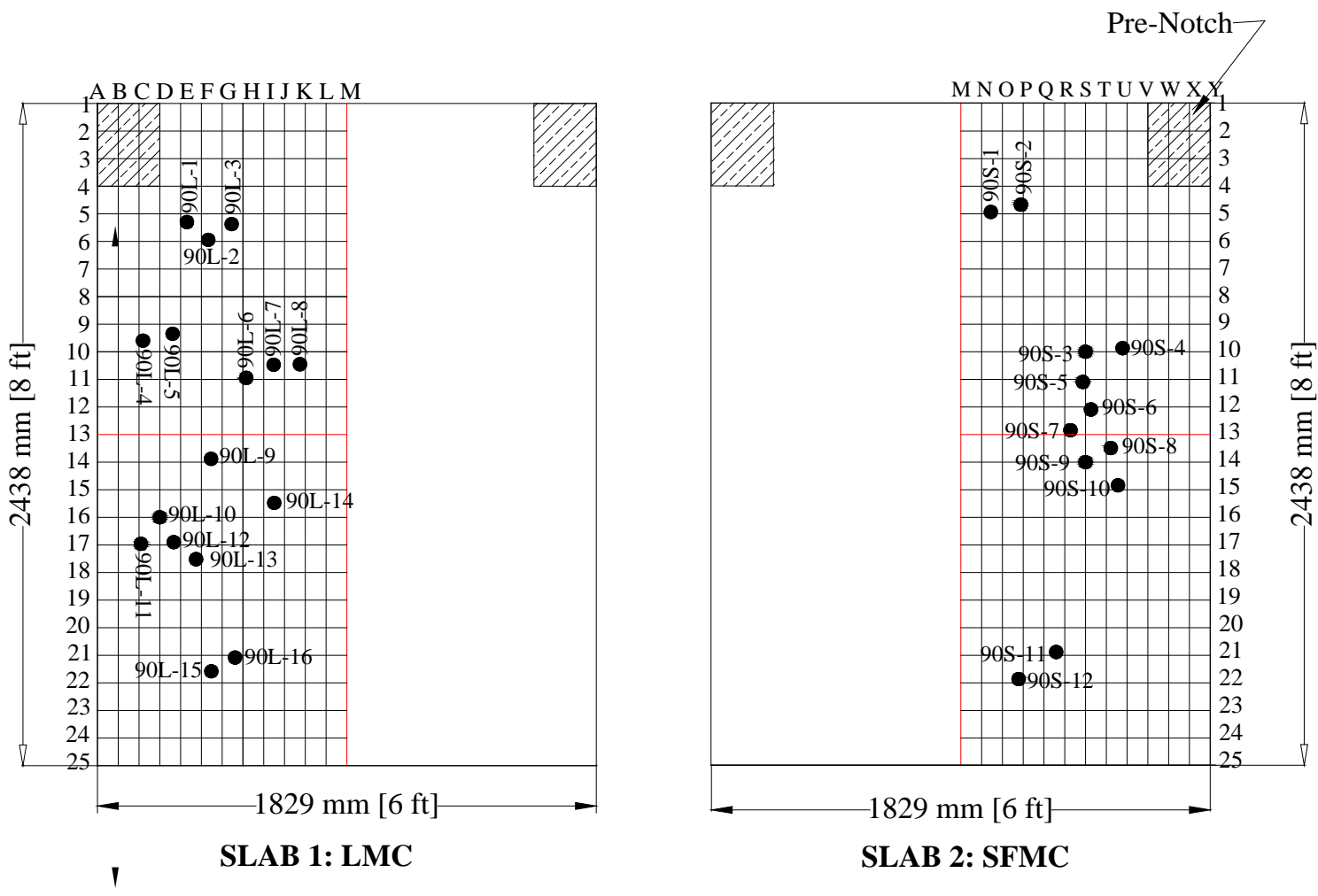

SLAB 2: SFMC

Figure A.11: 90 days pull-off locations on LMC and SFMC Slabs of Stage-II

Table A.11: 90 days pull-off data of LMC and SFMC slabs in Stage-II

\begin{tabular}{|c|c|c|c|c|}
\hline \multicolumn{5}{|c|}{ Slab 1-LMC } \\
\hline $\begin{array}{c}\text { Core } \\
\text { No. }\end{array}$ & $\begin{array}{c}\text { Failure } \\
\text { load } \\
\mathbf{( N )}\end{array}$ & $\begin{array}{c}\text { Area } \\
\mathbf{( m m}^{2} \mathbf{)}\end{array}$ & $\begin{array}{c}\text { Tensile } \\
\text { stress } \\
\mathbf{( M P a})\end{array}$ & $\begin{array}{c}\text { Mode } \\
\text { of } \\
\text { failure }\end{array}$ \\
\hline 90L-1 & 2856 & 1551.8 & 1.84 & $\mathrm{~s}$ \\
\hline 90L-2 & 2233 & 1551.8 & 1.44 & $\mathrm{~s}$ \\
\hline 90L-3 & 1948 & 1551.8 & 1.26 & $\mathrm{i}$ \\
\hline 90L-4 & 3661 & 1551.8 & 2.36 & $\mathrm{~s}$ \\
\hline 90L-5 & 3585 & 1551.8 & 2.31 & $\mathrm{~s}$ \\
\hline 90L-6 & 2598 & 1551.8 & 1.67 & $\mathrm{e}$ \\
\hline 90L-7 & 2180 & 1551.8 & 1.40 & $\mathrm{~s}$ \\
\hline 90L-8 & 1246 & 1551.8 & 0.80 & $\mathrm{i}$ \\
\hline 90L-9 & 1766 & 1551.8 & 1.14 & $\mathrm{i}$ \\
\hline 90L-10 & 0 & 1551.8 & 0.00 & $\mathrm{C}$ \\
\hline 90L-11 & 2882 & 1551.8 & 1.86 & $\mathrm{i}$ \\
\hline 90L-12 & 0 & 1551.8 & 0.00 & $\mathrm{C}$ \\
\hline 90L-13 & 1014 & 1551.8 & 0.65 & $\mathrm{i}$ \\
\hline 90L-14 & 1948 & 1551.8 & 1.26 & $\mathrm{i}$ \\
\hline 90L-15 & 1690 & 1551.8 & 1.09 & $\mathrm{i}$ \\
\hline 90L-16 & 1197 & 1551.8 & 0.77 & $\mathrm{i}$ \\
\hline
\end{tabular}

\begin{tabular}{|c|c|c|c|c|}
\hline \multicolumn{5}{|c|}{ Slab 2-SFMC } \\
\hline $\begin{array}{l}\text { Core } \\
\text { No. }\end{array}$ & $\begin{array}{c}\text { Failure } \\
\text { load } \\
(\mathrm{N}) .\end{array}$ & $\begin{array}{c}\text { Area } \\
\left(\mathrm{mm}^{2}\right)\end{array}$ & $\begin{array}{c}\text { Tensile } \\
\text { stress } \\
\text { (MPa) }\end{array}$ & $\begin{array}{c}\text { Mode } \\
\text { of } \\
\text { failure }\end{array}$ \\
\hline 90S-1 & 1508 & 1551.8 & 0.97 & $\mathrm{i}$ \\
\hline $90 \mathrm{~S}-2$ & 2598 & 1551.8 & 1.67 & $\mathrm{~s}$ \\
\hline $90 \mathrm{~S}-3$ & 129 & 1551.8 & 0.08 & i \\
\hline $90 \mathrm{~S}-4$ & 0 & 1551.8 & 0.00 & C \\
\hline $90 S-5$ & 0 & 1551.8 & 0.00 & C \\
\hline $90 \mathrm{~S}-6$ & 1299 & 1551.8 & 0.84 & $\mathrm{i}$ \\
\hline $90 \mathrm{~S}-7$ & 1819 & 1551.8 & 1.17 & $\mathrm{i}$ \\
\hline $90 \mathrm{~S}-8$ & 885 & 1551.8 & 0.57 & $\mathrm{i}$ \\
\hline 90S-9 & 596 & 1551.8 & 0.38 & $\mathrm{i}$ \\
\hline 90S-10 & 859 & 1551.8 & 0.55 & $\mathrm{i}$ \\
\hline 90S-11 & 1063 & 1551.8 & 0.69 & $\mathrm{i}$ \\
\hline 90S-12 & 1143 & 3832.0 & 0.30 & $\mathrm{i}$ \\
\hline
\end{tabular}


APPENDIX B 


\section{UPV Results}

\section{Stage-I}

\section{Data Aquisition \\ System}

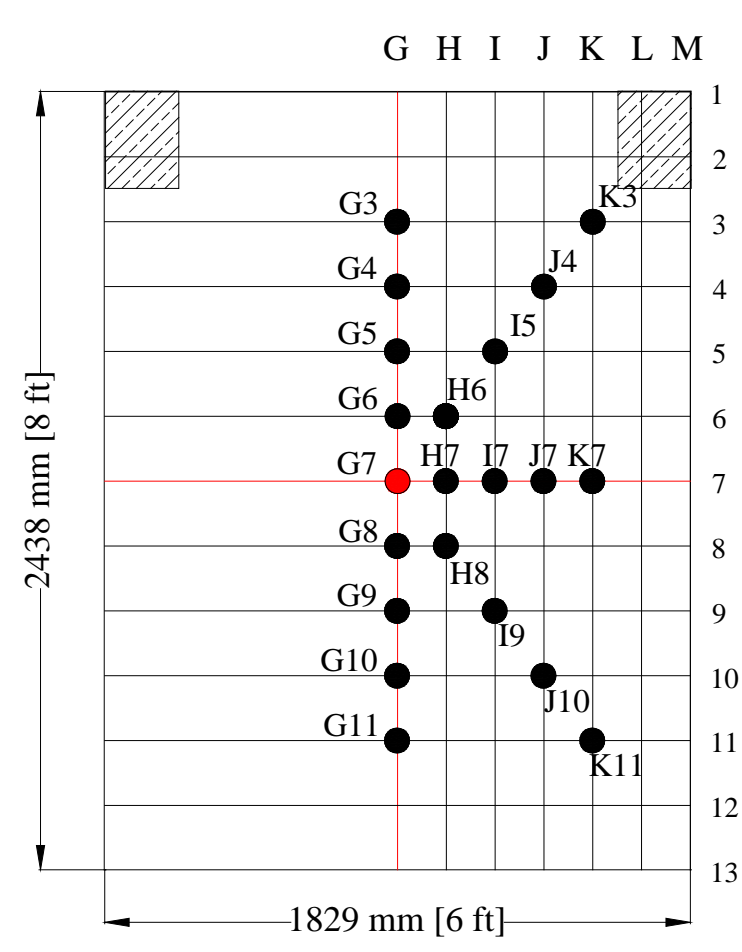

SLAB 1: LMC

(Without Bonding Slurry)

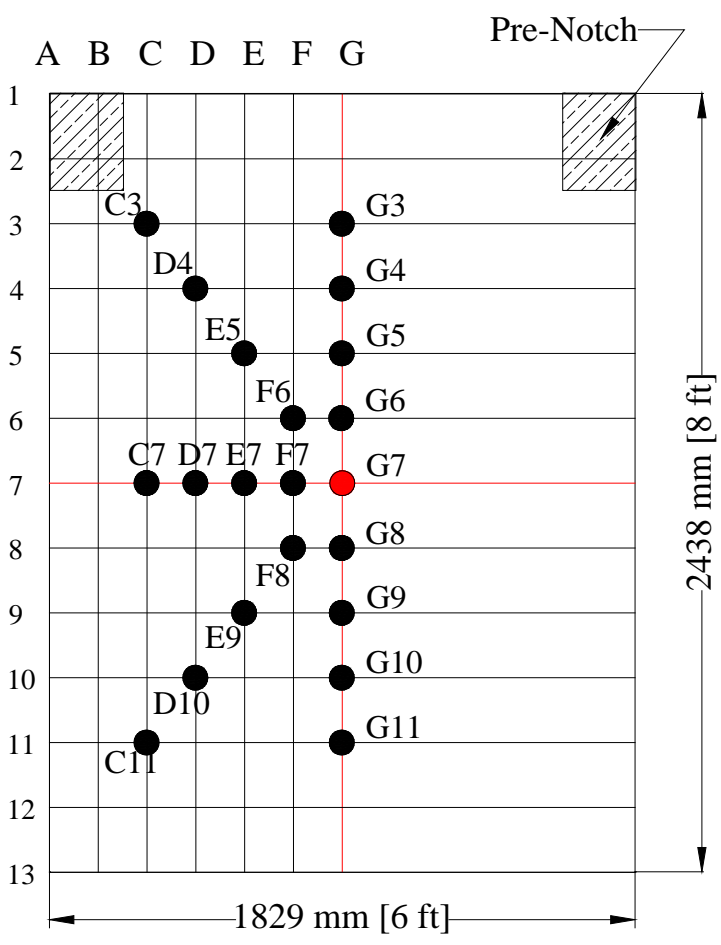

SLAB 2: LMC(S) (With Bonding Slurry)

Figure B.1: Locations at which indirect UPV was conducted

Table B.1: Indirect UPV table along: G7-M7 for LMC slab and G7-A7 for LMC-S slab

\begin{tabular}{|c|c|c|c|c|}
\hline \multicolumn{5}{|c|}{ SLAB 1: LMC } \\
\hline $\begin{array}{c}\text { Trans. } \\
\text { Location }\end{array}$ & $\begin{array}{c}\text { Rec. } \\
\text { Location }\end{array}$ & $\begin{array}{c}\text { Distance } \\
\text { (mm.) }\end{array}$ & $\begin{array}{c}\text { Time } \\
(\mu S)\end{array}$ & $\begin{array}{l}\text { Velocity } \\
\text { (Km/S) }\end{array}$ \\
\hline G7 & $\overline{\mathrm{H}} \overline{7}$ & $152 . \overline{4}$ & 35 & 4.35 \\
\hline G7 & I7 & 304.8 & 72.6 & 4.20 \\
\hline G7 & J7 & 457.2 & 161 & 2.84 \\
\hline G7 & K7 & 609.6 & 248 & 2.45 \\
\hline
\end{tabular}

\begin{tabular}{||c|c|c|c|c||}
\hline \multicolumn{5}{|c|}{ SLAB 2: LMC-S } \\
\hline \hline $\begin{array}{c}\text { Trans. } \\
\text { Location }\end{array}$ & $\begin{array}{c}\text { Rec. } \\
\text { Location }\end{array}$ & $\begin{array}{c}\text { Distance } \\
(\mathbf{m m})\end{array}$ & $\begin{array}{c}\text { Time } \\
(\boldsymbol{\mu} \mathbf{S})\end{array}$ & $\begin{array}{c}\text { Velocity } \\
(\mathbf{K m} / \mathbf{S})\end{array}$ \\
\hline- G7 $^{-}$ & F7 & 152.4 & 52.9 & 2.88 \\
\hline G7 & E7 & 304.8 & 119 & 2.57 \\
\hline G7 & D7 & 457.2 & 184 & 2.48 \\
\hline G7 & C7 & 609.6 & 251 & 2.43 \\
\hline
\end{tabular}


Table B.2: Indirect UPV table along G7-G1 and G7-G13 for both the slabs

\begin{tabular}{|c|c|c|c|c|}
\hline \multicolumn{5}{|c|}{ SLAB 1: LMC } \\
\hline $\begin{array}{l}\text { Trans. } \\
\text { Location }\end{array}$ & $\begin{array}{c}\text { Rec. } \\
\text { Location }\end{array}$ & $\begin{array}{c}\text { Distance } \\
\text { (mm.) }\end{array}$ & $\begin{array}{l}\text { Time } \\
(\mu S)\end{array}$ & $\begin{array}{l}\text { Velocity } \\
(\mathrm{Km} / \mathrm{S})\end{array}$ \\
\hline$--\frac{-}{\mathrm{G} 7}--$ & $-\overline{\mathrm{G} 6}$ & $-\frac{-}{203.2}$ & $-\overline{47.5}$ & $-\overline{4.28}-$ \\
\hline G7 & G5 & 406.4 & 113 & 3.59 \\
\hline G7 & G4 & 609.6 & 207 & 2.94 \\
\hline G7 & G3 & 812.8 & 269 & 3.02 \\
\hline & & & & 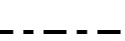 \\
\hline $\begin{array}{l}\text { Trans. } \\
\text { Location }\end{array}$ & $\begin{array}{c}\text { Rec. } \\
\text { Location }\end{array}$ & $\begin{array}{c}\text { Distance } \\
\text { (mm.) }\end{array}$ & $\begin{array}{l}\text { Time } \\
(\mu S)\end{array}$ & $\begin{array}{l}\text { Velocity } \\
(\mathrm{Km} / \mathrm{S})\end{array}$ \\
\hline$--\frac{-}{\mathrm{G} 7}=-$ & $-\frac{-}{\mathrm{G} 8}=-$ & $=\frac{-}{203.2}=$ & 81.3 & ---50 \\
\hline G7 & G9 & 406.4 & 167 & 2.44 \\
\hline G7 & G10 & 609.6 & 219 & 2.78 \\
\hline G7 & G11 & 812.8 & 298 & 2.73 \\
\hline
\end{tabular}

\begin{tabular}{|c|c|c|c|c|}
\hline \multicolumn{5}{|c|}{ SLAB 2: LMC-S } \\
\hline $\begin{array}{c}\text { Trans. } \\
\text { Location }\end{array}$ & $\begin{array}{c}\text { Rec. } \\
\text { Location }\end{array}$ & $\begin{array}{c}\text { Distance } \\
\text { (mm.) }\end{array}$ & $\begin{array}{l}\text { Time } \\
(\mu S)\end{array}$ & $\begin{array}{l}\text { Velocity } \\
(\mathrm{Km} / \mathrm{S})\end{array}$ \\
\hline$\overline{\mathrm{G}} 7$ & $--\overline{G 6}--$ & 203.2 & $-\overline{45.5}$ & 4.47 \\
\hline G7 & G5 & 406.4 & 105 & 3.87 \\
\hline G7 & G4 & 609.6 & 155 & 3.93 \\
\hline G7 & G3 & 812.8 & 225 & 3.61 \\
\hline $\begin{array}{c}\text { Trans. } \\
\text { Location }\end{array}$ & $\begin{array}{c}\text { Rec. } \\
\text { Location }\end{array}$ & $\begin{array}{c}\text { Distance } \\
\text { (mm.) }\end{array}$ & $\begin{array}{l}\text { Time } \\
(\mu S)\end{array}$ & $\begin{array}{l}\text { Velocity } \\
(\mathrm{Km} / \mathrm{S})\end{array}$ \\
\hline$-\bar{G} 7$ & $--\overline{G 8}=-$ & 203.2 & 72.2 & 2.81 \\
\hline G7 & G9 & 406.4 & 140 & 2.90 \\
\hline G7 & G10 & 609.6 & 225 & 2.71 \\
\hline G7 & G11 & 812.8 & 314 & 2.59 \\
\hline
\end{tabular}

Table B.3: Indirect UPV table along: G7-M1 and G7-M13 for LMC slab, and G7-A1 and G7-A13 for LMC-S slab

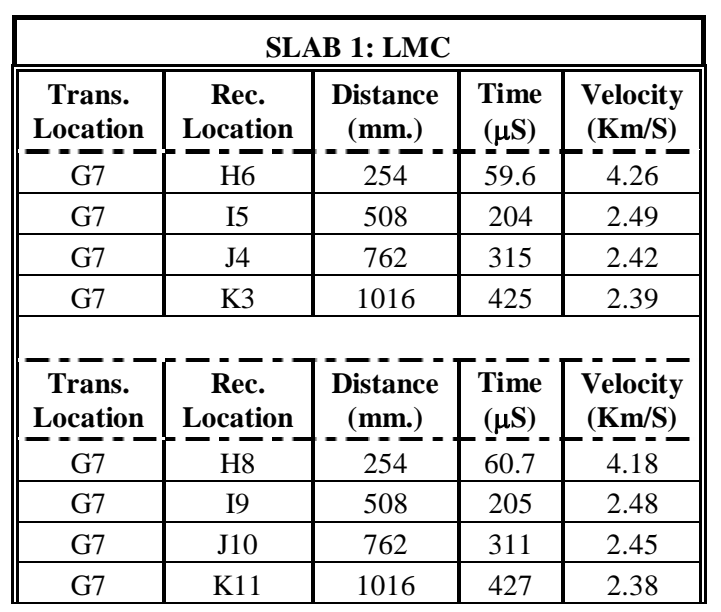

\begin{tabular}{|c|c|c|c|c|}
\hline \multicolumn{5}{|c|}{ SLAB 2: LMC-S } \\
\hline $\begin{array}{c}\text { Trans. } \\
\text { Location }\end{array}$ & $\begin{array}{c}\text { Rec. } \\
\text { Location }\end{array}$ & $\begin{array}{c}\text { Distance } \\
\text { (mm.) }\end{array}$ & $\begin{array}{l}\text { Time } \\
(\mu S)\end{array}$ & $\begin{array}{l}\text { Velocity } \\
\text { (Km/S) }\end{array}$ \\
\hline$\overline{\mathrm{G}} \overline{\mathrm{B}}$ & $\overline{\mathrm{F} 6}$ & $25 \overline{4}$ & 56.4 & 4.50 \\
\hline G7 & E5 & 508 & 116 & 4.38 \\
\hline G7 & D4 & 762 & 211 & 3.61 \\
\hline G7 & C3 & 1016 & 314 & 3.24 \\
\hline $\begin{array}{c}\text { Trans. } \\
\text { Location }\end{array}$ & $\begin{array}{c}\text { Rec. } \\
\text { Location }\end{array}$ & $\begin{array}{c}\text { Distance } \\
\text { (mm.) }\end{array}$ & $\begin{array}{l}\text { Time } \\
(\mu \mathrm{S})\end{array}$ & $\begin{array}{l}\text { Velocity } \\
(\mathrm{Km} / \mathrm{S})\end{array}$ \\
\hline G7 & F8 & 254 & 102 & 2.49 \\
\hline G7 & E9 & 508 & 217 & 2.34 \\
\hline G7 & D10 & 762 & 325 & 2.34 \\
\hline G7 & C11 & 1016 & 436 & 2.33 \\
\hline
\end{tabular}




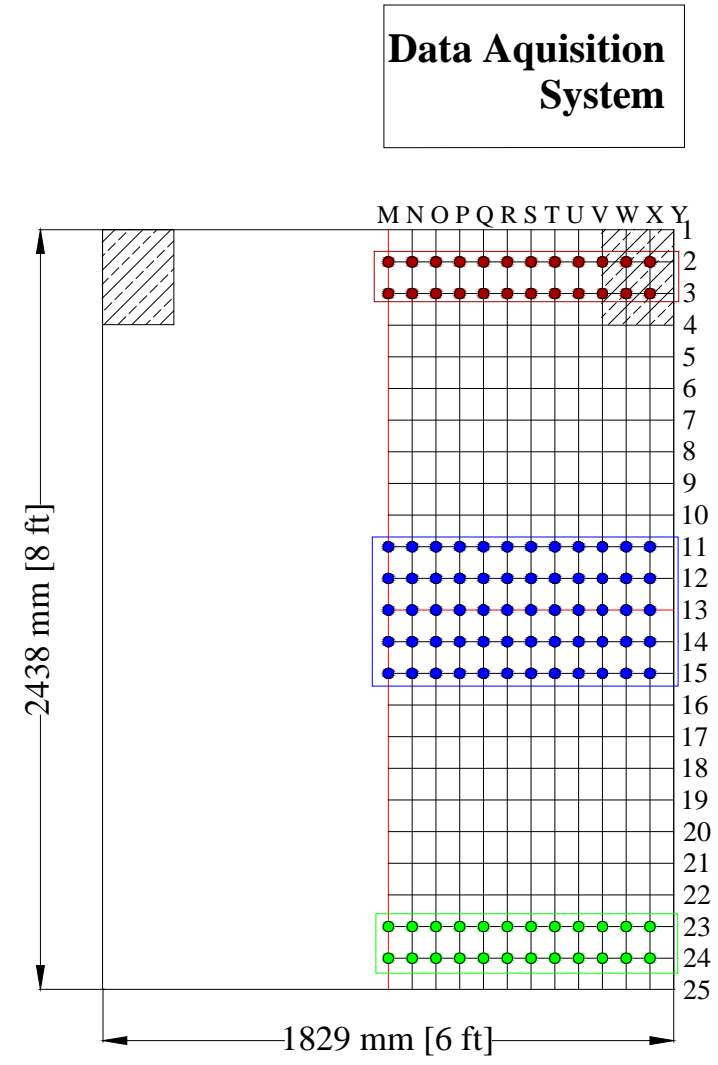

SLAB 1: LMC

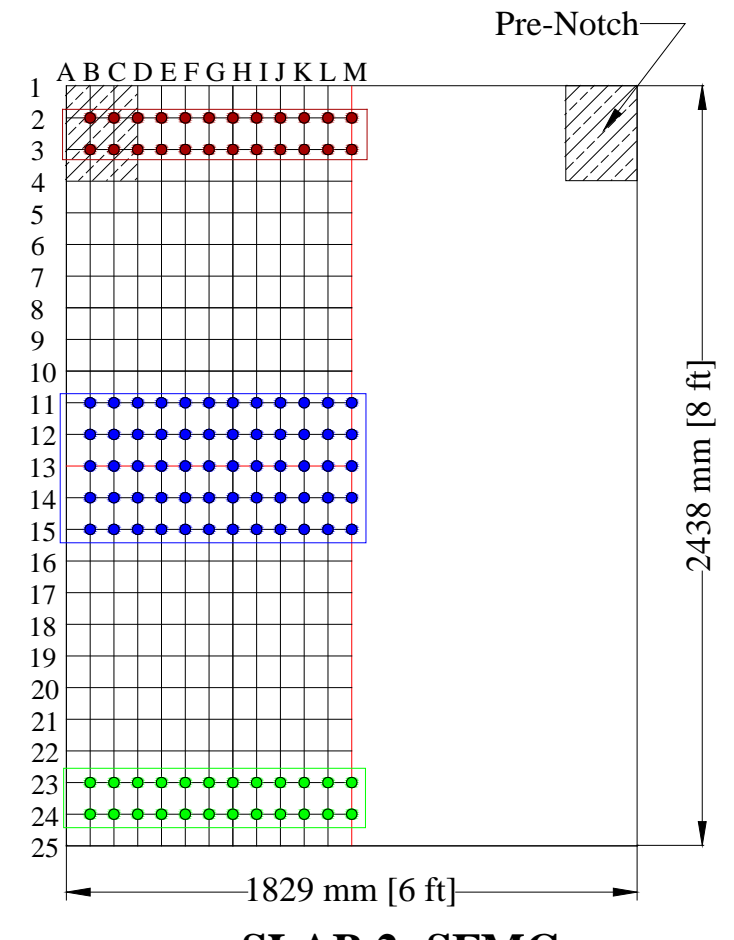

SLAB 2: SFMC

Figure B.2: Locations at which direct UPV testing was conducted (Stage-II) 
Table B.4: Direct UPV results at 28 days for LMC and SFMC overlays

\begin{tabular}{|c|c|c|c|c|c|c|c|c|c|}
\hline & \multicolumn{9}{|c|}{ Latex Modified Concrete (LMC) } \\
\hline & \multicolumn{2}{|c|}{$\begin{array}{c}\text { Delaminate } \\
\text { d Zone with } \\
\text { Pre-notch }\end{array}$} & \multicolumn{5}{|c|}{ Center Zone } & \multicolumn{2}{|c|}{$\begin{array}{c}\text { Delaminated } \\
\text { Zone without } \\
\text { Pre-notch }\end{array}$} \\
\hline & $2-2$ & $3-3$ & 11-11 & $12-12$ & 13-13 & 14-14 & $15-15$ & $23-23$ & 24-24 \\
\hline M-M & 3.07 & 3.34 & 3.36 & 3.28 & 3.32 & 3.02 & 3.30 & 3.49 & 3.32 \\
\hline $\mathbf{N}-\mathbf{N}$ & 3.05 & 3.23 & 3.67 & 3.51 & 3.56 & 3.48 & 3.50 & 3.46 & 3.31 \\
\hline O-O & 3.27 & 3.46 & 3.53 & 3.33 & 3.43 & 3.38 & 3.38 & 3.54 & 3.39 \\
\hline $\mathbf{P}-\mathbf{P}$ & 3.22 & 3.44 & 3.55 & 3.43 & 3.50 & 3.40 & 3.58 & 3.50 & 3.21 \\
\hline Q-Q & 3.16 & 3.44 & 3.55 & 3.43 & 3.50 & 3.40 & 3.58 & 3.50 & 3.21 \\
\hline R-R & 3.31 & 3.42 & 3.48 & 3.19 & 3.51 & 3.29 & 3.50 & 3.24 & 3.24 \\
\hline S-S & 3.31 & 3.50 & 3.54 & 3.42 & 3.46 & 3.35 & 3.50 & 3.47 & 3.26 \\
\hline T-T & 3.27 & 3.46 & 3.52 & 3.38 & 3.35 & 3.38 & 3.53 & 3.48 & 3.28 \\
\hline U-U & 3.09 & 3.34 & 3.39 & 3.21 & 3.31 & 3.20 & 3.41 & 3.38 & 3.25 \\
\hline V-V & 3.08 & 3.27 & 3.48 & 3.39 & 3.45 & 3.34 & 3.13 & 3.45 & 3.24 \\
\hline W-W & 1.82 & 2.82 & 3.34 & 3.26 & 3.40 & 3.26 & 3.35 & 3.49 & 3.16 \\
\hline $\mathrm{X}-\mathrm{X}$ & 1.24 & 1.70 & - & 1.78 & 3.08 & 3.13 & 3.21 & 3.29 & 1.91 \\
\hline $\mathbf{Y}-\mathbf{Y}$ & 1.23 & 1.49 & 1.31 & - & 1.41 & 2.90 & 2.91 & 1.96 & 1.05 \\
\hline
\end{tabular}

\begin{tabular}{|c|c|c|c|c|c|c|c|c|c|}
\hline & \multicolumn{9}{|c|}{ Silica Fume Modified Concrete (SFMC) } \\
\hline & \multicolumn{2}{|c|}{$\begin{array}{c}\text { Delaminate } \\
\text { d Zone with } \\
\text { Pre-notch } \\
\end{array}$} & \multicolumn{5}{|c|}{ Center Zone } & \multicolumn{2}{|c|}{$\begin{array}{c}\text { Delaminated } \\
\text { Zone without } \\
\text { Pre-notch } \\
\end{array}$} \\
\hline & $2-2$ & $3-3$ & 11-11 & 12-12 & 13-13 & 14-14 & 15-15 & 23-23 & 24-24 \\
\hline A-A & 1.72 & 1.39 & 1.40 & - & - & - & - & - & - \\
\hline B-B & 1.44 & 1.12 & 2.87 & 1.59 & 1.74 & 1.06 & 1.24 & 1.25 & 0.79 \\
\hline C-C & 1.58 & 2.64 & 3.05 & 2.60 & 2.91 & 2.96 & 3.19 & 1.89 & 0.64 \\
\hline D-D & 3.14 & 3.27 & 3.11 & 3.13 & 3.26 & 3.22 & 3.37 & 2.03 & 1.84 \\
\hline E-E & 3.25 & 3.28 & 3.21 & 3.07 & 3.19 & 3.23 & 3.29 & 1.85 & 1.87 \\
\hline F-F & 3.26 & 3.36 & 3.12 & 3.08 & 3.11 & 3.22 & 3.21 & 3.23 & 2.08 \\
\hline G-G & 3.17 & 3.37 & 3.29 & 3.18 & 3.04 & 3.11 & 3.18 & 3.27 & 2.59 \\
\hline H-H & 3.25 & 3.32 & 3.37 & 3.25 & 3.21 & 3.23 & 3.36 & 3.27 & 3.05 \\
\hline I-I & 3.28 & 3.34 & 3.33 & 3.27 & 3.35 & 3.21 & 3.29 & 3.35 & 1.98 \\
\hline $\mathbf{J}-\mathbf{J}$ & 3.21 & 3.30 & 3.32 & 3.20 & 3.12 & 3.22 & 3.30 & 3.30 & 3.08 \\
\hline K-K & 3.19 & 3.28 & 3.31 & 3.20 & 3.22 & 3.26 & 3.33 & 3.29 & 3.12 \\
\hline L-L & 3.08 & 3.28 & 3.33 & 3.20 & 3.25 & 3.27 & 3.37 & 3.28 & 3.05 \\
\hline M-M & 2.89 & 3.27 & 3.27 & 3.08 & 3.10 & 3.27 & 3.34 & 3.24 & 2.87 \\
\hline
\end{tabular}


Table B.5: Direct UPV results at 56 days for LMC and SFMC overlays

\begin{tabular}{|c|c|c|c|c|c|c|c|c|c|}
\hline & \multicolumn{9}{|c|}{ Latex Modified Concrete (LMC) } \\
\hline & \multicolumn{2}{|c|}{$\begin{array}{c}\text { Delaminate } \\
\text { d Zone with } \\
\text { Pre-notch } \\
\end{array}$} & \multicolumn{5}{|c|}{ Center Zone } & \multicolumn{2}{|c|}{$\begin{array}{c}\text { Delaminated } \\
\text { Zone without } \\
\text { Pre-notch } \\
\end{array}$} \\
\hline & $2-2$ & $3-3$ & 11-11 & $12-12$ & 13-13 & 14-14 & $15-15$ & $23-23$ & 24-24 \\
\hline M-M & 3.02 & 3.28 & 3.29 & 3.18 & 3.46 & 3.00 & 3.29 & 3.49 & 3.24 \\
\hline $\mathbf{N}-\mathbf{N}$ & 2.97 & 3.19 & 3.64 & 3.47 & 3.50 & 3.42 & 3.46 & 3.43 & 3.28 \\
\hline O-O & 3.26 & 3.44 & 3.44 & 3.28 & 3.41 & 3.31 & 3.37 & 3.51 & 3.37 \\
\hline $\mathbf{P}-\mathbf{P}$ & 3.24 & 3.38 & 3.60 & 3.48 & 3.37 & 3.37 & 3.64 & 3.34 & 3.30 \\
\hline Q-Q & 3.18 & 3.45 & 3.51 & 3.38 & 3.42 & 3.36 & 3.49 & 3.49 & 3.18 \\
\hline R-R & 3.30 & 3.41 & 3.44 & 3.14 & 3.45 & 3.32 & 3.43 & 3.40 & 3.20 \\
\hline S-S & 3.30 & 3.49 & 3.52 & 3.38 & 3.43 & 3.36 & 3.48 & 3.43 & 3.23 \\
\hline T-T & 3.25 & 3.41 & 3.48 & 3.34 & 3.32 & 3.32 & 3.49 & 3.45 & 3.25 \\
\hline U-U & 3.08 & 3.34 & 3.34 & 3.15 & 3.31 & 3.16 & 3.36 & 3.36 & 3.20 \\
\hline V-V & 3.16 & 3.26 & 3.40 & 3.32 & 3.39 & 3.28 & 3.31 & 3.42 & 3.20 \\
\hline W-W & 1.63 & 2.87 & 3.27 & 3.21 & 3.34 & 3.24 & 3.35 & 3.44 & 3.12 \\
\hline$X-X$ & 1.73 & 1.55 & 2.91 & 2.69 & 2.98 & 3.09 & 3.18 & 3.22 & 3.15 \\
\hline $\mathbf{Y}-\mathbf{Y}$ & 1.71 & 2.33 & 1.68 & 0.90 & 2.81 & 2.93 & 2.91 & 2.95 & 2.82 \\
\hline
\end{tabular}

\begin{tabular}{|c||c|c||c|c|c|c|c||c|c|}
\hline \multicolumn{1}{|c||}{} & \multicolumn{9}{c|}{ Silica Fume Modified Concrete (SFMC) } \\
\cline { 2 - 11 } & $\begin{array}{c}\text { Delaminate } \\
\text { d Zone with } \\
\text { Pre-notch }\end{array}$ & \multicolumn{9}{c||}{ Center Zone } & \multicolumn{2}{c|}{$\begin{array}{c}\text { Delaminated } \\
\text { Zone without } \\
\text { Pre-notch }\end{array}$} \\
\cline { 2 - 11 } & $\mathbf{2 - 2}$ & $\mathbf{3 - 3}$ & $\mathbf{1 1 - 1 1}$ & $\mathbf{1 2 - 1 2}$ & $\mathbf{1 3 - 1 3}$ & $\mathbf{1 4 - 1 4}$ & $\mathbf{1 5 - 1 5}$ & $\mathbf{2 3 - 2 3}$ & $\mathbf{2 4 - 2 4}$ \\
\hline A-A & 1.87 & 2.27 & 2.55 & 1.12 & 1.49 & 1.87 & 2.75 & 1.56 & 1.58 \\
\hline B-B & 1.72 & 1.72 & 3.50 & 1.67 & 2.39 & 1.95 & 3.29 & 1.63 & 1.26 \\
\hline C-C & 2.74 & 2.79 & 3.59 & 3.48 & 3.38 & 3.54 & 3.69 & 2.60 & 1.97 \\
\hline D-D & 3.29 & 3.41 & 3.72 & 3.62 & 3.86 & 3.71 & 3.97 & 3.74 & 2.31 \\
\hline E-E & 3.37 & 3.47 & 3.75 & 3.62 & 3.75 & 3.73 & 3.91 & 3.61 & 2.29 \\
\hline F-F & 3.42 & 3.55 & 3.85 & 3.81 & 3.84 & 3.92 & 4.00 & 3.72 & 2.81 \\
\hline G-G & 3.36 & 3.54 & 3.90 & 3.82 & 3.88 & 3.86 & 3.97 & 3.77 & 3.84 \\
\hline H-H & 3.43 & 3.53 & 3.97 & 3.83 & 3.77 & 3.81 & 3.95 & 3.86 & 3.53 \\
\hline I-I & 3.44 & 3.51 & 3.90 & 3.84 & 3.96 & 3.77 & 3.94 & 3.91 & 3.47 \\
\hline J-J & 3.39 & 3.49 & 3.90 & 3.74 & 3.62 & 3.82 & 3.88 & 3.86 & 3.49 \\
\hline K-K & 3.34 & 3.46 & 3.88 & 3.77 & 3.79 & 3.83 & 3.95 & 3.81 & 3.56 \\
\hline L-L & 3.21 & 3.45 & 3.88 & 3.75 & 3.81 & 3.85 & 3.98 & 3.83 & 3.53 \\
\hline M-M & 3.03 & 3.47 & 3.86 & 3.62 & 3.68 & 3.76 & 3.93 & 3.70 & 3.36 \\
\hline
\end{tabular}


Table B.6: Direct UPV results at 90 days for LMC and SFMC overlays

\begin{tabular}{|c|c|c|c|c|c|c|c|c|c|}
\hline & \multicolumn{9}{|c|}{ Latex Modified Concrete (LMC) } \\
\hline & \multicolumn{2}{|c|}{$\begin{array}{c}\text { Delaminate } \\
\text { d Zone with } \\
\text { Pre-notch } \\
\end{array}$} & \multicolumn{5}{|c|}{ Center Zone } & \multicolumn{2}{|c|}{$\begin{array}{c}\text { Delaminated } \\
\text { Zone without } \\
\text { Pre-notch }\end{array}$} \\
\hline & $2-2$ & $3-3$ & 11-11 & 12-12 & 13-13 & 14-14 & $15-15$ & $23-23$ & 24-24 \\
\hline M-M & 3.49 & 3.83 & 3.28 & 3.17 & 3.20 & 2.94 & 3.24 & 3.43 & 3.18 \\
\hline $\mathbf{N}-\mathbf{N}$ & 3.53 & 3.75 & 3.58 & 3.44 & 3.49 & 3.41 & 3.50 & 3.37 & 3.20 \\
\hline O-O & 3.77 & 4.09 & 3.39 & 3.24 & 3.42 & 3.27 & 3.36 & 3.47 & 3.30 \\
\hline $\mathbf{P}-\mathbf{P}$ & 3.77 & 4.05 & 3.56 & 3.44 & 3.37 & 3.39 & 3.61 & 3.31 & 3.23 \\
\hline Q-Q & 3.67 & 4.07 & 3.49 & 3.34 & 3.38 & 3.34 & 3.45 & 3.44 & 3.12 \\
\hline R-R & 3.81 & 4.00 & 3.43 & 3.10 & 3.43 & 3.21 & 3.41 & 3.33 & 3.18 \\
\hline S-S & 3.86 & 4.11 & 3.45 & 3.33 & 3.41 & 3.31 & 3.43 & 3.39 & 3.15 \\
\hline T-T & 3.81 & 4.07 & 3.45 & 3.30 & 3.32 & 3.31 & 3.47 & 3.38 & 3.17 \\
\hline U-U & 3.68 & 3.93 & 3.29 & 3.13 & 3.29 & 3.16 & 3.35 & 3.30 & 3.15 \\
\hline V-V & 3.72 & 3.82 & 3.37 & 3.32 & 3.39 & 3.27 & 3.32 & 3.38 & 3.16 \\
\hline W-W & 1.97 & 3.26 & 3.27 & 3.16 & 3.30 & 3.23 & 3.31 & 3.39 & 3.10 \\
\hline $\mathrm{X}-\mathrm{X}$ & 1.75 & 1.85 & 3.05 & 2.74 & 3.02 & 3.08 & 3.14 & 3.17 & 2.97 \\
\hline $\mathbf{Y}-\mathbf{Y}$ & 2.15 & 2.61 & 1.69 & 1.62 & 2.84 & 2.94 & 2.93 & 2.79 & 2.78 \\
\hline
\end{tabular}

\begin{tabular}{|c|c|c|c|c|c|c|c|c|c|}
\hline & \multicolumn{9}{|c|}{ Silica Fume Modified Concrete (SFMC) } \\
\hline & \multicolumn{2}{|c|}{$\begin{array}{c}\text { Delaminate } \\
\text { d Zone with } \\
\text { Pre-notch } \\
\end{array}$} & \multicolumn{5}{|c|}{ Center Zone } & \multicolumn{2}{|c|}{$\begin{array}{c}\text { Delaminated } \\
\text { Zone without } \\
\text { Pre-notch } \\
\end{array}$} \\
\hline & $2-2$ & $3-3$ & 11-11 & 12-12 & 13-13 & 14-14 & 15-15 & $23-23$ & 24-24 \\
\hline A-A & 1.85 & 2.31 & 1.69 & 0.70 & 1.06 & 1.54 & 2.43 & 1.14 & 0.70 \\
\hline B-B & 1.77 & 1.78 & 3.25 & 1.13 & 1.55 & 1.93 & 3.09 & 1.25 & 0.86 \\
\hline C-C & 2.30 & 2.99 & 3.53 & 3.08 & 3.26 & 3.26 & 3.62 & 1.89 & 1.98 \\
\hline D-D & 3.57 & 3.74 & 3.60 & 3.55 & 3.75 & 3.65 & 3.87 & 2.20 & 2.02 \\
\hline E-E & 3.68 & 3.80 & 3.67 & 3.53 & 3.67 & 3.72 & 3.80 & 3.05 & 2.05 \\
\hline F-F & 3.75 & 3.90 & 3.73 & 3.73 & 3.76 & 3.81 & 3.90 & 3.24 & 2.52 \\
\hline G-G & 3.68 & 3.90 & 3.78 & 3.72 & 3.75 & 3.79 & 3.90 & 3.25 & 3.22 \\
\hline H-H & 3.72 & 3.82 & 3.86 & 3.75 & 3.76 & 3.74 & 3.86 & 3.31 & 3.07 \\
\hline I-I & 3.74 & 3.86 & 3.81 & 3.74 & 3.85 & 3.70 & 3.82 & 3.35 & 3.07 \\
\hline $\mathbf{J}-\mathbf{J}$ & 3.70 & 3.86 & 3.81 & 3.69 & 3.54 & 3.74 & 3.81 & 3.31 & 3.02 \\
\hline K-K & 3.64 & 3.81 & 3.85 & 3.67 & 3.72 & 3.73 & 3.83 & 3.28 & 3.09 \\
\hline L-L & 3.49 & 3.79 & 3.84 & 3.65 & 3.72 & 3.79 & 3.93 & 3.30 & 3.07 \\
\hline $\mathbf{M}-\mathbf{M}$ & 3.32 & 3.80 & 3.81 & 3.59 & 3.55 & 3.70 & 3.86 & 3.18 & 2.95 \\
\hline
\end{tabular}

John

$\mathrm{H}$. 